\title{
Arbeitspolitischer Pragmatismus
}

\section{Eine vergleichende Fallstudienuntersuchung zur subjektiven Aneignung von KVP durch Produktionsbeschäftigte}

\author{
Dissertation \\ Zur Erlangung des sozialwissenschaftlichen Doktorgrades \\ der Sozialwissenschaftlichen Fakultät der \\ Georg-August-Universität Göttingen
}

vorgelegt von:

Barbara Splett

geboren in Kassel

Göttingen, 2021 
Betreuungsausschuss:

Erstgutachter: Prof. Dr. Jürgen Kädtler

Weitere Betreuer: $\quad$ Prof. Dr. Thomas Haipeter

Dr. Martin Kuhlmann

Tag der mündlichen Prüfung: 12.02.2019 



\section{Danksagung}

Die vorliegende Arbeit wurde 2018 als Dissertationsschrift an der GeorgAugust-Universität Göttingen angenommen. Sie wurde im Rahmen eines empirischen Begleitforschungsprojekts zu „Agilitätsrelevanten Ausprägung innovativer Arbeitspolitik" des Soziologischen Forschungsinstituts Göttingen erstellt. In der Abschlussphase wurde die Arbeit durch ein Stipendium des Niedersächsische Ministerium für Wissenschaft und Kultur gefördert, für deren finanzielle Unterstützung ich mich bedanke.

Mit der Veröffentlichung dieses Buches fällt der Blick erleichtert und dankbar zurück auf die vielen Wegbereiter und Wegbegleiter, die mich fachlich, finanziell, durch bereichernde Gespräche und emotionale Unterstützung begleitet haben und ohne die diese Arbeit nicht möglich gewesen wäre.

Der Dank richtet sich zunächst an die Beschäftigten der beiden österreichischen Betriebe, die wir über einen längeren Zeitraum bei ihrer Arbeit beobachten durften und selbst in schwierigen Zeiten eine große Bereitschaft gezeigt haben, sich auf die Interviews und Beobachtungen einzulassen.

Direkt daran anschließend, möchte ich mich bei meinen Kolleginnen und Kollegen im SOFI bedanken, die mich vor allem in der Forschungsperspektive "Arbeit - Organisation - Subjekt" durch fachlich kritische und anregende Diskussionen sowie wertschätzenden Ratschläge unterstützt haben. Besonderer Dank gilt Martin Kuhlmann, der nicht nur mein Projektleiter war und von dem ich das praktische Handwerkszeug der „SOFI-Feldarbeit“ gelernt habe, sondern der mir auch menschlich wie fachlich stets in der Dissertationszeit und Projektarbeit zur Seite stand und den Rücken freigehalten hat. Gleiches gilt für meinem Doktorvater Jürgen Kädtler: Danke für die große Geduld, anregenden Fragen und hilfreichen Ratschlägen. Ein Dank gebührt auch Lukas Underwood, der als studentische Hilfskraft in den viele Wochen der operativen Feldarbeit ein wertvoller Begleiter und Unterstützer war.

Und schließlich danke ich meinem Mann, der mit großem Herzen, Geduld und Zuversicht einen bedeuten Anteil am Gelingen dieser Arbeit hatte, und meiner Familie, die auf mich so manches Mal verzichten musste und deren Unterstützung unfassbaren Wert hat. ich mir immer sicher sein konnte. 



\section{Inhaltsverzeichnis}

1. Einleitung

2. Kontinuierlicher Verbesserungsprozess - im Spannungsfeld von Beschäftigten- und Betriebsinteressen.................................................. 27

2.1. KVP aus Perspektive des Transformationsproblems: Das

Potenzial und die Steuerung menschlichen

Arbeitsvermögens

2.2. Entwicklungslinien und Herausforderungen partizipativer Managementsysteme ………………............................................ 35

2.2.1. Vom Taylorismus bis zu neuen Produktionssystemen ... 36

2.2.2. Lean Production und Ganzheitliche Produktionssysteme ............................................................... 47

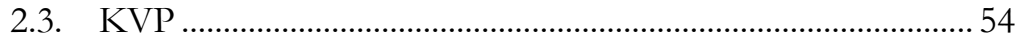

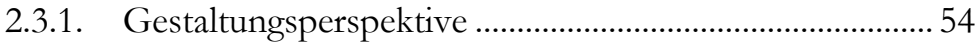

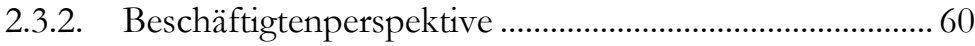

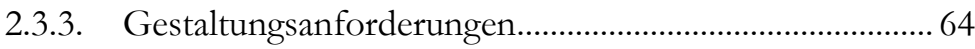

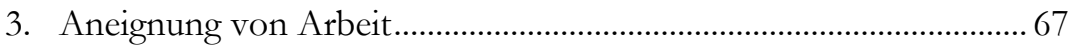

3.1. Aneignung als bewusstes oder unbewusstes, sinnliches Einlassen auf die Arbeitssituation ............................................... 68

3.2. Das Verhältnis von Struktur und Subjekt................................ 72

4. Untersuchungsdesign, Methodik, Auswertungskonzept .................. 81

4.1. Empirische Konzeptionalisierung............................................. 82

4.1.1. Analysekonzept der Aneignungsformen von KVP ........ 82

4.1.2. Analyse der objektiven Gestaltungspraktiken von KVP: Die arbeitspolitische Beteiligungskonstellation.... 92 
4.2. Vergleichende Fallstudie und Sampling .................................. 96

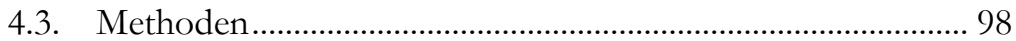

4.3.1. Problemzentrierte Interviews............................................ 99

4.3.2. Beobachtung von Arbeitsplätzen, Teambesprechungen und Workshops ............................. 100

4.3.3. Expertengespräche .......................................................... 101

4.3.4. Auswahl der Interviewten: Reflexion des Samplings ... 102

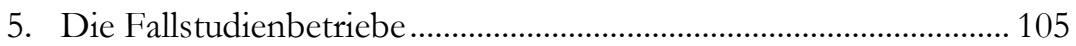

5.1. NF 106

5.1.1. Arbeitspolitische Vorgeschichte ...................................... 108

5.1.2. Arbeitspolitische Neuorientierung .................................. 110

5.1.3. KVP bei NF............................................................... 115

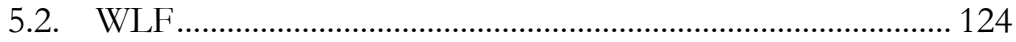

5.2.1. Arbeitspolitische Vorgeschichte ..................................... 125

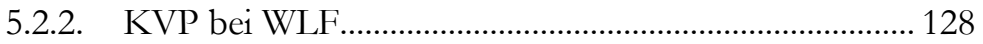

5.3. Vergleich der KVP-Konzepte .................................................. 134

6. Beteiligungsorientierungen ................................................................. 139

6.1. Auswertungskonzept................................................................. 140

6.2. Beteiligungsrelevante Motive und Dimensionen subjektiver Orientierungen.................................................. 144

6.3. Dimensionen subjektiver Orientierungen ............................. 147

6.3.1. Arbeitsverständnis ............................................................ 147

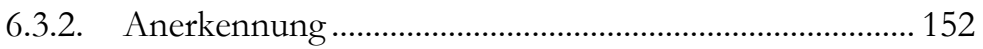

6.3.3. Arbeitskraftorientierung.................................................. 154

6.3.4. Erwerbsbiografische Perspektive .................................... 157

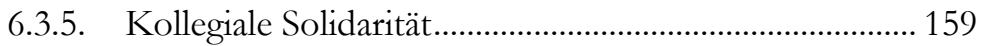

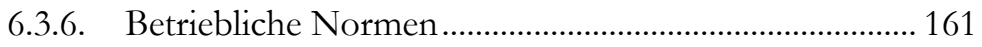


6.3.7. Rationalisierungsverständnis ............................................ 163

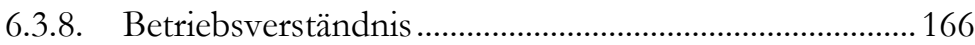

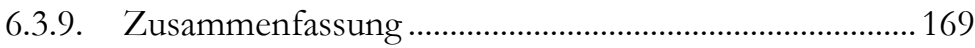

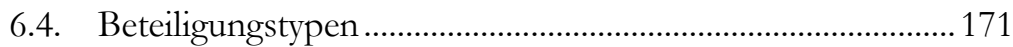

6.4.1. Mitgestaltungsorientierung ….......................................... 172

6.4.2. Karriereorientierung ........................................................ 177

6.4.3. Status-quo-Orientierung...................................................... 183

6.4.4. Zusammenfassung der Beteiligungstypen....................... 188

7. Arbeitspolitische Beteiligungskonstellationen: KVP-Praktiken aus der Perspektive struktureller Beteiligungsbedingungen ........... 193 7.1. Arbeitspolitische Beteiligungskonstellationen bei NF ......... 194

7.1.1. „Kampf um gute Arbeit“ (NF Montage F)..................... 195

7.1.2. „Im Stich gelassen“ (NF Montage K)............................. 199

7.1.3. KVP-Workshops bei NF ..................................................2 204

7.1.4. Bürokratisches Ideenmanagement bei NF...................... 210

7.1.5. Resümee: „Eine überforderte Betriebsorganisation“...212

7.2. Arbeitspolitische Beteiligungskonstellation WLF..................213

7.2.1. „Kooperative Beteiligungspartnerschaft“ (WLF

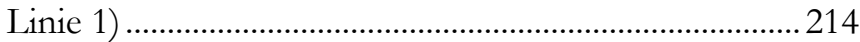

7.2.2. „Desintegrierte Arbeitsgruppe“ (WLF Linie 2)............. 219

7.2.3. Dezentrales Ideenmanagement bei WLF ........................ 225

7.3. Zwischen Restriktion und Partizipation................................... 228

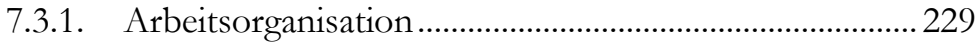

7.3.2. Rollenverständnis und Funktionszuschnitt der ersten Führungsebene .................................................................. 231

7.3.3. Prozessnahe Kommunikation und Kooperationsstrukturen zwischen Produktion und indirekten Bereichen 
7.3.4. Arbeitspolitische Ausrichtung von Optimierungsprozessen ................................................. 235

7.3.5. Finanzielle Gratifizierung ................................................ 238

7.3.6. Kohärenz betrieblicher Arbeitspolitik .......................... 239

7.3.7. Profile arbeitspolitischer Beteiligungskonstellationen.. 241

7.4. Betriebs- und Rationalisierungsverständnis............................ 243

7.4.1. Ausgeprägter Interessenantagonismus bei NF.............. 244

7.4.2. Zwischen latentem und bewusstem Interessenantagonismus bei WLF ................................... 248

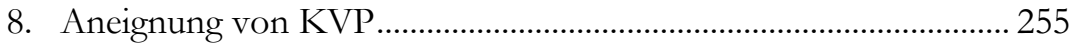

8.1. Keine Beteiligung.................................................................... 259

8.1.1. „Kein Beteiligungsangebot - keine Aneignung“.......... 259

8.1.2. „Interessiert mich nicht“ .................................................. 260

8.1.3. „Ich will nichts mit dem Betrieb zu tun haben.“ Demonstrative Ablehnung ................................................ 261

8.2. Pragmatisch gesteuerte Beteiligung ......................................... 264

8.2.1. „Nur wenn es gut für den Arbeiter ist“ Kontrollmodus bzw. abgesicherte, kontrollierende Beteiligung.......................................................................... 265

8.2.2. „Wir fordern unser Recht ein“ - Durchsetzung des Beteiligungsanspruchs: .................................................... 271

8.3. Aktive Beteiligung ................................................................... 276

8.3.1. „Wenn es uns gut geht, geht es dem Betrieb auch gut“ - Kooperative Optimierungspartnerschaft.................... 276

8.3.2. Beteiligung als Weiterentwicklungschance...................... 279

9. Zwischen arbeitspolitischem Konservativismus und Pragmatismus: Zusammenführung und Ausblick ........................... 283

9.1. Arbeitspolitischer Pragmatismus.............................................. 288 
9.2. Reziprozität der Anerkennungsverhältnisse: KVPBeteiligung im Spannungsfeld betrieblicher Anforderungen und subjektiver Orientierungen 291

9.3. Reichweite und Aussagekraft der Fallstudienergebnisse und weiterführende Perspektiven

Literatur. 301

Abbildungen 321

Tabellen 321

Anhang 323 



\section{Einleitung}

Unter dem Begriff „Kontinuierlicher Verbesserungsprozess“ - in deutschen Betrieben häufig auf das gängigere Akronym $\mathrm{KVP}^{1}$ verkürzt - stehen seit den 1990er Jahren beschäftigtengetragene Rationalisierungskonzepte im Fokus betrieblicher Managementstrategien. Ausgelöst von der Lean-Production-Welle und weitergetragen von Ganzheitlichen Produktionskonzepten (GPS), die Kaizen - japanisch für ständige Verbesserung - als ein Kernprinzip der japanischen Managementphilosophie populär machten, wurde die Mitwirkung aller Beschäftigten an stetig fortlaufenden betrieblichen Optimierungsprozessen als wichtiger Erfolgsfaktor für die Verbesserung von Produktqualität, Fertigungsprozessen und Produktionsabläufen betrachtet (Imai 1992; Howaldt 1994; Teufel 2003). In Gestalt unterschiedlicher Konzepte wird KVP insbesondere in Industriebetrieben praktiziert, die ihre Produktionsbeschäftigten als aktive Akteure von Problemlösungen und Prozessoptimierungen in das betriebliche

${ }^{1} \mathrm{KVP}$, Kaizen und beschäftigtengetragene Prozessoptimierungen werden in dieser Arbeit ungeachtet unterschiedlicher Differenzierungen und unternehmensphilosophischer Ansätze synonym verwendet. In der betrieblichen Praxis lässt sich nicht einfach von der Bezeichnung der beschäftigtengetragenen Optimierungsprozesse auf die Form und den Charakter der Beteiligung der Beschäftigten an Prozessoptimierungen schließen, sondern es bedarf immer eines genauen Blicks auf die jeweiligen Praktiken. Daher wird hier der Begriff KVP in einem weiten Verständnis benutzt, welches jegliche Beteiligung von Beschäftigten in Optimierungsprozessen einschließt. Im Folgenden wird KVP als Akronym und synonym mit dem Begriff beschäftigtengetragene Optimierungen verwendet. 
Rationalisierungsgeschehen einbeziehen wollen. Als Methodenkomplex, der unterschiedliche Instrumente zur ständigen Verbesserung umfasst, wie beispielsweise PDCA-Zyklus, 5S oder 7-Arten der Verschwendung ${ }^{2}$, findet KVP vor allem in von Trainern angeleiteten Workshops, in Gruppenarbeit mit integrierten Optimierungsaktivitäten und in unterschiedlichen Varianten eines modernisierten Ideenmanagements organisatorische Umsetzung (Teufel 2003; Kuhlmann et al. 2004).

Obgleich die arbeitssoziologische Debatte derzeit eher von boomenden Themen wie Mobilität, Digitalisierung und der sozial-ökologischen Transformation dominiert wird, ${ }^{3}$ sind in den Betrieben Sujets wie KVP und die Gestaltung von Arbeitsprozessen noch immer sehr aktuell und im Rahmen von systematischen Gestaltungskonzepten wie GPS oder Shopfloormanagement ${ }^{4}$ wiederzufinden. KVP ist seit 30 Jahren festes Gestaltungselement betrieblicher Rationalisierungsstrategien und von am Toyota-Produktionssystem orientierten Ganzheitlichen Produktionssystemen (Nordhause-Janz/Pekruhl 2000; Kuhlmann 2004; Kuhlmann et al. 2004; Schumann et al. 2004; Lay/Neuhaus 2005; Kirner et al. 2006; Gerst 2010; 2012b; Schwarz-Kocher et al. 2012; Jürgens, U. 2013; Dombrowski/Mielke 2015). ${ }^{5}$

Die Partizipation von Beschäftigten an der Gestaltung von Optimierungen zielt auf ihre aktive Einbindung und Verantwortungsübernahme im betrieblichen Rationalisierungsprozess ab (Moldaschl/Schultz-Wild 1994b). Mit diesem

2 PDCA-Zyklus bezeichnet die vier Phasen eines Qualitätsverbesserungszirkels (PlanDo-Check-Act), der mittlerweile Standard für die Steuerung von Verbesserungsprozesse ist. Dazu zählen auch die 5S-Methode. 5S - im Deutschen auch 5A genannt - umfasst Richtlinien für die Ordentlichkeit und Sauberkeit am Arbeitsplatz (Aussortieren, Aufräumen, Arbeitsplatzsauberkeit, Standardisierung und Ständige Verbesserung), Visualisierungsmethoden (Teufel 2003).

${ }^{3}$ Blickt man beispielsweise auf die Themen der WSI-Mitteilungen oder der Sektionstagungen der Arbeits- und Industriesoziologie, so stehen seit ca. 2015 Debatten um die industrielle Arbeitsgestaltung und die Auswirkung auf die Beschäftigten kaum noch im Fokus.

${ }^{4}$ Nähere Informationen zu Shopfloormanagement siehe Fußnote 64.

${ }^{5}$ Aktuelle Zahlen über die Verbreitung von KVP in Betrieben lassen sich nur schwer finden. Kirner et al. (2006) haben in einer Studie ermittelt, dass in 72 Prozent der Industrieunternehmen (68 Prozent in klein- und mittelständigen Unternehmen [KMU]; 88 Prozent in Großbetrieben) KVP genutzt wird, wobei die Anzahl der Betriebe, die KVP intensiv nutzen, in Großbetrieben bei 29 Prozent und in KMU lediglich bei 18 Prozent liegt (Kirner et al. 2006). 
arbeitspolitischen Ansatz werden spezifische, für Optimierungsleistungen benötigte subjektive Potenziale der Beschäftigten anvisiert. Sie sollen sich über ihre eigentliche, zu großen Teilen klar definierte Arbeitsleistung hinaus - freiwillig und selbstverständlich - mit zusätzlichem Engagement, eigenen Wissensbeständen und Erfahrungen sowie Kreativität in den Betrieb einbringen (Dörre et al. 1993; Howaldt 1994; Wolf 1994; Howaldt/Kopp 1997; Deutschmann 2002).

Diese gefragten Potenziale wie Kreativität, Erfahrungen und Engagement sind jedoch nicht immer leicht „abgreifbar“ und ihr Einsatz lässt sich vom Betrieb nicht einfach erzwingen - dies gilt insbesondere für Industriearbeiter, deren vorrangige Arbeitsaufgabe in der Verarbeitung und Produktion von materiellen Gütern besteht. Vielmehr ist ein Mindestmaß an Kooperationsbereitschaft der Beschäftigten nötig (Berger 1995; Deutschmann 2002), denn:

„Warum sollte der einzelne Arbeiter mebr als das Nötigste tun, wenn er das Recht auf den finanziellen Ertrag aus seiner Leistung und Kreativität von vornherein per Arbeitsvertrag an den Unternehmer abgetreten hat und wenn er mit ibr - vor allem wenn sie zur Rationalisierung des Arbeitsprozesses beiträgt - leicht seinen eigenen Arbeitsplatz. gefäbrdet?" (Deutschmann 2002: 99)

Gerade im besonderen Fall der Beteiligung der Beschäftigten an Optimierungen und Rationalisierungsprozessen ist es aber nicht selbstverständlich, dass Beschäftigte die Möglichkeit zur aktiven Beteiligung als Angebot verstehen und positiv darauf reagieren. Im Kontext tayloristisch geprägter Arbeitsbedingungen und Rationalisierungserfahrungen der 1980er Jahre zeigten sie gegenüber entsprechenden Ansätzen zunächst eine reservierte, widerständige Haltung, die als ,arbeitspolitischer Konservativismus" (Schumann et al. 1982) charakterisiert wurde. Diese konservative Haltung begründet sich in der Erfahrung der Beschäftigten, dass „technisch-organisatorische Veränderungen selbst dann, wenn sie zu Verbesserungen der materialen Konditionen der Arbeit führen, per Saldo auf der Leistungsseite doch wieder auf Kosten der Beschäftigten gehen." (Schumann et al. 1982: 555). Beschäftigte würden daher aufgrund ihres Status als abhängig Beschäftigte und Leidtragende der Rationalisierung ihre Teilnahme an Optimierungsmaßnahmen verweigern und die - sehr wohl vorhandenen - eigenen Optimierungsideen im Verborgenen umsetzen.

Im Vergleich zur tayloristischen Arbeitsgestaltung bieten posttayloristische, partizipative Organisationskonzepte Produktionsbeschäftigten Möglichkeiten, ihre (steigenden) Ansprüche an Arbeit zu verwirklichen (Baethge 1991), an der Gestaltung und Verbesserung der Arbeitsbedingungen 
und dem Produktionsprozess mitzuwirken und sich mit den eigenen Fähigkeiten und Kompetenzen als „aktive Problemlöser“ (Womack, J.P. et al. 1991; Jürgens, U. 2006b) einzubringen. Als arbeitspolitische Türöffner zur Nutzung der subjektiven Potenziale dienen die Rücknahme der direkten Kontrolle durch Erweiterung der Handlungs- und Entscheidungsspielräume, eine erweiterte Selbstorganisation durch Gruppenarbeit sowie Dezentralisierung der Hierarchie und Kooperationsstrukturen (Moldaschl/Schultz-Wild 1994a; Wolf 1994). Die Beschäftigten sollen auf diese Weise Teil einer neuen Rationalisierungsstrategie werden, in der das „Gold aus den Köpfen“ für die betriebliche Effizienzsteigerung genutzt wird und sie vom Objekt zum Subjekt der Rationalisierung werden.

Was sich zunächst nach einer günstigen Prognose für die Entwicklung von Industriearbeit anhörte - langersehnte Beschäftigtenansprüche nach qualitativ gehaltvollerer (Fach-)Arbeit wurden (endlich) von den Betrieben anerkannt und finden einen Platz in den immer komplexeren technisierten Produktionsprozessen (Kern/Schumann 1984; Schumann et al. 1994a) -, mündete in der betrieblichen Realität durch die Verbreitung partizipativer Managementmodelle in ein Spannungsfeld zwischen betrieblichen Anforderungen und subjektiven Ansprüchen der Beschäftigten (Marrs 2010; Moldaschl 2010). Nun kann nicht per se davon ausgegangen werden, dass Betriebe den normativen Forderungen nach Verwirklichung der Beschäftigten lediglich aus humanistischen Gründen Folge leisten; vielmehr wurden die „subjektiven“ Anteile der Arbeitskraft als weitere Ressource für die betriebliche Effizienz- und Leistungssteigerung betrachtet, die zum Zwecke der Rationalisierung eingesetzt wurden (Moldaschl 2002; Wolf 2003). Im Zuge der Rücknahme direkter Kontrolle wurden durch Kennzahlenorientierung und vorgegebene Rahmenbedingungen sowie vermarktlichte Arbeitsbeziehungen neue indirekte Steuerungsmechanismen eingeführt, die das Denken und Handeln der Beschäftigten kanalisieren und auf betriebliche Interessen an Produktivitätssteigerung und Kostenminimierung ausrichten sollten (Sauer/Döhl 1997). Mit veränderten Anforderungen und der Projektion der Leitvorstellung eines unternehmerisch denkenden, sich selbst optimierenden Beschäftigten intendierten die Betriebe, dass die Beschäftigten ihre subjektiven Potenziale im Sinne der betrieblichen Zielerfüllung ausrichten und einbringen (Moldaschl/Schultz-Wild 1994b; Voß, G./Pongratz 1998; Kocyba/Vormbusch 2000; Moldaschl 2002; Wolf 2003) und sich folglich auch bereitwillig an Kontinuierlichen Verbesserungsprozessen zum Wohle des Betriebs beteiligen. 
Doch bereits mit dem Aufkommen neuer partizipativer Managementkonzepte wurde deutlich, dass diese „doppelte Subjektivierung von Arbeit“ (Kleemann et al. 2002), u.a. infolge von KVP, für die Beschäftigten mit einem ambivalenten Verhältnis zwischen Partizipation und Selbstverwirklichung auf der einen und gestiegenen Anforderungen an Selbstregulierung und durch Leistungsintensivierung auf der anderen Seiten verbunden ist (Kleemann et al. 2002; Moldaschl/Voß 2002; Lohr/Nickel 2005; Sauer 2005b; Lohr 2017a). Insbesondere in Zeiten zunehmender globaler Konkurrenzkämpfe zwischen Hochlohn- und Niedriglohnproduktionsstandorten, konzerninternen Benchmarkwettkämpfen, international vernetzen Wertschöpfungsketten und Marktbeziehungen sowie arbeitsmarktlichen, betrieblichen und ökologischen Transformationsprozessen steigt der Druck auf Betriebe - und auch auf die Beschäftigten. Dieser Druck schlägt sich einerseits in der Sorge der Beschäftigten um den eigenen Arbeitsplatz und die Beschäftigungssicherheit nieder, andererseits gibt er Anlass zur Mitwirkung an der Wirtschaftlichkeit des Betriebs (Kuhlmann/Schumann 2000; Kuhlmann et al. 2004). Betriebe begegneten diesem Marktdruck oftmals mit einem arbeitspolitischen Rückfall in tayloristische Strukturen mittels Einschränkungen von Tätigkeitsumfängen und Handlungsspielräumen und rigider Standardisierung, die gemessen an den Gestaltungsanforderungen von partizipativen Managementsystemen eher schlechte Ausgangsvoraussetzungen für die Aufgabenerfüllung eines Problemlösers sind (Kuhlmann 2004; Sauer 2005a; Iwer et al. 2008; Jürgens, U. 2017).

Mit der Verbreitung partizipativer Managementmodelle und der Einführung von KVP wurden ab den 1990er Jahren Gestaltungsformen und Wirkungsmechanismen von KVP-Praktiken sowie darauf bezogene Haltungen der Beschäftigten im Rahmen von Gruppenarbeitsstudien und einzelnen branchenbezogenen Fallstudien erforscht (Dörre et al. 1993; Howaldt 1994; Moldaschl/Schultz-Wild 1994a; Schumann et al. 1994a; Gerst et al. 1995; Howaldt 1995; Howaldt/Kopp 1997; Gerst 1998b; Kocyba/Vormbusch 2000; Kuhlmann et al. 2004). In der betrieblichen Realität zeigte sich ein breites Spektrum von KVP-Ansätzen, die sich hinsichtlich der einbezogenen Akteure (Beschäftigtenbeteiligung vs. Expertendominanz), der organisatorischen Struktur (Workshop, als Gruppenarbeitsaufgabe oder im Rahmen eines modernisieren Ideenmanagements) sowie eingebetteter betrieblicher Strukturen und des tatsächlichen Beteiligungsgrads der Beschäftigten unterschieden. Dabei wurde deutlich, dass es für das „Funktionieren“ von KVP als beschäftigtengetragenen Optimierungsprozessen maßgeblich ist, diese nicht singulär zu betrachten und 
$\mathrm{zu}$ gestalten; vielmehr handelt es sich um einen sehr diffizilen Gestaltungsansatz, der nicht ohne eine tiefgreifendere Neuausrichtung der arbeitsorganisationalen und betrieblichen Strukturen auskomme (Wolf 1994; Howaldt et al. 1998; Kuhlmann 2004; Kuhlmann et al. 2004). Verkürzt betrachtet, erweise sich eine strukturinnovative Arbeitsgestaltung mit erweiterten Aufgabenzuschnitten, Handlungs- und Entscheidungsspielräumen, aktiver Unterstützung eines aufgewerteten Meisters und prozessnahen Koordinations- und Kommunikationsstrukturen, gepaart mit Absicherungsvereinbarungen gegen KVPbedingten Stellenabbau, als günstig für eine Beteiligung der Beschäftigten an KVP - während in strukturkonservativen, taylorisierten Formen der Arbeitsgestaltung die Beteiligungsbereitschaft weniger hoch ist (Kuhlmann et al. 2004).

Aus den Ergebnissen der Gruppenarbeitsforschung sticht eine empirische Auffälligkeit heraus, die bislang in diesem Kontext unzureichend thematisiert und analysiert worden ist und Anlass zu der vorliegenden Untersuchung gegeben hat: Ungeachtet der oben beschriebenen Beteiligungstendenzen innerhalb der jeweiligen Arbeitsgestaltung ist eine erhebliche Varianz des potenziellen Beteiligungsverhaltens der Beschäftigten erkennbar, die sich nicht allein auf die Gestaltung der Optimierungs- und Arbeitsprozesse zurückführen lässt.

\section{Tabelle 1: Beschäftigtengetragene Prozessoptimierung und Gruppenar- beit}

\begin{tabular}{|c|c|c|c|c|}
\hline & & \multicolumn{3}{|c|}{$\begin{array}{c}\text { Reorganisationsniveau der } \\
\text { Arbeitsorganisation } 6\end{array}$} \\
\hline & in $\%$ & niedrig & mittel & hoch \\
\hline \multirow{3}{*}{$\begin{array}{l}\text { "Durch KVP habe ich die Möglichkeit, } \\
\text { größeren Einfluss auf die Gestaltung } \\
\text { meines Arbeitsplatzes zu nehmen, } \\
\text { so dass ich auf jeden Fall bereit bin, } \\
\text { mich zu engagieren." }\end{array}$} & ja & 48 & 55 & 63 \\
\hline & $\begin{array}{r}\text { unent- } \\
\text { schieden }\end{array}$ & 27 & 33 & 30 \\
\hline & nein & 25 & 13 & 8 \\
\hline
\end{tabular}

Quelle: Kuhlmann et al. (2004)

Wie in Tabelle 1 dargestellt, ermittelten Kuhlmann et al. (2004) zwar, dass ein Gros der Beschäftigten in strukturinnovativen Arbeitsstrukturen positiver

6 Unterschiedliche Reorganisationsniveaus der Arbeitsorganisation ergeben sich in „Konzepte innovativer Arbeitspolitik“" (Kuhlmann et al. 2004: 301ff.) aus dem Grad der Selbstorganisation sowie des Aufgaben- und Funktionszuschnitts der Beschäftigten in Gruppenarbeit. 
gegenüber KVP und den jeweiligen betrieblichen KVP-Praktiken eingestellt ist als Beschäftigte unter strukturkonservativen Bedingungen, da erstere durch ihre Beteiligung Einfluss auf die Arbeitsgestaltung nehmen können. Aber selbst unter strukturinnovativen Bedingungen gibt es eine relevant hohe Anzahl von Beschäftigten, die eine Beteiligung ablehnen bzw. dieser unentschieden gegenüberstehen. Umgekehrt ist auch bei einer strukturkonservativen Arbeitsgestaltung ein großer Teil der Beschäftigten bereit, sich an KVP zu beteiligen. Obgleich diese Befragung selbst noch viele Fragen aufwirft, ${ }^{7}$ decken sich diese Ergebnisse mit den ersten offenkundigen Beobachtungen des Beteiligungsverhaltens von Beschäftigten in den Fallstudien, welche die empirische Grundlage dieser Arbeit bilden: Es zeigte sich, dass sich die Beschäftigten eines Arbeitsteams sehr unterschiedlich an KVP beteiligen. Während einige sehr aktiv sind, beteiligen sich andere unter gleichen strukturellen Bedingungen nur selektiv oder gar nicht und bringen mitunter deutlich eine skeptische bis ablehnende Haltung zum Ausdruck.

Kuhlmann et al. (2004) messen den arbeitspolitischen Gestaltungsbedingungen von beteiligungsorientierter Optimierung eine hohe Bedeutung für das Ausmaß und die Art und Weise der Beteiligung der Beschäftigten bei. Beteiligungshinderliche Gründe liegen demnach in der Furcht vor Arbeitsplatzabbau und -verlust (unabhängig von Optimierungs- und Rationalisierungspraktiken im Betrieb), in der Wahrnehmung, vom Betrieb nicht ernst genommen zu werden, sowie in unzureichenden Kenntnissen über betriebliche Entscheidungen und Optimierungskosten. Beteiligungsförderlich wirken hingegen der durch den fortwährenden Wettbewerb geschürte „Zwang“, sich am wirtschaftlichen Erfolg des Betriebs zu beteiligen, sowie die Zunahme interessanterer und qualifizierterer Arbeit. Die Beteiligung an KVP sei „nicht nur eine Reaktion auf bestehende Zwänge [...], sondern ist zugleich Ausdruck einer selbstbewussten, offensiven Definition der eigenen Kompetenzen und der eigenen Rolle im Produktionsprozess“ (Kuhlmann et al. 2004: 363f.). Diese Erkenntnisse spiegeln zwar bereits den komplexen Zusammenhang zwischen den

7 Zum Beispiel kann man die Fragelogik selbst infrage stellen, weil zwei Aussagen miteinander verknüpft werden, die für die Beschäftigten nicht unbedingt eindeutig zu beantworten sind. Denn der Einfluss auf die Gestaltung des Arbeitsplatzes durch KVP muss nicht zwingend dazu führen, dass die Beschäftigten in jedem Fall bereit sind, sich zu engagieren. Nichtdestotrotz dient diese Tabelle sehr anschaulich der Abbildung der Varianzen. 
Gestaltungsbedingungen der KVP-Praktiken und den subjektiven Orientierungen der Beschäftigten wider, verharren aber noch auf einer allgemeinen Ebene. Sie differenzieren weder zwischen unterschiedlichen Beteiligungshaltungen der Beschäftigten noch geben sie ausreichend Einblick darin, wie die Beschäftigten mit den Widersprüchen zwischen ihrem Interesse an qualifizierterer und interessanterer Arbeit einerseits und ihrem Interesse an Schutz vor Arbeitsplatzabbau und Leistungsintensivierungen andererseits umgehen. Worin genau sich die unterschiedlichen Beteiligungshaltungen begründen, bleibt somit vorerst offen.

\section{Fragestellung}

Anlass zu dieser Studie ist die - mit der beschriebenen widersprüchlichen und teils ungeklärten Befundlage verbundene - subjektorientierte Frage nach den Umgangs- und Aneignungsweisen der Beschäftigten mit bzw. von KVP. Mittels einer vergleichenden Fallstudienuntersuchung in zwei Industriebetrieben, in denen beschäftigtengetragene Optimierungsprozesse mit unterschiedlichen beteiligungsorientierten Gestaltungsansätzen eingeführt wurden, soll empirisch beleuchtet werden, wie Produktionsbeschäftigte mit der Aufforderung und dem praktischen Beteiligungsangebot, an Optimierungsprozessen mitzuwirken, umgehen. Besonderer Fokus liegt dabei auf einer differenzierenden Analyse des komplexen Zusammenhangs zwischen den objektiven Gestaltungsbedingungen von KVP im Betrieb und den subjektiven, handlungsleitenden Orientierungen der Beschäftigten. Folgt man zentralen Erkenntnissen der Subjektivierungsdebatte, so lassen sich die soeben beschriebenen Unterschiede in den Beteiligungshaltungen vor allem darauf zurückführen, dass die Beschäftigten ihre alltägliche Anforderungssituation, die sich aus den betrieblichorganisationalen Rahmenbedingungen von KVP ergibt, mittels und aufgrund ihrer subjektiven Potenziale, Ressourcen, Orientierungen, Ansprüche und Handlungsdispositionen jeweils unterschiedlich subjektiv verarbeiten und sich aneignen (Kleemann et al. 2002; Lohr 2017a).

Die Frage danach, ob und, wenn ja, in welcher Weise Beschäftigte sich an einzelnen KVP-Praktiken beteiligen, ist bislang gegenüber dem großen strukturationstheoretischen Debattenentwurf eines neuen Sozialtypus unter der Arbeiterschaft (Voß, G./Pongratz 1998; Faust 2002; Pongratz/Voß 2003) sowie den Fragen nach den Folgen subjektivierter, vermarktlichter Arbeitsstrukturen für die Beschäftigten weitgehend unterbelichtet (Moldaschl/Schultz-Wild 
1994b; Döhl et al. 2000; Kleemann et al. 2002; Moldaschl/Voß 2002; Kleemann/Voß 2010). Subjektorientierte Erklärungsansätze für das Beteiligungsverhalten der Beschäftigten operieren allerdings oftmals mit Hypothesen, die vorwiegend auf Kenntnissen über Anspruchsmuster und Interessenstrukturen von Industriearbeitern aus der Forschung der 1980er Jahre vor dem Hintergrund damaliger Gestaltungsbedingungen von Arbeit beruhen (Kudera et al. 1979; Brock/Vetter 1982; Schumann et al. 1982; Kuhlmann et al. 2004). Diese Bezüge und Schlussfolgerungen, die davon ausgehen, dass die vorhandenen Fähigkeiten und das Interesse an Optimierungstätigkeiten hinter dem Schutzbedürfnis vor körperlichem Verschleiß und Arbeitsplatzabbau zurückstehen, müssen nicht per se überholt oder falsch sein; angesichts veränderter betrieblicher Bedürfnisse und Verwertungsstrategien der Arbeitskraft sowie potenziell gewandelter arbeitsbezogener und lebensweltlicher Ansprüche und Interessen der Beschäftigten bedarf es jedoch eines aktuellen Blicks auf die subjektiven, handlungsleitenden Orientierungen der Beschäftigten in der betrieblichen Alltagspraxis.

Neuere Befunde legen nahe, dass für das Handeln der Beschäftigten Anerkennung seitens des Betriebs sowie Reziprozitätserwartungen zwischen den eigenen und betrieblichen Leistungen bedeutsam sind (Holtgrewe et al. 2000; Voswinkel 2005; 2017). Kratzer et al. (2015) rekonstruierten in einer breit angelegten Studie über Anspruchsmuster und Rationalitätsvorstellungen ein Spektrum „aktueller“ Ansprüche, die im Denken und Arbeitshandeln der Beschäftigten zum Ausdruck kommen: Leistungsgerechtigkeit, Fürsorge, Selbstverwirklichung, Beteiligung und Würde sowie Rationalitätsvorstellungen, die sich auf technisch-funktionale, bürokratische und ökonomische Vorstellungen von Arbeit beziehen. Durch die Hebung der normativen Ansprüche und Legitimitätsvorstellungen machen Kratzer et al. (2015) deutlich, dass in den Orientierungen der Beschäftigten bestimmte normative Wertvorstellungen und Ansprüche zum Tragen kommen, die über ein Handeln als reinen Austausch von Leistungen zwischen Betrieb und Beschäftigten oder als einseitige Nutzenmaximierung hinausgehen.

Gemein ist den aktuellen Studien, dass sie zwar auf ein Spektrum an vorhandenen Anspruchsmustern hinweisen, aber keine ausreichende Erklärung für die Varianzen im (Beteiligungs-)Handeln der Beschäftigten bieten. Es gibt nur wenige Arbeiten, die differenziert auf Unterschiede in den Orientierungen von Industriebeschäftigten blicken. Dazu gehören die Studien von Lacher et al. (1989), die mit Blick auf Weiterbildungserfahrungen die Orientierungen der 
Beschäftigten in vier biografisch begründete „Bildungstypen“ (Aufstiegstyp, Passivtyp, Handwerkstyp und Familientyp) einordnen, und die Arbeit von Kleinwechter (2014), in der untersucht wurde, inwiefern Produktionsmitarbeiter den an sie gestellten Anforderungen und Rollenerwartungen in Teamarbeit - unter anderem Selbstorganisation, Kooperation und Beteiligung an Verbesserungsprozessen - gerecht werden. Dabei wurden drei Haupttypen identifiziert: Progressive, Assimilierte und Separierte. Hinsichtlich der Beteiligung an betrieblicher Optimierung lassen sich grundlegende Unterschiede in deren Erwerbsorientierungen wiederfinden: Progressive suchen eigeninitiativ Optimierungsmöglichkeiten von Anlagen und Arbeitsprozessen sowie des eigenen Arbeitshandelns; Assimilierte fokussieren sichere Prozessverbesserungen, die keine negativen Folgen bewirken; separierte Erwerbsorientierte mit einem instrumentalistisch-materialistischen Arbeitsbezug stehen betrieblichen Veränderungen aufgrund drohender Leistungsintensivierung mit großer Skepsis gegenüber. Obgleich diese Erwerbstypen deutlich mögliche Unterschiede im Umgang mit betrieblicher Optimierungsangeboten erklären, konnte die Studie nur sehr wenige tatsächlich durchgeführte bzw. umgesetzte beschäftigtengetragene Optimierungen identifizieren. Neben Gründen der mangelhaften Unterstützung bei der Realisierung durch das betriebliche Ideenmanagement und Starrheit der betrieblichen Kooperationsstrukturen sei es den Beschäftigten nicht gelungen, sich auf die neuen Anforderungen, die mit der Beteiligung an KVP einhergehen, einzustellen, „sich ständig über den eigenen Arbeitsprozess Gedanken zu machen, [...] sich selbst in Frage zu stellen und sich damit der Kritik der Anderen preiszugeben“" (Kleinwechter 2014: 209).

Jenseits dieser Differenzierungen zeigte sich in den empirischen Untersuchungen, dass wesentliche handlungsleitende Bestimmungsmomente im Arbeits- und Betriebsverständnis der Industriebeschäftigten weiter dominant sind: die eigene Verortung der Arbeiter im „Unten“ des Betriebs, Leistungsbewusstsein, Körperlichkeit der Arbeit, fachlich-professionelle Orientierung und Absicherungsbedürfnis (Kuhlmann/Schumann 2000; Kleinwechter 2014; Heil/Kuhlmann 2016).

Der Fokus beteiligungsorientierter Optimierungsprozesse liegt auf der Nutzung der Subjektpotenziale der Beschäftigten. In dieser Studie wird es daher weniger darum gehen, welche spezifischen subjektiven (Handlungs-) 
Potenziale in KVP von den Beschäftigten eingesetzt und angewandt werden. ${ }^{8}$ Das Vorhandensein spezifischer subjektiver Handlungspotenziale, die für die Optimierung betrieblicher und arbeitsbezogener Abläufe nötig und nützlich sind, wird weitgehend vorausgesetzt. Im Mittelpunkt des Forschungsinteresses steht vielmehr die Frage, wie und warum diese subjektiven Potenziale im Aneignungsprozess zum Ausdruck kommen: Wie machen sich Beschäftigte die Beteiligungsstrukturen von KVP zu eigen (oder eben nicht) und welche Begründungsmuster stehen hinter einzelnen Aneignungsmodi? Dabei werden die unterschiedlichen KVP-Praktiken nicht isoliert, sondern im Kontext der jeweiligen Arbeits- und Beschäftigungsbedingungen und der Auswirkungen der KVP-Aktivitäten auf die Situation der Beschäftigten betrachtet. In der Rekonstruktion der Aneignungsmodi soll es nicht darum gehen, wie die Beschäftigten mit einzelnen konzeptionellen Vorstellungen, Idealvorstellungen oder Rollenbildern umgehen; ${ }^{9}$ auch wird auf einen theoretisch zu einschnürenden Überbau von Konzepten verzichtet, um nach dem Prinzip der Offenheit aus einer subjektorientierten Perspektive darauf zu blicken, wie die jeweiligen betrieblichen Anforderungen oder Handlungspraktiken aus Beschäftigtensicht wahrgenommen und angeeignet werden.

\section{Aufbau der Studie}

Während in der Managementliteratur und der öffentlichen Managementrhetorik die Einbindung aller Beschäftigten in einen Kontinuierlichen Verbesse-

8 Verschiedentlich wurde die Bedeutung erweiterter Konzepte subjektiver Handlungspotenziale für das Arbeitshandeln empirisch in Abgrenzung zum rein faktischen Wissen nachvollzogen. Für den Vollzug des Arbeitens sind je nach situativer Anforderung unterschiedliche Qualitäten des Handelns von Bedeutung, wie planvolles, situatives, erfahrungsgeleitetes, kooperatives Handeln (eine Übersicht gibt Böhle (2010).

9 Hiermit ist gemeint, dass man die konzeptionellen Bausteine einzelner Managementmodelle heranzieht und schaut, wie die Beschäftigten damit umgehen. Dies birgt die Gefahr, dass man die Erfahrungswelt und das alltagspraktische Handeln der Beschäftigten zu stark auf die Fragestellung einengt und die eigene Wahrnehmungen der Beschäftigten auf ein starres Interpretationsmuster reduziert, wie es beispielsweise bei der These des Arbeitskraftunternehmers (Pongratz/Voß 2003) der Fall war. Bedeutsam sind hingegen die Erfahrungs- und Erlebniswelt in der Wahrnehmung der Beschäftigten. Erst dadurch wird das Prinzip der Offenheit gewahrt und ermöglicht die eigene Relevanzsetzung der Beschäftigten. 
rungsprozess überwiegend als selbstverständlicher Akt dargestellt wird, soll in dieser Studie die Perspektive des strukturellen Interessenantagonismus zwischen Betrieb und Beschäftigten eingenommen werden. Daher findet im zweiten Kapitel zunächst eine theoretische Kontextualisierung von Aneignung aus der Perspektive des Transformationsproblems - der Problematik wie gekaufte Arbeit in tatsächliche geleistete Arbeit überführt werden kann - und eine Betrachtung des grundlegenden Spannungsverhältnisses zwischen Betrieb und Beschäftigten statt. Auf Basis dieser Ausführungen werden die eingangs skizzierten Entwicklungslinien partizipativen Managements und die geschilderten arbeitssoziologischen Debattenstränge eingeordnet. Besonderes Augenmerk liegt dabei auf der Gestaltungsperspektive von KVP sowie den arbeits- und betriebsorganisatorischen Rahmenbedingungen auf der einen und der Beschäftigtenperspektive auf der anderen Seite. Das dritte Kapitel leistet eine kurze theoretische Erarbeitung eines näheren Verständnisses von Aneignung als subjektivem Prozess der Auseinandersetzung der Beschäftigten mit ihrer Umwelt. Besonderes Augenmerk richtet sich dabei auf das Verhältnis zwischen den strukturellen Bedingungen des Arbeitsprozesses und dem Subjekt.

Das vierte Kapitel befasst sich mit dem Untersuchungsdesign und dem Auswertungskonzept der vergleichenden Fallstudienuntersuchung. Kontrastiv wurden pro Betrieb jeweils zwei Bereiche für die Analyse ausgewählt, die sich hinsichtlich der Beteiligungsintensität der Beschäftigten an KVP unterschieden. Mit einem Mixed-Methods-Ansatz wurden in den einzelnen KVP-Konstellationen Beobachtungen, leitfadengestützte Interviews mit Beschäftigten und Expertengespräche mit Führungskräften und anderen an KVP beteiligten Akteuren durchgeführt.

Die Untersuchung erfolgte in zwei österreichischen Industriebetrieben, die im Rahmen ihrer Produktionskonzepte jeweils teamförmig organisierte Optimierungsprozesse eingeführt haben. Im fünften Kapitel werden beide Betriebe (und die ausgewählten Untersuchungsbereiche) mit dem Fokus auf ihre KVPPraktiken, die in sie eingebetteten Produktionskonzepte sowie Arbeits- und Beschäftigungsbedingungen porträtiert.

Die Analyse der Aneignungs- und Umgangsformen der Beschäftigten erfolgt in einem Dreischritt; die Rekonstruktion der subjektiven Orientierungsmuster, der Analyse der Gestaltungs- und Kontextbedingungen der KVPPraktiken in vier Beteiligungskonstellationen und schließlich die Auswertung der einzelnen Aneignungs- und Umgangsformen der Beschäftigten mit KVP. Erstens werden im sechsten Kapitel die subjektiven Orientierungen der Beschäf- 
tigten rekonstruiert. Auf Basis von Motiven, welche die Beschäftigten mit ihrer Beteiligungsdisposition verbinden, werden hier einzelne Dimensionen der subjektiven Orientierungen identifiziert, die für die Beschäftigten in Bezug auf ihre potenzielle Beteiligung an KVP relevant sind: individuelle Orientierung an Fachlichkeit, gute und sinnvolle Arbeit, Beitragsorientierung, Anerkennung, Weiterentwicklungsperspektiven und Schutz der eigenen Arbeitskraft vor Verschleiß und Arbeitsplatzunsicherheit sowie soziale Normen des Betriebs und der kollegialen Verhaltenserwartungen, die sich insbesondere auf kollegiale Solidarität beziehen. Die einzelnen Orientierungsdimensionen können in unterschiedliche Cluster eingeteilt werden. Insgesamt lassen sich drei Beteiligungsorientierungen differenzieren: Mitgestaltungsorientierung, Karriereorientierung und Status-quo-Orientierung. In Kapitel 7 werden im zweiten Schritt die KVP-Praktiken als arbeitspolitische Beteiligungskonstellationen in den einzelnen Arbeitsbereichen analysiert. Dabei wird nicht nur in einem engeren Sinne die Gestaltung von KVP, sondern auch die betrieblichen und arbeitsorganisatorischen Rahmenbedingungen entlang der Analysekriterien strukturinnovativer und -konservativer Arbeitspolitik (Gerst et al. 1995; Kuhlmann et al. 2004) einbezogen. Das achte Kapitel befasst sich mit der Rekonstruktion der Aneignungsformen von KVP, die von Nichtbeteiligung aufgrund von Desinteresse, Widerstand und/oder Protest über selektive kontrollierte und kontrollierende Beteiligung bis hin zu proaktiver Beteiligung reichen. Dabei wird deutlich, dass die Beschäftigten aufgrund ihrer Beteiligungsorientierung jeweils unterschiedlich mit den arbeitspolitischen Rahmenbedingungen von KVP umgehen.

Im abschließenden Kapitel 9 wird mit der Zusammenführung der wichtigsten Befunde schließlich erläutert, dass die Haltung des arbeitspolitischen Konservativismus zwar noch immer vorhanden ist, mittlerweile aber mehrheitlich durch einen arbeitspolitischen Pragmatismus abgelöst worden ist. Die Arbeit schließt mit einer Diskussion der zentralen Ergebnisse und weiterführenden Perspektiven. 



\section{Kontinuierlicher Verbesserungsprozess - im Spannungsfeld von Beschäftigten- und Betriebsinteressen}

KVP bildet einen zentralen Kern partizipativer Managementkonzepte. Die arbeitspolitischen Maßnahmen, die zum Kanon posttayloristischer, LeanProduction-orientierter Arbeitsgestaltung gezählt werden, wie Abflachung von Hierarchien, Veränderung von Führungsstilen, Einführung von Gruppenarbeit sowie das neue Leitbild eines unternehmerisch handelnden Produktionsbeschäftigten, zielen letztendlich insgesamt darauf ab, die kreativen Produktivitätspotenziale - das „Gold in den Köpfen“ - der Beschäftigten zu fördern, um die Produktionsprozesse effizienter zu gestalten (Moldaschl/Schultz-Wild 1994b; Wolf 1994; Dörre/Neubert 1995; Kocyba/Vormbusch 2000; Dörre 2002). Wie in der Einleitung bereits kurz umrissen, ist die Gestaltung von KVP sehr voraussetzungsreich, möchte man eine breite und beständige Beteiligung 
der Beschäftigten fördern. Einerseits ermöglicht Partizipation, die mit Selbstorganisation und erweiterten Handlungsspielräumen einhergeht, Produktionsbeschäftigten, ihre Ansprüche an interessante Arbeit zu verwirklichen; andererseits führt die zielgerichtete Kopplung an Rationalisierung dazu, dass Beschäftigte Ressentiments gegenüber KVP hegen können.

Dieses Kapitel beginnt mit einer theoretischen-konzeptionellen Betrachtung von KVP und der arbeitspolitischen Ausgangssituation aus der Perspektive des Transformationsproblems und der Unvollständigkeit des Arbeitsvertrags. Diese begründen sowohl den strukturellen Interessenantagonismus zwischen Betrieb und Beschäftigten als auch die beiderseitigen Akteursperspektiven (2.1). Darauffolgend wird aus einer theoretisch-konzeptionellen Perspektive der Fokus auf Aneignung als subjektiven Auseinandersetzungsprozess mit der betrieblichen Wirklichkeit gelegt (3). Hierbei werden sowohl individuelle als auch sozial-strukturelle Aspekte der Aneignung beleuchtet.

Mithilfe dieser theoretisch-konzeptionellen Vorarbeiten werden die Entwicklungslinien partizipativer Managementkonzepte (2.2) in einzelnen Abschnitten vom Taylorismus zu neuen Produktionskonzepten (2.2.1) und von Lean Production zu Ganzheitlichen Produktionssystemen (2.2.2) vorgestellt. Das Kapitel endet mit der Anwendung des erarbeiteten Wissens auf das spezifische Anwendungsfeld KVP (2.3). Die Darstellung der Erkenntnisse erfolgt zumeist aus zwei Perspektiven: erstens aus der Gestaltungsperspektive, in der einerseits auf die Leitideen und Verwertungsstrategien des Betriebs und andererseits auf die betrieblichen Praktiken als Realisierung dieser Konzepte eingegangen wird. Zweitens werden diese Konzepte und Praktiken aus der Beschäftigtenperspektive betrachtet, in der der Fokus auf den subjektiven Wahrnehmungen, Bewertungen, Orientierungen und letztlich auf der Aneignung der Beschäftigten liegt. Kontext und Struktur auf betrieblicher Seite werden subjektiven Orientierungen der Beschäftigten gegenübergestellt.

\subsection{KVP aus Perspektive des Transformationsproblems: Das Potenzial und die Steuerung menschlichen Arbeitsvermögens}

Im Kontinuierlichen Verbesserungsprozess vergegenständlicht sich ein postfordistischer Grundmechanismus der kleinschrittigen, permanenten Verbesserung von Abläufen und Produkten, der in die Haltungen und Handlungen der 
Beschäftigten übergehen soll. Beschäftigte sind angehalten, Probleme als Verbesserungspotenziale zu begreifen und permanent die etablierten Prozessabläufe im eigenen Bereich nach Optimierungsmöglichkeiten zu hinterfragen und diese umzusetzen (Imai 1992; Teufel 2003).

\section{Subjektpotenziale - das menschliche Arbeitsvermögen}

Das KVP-Konzept operiert mit einem bestimmten Bild von Arbeitnehmern als Träger komplexer, objektivierbarer und nicht-objektivierbarer, individueller Dispositionen, Eigenschaften, Fähigkeiten und Fertigkeiten, die sie in die Lage versetzen, ,intentional zu handeln, den eigenen Arbeitsprozess selbst zu strukturieren, kreativ auszugestalten, innovativ zu sein und Verantwortung zu übernehmen" (Lohr 2017b: 282). KVP fokussiert darauf, diese Subjektpotenziale für die betrieblichen Ziele nutzbar zu machen. Subjektpotenziale stellen als lebendiges Arbeitsvermögen (Pfeiffer 2004) ein wichtiges Produktionsmittel im betrieblichen Verwertungsprozess dar. Das menschliche Arbeitsvermögen umfasst sowohl objektivierbare als auch subjektivierende Potenziale für die Bewältigung der übertragenen Aufgaben und Probleme des Produktionsprozesses - also jene Potenziale, die in der Transformation der Arbeitskraft für die Herstellung eines Produkt einen Gebrauchswert darstellen (Marx 1867; Pfeiffer 2004; 2007). Mit anderen Worten: Das menschliche Arbeitsvermögen ist lebendig (Pfeiffer 2004). Es drückt sich in den sedimentierten und aktuellen Erfahrungen aus, welche die Beschäftigten mit allen Sinnen und Subjektpotenzialen bei der Bewältigung der alltäglichen Probleme und Herausforderungen des Produktionsprozesses machen. Es formt sich aber auch permanent neu durch das Arbeitshandeln der Subjekte - etwa durch Zugewinn an Wissen, Kompetenzen, Reflektion, Erfahrungen, Gefühlen und Wahrnehmungen etc. und, kumulativ betrachtet, einen Zugewinn an Identität. ${ }^{10}$ Das Arbeitsvermögen bilde sich nicht nur in der Arbeit, sondern entstehe aus der ganzen lebensweltlichen Umwelt des Subjekts und übersteige daher immer das Mindestmaß, das für die Bewältigung der Arbeit vonnöten wäre. Aus dieser Perspektive wird deutlich, dass die Subjektpotenziale, deren Nutzung potenziell für das Management interessant ist, nur einen Teil des menschlichen Arbeits-

10 Pfeiffer versteht diesen Prozess der fortlaufenden Verausgabung und Neubildung des lebendigen „Arbeitsvermögen[s] als zur „Form gebrachte Aneignung“ (Pfeiffer 2004: 160). 
vermögens darstellen. Ob und unter welchen Umständen die „gewünschten“ Subjektpotenziale der Beschäftigten in Optimierungsprozessen zum Einsatz kommen, muss im Kontext des gesamten Arbeitsvermögens der Beschäftigten betrachtet werden.

Es gibt unterschiedliche Konzeptionen von Subjektivität und Subjektpotenzialen; ihnen gemein ist jedoch die Aussage, dass jenseits von formalisierbaren und objektiven Wissensbestandteilen im Arbeitssubjekt Potenziale vorhanden sind, die für die Arbeit und funktionierende Produktionsprozesse von erheblicher Bedeutung sind. ${ }^{11}$ So haben Böhle et al. (2004) mit ihrem Konzept des subjektivierenden Arbeitshandelns herausgearbeitet, dass neben formalfaktischem Wissen die Ebene des sinnlichen Erlebens einer Arbeitssituation, der persönlichen Erfahrung und der Intuition im Umgang mit Problemen und unvorhersehbaren Herausforderungen im Produktionsprozess eine bedeutende Rolle zukommt (Böhle et al. 2004; Böhle 2017). Im technisch-funktionalen Kontext eines Betriebs etwa sind für das praktische Handeln der Beschäftigten Subjektpotenziale bedeutsam, die „kompensatorisch“ und „gewährleistend“ die Unwägbarkeiten des Produktionsprozesses ausgleichen, zum Beispiel bei Störungen von Anlagen, „strukturierend“ die organisatorischen Abläufe von Produktionsprozess, Zusammenarbeit und Erreichung von Arbeitsziels regeln und „reklamierend“ das Handeln in Sinnstrukturen und Deutungsmuster einordnen (Deutschmann 2002; Kleemann et al. 2002; Lohr 2003). Für Optimierungs- und Gestaltungsaktivitäten der Beschäftigten wird dem spezifischen Erfahrungswissen der Beschäftigten großen Stellenwert beigemessen (Pfeiffer 2007)

Folgt man dem High-Performance-Work-System-Ansatz, welcher den Erfolg von Unternehmen auf eine spezifische Kombination von personalpolitischen Praktiken, Strukturen und Prozessen, welche die Qualifikation, das Engagement und die Beteiligung der Beschäftigten fördern, zurückführt (Appelbaum et al. 2000; Combs et al. 2006; Boxall 2012), so sind für die betriebliche Nutzung der Subjektpotenziale drei Gestaltungsperspektiven von großer Bedeutung, und zwar: Ability - Motivation - Opportunity. Die Beschäftigten müssen demnach nicht nur die Fähigkeiten besitzen, sich an Optimierungen zu

11 Gute Überblicke zu unterschiedlichen Konzeptionen und Systematisierungen von Subjektivität und Subjektpotenzialen geben Kleemann et al. (2002) und Lohr (2003). 
beteiligen, sondern sie müssen das auch wollen und vom Betrieb zudem die Gelegenheit dazu erhalten. Dieses Zusammenwirken zwischen lebendigen Arbeitsvermögen und dem betrieblichen Verwertungskonzept (Beteiligungsstrukturen) lässt sich mittels der interessentheoretische Perspektive des Transformationsproblems beleuchten.

\section{Das Transformationsproblem und die Unvollständigkeit des Arbeitsvertrags}

Die Arbeitsbeziehungen zwischen dem Betrieb als Arbeitgeber und den Beschäftigten als Arbeitnehmer sind dadurch gekennzeichnet, dass die Beschäftigten ihr Arbeitsvermögen (Gebrauchswert der Arbeit) für eine vertraglich bestimmte Zeit gegen Lohn (Tauschwert der Arbeit) überlassen (Marx 1867). In der Frage, wie die dem Betrieb vertraglich überlassene Arbeitszeit in Leistung transformiert wird, stehen sich Unternehmen und Beschäftigte mit zwei verschiedenen Interessanlagen gegenüber (Marx 1867; Edwards 1981; Deutschmann 2002). Unternehmen streben nach einer größtmöglichen Verwertung der gekauften Arbeitskraft, was sie mittels Anweisungen und Kontrolle der Arbeitsleistungen zu realisieren versuchen. Beschäftigte hingegen streben vorrangig nach einer für sie günstigen Lohn-Leistungs-Relation, dem Schutz vor dem Verschleiß und Dequalifizierung sowie nach Verbesserung ihrer Arbeitskraft (Braverman 1977). Neben den Reproduktionsinteressen sind arbeitsinhaltliche Ansprüche bedeutsam, wie die Suche nach Sinnhaftigkeit des eigenen Arbeitshandelns, Entfaltung der eigenen Fähig- und Fertigkeiten sowie Anerkennung (Schumann et al. 1982; Voß, G. Günter 2010; Nies 2015).

Die Arbeitsleistung und die für die Erzielung der Arbeitsleistung notwendigen Handlungs- und Übersetzungsschritte können - auch wenn sie mitunter höchst kleinteilige und für den Betrieb unökonomische Formen annehmen nicht bis in das letzte Detail vorgegeben werden (Deutschmann 2002; Marrs 2010). Aus dieser Unvollständigkeit des Arbeitsvertrags ergeben sich Interpretationsspielräume hinsichtlich der Art der Leistungsverrichtung, die für die Beschäftigten mit Handlungsspielräumen, für den Arbeitgeber aber eher mit der Ungewissheit verbunden sind, ob er auch die gewünschte (bestmögliche) Arbeitsleistung der Beschäftigten erhält. Der Arbeitgeber versucht im Rahmen der betriebliche Organisation von Arbeit diese Unsicherheitsräume durch Kontroll- und Steuerungsmechanismen zu beherrschen, kann sie aber niemals vollkommen kontrollieren. Beschäftigte hingegen können diese Spielräume auf 
ihre eigene Weise nach ihren Vorstellungen ausfüllen (Burawoy 1979; Edwards 1981).

Neben diesen antagonistischen Tendenzen sind beide Akteure bzw. Akteursgruppen jedoch zugleich elementar aufeinander angewiesen: der Betrieb auf die Kooperation der Beschäftigten und die Beschäftigten auf den Erhalt ihres Arbeitsplatzes (Braverman 1977; Edwards 1981; Thompson/Smith 2009). Spannungsfelder von Widerstand und Kooperation, Verfolgung der jeweiligen inhärenten Interessen der betrieblichen Akteure und die Notwendigkeit zur Kooperation bilden die Rahmenbedingungen für fortwährende Aushandlungsprozesse über die tatsächlichen Anforderungen, Erfüllungen von Arbeitsleistungen und Arbeitsbedingungen (Edwards 1981).

Während bei der Analyse betrieblicher Lösungen des Transformationsproblems der industriesoziologische Fokus vorwiegend auf Formen direkter oder technisch vermittelter Kontrolle durch tayloristische Arbeits- und Organisationsgestaltung lag (Braverman 1977; Edwards 1981), wies Friedman (1977) auf das Vorhandensein unterschiedlicher Managementstrategien im Umgang mit menschlicher Arbeitskraft hin. Das Spektrum möglicher Lösungen des Transformationsproblems entfalte sich zwischen zwei strategischen Polen: direkter Kontrolle und verantwortlicher Autonomie. Durch direkte Kontrolle mit engmaschiger Überprüfungen sowie standardisierten und hoch arbeitsteiligen Arbeitsstrukturen wird vonseiten des Managements versucht, eigensinne und unerwünschte Arbeitshandlungen sowie widerständige Leistungszurückhaltung zu reduzieren. Der Einsatz von (eigen-)verantwortlichem, „mitdenkenden“ Handeln bzw. von Subjektivität der Beschäftigten soll demnach so weit wie möglich eingeschränkt werden - denn wenn Beschäftigte Spielraum für solche Handlungen haben, würden sie diesen zu ihren Gunsten und nicht im Sinne des Betriebs nutzen. Direkte Kontrolle als Managementstrategie ist Ausdruck eines starken Misstrauens gegenüber den Beschäftigten.

Managementstrategien, die sich am Prinzip der verantwortlichen Autonomie orientieren, operieren mit einem diametralen Verständnis der Qualität und der Nutzbarkeit menschlicher Arbeitskraft. Durch Verantwortungsübertragung, Rücknahme von direkter Kontrolle und Maßnahmen zur Stärkung der Bindung der Beschäftigten an den Betrieb wird versucht, auf die Interessen der Beschäftigten einzugehen, so dass sie Leistungspotenziale und Kompetenzen einbringen, auf die der Betrieb angewiesen ist, wie Engagement, Eigenverantwortung, Kreativität, Kooperation und Qualifikation. Hierbei geht es vor allem um Kompetenzen, bei denen der Einsatz von direkter Kontrolle nicht mög- 
lich, unverhältnismäßig oder hinderlich ist. Die strategische Entscheidung des Betriebs, Kontrolllücken des Arbeitsvertrags nicht durch enge Kontrollmechanismen zu schließen, erfordert einen höheren Aufwand an vertrauensbildenden Maßnahmen, Sozialleistungen und Vermittlungsprozessen, der dazu führen soll, dass Beschäftigte ihre subjektiven Potenziale freiwillig im Sinne des Betriebs einsetzen (Friedman 1977; Deutschmann 2002; Marrs 2010). Genau an diesem Punkt setzt das Verwertungskonzept von KVP an.

Entlang der Pole der beschriebenen Managementstrategien ließen sich bis in die 1980er Jahre hinein die Arbeitsstrukturen verschiedener Beschäftigtengruppen relativ eindeutig zuordnen: direkte Kontrolle bei Arbeitern im Produktionsbereich - verantwortliche Autonomie im Angestelltenbereich (Deutschmann 2002).

Mit Friedmans Analyse der Managementstrategien wurden die Variabilität, Veränderbarkeit und strategischen Entscheidungsmöglichkeiten betrieblicher Arbeitspolitik hervorgehoben (Deutschmann 2002). So unterstreichen auch Schumann et al. (1982), dass die Frage, wie Betriebe die Arbeitskraft bzw. das Leistungsvermögen ihrer Beschäftigten einsetzen (wollen), Resultat betrieblicher Entscheidungen über Verwertungsstrategien und Funktionsgestaltung der Produktionsmittel ist (Schumann et al. 1982)..$^{12}$ Die Nutzung menschlicher Arbeitskraft ist von der betrieblichen Gestaltung des Produktionskonzepts abhängig, welches sich aus den stofflichen Bedingungen der Herstellung eines Produktes ${ }^{13}$, den zur Verfügung stehenden Produktionsfaktoren und dem „Produktionskalkül“ (54f.) ergibt. Unter Produktionskalkül verstehen Schumann et al. die strategischen Gestaltungsziele, an denen der technische und arbeitsorganisatorische Produktionsprozess ausgerichtet ist, um ein zuvor bestimmtes Produkt zu einem bestimmten (Mehr-)Wert herzustellen. ${ }^{14}$ Tätig-

12 Betriebliches Handeln findet nicht im luftleeren Raum statt. Es ist in hohem Maße vom marktlichen Gesamtgeschehen abhängig. So differenzieren Schumann et al. (1982) das Verwertungskonzept neben dem eigentlichen Produktionskonzept, welches bei der Betrachtung von arbeits- und betriebsorganisatorischen Analysen häufig im Vordergrund steht, weiter in Einkaufsmarktkonzept und Absatzmarktkonzept. Diese drei betrieblichen Handlungsfelder beeinflussen sich - besonders in der Frage der Verfügbarkeit von Kapital und Produktionsmitteln - gegenseitig.

${ }^{13}$ Hierbei sind die Mittel gemeint, die schlichtweg physisch oder technisch notwendig sind, um ein Produkt herzustellen.

14 So Schumann et al. (1982) in stiller Anlehnung an Marx (2009 [1872]). 
keitsstrukturen, Anforderungsprofile und die Gestaltung von Arbeitsplätzen sind vorwiegend Resultat dessen, wie Betriebe vor dem Hintergrund arbeitsund absatzmarktlicher Bedingungen den Produktionsprozess gestalten. ${ }^{15}$ Der gleiche technische Produktionsprozess kann bei unterschiedlicher strategischer Ausrichtung zu unterschiedlicher Gestaltung der Arbeitsorganisation und somit des Arbeitsplatzes führen. In diesem Sinne ist betriebliches Handeln Resultat des jeweiligen gewählten Verwertungskonzepts, welches Schumann et al. (1982) als Kapitalperspektive bezeichnen.

Die Beschäftigten sind mit dem betrieblichen Verwertungskonzept in Gestalt von Arbeits- und Betriebsorganisation, Kontroll- und Steuerungsmodi konfrontiert, auf welche sie mit einer Gegenstrategie reagieren. Diese Gegenstrategie geht immanent von der Arbeitskraft aus, weil diese

„im Gegensatz, zu den anderen bei der Produktion verwendeten Faktoren [...] immer mit Menschen verbunden ist, die eigene Interessen und Bedürfnisse baben und die an ibrem Widerstand dagegen festhalten, wie eine Ware behandelt zu werden. " (Edwards 1981: 21f.)

So zeigen Beschäftigte in Arbeitssystemen mit direkter Kontrolle oftmals Widerstandsformen in Form von kollektiver Leistungszurückhaltung (Bummeln, Bremsen) oder stiller Selbstoptimierungen zur verdeckten Erweiterung eigener Handlungsspielräume (Edwards 1981; Ackroyd/Thompson 1999; Deutschmann 2002). Währenddessen wird am anderen Ende des Friedman'schen Pols - der verantwortlichen Autonomie - eine zunehmende Kooperationsbereitschaft der Beschäftigten verortet. Wie im Laufe dieses Kapitels noch ausgeführt wird, ist bislang nicht abschließend geklärt, auf welche „Strategien" Beschäftigte in Arbeitssystemen mit verantwortlicher Autonomie zurückgreifen und ob bzw. wie sie sich auf den vom Betrieb anvisierten Verwertungszweck einlassen.

Aus der Perspektive des Transformationsproblems lassen sich für die vorliegende Fragestellung und die theoretische Aufarbeitung einige Implikationen mitnehmen. Erstens ist deutlich geworden, dass die Art und Weise, wie Beschäftigte mit betrieblichen Verwertungsstrategien - und im Folgenden spezifisch auch auf KVP - umgehen, nicht als reines Produkt der vorherrschenden

15 In fordistischer Massenproduktion mit standardisierten und verketteten Montageprozessen ist Einfacharbeit mit stark repetitiver und restriktiver Arbeitsgestaltung wahrscheinlicher als in flexibler, hochtechnisierter Produktion. 
Verwertungsstrategie zu betrachtet ist. Betrieb und Beschäftigte haben divergierende Interessen, die jeweils nicht vollständig gegenseitig subsumiert werden können, sondern miteinander interagieren. Betriebliche Verwertungsstrategien, die in Arbeitsorganisation, Kontroll- und Steuerungsmechanismen für die Beschäftigten konkrete Gestalt annehmen - wie tayloristische Arbeitsgestaltung oder partizipative Managementstrategien - bewirken eine spezifische „Antwort“ der Beschäftigten. Beide Perspektiven - die des Betriebs und die der Beschäftigten - müssen jeweils sowohl für sich in ihrer Eigenlogik als auch aufeinander bezogen betrachtet werden. Ausgehend von dieser Prämisse werden in dieser Studie die Entwicklungslinien des partizipativen Managements aus Gestaltungsperspektive sowie aus Beschäftigtenperspektive ${ }^{16}$ dargestellt.

\subsection{Entwicklungslinien und Herausforderungen partizipativer Managementsysteme}

In einer systematisierenden, eher historischen Betrachtung der Verwertungskonzepte werden im folgenden Abschnitt die Entwicklungslinien der Beschäftigtenbeteiligung an Rationalisierungs- und Optimierungsprozessen nachgezeichnet. Dabei geht es nicht nur darum, die Genese beteiligungsorientierter Gestaltungkonzepte nachzuzeichnen, sondern auch darum, vorzubereiten, dass Aneignungsmechanismen von tayloristischer Arbeitsgestaltung - wie in der Einleitung angedeutet - auch heute noch aktuell sein könnten. Beginnend mit dem Taylorismus wer-

16 Diese Systematisierung in Gestaltungs- und Beschäftigtenperspektive ist abgeleitet aus der sogenannten „Werftstudie“ (Schumann et al. 1982), die in ihrem Ansatz die analytische Trennung von Kapital- und Arbeiterperspektive vorgenommen hat. Mit dem Begriff Beschäftigtenperspektive wird sich hier von der dominierenden Begrifflichkeit des männlichen, hauptverdienenden Arbeiters verabschiedet, auf die sich die Arbeiterbewusstseinsforschung meist undifferenziert bezieht. Weibliche Beschäftigte wurden entweder im betrieblichen Kontext unterrepräsentiert, in weiten Teilen ignoriert oder nicht als gesellschaftlich relevante soziale Klasse wahrgenommen. Obgleich mit dem Begriff des Arbeiters und der Arbeiterschaft insbesondere aus historischer und gesellschaftspolitischer Sicht weit mehr als nur die maskuline Form des Arbeiters, wie etwa Arbeiterbewusstsein und angeschlossene Klassenidentitäten, verbunden ist und sich die meisten Befunde dezidiert auf Arbeiter beziehen, werden in dieser Arbeit die Begriffe Beschäftigte und Beschäftigtenperspektive verwendet, um den exklusiven Charakter aufzulockern. 
den wichtige arbeitspolitische Veränderungen in der Verwertungsstrategie (Gestaltungsperspektive) sowie der spezifische Umgang der Beschäftigten mit diesen Veränderungen (Beschäftigtenperspektive) betrachtet. Diese Ausführungen münden in eine Analyse der derzeitigen arbeitspolitischen widersprüchlichen Anforderungssituation für die Beschäftigten und eine Spurensuche möglicher Aneignungsformen.

\subsubsection{Vom Taylorismus bis zu neuen Produktionssystemen}

\section{Die Gestaltungsperspektive: Taylorismus}

In der soziologischen Debatte wird beteiligungsorientiertes Management zumeist vor dem Hintergrund der „anti-partizipativen“ Kontrastfolie des Taylorismus betrachtet. Das Verwertungskonzept von Taylors „Scientific Management" (Taylor 1995 (1913)) fußte auf der unternehmerischen Intention, den Arbeitskrafteinsatz im Produktionsprozess methodisch so zu strukturieren, dass Produktionsbeschäftigten Möglichkeiten zur Leistungszurückhaltung weitestgehend entzogen werden. Dazu werden die Produktionsprozesse so effizient wie möglich gestaltet, indem eine strikte Trennung von ausführenden und planerischen Tätigkeiten vorgenommen wird und eine akribische Zerlegung des Produktionsprozesses in kleinschrittige, leicht zu kontrollierende Einzeltätigkeiten erfolgt. Um die vom Betrieb gekaufte Arbeitskraft der Beschäftigten möglichst wirtschaftlich im Betrieb einsetzen zu können, werden monetäre Leistungsanreize gesetzt oder Lohnkürzungen bei Nichterfüllung der Leistung vorgenommen (Braverman 1977; Rammert 1983; Taylor 1995 (1913)). Die Grundstrukturen tayloristischer Arbeitsgestaltung verliehen der Industriearbeit den Charakter monotoner, wenig interessanter, gering qualifizierter Arbeit, die durch geringe Handlungs- und Entscheidungsspielräume, hohe körperliche Belastungen und eine große Arbeitsplatzunsicherheit geprägt war. Das Arbeitsbüro war die zentrale Instanz der Kontrolle und Rationalisierung der Arbeitsprozesse - vonseiten der Produktionsbeschäftigten wurde keine Einmischung gewünscht. Die Subjektivität der Beschäftigten und die in ihr begründeten Bedürfnisse, Interessen, Ansprüche und Fähigkeiten wurden hingegen misstrauisch als Störfaktor, als Quelle der Leistungszurückhaltung 
und des Widerstandes gegen das Management betrachtet (Edwards 1981). ${ }^{17}$ Das Management versuchte, jegliche Freiräume innerhalb der Arbeitszeit zu reduzieren, da Beschäftigte diese zur Leistungszurückhaltung nutzen würden.

\section{Die Beschäftigtenperspektive: Taylorismus, Instrumentalismus und „klassisches Arbeiterbewusstsein"}

Die Frage nach dem Umgang der Beschäftigten mit tayloristischen Arbeitsund Produktionsbedingungen ist mit der Herausforderung verbunden, die arbeits- und industriesoziologischen Befunde methodologisch und inhaltlich einzuordnen. Denn obwohl mit Marx (1867) und Popitz et al. (1957b) Konzepte zur Aneignung, etwa zur sinnlichen Auseinandersetzung der Beschäftigten mit dem Arbeitsprozess, der Einordnung und Bewertung der Erfahrungen sowie der individuellen, subjektiven Bezüge auf die stofflichen Bedingungen des Arbeitsprozesses, aus heutiger Sicht bereits sehr weit gediehen waren, wurden diese durch die Fokussierung auf das Arbeiterbewusstsein als Klassenund Lagebewusstsein in den Hintergrund gedrängt (Voß, G. 1984; Langfeldt 2009; Heil/Wolf 2017).

So wurde in der zeitgenössischen Industriesoziologie der 1960er/70er Jahre korrespondierend zur monotonen, repetitiven Arbeitsgestaltung und der Fokussierung auf extrinsische Leistungsanreize angenommen, dass Produktionsbeschäftigte (vorwiegend Montagearbeiter) eine rein instrumentelle Beziehung zu ihrer Arbeit hätten (Instrumentalismusthese). Charakteristisch sei die Haltung, dass die Beschäftigten ihre Arbeit lediglich zu dem Zweck leisten, Mittel zur Realisierung von Interessen außerhalb der Arbeit zu erwerben (Goldthorpe et al. 1968). Goldthorpe et al. (1968) interpretierten ihre Erhebungsdaten dahingehend, dass vor allem solche Arbeiter, die in hohem Maße an der Realisierung außerberuflicher Interessen interessiert sind und einer eher allgemeinen gesellschaftlichen Konsumorientierung folgen, die Beschäftigung als Fließbandarbeiter annehmen und somit ohnehin keine arbeitsinhaltlichen

17 Diese Interessengegensätze zwischen Management und Beschäftigten sind immer wieder Quell von Konflikten über die Arbeitsgestaltung, Arbeitsbedingungen und Organisation des Arbeitsprozesses. Trotz des Kontrollsystems und der engmaschigen Arbeitsgestaltung gibt es durch Veränderungen im Produktionsalltag unregulierte Schlupfwinkel, welche die Beschäftigten für ihre Interessen nutzen können, so dass Subjektivität - obgleich vom Management nicht erwünscht - nicht gänzlich ignoriert werden kann (Schienstock 1993). 
Ansprüche an ihre Arbeit richten. Aufgrund seiner materialistischinstrumentellen Orientierung und der hohen Bedeutung außerberuflicher Interessen bringe sich der Arbeiter somit nur begrenzt in die Arbeit ein. Rein instrumentalistische Orientierungen entsprächen somit der beschriebenen Haltung des tayloristischen Managements, dass Beschäftigte gar kein Interesse hätten, sich über die rein ausführende Arbeit hinaus zu beteiligen - und erst recht nicht an Optimierungs- und Rationalisierungsmaßnahmen.

Während Goldthorpe et al. den Instrumentalismus der Arbeiter als durch den gesellschaftlichen Wertewandel bestimmte Orientierung verstehen, stellen Kern/Schumann (1970) die Arbeitsorientierung in den Erfahrungszusammenhang der Produktionsbeschäftigten mit den unmittelbaren Arbeitsbedingungen. ${ }^{18} \mathrm{Im}$ Vergleich unterschiedlicher Tätigkeitszuschnitte in technisierten Produktionsprozessen (Produktionsarbeiter, Automationsarbeiter, Instandhaltungsarbeiter) stellen sie heraus, dass Produktions- und Automationsarbeiter, deren Arbeit durch Restriktionen, geringe Qualifikationsanforderungen und Monotonie bestimmt ist, eine instrumentelle Arbeitsorientierung annehmen, während in qualifizierten Facharbeitertätigkeiten mit ganzheitlichen Aufgabenzuschnitten (Instandhaltung) arbeitsinhaltliche Orientierungen, wie Arbeitsfreude und Identifikation mit der Arbeit, erkennbar sind. Kern/Schumann verknüpfen die Arbeitsorientierung direkt mit den unmittelbaren Arbeitserfahrungen. Solange die Arbeitssituation durch hohe körperliche Belastungen und restriktive Tätigkeitszuschnitte charakterisiert sei, gebe es keine identifikatorischen Bezugspunkte - also auch keine subjektiven Orientierungen, die sich aus den Arbeitsinhalten selbst ergäben.

Statt auf arbeitsinhaltliche Bezüge rekurrieren wesentliche Orientierungspunkte der Produktionsbeschäftigten auf ein - zuvor bereits von Popitz et al. (1957a) identifiziertes - typisches Arbeiterbewusstsein, das durch die drei Kernelemente spezifisches Leistungsbewusstsein, Produzentenstolz und ein dichotomes Interessenverständnis gekennzeichnet sei. Das spezifische Leistungsbewusstsein beziehe sich, so Popitz et al. (1957a), auf die handwerklich-körperlichen

${ }^{18}$ Die Forschungsergebnisse zu Arbeitsorientierungen der Beschäftigten lassen sich eher als Nebenergebnis der großen Arbeiterbewusstseinsstudien begreifen, in deren Kern vor allem die Frage nach dem gesellschaftlichen Bewusstsein der Arbeiter steht - und nach dem Konfliktpotenzial, welches von der sich selbst im gesellschaftlichen „Unten“ verortenden Arbeiterschaft ausgehen könnte (Voß, Gerd Günter 1980; Kudera et al. 1983). 
Aspekte menschlicher Arbeit. Der Arbeiter schafft durch seine körperliche Leistung - im Gegensatz zur geistigen Arbeit der Angestellten - die eigentlichen, materielle Werte, aus denen der Betrieb Profit schöpft. Das sinnstiftende Element ziehe der Arbeiter aber nicht allein aus seiner eigenen Tätigkeit heraus, sondern daraus, dass er als Teil der kollektiven Arbeiterschaft Werte schaffe, die gesellschaftlich anerkannt werden. Diesen Sinngehalt kann er als Produzentenstolz direkt auf seine Arbeit übertragen. Zugleich würden sich die Arbeiter auch innerhalb der Arbeiterschaft als Gruppe in einem antagonistischen Verhältnis gegenüber anderen betrieblichen - aber auch gesellschaftlichen - Gruppen abgrenzen und sich unter ihnen einordnen (dichotome Einteilung in ein Unten und Oben). Dies sei vor allem mit mangelnden Aufstiegschancen und einem Ohnmachtsgefühl verbunden, welche sie in ihrem Arbeitsalltag erfahren.

Das Arbeitshandeln der Beschäftigten wurde in der Industriesoziologie der 1960er Jahre vorwiegend im Kontext von Entfremdung gedeutet, die außer einer instrumentellen Arbeitsorientierung keine subjektiven Orientierungen der Arbeiter zuließ. Obwohl Popitz et al. (1957b) schon in „Technik und Industriearbeit" betonten, dass Arbeitsleistung nicht als abstrakte Arbeit, sondern als konkrete Handlung im Arbeitsprozess durch die Beschäftigten aktiv hergestellt werden muss, blieben solche subjektivierenden Aspekte in der arbeitssoziologischen Forschung bis Mitte/Ende der 1970er Jahre - und teilweise bis heute - stark unterbelichtet. Dies lässt sich zurückführen auf die Dominanz des Taylorismusnarrativs, welches die Negation der arbeitsinhaltlichen Kompetenzen und Ansprüche der Beschäftigten von betrieblicher Seite und die instrumentellen Arbeitsorientierung auf der Seite der Beschäftigten betont. Durch den gesellschaftspolitischen Fokus auf Arbeiterbewusstsein als Klassenbewusstsein und die konzeptionelle Engführung des Verhältnisses von Arbeitserfahrungen und Arbeitsorientierung wird weitgehend verkannt, dass auch einfache Produktionsarbeiter unter restriktiven Arbeitsbedingungen subjektive, nach Sinnhaftigkeit suchende, arbeitsinhaltliche Ansprüche an Arbeit stellen können (Voß, G. 1984; Langfeldt 2009; Nies 2015).

Ab Ende der 1970er Jahre befasste sich die deutsche Arbeiterbewusstseinsforschung im Kontext der zunehmenden Technisierung von Produktionsprozessen unter anderem wieder mit der Frage nach Subjektivität im Arbeitsprozess und ihr Verhältnis zu eher instrumentellen Arbeitsorientierungen. Erst mit Kudera et al. (1979) und etwas später Schumann et al. (1982) wurde die Instrumentalismusthese mit dem Befund relativiert, dass 
„Lohnarbeit nicht nur Einkommensquelle [ist], sondern als konkrete Tätigkeit immer auch Anwendung subjektiver Fäbigkeiten. Sie ist ein zweckmäßiger Prozess, in dem der Arbeiter als Produzent planvoll und zielbewußt bestimmte Arbeitsaufgaben bewältigt, um den Arbeitsgegenstand in der beabsichtigten Form zu gestalten. Dies gilt auch dort, wo infolge von extremer Arbeitszerlegung, Mechanisierung und Teilautomation die arbeitsinhaltliche Entscheidungskompetenzen des Arbeiters weitgehend unterböblt ist." (Kudera et al. 1979: 26f.)

Neben dem Bewusstsein, den Status eines abhängigen Lohnarbeiters zu haben und im Spannungsfeld der widersprüchlichen Interessen von Kapital und Arbeit den ökomischen Zwecken des Produktionsprozesses zu unterliegen, beziehen sich Arbeiter auf den Inhalt ihrer Arbeitstätigkeit unter dem Gesichtspunkt der Identifikation. Arbeiter haben ein eigenes, subjektives Verhältnis zu ihrer Tätigkeit und zu ihrem Können, das sie in den Arbeitsprozess einbringen. $\mathrm{Ob}$ und wie stark sie ihre eigenen Ansprüche an die Tätigkeit und ihre Fähigkeiten aber tatsächlich einbringen können, sei abgängig von der betrieblichen Organisation von Arbeit (Kudera et al. 1979). Die Arbeitsorientierung der Arbeiter ergebe sich aus einem Abwägen zwischen in der Lohnarbeit begründeten Ansprüchen und den eigenen arbeitsinhaltlichen Ansprüchen, die oftmals im Widerspruch zueinanderstehen, aus einem Austarieren von Arbeitsbedingungen, Belastung, Verschleiß und Verdienst. In ihrer Studie rekonstruieren die Autoren fünf unterschiedliche Orientierungstypen von Arbeitern: den identifikatorischen, den kompensatorischen, den resignativen, den negatorischen und den gleichgültigen Typus, die sich hinsichtlich des Grads arbeitsinhaltlicher Ansprüche und des Umgangs mit fehlenden identifikatorischen Bezugsmöglichkeiten unterscheiden. So finden sie identifikatorische Orientierungen vor allem in Tätigkeitsbereichen mit ganzheitlicherem Zuschnitt, in denen die Arbeiter weniger restriktiven Arbeitsprozesses ihre Qualifikationen („handwerkliches Produzentenbewußtsein“) (Kudera et al. 1979: 29) einbringen können. Unter sehr restriktiven Bedingungen sind die Orientierungen der Arbeiter durch identifikatorische Ersatzbezüge (Kompensation), wie eine starke kollektive Orientierung innerhalb der Arbeiterschaft, durch Aufgabe der Ansprüche (Resignation), durch Kritik und Widerstand (Negation) oder Gleichgültigkeit gegenüber den eigenen Ansprüchen geprägt. Diese „Gebrochenheit des Instrumentalismus" (Kudera et al. 1979) entstehe nach Kudera und Kollegen dadurch, dass die Beschäftigten sich aufgrund der durch Lohnarbeit bedingten Widersprüchlichkeiten nicht gänzlich instrumentell auf ihre Arbeit beziehen könnten. Verschleiß und Reproduktion der Arbeitskraft, Leistungs- und Lohnorientierung, Ansprüche auf Entfaltung des Arbeitsvermö- 
gens und Fremdbestimmung in der Arbeit, Konkurrenz unter den Arbeitern sowie Abhängigkeit vom unternehmerischen Erfolg des Betriebs stellen für die Arbeiter widersprüchliche Bedingungen dar. Der jeweilige Umgang mit diesen Widersprüchen sei vor dem Hintergrund der jeweiligen objektiven Bedingungen der Beschäftigung und Arbeitsgestaltung von individuellen Ressourcen, wie etwa Alter und Qualifikation, abhängig (Kudera et al. 1979: 118).

Kudera et al. (1979) haben mit ihrer Studie zum Arbeitsverständnis von Lohnarbeit einen wesentlichen konzeptionellen Beitrag zur Auflösung der zuvor kritisierten Interpretation des Arbeiterbewusstseins und von Handlungsorientierungen beigetragen, die zuvor entweder als rein gesellschaftlich bestimmt und von außen in die Arbeit hineingetragen oder als linear von den Arbeitsbedingungen abhängig betrachtet worden waren. Jedoch behandeln auch sie subjektive Orientierungen in der Arbeit und deren Verhältnis zu den objektiven Bedingungen relativ verkürzt. Obwohl arbeitsinhaltliche und leistungsbezogene Bestimmungsmomente sich als wichtig für die Arbeitsorientierungen der Arbeiter herausgestellt haben und dies vor allem am Bespiel identifikatorischer Orientierungen von Facharbeitern mit eher ganzheitlichen Aufgabenstrukturen verdeutlicht werden konnte, gehen sie nicht darauf ein, wie sich subjektive Orientierungen unter restriktiven Bedingungen konstituieren (Langfeldt 2009), sondern stellen sie aufgrund fehlender professioneller Anknüpfungspunkte schlichtweg als nicht vorhanden da.

\section{Der „Doppelte Bezug auf Arbeit“}

Noch viel deutlicher und systematisierender arbeiteten Schumann et al. (1982) den Stellenwert und einzelne Aspekte sinnhafter Bezugspunkte für die Subjekte in der Arbeiter heraus. Im Analyserahmen des „Doppelten Bezugs auf Arbeit", den die Autoren in der Studie „Rationalisierung, Krise, Arbeiter" über den Umgang von Werftarbeitern mit betrieblicher Rationalisierung entwickelten, wird die „Kapitalperspektive“ der „Arbeiterperspektive“ gegenübergestellt. Die Arbeiterperspektive greift grundlegende Bestimmungsmomente von Erwerbsarbeit auf, die konstitutiv für die arbeitsbezogenen Bedürfnisse, Interessen und Ansprüche der Subjekte sind: die Notwendigkeit des Erhalts der Arbeitskraft, die durch Belastungen durch den Arbeits- und Verwertungsprozess beansprucht werden (Arbeitskraftperspektive), und die Interessen des arbeitenden Subjekts, sich in der Arbeit als Person wiederzufinden und Anerkennung zu erfahren (Subjektperspektive). 
Aus der Arbeitskraftperspektive betrachtet das Subjekt seine Situation unter dem Aspekt „der Erhaltung bzw. Vernutzung seines Mittels Arbeitskraft“ (Schumann et al. 1982: 305) und materieller Interessen. Vernutzung oder auch Verschleiß der Arbeitskraft können durch physische und psychische Belastungen, den betrieblichen Leistungsanspruch und durch Entwertung nicht genutzter qualifikatorischer Potenziale entstehen. Inbegriffen sind nicht nur die Dimensionen des körperlichen Verschleißes, der durch die stofflichen Anforderungen des Arbeitsprozesses bedingt ist, sondern auch der räumliche, zeitliche und organisationale Einsatz der Arbeitskraft, wie zum Beispiel Schichtarbeit, das durch qualitative und quantitative Leistungsanforderungen bedingte Arbeitspensum, Formen der Überwachung und Kontrolle sowie technik- oder ablaufbedingte „Zwänge“. Gerade in Bezug auf den betrieblichen Leistungsanspruch stellten individuelle und kollektive Handlungsspielräume wichtige Ressourcen dar, mit deren Hilfe die Beschäftigten Strategien zum Umgang und zur Regulation der Arbeitskraftvernutzung entwickeln könnten.

In Subjektperspektive spiegeln sich diejenigen Bestimmungsmomente wider, ,mittels derer sich der Arbeiter auf seine Arbeit als eine subjektive und sinnhafte Tätigkeit bezieht und darin Selbstbestätigung und Selbstbewertung sucht“ (Schumann et al. 1982: 27). Die Subjektperspektive lässt sich in zwei Dimensionen unterteilen: zum einen in subjektive Bestimmungsmomente, bei denen es um die Wiedererkennung des eigenen Selbst durch die Einbringung eigener Fähigkeiten und Fertigkeiten in den Arbeitsprozess geht. Zum anderen sind Bestimmungsmomente bedeutsam, durch die sich die Beschäftigten in Beziehung zu anderen setzen und nach Anerkennung streben. Hierbei steht im Vordergrund, inwiefern die Beschäftigten durch ihre Arbeit die sozialen Erwartungen erfüllen. An dieser Stelle lässt sich wiederum weiter ausdifferenzieren in unterschiedliche Ausprägungen und Adressaten der Anerkennung ${ }^{19}$.

Die Auseinandersetzung mit der Arbeitssituation erfolgt immer unter beiden Bezugsweisen sowohl der Arbeitskraft- als auch der Subjektperspektive, wobei durchaus Konflikte zwischen einzelnen Dimensionen entstehen können. Bezugsweisen können sich durch individuelle Relevanzsetzungen der Subjekte in ihren Ausprägungen unterscheiden und zusammengefasst als Muster von Orientierungen verstanden werden. In ihrer Untersuchung konnten

${ }^{19}$ Weitere Ausführungen dazu erfolgen in der empirischen Konzeptionalisierung in Kapitel 4.1.1. 
Schumann et al. keine eindeutigen Muster von Orientierungen ausmachen, die sich entweder rein auf die Subjekt- oder ausschließlich auf die Arbeitskraftperspektive beziehen. In Bezug auf betriebliche Rationalisierung und der Möglichkeit, sich daran zu beteiligen, arbeiten Schumann et al. (1982) heraus, dass mangelnde Beteiligungsbereitschaft nicht auf Desinteresse an der inhaltlichen Aufgabe oder mangelnde Kreativität zurückzuführen sei. Vielmehr sei sie in einem interessenbewussten Arbeitsverständnis und -bewusstsein begründet, in dem sich die Erfahrungen betrieblicher Rationalisierungsmaßnahmen widerspiegeln. Aufgrund der Wahrnehmung, dass betriebliche Rationalisierung und die Teilnahme daran sich letztlich negativ auf die Beschäftigten auswirkt, verweigern diese ihre Beteiligung (Schumann et al. 1982: 554).

In den 1980er Jahren verschob sich der Forschungsfokus weg von der Klassenfrage hin zu einer von der zunehmenden Individualisierung geleiteten Subjektorientierung sowie auf Fragen der Arbeits- und Lebensorientierung (Kleemann/Voß 2010). Auch retrospektiv wurden neuere Forschungsperspektiven an die Zeit des Taylorismus herangetragen, unter subjektorientierten Fragestellungen beleuchtet und teils neu bewertet.

So verdeutlicht Wolf (1999), dass Selbsttätigkeit der Beschäftigten als Ausdruck eines von den betrieblichen Verhaltenserwartungen abweichendes Verhalten bereits unter tayloristischen Arbeitsbedingungen stattfindet. Denn hinter dem - auch heute noch - stilisierten Bild des durch wissenschaftliche Betriebsführung perfekt strukturierten und organisierten tayloristischen Produktionsprozesses verbarg sich in der betrieblichen Realität ein eher unvollkommener Produktionsprozess mit täglichen Störungen sowie technischen und organisatorischen Ineffizienzen. Diese Mängel fordern die Selbsttätigkeit der Beschäftigten heraus, diese Lücken mit ihrem spezifischen erfahrungsgesättigten Produktionswissen kompensatorisch auszufüllen, da der Produktionsprozess anderenfalls kollabieren würde. Die zweite Aktivierungsquelle von Selbsttätigkeit sieht Wolf darin, dass die Arbeits- und Beschäftigungsbedingungen den eigenen Interessen der Beschäftigten zuwiderlaufen und diese sich individuell oder kollektiv Ressourcen schaffen, um mit der Situation umzugehen. Die selbsttätigen Arbeitshandlungen können dabei von betriebskonformer Kompensation bis hin zu „subversiven', den betrieblichen Normen direkt widersprechenden Tätigkeiten“ (Wolf 1999: 81). Die Selbsttätigkeit stelle in tayloristischen Produktionssystemen stets eine illegale, offiziell nicht erwünschte Arbeitshandlung dar, welche die Beschäftigten vor einen inneren Wider- 
spruch stelle, der eigentlich nur dann gelöst werden könne, wenn der Betrieb die durch die Notwendigkeit von Selbsttätigkeit begründeten Ansprüche auf Selbstbestimmung im Produktionsprozess anerkenne. Stattdessen regieren tayloristische Arbeits- und Produktionssysteme mit einer Intensivierung der Regulierung und Kontrolle von oben. Trotz der wahrgenommen - teils untersagten, teils geduldeten - Selbsttätigkeit der Beschäftigten war es im tayloristischen Mainstream nicht vorstellbar, dass sich einfache Produktionsbeschäftigte an selbstgetragenen Optimierungen beteiligen könnten oder wollten. Dies war allenfalls für Beschäftigte in höherwertigen handwerklichen Tätigkeiten denkbar. ${ }^{20}$

\section{Partiripative Episoden im 20. Jabrhundert}

Obgleich die tayloristische Arbeitsgestaltung, die in Deutschland erst nach dem Zweiten Weltkrieg eine flächenmäßige Ausbreitung als tayloristischfordistisches Produktionssystem gefunden hat (Schmidt 2017), die dominante Form der Arbeitsgestaltung in der industriellen Produktion war, gab es in einzelnen Betrieben Formen von Beteiligung der Produktionsbeschäftigten. Seit Beginn der Taylorismuswelle waren alternative Gestaltungsformen, die ein höheres Maß an Beteiligung der Beschäftigten vorsahen, Teil der wissenschaftlichen Debatte (Wolf 2003). Als managementorientierter Ansatz befasste sich die Human-Relations-Bewegung schon in den 1930er Jahren mit der leistungssteigernden Wirkung sozialer Beziehungen im Betrieb (zwischen den Beschäftigten untereinander und zwischen Beschäftigten und ihren Führungskräften) als Gegenprogramm zum auf monetären Anreizen basierenden Leistungslohnsystem des Taylorismus (Mikl-Horke 2007). In den 1960er/70er Jahren wurde unter dem Einfluss zunehmender Technisierung in Bergwerksbetrieben der Soziotechnische Ansatz aus der empirischen Beobachtung entwickelt, dass direkte Partizipation der Beschäftigten bei der Einführung neuer Technik oder Veränderungen im Arbeitsprozess nicht nur zu mehr Handlungsspielräumen und Einflussnahme auf die eigene Arbeitssituation führt, sondern auch gute Lösungen für die technische Systemgestaltung hervorbringen kann. Mensch, Technik und Organisation (M-T-O) seien demnach nicht als starre Systeme $\mathrm{zu}$ betrachten, sondern müssten sich aufeinander beziehen und aneinander

20 So auch Kern/Schumann (1970). 
angepasst werden (Emery/Thorsrud 1982). Der Soziotechnische Ansatz geht davon aus, dass der Arbeitende als Faktor Mensch grundlegende

„Bedürfnisse nach interessanter, abwechslungsreicher Tätigkeit, nach Lernen am Arbeitsplats, nach Verantwortung und Spielraum für eigene Entscheidungen, nach Anerkennung und Einbeziebung der Arbeit in das soziale Leben der Menschen und ein Streben nach persönlicher Entfaltung und Entwicklung" (Mikl-Horke 2007: 155)

hat. Umsetzung fand die soziotechnische Systemgestaltung in einer Reihe von skandinavischen Betrieben in Form von teilautonomen Arbeitsgruppen mit Aufgabenintegration von planerischen Tätigkeiten und Aufgabenrotation (Emery/Thorsrud 1982; Sydow 1985; Mikl-Horke 2007).

Diese Entwicklungen hatten auch Einfluss auf die sich in den 1960er/70er Jahren in Deutschland entwickelnde HdA-Bewegung (Humanisierung der Arbeitswelt). In Folge der prosperierenden, stark tayloristisch strukturierten Nachkriegswirtschaft forderte die Arbeiterbewegung mehr Demokratie und Autonomie. Damit verbunden war der Wunsch nach mehr Freiräumen, Selbstbestimmtheit in der Arbeit, Verbesserungen der Arbeitsmittel sowie der ergonomischen Bedingungen. Diese Debatte mündete unter anderem in die Novellierung des Betriebsverfassungsgesetzes 1972 mit einer Erweiterung der betrieblichen Mitbestimmung von Interessenvertretungen und in das staatliche Aktions- und Forschungsprogramm zur Humanisierung des Arbeitslebens (Matthöfer 1980).

Diese Partizipationsbewegungen hatten allerdings gegenüber den „ökonomischen Krisen und Stagnationstendenzen, Entpolitisierungs- und Individualisierungstrends sowie de[m] aufkommende[n] Neoliberalismus“" (Wolf 2003: 14) nur geringe Durchsetzungschancen.

\section{Neue Produktionskonzepte - die Entdeckung der Verwertbarkeit der Subjektpotenziale}

Mit dem empirischen Befund der "neuen Produktionskonzepte“ (Kern/Schumann 1984) wurden ab Mitte der 1980er Jahre in der deutschen Industriesoziologie erneut alternative, aufgewertete Formen der Organisation von Arbeit diskutiert. Der Unterschied zu den vorangegangenen Debatten und politischen Forderungen bestand darin, dass das menschliche Arbeitsvermögen als wichtige Produktivkraft besonders in stark technisierten Produktionen genutzt werden sollte. Die Notwendigkeit des menschlichen Arbeitskraftein- 
satzes gerade in automatisierten Produktionen ergebe sich daraus, dass die zunehmende Komplexität technischer Produktionsprozesse mit einem hohen $\mathrm{Maß}$ an Störungen und Unwägbarkeiten einhergehe, was ein kompensatorisches Eingreifen qualifizierter Produktionsarbeiter erfordere. Dies sei vor allem in Arbeitssystemen relevant, die auf Reprofessionalisierung durch Verbreiterung der Tätigkeitszuschnitte, Aufgabenintegration von planerischen Tätigkeiten sowie Ausweitung der Entscheidungsspielräume der Produktionsbeschäftigten setzen. Der nunmehr veränderte Umgang mit Arbeitskraft sei, so Kern/Schumann (1984), wichtig für eine flexible und kundenorientierte Produktion hochwertiger Produkte und somit unerlässlich für den langfristigen Unternehmenserfolg in westlichen Hochlohnwirtschaften. Angesichts dieser Entwicklungen sei anzunehmen, dass die tayloristische Arbeitsgestaltung in technisierten Bereichen zunehmend von neuen Produktionskonzepten verdrängt werde. ${ }^{21}$

Mit dem Aufkommen neuer Produktionskonzepte, neuer industrieller Tätigkeitstypen - beispielsweise des Systemregulierers (Kern/Schumann 1984) und der Zunahme sowie steigenden Akzeptanz von Selbstverwirklichungsansprüchen prognostizierte Baethge (1991), dass Beschäftigte ihrer Arbeit im Betrieb mit mehr und höheren subjektorientierten Ansprüchen, etwa nach Spaß, Selbstverantwortung sowie Anwendung und Entfaltung der eigenen Fähigkeiten und Kompetenzen, nachgehen würden. Dieser normative Subjektivismus sei nicht nur traditionell in der Beschäftigtengruppe der hochqualifizierten Angestellten zu verorten, sondern lasse sich zunehmend in der Breite der Arbeitswelt wiederfinden. Im Sinne dieser Prognosen könnten Beschäftigteninteressen und Unternehmensinteressen in neuen beteiligungsorientierten Produktionskonzepten stärker vereint werden einander begegnen. Wie sich die Beschäftigten aber in die neue betriebliche Verwertungsstrategie einfügen, wie sie sich diese aneignen und in welchem Verhältnis die Haltung des arbeitspolitischen Konservatismus zu der normativen Subjektivierung steht, ist noch offen.

21 Wobei Kern/Schumann (1984) in ihrer Prognose der Verdrängung des Taylorismus hier die Einschränkungen vornehmen, dass die Reprofessionalisierung der Produktionsarbeit parallel zu Dequalifizierungsprozessen verlaufe. Als Erweiterung und Aktualisierung der in „Industriearbeit und Arbeiterbewußtsein“ (Kern/Schumann 1970) formulierten Polarisierungsthese verweisen Kern und Schumann darauf, dass eine Segmentierung zwischen Beschäftigtengruppen und Branchen stattfinde. 
2.2.2. Lean Production und Ganzheitliche Produktionssysteme

\section{Die Gestaltungsperspektive - Partizipative Wende?}

Der eigentliche Durchbruch und „Paradigmenwechsel“ von partizipativen Rationalisierungs- und Produktionssystemen erfolgte unter dem Einfluss der Lean-Production-Welle zu Beginn der 1990er Jahre, die durch die MIT-Studie „The Machine That Changed The World“ (Womack, J. et al. 1990) ausgelöst wurde. In dieser Studie beschäftigten sich Womack, J. et al. (1990) mit der Charakterisierung von Produktionssystemen der zu jener Zeit erfolgreichen japanischen Automobilindustrie. In der Folge orientierten sich viele westliche Betriebe am Vorbild des schlanken (Toyota-)-Produktionssystems. In einer ersten Welle wurden in Anlehnung an das japanische Ideal Prinzipien und Methoden von Lean Production wie Kaizen (Imai 1992), KVP und andere Methoden zur Optimierung und Standardisierung des Arbeits- und Produktionsablaufs (unter anderem Andon, Just-in-Time, TPM und Standardisierung) als Teil umfangreicher Restrukturierungs- und Reorganisationsmaßnahmen umgesetzt. Oftmals war dies begleitet von Formen selbstorganisierter Gruppenarbeit, Dezentralisierung betrieblicher Organisations- und Entscheidungsstrukturen sowie Abflachung der Führungsstrukturen (Moldaschl/SchultzWild 1994b; Wolf 1994; Howaldt/Kopp 1997; Kocyba/Vormbusch 2000; Teufel 2003; Wolf 2003) 22 .

Der wesentliche Unterschied zu den vorangegangenen Partizipationsbewegungen, die vor allem von Gewerkschaften und Wissenschaftlern vorangetrieben worden waren, bestand darin, dass das Management, das zuvor eine Beteiligung der Beschäftigten an der Gestaltung von Arbeits- und Produktionsprozessen eher abgelehnt hatte, nun das produktive Arbeitsvermögen der Beschäftigten anerkannte und gezielt nutzen möchte. Anstelle des Misstrauens, welches der Betrieb den Beschäftigten hinsichtlich potenzieller Leistungszurück-

22 Lean-Production-Gestaltungsansätze beziehen sich nicht nur auf die innerbetriebliche Restrukturierung der Arbeits-, Betriebs- und innerbetrieblichen Fertigungsorganisation. Vor allem Anfang der 1990er Jahre werden diese in der Automobilindustrie durch zentrale Veränderungen in der Zuliefererstruktur, durch starke Kostensenkungsmaßnahmen und Standortdebatten begleitet, wie weitreichende Reduzierung der Wertschöpfungstiefe durch Zentralisierung und Outsourcing sowie die Zunahme von prekären Beschäftigungsformen (Leiharbeit) (Kuhlmann 2004; Jürgens, U. 2017). 
haltung entgegengebracht hatte, sollten nun Verantwortungsübergabe und Vertrauen treten. Der Wunsch der Beschäftigten nach Beteiligungsmöglichkeiten und Erweiterung von realen Handlungs- und Entscheidungsspielräumen wurde vom Management aufgegriffen und in eine normative Erwartungshaltung gegenüber den Beschäftigten gewendet: Die Beschäftigten sollten sich nun produktiv in Arbeits- und Optimierungsprozesse einbringen (Kocyba/Vormbusch 2000; Wolf 2003).

Zentrales Charakteristikum der partizipativen Rationalisierungs- und Optimierungskonzepte ist die gezielte Nutzbarmachung der Wissens-, Kreativitäts- und Erfahrungspotenziale der Beschäftigten durch das Management. Dies geschieht durch die Implementierung von partizipativen Gestaltungskonzepten und durch die ,Zuweisung neuer Rollenmuster im Sinne selbstverantwortlicher, mitdenkender und initiativer Arbeitskräfte" (Kocyba/Vormbusch 2000: 14). Rationalisierung und Optimierung von Arbeits- und Produktionsprozessen wird nun nicht mehr, wie in tayloristisch geprägten Arbeitssystemen, ausschließlich von Experten vorangetrieben. Die Produktionsbeschäftigten können und sollen in Zusammenarbeit mit den indirekten Planungsbereichen selbst kleine Verbesserungen vornehmen, also „Rationalisierung in begrenzter Eigenregie" (Dörre et al. 1993: 16) durchführen und somit zum Rationalisierungssubjekt werden (Dörre et al. 1993; Howaldt 1993; Wolf 1994). Um die Beteiligung der Beschäftigten zu fördern, werden größere Entscheidungs- und Gestaltungsspielräume, Aufgabenerweiterung, Funktionsintegration (häufig teamförmig organisiert), Einschränkung der direkten Kontrolle des Managements und teilweise auch finanzielle Anreize geschaffen. Mit diesen arbeitspolitischen Veränderungen verbinden Unternehmen - im Sinne der verantwortlichen Autonomie Friedmans - einen auf sozialen Austausch, Konsens und Vertragen setzenden Führungsstil und Programme zum Werte- und Kulturwandel, deren zentraler Bestandteil das Leitbild eines selbstverantwortlichen, loyalen und flexiblen Mitarbeiters ist (Moldaschl/Schultz-Wild 1994b; Wolf 1994; Marrs 2010). Auf diese Weise beinhalten partizipative Managementkonzepte das Potenzial, ,sich über den alten Rationalisierungsrahmen hinaus [zu bewegen]. Sie implizieren, dass ein neuer Rahmen, eine neue Rollenverteilung, eine neue soziale Infrastruktur der Rationalisierung entsteht.“ (Wolf 1994: 250) 


\section{Spielfeld arbeitspolitischer Gestaltungskonzepte}

Die an Lean-Production-Methoden und Prinzipien orientierten Managementkonzepte entfalteten sich in einer Reihe innerbetrieblicher Restrukturierungen der Arbeits-, Betriebs- und innerbetrieblichen Fertigungsorganisation. Das arbeitspolitische Programm der 1990er Jahre wurde insbesondere in der Automobilindustrie aufgrund zentraler Veränderungen in der Zuliefererstruktur von drastischen Kostensenkungsmaßnahmen und erhitzten Standortdebatten begleitet, was sich etwa in der weitreichenden Reduzierung der Wertschöpfungstiefe durch Zentralisierung und Outsourcing sowie in der Zunahme von prekären Beschäftigungsformen wie Leiharbeit und Befristungen niederschlug (Kuhlmann 2004; Jürgens, U. 2017). In Deutschland fand Lean Production vor allem Ausdruck in der Einführung von Gruppenarbeit, die zunächst gemeinsam mit den genannten arbeits- und betriebsorganisatorischen Veränderungen als Bestätigung und Weiterentwicklung der neuen Produktionssysteme gedeutet wurde (Schumann et al. 1994a; Kißler 1996).

Nach einer Phase der Implementierung von Gruppenarbeit mit unterschiedlichen Konzeptelementen von teilautonomer Gruppenarbeit, die in unterschiedlichem Maße mit der Integration von indirekten Tätigkeiten, höheren Anteilen an Selbstorganisation, Gruppensprechern und KVP-Aufgaben operierten, zeichnete sich bereits ab Mitte der 1990er Jahre in Teilen eine Rekonventionalisierung der Arbeitsstrukturen und -organisation ab (Jürgens, U. 1997; Kuhlmann 2004; Kuhlmann et al. 2004; Kötter 2007; Sauer 2008). ${ }^{23}$ Diese oftmals als „arbeitspolitisches Rollback“ beschriebene Retaylorisierungstendenzen führten dazu, dass den Beschäftigten nur wenig Handlungs- und Gestaltungsspielräume blieben, um die ihnen zugedachte „aktive Rolle des Problemlösers“ (Jürgens, U. 2006b) im für Störungen anfälligen Produktionssystem wahrnehmen zu können. Der steigende Kostendruck und die nach wie vor hohe Bedeutung von marktorientierten Kontroll- und Steuerungsformen (Peters, K./Sauer 2005; Kratzer et al. 2008) ließen Gruppenarbeitskonzepte, in

23 Diese Entwicklung resultierte häufig daraus, dass bei einer hohen Reorganisationsdynamik verschiedene Konzeptelemente von Lean Production oftmals eher uneinheitlich und ungleichzeitig eingeführt wurden. Halbherzig konzipierte und praktizierte Reorganisationskonzepte scheiterten und erfolgreiche (strukturinnovative) Konzepte fanden wenig Nachahmer (Kuhlmann et al. 2004; Kötter 2007; Sauer 2008). 
denen Beschäftigte günstige Gelegenheitsstrukturen für die Beteiligung an Optimierungs- und Rationalisierungsprozessen finden, in die arbeitspolitische Defensive rücken (Lay et al. 1996; Springer 1999a; Wolf 2003). Die Durchsetzungschancen für partizipative Gestaltungskonzepte wurden für Betriebe mit hohem Technisierungsgrad oder mit einer beteiligungsorientierten arbeits- und interessenpolitischen Tradition höher eingeschätzt als für Betriebe mit einem hohen Anteil manueller Tätigkeiten (Moldaschl/Schultz-Wild 1994b; Kocyba/Vormbusch 2000; Wolf 2003).

Aufwind erhielten arbeitspolitische Gestaltungsthemen durch die zweite Lean-Production-Welle ab Ende der 1990er Jahre, die mit der Verbreitung Ganzheitlicher Produktionskonzepte einherging (Liker 2004). Wiederum das Toyota-Produktionssystem als Vorbild nehmend, integrierten Unternehmen die bereits bekannten Lean-Production-Konzepte zu einem aufeinander abgestimmten und an den Bedingungen und Strategien des Unternehmens orientierten Produktionssystem (Lay/Neuhaus 2005; Gerst 2012a; Jürgens, U. 2013). Im Vergleich unterschiedlicher Produktionskonzepte lassen sich oftmals die gleichen Gestaltungsprinzipien und -methoden erkennen: kundenorientierte Ausrichtung der Fertigungsprozesse, Total Quality Management (TQM), beständige und standardisierte Arbeitsprozesse, geringe Lagerbestände und Just-in-Time-Zulieferung, Gruppenarbeit und Kontinuierlicher Verbesserungsprozess (Dombrowski et al. 2006; Pfäfflin et al. 2011; Dombrowski/Mielke 2015). In der betrieblichen Praxis lassen sich sowohl zwischen den Betrieben als auch innerhalb eines Betriebs unterschiedliche Akzentuierungen einzelner Inhalte der Produktionssysteme feststellen (Heil et al. 2014):

- Die Supply-Chain-Perspektive zielt auf den Materialfluss des Produktionsprozesses: auftrags- und kundenorientierte Ausrichtung der Produktion mit transparenten, leicht steuerbaren Produktionsprozessen und mit klar strukturierten und vorab definierten Produktionsreihenfolgen. Hierbei wird vor allem auf die Optimierung der Kosten-Termintreue-Relation wertgelegt.

- Die Qualitätsmanagement-Perspektive fokussiert mittels transparenter und standardisierter Prozesse die Rückverfolgbarkeit von Fehlerquellen und die Eigenverantwortung der Produktionsbereiche für die Vermeidung von Fehlern (Null-Fehler-Prinzip). Standar- 
disierung und Eigenverantwortlichkeit können oftmals dabei in Widerspruch geraten. Zielgröße hierbei ist die Verbesserung des Verhältnisses von Qualität und Kosten.

- Die Industrial-Engineering-Perspektive umfasst die - in dieser Arbeit interessierende - Fokussierung auf die kontinuierliche Verbesserung der Produktions- und Arbeitsprozesse durch Reduzierung nicht wertschöpfender Tätigkeiten und Optimierung der Abläufe mittels Anwendung verschiedener Methoden (unter anderem 5SMethode, Verschwendungsvermeidung) sowie die konsequente Einbeziehung von Beschäftigten.

- Die arbeitspolitische Perspektive setzt auf die Nutzung des menschlichen Arbeitsvermögens durch die Schaffung von Handlungsspielraumen, Aufgabenerweiterung und Beteiligungsmöglichkeiten.

Die meisten Ganzheitlichen Produktionskonzepte (GPS) operieren mit diesen Perspektiven in jeweils unterschiedlicher Intensität. Vor allem ist die Akzentsetzung bei der Umsetzung von GPS aber in hohem Maße davon abhängig, welche Akteursgruppen für die Konzeption auf Unternehmensebene und für die Realisierung im Betrieb verantwortlich sind (Heil et al. 2014). Die unterschiedliche Akzentuierung einzelner Perspektiven durch verschiedene Akteursgruppen im Betrieb kann zu Irritationen und Widersprüchen führen. Die Spannbreite der Gestaltungsfelder ist also sehr weit; einige zentrale Aspekte wie Standardisierungen und Kontinuierlicher Verbesserungsprozess spielen heutzutage jedoch in allen GPS eine bedeutende Rolle.

\section{Doppelte Subjektivierung, Vermarktlichung und indirekte Steuerung}

Mit den seit den 1990er Jahren einsetzenden Reorganisations- und Restrukturierungsmaßnahmen veränderte sich das arbeitspolitische Koordinatensystem beträchtlich. In der Frage des veränderten Stellenwerts des Subjekts sowie der Organisation und Steuerung von Arbeit in einem arbeitsteiligen Produktionsprozess haben sich zentrale Entwicklungslinien herausgebildet, die den Wandel der Arbeit in Betrieben maßgeblich prägen. Die Auswirkungen der Entwicklungen, die im Folgenden näher als Phänomene der Subjektivierung, indirekten 
Steuerung, Informatisierung und Vermarktlichung thematisiert werden, treten nicht überall in gleichem Maße zutage, bedingen aber sich in hohem Maße gegenseitig und bilden wichtige Rahmenbedingungen für die Frage nach dem Umgang der Beschäftigten mit KVP.

Mit der oben geschilderten veränderten Perspektive auf die Nutzung menschlicher Arbeitskraft und die gewandelten Arbeitsstrukturen mit Rücknahme der direkten Kontrolle und Gewährung von größeren Handlungsspielräumen wird den Beschäftigten eine neue Rolle zu geschrieben. Subjektivierung der Arbeit realisiert sich durch das Arbeiten in selbstorganisierten Gruppen mit umfassenderen Tätigkeitsspektren sowie höheren Handlungs- und Entscheidungsspielräumen, der Gelegenheit zur Entwicklung von Optimierungsideen und Mitarbeit an der Realisierung dieser Optimierungen und ermöglicht, etwaige Ansprüche an inhaltlich-fachlich höherwertige und sinnstiftendere Arbeit zu verwirklichen (Baethge 1991; Lohr 2003; Nickel et al. 2008; Kleemann 2012; Lohr 2017b). Die Beschäftigten können ihre Subjektpotenziale einbringen und die gewandelten Arbeitsstrukturen erfordern ein höheres $\mathrm{Maß}$ an subjektiven Kompetenzen: Statt Fremdstrukturierung und -kontrolle sind Eigenstrukturierungsleistungen (Selbstregulierungen) sowie ein höheres $\mathrm{Maß}$ an Kooperations- und Kommunikationsfähigkeiten gefragt.

Direkte Kontrollformen und Detailsteuerung von Arbeitsprozessen werden seit den 1990er Jahren zunehmend von marktorientierten Steuerungs- und Koordinationsmechanismen abgelöst (Sauer/Döhl 1997; Moldaschl/Sauer 2000; Dörre 2001a). Verbunden mit der Dezentralisierung der Unternehmensstrukturen, also der Verlagerung von Entscheidungen und Kostenverantwortlichkeiten von übergreifenden Unternehmensbereichen auf einzelne Abteilungen (Kostenstellen), wurden Unternehmensziele und Produktionsprozesse immer stärker auf die Erreichung von marktorientierten Zielen ausgerichtet (Vermarktlichung) (Sauer 2010). Kundenorientierung - sowohl auf den Endkunden als auch auf den innerbetrieblichen „Kunden“ im nachgelagerten Produktionsprozess - und Kennzahlenorientierung bilden die wesentlichen Steuerungsmechanismen, die operative Funktionen bis hinunter auf die Arbeitsebene erfüllen. Die Kennzahlen „als tägliche Erinnerung an den Markt, die Entwicklung der Preise und der Lage des Unternehmens" (Sauer 2010: 555) werden zum Fixpunkt der Steuerung des Produktionsprozesses und fungieren als Rechtfertigung für betriebliche Rationalisierungsaktivitäten, wie beispielsweise Leistungsintensivierung, und als Handlungsaufforderung an die Beschäftigten, im Sinne des marktgebundenen Unternehmens zu handeln. Die indirekte Steue- 
rung operiert mittels Vorgaben von meist kennzahlenorientierten Zielen und der Bereitstellung von technischen, organisationalen und personellen Rahmenbedingungen. Sie nimmt die Beschäftigten in die Verantwortung, die Transformation des eigenen Arbeitsvermögens in Leistung durch Selbststeuerung und -regulierung sowie den Einsatz von fachlichen, kooperativen und kommunikativen Kompetenzen zu vollziehen (Sauer/Döhl 1997; Moldaschl/Sauer 2000; Tullius 2004; Sauer et al. 2005; Sauer 2010). ${ }^{24}$ Problematisch ist hierbei, dass durch Vorgabe von marktorientierten Kennzahlen die Leistungszielen tendenziell von den Bedingungen des Produktionsprozesses entkoppelt sind und die Zielerreichung die Beschäftigten vor widersprüchliche bis tendenziell unlösbare Herausforderungen stellen kann.

\section{Die Prognose eines neuen Typus des Produktionsbeschäftigten}

Mit dem Aufkommen partizipativer Managementstrategien und dem Wandel betrieblicher Rationalisierungsstrategien - unter anderem als partizipativarbeitskraftzentrierte Managementkonzepte (Dörre et al. 1993) oder arbeitsorientierte Rationalisierung (Moldaschl/Schultz-Wild 1994a) bezeichnet - entfalteten sich intensive Debatten darüber, inwiefern und unter welchen Bedingungen es sich dabei um einen substanziellen Wandel im Umgang mit menschlicher Arbeitskraft handelt. Die veränderten, subjektivierten Arbeitsstrukturen und Arbeitsanforderungen erfordern und fördern ein höheres Maß an Kompetenzen, als es unter tayloristischer Arbeitsgestaltung der Fall war. Arbeiten in selbstorganisierten Gruppen mit weiteren Tätigkeitsspektren sowie größeren Handlungs- und Entscheidungsspielräumen und der Gelegenheit zur Entwicklung von Optimierungsideen sowie Mitarbeit an der Realisierung dieser Optimierungen bedienen nicht nur etwaige Ansprüche an inhaltlich-fachlich höherwertige und sinnstiftende Arbeit, sondern verlangen statt Fremd- nun Eigenstrukturierungsleistungen (Selbstregulierung) und soziale Kompetenzen. Unter der Annahme, dass Beschäftigte die unternehmerischen Ziele, die

${ }^{24}$ In diesem Zusammenhang lässt sich die zunehmende Informatisierung ergänzen. Informations- und Kommunikationstechnologien durchdringen den Produktionsprozess, sodass auf der digitalen Steuerungsebene Prozesse zentral erfasst und gelenkt werden können. Mittels dieser Technologien werden die einzelnen Produktionsbereiche stärker vernetzt und im gesamten Wertschöpfungsprozess weitere Rationalisierungspotenziale zu ermitteln versucht (Boes/Pfeiffer 2005; Pfeiffer 2010; Sauer 2010). 
marktorientierten, indirekten Steuerungsmechanismen (Orientierung an Kennzahlen wie Qualität, Kosten und Zeit) und partizipativen Arbeitsstrukturen antizipieren, wurden zeitlich mit dem Aufkommen neuer „posttayloristischen“ Managementkonzepte diskutiert, inwiefern sich aus diesen als „doppelte Subjektivierung" bezeichneten neuen Arbeitszusammenhängen und den gesamtgesellschaftlichen Entwicklungstendenzen der Individualisierung sowie veränderten Ansprüchen an die Vereinbarkeit von Arbeit und Leben (Work-LifeBalance) neue Typen von Arbeitnehmern herausbilden. Skizziert wurden Leitbilder oder neue Orientierungsmuster von Arbeitnehmern, die sich in ihrem Handeln an für Unternehmer typischen Zielen und Handlungsweisen ausrichten und vom bislang typischen abhängigen Lohnarbeiter unterscheiden (Kuhn 1997; Kleemann/Voß 2010). So werde sich nach Kuhn (1997) aus dem „maschinenhaften“, „vom unternehmerischen Denken befreiten“ Arbeitnehmer ein unternehmerisch handelnder "Mitunternehmer" herausbilden. Voß, G./Pongratz (1998) prognostizierten, dass sich die dominante Arbeitnehmerorientierung eines fordistisch geprägten, detailgesteuerten, „verberuflichten Arbeitnehmers" zu der eines Arbeitskraftunternehmers entwickle, der die Funktionen der Kontrolle, der wirtschaftlichen Verwertbarkeit und Entwicklung der Arbeitskraft sowie der Optimierung des Handelns in die eigenen Hände nimmt (Pongratz/Voß 2003). Etwas schärfer formuliert Bröckling (2007) als „unternehmerisches Selbst" das neoliberale Leitbild eines rundum selbstzentrierten und -optimierten Individuums, das sich in seinem Handeln danach ausrichtet, möglichst erfolgreich am (Arbeits-)Markt zu sein. Ob und in welcher Weise diese neue Arbeitsstrukturen sich in veränderte Aneignungsformen von Arbeit niederschlagen, wird im Kontext der Beschäftigtenperspektive auf KVP näher betrachtet.

\subsection{KVP}

\subsubsection{Gestaltungsperspektive}

Während es zu Wirkungsweisen von posttayloristischer Arbeitsgestaltung der frühen Lean-Production-Phase eine breite Befundlage gibt, die vorwiegend aus Gruppenarbeitsstudien und Subjektivierungsforschung hervorgegangen ist, sind die wissenschaftlichen Forschungsarbeiten zu Gestaltung und Wirkungs- 
weisen von KVP auf die Beschäftigten jenseits konzeptioneller Managementberatungsliteratur sehr überschaubar.

Kontinuierliche Verbesserungsprozesse als beschäftigtengetragener Optimierungsprozess lassen sich in Industriebetrieben in unterschiedlichen konzeptionelle Ausrichtungen und Gestaltungsformen finden (Howaldt 1995; Howaldt/Kopp 1997; Teufel 2003; Kuhlmann et al. 2004; Kirner et al. 2006; Schwarz-Kocher et al. 2012).

Gängige KVP-Gestaltungsformen sind:

- feste, zumeist in ein Gruppenarbeitskonzept integrierte Arbeitsteams, deren zugewiesene Aufgabe darin besteht, dass die Beschäftigten in ihrer Gruppe selbstständig Verbesserungspotenziale am eigenen Arbeitsbereich anregen, diskutieren und - ggf. mit Hilfe von Vorgesetzten und indirekten Servicebereichen - selbst durchführen;

- methodisch angeleitete Workshops oder Qualitätszirkel: temporäre, interdisziplinäre Teams bestehend aus Produktionsbeschäftigten, Planungsexperten, Führungskräften und Moderatoren;

- Formen des Vorschlagswesens/Ideenmanagement (Kuhlmann et al. 2004; Schwarz-Kocher et al. 2012).

Der inhaltliche Fokus von KVP richtet sich auf „Verbesserung der Arbeitsabläufe, Beseitigung der Störeinflüsse, Verbesserung der Arbeitssicherheit, Anreicherung der Arbeitsinhalte und Verbesserung von Qualität, Kosten und Zeit" (Teufel 2003: 509). KVP geht meist mit der Einführung standardisierter Kaizen-Methoden zur Entdeckung von Verbesserungspotenzialen (zum Beispiel Verschwendungsschulungen) und Problemlöseprozessen (beispielsweise PDCA-Zyklus, DMAIC) und zur Arbeitsplatzgestaltung (zum Beispiel 5SMethoden) nach dem Vorbild des Toyota-Produktionskonzepts einher (Teufel 2003). ${ }^{25}$

25 Im Mittelpunkt des Kaizen-Optimierungsansatzes stehen die Vermeidung von Verschwendungen jeglicher Art und die Standardisierung von Abläufen - daher auch die von Womack, J.P. et al. (1991) gewählte Bezeichnung „lean production“. Als zu vermeidende Verschwendungen (in der Toyotasprache als „muda“ bezeich- 
In der betrieblichen Praxis lassen sich zwischen KVP-Konzepten und ihren gelebten Praktiken starke Divergenzen hinsichtlich des Grads der tatsächlichen Einbindung der Beschäftigten, der Priorisierung von Optimierungsinhalten und der organisatorischen Gestaltung erkennen (Gerst et al. 1995; Howaldt 1995; Howaldt/Kopp 1997; Schwarz-Kocher et al. 2012; Schwarz-Kocher, M. et al. 2016). Die Unterschiede reichen von aktiver Einbindung in Planungsprozesse über selbstinitiierte Optimierungen, welche dezidiert die Verbesserung der Arbeitsbedingungen inkludieren und von den Beschäftigten in Kooperation mit den indirekten Bereichen erarbeitet und umgesetzt werden, bis hin zu KVP-Gestaltungsformen, in denen Beschäftigte zwar formal beteiligt sind, aber eine starke Dominanz von Planungsexperten ausgeht. Letztere orientieren sich meist eng an betrieblichen Rationalisierungs- und Leistungskennzahlen, wie Kosten, Qualität und Liefertreue, und nutzen KVP - insbesondere in Form von Workshops -, um die vom Management vorgegebenen Standardisierungskonzepte zu realisieren (Gerst et al. 1995; Kuhlmann 2004; Kuhlmann et al. 2004).

In der deutschen Arbeitssoziologie konzentriert sich die Diskussion um KVP vorwiegend auf die neue Arbeitsorganisation der Gruppenarbeit (Gerst et al. 1994; Moldaschl/Schultz-Wild 1994b; Schumann et al. 1994b; Kocyba/Vormbusch 2000; Nordhause-Janz/Pekruhl 2000). Jenseits konzeptioneller Ausrichtungen einzelner KVP-Ansätze ist von Bedeutung, wie die Konzepte in die Praxis überführt werden und welche Ausrichtung die Realisierung erhält. So hat Dörre (2002) in der Analyse betrieblicher Praktiken von Beteiligungskonzepten zwischen zwei Wegen unterschieden: einerseits einer „High Road“ hoher Partizipation der Beschäftigten in kompromissgetragenen Kooperationsstrukturen und andererseits einer „Low Road“ stark eingeschränkter und selektiver Partizipation, die durch rigide, stark an kurzfristiger

net) gelten Überproduktion, Bestände, Transport, Wartezeit, Arbeits- und Herstellungsprozess, Bewegung und Fehler. PDCA („Plan - Do - Check - Action“) und DMAIC („Define - Measure - Analyse - Improve - Control“), eine Kernmethodik aus dem Managementsystem Six Sigma), sind Instrumente zur Planung und Kontrolle von definierten Problemlöseprozessen (Birkmayer et al. 2009). 5S bzw. 5A (im Deutschen) ist eine Methodik zur standardisierten Gestaltung von Arbeitsplätzen und Fertigungsbereichen durch Ordnung und Sauberkeit (Teufel 2003). 
Kostensenkung orientierter Arbeitspolitik geprägt ist. ${ }^{26}$ Auf Gruppenarbeit bezogen zeigten sich auch hier zwei Fluchtpunkte der Konzepte und insbesondere der realisierten Praxis. Gerst et al. (1995) ${ }^{27}$ systematisierten unterschiedliche Gruppenarbeitspraktiken in strukturinnovativ und strukturkonservativ. 28

Strukturinnovative bzw. selbstorganisierte Gruppenarbeit zeichnet sich durch ein hohes Maß an Selbstorganisation, Aufgabenerweiterung und reale Entscheidungs- und Handlungsspielräume aus. Um das zu gewährleisten, erhalten die Gruppen zum einen Ressourcen in Form von selbstgewählten Sprechern, Zeit für die Koordination der Gruppenaufgaben - Optimierungen zählen dezidiert dazu -, zum anderen erhalten die Gruppen Unterstützung durch Führungskräfte und fertigungsnahen, indirekten Bereiche ${ }^{29}$. Notwendig dafür ist der Abbau hierarchischer Strukturen, ein Rollenwandel der unteren Führungsebenen sowie die dezentrale Ausrichtung der Fachfunktionen (zum Beispiel Planung, Instandhaltung) und prozessnahe Koordinations- und Kommunikationsstrukturen. Strukturinnovative Gruppenarbeit orientiert sich

${ }^{26}$ Hierbei identifiziert (Dörre 2002) „sozialintegrative Beteiligung im Kleinfirmensetz“ sowie „selbstgesteuerte Partizipation in teilautonomen Arbeitsgruppen“ auf der High Road und „erzwungene Partizipation im desintegrierten Firmennetz“ und „gelenkte Partizipation in der gestrafften Firmenbürokratie“ auf der Low Road. Als dominante Form partizipativer Arbeitsgestaltung stellt Dörre die gelenkte Partizipation heraus, bei der die Beschäftigten weiterhin in eine bürokratische Betriebshierarchie und Kontrollsysteme eingebunden sind und nur über geringe Entscheidungsspielräume verfügen, ihre Aufgaben sind ebenfalls nur geringfügig erweitert. Die Beteiligung an Optimierungsaktivitäten sei hierbei auf kleine Maßnahmen begrenzt (Dörre 2001b; 2002).

27 Daran anknüpfend arbeiteten Kuhlmann et al. (2004) im Projekt „Konzepte innovativer Arbeitspolitik" mit diesem Analyserahmen weiter.

${ }^{28}$ Mit Bezug auf die ähnlichen Kriterien der Aufgabenerweiterung, Funktionsintegration sowie Handlungs- und Entscheidungsspielräume werden empirische Erscheinungsformen der Gruppenarbeit auch in qualifizierte Gruppenarbeit (Lutz 1988), teilautonome Gruppenarbeit oder selbstorganisierte Gruppenarbeit (Gerst 1998b) in Abgrenzung zu strukturkonservativen bzw. taylorisierten Gruppenarbeit differenziert. Für einen Überblick über unterschiedliche Formen von Gruppenarbeit in Unternehmen siehe (Antoni 1994).

${ }^{29}$ Unter indirekte Bereiche werden allgemein die Funktionsbereiche bezeichnet, die nicht direkt zur Produktion zählen, wie produktionsnahe Bereiche der Instandhaltung, Logistik und Planung (Produktions- und Anlagenplanung sowie Arbeitsvorbereitung) sowie produktionsfernere Abteilungen wie u.a. Personalwesen und Controlling. 
stärker an einem Konsens bzw. an Aushandlungsprozessen zwischen Beschäftigten- und Betriebsinteressen. Im Gegensatz dazu bilden strukturkonservative bzw. taylorisierte Formen eine Weiterführung rigider Arbeitsstrukturen, die durch standardisierte Aufgabenzuschnitte, geringe Handlungs- und Entscheidungsspielräume, Trennung zwischen ausführenden und dispositiven Tätigkeiten und die Beibehaltung hierarchischer und funktionaler Spezialfunktionen bestimmt sind.

In strukturkonservativer Gruppenarbeit erhöht sich durch Rotation und sehr begrenzte Selbstorganisation zwar ihre Einsatzflexibilität, aber „die Arbeitsrealität der Beschäftigten [ist] wie bisher durch genaue, verhaltensorientierte Vorgaben, geringe inhaltliche Anforderungen und in erster Linie disziplinierende Rahmenbedingungen geprägt" (Gerst et al. 1995: 43). Die Beteiligung an Optimierungsprozessen wird bei strukturkonservativer Gruppenarbeit vom Management nicht prinzipiell ausgeschlossen, doch werden meist nur einzelne Beschäftigte in Spezialfunktionen oder vom Betrieb bestimmte Teamleader in Optimierungsprozesse und Workshops einbezogen, während die breite Masse der Beschäftigten kaum eingebunden wird (Kuhlmann et al. 2004). Diese Differenzierung in strukturkonservativ und -innovativ (bzw. in geringen und hohen Realisierungsgrad der Arbeitsorganisation) ist insofern relevant, als Beschäftigte in strukturinnovativer Gruppenarbeit bzw. mit hohem Realisationsniveau von Aufgabenzuschnitt, Selbstorganisation und Funktionsintegration eine höhere Bereitschaft zur Beteiligung an betrieblicher Optimierung zeigen als Beschäftigte in Gruppen mit niedrigem Realisierungsniveau (Kuhlmann et al. 2004). Als Gründe hierfür verweisen Beschäftigte darauf,

„dass man die Möglichkeit hat, gute Ideen auch umzusetzen [...], dass die eigene Tätigkeit durch KVP-Aktivitäten interessanter und qualifizierter wird und dass man bereit ist[]] sich zu engagieren, weil man größeren Einfluss auf die Gestaltung des eigenen Arbeitsplatzes nebmen kann" (Kublmann et al. 2004: 366).

Darüber hinaus spielen in diese Bewertung die Wirkungen strukturinnovativer bzw. selbstorganisierter Gruppenarbeit hinein: Durch breitere Aufgaben- und Funktionszuschnitte wird die Arbeit von den Beschäftigten insgesamt als interessanter wahrgenommen, durch Selbstorganisation und Zusammenarbeit innerhalb der Gruppe sowie durch Unterstützung der Führungskraft und eine engere Kooperation mit den Planungsbereichen verbessern sich die Bewältigungsmöglichkeiten der Leistungsvorgaben und erhöhen sich Einflussmöglichkeiten auf betriebliche Entscheidungen und Gestaltungsprozesse (Kuhlmann et al. 2004). 
Mit der Retaylorisierungstendenz der Arbeitsstrukturen stand auch die Zukunft der innovativen Gruppenarbeit infrage. Angesichts des hohen betrieblichen Aufwands, des starken Kostendrucks der Betriebe und des geringen Nutzens würden sich eher Formen der standardisierten oder tayloristischen Gruppenarbeit als „eine Synthese aus der teilautonomen Gruppenarbeit und der repetitiven Teilarbeit" durchsetzen . Die Beteiligung der Beschäftigten an Optimierungsprozessen ist dabei durch die Führungskräfte gelenkt und auf Unternehmensziele und Produktivitätskennzahlen ausgerichtet. Hierbei geht es vor allem darum, die Mitarbeiterpotenziale zu kanalisieren, also eher einseitig zu nutzen; Interessenausgleich und Kooperation, die in strukturinnovativen Gruppenarbeitskonzepten von hoher Bedeutung sind, werden hingegen eher vernachlässigt (Gerst 1999b).

Obgleich bislang keine quantifizierenden Aussagen über die Verbreitung von Gruppenarbeit getroffen werden konnten, ${ }^{30}$ fanden sich in unterschiedlichen Unternehmen, aber auch innerhalb eines Betriebs sowohl strukturkonservative als auch -innovative sowie standardisierte Gruppenarbeitsformen nebeneinander (Minssen 1999). ${ }^{31}$ In der praktischen Anwendung von KVP zeigen sich ebenfalls deutliche Unterschiede, die von gescheiterten oder stagnierenden Einführungsprozessen bzw. stark expertengetriebenen Formen der Prozessoptimierungen, die meist mit einer Rückkehr zu konventioneller Arbeitsgestaltung einhergingen, bis hin zu beschäftigtengetragenen Prozessoptimierungen reichen (Heil/Kuhlmann 2013; Kötter et al. 2016; Seibold et al. 2016).

In der betrieblichen Praxis weisen Gestaltungsformen von KVP starke Unterschiede im Grad der tatsächlichen Einbindung der Beschäftigten, des inhaltlichen Fokus auf Optimierungspotenziale und der Art und Weise der organisatorischen Gestaltung auf (Gerst et al. 1995; Howaldt 1995; Howaldt/Kopp 1997). Die Unterschiede reichen von aktiver Einbindung in Planungsprozesse

${ }^{30}$ Die wissenschaftlichen Erkenntnisse zu partizipativen Managementkonzepten basieren vorwiegend auf einzelnen Fallstudien.

${ }^{31}$ Die arbeitspolitische Debatte darüber, welche Formen der Arbeitsorganisation und insbesondere der Gruppenarbeit sich durchsetzen, ob es also zu einer breiten Einbindung in Optimierungs- und Rationalisierungsprozesse und so zu einem grundlegenden Strukturwandel in den Betrieben kommen wird, wurde Mitte der 1990er Jahre in arbeitssoziologischen und gewerkschaftlichen Kreisen sehr intensiv geführt (Gerst 1999a; Springer 1999b; Gerst 2000). 
und selbstinitiierten Optimierungen, in die dezidiert die Verbesserung der Arbeitsbedingungen eingeschlossen ist und die die Beschäftigten in Kooperation mit den indirekten Bereichen erarbeiten und umsetzen, bis hin zu Gestaltungsformen, in denen Beschäftigte zwar formal beteiligt sind, aber eine starke Dominanz von Planungsexperten ausgeht und die eng an betrieblichen Rationalisierungs- und Leistungskennzahlen, wie Kosten, Qualität und Liefertreue, orientiert sind. (Gerst et al. 1995; Kuhlmann 2004; Kuhlmann et al. 2004; Schwarz-Kocher et al. 2012). Aufgrund der Varianzen von praktizierten KVPAnsätzen ist es kaum verwunderlich, dass KVP in der Wahrnehmung der Betriebsräte mit unterschiedlichen betrieblichen Rationalisierungsstrategien verbunden wird: KVP als jährliches Einsparungsprogramm, als Neuauflage des Ideenmanagements oder als beteiligungsorientierter Prozess, in dem auch den Beschäftigten die Möglichkeit gegeben wird, ihre Arbeitsabläufe und Produktionsprozesse mitzugestalten und Verbesserungen ihrer Arbeitsbedingungen zu bewirken (Howaldt/Kopp 1997; Schwarz-Kocher et al. 2012).

\subsubsection{Beschäftigtenperspektive}

Der Frage, wie die Beschäftigten mit der neuen Gestaltung von Arbeit und der Verhaltenserwartung des Betriebs, sich an KVP zu beteiligen, umgehen, steht eine relativ überschaubare Befundlage gegenüber. ${ }^{32}$ Quantitative Untersuchungen von Produktionsbeschäftigten des Projektes „Konzepte Innovativer Arbeitspolitik“ (Kuhlmann et al. 2004) ergaben, dass Beschäftigte grundsätzlich eine hohe Bereitschaft zeigen, sich an KVP zu beteiligen. Dies resultiere aus der Erwartung einer interessanteren Arbeit, erhofften höheren Einflussmöglichkeiten auf die Gestaltung der Arbeit sowie der Aussicht, einen Beitrag zur Verbesserung der Wirtschaftlichkeit zu leisten. Kratzer et al. (2015) setzen die hier angedeutete Motivation zur Mitwirkung an KVP noch viel deutlicher in einen Zusammenhang mit dem Beteiligungsanspruch der Beschäftigten, der sich zum einen aus dem eigenen Rollenverständnis als Experten des eigenen

32 Die meisten subjektorientierten Befunde zu KVP wurden mittels Interviews und Fragebogenbefragungen von Betriebsräten (Howaldt 1994; Howaldt/Kopp 1997) sowie Expertengesprächen mit Vertretern des Managements (Kirner et al. 2006) ermittelt. In dem Sample der Untersuchung von Kocyba/Vormbusch (2000) waren einfache Produktionsbeschäftigte deutlich unterrepräsentiert und die breitangelegte Befragung konnte nur eingeschränkt durchgeführt werden. 
Arbeitsbereichs speise. Die Beschäftigten orientieren sich dabei an den stofflichen Besonderheiten des Produktionsprozesses (technisch-funktionale Rationalität). Eine Anhörung der Produktionsbeschäftigten bzw. ihr Einfluss auf die Gestaltung des Arbeitsbereichs sei aus ihrer Sicht wesentlich für das Funktionieren des Produktionsprozesses. Zum anderen begünstigen die Mitgestaltungsmöglichen die Bedingungen der Leistungserbringung der Beschäftigten. Die Erreichung des selbstgesteckten oder auch auferlegten Arbeitsziels beschreibt Nies (2015) als wichtige Arbeitsorientierung der Beschäftigten, mit der diese - ähnlich wie es Kratzer et al. (2015) formulieren - ihren Anspruch auf Beteiligung legitimieren.

Dem gegenüber stehen einerseits bisherige Erfahrungen der Beschäftigten mit betrieblicher Rationalisierung und die Angst vor Leistungsintensivierung und Arbeitsplatzabbau (Kuhlmann et al. 2004). Diese Bedenken bzw. realen Erfahrungen seien mit dem Lohnarbeiterbewusstsein verbunden, dass Beschäftigte letztlich die Folgen von Rationalisierungsmaßnahmen tragen müssen, und führten dazu, dass Beschäftigte die Haltung des arbeitspolitischen Konservativismus einnehmen. Andererseits erwachsen Hinderungsgründe daraus, dass Beschäftigte im Arbeitsalltag zu wenig ernstgenommen und ihre Optimierungsideen nicht oder zu langsam umgesetzt werden sowie daraus, dass die Beschäftigten zu wenig Informationen über Kosten und Planungsaktivitäten des Betriebs haben (Kuhlmann et al. 2004; Schwarz-Kocher et al. 2012; Kratzer et al. 2015). ${ }^{33}$

Tatsächlich lassen sich pauschale Aussagen über Reaktionen oder Aneignungsformen von KVP nicht ohne die Berücksichtigung der betrieblichen Bedingungen und KVP-Praktiken treffen. Wie die Beschäftigten mit dem betrieblichen Beteiligungsangebot KVP umgehen, hängt in hohem Maße davon an, wie diese Maßnahmen in ihrem betrieblichen Arbeitsalltag umgesetzt werden. Denn die realen Erfahrungen mit KVP-Abläufen und den Veränderungen der Arbeit scheinen eine bedeutende Rolle zu spielen. Unter Bedingungen von strukturinnovativer Gruppenarbeit und prozessnaher Kooperationsund Kommunikationsstrukturen berichten Beschäftigte, ein höheres Maß an ernstgenommener Zusammenarbeit, Umsetzung ihrer Ideen und Einfluss auf die Gestaltung ihres - zudem interessanteren - Arbeitsplatzes erfahren zu haben. Diese positiven Erfahrungen eines überwiegenden Teils der Beschäftig-

33 So auch Schwarz-Kocher et al. (2012). 
ten in strukturinnovativer Arbeitsumgebung sind aus Sicht von Kuhlmann et al. (2004) ein bedeutender Unterschied gegenüber den Beschäftigten in konservativen Strukturen. Durch das Ernst-genommen-Werden und die kooperativen Beziehung zu anderen betrieblichen Gruppen fühlten Beschäftigte sich in ihrer sozialen Stellung im Betrieb aufgewertet. Unter diesen Bedingungen betrachten sehr aktive Beschäftigte Optimierungsaufgaben als selbstverständlichen Teil des eigenen Aufgabenverständnisses (Gerst 2004; Kratzer et al. 2015), welches sie unter Umständen auch gegen die Interessen von Kollegen durchsetzen (Kratzer et al. 2015). Häufig kritisierten Beschäftigte eine PseudoBeteiligung, bei der sie zwar rhetorisch aufgefordert werden, an Optimierungen mitzuwirken, die betrieblichen Umsetzung aber so gestaltet ist, dass eine echte Beteiligung nicht möglich ist bzw. als unerwünscht wahrgenommen wird. Von den anderen betrieblichen Akteuren zu spät in betriebliche Entscheidungen einbezogen und wenig oder nicht bei Optimierungsideen unterstützt, erfahren Beschäftigte, dass sie nicht ernst genommen werden.

Nicht nur die Gestaltung von KVP an sich, sondern auch die kumulierten Erfahrungen mit dem alltäglichen Arbeitsprozess spielen für die Beschäftigten eine wichtige Rolle. So treffen dort oftmals Anforderungen unterschiedlicher Optimierungsmaßnahmen, -methoden und -prinzipien gleichzeitig aufeinander. Kaizen, Andon, Standardisierung, Visualisierung, Flexibilisierung etc. werden im Betrieb von unterschiedlichen betrieblichen Gruppen (Führungskräfte, Arbeitsvorbereitung, Qualitätssicherung etc.) vorangetrieben und können zu divergierenden Handlungs- und Anforderungsbedingungen führen. Diese gegensätzlichen Ziele und Leistungsanforderungen entstehen durch konträre Priorisierungen: hohe Flexibilität sowie stabile Produktionsprozesse und Qualität einerseits, Leistungsintensivierung und Zeitdruck andererseits sowie Einforderung aktiver Beteiligung bei gleichzeitiger Beschränkung der Beteiligungs-, Mitsprache- und Entscheidungsmöglichkeiten. Durch ein solches System von Optimierungsmethoden würden fortwährend Krisensituationen heraufbeschworen, während die Beschäftigten eher nach Stabilisierung der Arbeits- und Produktionsabläufe strebten. Diese Diskrepanzen können zu Irritationen, Widerstand, Überforderungen und einem Verharren im „alten“, tayloristisch geprägten Rationalisierungsverständnis und demnach zu fehlender Bereitschaft, sich an betrieblicher Optimierung zu beteiligen, führen (Dörre et al. 1993; Pardi 2007).

Die neue Arbeits- und Anforderungssituation führe insgesamt nicht zu einer generellen Minderung der Skepsis gegenüber den Folgen der betrieblichen 
Rationalisierungsmaßnahmen - insbesondere dann nicht, wenn der Arbeitsalltag von Konflikten über Vorgabezeiten und Leistungserfüllung geprägt ist. Im persönlichen Austarieren zwischen dem eigenen Anspruch an Beteiligung und dem Misstrauen gegenüber dem Betrieb selektieren Beschäftigte bewusst zwischen Optimierungspotenzialen, die ihre Arbeitssituation verbessern, und Rationalisierungspotenzialen, die zu Leistungsintensivierung und Arbeitsplatzverlust führen (Gerst 2004; Kuhlmann et al. 2004; Frerichs 2014).

Dies gilt auch und insbesondere für vom Betrieb initiierte KVPWorkshops, bei denen unter Beteiligung von Produktionsbeschäftigten, KVPModeratoren, Betriebsrat und oftmals auch Führungskräften und Arbeitsvorbereitung alle Arbeits- und Prozessschritte eines vorabdefinierten Produktionsabschnitts systematisch auf die Reduzierung von Verschwendung und Optimierungspotenzialen überprüft werden. Oftmals sind KVP-Workshops, wie in der Fallstudie von Frerichs (2014) zu Machtkonfigurationen innerhalb von KVP-Workshops verdeutlicht, mit klaren Kosteneinsparungszielen (teils auf genaue Einsparungsquoten festgesetzt) verbunden, die in Frerichs Betriebsfall aber vordergründig durch das Narrativ der ,gemeinsamen Verbesserungsaktivitäten“ verschleiert werden. Obwohl die Beschäftigten dieses „Spiel“ schnell durchschauen und letztlich die Erfahrung machen, dass das Workshopergebnis auch mit ihrem Zutun überwiegend zugunsten des Betriebs ausfällt, begründen die Beschäftigten ihre Teilnahme am KVP-Workshop damit, die Veränderungen, Entscheidungen und Maßnahmen mitzusteuern und den angebotenen Handlungsspielraum nicht gänzlich ungenutzt zu verschenken. Die am Workshop teilnehmenden Beschäftigten (meist Teamsprecher und Vertrauensleute) stehen aufgrund der Veränderungsmaßnahmen oftmals stark in der Kritik und werden als Erfüllungsgehilfe des Betriebs verortet, wenn es ihnen nicht gelingt, ihren Kollegen die Maßnahmen zu vermitteln. Im Verlauf dieses Fallbeispiels machten die Beschäftigten häufig die Erfahrung, dass der Betrieb nicht nur die Spielregeln der Beteiligung an Optimierungen festlegt, sondern diese im Bedarfsfall auch selbst bricht, wenn im Workshopverlauf nicht die gewünschten Ergebnisse erarbeitet werden. Widersprüche und Eskalationen (wie der Abbruch des Workshops einiger Beschäftigter) werden teils übergangen; außerdem werden für Beschäftigte günstige Optimierungspotenziale entgegen den Absprachen im Workshop nicht oder nur sehr langsam umgesetzt.

Als einen wichtigen Mechanismus arbeitet Frerichs in ihrer Figurationsanalyse von KVP-Workshops die Akteurskonstellationen heraus. Dabei fokussiert 
sie auf die Rolle und Interessen der KVP-Moderatoren. Neben den Interessen des Betriebs und der Beschäftigten verfolgten, so Frerichs, KVP-Moderatoren ihre eigenen Interessen. Funktion, Leistungsziel und letztlich Daseinsberechtigung der Moderatoren ist unmittelbar die erfolgreiche Durchführung der Workshops mit Erreichen der Einsparungsziele, die am Ende des Workshops betriebsöffentlich vor dem Abteilungsleiter präsentiert werden. Langfristig stellt in diesem Betrieb die Rolle des KVP-Moderators im günstigen Fall eine Aufstiegsetappe dar. Aufgrund des hohen persönlichen Interesses der KVPModeratoren, diesen Workshop zum „Erfolg“ zu führen, treten diese einerseits stark für die Vermittlung von Kompromissen zwischen Beschäftigten und Betrieb ein, andererseits drehen sie die Workshopergebnisse in den Präsentationen so, dass sie sie letztlich als eigenen Erfolg darstellen können.

\subsubsection{Gestaltungsanforderungen}

Die vorangegangenen Ausführungen verdeutlichen, dass die Konzeption und praktische Gestaltung von KVP mit großen Herausforderungen verbunden ist. Obgleich mit den einzelnen KVP-Formen im Detail unterschiedliche Gestaltungsanforderungen verbunden sind, identifizierten Kuhlmann et al. (2004: 371) zentrale Grundlinien, die eine langfristige Beteiligung der Beschäftigten an beschäftigtengetragenen Prozessoptimierungen begünstigen:

- Absicherung als grundlegende Prämisse für die Beteiligung der Beschäftigten, etwa in Form von KVP-Betriebsvereinbarungen: kein Arbeitsplatzabbau durch KVP, Sicherung von Qualifikation und Entgelt; 34

- Schutz vor Arbeitsverschlechterung: Optimierung von Abläufen statt Leistungs- und Belastungsintensivierung;

- Doppelte Zielsetzung: Kopplung von Verbesserung der Wirtschaftlichkeit an Verbesserung der Qualität der Arbeit;

34 Siehe hierzu auch Schwarz-Kocher et al. (2012). Das IGM-Institut hat dezidierte Handlungs- und Gestaltungshilfen für Betriebsräte erstellt, die unter anderem auch eine zyklische Kontrolle der Ergebnisse von KVP und ihrer Wirkungen auf die Arbeitsqualität der Beschäftigten vorsehen. 
- Reguläre und definierte Gruppenaufgabe mit festen zeitlichen Ressourcen;

- Regulärer und frühzeitiger Einbezug der Beschäftigten in Planungsprozesse: arbeitsprozessnahe Planung und Kooperation;

- Kohärenz der einzelnen arbeits- und betriebsorganisatorischen Gestaltungselemente;

- Honorierung.

Diese Befunde verweisen auf beteiligungshinderliche und -förderliche Bedingungen der Gestaltung von KVP und betonen, dass für eine ernsthafte Beteiligung der Beschäftigten in einem Kontinuierlichen Verbesserungsprozess nicht allein die organisatorische Gestaltung einzelner Beteiligungsinstrumente (Gruppenarbeit, Workshops, Ideenmanagement) relevant ist, sondern es vor allem darum geht, wie sich die einzelnen arbeitspolitischen Gestaltungselemente - Arbeitsorganisation, Hierarchie, Rollen- und Funktionszuschnitt von Führungskräften und betrieblichen Planungsexperten, Leistungspolitik sowie Kontroll- und Steuerungsformen - zu einem arbeitspolitischen Gesamtkonzept verbinden (Appelbaum et al. 2000; Kuhlmann 2004; Lazonick 2006). Beteiligungsförderlich wirken beschäftigtengetragene Prozessoptimierungen vor allem dann, wenn sie in strukturinnovative Arbeitsgestaltung mit prozessnahen Kooperations- und Kommunikationsstrukturen eingebettet sind und den Beschäftigten eine reale Mitgestaltungsmöglichkeit ermöglichen. Als beteiligungshinderlich wird überwiegend die konkrete oder potenzielle Bedrohung durch Rationalisierungsfolgen angesehen. Bislang wurde allerdings vernachlässigt, wie sich Beschäftigte konkret im Spannungsfeld von Mitgestaltung und Bedrohung subjektiv mit der Anforderung des Betriebs auseinandersetzen, sich an KVP zu beteiligen, wie sie sich in ihrem Arbeitsalltag die Beteiligungs- und Mitsprachemöglichkeiten aneignen und unter welchen Bedingungen dieses stattfindet.

KVP erfordert Kooperation innerhalb und zwischen den einzelnen Arbeitsteams, mit Führungskräften und den betrieblichen Rationalisierungsexperten. Die konkrete Ausgestaltung der Kooperationsbeziehungen ist Produkt von Kompromissen und abhängig vom Status, der Position, des Einflusses und der Rollenverständnisse der einzelnen Akteure. Aufgrund der strukturell unausgewogenen Machtverteilung, der unterschiedlichen, teils hierarchisch 
geprägten Sozialbeziehungen und Interessengegensätze im Betrieb können sich diese Aushandlungsprozesse schwierig gestalten und erfordern ein Mindest$\mathrm{maß}$ an geteilten Erfahrungen, Methodenwissen, Vertrauen und Respekt (Pardi 2007). Die Förderung von beschäftigtengetragenen Prozessoptimierungen bedarf somit eines „kleinsten gemeinsamen Nenners“ an geteiltem Wissen über den Produktionsprozess und Optimierungsmethoden zwischen den Produktionsbeschäftigten und den anderen betrieblichen Akteursgruppen (Appelbaum et al. 2000; Dörre 2002; Pardi 2007). 


\section{Aneignung von Arbeit}

Für die Beantwortung der Frage, wie Beschäftigte mit den vom Betrieb praktizierten Verwertungsstrategien umgehen und wie sie sich letztlich mit ihrem Subjektpotenzial in einen beschäftigtengetragenen Optimierungsprozess einbringen, ist ein genauerer Blick hilfreich darauf, was Aneignung ist und welche Dimensionen und Wirkmechanismen im Prozess der Aneignung eine Rolle spielen.

Aneignung - „sich etwas zu eigen machen“ - bezieht sich in der arbeitswissenschaftlichen Literatur zum einen in einem materiellen Sinne auf die Aneignung von Dingen und Gegenständen, zum anderen auf die Aneignung von Abstraktem, wie Ideen, Wissen und Fähigkeiten ${ }^{35}$ (Frey 2009; Poppitz

35 Der Begriff der Aneignung ist vor allem durch einen eher unterdefinierten Alltagsgebrauch bestimmt. Vor allem in der Rechtsphilosophie gibt es eine lange Tradition, die sich insbesondere mit Aneignung als Verinnerlichung und Übernahme geistigen 
2009). Im Folgenden werden einige ausgewählte Ansätze zu Aneignung vorgestellt, die später die theoretisch-konzeptionelle Grundlage für die Analyse der Aneignungsform von KVP bilden.

\subsection{Aneignung als bewusstes oder unbewusstes, sinnliches Einlassen auf die Arbeitssituation}

In seinen Frühwerken beschreibt Marx (1844) Aneignung als einen identitätsstiftenden Prozess des Menschen durch die sinnliche Auseinandersetzung mit der Wirklichkeit, welcher der Entfremdung des Menschen entgegensteht:

„Der Mensch eignet sich sein allseitiges Wesen auf eine allseitige Art an, also als ein totaler Mensch. Jedes seiner menschlichen Verbältnisse zur Welt, Sehn, Hören, Riechen, Schmecken, Füblen, Denken, Anschauen, Empfinden, Wollen, Tätigsein, Lieben, kurz, alle Organe seiner Individualität, wie die Organe, welche unmittelbar in ihrer Form als gemeinschaftliche Organe sind, sind ihn ihrem gegenständlichen Verbalten oder in ibrem Verhalten zum Gegenstand die. Aneignung desselben. Die Aneignung der menschlichen Wirklichkeit, ibr Verbalten zum Gegenstand ist die Betätigung der menschlichen Wirklichkeit [...]." Marx 1844: 313)

Hierin drücken sich zwei zentrale Dimensionen von Aneignung aus: die sinnliche Wahrnehmung der Umwelt und das zweckgerichtete Handeln des Individuums. Mittels Aneignung bringt der Arbeitende seine Fähigkeiten in den Arbeitsprozess ein. Dabei werden nicht nur Gebrauchswerte hergestellt, sondern dieser Vorgang ist auch Teil der Subjektwerdung bzw. der Identitätsstiftung des Arbeitenden (Frey 2009). Durch das kapitalistische Verwertungskonzept des arbeitsteiligen Produktionsprozesses kann der Mensch jedoch nie sein vollständiges Arbeitsvermögen einbringen. Der Mensch werde durch die Enteignung von Produktionsmitteln, Tätigkeitsfeldern und Arbeitsergebnissen in der Aneignung seiner Arbeit behindert (Marx 1844: 360). Infolgedessen bezögen sich die Beschäftigten weniger auf den Gebrauchswert ihrer Arbeit, sondern auf den Tauschwert (Lohn) ihrer Arbeit, so dass besonders in restriktiven, stark arbeitsteiligen Arbeitssystemen Beschäftigte sich weniger auf arbeitsin-

Eigentums und später mit Fragen der Legitimität von Besitz im Sachenrecht befasst (Poppitz 2009). 
haltliche Interessen, sondern mehr auf instrumentelle Interessen beziehen (Goldthorpe et al. 1968).

Obgleich Aneignung und Entfremdung Schlüsselkategorien in Marx’ Arbeiten sind, weist Marx kein geschlossenes Aneignungskonzept aus. Fragen nach konkreten Formen der Aneignung der Arbeit, nach wichtigen Bezügen für die subjektive Auseinandersetzung mit der Arbeit oder der Rolle von überindividuellen, sozialen Bezügen - jenseits von Klassenbezügen von Arbeitern , die für die Auseinandersetzung der Subjekte mit der Wirklichkeit bzw. mit dem Arbeits-, Produktions- und Verwertungsprozess von Bedeutung sind, bleiben unbeantwortet.

Popitz et al. (1957b) befassen sich in ihrer Studie „Technik und Industriearbeit" explorativ mit der Herstellung der menschlichen Leistung und dem Arbeitshandeln, welches nicht allein als „passive Reaktion auf eine unveränderliche Zwangsreaktion gedeutet werden“ (Popitz et al. 1957b) kann. Dabei betrachten sie Aneignung unter den stofflichen Bedingungen ${ }^{36}$ des Produktionsprozesses und der Kooperationserfordernisse der Arbeitsteilung. Diese prägten zwar maßgeblich die Leistungsanforderungen („,Mit diesen Bedingungen müssen sie irgendwie fertig werden“, S. 175), das menschliche Handeln, welches der Erfüllung der Anforderungen dient, gehe aber nicht allein darin auf.

„[... so so ist er doch nicht nur halb, sondern gan₹, als Person durch einen Leistungsanspruch herausgefordert. Er kann sich vielleicht bewußt, wenn dies der Leistungsanspruch gestattet, teilwveise wieder, zurückziehen, er kann träumen und Pläne machen. Aber was er tut, bleibt eine menscbliche Leistung, die als Handlung eines Menschen und nicht potenziell als maschinelles Funktionieren zu verstehen ist. [...] Der Leistungsanspruch fordert ibn [den Arbeiter, B.S.] zu einer menscblichen Leistung heraus: das heißst vor allem, daß er geanungen ist, umfassender zu antworten, als er gefragt worden ist. [...] Die berausgeforderte Intelligen ₹, kann nicht nur [hervorgehoben im Original] auf die spezielle Arbeitsaufgabe antworten. Sie muß, um die sperielle Arbeitsaufgabe leisten zu können, jeweils umfassender sein, - der Horizont der Erfabrungen und der Denkfäbigkeit muß weiter sein als die jeweilige intelligente Antwort." (Popitz, et al. 1957b: 176).

Popitz et al. (1957b) betonen, dass die Beschäftigten sich mit den stofflichen Bedingungen, also mit dem konkreten Wesen (der technischen Funktionalität, dem Verarbeitungs- und Herstellungsprozess) und den Anforderungen des Arbeitsprozesses sowie der Organisation von Arbeit, auseinandersetzen müs-

36 Popitz und Kollegen verwenden hierbei aber noch nicht den Begriff Stofflichkeit, sondern beziehen dies auf die von Maschinen und Kooperationsbedingungen geprägten Arbeitssituationen. 
sen. Um Leistung im Sinne der Verausgabung ihrer Arbeitskraft zu erbringen, seien die Beschäftigten gefordert, ihre Fähigkeiten und Fertigkeiten einzusetzen und sich so die Arbeit und Arbeitsbedingungen anzueignen. Im Verständnis von Popitz et al. (1957b) lässt sich erkennen, dass Aneignung sich nicht nur auf eine bestimmte - vom Betrieb gewünschte - Form der Aneignung begrenzen lässt, sondern dass grundsätzlich unterschiedliche Aneignungsformen möglich sind und aufgrund der kognitiven Auseinandersetzung mit den stofflichen Bedingungen des Arbeitsprozesses gleichsam ein Überschuss an Subjektivität vorhanden ist, der sich nicht allein auf die Erfüllung von Leistungsvorgaben oder betrieblich nützliche Verhaltenserwartungen begrenzen lässt.
„Der Begriff der aktiven Anpassung verweist auf ein Strukturmoment der Arbeitssitua- tion, ja der praktischen Situation überbaupt. Unter Arbeitssituation verstehen wir die Bedingungen eines Arbeitsvollzugs und den Volliug selbst, insoweit und in der Weise, wie sie dem Bewußtsein gegeben sind. In dem Ausdruck, aktive Anpassung' liegt nun allerdings mehr als diese Grundstruktur jeder praktischen Situation. Zwar verlangt jede Situation eine Einstellung des Subjekts, jede veränderte Situation eine Veränderung der Einstellung, d.h. ein Sich-Einstellen, - wenn man will, also auch ein Sich-Anpassen. Aber dieses Sich-Einstellen braucht keine Aktivität zu enthalten; es kann ein Sich- Überlassen, ein unmerkliches Hineingleiten in ein Verbalten sein, in dem sich das Sub- jekt dann als ,eingestellt' vorfindet. Sein Beitrag zu den Gegebenheiten kann unartiku- liert oder entfremdet sein. Anpassung heißt dann: es hat sich vereinnabmen lassen. Ak- tive Anpassung wird dagegen ausdrücklich und wiederbolt vollzogen und ist als etwas Eigenständiges - ₹.B. als Durchbalten gegen etwas - dem Verbalten mitgegeben. Diese Eigenständigkeit drückt sich aber auch aus in einer inhaltlichen Andersartigkeit, die gleichwobl die Angepaßtheit nicht aufhebt." (Popitz, et al. 1957b: 106).

Hierbei wird deutlich, dass Aneignung bzw. Anpassung sich innerhalb eines weiten Spektrums von bewusst-strategischen bis zu unbewussten, unartikulierten Handlungen vollziehen können. In welchem direkten Zusammenhang Aneignung mit für Arbeiter relevanten Handlungsorientierungen steht, versuchen in ihrer Parallelstudie „Das Gesellschaftsbild der Arbeiter“ zu erkunden. Sie zeichneten nach, dass spezifische politische und gesellschaftliche Vorstellungen (Gesellschaftsbilder), ${ }^{37}$ welche die Arbeiter als Deutungen und Orientierung in die Arbeit herantragen, beeinflussen, wie sie sich mit den Anforderungen des Arbeitsprozesses auseinandersetzen. ${ }^{38}$ Ein gemeinsames Bestim-

37 beziehen ihre Typologie der Gesellschaftsbilder zwar vorwiegend auf die Deutungen gesamtgesellschaftlicher Verhältnisse, in ihren Ausführungen spiegeln sich allerdings deutliche Bezüge auf die eigene Arbeit der Beschäftigten wider.

38 Die Darstellung der sechs Gesellschaftsbilder verdeutlicht, dass spezifische Vorstellungen das Denken und Handeln der Arbeiter prägen. In ihrer Untersuchung der 
mungsmoment der identifizierten Gesellschaftsbilder von Arbeitern eines Hüttenwerks stellt zum einen eine dichotome Gesellschaftswahrnehmung dar, in der Arbeiter zwischen einem privilegierten „Oben“ und einem „Unten“ unterscheiden und sich selbst im Unten verorten. Zum anderen lokalisierten Popitz et al. bei den Arbeitern ein spezifisches Leistungsbewusstsein, das durch körperliche Leistungsverausgabung und gesellschaftlich-produktive Wertschöpfung bestimmt ist.

Durch die Arbeiten von Popitz et al. wird der Aneignungsbegriff in Bezug auf Arbeit erweitert. Ebenso wie Marx verweisen Popitz et al. auf die gegenständliche und sinnliche Auseinandersetzung der Beschäftigten mit den stofflichen Bedingungen des Produktionsprozesses und der Kooperationserfordernisse von betrieblicher Arbeitsteilung. Popitz et al. verstehen Aneignung aber als notwendigen Prozess der subjektiven Auseinandersetzung, der unabhängig von strukturellen Bedingungen der Arbeit zur Erfüllung der Arbeitsaufgabe und Leistungsanforderungen notwendig ist. Das bedeutet, dass selbst unter Entfremdung Aneig-

Gesellschaftsbilder von Arbeitern eines Hüttenwerks arbeiten Popitz et al. heraus, dass sich ein Teil der Arbeiter auf Gesellschaft und Betrieb als ein legitimes und sinnhaftes Ordnungssystem (statisches und progressives Ordnungssystem) beziehen, mit dem sie zufrieden sind und von dem sie sich nicht bedroht fühlen. Das beinhaltet auch die Anerkennung der Arbeitsteilung und sozialen Gefüge im Betrieb. Progressiv orientierte Arbeiter betrachten dabei die Verhältnisse als Entwicklungsstufen zur persönlichen Weiterentwicklung. Davon unterscheiden Popitz et al. das Gesellschaftsbild der unabwendbaren Dichotomie, das dadurch gekennzeichnet ist, dass die Arbeiter ihren sozialen Status im Betrieb (und Gesellschaft) als unentrinnbare Position am unteren Rand der Gesellschaft wahrnehmen. Dies äußert sich in einer pessimistischen Sicht auf die Verhältnisse und Misstrauen gegenüber „Höhergestellten“. Ein kleinerer Teil weist ein Gesellschaftsbild auf, dass sich an stärker an gesamtgesellschaftlichen Strukturen und Klassenlagen orientiert, welche es - durch mehr oder weniger radikale Mittel - zu überwinden gilt (Popitz et al. 1957a). Im Zusammenhang dieser Arbeit ist interessant, dass Popitz et al. auch Arbeiter ohne klar artikuliertes Gesellschaftsbild identifizierten. Diese Arbeiter zeigen keine über den eigenen unmittelbaren Erfahrungsbereich der täglichen Arbeit hinausgehende abstrakte Vorstellungsmuster. Zu dieser Gruppe gehören Arbeiter mit einem „souveränen Nichtwissen“ (S. 227), sehr junge Arbeiter, die von den gesellschaftlichen Verhältnissen noch eher unberührt sind und diese hinnehmen, sowie Arbeiter, die in ihren Aussagen nur auf die unmittelbare Erfahrung des Arbeitsalltags beziehen, ohne diese in größere Sinnzusammenhänge einzuordnen. Gleichwohl kann man davon ausgehen, dass all dies Formen der Aneignung der alltäglichen Wirklichkeit darstellt. 
nung durch die Subjekte stattfindet. In diesem Verständnis von Aneignung sind nicht nur bewusst zweckgerichtete, sondern auch unbewusste Handlungen inbegriffen. Darüber hinaus weisen Popitz et al. darauf hin, dass Denken und Handeln durch verfestigte, subjektive Vorstellungen geprägt sind.

\subsection{Das Verhältnis von Struktur und Subjekt}

Dieser Perspektive, also der Frage, wie - und nicht $o b$ - größere Gesamtzusammenhänge des Arbeiterbewusstsein im Kontext des gesellschaftlichen Klassenbewusstseins Einfluss auf die handlungsleitenden Orientierungen der Beschäftigten haben, widmete sich insbesondere die Arbeits- und Industriesoziologe der 1950er bis frühen 1980er Jahre (Voß, G. 1984; Langfeldt 2009). Dabei haben sich bezüglich der Wirkmechanismen von Subjekt und Umwelt theoretisch-konzeptionell drei Stränge herausgebildet (Voß, G. 1984; Kleemann/Voß 2010): Arbeiterbewusstsein sei demnach zu verstehen als

- als arbeitserfahrungsfernes, durch kollektive und gesellschaftliche Wertvorstellungen und soziale Lage geprägtes Bewusstsein, welches die Arbeiter in den Betrieb und ihre Arbeit hereintragen;39

- als Produkt unmittelbarer Arbeits- und Gesellschaftserfahrungen ${ }^{40}$

- oder als Folge eines mehrdimensionalen Wechselwirkungen zwischen gesellschaftlichen Wertvorstellungen und Produktionsbedingungen sowie individueller Auseinandersetzung mit den alltäglichen Bedingungen der Arbeit.41

${ }^{39}$ Wie z.B. „Das Gesellschaftsbild des Arbeiters“ von ; , ,The Affluent Worker: Industrial Attitudes and Behaviour“"von Goldthorpe et al. (1968); (1970).

40 Wie z.B. „Industriearbeit und Arbeiterbewusstsein“ von Kern/Schumann (1977); „Das Bewußtsein der Arbeiter“ Arbeiterbewusstsein, Klassenzusammenhang und ökonomische Entwicklung"von (Deppe 1971).

${ }^{41}$ Einen genaueren Überblick und eine ausführlichere Erörterung unterschiedlicher konzeptioneller Ansätze zum Arbeiterbewusstsein gibt Voß, G. (1984). 
In den wissenschaftlichen Bewusstseinsstudien, welche die beiden ersten Ansätze verfolgten, wurden das Subjekt und die subjektive Auseinandersetzung mit den Bedingungen seiner Arbeit weitgehend außer Acht gelassen und Handeln sowie Orientierung der Beschäftigten eng an Klassenlagen und Produktionsbedingungen geknüpft. Die Bedeutung der herrschenden Produktions- und Arbeitsbedingungen sowie der Erfahrung der Arbeiterschaft mit diesen wurden entweder überbetont oder zugunsten des Einflusses klassenbezogenen Wertvorstellungen vernachlässigt. Dies lässt sich beispielsweise an der lange Zeit dominanten Debatte um die Instrumentalismusthese nachzeichnen, die zunächst von Goldthorpe et al. (1968); (1970) in ihrer Studie „The Affluent Worker" formuliert wurde. Nach der Instrumentalismusthese ist die Arbeitsorientierung der Arbeiter auf den reinen Zweck des Erwerbs von Mitteln gerichtet, die der Realisierung außerbetrieblicher, privater Interessen dienen. Dies führten Goldthorpe et al. weniger auf mangelhafte Realisierungsmöglichkeiten etwaiger arbeitsinhaltlicher Bezüge in den konkreten Bedingungen der Arbeitssituation einer restriktiv gestalteten Fließbandfertigung zurück, wie es Kern/Schumann (1970) taten, sondern primär auf eine wachsende gesellschaftliche Konsumorientierung. Goldthorpe et al. (1968) interpretierten ihre Daten in der Studie dahingehend, dass diejenigen Arbeiter, die in hohem Maße an der Realisierung außerbetrieblicher Interessen orientiert sind, die Beschäftigung als Fließbandarbeiter annehmen und somit ohnehin keine arbeitsinhaltlichen Ansprüche an ihre Arbeit stellen. Aufgrund der materialistischinstrumentellen Orientierung der Arbeiter und der hohen Bedeutung außerberuflicher Interessen bringe sich der Arbeiter nur begrenzt in die Arbeit ein. Den auf individuelle Maximierung des Erwerbsertrags basierenden Orientierungen stehen nach Goldthorpe et al. solidarische Orientierungen an sozialen Gruppennormen entgegen. Reine instrumentalistische Orientierungen würden also mit der beschriebenen Haltung des tayloristischen Managements korrespondieren, dass Beschäftigte kein Interesse haben, sich über die reine ausführende Arbeit hinaus - und erst recht nicht an Optimierungs- und Rationalisierungsmaßnahmen - zu beteiligen.Während Goldthorpe et al. den Instrumentalismus der Arbeiter als durch gesellschaftlichen Wertewandel bestimmte konstante Orientierung verstehen, ist bei Kern/Schumann (1970) die Arbeitsorientierung durch die Erfahrungen der Arbeiter mit den unmittelbaren Arbeitsbedingungen geprägt. Im Vergleich unterschiedlicher Tätigkeitszuschnitte in technisierten Produktionsprozessen (Produktionsarbeiter, Automationsarbeiter, Instandhaltungsarbeiter) stellen sie heraus, dass Produktions- und Automa- 
tionsarbeiter, deren Arbeit durch Restriktionen, geringe Qualifikationsanforderungen und Monotonie bestimmt ist, eine instrumentelle Arbeitsorientierung annehmen, während in qualifizierten Facharbeitertätigkeiten mit ganzheitlichen Aufgabenzuschnitten (Instandhaltung) arbeitsinhaltliche Orientierungen, wie Arbeitsfreude und Identifikation mit der Arbeit, erkennbar sind. Durch die konzeptionelle Engführung des Verhältnisses von Arbeitserfahrungen und Bewusstsein der Arbeiter verkennen Kern/Schumann allerdings, dass auch Arbeiter unter restriktiven Arbeitsbedingungen subjektive, sinnorientierte Orientierungen haben können (Voß, G. 1984; Langfeldt 2009; Nies 2015).

Mit der Kritik, „den Eigensinn subjektiven Handelns nicht zugänglich erfassen“ (Schumm 1988: 3) zu können, wurden ab den 1970er Jahren in Abgrenzung dazu mehrdimensionale Konzeptionen entwickelt, die dem Subjekt in Auseinandersetzung mit seiner Umwelt und den Bedingungen seiner Arbeit mehr Raum für individuelle Umgangs- und Aneignungsformen geben, diese gleichwohl in sozial-normative Bezüge einbetten. Zu nennen sind hier etwa der Ansatz der „doppelten Konstitution sozialer Realität“ von (Hack et al. 1972) oder der Deutungsmusteransatz (Neuendorf/Sabel 1976).

\section{Relevanzstrukturen und Deutungsmuster: Aneignung als multidimensionaler Prozess}

Hack et al. (1972) ${ }^{42}$ greifen die Überlegungen von Popitz und Kollegen zu Aneignung auf und gliedern sie in ihre Konzeption des Verhältnisses von Subjekt und Umwelt ein. Sie verstehen Bewusstsein und Handlungsmuster als einen Prozess der „doppelten Konstitution sozialer Realität“. Einerseits seien Verhaltensweisen von Subjekten durch die objektiven Bedingungen ihrer sozialen Lage, gesellschaftlicher Produktionsstrukturen sowie Verwertungsstrategien und Arbeitsprozesse bedingt. Besonders prägend für Industriearbeit seien dabei die Bedingungen der Einfachheit der Tätigkeiten und die Erfahrungen des Berufslebens, Machtlosigkeit aufgrund geringer Verhandlungsmacht sowie Verzicht und Unsicherheit, die letztlich im fehlenden Zugang zu nötigen Ressourcen zur Erfüllung eigener Bedürfnisse begründet seien (Hack et al. 1972: 17). Andererseits ließen sich von den objektiven Bedingungen nicht die subjektive Wahrnehmung und Handlungsweisen theoretisch ableiten, da die soziale

42 In „Leistung und Herrschaft“ untermauern Hack et al. (1979) ihre 1972 erstellte Konzeption mit ausführlichen empirischen Analysen von Jungindustriearbeitern. 
Realität der Betroffenen eine situative Interpretation der gegebenen Bedingungen darstelle. Der doppelte Konstitutionsprozess sozialer Realität bestehe darin, dass Subjekte die objektiven Bedingungen subjektiv interpretieren und verarbeiten. ${ }^{43}$ Die soziale Realität sei - „immer nur als interpretierte in ihrer subjektiven Bedeutung handlungs- und bewußtseinsrelevant" (Hack et al. 1972: 25) und stelle eine Form der Aneignung dieser dar. Das bedeutet, dass die Interpretation und Einordnung des Erlebten bzw. der subjektiv wahrgenommenen Realität und der Folgenabschätzung eigener Handlungen nicht in einem luftleeren Raum stattfindet, sondern

„vorgeformt durch die in primärer und sekundärer Sozialisation erworbenen Orientierungs- und Bewertungsmuster [...]. Diese (sub-)kulturellen Vorstellungen, Werte und Interpretationsmusterverveisen über den in ibnen enthaltenen Bestand an gemeinsamer typischer Erfahrung zurück auf die Bedeutung der objektiven Grundstruktur, der die in diesen Erfabrungen verarbeiteten sozialen Tatbeständen zugehören. "(Hack et al. 1972. 25) 44

Die Orientierungsmuster werden durch das Handeln der Subjekte in sozialen Situationen werden teils tradiert und reproduziert, teils verändert, zudem durch die herrschenden objektiven Bedingungen gerahmt und begrenzt. Die subjektiven Deutungen der sozialen Realität - von Hack et al. (1979) als subjektive Relevanzstrukturen bezeichnet - stellen sowohl Vermittlungsprozess als auch Ergebnis der Verarbeitung, Interpretation und Aneignung der gegebenen objektiven Bedingungen der Produktionsverhältnisse dar. Subjektive Relevanzstrukturen dokumentieren die Sinnkonstruktionen der Subjekte, die sich im Laufe der (Erwerbs-)Lebens verfestigen, und führen zu Handlungsorientierungen - sei es als planvolle, strategische Handlungen oder als unbewusste Handlungszwänge. ${ }^{45}$ Diese subjektiven Relevanzstrukturen verstehen Hack et al.

43 „Die sinnliche Wirklichkeit der sozialen Tatsachen ist ebenso Produkt der allgemeinen gesellschaftlichen Praxis wie die begrifflichen Ordnungen, in denen sie interpretiert werden. Zugleich gilt aber auch, daß das, was bereits produziert und objektiviert ist, je individuell noch einmal eingeholt werden muß, sowohl als Problem der historischen Abfolge als auch vor allem in der von MARX analysierten Perspektive der unbegrifflichen Verselbstständigung des Produzierten." (Hack et al. 1972: 25).

${ }^{44}$ Diese Werte und Normen definieren Hack et al. (1972) als gemeinsame Lösungsstrategie für die spezifische Problemlage der Arbeiterschaft, die sich vor allem aus der Deprivation und Unsicherheit der Arbeiter ergibt.

45 In ihrer empirischen Untersuchung „Leistung und Herrschaft“ (Hack et al. 1979) versuchten sie ihr Konzept der doppelten Konstitution sozialer Realität anhand der 
aber nicht als „relativ autonom“, sondern sie sehen darin Variationen von Deutungsmustern, die an strukturelle Bedingungen gebunden sind.

Neuendorf/Sabel (1976) messen in ihrer Konzeption „Zur relativen Autonomie der Deutungsmuster" den Subjekten eine höhere, aber relative Eigenständigkeit gegenüber den durchaus vorhandenen sedimentierten Orientierungsmustern bei. Subjekte eignen sich Deutungsmuster an, die „ein konsistentes Gefüge von Interpretationsregeln [geprägt, B.S.], die ihrer eigenen Logik gemäß die Erfahrungen der Subjekte sinnvollen, ihre Relevanzbereiche bestimmenden Wirklichkeit ordnen“ (Neuendorf/Sabel 1976: 842). Diese Deutungsmuster bezögen sich strukturell auf die für die Subjekte relevanten und aktuell gegebenen Bedingungen. Sie werden als intersubjektive und soziokulturelle Alltagstheorien innerhalb von (Teil-)Kollektiven ${ }^{46}$ herausgebildet, in der die Alltagserfahrungen verarbeitet werden und Handlungsorientierungen zur Bewältigung von Problemlagen und der Aufrechterhaltung der eigenen sozialen Identität geben. Die Deutungsmuster seien zwar durch die objektiven Bedingungen der

subjektiven Relevanzsysteme von Arbeitern und Angestellten nachzuzeichnen. Dabei verfolgten sie vor allem den Nachweis von in sich konsistenten Relevanzstrukturen. Von den Subjekten benannte Widersprüchlichkeiten wurden von ihnen zwar benannt, aber nicht in Bezug zueinander gestellt, sondern vielmehr im Rahmen sehr differenzierter und spezialisierter, widerspruchsfreier Typen sozialer Relevanzstrukturen erfasst, die in sich konsistente Strukturen aufwiesen (Langfeldt 2009; Menz 2009). Trotz des bedeutenden inhaltlichen und konzeptionellen Beitrags von Hack et al. (1979) zum Verhältnis von Subjekt und Umwelt wird an der Studie vielseitig kritisiert, dass für die Autoren methodisch eher der Nachweis ihrer Theorie - der doppelten Konstitution sozialer Realität - im Vordergrund stand und weniger die strukturellen Gegebenheiten des Forschungsgegenstands, so dass wichtige Momente subjektiver Verarbeitung, wie gleichzeitig bestehende subjektive Widersprüche, außer Acht gelassen wurden (Langfeldt 2009; Menz 2009). Wichtige Ergebnisse dieser Studie sind die Relativierung der instrumentalistischen Arbeitsorientierung, die Hervorhebung intrinsischer Motivation und eine relativ hohe Akzeptanz des Leistungsprinzips. Angesichts der methodischen Fixierung auf den Nachweis subjektiver Relevanzstrukturen und der doppelten Konstitution sozialer Realitäten wird an Hack et al. kritisiert, dass sie methodisch zu stark auf die Reproduktion gegebener Strukturen bzw. objektiver Bedingungen fokussieren und wenig Raum für die Entstehung von Neuem (Deutungsmustern und Handlungsweisen) lassen (Menz 2009)

46 Neuendorf/Sabel sprechen hierbei von Fraktionierung der Arbeiterklasse in Untergruppen mit gleichen Problemlagen und Relevanzstrukturen (Neuendorf/Sabel 1976). 
gesellschaftlichen Produktionsverhältnisse geprägt und an die Lage der Subjekte innerhalb der spezifischen Beschäftigtengruppe gebunden, ließen sich aber nicht aus diesen Bedingungen direkt ableiten. Sie bildeten im Rahmen der vorgegebenen Bedingungen jedoch eine eigenständige - eben relativ autonome - Wahrnehmung der Wirklichkeit, die durch individuelle Relevanzen der Subjekte bzw. (Teil-)Kollektive variieren könne. So könnten sich bei gleichen Bedingungen unterschiedliche Deutungsmuster oder aber gleiche Muster bei verschiedenen Handlungsproblemen herausbilden. Deutliche Differenzierungslinien können sich aus den betrieblichen und sektoralen Arbeitsmarkstrukturen sowie der eigenen wahrgenommen sozialen Stellung ergeben, was sich in typischen Fraktionierungen der Arbeiterschaft niederschlägt. ${ }^{47}$

Grundsätzlich seien Deutungsmuster stabile Vorstellungen von der Wirklichkeit:

„Jedoch können objektive Entwicklungen in Wechselwirkung mit den Erfabrungen dieser Entwicklungen, die mit dem Deutungsmuster nicht in Einklang zu bringen sind und doch auch nicht mehr vollständig verleugnet werden können, zu Umdeutungen der Wirklichkeit fübren, die das tradierte Deutungsmuster transformieren, weil die bisher von ihm verkannten Aspekte der Wirklichkeit auf denen gerade die Übereinstimmung von Deutungsmuster und Wirklichkeit berubt, dauerbaft nicht mehr ausgeblendet werden können." (Neuendorf/Sabel 1976: 858)

${ }^{47}$ Neuendorf/Sabel verdeutlichen dies an Arbeiterbauern, Ghettoarbeitern mit unterschiedlichen Qualifikationstypen von aufgewerteten und normierten Facharbeitern, sowie angelernten Montagearbeitern. So zeigen sie beispielhaft, dass „Arbeiterbauern“ ihre soziale Identität weiterhin in der bäuerlichen Lebenswert verorten - auch wenn sie schon jahrelang hauptsächlich vom Erwerbseinkommen der Industriearbeit leben - und die Industriearbeit als Zwischenstation bzw. Übergangslösung betrachten. Durch dieses Deutungsmuster liege den Arbeiterbauern ein instrumentalistisches Arbeiterbewusstsein nahe, welches ohne Identifikation mit der eigenen Erwerbsarbeit auskomme. „Die Inhaltslosigkeit seiner Arbeit erkennt und erträgt der Arbeiterbauer, weil er sich der Illusion hingibt, daß er sich in einem zeitlich vorübergehenden Zustand befindet. Seine in der bäuerlichen Lebenswelt verankerte stellungsspezifische Selbstdefinition als Nicht-Industriearbeiter ermöglicht es ihm, das für ihn subjektiv unerträgliche Moment seiner Lage - ihren Dauerzustand - zu verdrängen." (Neuendorf/Sabel 1976: 855). Im Gegensatz dazu würden Ghettoarbeiter - aus Mangel an alternativen Deutungsmustern - eine arbeitsinhaltliche Orientierung haben, die sich am aufgestiegenen Facharbeiter, an der gesellschaftlichen Bedeutung eines wertschöpfenden Mitglieds des Produktionssystems und der Hoffnung des betrieblich und sozialen Aufstiegs orientiert. 
Diese Veränderungen stünden zwar in einem Zusammenhang mit objektiven Wandlungsprozessen, wirkten aber nicht zwingend auf die Deutungsmuster ein. Im Verständnis von Neuendorf et al. befinden sich Deutungsmuster in einem relativen, aber nicht zwingend kausalen Verhältnis zur Wirklichkeit und bilden eine „eigenständige Strukturebene objektiver Bedeutungen“ (Matthiesen/Neuendorf 1989), die methodologisch unabhängig von der objektiven Ebene der gesellschaftlichen Lage und Produktionsbedingungen der Subjekte betrachtet werden muss.

Trotz Hervorhebung der subjektiven Deutungsmuster als eines bedeutsamen konzeptionellen Bausteins der empirischen Betrachtung von Arbeiterbewusstsein und Handlungsorientierungen von Subjekten fehlen in den Arbeiten von Hack et al. und Neuendorf empirische Nachweise der Eigenständigkeit von Deutungsmustern gegenüber strukturellen, objektiven Bedingungen. ${ }^{48}$

48 Dies hänge - so Voß, G. (1984) - insbesondere auch davon ab, dass sich in ihre empirischen Arbeiten ein „latenter Objektivismus“ eingeschlichen habe. Die empirischen Arbeiten von Hack et al. (1979) mündeten in einer weitschweifigen und komplexen Typologie von subjektiven Relevanzsystemen, die weniger die subjektive Konstitution der Wirklichkeit von Arbeitern waren, sondern „Ausdruck objektiver Problemlagen“ (Kleemann et al. 2002) darstellten. Nichtdestotrotz bilden diese Arbeiten die Grundlage für Forschungsansätze, die das Subjekt in der Auseinandersetzung mit seiner Umwelt in den Mittelpunkt der Betrachtungen stellen. Dabei haben sich innerhalb der Arbeitssoziologie Forschungslinien herausgebildet, in denen Subjekte einerseits intensiver in Auseinandersetzung mit den stofflichen Bedingungen des Arbeitsprozesses und (gewandelter) betrieblicher Verwertungsstrategien betrachtet werden. Hierzu zählen vor allem die Arbeiten von Kudera et al. (1979); Schumann et al. (1982), bis hin zu theoretischen Debatten und Forschungsarbeiten der 2000er Jahre zum Wandel der Arbeit sowie zu den Arbeitsorientierungen und dem Bewusstsein von Arbeitnehmern vor dem Hintergrund veränderter Verwertungsstrategien von Arbeitskraft; zu nennen sind bspw. die Arbeiten zu Subjektivierungs- und Entgrenzung (Kleemann et al. 1999; 2002) sowie die Debatten über das Leitbild der Arbeitskraftunternehmers (Pongratz/Voß 2003), Fragen der Anerkennung (Voswinkel 2002) und neuere Fragen nach dem Bewusstsein von Arbeitnehmern (Dörre et al. 2013; Kleinwechter 2014; Kratzer et al. 2015). Zum anderen entwickelte sich eine Forschungslinie, die sich mit den über die eigentliche Arbeit hinausgehenden Reproduktionsbedingungen und -interessen der Subjekte im Kontext von subjektiver Verarbeitung von Erwerbsbiografien befasst, hingewiesen sei hier auf z.B. auf die Werke „Alltägliche Arbeiterexistenz“ (Brock/Vetter 1982) und „Arbeitskraft und Lebenskraft“ (Jürgens, K. 2006a). 
Mit Blick auf die in dieser Arbeit fokussierten Fragen, ob und inwiefern unter gleichen strukturellen Bedingungen unterschiedliche Formen der Aneignung von Beschäftigten möglich sind, geben die theoretischen Konzeptionen der Deutungsmuster entscheidende Hinweise auf den Vermittlungsprozess zwischen den objektiven Strukturen und dem Handeln der Beschäftigten; daher lassen sich aus den soeben vorgestellten Arbeiten folgende theoretische und methodische Implikationen ableiten:

- Subjekte setzen sich subjektiv mit den gegebenen Bedingungen des Produktionsprozesses auseinander.

- Denken und Handeln der Subjekte in der subjektiven Auseinandersetzung wird durch relativ stabile Deutungsmuster beeinflusst, welche sich im Laufe der Erwerbsleben durch subjektive Erfahrungen der Beschäftigten mit ihrer Arbeitssituation und ihre erwerbsbiografische Sozialisation als sedimentierte Orientierungen verfestigt haben. Diese Verarbeitung subjektiver Erfahrungen ist von individuellen Relevanzen der Subjekte abhängig.

- Die Ermittlung wesentlicher Bestimmungsmomente des Verhaltens und der Aneignung der Beschäftigten erfordert sowohl eine empirische Analyse der objektiven Bedingungen als auch eine eigenständige Rekonstruktion subjektiver Deutungsmuster der Arbeiter in ihren jeweiligen sozialen Kontexten; es bedarf also einer Rekonstruktion von Deutungsmustern, die sich auf objektive Bedingungen beziehen, aber sich nicht von ihnen abgeleitet werden.

Die behandelten Aneignungskonzepten eint, dass sie das Subjekt als aktiv handelndes Individuum betrachten, welches sich in einem mehrdimensionalen Prozess mit den Anforderungen der es umgebenden Bedingungen auseinandersetzt und durch zweckgerichtetes Handeln auf seine Umwelt und die Bedingungen einwirkt. Unterschiede lassen sich hierbei in der Frage erkennen, wie groß die gedanklichen und praktischen Aneignungsspielräume der Beschäftigten sind, da die Beschäftigten einerseits durch tradierte Deutungsmuster, andererseits durch die Bedingungen des Arbeits- und Verwertungsprozesses in ihrem autonomen Handeln eingeschränkt sind. Nichtdestotrotz beinhaltet die subjektive Auseinandersetzung Momente relativer Eigenständigkeit und 
ermöglicht sowie begründet „eine aktive und eigensinnige Subjektwerdung der Individuen in und durch Arbeit" (Frey 2009: 67).

Mit dieser zweifach doppelten Perspektive, die einerseits das interessenorientierten Verhältnis zwischen Verwertungsstrategie des Betriebs und Beschäftigteninteressen sowie andererseits den multidimensionalen Auseinandersetzungsprozesses der Beschäftigten mit ihrer Arbeitsumwelt im Blick hat, werden im folgenden Methodenkapitel die empirische Konzeptionalisierung vorgenommen. 


\section{Untersuchungsdesign, Methodik, Auswertungskonzept}

Im Mittelpunkt dieser Studie steht die Frage danach, wie Beschäftigte sich mit dem konkreten betrieblichen Beteiligungsangebot im betrieblichen Arbeitsalltag auseinandersetzen und sich dieses aneignen. Ausgangspunkt dieser Untersuchung ist die empirisch beobachtete Heterogenität der Beteiligungsbereitschaft von Produktionsbeschäftigten in beschäftigtengetragenen Optimierungsprozessen. Weder durch die Gestaltungspraktiken noch anhand subjektiver Orientierungen der Beschäftigten lassen sich unterschiedliche Formen der Beteiligung bzw. Aneignung von KVP befriedigend erklären.

Ziel der Untersuchung ist die Rekonstruktion der Aneignungsformen und ihrer Begründungszusammenhänge im Kontext arbeitspolitischer Gestaltungspraktik von KVP. Zur Realisierung des Forschungsinteresses wird ein vergleichender Fallstudienansatz gewählt: Betrachtet werden zwei Industriebetriebe mit unterschiedlichen beteiligungsorientierten KVP-Konzepten, die ihre Beschäftigten in Optimierungsprozesse einbinden wollen. Es soll nicht vorrangig darum gehen, ob Beteiligung der Beschäftigten an KVP „funktioniert“ oder nicht. Vor allem soll explorativ darauf geschaut werden, welche Aneignungsformen identifizierbar sind und wie sich diese zum einen aus den spezifischen KVP-Praktiken und den jeweiligen arbeitspolitischen Kontexten und zum anderen aus den subjektiven Orientierungen der Beschäftigten rekonstruieren lassen. Auf diese Weise soll ermittelt werden, ob Aneignungsformen jenseits 
des „sich Wegduckens“ des arbeitspolitischen Konservativismus zu identifizieren sind und auf welche Bestimmungsmomente sich diese zurückführen lassen. Dazu wird in diesem Kapitel zunächst ein genauerer Blick darauf geworfen, mit welchen empirischen Konzeptionalisierungen die Fallstudie operiert, um anschließend Forschungsmethodik, Sampling sowie die Auswertungsmethodik zu erläutern.

\subsection{Empirische Konzeptionalisierung}

Der Fokus der zentralen Fragestellung liegt auf der Aneignung von KVP durch die Beschäftigten. Aneignung als subjektiver Auseinandersetzungsprozess bildet den Fall innerhalb des vergleichenden Fallstudiendesgins (Yin 2003; Pflüger et al. 2010). Beschäftigte zeigen gegenüber KVP unterschiedliche Beteiligungsdispositionen, so dass von unterschiedlichen, voneinander differenzierbaren Aneignungsformen auszugehen ist. Ungeachtet der Multidimensionalität der Subjektivität auf der einen und der arbeitspolitischen Gestaltungsbedingungen von KVP auf der anderen Seite wird in dieser Untersuchung davon ausgegangen, dass sich die Ausprägungen der einzelnen Aneignungsformen aus unterscheidbaren Bestimmungsmomenten (Kontextfaktoren) rekonstruieren lassen. Diese Untersuchung operiert mit dem Verständnis von Aneignung als einem mehrdimensionalen Auseinandersetzungsprozess des Subjekts mit seiner Umwelt. Obgleich sich weder das Subjekt selbst noch die objektiven Rahmenbedingungen der Gestaltung von KVP und Arbeit präzise von Aneignung trennen lassen, werden diese im Folgenden aus analytischen Gründen als zentrale Kontextfaktoren von Aneignung behandelt.

Aus dieser Fallkonstruktion ergibt sich für die Analyse eine Zweiteilung: Erstens in Aneignung und subjektive Orientierungen und zweitens in arbeitspolitische Beteiligungskonstellation, die sowohl die KVP-Praktiken als auch die für die Beteiligung an KVP relevanten arbeits- und betriebsorganisatorischen Gestaltungselemente beinhalten. Im Folgenden werden die einzelne Konzepte für die empirische Untersuchung operationalisiert.

\subsubsection{Analysekonzept der Aneignungsformen von KVP}

Zur Rekonstruktion der KVP-bezogenen Aneignungs- und Umgangsformen der Beschäftigten von KVP wird auf das interaktionistische Aneignungskonzept der Alltagspraxis von Leu (1989) zurückgegriffen, welches Aneignung als 
multidimensionalen Prozess von Wahrnehmungen, Verstehen und Handeln in spezifischen situativen Kontexten fasst. In diesem Modell vereint Leu die bereits in Kapitel 3 angesprochenen Dimensionen der sinnlichen Wahrnehmung der Stofflichkeit des Produktionsprozesses sowie von Sinnstrukturen und Deutungsmustern mit der Handlungsebene von wahrgenommenen Handlungsressourcen und Realisierungschancen eigener Interessen. Der Fragestellung folgend geht es hierbei um diejenigen Situationen des betrieblichen Alltags, in denen die Beschäftigten damit konfrontiert sind, sich an Optimierungen zu beteiligen.

Leu (1989) systematisiert Aneignung in drei Ebenen: sinnlich-erfahrende Wahrnehmung, mental-symbolische Aneignung und praktisch-handelnde Aneignung (Leu 1989: 44f.). Die einzelnen Aneignungsebenen werden im Folgenden für die empirische Untersuchung operationalisiert.

\section{Sinnlich-erfahrende Aneignung}

Mit sinnlich-erfahrender Aneignung meint Leu die Ebene der unmittelbaren Wahrnehmung der spezifischen Situation. Wie ein Subjekt die Situation, die konkreten stofflichen Bedingungen des Arbeits- und Verwertungsprozesses in ihrer Körperlichkeit mittels der eigenen Sinne erfährt, komme nach Leu durch positives oder negatives Empfinden (angenehm, unangenehm, belastend, freudig, leidend usw.) zum Ausdruck. Leus Auffassung bezieht sich auf die unmittelbaren physischen Empfindungen der körperlichen Beanspruchung und Belastung. Wenn eine Situation aber als angenehm oder unangenehm, als Spaß oder Mühsal, als interessant oder langweilig erfahren wird oder man Anerkennung oder Ablehnung erfährt, so geht dies über die reine Körperlichkeit hinaus. Das, was von den Subjekten als angenehm oder unangenehm empfunden wird, ist abhängig davon, was sie jeweils in ihrer eigenen Relevanzsetzung als bedeutsam und wichtig, als relevante soziale Regeln und Verhaltenserwartungen betrachten.

Als Analysedimension geht es in dieser Arbeit auf der Ebene der sinnlicherfahrenden Aneignung die Wahrnehmung betrachtet, inwiefern die Subjekte ibre Bedürfnisse, Ansprüche und Interessen an Arbeit in der sperifischen Situation realisiert oder verletz: sehen.

Daran knüpfen weitere Fragen an, etwa: Wird die unmittelbare Beteiligung an einzelnen KVP-Formen im Betrieb, die Aufforderung zur Beteiligung oder auch die Beteiligung anderer Beschäftigter, zum Beispiel als Vertreter der Ar- 
beitsgruppe, von nicht teilnehmenden Beschäftigten als etwas Positives oder Negatives erlebt und worauf beziehen sich diese sinnlichen Wahrnehmungen?

\section{Mental-symbolische Aneignung}

Sinnlich erfahrene Aneignung ist eng mit individuellen Vorstellungen von Arbeit, Ansprüchen und Bedürfnissen der Subjekte sowie sozial geltenden Normen und Wertvorstellungen verbunden. Unter mental-symbolischer Aneignung fasst Leu das Verstehen von übergreifenden Zusammenhängen, die sich durch individuelle Erfahrungen der Subjekte im Laufe der Erwerbsbiografie und Sozialisation zu gefestigten, subjektiven Sinnzusammenhängen und Vorstellungen der sozialen Wirklichkeit konstituieren. Diese subjektiven Orientierungen bzw. Deutungsmuster prägen die Wahrnehmung der jeweiligen spezifischen Situation und stellen als handlungsleitende Orientierungen einen Rahmen für das Handeln der Beschäftigten dar (Neuendorf/Sabel 1976; Hack et al. 1979; Leu 1989).

Im Fokus der Untersuchung stehen hier Orientierungen, die für Aneignung der Beschäftigten mit KVP relevant sind, das heißt etwa diejenigen Orientierungen und Sinnstrukturen, die sich auf die Arbeit als gegenständliche Tätigkeit und Erwerbsform (Arbeitsorientierung), auf den Betrieb als sozialen Ort der Organisation von Arbeits- und Verwertungsinteressen (Betriebsverständnis) und auf Rationalisierung (Rationalisierungsverständnis) beziehen.

Kurzum, es geht um die Frage: Welche subjektiven Orientierungen und Sinnstrukturen bilden relevante handlungsleitende Orientierungen für das Beteiligungsverbalten der Beschäftigten in KVP?

\section{Praktisch-handelnde Aneignung}

Die praktisch-handelnde Aneignung versteht Leu als Ebene der aktiven Beeinflussung von Situationen gemäß den eigenen Bedürfnissen und Wertvorstellungen. Die aktive Gestaltung von Situationen erfolge unter Bezugnahme auf zur Verfügung stehende Mittel und ein zweckgerichtetes Handeln. Die praktisch-handelnde Aneignung impliziert einen Abwägungsprozess zwischen den wahrgenommenen Ressourcen und Handlungsspielräumen auf der einen und den subjektiv eingeschätzten Chancen, die eigenen Interessen und Bedürfnisse tatsächlich zu realisieren, auf der anderen Seite. 


\section{Operationalisierung des Aneignungskonzepts}

Die wahrgenommenen Ressourcen und Handlungsspielräume rekurrieren im Wesentlichen auf mindestens drei Ressourcen- und Handlungsebenen. Erstens auf die Selbstressourcen, in Form der Subjektpotenziale (eigene Fähigkeiten, Fertigkeiten, technische, sozialkommunikative Kompetenzen, Intuitionen etc.); zweitens auf die sozial-kooperativen Ressourcen in Form von praktischer Unterstützung durch Kollegen im Arbeitsteam, Vorgesetze und Vertreter indirekter Servicebereiche; und drittens auf formal-bürokratische/prozessuale Ressourcen, die sich aus dem KVP-Konzept, der Arbeits- und Betriebsorganisation ergeben. Entscheidend ist hierbei sowohl die konzeptionelle Ebene, die häufig formal und öffentlich, beispielsweise mittels Teamarbeitshandbüchern, KVP-Betriebsvereinbarungen oder Ideenmanagementprozessen, im Betrieb festgehalten werden, als die Interpretation und Auslegung dieser Konzepte von den verschiedenen betrieblichen Akteuren im betrieblichen Alltag. Entscheidend ist hierbei die Frage, ob die Beschäftigten, die mittels Subjektpotenzialen Optimierungsideen generieren, die nötige Unterstützung erfahren, um diese auch tatsächlich zu realisieren.

Diese analytisch getrennten Dimensionen der Aneignung können sich gleichzeitig bewusst und - zumeist - unbewusst vollziehen und sich zyklisch und überlappend wiederholen: Handeln $\rightarrow$ sinnliche Wahrnehmung $\rightarrow$ Bewertung und Einordnung in Sinnzusammenhänge und Erwartung künftiger Ereignisse $\rightarrow$ Wahrnehmung der Handlungsspielräume und Chancen-RisikoEinschätzung für die Realisierung eigener Interessen und Ansprüche $\rightarrow$ eigenes Handeln $\rightarrow$ Erfahrungen $\rightarrow$ Verarbeitung dieser Erfahrungen $\rightarrow$ usw. Im Mittelpunkt dieser Konzeption von Aneignung stehen Bedürfnisse, Interessen und Ansprüche der Beschäftigten, die als subjektive Referenzfolie des subjektiven Abwägungsprozesses dienen.

Da Leu den empirischen Zugriff auf Aneignung eher offen lässt, bietet sich dazu der „Doppelte Bezug auf Arbeit“ nach Schumann et al. (Schumann et al. 1982) an. In dieser Konzeption kann der Doppelte Bezug auf Arbeit als Analyseinstrument sowohl zur Ermittlung von Bedürfnissen und Interessen, die für die Beschäftigten in spezifischen Situationen relevant sind, als auch für die Rekonstruktion von Arbeitsorientierungen verwendet werden. Dieser Ansatz greift grundlegende Bestimmungsmomente von Erwerbsarbeit auf, welche die arbeitsbezogenen Bedürfnisse, Interessen und Ansprüche der Subjekte begründen: die Notwendigkeit des Verkaufs, Erhalts und der Weiterentwick- 
lung der Arbeitskraft (Arbeitskraftperspektive) und das Interesse des Subjekts, sich in der Arbeit als Person wiederzufinden und Anerkennung zu erfahren (Subjektperspektive).

Schumann et al. (1982) leiten aus der Arbeitskraftperspektive drei zentrale Interessen ab (196ff.):

- an Erhalt der Arbeitskraft und Schutz vor Verschleiß,

- an der Erhaltung und Erweiterung des Qualifikationspotenzial

- an Lohn und Erhalt des Arbeitsplatzes.

Die Subjektperspektive lässt sich zum einen im Interesse, sich selbst in seiner Arbeit wiedererkennen zu können. Die Interessen richten sich auf (302ff.):

- Interessantheit und Abwechslungsreichtum der Arbeit,

- Realisierung eigener professioneller Standards,

- die eigenen Gestaltungsmöglichkeiten im Arbeitsvollzug,

- Einbringung eigener Fähigkeiten und Fertigkeiten.

Zum anderen richten sich die Interessen des Subjekts auf die Erfüllung sozialer Erwartungen und die Suche nach Anerkennung:

- Interesse an Anerkennung der eigenen Person durch Kollegen und Vorgesetzte (individuell),

- Interesse an Anerkennung der Leistung und Fähigkeiten sowie Erfüllung sozialer Standards (betrieblich),

- Interesse an Anerkennung über Prestige des Produkts und des eigenen Arbeitsplatzes (gesellschaftlich).

Arbeitskraft und Subjektperspektive sind immer zugleich wichtige Bestimmungsmomente der Auseinandersetzung des Subjekts mit der Arbeit. Sie könne in Konflikt zueinanderstehen, wie die Abhängigkeit ein Lohnarbeitsverhältnis aus dem Reproduktionsinteresse eingehen zu müssen, in welchem man nur wenig Handlungsspielraum hat, um selbstbestimmt seinen Interessen nachzugehen. 
Ein von der arbeitssoziologischen Forschung meist ausgeblendeter Aspekt der Konzeption von Schumann et al. (1982) ist der dezidierte Einbezug von wichtigen subjektiven Bezügen auf die eigene Erwerbsbiografie. Aufgrund von Erfahrungen mit formalisierter beruflicher Bildung, die „mit der Verinnerlichung berufsspezifischer Standards und mit der ausgeprägten Erwartung qualifizierter Arbeitsplätze“ (Schumann et al. 1982: 308) verbunden ist, Erfahrungen mit der möglichen Begrenztheit des eigenen Berufswegs sowie Erfahrungen mit Kontinuität oder Brüchen der eigenen beruflichen Laufbahn können die eigenen Relevanzsetzungen und Deutungsmuster der Beschäftigten jenseits der konkreten Arbeitssituation stark variieren.

Schumann et al. (1982) behandeln die erwerbsbiografischen Dimension eher retrospektiv. In der arbeitssoziologischen Forschung wurden immer wieder Orientierungsmuster von Beschäftigten identifiziert, wie beispielsweise Untersuchungen der Aufstiegstyp von Lacher et al. (1989) oder der ambitionierte-assimilierte Typ als Erwerbsorientierung im Kontext von Teamarbeit von Kleinwechter (2014). Die Präsenz dieser Typen legen nahe, dass die beruflichen Ambitionen und Aspirationen einen distinktiven Einfluss auf die Ausrichtung der eigenen Handlungsorientierungen haben.

Mit Blick auf die Forschungsfrage stellen die Analysekriterien des Doppelten Bezugs auf Arbeit wesentliche Dimensionen für die situative Auseinandersetzung der Beschäftigten mit den Bedingungen von beschäftigtengetragenen Prozessoptimierungen dar. Zugleich verweisen diese Analysekriterien auf Elemente subjektiver Orientierungen von Beschäftigten, die Leu unter mentalsymbolische Anerkennung fassen würde.

\section{Erweiterungsperspektiven für den Doppelten Bezug auf Arbeit}

Neuere Erkenntnisse der arbeitssoziologischen Forschung, die sich mit Anspruchsmustern und Interessen von Beschäftigten in postfordistischen Arbeitssystemen befassen, geben Anlass, einzelne Dimensionen des doppelten Bezugs auf Arbeit weiterzuentwickeln. Diese einzelnen empirischen Befunde dienen hier allerdings weniger der strukturellen Weiterentwicklung des Analysekonzepts, sondern werden in dieser Studie eher als heuristische Konzepte und Interpretationsräume behandelt, innerhalb derer die Ergebnisse eingeordnet und diskutiert werden. 
In Bezug auf Anerkennung hat sich in den letzten Jahren ein eigenes arbeitssoziologisches Forschungsfeld entwickelt, in der aus einer identitätstheoretischen Perspektive gesellschaftliche und betriebliche Strukturen als institutionalisierte Anerkennungsverhältnisse betrachtet werden (Holtgrewe et al. 2000; Voswinkel 2002). Anerkennung wird hierbei als wichtiges Element für die subjektive Sinnhaftigkeit der eigenen Identität verstanden. Anerkennung erfolgt nicht nur durch die direkte soziale Interaktion, sondern hat sich auch in gesellschaftlichen und betrieblichen Strukturen, Symbolen und institutionellen Regularien verfestigt (Voswinkel 2017). Auf betrieblicher Ebene lassen sich Anerkennungsstrukturen sowohl in Verträgen, Entgeltsystemen, Arbeitsteilung sowie Kooperations- und Partizipationsstrukturen als auch in symbolischen Akten, wie öffentlichen Ehrungen besonderer Arbeitsleistungen, Würdigung von Betriebszugehörigkeiten oder Betriebsfeiern, wiederfinden. Anerkennung - oder aber auch der Konterpart Missachtung - bezieht sich hierbei sowohl auf soziale Gruppen als auch auf individuelle Anerkennung, die insbesondere in Formen des sozial-kommunikativen Umgangs zwischen Kollegen, gegenüber dem Vorgesetzten und Vertretern anderer betrieblicher Gruppen deutlich wird (Voswinkel 2002; Frey 2009; Voswinkel/Wagner 2013; Voswinkel 2017). Insbesondere bei der Frage nach der Beteiligung von Beschäftigten an KVP kann Anerkennung, fehlende Anerkennung oder gar Missachtung aus anerkennungstheoretischer Sicht mit normativen Reziprozitätserwartungen verbunden sein (Voswinkel 2008). Wenn Beschäftigte Leistungen erbringen, die aus ihrer Sicht über die eigentlichen vertraglichen Vereinbarungen hinausgehen, wie kostenlose Überstunden, besonderes Engagement - die Beteiligung an KVP oder hohe Identifikation mit dem Betrieb, verbinden sie diese mit der Erwartung von Gegenleistungen des Betriebs. Diese „Belohnungen“ können Aufstiegsmöglichkeiten, Sicherheit vor Arbeitsplatzverlust oder zusätzliche Gratifizierungen sein (Voswinkel 2005). Das Fehlen von Anerkennung oder gar (wiederholte) Missachtungserfahrungen sind auf der sinnlich-erfahrenden Ebene unmittelbar negativ spürbar. Individuelle oder kollektive Abwertung, Missachtung als Kompetenzträger (nicht ernstgenommen werden) und Verortung im betrieblichen Unten (Popitz et al. 1957a), als Spielball betrieblichen Handelns, können als negative Erfahrung in das Betriebsverständnis der Beschäftigten eingehen. Diese Erfahrungen können dazu führen, dass Beschäftigte Arbeitsleistungen verweigern, die über die „eigentliche“ Arbeit hinausgehen (Arbeitshandeln im Sinne „Dienst nach Vorschrift“), oder auch innerlich oder gar offiziell kündigen. Positiv gewendet kann die betriebliche Anerkennung der 
Leistungen und Fähigkeiten des Beschäftigten hingegen das Engagement und die Einsatzbereitschaft starken (Friedman 1977).

Anerkennung bzw. Missachtung verknüpft sich besonders in sozialkommunikativer Hinsicht mit normativen und moralischen Wertvorstellungen, wie geteilten Vorstellungen eines würdevollen und „menschengerechten“ Umgangs miteinander (Kratzer et al. 2015; Hürtgen/Voswinkel 2016). Nach Kratzer et al. (2015) handelt es sich bei dem Anspruch, mit Würde und Respekt („Würdeprinzip") behandelt zu werden,

„um einen generalistischen Anspruch auf Wahrung körperlicher und seelischer Unversebrtheit, auf Einhaltung angemessener Distan₹, gegenüber der Person, basaler Normen der Höflichkeit und des "Anstands", auf Befriedigung menschlicher Grundbedürfnisse" (99).

Das Würdeprinzip stellt somit einen basalen und universalistischen Anspruch dar, dessen Erfüllung Voraussetzung dafür ist, dass weitergehende Interessen und Bedürfnisse überhaupt erst wirksam eingefordert werden können.

Bei der Frage nach Legitimitätsvorstellung von abhängigen Beschäftigten identifizierten Kratzer et al. vier weitere Ansprüche, die für Beschäftigte in Zeiten postfordistischer, neoliberaler Arbeitsstrukturen bedeutsam sind: Leistungs-, Fürsorge-, Beteiligungs- und Selbstverwirklichungsansprüche. Der Leistungsanspruch bezieht sich darauf, dass für eine erbrachte Leistung eine Gegenleistung des Betriebs zu erwarten ist. Der Fürsorgeanspruch beinhaltet, dass Menschen gemäß ihrer individuellen Bedürfnisse und „Besonderheiten“ behandelt werden. Mit Beteiligungsanspruch ist die Erwartung an Entscheidungen des betrieblichen Alltags verbunden. Als Selbstverwirklichungsbedürfnis werden Erwartungen der Beschäftigten an eine subjektivierende' Arbeitsgestaltung, oft im Sinne von Interessantheit, Abwechslungsreichtum, Kreativität und Gestaltungsfreiräumen (in) der Arbeit“ (Tullius/Wolf 2016: 497), verstanden. Verletzungen der Ansprüche können zu Konflikten auf gesellschaftlicher und betrieblicher Ebene führen - mit oben beschriebenen Folgen. Darüber hinaus verweist die Studie von Kratzer et al. (2015) auf drei Rationalitätsansprüche, die sich direkt auf die Organisation von Arbeit und Verwertungsprozesse beziehen: Als technisch-funktionale Rationalität werden Vorstellungen gefasst, sie sich auf die stofflichen Bedingungen des Produktionsprozesses (Herstellungsprozess, Ablauf, Kooperationserfordernisse) unter dem Aspekt der Funktionalität und Effektivität beziehen. Bürokratische Rationalität bezeichnet die Orientierung an Verfahrensweisen, Regeln und Hierarchien. Zuletzt werden Wirtschaftlichkeit und Wettbewerbsfähigkeit als Ansprüche der ökomi- 
schen Rationalität gefasst. Technisch-funktionale Rationalisierungsansprüche sind insbesondere bei Facharbeitern (in der Industrie) zu finden. Sie speisen sich aus der Fachlichkeit und dem Erfahrungswissen der Beschäftigten, begründen normativ die Kritik an betrieblichen Entscheidungen und Dysfunktionalitäten und können dadurch in besonderer Weise Beteiligungsansprüche legitimieren.

Die thematisierten Studien haben vorwiegend entweder die Wirkungen struktureller Bedingungen auf die Beschäftigten erarbeitet oder eine Art Landkarte relevanter Ansprüche und Handlungsorientierungen gezeichnet. Die Beschäftigten werden also entweder als Opfer - nicht als Akteure - der strukturellen Arbeits- und Beschäftigungsbedingungen behandelt (Lohr 2003) oder in ihren subjektiven Ansprüchen ohne ausreichende Kontextualisierung in spezifische Bedingungen analysiert. Diese Leerstelle soll durch diese empirische Studie ein Stück gefüllt werden.

\section{KVP - ein Aneignungsmodell}

KVP als interaktionistischer, beschäftigtengetragener Optimierungsprozess beinhaltet vier zentrale Prozessschritte: Genese der Optimierungspotenziale, Artikulation gegenüber dem Betrieb, die Rezeption der Idee durch den Betrieb und schließlich die Realisation der Idee. Die Genese und die Artikulation von Optimierungspotenzialen liegen in der natürlichen Sphäre des Subjekts bzw. des Beschäftigen. Die Entdeckung von Problemen und ihre Lösungen sind unmittelbar mit den Subjektpotenzialen - den Kompetenzen, Erfahrungen und Interessen - verbunden. Vor allem ist es eine bewusste Entscheidung, ob und inwiefern die Beschäftigten diese Optimierungspotenziale dem Betrieb offiziell im Rahmen eines KVP zur Verfügung stellen. Sie können sich grundsätzlich zwischen folgenden Möglichkeiten entscheiden: ihre Optimierungsidee gar nicht zur Sprache zu bringen, sie nur einzelnen Kollegen oder dem Team kundzutun oder betriebsöffentlich preiszugeben. Bei ihrer Entscheidung spielen wiederum die subjektiven Orientierungen, das Betriebs- und Rationalisierungsverständnis sowie die Erfahrungen der Beschäftigten eine bedeutende Rolle.

Wenn Beschäftigte ihre Optimierungspotenziale artikulieren, müssen diese vom Betrieb rezipiert und schließlich umgesetzt werden. Hierbei sind vor allem die betrieblichen Gestaltungsbedingungen relevant. 


\section{Abbildung 1: Aneignungsmodell von KVP}

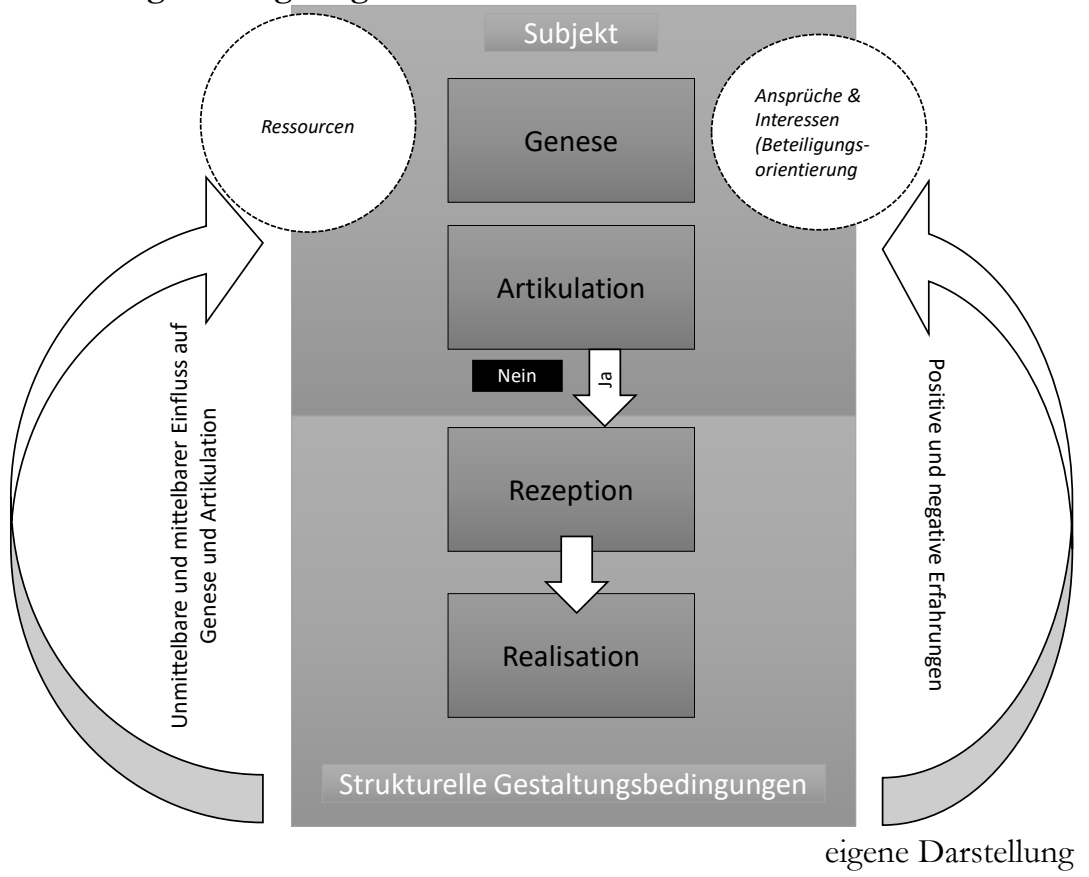

Diese strukturellen Gestaltungsbedingungen nehmen aber auch mittelbar Einfluss auf Genese und Artikulation von Optimierungsideen, da sie die Rahmenbedingungen für die Nutzungsmöglichkeiten der Subjektpotenziale setzen sowie die Handlungs- und Entscheidungsspielräume in Form von Handlungsressourcen (Selbstressource, sozial-kooperative und prozessuale Ressourcen) bestimmen.

Die (sinnlichen) Erfahrungen, welche die Beschäftigten im Zuge des KVP machen, fließen über Deutungen des Erlebten und in Auseinandersetzung mit den konsolidierten Ausrichtungen der Arbeitsorientierung sowie des Betriebsund Rationalisierungsverständnisses wieder in den KVP-Prozess ein. Das Modell geht davon aus, dass die Beschäftigten ihre Artikulationsentscheidungen relativ eigenständig treffen und damit über eine gewisse Autonomie gegenüber den betrieblichen Bedingungen, die wiederum das Handeln der Beschäftigten rahmen, verfügen. 


\subsubsection{Analyse der objektiven Gestaltungspraktiken von KVP: Die arbeitspolitische Beteiligungskonstellation}

Gegenstand der Analyse der objektiven Gestaltungsbedingungen sind zum einen die jeweiligen Gestaltungskonzepte und -praktiken von KVP und zum anderen die Gestaltung der Arbeits- und Betriebsorganisation, in die KVP jeweils eingebettet ist. Die Differenzierung von Gestaltungskonzepten und Praktiken ist in dieser Arbeit deswegen bedeutsam, da innerhalb eines Betriebs, in dem ein Gestaltungskonzept eingeführt wird, sich in einzelnen Bereichen unterschiedliche Praktiken im betrieblichen Alltag entwickeln können. Gründe für die Etablierung unterschiedlicher Praktiken innerhalb eines Betriebs können in den unterschiedlichen arbeitspolitischen Ausgangssituationen (SchwarzKocher, M. et al. 2016) sowie in den Wechselwirkungen zwischen den jeweiligen Interpretationen des Konzepts durch die beteiligten Akteure liegen. Trotz er der unterschiedlichen Auslegung sind die formalen Konzepte nicht obsolet, sondern stellen als Regelwerk oder gar normativ-objektive Instanz eine wichtige Referenz für die Legitimität des eigenen Handelns dar.

Diese Analyse der arbeitspolitischen Beteiligungskonstellationen dient der Rekonstruktion der stofflichen Bedingungen des Arbeits- und Verwertungsprozesses, mit dem sich die Beschäftigten bei KVP und insgesamt in ihrer Arbeit auseinandersetzen. Wie die Ausführungen zu beteiligungsförderlichen und -hinderlichen Gestaltungsbedingungen (strukturinnovative und konservative Arbeitsgestaltung) zeigten, ist KVP nicht als isoliertes Gestaltungsinstrument zu betrachten. Vielmehr gilt es in einer fundierten Analyse genauer zu betrachten, wie KVP mit der jeweiligen Arbeits- und Betriebsorganisation verwoben ist (Appelbaum et al. 2000; Kuhlmann et al. 2004).

KVP, als beschäftigtengetragener Optimierungsprozess, umfasst unterschiedliche konzeptionelle Ausrichtungen und Gestaltungsformen, die primär Optimierungsaufgaben in festen Arbeitsteams (innerhalb von Gruppenarbeit), Workshops (zeitlich begrenzt und interdisziplinär zusammengesetzt) und neue Formen des Vorschlagswesens beinhalten (Kuhlmann et al. 2004). Gegenstand der Analyse der Beteiligungskonstellation soll sowohl das offizielle KVPKonzept als auch dessen Realisierung in der betrieblichen Praxis sein, letztere wird im Folgenden als KVP-Praktiken bezeichnet.

Hinsichtlich der Gestaltung wurden wichtige Differenzierungslinien in der Gestaltung von KVP für die Beteiligung der Beschäftigten identifiziert (Kuhlmann et al. 2004; Schwarz-Kocher et al. 2012): 
- Absicherung als grundlegende Prämisse für Beteiligung der Beschäftigten, etwa in Form von KVP-Betriebsvereinbarungen: kein Arbeitsplatzabbau durch KVP, Sicherung von Qualifikation und Entgelt, ${ }^{49}$

- Schutz vor Arbeitsverschlechterung: Optimierung von Abläufen statt Leistungs- und Belastungsintensivierung,

- Optimierung als reguläre Gruppenaufgabe mit festen zeitlichen Ressourcen,

- Zeitpunkt und Grad der realen Einbeziehung von Beschäftigten in Optimierungsprozesse,

- Prozessnahe Planungs- und Kooperationsstrukturen,

- Grad der Dominanz von betrieblichen Planungsexperten,

- Fokus der Optimierungsaktivitäten: nur reine Kosteneinsparungen oder auch Verbesserung von Prozessabläufen und Arbeitsbedingungen,

- Honorierung,

- Kohärenz der arbeitspolitischen Gestaltungskonzepte.

Insbesondere in dem als Kohärenz bezeichneten letzten Aspekt der Gestaltung von KVP drückt sich die Verwobenheit von KVP-Formen mit der Arbeitsund Betriebsorganisation, die wichtige Kontextbedingungen der Fallstudie bilden, aus.

Die Analyse der Beteiligungskonstellationen erfolgt in Anlehnung an Konzepte innovativer Arbeitspolitik (Kuhlmann et al. 2004) und der Gruppenarbeitsforschung (Gerst et al. 1995) und bezieht sich auf die Kategorien Arbeitsorganisation, Betriebsorganisation und beschäftigtengetragene Prozessoptimierungen. Ergänzt werden diese durch die Kategorien Beschäftigungsbedingungen und Arbeitssituation. Dabei werden die einzelnen arbeitspolitischen Gestaltungsdimensionen unter der Perspektive betrachtet, wie sie mit KVP zusammenwirken.

${ }^{49}$ Siehe hierzu auch Schwarz-Kocher et al. (2012). Das IGM-Institut hat dezidierte Handlungs- und Gestaltungshilfen für Betriebsräte erstellt, die unter anderem auch eine zyklische Kontrolle der Ergebnisse von KVP und ihrer Wirkungen auf die Arbeitsqualität der Beschäftigten vorsehen. 
In Anlehnung an die von Gerst et al. (1995) entwickelte Dichotomie arbeitspolitischer Gestaltungskonzepte von „strukturinnovativ“ vs. „strukturkonservativ“"werden die Realisierungsgrade der Gestaltungsbedingungen hinsichtlich ihrer Wirkung auf Beteiligungsmöglichkeiten der Beschäftigten als „partizipativ“ oder „restriktiv“ verortet (Tabelle 2).

\section{Tabelle 2: Strukturkonservative und -innovative Gestaltung}

\begin{tabular}{|c|c|}
\hline Strukturkonservative Arbeitspolitik & Strukturinnovative Arbeitspolitik \\
\hline $\begin{array}{l}\text { Arbeitsorganisation: } \\
\text { Kurzzyklische, taktgebundene Tätigkeiten } \\
\text { begrenzte Rotation } \\
\text { geringes Maß an Aufgabenerweiterung } \\
\text { geringes Maß an qualifikatorischen und } \\
\text { qualifizierende Aufgaben } \\
\text { geringe Handlungs- und Entscheidungsspiel- } \\
\text { räume } \\
\text { geringes Maß an Selbstorganisation } \\
\text { geringe Arbeitseinsatzflexibilität } \\
\text { geringes Maß an Ressourcen (Zeit) } \\
\text { rigide Standardisierung }\end{array}$ & $\begin{array}{l}\text { Arbeitsorganisation: } \\
\text { Aufgabenerweiterung und Funktionsintegra- } \\
\text { tion (durch Übernahme indirekter Tätigkei- } \\
\text { ten) } \\
\text { Rotation } \\
\text { Qualifizierende Tätigkeiten } \\
\text { Handlungs- und Entscheidungsspielräume } \\
\text { Selbstorganisation } \\
\text { Hohe Arbeitseinsatzflexibilität } \\
\text { Zeitliche Ressourcen } \\
\text { Flexible Standardisierung }\end{array}$ \\
\hline $\begin{array}{l}\text { Betriebsorganisation: } \\
\text { Direkte Kontrolle und Steuerung durch den } \\
\text { Meister } \\
\text { Hierarchische, bürokratische Strukturen } \\
\text { Zentralisierung von Spezialfunktionen } \\
\text { Geringe Integration einzelner Funktionen } \\
\text { Vorgabeorientierte, Top-down- } \\
\text { Steuerungssysteme }\end{array}$ & $\begin{array}{l}\text { Betriebsorganisation: } \\
\text { Meister als Unterstützer und Koordinator } \\
\text { Meister mit erweitertem Funktionszuschnitt } \\
\text { Dehierarchisierung } \\
\text { Dezentralisierung von Angestelltentätigkei- } \\
\text { ten und Spezialfunktionen } \\
\text { Hierarchie- und funktionsübergreifende } \\
\text { Kooperations- und Kommunikationsstruktu- } \\
\text { ren }\end{array}$ \\
\hline
\end{tabular}




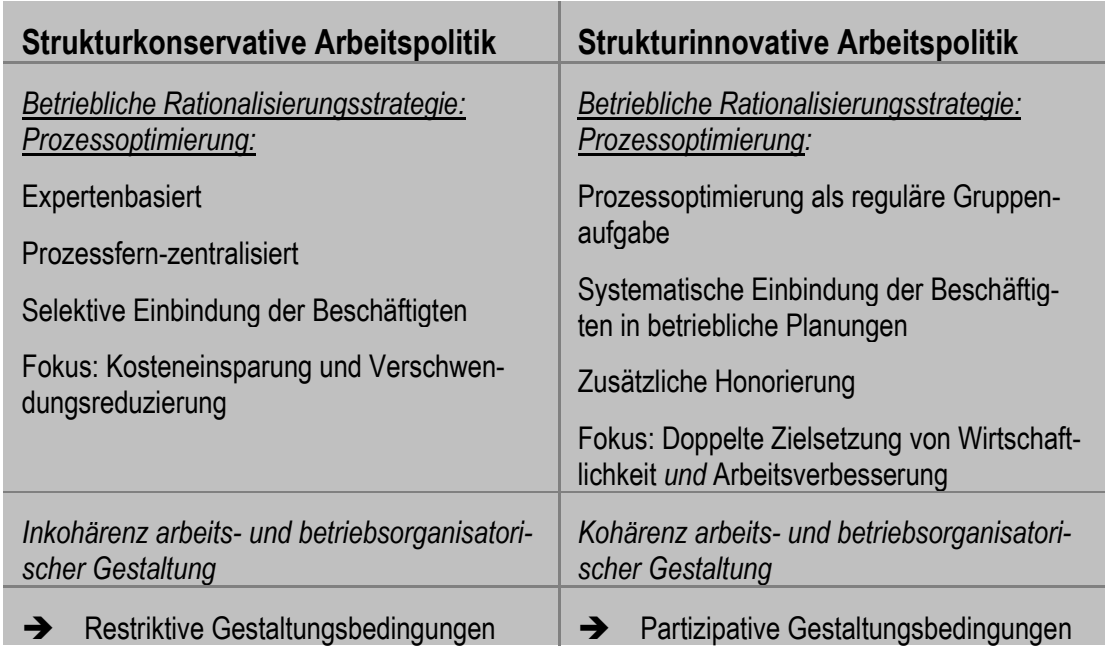

eigene Darstellung nach Gerst et al. (1995) und Kuhlmann et al. (2004)

Da der Schutz des eigenen Beschäftigungsverhältnisses, der Arbeitskraft und materieller Interessen zentral für Erwerbsarbeit ist, wird dieses Analyseschema durch die Dimensionen allgemeiner Beschäftigungs- und Arbeitsbedingungen ergänzt:

- Beschäftigungssicherung, Personalpolitik,

- Leistungs- und Belastungssituation,

- Entgelt.

Das Ziel dieses Analyseschritts besteht darin, im Vergleich unterschiedlicher Gestaltungsbedingungen zentrale Dimensionen einer beteiligungsorientierten Gestaltung von KVP herauszuarbeiten. Die jeweiligen spezifischen Bedingungen werden als arbeitspolitische Beteiligungskonstellationen analysiert. Dies stellt die Basis für die späteren Auswertungsschritte dar, in denen genauer auf die situativen, subjektiven Wahrnehmungen und Bewertungen der objektiven Gestaltungsbedingungen durch die Beschäftigten eingegangen wird. 


\subsection{Vergleichende Fallstudie und Sampling}

Vorrangiges Erkenntnisinteresse dieser Studie ist die Rekonstruktion des Spektrums von Aneignungsformen von KVP durch Produktionsbeschäftigte sowie der objektiven und subjektiven Bestimmungsmomente, durch die sich die einzelnen Aneignungsformen differenzieren. Zur Bearbeitung der Fragestellung wurde ein vergleichender Fallstudienansatz (Yin 2003; Pflüger et al. 2010) gewählt, der die Analyse „unterschiedlicher Ausprägungen und Wechselwirkungen eines sozialen Phänomens durch vergleichende Analysen unterschiedlicher Fälle“ (Pflüger et al. 2010: 45) und ihrer jeweiligen Kontextbedingungen ermöglicht. Die Fallstudien sind jeweils in einem dreistufigen qualitativen Mehrebenendesign (Nohl 2013) angelegt, das die Ebenen Subjekt (Aneignung), Arbeitsbereich (arbeitspolitische Beteiligungskonstellation) und Betrieb (übergreifende arbeits- und beschäftigungspolitische Gestaltung) umfasst.

Das empirische Material der vorliegenden Arbeit wurde im Rahmen des SOFI-Begleitforschungsprojekts AGTIL ${ }^{50}$ (2010-2014) erhoben. In diesem Projekt wurden Konzeptelemente und Wirkungsweisen innovativer Arbeitspolitik und agilitätsrelevanter Arbeitsgestaltung in mehreren österreichischen Betrieben erforscht. Bei den beiden Untersuchungsbetrieben wurden einerseits die Einführung von Teamarbeit und betrieblicher Reorganisation bei NF, andererseits problemorientiertes Abweichungsmanagement bei WLF begleitend untersucht.

\section{Sampling: Betrieb}

Die Untersuchung wurde in zwei österreichischen Industriebetrieben durchgeführt, deren Arbeitspolitik dezidiert auf die aktive Beteiligung der Beschäftigten an Optimierungsprozessen abzielt, deren Konzepte sich aber hinsichtlich der Ablauforganisation, der strukturellen Ausrichtung und der Verwirklichung unterscheiden. Obgleich sich die beiden Betrieben nicht nur hinsichtlich der KVP-Ansätze und arbeitspolitischen Gestaltungspraktiken, sondern auch in wichtigen Charakteristika, wie Betriebsgröße und Fertigungs- und teilweise Tätigkeitsstrukturen, deutlich unterschieden, bot sich die Auswahl dieser Betriebe an, um ein reichhaltiges Spektrum an unterschiedlichen Aneignungsfor-

50 Im Rahmen des AGTIL-Forschungsprojekts wurden folgende Beiträge publiziert: (Heil/Kuhlmann 2013; Heil et al. 2014; Heil/Kuhlmann 2016). 
men ausfindig zu machen. So wurde schon früh in der Erhebung deutlich, dass WLF und NF in der Frage der Beteiligung der Beschäftigten an KVP qualitativ unterschiedliche Beteiligungsansätze wählten. Während NF mit der Einführung von selbstorganisierter Gruppenarbeit mit Optimierungsaufgaben und KVP-Workshops gleichsam dem Mainstream der methodenorientierten Ganzheitlichen Produktionssysteme folgte, implementierte WLF beschäftigtengetragene Problemlöseprozesse. Diese Problemlöseprozesse wurden von externen Moderatoren moderiert, setzten an den alltäglichen Hürden und Abweichungen im Produktionsprozess an und führten offensichtlich in vielen Bereichen zu einer hohen Beteiligung der Beschäftigten an betrieblichen Optimierungsprozessen. Aus diesen Gründen bot sich an, für die vergleichende Fallanalyse nicht nur zwei KVP-Praktiken mit unterschiedlicher Beschäftigtenbeteiligung innerhalb eines Betriebs zu untersuchen, sondern dieses Sample um zwei Konstellationen aus einem anderen Betrieb, der einen strukturell anderen Zugang zur Nutzung der Subjektpotenziale wählte, zu ergänzen. Die große Variation der Kontextbedingungen erhöht zwar die Komplexität der Rekonstruktion, wird aber in der Analyse stets mit reflektiert.

\section{Sampling: Arbeitspolitische Beteiligungskonstellation (Arbeitsbereich)}

Innerhalb der Betriebe wurden jeweils zwei natürliche Arbeitsbereiche für die Rekonstruktion der arbeitspolitischen Beteiligungskonstellation nach dem Prinzip der maximal kontrastierenden Fallauswahl ausgewählt (Glaser/Strauss 1998; Kelle/Kluge 2010). Die Auswahl der arbeitspolitischen Beteiligungskonstellationen wurde von der Frage geleitet, wie das vom Betrieb initiierte KVP-Konzept in den einzelnen Bereichen umgesetzt wurde. Zum einen wurden Bereiche mit KVP-Praktiken für die Untersuchung ausgewählt, von denen eine eher hohe Beteiligungsaktivität erkennbar war; zum anderen kontrastierend dazu Bereiche mit einer wenig lebhaften KVP-Praxis. Die Auswahl der beiden arbeitspolitischen Beteiligungskonstellationen je Betrieb wurde jeweils nach eingehenden Expertengesprächen mit Führungskräften, Betriebsräten sowie nach eigener Begehung der Produktionsbereiche getroffen.

\section{Sampling: Subjekt}

Auf der Subjektebene wurden aus den einzelnen Bereichen Beschäftigte ausgewählt, die sich in unterschiedlichem Grad an KVP und Optimierungen beteiligten. Berücksichtigt wurden hierbei sowohl die Intensität der Beteiligung 
als auch die Wahl der KVP-Form. Durch die kontrastive Auswahl auf der Subjektebene soll ermittelt werden, worauf sich die Unterschiede der Aneignung durch die Beschäftigten zurückführen lassen.

In der Auswahl der Beschäftigten wurde, wenngleich etwas untergeordnet auch das Alter berücksichtigt. Subjektive, handlungsleitende Orientierungen können sich in Abhängigkeit vom Alter und der Lebensphase verändern. Beispielsweise ist denkbar, dass jüngere Beschäftigte andere Weiterbildungs- und Karriereaspirationen als Ältere haben.

In der Gesamtschau wurden somit Beschäftigte in jeweils zwei arbeitspolitischen KVP-Konstellationen von zwei Betrieben mit unterschiedlichen KVPKonzepten miteinander verglichen. Die Vorstellung der Fallbetriebe erfolgt unter besonderer Berücksichtigung der KVP-Ansätze und arbeitssituativer sowie betriebsorganisatorischer Gestaltungsbedingungen erfolgt in Kapitel 5 und die Analyse der Beteiligungskonstellationen in Kapitel 7.

\subsection{Methoden}

Innerhalb jeder Fallkonstellation kam ein Ensemble aus verschiedenen Erhebungsmethoden zum Einsatz, die sowohl im Erhebungsprozess als auch in der Auswertung miteinander trianguliert wurden. Die Mehrebenenanalyse erfolgte nicht allein auf Basis der qualitativen Interviews, wie bei Nohl (2013), indem man ausgehend vom Vergleich der Orientierungsrahmen auf gemeinsam wirkende soziogenetische Elemente schließt. In diesem Untersuchungsdesign hingegen wurden komplementäre Erhebungsmethoden eingesetzt, um umfassende Erkenntnisse über die objektiven Gestaltungsbedingungen von KVP auf der Ebene der Fallkonstellationen und jener des Betriebs zu erlangen. So wurden je arbeitspolitischer Beteiligungskonstellation folgende Erhebungsmethoden angewandt (siehe Tabelle 3). ${ }^{51}$

51 Darüber hinaus wurden quantitative Befragungen aller Beschäftigten in den ausgewählten Produktionsbereichen durchgeführt, die in das Erhebungsprogramm des SOFI-Begleitforschungsprojekts eingebunden waren. Aufgrund der Komplexität und qualitativen Ausrichtung der Fragestellung wird in dieser Arbeit zunächst auf eine Auswertung der quantitativen Daten verzichtet und als Option für mögliche nachfolgende Veröffentlichungen aufgehoben. 


\begin{tabular}{|c|c|c|}
\hline & NF & WLF \\
\hline $\begin{array}{l}\text { Problemzentrierte, } \\
\text { leitfadengestützte } \\
\text { Interviews }\end{array}$ & $\begin{array}{l}11 \\
\text { (5 in Montage F; } 6 \text { Mont K) }\end{array}$ & $\begin{array}{l}9 \\
\text { (4 in Linie 1; } 5 \text { in Linie 2) }\end{array}$ \\
\hline Beobachtungen & $\begin{array}{l}\text { Arbeitsplatzbeobachtungen, } \\
\text { Wöch. Teamgespräche; } \\
3 \text { KVP-Workshops }\end{array}$ & $\begin{array}{l}\text { Arbeitsplatzbeobachtungen, } \\
\text { Tägl. und wöch. Abwei- } \\
\text { chungsgespräche, Produk- } \\
\text { tionsbesprechungen }\end{array}$ \\
\hline Expertengespräche & 25 & 13 \\
\hline
\end{tabular}

\subsubsection{Problemzentrierte Interviews}

Problemzentrierte Interviews zielen auf eine möglichst offene Ermittlung subjektiver Bezugsweisen und Ansichten vor dem Hintergrund theoriegeleiteten Vorwissens und dienen als „heuristisch-analytischer Rahmen für Frageideen im Dialog zwischen Interviewern und Befragten“" (Witzel 2000: 3). Durch die Problemzentrierung wird das Interview auf die fokussierte Fragestellung - hier den Umgang mit den Anforderungen und Gestaltungsbedingungen von KVP - gelenkt. Zugleich werden die Befragten durch offene, erzählgenerierende Fragen zu Narrationen angeregt, in denen eigene Relevanzsetzungen möglich sind (Offenheitsprinzip).

Mit freiwilligen, aber ausgewählten Produktionsbeschäftigten wurden problemzentrierte Interviews (Witzel 1989; 2000) durchgeführt, die in der Regel ca. 1,5 bis 3 Stunden dauerten. Der Leitfaden ${ }^{52}$ orientierte sich an folgenden thematischen Schwerpunkten:

- Beruflicher Werdegang (Erwerbsbiografie, Qualifikationshintergrund, erwerbsbiografische Perspektive),

- Arbeitsorientierung (Was ist wichtig an Arbeit?),

- Arbeit und Arbeitssituation (Arbeitsorganisation, Leistungssituation, Belastung, Führung, Kooperationspartner),

52 Der Grundleitfaden für die Interviews, der jeweils in den einzelnen Spezifika des Betriebs angepasst wurde, befindet sich im Anhang. 
- Erfahrung mit und Bewertung von beschäftigtengetragenen Optimierungsprozesse

○ im Rahmen der Arbeitsorganisation (Teamarbeit bzw. ProduktionsExzellenz) ${ }^{53}$

○ Workshops

$\circ$ Ideenmanagement,

- Rationalisierungsverständnis (Wie nehmen die Beschäftigten Rationalisierung im Betrieb wahr und welche Rolle kommt ihnen dabei zu?),

- Betriebsverständnis (Wie nehmen die Beschäftigten ihre Rolle im Betrieb wahr?).

Während der Durchführung der Interviews wurde darauf geachtet, einzelne thematische Bezüge zunächst mit erzählgenerierenden Fragen einzuleiten, um anschließend immanent nachfragend einzelne Punkte zu intensivieren. Die Interviewführung zielte darauf ab, die subjektive Wahrnehmung der Beschäftigten ihrer Arbeits- und Betriebssituation sowie ihre Erfahrungen mit beschäftigtengetragenen Optimierungsprozessen offenzulegen. Der Leitfaden wurde ihm Rahmen des SOFI-Begleitforschungsprojektes relativ detailliert konstruiert, dennoch wurde bei der Interviewführung darauf geachtet, ihn nach dem Prinzip der Offenheit flexibel und „unbürokratisch“ einzusetzen und den Interviewten möglichst viel Raum für eigene Themeninitiierung, Erzählpassagen und Ausführungen zu überlassen (Hopf 2009).

\subsubsection{Beobachtung von Arbeitsplätzen, Teambesprechungen und Workshops}

Für die Fallkonstellationen wurden im Vorfeld und während der qualitativen Interviews mehrtägige Beobachtungen der Arbeitsplätze und Teambesprechungen in unterschiedlichen Schichten und Arbeitsteams vorgenommen. Zusätzlich fanden Beobachtungen während mehrerer KVP-Workshops in den ausgewählten Produktionsbereichen statt. Die Beobachtungen wurden im Sinne des Beobachtungsinterviews (Kuhlmann 2002; Wittemann et al. 2010) durchgeführt, um die arbeitssituativen und betrieblichen Charakteristika und

${ }^{53}$ Um den Namen ProduktionsExzellenz handelt es sich um den anonymisierten $\mathrm{Na}$ men des problemorientierten Abweichungsmanagements. 
Anforderungen an die Beschäftigten zu ermitteln. Das Beobachtungsinterview bildet eine Kombination von Beobachtungen und auf das Verstehen der beobachteten Aktivitäten bezogenen Rückfragen an die Beschäftigten (Kuhlmann 2002). Hier ging es vorwiegend darum, die Arbeitsvorgänge, -strukturen und Handlungszusammenhänge in den jeweiligen Produktionsbereichen verstehend nachzuvollziehen.

Beobachtungen stellen ein eigenes Erhebungsinstrument zur Erfassung der objektiven Gestaltungsbedingungen von Arbeit dar; darüber hinaus bilden sie eine wichtige Basis für die qualitativen Interviews, weil sich Interviewer*innen im Interview ausführlicher auf beobachtete Handlungsvorgänge und Ereignisse (zum Beispiel aus Teamgesprächen oder Workshops) beziehen und konkrete Situationen im KVP ansprechen können. Auf diese Weise eröffnet sich ein im Interview besserer und direkter Zugang zum Handlungswissen und zu Orientierungen der Beschäftigten im Interview . Durch die umfangreichen Beobachtungen kann eine gezieltere Auswahl von potenziellen Interviewkandidaten nach den Auswahlkriterien des Beteiligungsverhaltens und Alters getroffen werden.

\subsubsection{Expertengespräche}

Die Expertengespräche (Liebold/Trinczek 2002) wurden mit Führungskräften der ausgewählten Produktionsbereiche, den darüberliegenden Führungsebenen (Abteilungsleiter und Produktionsleiter), den betrieblichen Fachexperten für KVP (Koordinatoren und Stabsfunktionen KVP und Ideenmanagement) sowie den für die ausgewählten Produktionsbereiche zuständigen Vertretern der wichtigsten indirekten Bereiche (Planung, Arbeitsvorbereitung, Logistik und Instandhaltung) geführt. Thematisch fokussierten sich die Gespräche jeweils auf die Arbeit der einzelnen Funktionen in Bezug auf die Produktionsbereiche. Es wurde beleuchtet, welche Erfahrungen die Vertreter der einzelnen Funktionen mit der Zusammenarbeit mit den Produktionsbereichen und beschäftigten haben, welchen Einfluss die neue Arbeitsorganisation im Produktionsbereich auf ihre Arbeite hat und wie ihre jeweilige Arbeit im Kontext der betrieblichen Arbeitsteilung strukturiert ist. Dadurch wurde näheres Kontextwissen über betriebliche Kooperationsstrukturen und Arbeitszusammenhänge generiert, das für die Fragestellung von Bedeutung ist.

In den Expertengesprächen mit Vertretern des Managements und Betriebsräten wurde vor allem über die prägenden Leitbilder der betrieblichen 
Arbeitspolitik, die konkreten Gestaltungskonzepte sowie deren aktuelle Umsetzung im Betrieb gesprochen. Ergänzend zu Beobachtungen und qualitativen Interview mit Produktionsbeschäftigten bilden Expertengespräche eine Grundlage für die Rekonstruktion betrieblicher Strukturen und Gesamtzusammenhänge, die sich für die Realisierung beschäftigtengetragener Prozessoptimierungen als wichtige Einflussgrößen erwiesen haben.

\subsubsection{Auswahl der Interviewten: Reflexion des Samplings}

Die Interviews wurden während der Arbeitszeit in einem geschlossenen Raum durchgeführt. Die Teilnahme am Interview erfolgte freiwillig. Die Durchführung einzelner vereinbarter Interviews war nicht selten schwierig, da diese häufig kurzfristig aufgrund des Personalmangels an den Linien verschoben werden mussten. Eine große Herausforderung stellte die Durchführung des anvisierten Samplings dar, das dezidiert auf Beschäftigte mit unterschiedlichen Beteiligungsverhalten ausgelegt war. Während sehr aktive und kommunikative Beschäftigte sehr stark im Sample vertreten sind, waren insbesondere diejenigen Beschäftigten, die in den Gesprächssequenzen während der Beobachtungen gegenüber den Forschenden relativ „kurz angebunden“ waren und die dem Forschungsprojekt - dem ersten Eindruck nach - skeptisch gegenüberstanden, nur selten bereit, ein Interview zu geben. Dies galt insbesondere für eine Fallkonstellation (Montage K) bei NF. Im Verlauf der Feldphase wurde deutlich, dass dies vor allem Beschäftigte waren, die auch der betrieblichen Reorganisation der Arbeitsorganisation und Rationalisierung besonders kritisch gegenüberstehen und sich gegenüber dem betrieblichen Beteiligungsangebot passiv verhalten. Gerade diese Beschäftigten aber sind für eine ausgewogene und genaue Rekonstruktion subjektiver Umgangs- und Aneignungsformen sehr wichtig.

Umgekehrt haben sich sehr häufig besonders engagierte Arbeiter, die sich sowohl im Betrieb als auch in ihrer Freizeit als sehr engagiert und aktiv in beschäftigtengetragenen Prozessoptimierungen dargestellt haben, zur Teilnahme an Interviews bereit erklärt. Besonders bemerkenswert ist, dass sich diese engagierten Arbeiter häufig vehement vom Typus des passiven, engagementlosen und desinteressierten Arbeiter abgrenzen und diesen als negative Referenzfolie für ihr Arbeitsverständnis heranziehen.

Obgleich im Untersuchungssample drei Beschäftigte vertreten sind, die KVP eher ablehnend gegenüberstehen, muss kritisch berücksichtigt werden, 
dass im Ergebnis dieser Studie nicht das gesamte Spektrum möglicher Aneignungsformen vollständig erfasst werden konnte - insbesondere die passiv, verweigernden Formen sind wenig vertreten. Diese Verzerrung im Sample muss bei der Auswertung unbedingt berücksichtigt werden.

$\mathrm{Da}$ im Betrieb WLF nicht ausreichend viele Beschäftigte für Interviews freigestellt werden konnten, wurden hier Interviews mit Beschäftigten aus anderen Produktionslinien des Betriebs mit vergleichbaren arbeitspolitischen Beteiligungskonstellationen in das Auswertungssample hinzugenommen. Für einen detaillierten Überblick über das ausgewählte Sample der Interviews mit den Produktionsbeschäftigten sowie deren Führungskräften siehe Anhang.

Die Interviews und Expertengespräche wurden unter Einverständnis der Interviewten aufgenommen und vollständig transkribiert. Da die Produktionsbeschäftigten teilweise einen starken österreichischen Dialekt sprechen, wurden die Interviews von orts- und sprachvertrauen studentischen Hilfskräften transkribiert. Die Beobachtungen von Arbeitsbereichen, Teamgesprächen und Workshops wurden als Feldprotokoll festgehalten. Für die Analyse wurden alle Angaben, die auf einzelne Personen hindeuten, anonymisiert. Für den Beleg wichtiger Befunde dieser Arbeit wurden den Interviewten aus Gründen der Ästhetik und besseren Lesbarkeit und des Wiedererkennens zufällig Nachnamen alphabetisch (Auer, Berger, Czerni, D... usw.) zugeordnet, die für diesen Kulturraum typisch erschienen. 



\section{Die Fallstudienbetriebe}

Die vergleichende Betriebsfallstudie wurde in zwei österreichischen Industriebetrieben durchgeführt, die am selben Standort angesiedelt sind und zu den angesehenen Arbeitgebern der Region zählen. NF und WLF stammen beide aus einem österreichischen, metallverarbeitenden Mischkonzern und wurden Anfang der 1990er Jahre jeweils als Produktionsstandorte von zwei renommierten, international agierenden Konzerne übernommen. ${ }^{54}$

${ }^{54}$ Beide Betriebe verbindet sie eine gemeinsame Geschichte, die auch nach über 20 Jahren im Bewusstsein der älteren Beschäftigten meist noch sehr präsent ist. NF war der prestigeträchtige Hauptbetrieb, während WLF als Komponentenwerk innerhalb der Konzernbetriebe an diesem Standort eine eher weniger angesehene Betriebsstätte war. Aufgrund der Informationen zum Forschungsprojekt war vielen Beschäftigten bekannt, dass in beiden Betrieben geforscht wurde. Insbesondere die Beschäftigten von WLF wiesen immer wieder darauf hin, welches historische Verhältnis zwischen dem Hauptwerk und dem kleineren Nebenbetrieb existierte und 
Beide Betriebe haben neben einem bereits etablierten Ideenmanagement jeweils in Anlehnung an ihr Konzern-Produktionssystem ein eigenes betriebliches Gestaltungskonzept zur Beteiligung ihrer Beschäftigten an Optimierungsprozessen entwickelt und realisiert. In Betrieb NF wurden 2011 Teamarbeit als Arbeitsorganisation (TA) sowie 2012 KVP-Workshops mit Beteiligung von Produktionsmitarbeitern eingeführt. In Betrieb WLF wurde 2010 ein prozessorientiertes Abweichungsmanagement namens ProduktionsExzellenz ${ }^{55}$ initiiert. Beide Betriebe werden im Folgenden kurz porträtiert - die Angaben beziehen sich, wenn nicht anders angegeben, auf den Zeitraum der Dauer des Projekts von 2010 bis 2013.

\subsection{NF}

NF ist ein traditionsverbundener Endmontagebetrieb, in dem neben fertigen Fahrzeugen auch Komponenten für andere europäische Werke des Konzerns gefertigt werden. Als Kern einer wirtschaftlich eigenständigen österreichischen Gesellschaft ist der Betrieb als Komponenten- und Endmontagewerk über die Produktionskette eng mit anderen europäischen Konzernbetrieben verbunden. Betriebsorganisatorisch ist NF neben der Produktion in die Bereiche Qualitätssicherung, Logistik und Planung (Industrial Engineering), Personal und Produktion unterteilt. Die Produktion gliedert sich in die zwei Großbereiche Fahrzeugfertigung und Komponentenfertigung ${ }^{56}$ auf.

NF ist der einzige europäische Produktionsstandort für zwei Modellreihen von Nutzfahrzeugen, an dem die Komponenten für die eigene Endmontage sowie für andere Standorte des Konzerns gefertigt werden. NF ist sehr eng in die Produktionslieferkette des Konzerns eingebunden und steht dadurch insbesondere hinsichtlich Qualität und Liefertreue der Produkte unter Druck. Betriebsangaben zufolge hat NF im Jahr 2011 über 19.000 Lastkraftwagen und

welche unterschiedliche Entwicklungen beide Betriebe nach der Zerschlagung genommen haben. Dabei waren die Erzählungen der Beschäftigten von WLF vom stolzen Bild des „hässlichen Entleins“ geprägt. WLF sei von einem vernachlässigten und schmutzigen Betrieb zu einem selbstbewussten und attraktiven Betrieb avanciert, während NF betriebsorganisatorisch erst viel später alte Strukturen des Großbetriebsgefüges aufgebrochen habe.

55 Anonymisiert.

${ }^{56}$ Bestehend aus den Produktionsbereichen Rohbau, Lackierung und Montage. 
über 33.000 Komponenten mit ca. 2.500 Mitarbeitern (davon ca. 2000 gewerblich Beschäftigte) produziert. Bei NF arbeiten in der Produktion weit überwiegend männliche ${ }^{57}$ Facharbeiter mit einem metallverarbeitenden oder handwerklichen Hintergrund in zwei Schichten.

Die Auftragslage unterlag in den 2000er Jahren starken Schwankungen, die innerhalb kurzer Zeiträume wiederholt personalpolitische Maßnahmen zur Folge hatten, wie den Einsatz und die Entlassung von Leiharbeitnehmern, die Entsendung von Beschäftigten in Qualifizierungsmaßnahmen, Kurzarbeit und Überstunden durch Sonderarbeit, was bei den Beschäftigten teils starke Verunsicherungen über die Zukunft ihrer Arbeit am Standort hervorgerufen hatte. Aufgrund der starken Auftragsschwankungen, der Banken- und Wirtschaftskrise ab 2008, der Eurokrise ab 2010 und im Zuge der unternehmensinternen Restrukturierungen wurden mit der Konzernzentrale Verträge $(2007,2012)$ zur Standortsicherung abgeschlossen und Investitionszusagen zur Modernisierung der Produktionsbereiche getroffen. Insgesamt hat NF diese Krisenzeiten mittels verschiedener personalpolitischer Maßnahmen gut überstanden. Die regelmäßige Reorganisation von Konzernstrukturen und fortwährende Neuordnungen von Produktionsstandorten prägen zum Zeitpunkt der Untersuchung ein Klima der Anspannung und Verunsicherung im Betrieb NF.

Während NF einst das Zentrum eines angesehenen Mischkonzerns mit vielen unterschiedlichen Produktionszweigen und Fertigungen war, haben sich die strategische Ausrichtung sowie die Bedeutung des Betriebs innerhalb der letzten 25 Jahre stark gewandelt. Während kurz nach der Übernahme beispielsweise noch Motorenfertigungen am Standort angesiedelt waren, wurde der Betrieb im Rahmen großer, investitionsintensiver Reorganisationen und Anlageninnovationen schrittweise zu einem reinen Montagewerk umgebaut. Um 2010 lässt sich eine große arbeitspolitische Umbruchphase nachzeichnen, die von einer deutlichen personellen Einflussnahme des Konzernmanagements (in Form eines häufig wechselnden Konzernmanagement im oberen Management von NF) und - mit der Einführung des konzernweiten Produktionssystems (NF-PS) - einer noch stärkeren Ausrichtung an arbeits- und produktionsorganisatorischen Gestaltungskonzepten der Automobilindustrie gekennzeichnet war.

${ }^{57}$ Der Anteil der Frauen in der Produktion liegt schätzungsweise bei unter zehn Prozent. 


\subsubsection{Arbeitspolitische Vorgeschichte}

Das Management und die betriebliche Führung waren bis 2010 durch eine hohe personelle Kontinuität geprägt. Während Führungspositionen bis zu diesem Zeitpunkt traditionell betriebsintern besetzt wurden, dominierten seit Ende der 2000er kurzzyklische Wechsel der Managementpositionen durch Konzernführungskräfte. ${ }^{58}$ Kontinuität bildete bei NF eher der Betriebsrat, der durch einen in Industriebetrieben typisch hohen Organisationsgrad einen hohen Stellenwert hat und über umfangreiche Verhandlungsmacht im Betrieb verfügt. Diese setzte der Betriebsrat nicht nur intensiv bei mitbestimmungspflichtigen Vereinbarungen ein, sondern er wirkte auf der Ebene der Bereichsbetriebsräte in den Arbeitsgruppen zur Entwicklung und Einführung betrieblicher Veränderungen auch aktiv gestalterisch. ${ }^{59}$ Insgesamt hatte der Betriebsrat bei NF eine starke Position, die er immer wieder in Verhandlungen um Beschäftigungssicherheit, Leistungspolitik und Arbeitsgestaltung einbrachte, einzelne betriebliche Entscheidungen blockierte, andere wiederum vorantrieb.

Die betriebliche Führungsstruktur in der Produktion war bis 2010 traditionell durch eine hierarchische Meister-Vorarbeiter-Struktur geprägt. Der Meister, der in der Tagschicht arbeitete, hatte die disziplinarische Personalverantwortung für ca. drei Produktionsabschnitte mit durchschnittlich ca. 60 Arbeitern pro Schicht, die von schichtgängigen Vorarbeitern operativ (Arbeitseinteilung, Urlaubsplanung) geführt wurden. Die betrieblichen Kooperationsformen waren durch ein hierarchisches Vorgesetztenmodell geprägt. Schon bei kleineren Problemen in der Produktion wurde der Vorarbeiter verständigt, der sich

58 Allein während des Untersuchungszeitraums von 2010 bis 2014 wurde die Position des Produktionsleiters von vier verschiedenen Managern besetzt. Die in jüngerer Vergangenheit eingesetzten und oftmals aus Deutschland stammenden Manager wurden von den Beschäftigten durch ihren „übersachlichen Führungsstil“ teils als „kulturfremd“ empfunden und als personifizierte Hegemonie des Mutterkonzerns verstanden.

59 In Österreich ist die Interessenvertretung sowohl betrieblich als auch gewerkschaftlich in Arbeiter- und Angestelltenvertretungen geteilt. Obwohl sie als ein Gremium mit der Betriebsführung verhandeln, haben Arbeiter- und Angestelltenbetriebsrat sowohl eigene Bezugsgruppen als auch eigene Agenden. Der Vorstand des Arbeiterbetriebsrats fokussiert sich in seiner Arbeit eher auf traditionelle interessenpolitische Verhandlungsthemen wie Arbeitszeit- und Leistungspolitik. 
mit den Servicebereichen oder mit den Vorarbeitern anderer Produktionsabschnitte in Verbindung setzte. Eine direkte betriebliche Kooperation zwischen den Arbeitern und anderen Abteilungen zwecks Problembehandlung fand eher selten statt. Die indirekten Bereiche Logistik, Industrial Engineering, Instandhaltung, Qualitätssicherung und Personal waren zwar fachlich auf einzelne Produktionsbereiche bezogen, aber dennoch zentral für sich organisiert und nicht in die Produktionsbereiche integriert.

Der Produktionsprozess in den Montagebereichen war durch eine hohe Anzahl verschiedener Fahrzeug- bzw. Komponentenvarianten in Fließfertigung mit einer über die Varianten gemittelten Vorgabezeit (Takt) geprägt. Im ersten Untersuchungsbereich der Fahrzeugmontage (Montage F) gibt es eine flexible Taktbindung mit Pufferungsmöglichkeiten, da die Beschäftigten die Fahrzeuge selbst an die nächsten Produktionsabschnitte befördern. In der Komponentenmontage (Montage $\mathrm{K}$ ) erfolgt die Taktbindung durch ein automatisches Beförderungssystem. Alle Montagebereiche sind durch die Produktionslinie unmittelbar miteinander verkettet.

Vor Einführung der Teamarbeit war die Arbeit in der Montage tayloristisch organisiert. In einem wöchentlich erstellten Arbeitseinsatzplan wurden nach Produktionsprogramm und Stückzahl die Arbeitsumfänge der einzelnen Arbeitsplätze vom Meister oder Vorarbeiter definiert und den Arbeitern zugewiesen. Die Inhalte der einzelnen Arbeitsplätze variierten je nach Stückzahl und Modellmix pro Schicht, wobei die vorgegebene Taktzeit über die Schicht ohne Berücksichtigung des Modellmixes gemittelt wurde und in der LKWMontage ca. 9,5 bis 11 Minuten, in der Komponentenmontage 4,5 bis 7 Minuten betrug. ${ }^{60}$ In der Regel wurden die Arbeitsplätze nicht während der Schicht und oftmals auch über Wochen kaum gewechselt. Für die kurzfristige Vertretung von Abwesenheitszeiten einzelner Arbeiter während der Arbeitszeit gab es einen Verteilzeitspringer. In der Praxis wurde diese Position häufig so eingesetzt, dass der Verteilzeitspringer durch den Montageabschnitt roulierte, so dass jeder Mitarbeiter regelmäßig ca. 5-10 Minuten Pause zusätzlich zu den betrieblichen Pausen hatte.

Der Tätigkeitszuschnitt beinhaltete meist nur direkte Montagetätigkeiten. Die Arbeit war als sehr belastend einzustufen. Heben und Montieren schwerer

${ }^{60} \mathrm{Je}$ mehr Fahrzeuge pro Schicht produziert wurden, umso geringer die Taktzeit und umso kleiner die Arbeitsumfänge. 
Anbauteile, Arbeiten in gebückter Haltung sowie teilweise der Transport der Montageträger, auf denen das Fahrzeug in den ersten beiden Montageabschnitten der Montage F von Hand weitergeschoben wird, hoher Lärmpegel durch Druckluftschrauber und hohe Konzentrationsanforderungen beim Bedienen von Hebekränen, um schwere Anbauteile zum Fahrzeug zu transportieren, bedeuten eine hohe physische Belastung. Vor der Einführung von Teamarbeit wurde zwischen den einzelnen Arbeitsplätzen nicht systematisch rotiert, obwohl viele Arbeiter die Aufgaben an den meisten Arbeitsplätzen ausüben können. Zusätzlich herrscht durch die Vorgabezeiten ein für Montagearbeiter typischer Zeitdruck. Dieser ist insbesondere dann sehr hoch, wenn mehrere Fahrzeugmodelle mit höherem Arbeitsaufwand nacheinander montiert werden müssen. Diese Situation wurde und wird von den Beschäftigten häufig stark kritisiert und gibt immer wieder Anlass zur Hinterfragung der geplanten Vorgabezeit und der Austaktung der Produktionsreihenfolge. Der wahrgenommene Zeitdruck kann durch (inoffizielle) kollektive Arbeitsnormen, die zwischen den Arbeitern gelten, noch verschärft werden. Häufig wird in einzelnen Montageabschnitten gemeinsam schneller montiert, um sich zusätzliche Pausen zu verschaffen. ${ }^{61}$ Dies kann nur funktionieren, wenn die meisten Beschäftigten eines Bereiches sich dieser Arbeitsweise anschließen.

\subsubsection{Arbeitspolitische Neuorientierung}

Ausgelöst durch einen Wechsel im Vorstand von NF 2008 sowie durch die Einführung eines konzernweiten Produktionssystems (im Weiteren NF-PS genannt) ab 2007 wurde bei NF eine auf Ganzheitlichkeit orientierte, arbeitsund rationalisierungspolitische Neuausrichtung eingeleitet.

„Der wachsende Wettbewerbsdruck erfordert es, selbst in einem turbulenten Umfeld Möglichkeiten sofort zu erkennen, um wirtschaftlich zu arbeiten. Ganz wesentlich ist es dabei, dass wir uns alle auf dieselben Werte und Prinzipien als Richtschnur des Unternehmens verlassen und uns daran orientieren können. "(Auszug aus NF 2007: 6)

${ }^{61}$ In dem Montagebereich F war dieses Verhalten besonders auffällig. Die Arbeiter schoben den Produktionsträger selbstständig zu den nächsten Taktstationen weiter und waren somit nicht von einem automatischen Fördersystem abhängig. Beschäftigte konnten durch schnelleres Vorarbeiten besonders in Montage F einen Puffer aufbauen, der ihnen ermöglichte, eigene Freiräume zu erarbeiten. 
Mit der Einführung des NF-PS folgt NF beispielhaft dem Trend der Fahrzeugbranche, Ganzheitliche Produktionssysteme nach dem Beispiel des Produktionssystem-Pioniers Toyota (Ohno 1993) einzuführen. Im Zentrum des NF-PS stehen die Ausrichtung der Unternehmens- und Produktionsorganisation sowie aller Mitarbeiter auf Steigerung der Wertschöpfung, marktorientierten Flexibilisierung und Kundenorientierung. Das NF-PS stellt den Mitarbeiter als verantwortlich handelnden Akteur in den Mittelpunkt des Unternehmenserfolgs. Es entwirft ein Leitbild des Idealbeschäftigten, der stets aus seinen Fehlern lernt, verantwortlich mit den Produktionsanlagen umgeht, auf hohe Qualität achtet, Prozesse standardisiert, diese Prozesse einhält sowie permanent verbessert und mit konstruktiver Kritikfähigkeit mit den Kollegen und Vorgesetzten Probleme und Fehler kommuniziert.

„Mit dem [NF-PS, B.S.] wollen wir erreichen, dass alle Mitarbeiter mit Begeisterung ibrer Arbeit nachgehen können, dass sie sich sicher und unbeschwert unseren Produkten widmen können und dass sich jeder Einzelne bewusstwird, dass er einen wichtigen Beitrag für die Zufriedenheit unserer Kunden leistet. " (NF 2007: 11)

Zugleich werden den Beschäftigten zur Orientierungen des „richtigen Handelns" 62 normative Handlungsprinzipien, Gestaltungskonzepte und Methoden vorgegeben: Konzepte zur Materialflusssteuerung, Vermeidung von Verschwendungen jeglicher Art im Produktionsprozess, Null-Fehler-Strategie, Steigerung der Wertschöpfungsanteile, ständige Verbesserung, KAIZEN, Standardisierung, visuelles Management und auftragsgesteuerte Produktion. ${ }^{63}$

„Unsere innovativen Ansätze liegen bier vor allem in der Ausschöpfung der Kreativität jedes einzelnen Mitarbeiters. Dafür muss es richtige Strukturen und Instrumente geben. Und die haben wir [bier, B.S.] perfektioniert. Top ausgebildete Mitarbeiter nützen dem Betrieb nur, wenn sie ibr Wissen auch in ibrem Bereich anwenden können. Wenn sich die Arbeitskräfte bei dem, was sie tun, woblfüblen, dann kommen auch die guten Ideen, die den Betrieb weiterbringen." (Presseinterview Automobil-Cluster: Interview mit dem Vorstand NF 2012)

62 In dem als Haus dargestellten NF-PS-Produktionssystem werden die Handlungsprinzipien, Mitarbeiterorientierung und Materialflussbedarf als die drei tragenden Säulen dargestellt.

63 All diese Leitbilder, Gestaltungsprinzipien und Handlungsorientierungen sind in einem Handbuch des NF-PS ausführlicher dargestellt und zusammengefasst. 


\section{Konzeptionelle Ausricbtung}

Um diesen Anspruch organisatorisch zu verwirklichen, wurden eine Reihe von Strukturprojekten am NF-Standort initiiert, welche die arbeitspolitischen Rahmenbedingungen für die Beteiligungsorientierung der Beschäftigten schaffen sollten. Die vormals hierarchisch und tayloristisch organisierte Fabrik wurde fortan durch Einführung von Teamarbeit, durch KVP-Workshops mit Beteiligung der betroffenen Montagearbeiter, durch Dezentralisierung der indirekten Bereiche sowie durch Neuorganisation der Führungsstruktur in der Produktion neu ausgerichtet. Dazu wurden mehrere eigene standortbezogene Strukturprojekte zur konzeptionellen Ausarbeitung der Reorganisation des Betriebs durchgeführt, an denen Mitarbeiter aus unterschiedlichen Hierarchieebenen der Produktion, Fachfunktionen und des Betriebsrates beteiligt wurden. Folgende Restrukturierungs- und Reorganisationskonzepte wurden entwickelt und im Zeitraum von 2010 bis 2013 umgesetzt:

- Reorganisation der Hierarchiestrukturen in der Produktion mit Shopfloormanagement ${ }^{64}$,

- Dezentralisierung der indirekten Service-Bereiche (stärkere Integration der Bereiche Logistik, Instandhaltung, technischer Service, Prozessentwicklung, Arbeitsvorbereitung, Produktentwicklung in die Produktion),

- Teamarbeitsorganisation in den gewerblichen Bereichen, ${ }^{65}$

- KVP-Workshops mit systematischer Beteiligung von Arbeitern sowie

- Neuregelung des Ideenmanagements.

64 Shopfloormanagement bezeichnet ein aus der Lean-Production-Strömung entwickeltes Führungs- und Steuerungsinstrument. Beschäftigte und Führungskräfte besprechen am „Ort der Wertschöpfung“ (Peters, R. 2009) täglich wesentliche Kennzahlen und aufkommende Probleme und leiten bei Bedarf entsprechende Maßnahmen zur Kennzahlenerfüllung ein. Die Gespräche finden gestaffelt auf unterschiedlichen Hierarchieebenen bis hin zum Produktionsleiter statt. Je nach betrieblichem Zuschnitt des Konzepts werden auch Vertreter der indirekten Servicebereiche unmittelbar eingebunden (Schwarz-Kocher, M./Salm 2016: 12 f.).

65 Nach der Einführung der Gruppenarbeitsorganisation in den gewerblichen Bereichen wurden - hauptsächlich angetrieben durch den Betriebsrat - Versuche unternommen, die Angestelltenbereiche ebenfalls in Teamarbeitsstrukturen zu reorganisieren. 
Bei der Konzeption und Implementierung war es dem Betrieb besonders wichtig, dass die einzelnen Veränderungen komplementär zueinander wirkten. Die Entwicklung der Strukturprojekte und ihre Implementierung erfolgten allerdings nicht immer synchron. Einführung und Ausrollung der Teamarbeitsorganisation in den Produktionsbereichen verzögerten sich teilweise stark, weil die Veränderung der Hierarchiestrukturen erst kurz nach dem Start des Pilotbereichs vorgenommen wurde. Zudem wurde die stufenweise Implementierung von Teamarbeit auch aus betriebspolitischen Gründen aufgeschoben, da betriebsratsseitig unzureichende Rahmenbedingungen, wie eine ungenügende Personalbemessung und eine mangelhafte Infrastruktur im Pilotbereich (Teamarbeitsinseln), kritisiert wurden. Die Behebung der Mängel wurde an den Fortgang der Teamarbeit geknüpft. Diese Verzögerungen setzten sich im Einführungsprozess fort und wurden von den Beschäftigten häufig sehr stark kritisiert.

Parallel zur den Reorganisationsmaßnahmen wurden zentral durch die Werkführung weitreichende Standardisierungs-, Ordnungs- und Transparenzmaßnahmen in der Fabrik- und Fertigungsgestaltung vorgenommen. Diese umfassten: Aufteilung des Produktionsbereichs in Montage- und Bereitstellungszonen (Werkzeug und kommissionierte Anbauteile), größere Transparenz der Montagebereiche durch Entfernung von Sichtbarrieren, Eneuerung von Böden sowie Einrichtung von standardisierten Werkzeugtafeln und Arbeitsplätzen. Diese Maßnahmen wurde besonders durch KVP-Workshops vorangetrieben und mittels der 5S-Methode von den Mitarbeitern im Rahmen ihrer Teamaufgaben durchgeführt. Die Beschäftigten waren somit in Teilen selbst an diesen Maßnahmenbeteiligt.

Die hohe Reorganisationsintensität bei NF wurde flankiert von einem hohen Innovations- und Rationalisierungsdruck seitens des Konzerns, der zusätzliche beschäftigungspolitische Maßnahmen bewirkte. So wurden Anpassungen des Arbeitszeitmodells in der Produktion vorgenommen, Arbeitszeitkonten eingeführt sowie neue Zeitbemessungsregelungen vorbereitet. In Summe bedeuten die zahlreichen arbeitspolitischen Veränderungen einen radikalen Bruch in der Arbeitspolitik von NF, dem die Beschäftigten mit großer Unsicherheit und starker Kritik begegneten. 


\section{Strukturelle Veränderungen der Arbeits- und Betriebsorganisation}

Eine der ersten Veränderungen der arbeitspolitischen Neuausrichtung war die dezentrale funktionale und räumliche Zuordnung der indirekten Bereiche. Die indirekten Bereiche der operativen Logistik, Prozesstechnik, dezentralen Instandhaltung, Industrial Engineering mit Arbeitsvorbereitung (Prozessplanung) und Anlagenplanung sowie NF-PS-Trainer, Qualitätssicherung und Materialmanagement sowie der Bereichsbetriebsrat wurden räumlich direkt neben der operativen Führung der Montage (Abteilungsleitung und Meister) in der Nähe der Produktionslinie angesiedelt. ${ }^{66}$ Die räumliche Heranführung sollte eine direkte und spontane Kommunikation und größere Transparenz zwischen den einzelnen Funktionen bewirken.

Durch die Hierarchie-Restrukturierung wurde die traditionelle MeisterVorarbeiter-Struktur aufgehoben und eine erste Führungsebene eingerichtet, die schichtgebunden die Personal- und Bereichsverantwortung innehatte. Komplementär zum Teamarbeitskonzept, welches die Übernahme von organisatorischen Funktionen (Arbeitseinteilung, Organisation des Urlaubs, Qualifizierung, Wartung, Qualitätssicherung) durch die Teams vorsah, war die neue Meisterfunktion darauf ausgerichtet, die Rahmenbedingungen für eigenverantwortliches Arbeiten der Teams zu schaffen und nur im Eskalationsfall in teaminterne Belange einzugreifen. Darüber hinaus beinhaltete die neue Meisterfunktion die Verantwortung für die Zielerreichung vor allem bezüglich Qualität, Liefertermintreue, Gesundheitskennzahlen und Kosten. ${ }^{67}$ Der Zuständigkeitsbereich der neuen Meisterfunktion umfasste die Produktionsabschnitte von drei Teams (Führungsspanne ca. 30 Mitarbeiter).

Im Rahmen des neuen Shopfloormanagements finden zu Beginn jeder Schicht auf jeder Hierarchieebene, beginnend mit der ersten Führungsebene bis hin zur Werksleitung, kurze Regelgespräche in der Produktion statt, bei denen mit

${ }^{66}$ In der Komponentenmontage wurde dies zum Zeitpunkt der Untersuchung im Rahmen eines Großraumbüros bereits umgesetzt. Die Realisierung in anderen Produktionsbereichen wurde aufgrund von Platzgründen häufig verschoben und im Laufe der Zeit lediglich in einer reduzierten Variante (nur ein gemeinsames Büro für Meister - die anderen Funktionen wurden räumlich näher an die Produktionslinie verlagert) umgesetzt.

$67 \mathrm{Da}$ die Meisterfunktion primär auf die Führung der Produktionsteams zugeschnitten war, wurden dem Meister für die technische Weiterentwicklung und Problemlösung produktions- und prozessnahe Fachkräfte zur Verfügung gestellt. 
der jeweiligen direkten Vorgesetztenebene Produktions- und Ablaufprobleme anhand von Kennzahlen besprochen werden. Teilweise werden dabei auch auf der zweiten und dritten Hierarchieebene einzelne indirekte Funktionen (Logistik, Instandhaltung und Prozesstechnik) direkt eingebunden.

Die Arbeitsorganisation der Produktion sollte durch die Einführung von Teamarbeit schrittweise von einer hierarchisch, tayloristisch geprägten Produktionsorganisation hin zu einer durch Selbstorganisation und Mitgestaltung geprägten Arbeitsorganisation verändert werden. Auf die Veränderungen der Arbeitsorganisation durch Teamarbeit als zentrales Gestaltungselement von beschäftigtengetragenen Optimierungsprozessen bei NF wird im nächsten Abschnitt genauer eingegangen.

\subsubsection{KVP bei NF}

NF hat in seinem Produktionskonzept den Anspruch formuliert, Beschäftigte in Optimierungsaktivitäten einzubeziehen und deren fachliche Kompetenzen zu nutzen. Dies konkretisiert sich bei NF in zwei zentralen Gestaltungskonzepten: erstens in der Einführung von selbstorganisierter Teamarbeit in der Produktion, in deren Zuge die Beschäftigten nach einer Eingewöhnungsphase selbst Optimierungsaktivitäten anstoßen und umsetzen sollen. Zweitens in der systematischen Einbeziehung der Produktionsbeschäftigten in flächendeckende und funktionsintegrierte Workshops. Diese Konzepte „Optimierung in Teamarbeit" und „Workshops" werden durch eine Neuorganisation des bereits zuvor über viele Jahre existierenden Ideenmanagements ergänzt, um herausragende Ideen, die eigentlich nicht zur Arbeitsaufgabe zählen, zu prämieren.

\section{a) Optimierung in Teamarbeit}

Erklärter Anspruch und Ziel der Einführung von Teamarbeit war die Erzielung höherer Produktivität und Flexibilität durch verbesserte Zusammenarbeit der qualifizierten Beschäftigten in der Produktion.

„Unsere Teamarbeit versucht in hohem Maße die Kreativität, das Engagement und das Können der Menschen, aber auch des Kollektivs, der Gemeinschaft, besser auszunutzen. " Vorstand von NF in der Unternehmenszeitschrift Juli-September 2011)

Das Teamarbeitskonzept von NF umfasst die schrittweise Übertragung von Verantwortung und Entscheidungsbefugnissen von den Führungskräften an die Teams durch mehr Selbstorganisation und größere Handlungsspielräume 
im Sinne einer innovativen Arbeitspolitik (Kuhlmann et al. 2004). Neben der Verantwortungsübernahme wird dezidiert auch die Verbesserung der Arbeitsbedingungen für die Beschäftigten angestrebt. Das Teamarbeitskonzept umfasst folgende, komplementär und integrativ konzipierte Gestaltungselemente:

\section{Teamaufgaben}

Die Implementierung der Teamarbeit erfolgte in einem dreistufigen Prozess, in dem die Teams die vorgesehenen Aufgaben schrittweise bis zur eigenständigen Ausführung übernahmen. In der ersten Implementationsphase wurden organisatorische Aufgaben an die Beschäftigten übertragen, wie eigenständige Arbeitseinteilung, Organisation des Urlaubs und Qualifizierung der Mitarbeiter mit dem Ziel, dass jeder Arbeiter an jedem Arbeitsplatz arbeiten kann. Die Beschäftigten werden mittels Visualisierung an Teamtafeln mit den Qualitätskennzahlen vertraut gemacht. In den Phasen 2 und 3 werden diese Aufgaben um die Übernahme und eigenständige Initiierung von Optimierungen und Weiterentwicklungen des Produktionsabschnitts der Teams ergänzt. In Phase 3 der Einführung von Teamarbeit sollen die Teams gemeinsam mit ihren Führungskräften Ziele bezüglich der Qualität, Kosten, Durchlaufzeiten und Liefertreue entwickeln. Gemeinsam mit betrieblichen Experten sollen die Teams Maßnahmen zur Verbesserung der Arbeitsplätze, Ergonomie, Maschinen und Anlagen erarbeiten und ihre Umsetzung verfolgen.

\section{Team, Teamsprecher und Teamgespräch}

Die Teamgröße umfasst in der Regel acht bis zwölf Beschäftigte, die über drei meist zusammenhängende Takte verteilt sind. ${ }^{68}$ Je Team wird ohne Mitspracherecht der Führungskräfte oder des Betriebsrates ein Teamsprecher gewählt, der als zentraler Ansprechpartner für Team, Vorgesetzte und für die Kommunikation sowie Konfliktlösung innerhalb und zwischen den Teams zuständig ist. Die Teamsprecher erhalten nach ihrer Wahl eine Schulung. Neben Moderation der Teamgespräche zählen Organisationsaufgaben wie Verwaltung und Bestellung von Arbeitsmitteln, Anwesenheitsplanung, Pflege von Kennzahlentabellen und Qualifikationsmatrix, Absprachen mit dem Vorgesetzten und

68 Die Personalbemessung eines Bereichs beinhaltet die Montagezeiten, individuelle Pausenzeiten und Urlaubsvertretung. 
anderen Teamsprechern sowie die Vermittlung betrieblicher Entscheidungen zu den Aufgaben des Teamsprechers. Der Teamsprecher ist nicht freigestellt, erhält für die Erledigung seiner Aufgaben allerdings zusätzliche kleinere Zeitanteile.

Für die Selbstorganisation der Teamaufgaben und die Besprechung von Problemen, Lösungen und Verbesserungsmöglichkeiten erhalten die Teams wöchentlich dreißig Minuten Zeit für eigenverantwortliche Teamgespräche, die in ihren eigens errichteten Teambereichen abgehalten werden. Zu den Teamgesprächen, die vom Teamsprecher moderiert werden, können je nach Thema Vorgesetzte und Vertreter aus den Fachbereichen zur Problembehebung und zum Informationsaustausch eingeladen werden. Zusätzlich gibt es täglich fünfminütige Gespräche zum Schichtende, in denen aktuelle Informationen und Probleme ausgetauscht werden.

Die Kompetenzen und Aufgaben wurden vom Projektteam Teamarbeit in einem Handbuch festgehalten, welches ein wichtiger Bezugspunkt für Arbeiter, Betriebsrat und Führungskräfte ist. Insbesondere bei Unklarheiten von $\mathrm{Zu}$ ständigkeiten wird das Handbuch zurate gezogen. Die Einführung und Umsetzung der Teamarbeit wurde von Teamarbeitsbegleitern organisiert und begleitet. Als für diesen Zweck freigestellte Experten vermitteln sie vor allem in Konfliktsituationen zwischen den Teams und Führungskräften. Die Teamarbeitsbegleiter sollen helfen, die Beschäftigten, vor allem die Teamsprecher, an die Übernahme von Organisationsaufgaben heranzuführen und bei der produktionsbereichsübergreifenden Urlaubsplanung zur Seite stehen.

\section{Qualitätsregelkreismitarbeiter}

Parallel zur Teamarbeit wurde in jedem Team die Funktion eines freigestellten Qualitätsregelkreismitarbeiters (QRK) eingeführt, der in jedem Teamabschnitt für das Anlernen von Beschäftigten, die Qualitätskontrolle, gegebenenfalls Nacharbeit sowie kurzfristige Abwesenheitsvertretung einzelner Teammitglieder zuständig ist. Diese Position wird meist mit sehr erfahrenen Arbeitern besetzt. Zu Beginn der Teamarbeit war diese Funktion noch in der Gruppe integriert, konzeptionell soll sie jedoch von der Teamstruktur losgelöst werden und mit reduzierter Mitarbeiterzahl ihre Aufgaben im gesamten Produktionsabschnitt ausüben. Vor dem Hintergrund des Wegfalls des Verteilzeitspringers zugunsten einer persönlichen Verteilzeitregelung der Beschäftigten in der Gruppe und der neuen Personalbemessung bei Teamarbeit gab es zwischen 
den Beschäftigten und dem Betriebsrat einerseits und den Führungskräften und Management andererseits immer wieder Debatten darüber, ob und wie lange die Beschäftigten den Qualitätsregelkreismitarbeiter „für sich“ oder die Führungskräfte diesen „,ür den Betrieb“ einsetzen könnten. Im Produktionsalltag wurde der QRK trotz aller Debatten meist - besonders zu Beginn der Teamarbeit - eher vom Meister bestimmt und zur Personalabdeckung genutzt.

Der QRK trifft sich regelmäßig mit anderen QRKs aus dem Produktionssegment. In dieser Runde werden Qualitätsmängel und Neuerungen in der Produktion besprochen, welche die QRK-Mitarbeiter in ihre Teams kommunizieren. Ablauf- und Qualitätsprobleme besprechen die QRK-Mitarbeiter und Teamsprecher zunehmend direkt miteinander, ohne dass die Vorgesetzten benachrichtigt werden müssen. ${ }^{69}$

\section{Implementierung von Teamarbeit}

$\mathrm{Zu}$ Beginn der Implementierung von Teamarbeit bei NF wurden alle Beschäftigten über zwei Tage in ihren Gruppen geschult. Die Schulung wurde aufgrund der Verzögerungen bei der Einführung teilweise mehrere Monate vor dem eigentlichen Start der Teamarbeit durchgeführt. Die Teamarbeitsorganisation wurde im Frühjahr 2011 nach der Restrukturierung der Hierarchie in einem Pilotbereich in der Endmontage eingeführt. Ein halbes Jahr später erfolgte das Ausrollen der Teamarbeit in der gesamten Fahrzeugmontage (November 2011). wurde Mit einer Verzögerung von einem Jahr fand die Implementierung in der Komponentenmontage statt (Dezember 2012). Die Einführung von Teamarbeit war durch zahlreiche, bei vielen Teamarbeitsbereichen wiederkehrende Probleme gekennzeichnet. So wurde von den Teams zu Beginn die aus ihrer Sicht zu enge Personalbemessung und die damit zusammenhängenden fehlenden Möglichkeiten zum Anlernen der Teammitglieder und

69 Zusätzlich zur Funktion des QRKs wurde pro Produktionsabschnitt die Funktion des Prozessregelkreismitarbeiters (PRK) installiert. Diese Funktion steht außerhalb der Teamarbeitsorganisation und beinhaltet eine Freistellung des Mitarbeiters, der sich in Zusammenarbeit mit dem Meister um die Behebung von Problemen innerhalb des Fertigungsprozesses (Anlagen, Werkzeuge, Anlieferung beschädigter Teile etc.) kümmert. Die Aufgabe des PRKs haben meist sehr erfahrene Mitarbeiter übernommen. Diese wurden (auch als eine Art Potenzialkandidat) vom Meister ausgewählt. 
Abwesenheitsplanung sowie die Unklarheiten über den Einsatz des Qualitätsregelkreismitarbeiters stark kritisiert. Der Betriebsrat, der auch maßgeblich an der Konzeptentwicklung und Implementierung beteiligt war, forderte offiziell die nachhaltige Klärung und Nachbesserung der benannten Probleme und machte dies zur Voraussetzung für die Einführung der Teamarbeit in weiteren Bereiche. Gemeinsam mit den konjunkturellen Schwankungen der Auftragszahlen des Betriebs trug dies dazu bei, dass sich die Einführung von Teamarbeit im Betrieb NFZ über einen Zeitraum von drei Jahren hinzog und von schwierigen Rahmenbedingungen begleitet war.

\section{b) KVP-Workshops}

Das zweite zentrale Instrument der partizipativen Optimierungsprozesse bilden Workshops, die entweder als regelmäßig durchgeführte KVP-Workshops zur systematischen Optimierung einzelner Produktionsabschnitte oder als Problemlöse-Workshops zur Bearbeitung genau definierter und dringlicher Probleme durchgeführt werden. In KVP-Workshops werden nacheinander jegliche Verbesserungspotenziale durch eine interdisziplinäre Workshopgruppe überprüft. Die Suche nach Verbesserungspotenzialen wird durch die im NFPS festgehaltenen Prinzipien und Methoden angeleitet. Das Ziel der Workshops besteht darin, aufkommende Probleme im Produktionsbereich zu lösen und einzelne Bereiche schrittweise zu optimieren. Im Zentrum stehen vor allem die Standardisierung und Verbesserung von Abläufen, Arbeitsplätzen und Arbeitsumgebung (Werkzeuge, Hilfsmittel) im Sinne des Produktionssystems.

Im Untersuchungszeitraum wurden die KVP-Workshops mit einem $\mathrm{MTM}^{70}$-Workshop verbunden, so dass neben der Ermittlung von Optimierungsmaßnahmen und Problemlösungen auch die Arbeitsumfänge aller Arbeitshandlungen in dem betreffenden Produktionsabschnitt von den teilnehmenden Arbeitern überprüft und ins MTM-Datensystem eingetragen wurden.

70 MTM steht für „Methods-Time Measurement“ und ist ein Verfahren zur Planung und Berechnung manueller Tätigkeitsabläufe. Es geht von dem Ansatz aus, jegliche Arbeitsbewegungen („Hinlangen, Greifen, Gehen, Bücken“) zu definieren und auf Basis zuvor festgelegter Zeitwerte die Vorgabezeiten einzelner Arbeitstätigkeiten zu ermitteln (Bokranz/Landau 2012). 
Im Vorfeld der Einführung von KVP-Workshops wurde eine KVPBetriebsvereinbarung zwischen Unternehmensleitung und Betriebsrat verabschiedet, die zusichert, dass kein Mitarbeiter infolge eines KVP-Workshops seine Stellung verliert. Falls Arbeitsplatzrationalisierung durch einen Workshop angestoßen werden sollte, werden die betroffenen Mitarbeiter in andere Bereiche versetzt und/oder durch Qualifizierungsmaßnahmen fachlich weiterentwickelt. Diese Betriebsvereinbarung schließt Rationalisierungsmaßnahmen als Ergebnis der Workshops somit nicht aus, soll den Mitarbeitern aber Beschäftigungssicherheit garantieren.

\section{Standardisierter Ablauf eines KVP-(MTM-)Workshops}

Für den Ablauf von Workshops ${ }^{71}$ in der Produktion und in produktionsnahen Bereichen, deren Maßnahmen einen Einfluss auf die Arbeit der Beschäftigten haben, gibt es ein standardisiertes Workshopkonzept, dass die Beteiligung von mindestens einem Beschäftigten pro Schicht aus diesem Bereich vorsieht. Nach Implementierung der Teamarbeit ist dafür der Teamsprecher oder ein Mitarbeiter vorgesehen, der von der Gruppe dafür ausgewählt wird. Diese Beschäftigten sind für den Zeitraum des Workshops von ihren Produktionsaufgaben freigestellt. Verantwortlich als „Auftraggeber“ des KVP-Workshops ist die jeweilige Führungskraft (Meister) des Produktionsabschnitts. Die Durchführung, Moderation und Kurzschulungen werden jedoch von einem speziell für die Methoden des Produktionssystems spezialisierten Prozesstrainer durchgeführt. Feste Mitglieder des Workshopteams sind darüber hinaus ein Betriebsrat und der für den Produktionsabschnitt zuständige Planer. Aufgrund der Ausrichtung der Workshops auf die MTM-Vorgabezeiten gehören zusätzlich spezialisierte Planer aus der Arbeitsvorbereitung zu den Workshopgruppen. Da die Meister operative Verpflichtungen in der Produktion haben, sind sie in der Regel nicht während des gesamten Workshops anwesend, sondern erkundigen sich meist zwei- bis dreimal täglich über den Fortschritt des Workshops, diskutieren die Ideen und Problemlösungen mit dem Workshopteam und entscheiden über die Umsetzung.

71 Neben den KVP-Workshops gibt es Workshops, in denen besondere Probleme im Produktionsablauf, wie zum Beispiel Fehler an Anlagen oder Qualitätsprobleme, bearbeitet werden. An diesen Workshops sind ebenfalls Arbeiter beteiligt. 
Der Ablauf dieser Workshops (sowohl von KVP- als auch von Problemlöse-Workshops) ist anhand eines standardisierten Prozesses vorgegeben, der in der Regel auf fünf Tage konzipiert ist. Zu Beginn werden die Teilnehmer in die Grundlagen des MTM-Verfahrens ${ }^{72}$ eingewiesen und erhalten eine „Verschwendungsschulung“, die die „7 Arten der Verschwendung“ beinhaltet: unnötige Wartezeit, Materiallagerung, Transportarbeiten, Arbeitsschritte und Arbeitswege sowie Reparaturen durch mangelhafte Wartung bilden Anknüpfungspunkte für Optimierungen und Standardisierungspotenziale. AnschlieBend findet eine Begehung von allen Teilnehmern des zu optimierenden Bereichs statt. Dabei sollen bei den einzelnen Arbeitsschritten und -prozessen nach Verschwendungsquellen gesucht und Optimierungspotenziale entdeckt werden. In den folgenden ein bis drei Tagen werden die Optimierungsmöglichkeiten im Workshopraum gesammelt, konkrete Umsetzungsmöglichkeiten diskutiert und priorisiert. Um die Umsetzungsmöglichkeiten überprüfen zu können, können Spezialisten aus anderen Abteilungen (Instandhaltung, Logistik, Planer) hinzugezogen werden. Am vierten bzw. fünften Tag werden die Optimierungspotenziale umgesetzt. Am letzten Tag findet eine Abschlusspräsentation statt, an der der Abteilungsleiter der Montage und die Werksleitung teilnehmen. Bei dieser Präsentation stellen die Arbeiter die Optimierungspotenziale, die durchgeführten Veränderungen und die zukünftigen Maßnahmen selbst vor. Optimierungspotenziale, die zwar genehmigt, aber während des Workshops aufgrund des hohen Aufwandes nicht umgesetzt werden können, werden auf einem Maßnahmenplan notiert und sollen in der Verantwortung des Meisters innerhalb weniger Wochen umgesetzt werden.

Das Workshopkonzept sieht jenseits der allgemeinen Information über das Stattfinden des Workshops keinen systematischen Austausch mit den Arbeitern des Bereichs vor. Da mindestens ein Teamsprecher am Workshop teilnimmt, wird implizit davon ausgegangen, dass mögliche Veränderungen bzw. Optimierungspotenziale im Vorfeld und während des Workshops mit den Teammitgliedern diskutiert und die Ergebnisse anschließend im Team bekannt gegeben werden. Somit wird es dem Rollenverständnis des teilnehmenden Beschäftigten überlassen, inwiefern die übrigen Teammitglieder in den KVPWorkshop eingebunden werden.

72 Die am MTM-Workshop teilnehmenden Beschäftigten haben im Vorfeld zudem eine gesonderte MTM-Schulung erhalten. 
Im MTM-Teil des Workshops werden im Workshopraum die einzelnen, vom Planer in der MTM-Software bereits vorbereitete Arbeitshandlungen durchgegangen, welche die Basis für die Berechnung der Vorgabezeiten bilden. In der Workshoprunde, bei der auch der Betriebsrat anwesend ist, werden alle Montagehandlungen (wie Hinlangen, Greifen, Drehen etc.) nacheinander überprüft. Die Aufgabe der Produktionsbeschäftigten besteht darin, genau zu kontrollieren, ob alle Handlungen vollständig erfasst sind. Diese Aufgabe erfordert insbesondere von den Arbeitern hohe Konzentration - zumal sie sich in hohem Maße von ihrer eigentlichen Arbeit unterscheidet. Aus diesem Grund sind die MTM-Arbeitsphasen je nach Aufwand auf zweimal ein bis zwei Stunden pro Tag begrenzt.

\section{c) Ideenmanagement}

Das Ideenmanagement bildet ein eigenes System für die Sammlung von Mitarbeiterideen zur Verbesserung der Produktion und der Arbeitsbedingungen, welches in keinem direkten Zusammenhang zu eigentlichen Arbeit steht. Obwohl auch Ideen aus Workshops gemeinsam eingereicht werden können, steht es eher gleichsam zusammenhangslos als zusätzliche Quelle zur Wirtschaftlichkeitssteigerung neben den expertengetriebenen Optimierungen und systematischen Weiterentwicklungsaktivitäten der Produktionsbereiche.

Bis zur Einführung von Teamarbeit in der Produktion wurden betriebliche Optimierungs- und Rationalisierungsaktivitäten vorwiegend von betrieblichen Experten oder Beratungsunternehmen meist im Rahmen größerer fertigungsorganisatorischer Restrukturierungen und Modernisierungen betrieben. Produktionsbeschäftigten wurde über das Instrument des Ideenmanagements die Möglichkeit gegeben, Verbesserungsideen offiziell - entweder direkt in den Briefkasten oder über die eigene Führungskraft (Dritteinreicher) - als Vorschläge einzureichen. Die Vorschläge wurden in Fachabteilungen auf ihren „Nutzen“ für die Arbeitssicherheit sowie für die Wirtschaftlichkeit des Unternehmens begutachtet und im positiven Fall umgesetzt. Die Beschäftigten waren an diesem Prozess meist nicht direkt beteiligt und die Bearbeitung dauerte teilweise sehr lange. Als Beteiligungsanreiz wurden bei Annahme des Vorschlags je nach Einsparungspotenzial nach einem klar definierten Kriterienkatalog finanzielle Prämien in Form kleiner Anerkennungsbeträge (ca. 40, 70, 180, 390 Euro) in Aussicht gestellt. 
Mit der Einführung von Teamarbeit und mitarbeitergetragenen Workshops wurde auch das herkömmliche System des Ideenmanagements angepasst. In diesem Zuge sollten bisherige Defizite, wie mangelnde Transparenz über die Bearbeitungsprozesse und lange Bearbeitungszeiten, behoben werden. Die neuen, ab 2012 gültigen Regelungen des umgestalteten Ideenmanagements sehen vor, dass nur noch „herausragende“ Ideen prämiert werden, die nicht Teil der Teamaufgaben oder Gegenstand des Workshops sind und eine Mindesteinsparung von 2500 Euro umfassen. Ausgangsüberlegung dieser Neuregelung ist, dass ergonomische, arbeitsschutztechnische, ablauforientierte und wirtschaftliche Optimierungen des Arbeitsbereichs eine selbstverantwortliche Teamaufgabe der Arbeiter sind, die sie mit Unterstützung der Führungskraft und den Servicebereichen anstoßen und vorantreiben. Im neuen Ideenmanagement werden also nur noch Ideen anerkannt, die über die eigentlich „zu erwartende" Arbeit der Beschäftigten hinausgehen und dem Betrieb einen signifikanten wirtschaftlichen Nutzen bringen. Um allerdings weiterhin die Bereitschaft der Beschäftigten zu fördern, werden als Anreize zur Ideeneinreichung mitunter kleinere oder größerer Sachgeschenke verlost.

\section{Zusammenfassung}

Das KVP-Konzept von NF mit unterschiedlichen Zugängen und Beteiligungsmöglichkeiten an betrieblichen Optimierungsprozessen setzt auf einen sehr breiten Zugriff auf die Subjektpotenziale der Beschäftigten, verknüpft sie aber deutlich mit der Zielsetzung der Wirtschaftlichkeit des Unternehmens. Neben dem organisatorischen Gestaltungsprozess setzte NF auf die Facharbeiterkompetenzen seiner Produktionsbeschäftigten. Von den Beschäftigten selbst generierte Ideen und Probleme können in Teamgesprächen untereinander diskutiert und mit dem Meister und den Fachfunktionen bearbeitet werden. Bei Problemlösungen, die einen höheren Zeit- und Gestaltungsaufwand bedeuten, können Workshops veranstaltet werden, bei denen zwei freigestellte Teamvertreter die Beschäftigten bei der Umsetzung unterstützen. Gleichzeitig wird durch die betriebsseitig initiierten KVP-Workshops den Beschäftigten die Möglichkeit gegeben, die Optimierungsaktivitäten mitzugestalten. Das neustrukturierte Ideenmanagement verstärkt die Fokussierung auf bereichsbezogene Optimierungsaktivitäten, ermöglicht aber gleichzeitig die finanzielle Gratifikation von überragenden Ideen, die eine signifikante Kostenersparnis erzielen. 
Grundsätzlich sind die einzelnen Gestaltungskonzepte integrativ angelegt und stärken die Handlungsspielräume der Beschäftigten sowie ihre Beteiligung an Optimierungsprozessen. Das hier angestrebte arbeitspolitische Konzept und die Gestaltung von KVP lassen sich anhand der oben beschrieben Gestaltungskriterien insgesamt als „strukturinnovativ“ einordnen. Die mitarbeiterorientierten Gestaltungskonzepte zur Selbstorganisation und kontinuierlichen Verbesserung sehen eine breite Partizipation der Beschäftigten an Optimierung und Gestaltung des eigenen Bereichs vor.

Aufgrund des Ausmaßes der betrieblichen Veränderungen stellt die Realisierung der Konzepte eine hohe Herausforderung für die Gesamtorganisation des Betriebs dar. Bis zum Ende des Untersuchungszeitraumes waren die Einführung und stufenförmige Entwicklung von Teamarbeit noch nicht vollends abgeschlossen.

\subsection{WLF}

WLF ist Produktionsstandort eines international agierenden EngineeringUnternehmens und klassischer Zulieferer im Feld der Antriebstechnik für Maschinenbau und Automotive-Betriebe. Am Betriebsort produziert WLF komplexe Zulieferprodukte in hoher Variantenvielfalt und unterschiedlich großen Losgrößen sowie Sonderanfertigungen. ${ }^{73}$ WLF (Betriebsstandort) hat im Jahr 2010 mit ca. 800 Mitarbeitern einen Umsatz von ca. 290 Mio. mit der Produktion, dem Verkauf und der Reparatur ihrer Produkte erzielt. ${ }^{74}$ In der Produktion selbst arbeiten ca. 580 überwiegend männliche Beschäftigte mit einem metallverarbeitenden Facharbeiterabschluss (Dreher, Schlosser, Zerspanungsmechaniker etc.). Nachdem WLF Ende der 1980er Jahre übernom-

73 WLF ist eine wirtschaftlich eigenständige Aktiengesellschaft und produziert für eine Produktsparte des Konzerns, wobei WLF nicht der einzige Standort ist, an dem diese Produkte hergestellt werden. Die Fertigung von WLF hat eine große Fertigungstiefe und gliedert sich in zwei große Produktionsbereiche (Weich- und Hartbearbeitung mit Montage), Qualitätssicherung, Supply Chain, Prozessentwicklung, technischer Service mit Instandhaltung und Produktentwicklung sowie die kaufmännischen Bereiche Personal, Controlling und Rechnungswesen. Neben der Produktion gehören - teils als eigene Geschäftsbereiche - der Verkauf, die Messtechnik sowie der Aftersales-Kundenservice zum Standort.

74 Diese Angaben beziehen sich auf unternehmenseigene Präsentationen. 
men worden war, wurde der Betrieb schrittweise fertigungstechnisch, organisatorisch und infrastrukturell modernisiert. Vor der Zergliederung des Großkonzerns hatte der Betrieb eine eher untergeordnete und vernachlässigte Stellung im ursprünglichen Mischkonzern. So berichteten die älteren Beschäftigten von zuvor herrschenden miserablen Arbeitsbedingungen, der sehr dreckigen Produktionsstätte sowie der allgemeinen Vernachlässigung und fehlenden Investitionen. Insbesondere in der Wahrnehmung der Beschäftigten wird die Übernahme durch den WLF-Konzern als eine sehr positive Entwicklung (,Zeitenwende") für den Betrieb angesehen.

\subsubsection{Arbeitspolitische Vorgeschichte}

Im Gegensatz zur NF hat es bei WLF direkt nach der Übernahme sehr weitreichende Veränderungen auf der Führungsebene und im Managementstil gegeben. Es wurde ein neuer Produktionsleiter eingesetzt, der insbesondere durch einen mitarbeiterorientierten, kooperativen Managementstil einen Wandel der bis dahin sehr hierarchischen, statusorientierten Führungskultur und bürokratisierten Abläufe bewirkte. Produktionsorganisatorisch wurde die Fabrik von einer tayloristisch, durch Einzelakkord geprägten Fertigung mit hohem Frauenanteil von ca. 75 Prozent der Produktionsbeschäftigten und wenigen technisch höherwertigen Arbeitsplätzen (Einstellertätigkeiten) zu einer kanalisierten, flussorientierten Serienproduktion umstrukturiert. Die Serienfertigung und die Verkettung der Anlagen bedeuteten zum einen transparentere Prozessabläufe, zum anderen qualifiziertere Tätigkeitsstrukturen für die Beschäftigten. Zum Zeitpunkt der Untersuchung umfasste die mechanische Fertigung und Montagearbeit sowohl automatisierte als auch manuelle Tätigkeiten. Um eine höhere Personaleinsatzflexibilität zu erreichen, wurden die einfachen Einlege- und Messtätigkeiten systematisch um qualifikatorisch höherwertige Rüst- und Einstellertätigkeiten am Gros der Produktionsarbeitsplätze erweitert, an denen nur noch Beschäftigte mit einem metallverarbeitenden Facharbeiterabschluss eingesetzt wurden. Den älteren Beschäftigten wurde ermöglicht, die Facharbeiterausbildung nebenberuflich mit Unterstützung des Betriebs nachzuholen. Das führte insgesamt zu einer Veränderung der Belegschaftsstruktur mit einem zum Zeitpunkt der Untersuchung über 80 Prozent betragenden Facharbeiteranteil und weniger als fünfprozentigen Anteil von 
Frauen in Produktion. ${ }^{75}$ Der Altersdurchschnitt der Beschäftigten beträgt zum Zeitpunkt der Untersuchung ca. 43 Jahre. In der Produktion werden - mit einem Anteil von unter zehn Prozent der Stammbelegschaft - auch Leiharbeitskräfte eingesetzt. Der Einsatz von Leiharbeitskräften wird von WLF nicht nur als personalpolitische Flexibilisierungsmaßnahme genutzt, sondern vor allem als gezielte Personalrekrutierungsstrategie. ${ }^{76}$

WLF verfolgt seit der Übernahme eine Managementstrategie, die traditionelle Top-down-Organisationsstrukturen - zumindest auf der Selbstdarstellungsebene - ablehnt und eine kooperative und unterstützende Führungsstruktur fördert, die den Mitarbeiter als wichtigen Bestandteil des Unternehmenserfolgs bewusst einbezieht und um den man sich durch nachhaltiges Personalmanagement als Betrieb kümmert. In den letzten Jahren wurden nacheinander eine Reihe von Managementinitiativen und Produktionsleitlinien eingeführt, wie Qualitätsmanagement, Zero Accidents, „Gesundes Unternehmen“, NullFehler-Konzept, TQM, Mitarbeiterbeteiligung oder Six Sigma ${ }^{77}$, welche nicht nur permanente Produktionsverbesserungen vorwiegend auf Ebene des Managements bewirken, sondern zugleich auch Arbeitssicherheit und Ergonomie an den Arbeitsplätzen erhöhen sollen.

Die Fertigung ist nach dem Linienprinzip strukturiert. An mehr als $15 \mathrm{Li}-$ nien werden jeweils verschiedene Produktvarianten in unterschiedlich großen Chargen produziert. Die Produktion ist in zwei zentrale Bereiche, die Weichbearbeitung (Schneiden, Fräsen, Drehen, Härten) und die Hartbearbeitung der Endfertigung (Schleifen und Montage), unterteilt. Obgleich sich die einzelnen Bearbeitungsschritte bei Schleif- und Montageprozessen sehr ähneln, sind die Linien fertigungsorganisatorisch aufgrund der unterschiedlichen Größe der Produkte, Umfänge der Bearbeitungsschritte und Anlagen sehr unterschiedlich

75 Wobei die meisten Frauen nicht direkt in der Produktion, sondern überwiegend im Messlabor oder in der Verpackung arbeiten.

76 Diese strategische Ausrichtung zeigte sich besonders in der Krisenzeit 2008, in der die Leiharbeitskräfte nicht entlassen wurden, um durch die eingearbeiteten und fachlich qualifizierten Beschäftigten eine angestrebte Flexibilität zu erhalten.

77 Six Sigma ist ein Managementinstrument zur Verbesserung von Prozessen und Qualitätsmanagement. Das methodische Vorgehen orientiert sich an DMAIC (Problemdefinition - Messung - Analyse - Verbesserung - Überwachung bzw. Engl. Define - Measure - Analyze - Improve - Control) von Geschäftsvorgängen mit statistischen Mitteln (Dahm/Haindl 2009). 
aufgebaut. Kleinere Typen werden in hoher Stückzahl in verketteten, höher automatisierten Anlagen (Volumenlinien) produziert, während größere Typen in Linien mit größtenteils nicht verketteten Anlagen (Flexi-Linien) produziert werden. Die Personalstruktur besteht aus schichtgängigen Beschäftigten und Schichtführern, die von einem schichtgängigen, für zwei bis drei Linien verantwortlichen Vorarbeiter und dem Meister geführt werden. Die in drei Schichten arbeitenden Beschäftigten sind je nach Produktionslinie in Arbeitsgruppen von 8 bis 30 Mitarbeitern organisiert. Aufgrund der unterschiedlichen Gruppengrößen variiert die Führungsstruktur.

Der Vorarbeiter hat die disziplinarische Verantwortung für seine Beschäftigten, organisiert den Arbeitseinsatz und kümmert sich um die Rahmenbedingungen (Bestellung von Werkzeugen/Rüstmaterialien, rechtzeitige Bereitstellung der Werkstücke, Kontakt zur Logistik). Er ist teilweise bereits in Projekte eingebunden, die seinen Bereich betreffen. Die Vertretung des Vorarbeiters übernimmt der Schichtführer, der vom Betrieb als Potenzialkandidat ausgewählt wird. ${ }^{78}$ Oberhalb des Vorarbeiters ist der Meister positioniert, der je nach Umfang eines Kanals eine oder mehrere Produktionslinien verantwortet. $\mathrm{Zu}$ den Aufgaben des Gruppenmeisters gehören neben Unterstützung der Produktionsbereiche bei Problemlösungen auch Kostenverantwortung und Personalplanung, Erfüllung von Zielen hinsichtlich der Produktivität, Liefertreue sowie Ausschussreduktion.

Die indirekten Bereiche, wie Planung, Instandhaltung und Logistik, sind zentral organisiert, arbeiten aber eng mit den Führungskräften und Produktionsbeschäftigten zusammen. Ähnlich der variablen Führungsorganisation gibt es auch hinsichtlich der Arbeitsorganisation und -situation in den einzelnen Produktionslinien einige Unterschiede, zum Beispiel hinsichtlich der Gruppengröße. Alle Beschäftigten können aber umfänglich und eigenverantwortlich zumindest an einem Arbeitsplatz arbeiten. Neben Einlege- und Entnahmetätigkeiten zählt zu den Aufgaben der Beschäftigten das Rüsten, Einstellen und

78 Mit Potenzialkandidat ist hier gemeint, dass der Betrieb nach Produktionsbeschäftigten sucht, die sowohl qualifiziert und engagiert als auch in der Lage sind, die Interessen des Betriebs gegenüber ihren Kollegen durchzusetzen. In der Regel verfügen die Schichtführer über eine Werkmeisterausbildung und dadurch grundsätzlich über das Potenzial, betrieblich Karriere zu machen. Die Gruppenleiter (Meister) und Vorarbeiter suchen die Schichtführer gezielt aus der Belegschaft aus. 
Kontrollieren der Anlagen. Durch das Erlernen weiterer Arbeitsplätze (=Anlagen) können die Beschäftigten ihr Tätigkeitsspektrum steigern.

Das Entgelt ist von der Art der Tätigkeit und Anzahl der beherrschten Arbeitsplätze abhängig. Für jeden Beschäftigten gibt es eine Qualifikationsmatrix, an der sich neben dem Qualifikationsbedarf auch die Entlohnung orientiert.

\subsubsection{KVP bei WLF}

a) „ProduktionsExzellenz“ (PE)

2007 wurde von der Konzernzentrale ein Produktionssystem namens „ProduktionsExzellenz “79 zusammengestellt, welches die zuvor eingeführten Managementinstrumente zu einem Produktionssystem integriert und im besonderen Maße die Produktionsbeschäftigten in den Mittelpunkt des Wertschöpfungsprozesses von WLF stellt.

„Das Handeln auf Aktivitäten zu richten, welche Kundennutzen schaffen, ist der Scbliussel zu unserem gewinnbringenden Wachstum; dies erfordert Zusammenarbeit und gemeinsames Handeln [...]. Wir müssen unseren persönlichen Einsatz und unseren Enthusiasmus für diese Initiative zeigen, uns engagiert einbringen, gan₹, gleich, in welcher Funktion, Tätigkeit, Region oder Position wir tätig sind." (Vorwort des CEOs von WLF in der PE-Broschüre)

PE stellt eine kontinuierliche Weiterentwicklung bisheriger Reorganisationsund Restrukturierungsprozesse zur Kundenausrichtung und permanenten betrieblichen Optimierung mittels direkter Beteiligung der Beschäftigten dar. Ebenso wie NF unterstreicht auch WLF mit dem Produktionssystem die Bedeutung des Mitarbeiters und stellt ihn explizit als verantwortungsvollen und gestaltungsfähigen Akteur in den Mittelpunkt des betrieblichen Erfolgs. Das Produktionssystem operiert mit sehr ähnlichen Methoden, Gestaltungsansätzen Grundsätzen und Handlungsprinzipien wie das KVP bei NF und andere Ganzheitliche Produktionssysteme, die sich am Toyota-Produktionsmodell orientieren: Standardisierung, verantwortlichem und problemlösendem Handeln, Kundenorientierung und ständigen Verbesserungen.

Obgleich Produktionsarbeiter bereits zuvor mit einzelnen der managerialen Optimierungsansätze und Produktionsleitlinien in Berührung gekommen waren, stellt die Einführung von PE für die Beschäftigten eine qualitative Verän-

79 Der Originalname des Produktionssystems wurde anonymisiert. 
derung im Umgang mit Optimierungsprozessen und der Produktionsorganisation dar. Beschäftigte werden durch PE in ihrem Arbeitsalltag nun direkt und systematisch in Problemlöseprozesse eingebunden.

Als Gestaltungskonzept beinhaltet PE einen stufenförmigen Bottom-upProzess von regelmäßigen Treffen der Produktionsteams, bei denen über tägliche Abweichungen im Fertigungsprozess gesprochen und nach Lösungen für diese Abweichungen und Probleme gesucht wird. Probleme, welche die Arbeitsteams nicht selbst beheben können, werden mit Fachabteilungen besprochen und können auf der nächsten Hierarchieebene eskaliert werden, auf der ebenfalls regelmäßige Abweichungsgespräche stattfinden. Zusätzlich können die Beschäftigten Verbesserungsvorschläge beim Ideenmanagement einreichen.

\section{Geschichte und Konzeptentwicklung von ProduktionsExzellenz.}

Das Konzept PE entstand im Mutterkonzern nach einer intensiven Auseinandersetzung mit dem Toyota-Produktionssystem sowie der Begehung von Toyota-Fabriken und dem Erfahrungsaustausch mit anderen Unternehmen, die ähnliche mitarbeiterorientierte Produktionssysteme eingeführt hatten. Die Betriebsführung am österreichischen Standort von WLF stand diesem Konzept erst zögerlich gegenüber, hat 2009 dann jedoch aktiv eine eigene Adaption des PE-Konzepts entwickelt und dessen organisatorische Realisierung mit dem Entschluss begonnen, dieses Konzept nicht nur in der Produktion, sondern auch in allen betrieblichen Bereichen der Fabrik einzuführen und zentral in der Führungsstruktur zu verankern. Der österreichische Standort innerhalb des Konzerns hat sich als so erfolgreich bei der konzeptionellen Ausarbeitung und konsequenten Implementierung des PE-Konzepts erwiesen, dass er eine Vorbildfunktion genießt. Einzelne Mitarbeiter des Managements bieten konzernintern PE-Schulungen und Begehungen von anderen (konzernfremden) Betrieben an.

Zur organisatorischen Realisierung der Methoden und Handlungsprinzipien von ProduktionsExzellenz wurde ein dreistufiges Bottom-upEskalationsmodell konzipiert. Die Basis des Kontinuierlichen Verbesserungsprozesses bilden auf der Stufe der Produktionsbeschäftigten kurze tägliche Abweichungsgespräche (fünf bis zehn Minuten) sowie wöchentliche Verbesserungsgespräche (20 Minuten) der Arbeitsteams in den einzelnen Linien, die auch Practice Grounds genannt werden. Während in den täglichen Abweichungs- 
gesprächen kurze Informationen und Probleme - bei WLF wird hierbei von "Abweichungen" gesprochen - thematisiert werden, werden in den wöchentlichen Gesprächen ausführlich Störungen im Produktionsablauf und dem Arbeitsbereich der Beschäftigten erörtert, mögliche Lösungsansätze diskutiert und auf einer Tafel dokumentiert. Zur Unterstützung können in den PracticeGround-Gesprächen wichtige Ansprechpartner aus anderen Produktions- oder den indirekten Bereichen hinzugezogen werden. Um den Fortschritt der Problemlösungen genau zu verfolgen und voranzutreiben, wird von der Gruppe je ein Mitarbeiter pro Thema als „Kümmerer“ bestimmt, der diese Aufgabe im Rahmen seiner Arbeit innerhalb seiner Arbeit ausführt. Darüber hinaus wird in nachfolgenden Abweichungsgesprächen zyklisch der aktuelle Stand der Umsetzung überprüft. Die wöchentlichen Gespräche werden von einem ehrenamtlichen PE-Begleiter (insgesamt 25 im ganzen Betrieb) moderiert, der aus einem anderen Bereichen des Betriebs stammt und diese Aufgabe neben seinen eigentlichen Arbeitsaufgaben übernimmt.

Auf der zweiten betrieblichen Ebene treffen sich die Vorarbeiter täglich mit ihrem Meister sowie mit Vertretern der Service-Bereiche und auf der dritten Ebene die Meister mit dem Produktionsleiter sowie den Abteilungsleitungen der Service-Bereiche, um nach der beschriebenen Logik Probleme und Abweichungen jeweils gemeinsam zu besprechen. Der Eskalationsprozess ist so konzipiert, dass ungelöste Probleme tagesaktuell auf der jeweils nächsthöheren Ebene eskaliert werden können. Einmal im Monat findet ein „Brücken“Treffen zwischen Geschäftsführung, Produktionsleitung und Betriebsrat statt. Auf diese Weise soll ein schneller, systematischer und lösungsorientierter Informationsaustausch zwischen den einzelnen Hierarchiestufen und Abteilungen der Fabrik gewährleistet werden.

\section{Implementierung}

Der Betriebsleitung und dem PE-Konzeptionsteam war es wichtig, das PEKonzept im Betrieb breit zu verankern. So wurden als erster Schritt zur Implementierung alle Manager der oberen Führungsebenen geschult. AnschlieBend erhielten alle Beschäftigten in interdisziplinären und hierarchieübergreifenden Kleingruppen die PE-Schulung. Inhalt der Schulungen war die neue Struktur der PE-Organisation sowie die detaillierte Erarbeitung der im Produktionssystem verankerten Werte, Handlungsprinzipien und Methoden. In die Schulung wurde ein Planspiel integriert, das den Beschäftigten die grundlegen- 
de Managementlogik von WLF und die engen Beziehungen zwischen Lieferund Produktionsketten bewusst machen soll. Die heterogene Zusammenstellung der Gruppen wurde von den Beschäftigten sehr positiv bewertet, da sich Mitarbeiter aus unterschiedlichen Bereichen kennenlernen konnten. Diese interfunktionalen und bereichsübergreifenden Schulungen sind beispielhaft für ähnliche Aktivitäten des Betriebs. Durch das Zusammenkommen von Beschäftigten unterschiedlicher Bereiche beabsichtigt die Führung von WLF die Verbesserung und Stärkung von Kooperationsbeziehungen innerhalb des Betriebs.

Nach den PE-Schulungen wurden im Betrieb freiwillige und ehrenamtliche Mitarbeiter gesucht, die zusätzlich zu ihrer eigenen Tätigkeit als PE-Begleiter die Moderation der Practice Grounds übernehmen. ${ }^{80}$ Der Einsatz von bereichsfremden PE-Begleitern sichert der Betriebsführung nicht nur die Vertiefung und Anwendung der PE-Prinzipien, -methoden und -werte, sondern kann die Vernetzung einzelner Bereiche und den familiären Umgang im Betrieb fördern. Die ca. 25 PE-Begleiter aus unterschiedlichen Bereichen des Betriebs treffen sich regelmäßig (alle vier bis sechs Wochen) zum Erfahrungsaustausch und werden von einem Kernteam aus PE-Experten begleitet, welches die Implementierung und Weiterentwicklung von PE beobachtet und steuert. Auf diese Weise wird versucht, neue Impulse in die Arbeitsteams hineinzutragen und die Grundprinzipien von ProduktionsExcellenz bei den Mitarbeitern inkrementell zu verankern.

Anfang 2010 wurde in 14 Practice Grounds (Linien) der Produktion gleichzeitig mit der Implementierung des PE-Konzepts begonnen. Kurz drauf folgten die angrenzenden Angestelltenbereiche, unter anderem Logistikplanung, Industrial Engineering, Qualitätssicherung. Die Arbeitsteams der Practice Grounds wurden aus den bestehenden Produktionslinien zusammengestellt, so dass sich die Gruppengröße, die Arbeitsaufgaben und Tätigkeitsstrukturen der Beschäftigten im Wesentlichen unverändert blieben. Durch die Einführung von PE wurden den Beschäftigten in ihrer jeweiligen Gruppe Wege

80 Die Übernahme von zusätzlichen, freiwilligen Aufgaben neben der eigentlichen Tätigkeit ist eine schon seit Jahren ausgeführte Praxis bei WLF. So übernehmen einzelne gewerbliche wie angestellte Beschäftigte regelmäßig Funktionen, wie Ersthelfer oder Sicherheitsbeauftragter, oder zusätzliche Mitarbeit in unterschiedlichen Arbeitsgruppen, etwa zum Gesundheitsmanagement. Dies wird bei WLF als „Ehrenamt" bezeichnet und in dieser Arbeit entsprechend als Begriff verwendet. 
innerhalb der betrieblichen Organisation eröffnet, Probleme und Störungen im Produktionsablauf systematisch zu thematisieren und Lösungen einzuleiten.

Insgesamt verlief die Realisierung des PE-Konzepts in den Produktionsbereichen unproblematisch. Aufgrund unterschiedlicher arbeitsorganisatorischer Voraussetzungen wurden jedoch, wie sich in den Auswertungen zeigen wird, die Gestaltungselemente von PE unterschiedlich gut von den Beschäftigten angenommen und umgesetzt.

\section{b) Ideenmanagement}

Das Ideenmanagement ist - wie bei NF - seit Jahren fester Bestandteil der beschäftigtenbezogenen Optimierungen. Auch hier können Beschäftigte selbstständig oder über den Vorgesetzten Vorschläge zur Verbesserung einreichen, die zentral im Ideenmanagement registriert und gratifiziert werden. Die Verfahrensweise des derzeitigen Ideenmanagementsystems unterscheidet sich aber deutlich von der Praxis bei NF. Grundsätzlich werden bei WLF keine Klassifizierung von Ideen nach Ergonomie, Arbeitssicherheit, Prozessoptimierung und Wirtschaftlichkeit vorgenommen. Von den Beschäftigten generierte Ideen werden mit dem für den Bereich zuständigen Vorgesetzten informell vorbesprochen. Im Fall eines positiven Feedbacks notieren die Beschäftigten ihre Idee mit ihrem Namen auf einem Ideenmanagement-Flipchart. Ehrenamtliche Koordinatoren - meist die integrierten Instandhalter der Produktionslinien - tragen die akzeptierten Ideen in eine Datenbank ein. Die Ideen werden von den Führungskräften aufgegriffen und in Zusammenarbeit mit dem Ideengeber oder betroffenen Beschäftigten sowie mit dem Instandhalter umgesetzt.

Auch die im Rahmen von PE generierten Ideen können als Gruppen- oder Einzelideen aufgenommen werden. Das Gratifikationssystem sieht vor, dass die Ideen nicht einzeln gratifiziert, sondern im Verlauf des Jahres gesammelt werden. Wenn ein Beschäftigter bis zu zehn Ideen eingereicht hat, erhält er auf der Weihnachtsfeier eine kleine, ab zehn eingereichten Ideen eine größere Goldmünze, die sich im Wert am Steuerfreibetrag bemisst (186 Euro). Der für das Ideenmanagement zuständige Mitarbeiter begründet die Prämierungspraxis wie folgt:

„Es geht um die Anerkennung der Beteiligung. Belohnung macht süchtig. Wo fängt man an, wo bört man auf. [...] Wenn man sagt, die Belobnung ist eine ebrliche Anerkennung, dann passt das. "(Ideenmanager WLF Min. 54) 
Ideen, die für das Unternehmen beträchtliche nachrechenbare Kosten sparen, werden gesondert behandelt. In einem Prämierungsausschuss, bestehend aus Produktionsleiter, Personalchef, Betriebsräten und Ideenmanager, wird individuell entschieden, wie hoch die Prämierung ausfällt. Gewöhnlich umfasst die Prämie 10-30 Prozent der Einsparungen für drei bis fünf Jahre nach Abzug der Kosten. ${ }^{81}$

Vorrangiges Ziel des Managements ist, mit dem Ideenmanagement und mit PE viele kleine Ideen der Beschäftigten zu fördern, um somit eine Veränderungskultur zu etablieren.

„Mit dem, dass man es zulässt, auch über kleine Ketten, über kleinste Verbesserungen nachzudenken, und es ist auch gewollt, dass die Mitarbeiter es vorbringen, mit dem etabliere ich eine sehr gesunde und gute Veränderungskultur. [...] Mit dieser Veränderungskultur kommen dann auch mit der Zeit größere und ganz große Ideen. Da babe ich meinen Benefit, auch obne, dass ich alles nacbrechne. "Ideenmanager WLF Min. 62f.)

Genaue Berechnungen von Durchlaufzeiten und Kosteneinsparungen sowie ein Festhalten an strikten bürokratischen Regelungen lehnt das Management bewusst ab, da dieses kalkulative Moment die Veränderungskultur im Betrieb und die pragmatischen Verfahrensweisen des Ideenmanagements zerstöre.

Das Ideenmanagement bei WLF ist bewusst sehr niederschwellig angelegt und basiert auf der direkten und regelmäßigen Kommunikation zwischen Führungskräften und Beschäftigten. Dadurch ist es in den Produktionsalltag integriert. Zwischen Ideen, die in den Practice Grounds erarbeitet werden, und dem Ideenmanagement werden keine Unterschiede gemacht. Die beiden Systeme greifen ineinander.

Während bei NF der bürokratische Aufwand und die Bearbeitung der Ideen im Ideenmanagement deutlich im Vordergrund stehen, zeigt sich bei WLF, dass die Veränderungskultur und die Aktivierung der Mitarbeiter die dominanten Kriterien der Gestaltung des Ideenmanagements sind.

81 Bei der Festlegung der Prämie wird berücksichtigt, wer der Einreicher ist. So werden Unterschiede hinsichtlich der Person und Funktion gemacht. Es wird danach gefragt, ob es sich um eine Führungskraft handelt, von der man erwarten kann, dass Verbesserungen zum eigenen Aufgabenbereich gezählt werden, oder ob der Einreichende ein Produktionsbeschäftigter ist. 


\subsection{Vergleich der KVP-Konzepte}

Wie deutlich wurde, forcieren sowohl NF als auch WLF mit ihrer konzeptionellen Ausrichtung der Arbeitspolitik die Beteiligung der Beschäftigten an der Optimierung ihrer Arbeitsbereiche hinsichtlich Ergonomie, Arbeitsabläufen, Fertigungsprozessen und Kostenersparnis. In beiden Betrieben lassen sich die arbeitspolitischen Gestaltungsansätze zunächst als strukturinnovativ charakterisieren. Durch Aufwertung der Arbeit, Bereitstellung von zeitlichen Ressourcen für die Arbeitsteams, Dezentralisierung und Prozessorientierung der indirekten Bereiche sollten Beteiligungsstrukturen geschafften werden, die beschäftigtengetragene Prozessoptimierungen ermöglichen.

Tabelle 4: Vergleich der KVP-Konzepte von NF und WLF

\begin{tabular}{|c|c|c|}
\hline $\begin{array}{l}\text { Konzeptionelle } \\
\text { Ausrichtung von } \\
\text { KVP }\end{array}$ & NF & WLF \\
\hline \multirow[t]{7}{*}{$\begin{array}{l}\text { Prozessoptimierung } \\
\text { als Gruppenaufgabe }\end{array}$} & Teamarbeit: & $\begin{array}{l}\text { moderierter Problemlösepro- } \\
\text { zess (ProduktionsExzellenz) }\end{array}$ \\
\hline & Unterstützung durch Vorgesetz- & $\begin{array}{l}\text { Regelmäßige Abweichungs- } \\
\text { und Verbesserungsgespräche }\end{array}$ \\
\hline & & $\begin{array}{l}\text { an standardisierten Methoden } \\
\text { orientiert (inkrementelle Einfüh- } \\
\text { rung) }\end{array}$ \\
\hline & & $\begin{array}{l}\text { Hierarchieübergreifender } \\
\text { Eskalationsprozess }\end{array}$ \\
\hline & & $\begin{array}{l}\text { Unterstützung durch Vorgesetz- } \\
\text { te und Indirekte }\end{array}$ \\
\hline & $\begin{array}{l}\text { Optimierungsinhalte: Ergono- } \\
\text { mie, Arbeitssicherheit, Abläufe, } \\
\text { Arbeitsmittel }\end{array}$ & $\begin{array}{l}\text { Optimierungsinhalte: alle im } \\
\text { Arbeitsprozess auftretenden } \\
\text { Probleme und Verbesserungs- } \\
\text { ideen }\end{array}$ \\
\hline & $\begin{array}{l}\rightarrow \text { basierend auf Selbstorga- } \\
\text { nisation der Gruppe }\end{array}$ & $\begin{array}{l}\rightarrow \text { basierend auf alltäglichen } \\
\text { Probleme des Arbeits- und } \\
\text { Produktionsprozesses }\end{array}$ \\
\hline
\end{tabular}




\begin{tabular}{|c|c|c|}
\hline $\begin{array}{l}\text { Konzeptionelle } \\
\text { Ausrichtung von } \\
\text { KVP }\end{array}$ & NF & WLF \\
\hline KVP-Workshops & $\begin{array}{l}\text { Interdisziplinär zusammenge- } \\
\text { setzt, moderiert, an standardi- } \\
\text { sierten Methoden orientiert } \\
\text { Freistellung von zwei Produkti- } \\
\text { onsbeschäftigten }\end{array}$ & keine ${ }^{82}$ \\
\hline Ideenmanagement & $\begin{array}{l}\text { Bürokratisch-zentralisiert (Be- } \\
\text { gutachtungsverfahren) } \\
\text { nur wirtschaftliche Ideen ab } \\
2500 € \text { Ersparnis akzeptiert }\end{array}$ & $\begin{array}{l}\text { dezentral organisiert (in direkter } \\
\text { Zusammenarbeit mit Instand- } \\
\text { haltung und Vorarbeiter) } \\
\text { weites Verständnis von „Ideen“ } \\
\text { (Ergonomie, Gesundheit, } \\
\text { Prozessoptimierung) }\end{array}$ \\
\hline Gratifizierung & nur über das Ideenmanagement & $\begin{array}{l}\text { Über Ideenmanagement: } \\
\text { niederschwellige, egalitäre } \\
\text { Honorierung + Beteiligung an } \\
\text { Ersparnis bei sehr wirtschaftli- } \\
\text { chen Ideen } \\
\text { Prämienlohn: Verlustzeitopti- } \\
\text { mierung (gemessen an Stück- } \\
\text { zahl pro Zeit + Ausschussre- } \\
\text { duktion) }\end{array}$ \\
\hline
\end{tabular}

Beide Betriebe unterscheiden sich hinsichtlich ihres konzeptionellen Zugangs zu Kompetenzen und Engagement ihrer Beschäftigten. NF setzt durch die Einführung von Teamarbeit in der Produktion auf die Nutzung des Selbstorganisationspotenzials der Teammitglieder. Durch die Teamgespräche werden den Teams zeitliche Ressourcen zur Verfügung gestellt, mit denen die Arbeiter

82 Bei WLF gibt es keine KVP-Workshops im eigentlichen Sinne. Gleichwohl existieren es interdisziplinär zusammengesetzte Arbeitsgruppen, etwa im Rahmen des Gesundheitsmanagements oder einzelner Projekte in der Produktion, beispielsweise zur Standardisierung von Rüstvorgängen. 
neben Aufgaben der Qualifizierung, Arbeitseinteilung und Urlaubsplanung eigenständig Probleme des Arbeitsalltags erörtern und Optimierungen initiieren sollen. Optimierungsaktivitäten bilden bei NF eine von vielen Aufgaben der Beschäftigten. Für die Verwirklichung des Konzepts „Optimierung in Teamarbeit" ist maßgeblich, dass die Beschäftigten aus der Gruppenselbstorganisation heraus eigenständig Ideen thematisieren und sich für ihre Umsetzung engagieren. Gleichsam als Ergänzung, aber auch Korrektiv der unbeherrschbaren Gruppenselbstorganisation sowie zur Unterstützung von Optimierungen wurden bei NF parallel KVP-Workshops mit Mitarbeiterbeteiligung eingeführt.

WLF hingegen wählte einen problemorientierten Zugang zum beschäftigtengetragenen Optimierungsprozess (PE als moderierter Problemlöseprozess). In den wöchentlichen, moderierten Practice-Ground-Gesprächen stehen weniger die Gruppenselbstorganisation als vielmehr die alltäglichen Probleme des Arbeits- und Produktionsprozesses im Mittelpunkt. Angeleitet, aber zugleich nicht thematisch fokussiert, sollen die Beschäftigten Probleme thematisieren, Lösungswege erörtern und sich zugleich um die Behebung von Problemen kümmern. Die systematische Kommunikation innerhalb der Practice Grounds und über einzelne Eskalationsstufen verstärkt die Fokussierung von Problemlösungen. Aspekte der Arbeitsorganisation und Arbeitseinteilung spielen bei WLF eine eher untergeordnete Rolle.

NF und WLF unterscheiden sich nicht nur hinsichtlich der Fertigungssysteme (Montage vs. mechanische Fertigung) deutliche Unterschiede, sondern auch in der Ausgangssituation der Arbeits- und Betriebsorganisation vor Einführung von KVP und Teamarbeit. Während bei NF vor der Reorganisation die Arbeit und Strukturen tayloristisch gestaltet waren, zeigte sich bei WLF durch Aufwertung der Tätigkeiten und des Funktionszuschnitts sowie durch Dezentralisierung und kooperativen Führungsstil in den letzten Jahren eine Entwicklung hin zur strukturinnovativen Organisationsgestaltung.

Die Ausgangsvoraussetzung für die Einführung von KVP fallen somit in den beiden Betrieben höchst unterschiedlich aus. Die Reorganisation bei NF markiert einen deutlichen Umbruch der etablierten Strukturen der Arbeitsteilung im Betrieb und des damit einhergehenden Rollenverständnisses. 
Tabelle 5: Vergleich der arbeitspolitischen Ausgangsbedingungen

\begin{tabular}{|c|c|c|}
\hline & NF & WLF \\
\hline $\begin{array}{l}\text { Fertigungs- } \\
\text { organisation }\end{array}$ & $\begin{array}{l}\text { Montagearbeit } \\
\text { Taktgebundene Fließfertigung }\end{array}$ & $\begin{array}{l}\text { Mechanische Fertigung } \\
\text { Linienfertigung }\end{array}$ \\
\hline $\begin{array}{l}\text { Arbeitsorganisation } \\
\text { (Vorsituation) }\end{array}$ & $\begin{array}{l}\text { Repetitive, taktgebundene } \\
\text { Tätigkeiten (hohe Taktzeiten) } \\
\text { Einfache Tätigkeiten } \\
\text { Keine Funktionsintegration } \\
\text { Geringes Maß an Selbstorgani- } \\
\text { sation } \\
\text { Geringes Maß an Handlungs- } \\
\text { und Entscheidungsspielräumen } \\
\text { Hohe Einsatzflexibilität }\end{array}$ & $\begin{array}{l}\text { Anlagenbetreuung (Einlegen, } \\
\text { Entnehmen, Rüsten, Quali- } \\
\text { tätskontrolle) } \rightarrow \text { hohes Maß } \\
\text { an Funktionsintegration (auch } \\
\text { durch Einbindung der Be- } \\
\text { schäftigten in die jährliche } \\
\text { Instandhaltung) } \\
\text { Maß an Selbstorganisation } \\
\text { und Handlungsspielräumen } \\
\text { unterschiedlich } \\
\text { Einsatzflexibilität nach Linien } \\
\text { unterschiedlich }\end{array}$ \\
\hline \multirow[t]{2}{*}{$\begin{array}{l}\text { Betriebsorganisation } \\
\text { (Vorsituation) }\end{array}$} & $\begin{array}{l}\text { Hierarchische Organisation } \\
\text { Starke Kontrolle und Steuerung } \\
\text { durch Meister und Vorarbeiter } \\
\text { Zentralisierte indirekte Bereiche } \\
\text { Top-down- } \\
\text { Kooperationsstrukturen }\end{array}$ & $\begin{array}{l}\text { Kooperativer Führungsstil } \\
\text { Dezentralisierte indirekte } \\
\text { Bereiche (nur teils räumlich } \\
\text { zugeordnet), integrierte In- } \\
\text { standhaltung } \\
\text { (teils) prozessorientierte } \\
\text { Steuerungsformen und Ko- } \\
\text { operationsstrukturen }\end{array}$ \\
\hline & $\rightarrow$ Tayloristische Organisation & $\begin{array}{l}\rightarrow \text { Entwicklung in Richtung } \\
\text { strukturinnovative Organisati- } \\
\text { onsgestaltung }\end{array}$ \\
\hline
\end{tabular}

Bei WLF wurde dieser Umbruch in den letzten Jahren inkrementell bereits vollzogen. Die Produktionsbeschäftigten, die Führungskräfte und Angestellten der indirekten Bereiche verfügen bei WLF daher über ein höheres Maß an arbeitspolitischen Erfahrungen mit posttayloristischen Formen der Arbeitsgestaltung. Allerdings ist zu bedenken, dass diese Erfahrungen nicht für alle Beteiligten positiv besetzt sein müssen. 



\section{Beteiligungsorientierungen}

Das praktische Beteiligungsverhalten der Beschäftigten in Kontinuierlichen Verbesserungsprozessen erstreckt sich aufsteigend von keiner Beteiligung über eine bewusst dosierte, selektive bis hin zu (pro)aktiver Beteiligung. Diese Beteiligungsformen ließen sich in Grundzügen in allen Untersuchungsbereichen erkennen. Zur Rekonstruktion der Beteiligungsorientierungen werden zunächst die von den Beschäftigten genannten Beteiligungsmotive eingehend analysiert . Insgesamt lässt sich im Arbeits-, Betriebs- und Rationalisierungsverständnis der Beschäftigten ein breites Spektrum unterschiedlicher Bezugspunkte für die Beteiligung an KVP erkennen - die allerdings von den Befragten sehr unterschiedlich gewichtet werden.

Dieses Kapitel befasst sich nach einer Erläuterung des Auswertungskonzepts, in welcher der empirische Zugriff auf die subjektiven Orientierungen erklärt wird, mit den beteiligungsrelevanten Motiven der Beschäftigten - zunächst in Form separater Betrachtungen einzelner subjektiver Orientierungen. Dazu findet zunächst eine inhaltliche Betrachtung der Motive und anschlieBend eine strukturelle Analyse der handlungsleitenden Orientierungen statt. Diese Analyse mündet in eine Kategorisierung von Beteiligungstypen, die sich hinsichtlich der persönlichen Gewichtung der Einzelorientierungen, des Arbeitsverständnisses, der wahrgenommenen Anerkennung, der Arbeitskraftorientierung, der erwerbsbiografischen Perspektive, der kollegialen und betrieblichen Normen sowie im Betriebs- und Rationalisierungsverständnis unterscheiden. Als Idealtypen der Beteiligungsorientierung emergieren drei klar differen- 
zierbare Beteiligungstypen: Mitgestaltungsorientierte, Karriereorientierte und Statusquo-Orientierte.

Im Folgenden wird eher von Beteiligungsdispositionen gesprochen, da mit den eingesetzten Methoden das konkrete Beteiligungshandeln und die dahinterliegenden Bestimmungsmomente nur selten direkt beobachtetes Beteiligungshandeln beschrieben werden kann. ${ }^{83}$

\subsection{Auswertungskonzept}

Die Auswertung der qualitativen Beschäftigteninterviews erfolgte in Anlehnung an die dokumentarische Methode nach Bohnsack (Bohnsack 2001; 2003) und Nohl (2006). Diese Methode ist ein mehrstufiges Verfahren zur Rekonstruktion von Orientierungen bzw. Orientierungsrahmen, die das Denken und Handeln der Menschen in ihrem jeweiligen, durch soziale Strukturen und Erfahrungen geprägten Kontext bestimmen. ${ }^{84}$ Die Herausforderung für die sozialwissenschaftliche Forschung besteht darin, diese subjektiven Bestimmungsmomente menschlichen Handelns, welche für das Subjekt selbst oftmals nicht in vollem Umfang als bewusstes, kognitiv fassbares und artikulierbares Wissen zugänglich sind, zu identifizieren.

Methodologisch basiert Bohnsacks dokumentarische Methode auf der Wissenssoziologie von Mannheim (1980), der für die Rekonstruktion des menschlichen Handelns die analytische Trennung zwischen kommunikativem und konjunktivem Wissen vorgenommen hat (Bohnsack 2003). Einerseits gibt Elemente des alltäglichen Handels, welches explizierbar und reflexiv zugänglich ist (kommunikatives Wissen), und andererseits Wissen, das den Akteuren

83 In den Interviews wird retrospektiv oder hypothetisch das Beteiligungshandeln als Konstruktion ersten Grades (Schütz 1971) dargelegt, in denen schon von den Beschäftigten Deutungen und Sinngebungen vorgenommen wurden. Da konkretes Handeln in spezifischen Situationen auch unbewusste Bestimmungsmomente enthält, lassen die Interviewpassagen nicht vollends auf das tatsächliche Beteiligungshandeln schließen.

${ }^{84}$ Ursprünglich wurde die dokumentarische Methode von Bohnsack als Analysemethode zur Interpretation von Gruppendiskussionen entwickelt, in denen in besonderem Maße kollektiv geteilte Orientierungen dokumentiert sind. Nohl (2006) hat diese Methode für die Auswertung von narrativen und leitfadengestützten Interviews weiterentwickelt. 
unbewusst ist (konjunktives Wissen). Daher können die Orientierungen nicht allein auf Basis von explizierbarem, kommunikativem Wissen rekonstruiert werden. Die un- oder vorbewussten handlungsleitenden Orientierungen liegen meist in Form von konjunktivem, impliziten und atheoretischen Wissen des Subjekts in einem spezifischen Erfahrungsraum vor. Den spezifischen Erfahrungsraum bilden in dieser Untersuchung KVP und die betriebliche Organisation von Arbeit. Das konjunktive, handlungspraktische Wissen drückt sich darin aus, wie Subjekte von der eigenen Handlungspraxis erzählen und sie beschreiben, also in der Art und Weise, wie Subjekte einzelne Themen oder artikulierte Probleme bearbeiten (Nohl 2006). Es ist somit in dem Gesagten der Subjekte dokumentiert und kann durch die Rekonstruktion des immanenten Sinngehaltes in Interviews zugänglich gemacht werden.

Bei der Auswertung der Beschäftigteninterviews zählt neben den reflexiv zugänglichen, explizit genannten Inhalten ( $W$ as wird gesagt?) vor allem die Analyseperspektive, das heißt die Frage, wie sich die Beschäftigten auf die einzelnen Gestaltungsformen der beschäftigtengetragenen Optimierungsprozesse, auf betriebliche Rationalisierungsaktivitäten, auf ihre Arbeit, ihr Verhältnis zum Betrieb sowie auf Optimierungsaktivitäten im Allgemeinen beziehen. Durch die Analyse des „Was“ und des „Wie“ können die Bestimmungsmomente, für die den Umgang der Beschäftigten mit beschäftigtengetragenen Optimierungsprozessen relevant sind, rekonstruiert werden.

Sinnliche Erfahrungen der Beschäftigten in spezifischen zeitlichen und situativen Konstellationen sind den Forschenden kaum direkt zugänglich. Selbst wenn ein Forscher, wie in Beobachtungsinterviews, in der Situationen unmittelbar anwesend ist, kann mit sozialwissenschaftlichen Methoden die sinnliche Wahrnehmung des Subjekts nur unzureichend erfasst werden. In Interviews und mögen sie noch so unmittelbar an die Situation anschließen - findet in Beschreibungen und Bewertungen der jeweiligen Situation in gewissem Sinne bereits eine Überführung in größere Zusammenhänge, subjektive Interpretationen und Sinnbezüge (wie etwa Deutungsmuster und subjektive Orientierungen) statt. Es gilt also zu beachten, dass die Darstellungen der Handlungen und subjektiven Wahrnehmung immer schon Konstruktionen bzw. Interpretationen ersten Grades sind, die durch die Rekonstruktion des Forschenden (Konstruktion zweiten Grades) in Typen und Theorien überführt werden (Schütz 1971).

Um den subjektiven Umgang der Beschäftigten mit KVP zu rekonstruieren, wird der analytische Zugang über die Identifizierung von Motiven ge- 
wählt, die von den Beschäftigten bezüglich ihrer Beteiligung an KVP in der vorliegenden Untersuchung implizit oder explizit artikuliert werden. Motive sind als stabile, konsolidierte Intentionen des Handelns zu verstehen, die subjektives Handeln in einen Sinn oder Motivzusammenhang stellen (Weber 1921, 1972). Es sind also diejenigen Bestimmungsmomente, durch die Subjekte ihrem Handeln einen subjektiven Sinn verleihen. Schütz versteht Handeln als „einen ablaufenden Prozess menschlichen Verhaltens [...], der vom Handelnden vorgezeichnet wurde, anders gesagt, der auf einem vorgefassten Entwurf beruht." (Schütz 1971: 77). Die Sinnhaftigkeit entsteht dadurch, dass die Subjekte diese ihr Handeln auf eine gewisse Regelhaftigkeit beziehen, die sich in einem impliziten oder expliziten (biografischen) Entwurf konkretisiert. Diese Regelhaftigkeit wird von Bohnsack und Nohl als „Orientierungsrahmen“85 subjektiver Handlungen bezeichnet (Bohnsack 2003). Die Art und Weise, wie Subjekte sich in ihrem Handeln auf bestimmte Motive beziehen, gibt Auskunft darüber, welche Bestimmungsmomente bzw. Orientierungen für ihren Handlungsentwurf relevant sind. Die Rekonstruktion der handlungsleitenden Orientierungen wird dadurch möglich, dass man in der Analyse nicht nur die Art der Motive erfasst, sondern auch danach fragt, wie diese von den Subjekten konstruiert und in Zusammenhang zum Handlungs- und Lebensentwurf gesetzt werden. Kurzum: Anhand von Beschreibungen von Handlungen und Erfahrungen der Beschäftigten lassen sich die Motive und die zugrundeliegenden Orientierungen rekonstruieren.

Schütz differenziert zwei Motivgruppen, um die subjektiven Handlungsentwürfe von Subjekten genauer zu analysieren: Um-zu-Motive und Weil-Motive (Schütz 1932: 93ff.). Die Um-zu-Motive eines Subjekts beziehen sich auf das Eintreten von angestrebten Ereignissen in der Zukunft. Im Sinne der Biografieforschung können damit beispielsweise längerfristige Lebenspläne eines Subjekts gemeint sein; die Ziele lassen sich aber auch kurz- oder mittelfristig auf einzelne Teilbereiche des Lebens beziehen, etwa auf die Arbeit oder die Freizeit. Um-zu-Motive verdeutlichen instrumentelle Zweck-Mittel-Relationen des eigenen Aspirationen und sind Ausdruck strategischer Handlungsentwürfe. Ein auf diese Arbeit bezogenes Beispiel für ein Um-zu-Motive ist:

85 In der dokumentarischen Methode ist auch von „Prozessstruktur“ die Rede. 
„Ich beteilige mich an Optimierungen, um dem Betrieb zu zeigen, dass ich ein fähiger und kompetenter Mitarbeiter bin. "86

Dieses Motiv kann - verkürzt betrachtet - dahingehend interpretiert werden, dass der Beschäftigte Anerkennung für seine Leistung sucht, indem er seine positive Leistung herausstellt. In diesem Um-zu-Motiv können auch Aspirationen des Beschäftigten dokumentiert sein, sich innerhalb des Betriebs weiterentwickeln und durch die Beteiligung an KVP von der breiten Masse der Beschäftigten herausheben zu wollen - als indirekte Bewerbung für einen beruflichen Aufstieg.

Die Weil-Motive hingegen beziehen sich auf die eigenen Erfahrungen in der Vergangenheit. Das eigene Tun wird also darauf bezogen, welche Erfahrungen gemacht wurden und welche Bedeutung diese für die eigene Biografie (oder Lebensbereiche) hatten bzw. haben. Sie verweisen auf Bestimmungsgründe des Handelns, die sich als sedimentierte Erfahrungen und subjektive Orientierungen habituell im Subjekt manifestieren und in Form von Handlungsentwürfen in Erscheinung treten. Diese Handlungsentwürfe beinhalten einerseits die Vorwegnahme erwarteter, zukünftiger Ereignisse, die auf Erfahrungen und Erlebnissen in der Vergangenheit beruhen. Mit anderen Worten: Weil man bestimmte Erfahrungen gemacht hat, tut man etwas oder unterlässt es (Schütz 1932; Esser 1991; Bohnsack 2003). Andererseits bringen sie auch ein identitätsbezogenes Selbstverständnis zum Ausdruck.

„Ich beteilige mich an betrieblichen Optimierungen, weil durch die Beteiligung der Arbeiter oft bessere Veränderungen erzielt werden und vermeintliche Verschlecbterungen abgewendet werden können."

Für dieses Motiv der Arbeitserleichterung spielt im Hintergrund die Erfahrung eine zentrale Rolle, dass in der Vergangenheit Veränderungen oftmals von Planern durchgeführt wurden, ohne dass die Beschäftigten einbezogen worden wären. Darüber hinaus spiegelt dieses Motiv das Bewusstsein der Beschäftigten wider, dass sie nicht nur über ausreichend fachliche Kompetenz und Erfahrungen mit den Anforderungen ihres Arbeitsbereichs verfügen, um sich beteiligen zu können, sondern dass sie sich auch beteiligen sollten, da nur dadurch ein reale eine Verbesserung der Arbeitssituation erzielt werden könne.

${ }^{86}$ Hierbei handelt sich um aus der vorliegenden Empirie sinngemäß rekonstruierte Motive der Beschäftigten. 
Handlungsentwürfe sind nicht als starre Vorstellungen, sondern vielmehr als eine Auswahl zwischen verschiedenen möglichen, das heißt ausführbaren Handlungsoptionen zu verstehen. Wie ein Subjekt die Ausführbarkeit seiner Entwürfe einschätzt und welche Entwürfe nach dem (bewussten oder unbewussten) Abwägen letztlich handlungsrelevant werden, ist durch das Wissen aus früheren Erfahrungen über Handlungsmöglichkeiten und Restriktionen, die Bedeutung des angestrebten Zielzustands sowie die Wahrscheinlichkeit, mit der ein angestrebter Zielzustand für das Subjekt erreichbar erscheint, bestimmt (Schütz 1971; Esser 1991).

Wenn man sowohl die instrumentellen Um-zu- als auch die Weil-Motive, die durch sedimentierte Erfahrungen geprägt sind, in das Zentrum der Analyse stellt, ist es möglich, nicht nur den mit einer Handlung unmittelbar verbundenen Zweck (Interessen und Bedürfnisse) zu identifizieren, sondern auch zu ermitteln, welche subjektiven Orientierungsrahmen für den Umgang der Beschäftigten mit beschäftigtengetragenen Optimierungsprozessen bedeutsam sind (Bohnsack 2003). Vergleicht man die unterschiedlichen Motive der Beschäftigten miteinander, lassen sich somit unterschiedliche Typen von subjektiven Orientierungen rekonstruieren.

\subsection{Beteiligungsrelevante Motive und Dimensionen subjektiver Orientierungen}

Die beteiligungsrelevanten Motive beinhalten ein breites Spektrum unterschiedlicher Bezüge für die Beteiligung bzw. Nicht-Beteiligung an KVP. Die Beschäftigten verorteten ihre Beteiligungsmotive keineswegs nur in den - in der arbeitssoziologischen Debatte zuhauf thematisierten - subjektiven Ansprüchen nach mehr Fachlichkeit und Interessantheit sowie Mitgestaltungsmöglichkeiten ihrer eigenen Arbeit - oder im Fall der Nicht-Beteiligung nicht ausschließlich im reinen Schutzinteresse. Bei der Betrachtung der beteiligungsrelevanten Motive (Abb. 1), die für die Analyse hauptsächlich den Beschäftigteninterviews entnommen und aus den Beobachtungsinterviews sinngemäß rekonstruiert wurden, zeigt sich deutlich, dass es ein breites Spektrum möglicher motivationaler Bezüge für die Beteiligung bzw. Nicht-Beteiligung der Beschäftigten gibt:

- Sinnhaftigkeit des eigenen Arbeitshandelns 
- Fachlichkeit

- Selbstwirksamkeit (sich in der Arbeit im weiten Sinne - auch durch Prozessgestaltung und sozialen Zusammenhang - wiederfinden)

- Anerkennung (der eigenen Person, der Arbeitsleistung, als Teil des Betriebs)

- Verbesserung der Arbeitssituation/Vermeidung einer Verschlechterung der Arbeitssituation

- Kontrolle über die Veränderungs- und Rationalisierungsmaßnahmen des Betriebs

- Verbesserung der Wirtschaftlichkeit des Betriebs

- Sicherung des eigenen Arbeitsplatzes

- Erhöhung der Aufstiegschancen

- Eigenes Rollenverständnis/Aufgabenverständnis

- Abwehr von/Schutz vor negativen Folgen

- Kollegialer Solidarität

- Betriebliche Normen

Die inhaltliche Betrachtung der Motive zeigt, dass die Beteiligungsdispositionen auf sehr unterschiedliche Bezugspunkte gerichtet sind. Neben den Auswirkungen der Beteiligung auf die Arbeitssituation stehen eigene arbeitsinhaltliche Orientierungen, normative Verhaltenserwartungen, Karriereaspirationen, Anerkennung, die eigene wahrgenommene Rolle im Betrieb (Betriebsverständnis), das Rationalisierungsverständnis, Gratifizierungen und das Schutzinteresse vor Leistungsintensivierung und Arbeitsplatzverlust. 


\section{Abbildung 1: Heuristisches Modell beteiligungsrelevanter Motive}

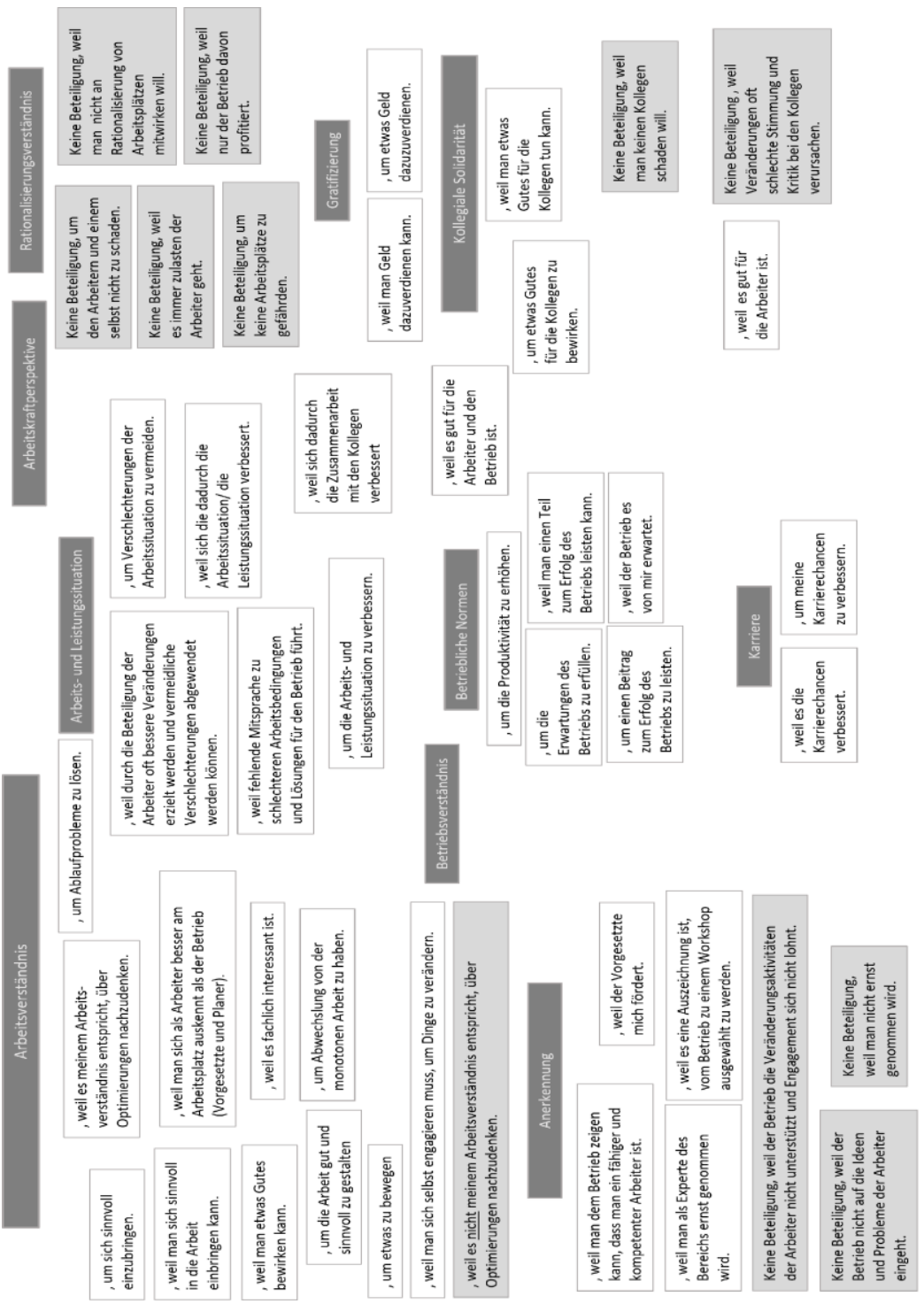




\subsection{Dimensionen subjektiver Orientierungen}

Die Motive zur Beteiligung der Beschäftigen an KVP lassen sich zentralen Orientierungsclustern zuordnen, wobei einige Motive Ausdruck unterschiedlicher subjektiver Orientierungen zugleich sein können. Die Cluster sind: Arbeitsverständnis, Anerkennung, Arbeitskraftorientierung, erwerbsbiografische Perspektive, kollektive Normen (kollegiale Solidarität und betriebliche Normen), Betriebs- und Rationalisierungsverständnis. Die einzelnen subjektiven Orientierungen lassen sich grundsätzlich bei allen Beschäftigten als wichtige Bezugspunkte wiederfinden, werden aber individuell jeweils unterschiedlich gewichtet.

\subsubsection{Arbeitsverständnis}

„Ich beteilige mich, ...

... weil es meinem Arbeitsverständnis entspricht, über Optimierungen nachrudenken."

... weil man sich als Arbeiter besser am Arbeitsplatz auskennt als der

Betrieb."

... weil durch die Beteiligung der Arbeiter oft bessere Veränderungen

erzielt werden und vermeidliche Verscblechterungen abgewendet werden können.

... weil es facblich interessant ist."

... weil man sich selbst engagieren muss, um Dinge zu verändern."

... weil man sich sinnvoll in die Arbeit einbringen kann."

... weil man etwas Gutes bewirken will."

... um etwas zu bewegen. "

... um sich sinnvoll ein₹ubringen."

... um die Arbeit gut und sinnvoll zu gestalten. "

... um Ablaufprobleme zu lösen."

... um Abwechslung von der monotonen Arbeit zu baben."

... um einen Teil zum Erfolg des Betriebs zu leisten."

... weil man einen Teil zum Erfolg des Betriebs leisten kann."

„Ich beteilige mich nicht, ...

... weil es nicht meinem Arbeitsverständnis entspricht, über Optimierungen nachzudenken."

Das Arbeitsverständnis umfasst alle beteiligungsrelevanten Motive, die als arbeitsinhaltliche Orientierungen verstanden werden können. Diese arbeitsin- 
haltlichen Ansprüche umfassen drei Bestimmungsmomente: Sinnhaftigkeit, Fachlichkeit/Erfahrung und Selbstwirksamkeit.

Im Arbeitsverständnis der meisten Beschäftigten spiegelt sich wider, dass das Nachdenken über Optimierungen als selbstverständlicher Teil des eigenen Aufgabenverständnisses betrachtet wird.

„Der optimale Arbeitsplatz, sollte für mich so sein, dass er sich ein bisschen verändert, dass ich mich, ich sage mal, geistig darauf einstellen muss, dass ich denken muss bei dem, was ich mache. Nicht so wie jetzt: schauen, nehmen, bineintragen, fertig. Sondern dass ich wirklich mal überlegen muss, was mache ich oder könnte ich das vielleicht anders machen oder so was, das wäre nicht schlecht." (Auer, NF Montage K T2A, 57ff.)

"Ich bin so und so immer gewesen. Ich habe selber einen Betrieb gehabt. Da ist KVP kein Thema, das ist das, was ein Betrieb ausmacht. Wo kann ich was verbessern, wo kann ich die Umstände ändern, das war für mich immer Thema von Haus aus, das begleitet mich sowieso schon seit Jahren. "(Eder, NFZ Montage K T2B, 1361ff.)

In diesem Fall wird KVP nicht nur als Bereicherung der eigentlichen Arbeit angesehen, sondern auch als wichtiges Instrument, sich sinnvoll und wirksam in den Arbeits- und Betriebsablauf einzubringen.

Konträr dazu gibt es Beschäftigte, welche die Beteiligung an Optimierungen dezidiert ausschließen, da dies für sie nicht zu ihrem Arbeitsverständnis gehört. Was vordergründig den Eindruck vermittelt, dass man die Beteiligung an KVP nicht als eigene Aufgabe annimmt, lässt sich auch dahingehend deuten, dass diese Beschäftigten die offizielle Verantwortung für Optimierungen nicht in der Sphäre der Beschäftigten, sondern in jener des Betriebs verorten. Denn auch bei diesen Beschäftigten lassen sich Orientierungen nach guter und sinnvoller Arbeit finden.

Sich sinnvoll und gut in die Arbeit einzubringen, wird bei der Frage nach Gründen für Beteiligung als eines der wichtigsten Motive angegeben. Im Zentrum guten und sinnvollen Arbeitens steht die Erreichung der Arbeitsaufgaben durch einen möglichst reibungslosen, gut funktionierenden Fertigungs- und Produktionsprozess. Beschäftigte beziehen sich dabei nicht nur auf Optimierungspotenziale im engeren Sinn, sondern auch auf ihre tagtägliche Arbeit und die Leistungserfüllung. Sie wollen ihre Arbeit gut und sinnvoll gemäß der von ihnen erwarteten Arbeitsleistung in Arbeitszeit und erwarteten Qualität erledigen.

„Das ist eh unsere Sache, wie wir die Arbeit machen, oder. Machen müssen wir es, in der vorgelegten Zeit, und die Qualität muss passen. Wenn das passt, warum soll irgendwer was dagegen [gegen die Beteiligung der Beschäftigten, B.S] haben." (Dauner, NF Montage K T2B, 698ff.) 
„Ja Spaß, Spaß, Spaß kann man nicht sagen. Bestätigung halt ja, wenn man das macht und es ist gut gelaufen und man sieht am nächsten Tag, man hat, weil am nächsten Tag sehen wir, wie viele Fehler wir haben und das ist alles im Rabmen, sagen wir, ein paar Febler und dies und das, dann passt das, dann ist man zufrieden, sagen wir so" (Gruber, NF Montage F T2B, 178ff.)

Aus Perspektive der arbeitsinhaltlichen Ansprüche stellt das Erreichen des Arbeitsziels ein wichtiges Element für die Zufriedenheit mit und Bestätigung in der Arbeit dar. Man ist mit der Arbeit zufrieden, wenn die Aufgaben gut erledigt werden können und damit auch der Produktionsprozess reibungslos abläuft. Was die Beschäftigten dabei als zu erreichendes Ziel betrachten, kann variieren. Mitunter werden betrieblich vorgegebene Ziele (Kosten, Qualität und Zeit) übernommen, mitunter offenbart sich ein ganz eigenes Verständnis des Leistungsziels handeln.

In Grubers Zitat wird deutlich, dass die Zielerreichung als arbeitsinhaltliche Orientierung eine stärkere Kraft zu entfalten scheint als das Erleben der Arbeit („Spaß haben“) an sich. ${ }^{87}$ Nichtdestotrotz kann die Beteiligung an KVP die Interessantheit der eigenen Arbeit steigern und durch die Vielseitigkeit der Optimierungsinhalte und Kooperationen eine willkommene Abwechslung darstellen.

Die Sinnhaftigkeit der eigenen Beteiligung an Optimierungen bemisst sich für die Beschäftigten daran, inwiefern Veränderungsaktivitäten einer verbesserten Ausführung ihrer eigenen Arbeit und dem Arbeitsprozess dienen. Maßnahmen und Regelungen, die tatsächlich die bestmögliche Erfüllung ihrer Arbeit fördern, werden grundsätzlich begrüßt und als sinnhaft erachtet.

"Ja, es gibt ja immer wieder so Sachen auch im Ablauf, wo ich denke, naja, wenn ich das jetżt besser oder anders machen würde, könnte es besser laufen und schneller sein und die Qualität besser sein."(Oswald, WLF Linie 1, 661ff.)

Besonders deutlich wird das Streben nach Sinnhaftigkeit, wenn die Beschäftigten den Mangel daran in ihrer Arbeit und insbesondere in der betrieblichen Organisation kritisieren.

${ }^{87}$ So argumentiert auch Nies (2015), die arbeitsinhaltliche Ansprüche dezidiert auf die Arbeitsergebnisse und die wahrgenommene Nützlichkeit der eigenen Arbeitsleistung für andere bezieht. Beschäftigte würden sich, selbst wenn das Erleben der Arbeit keinen Spaß, keine Freude bereitet, dennoch bei schwierigen Aufgaben am Erreichen des Ziels orientieren. Hierbei gehe es nicht um den Selbstzweck, sondern um den sozialen Bezug der Orientierung an der Erfüllung der Ziele. 
„Vergessen darf man nicht, das ist Bandarbeit und das ist Akkord, ich meine, Akkord in dem Fall nicht mehr so, es ist aber stumpfsinnige Arbeit. Es wird immer groß gesprochen von wegen, man soll sich einbringen, innovativ sein, man soll mitarbeiten und letztendlich lassen sie aber auch nicht mitarbeiten. " (Eder, NF Montage K T2B, 41ff.)

„Ja schon teilweise daran scheitert es [bilfreiche Veränderung, B.S.] oft auch, weil es ist Standard so, ob es Sinn macht oder nicht. Und das merkt man auch am Werkzeng. Ja es ist beim Standard haben wir jetzt die Nietmaschinen, aber die sind zum Schweißen, dann sag ich: ,Ich will meine alten haben', Ja, aber Standard ist das und das, und aus. " (Eder, NF Montage K T2B, 1017ff.)

Die Beschäftigten verbinden mit dem Begriff der Sinnhaftigkeit ihrer Arbeit genaue Vorstellungen darüber, wie Arbeit und Abläufe - jenseits von präzisen Arbeitsanweisungen - zu verrichten sind. Diese Vorstellungen fokussieren zumeist auf anvisierte Arbeitsziele. So entwickeln Beschäftigte aus ihrem fachlichen Verständnis, ihren arbeitsalltäglichen, aber auch lebensweltlichen Erfahrungen heraus spezifische Ansichten, wie die „Dinge im Betrieb ablaufen sollten“. Dazu zählt auch ein Problembewusstsein für Störungen im eigenen Arbeitsprozess, zum Beispiel aufgrund nicht funktionierender Werkzeuge oder weil Abläufe in ihren Augen zu umständlich gestaltet sind. Dies beinhaltet nicht nur fachliche Überlegungen, die sich auf den stofflichen Arbeitsprozess beziehen, sondern auch ökonomische Aspekte, wie in Fischers Aussage oben deutlich wird.

Diese im Arbeitsverständnis der Beschäftigten erkennbaren fachlichen und ökonomischen Orientierungen lassen sich mit den von Kratzer et al. (2015) beschriebenen technisch-funktionalen, bürokratischen und ökonomischen Rationalitätsansprüchen vergleichen, an denen die Beschäftigten die Gestaltung ihrer Arbeit sowie die betrieblichen Abläufe und Entscheidungen messen. Nicht selten sind große Diskrepanzen zwischen den vom Betrieb praktizierten Abläufen und definierten Standards auf der einen Seite und Rationalitätsvorstellungen im Arbeitsverständnis der Beschäftigten auf der anderen Seite zu erkennen.

Diese technisch-funktionalen Vorstellungen sind oftmals durch das berufssozialisatorisch erworbene Fachwissen der Beschäftigten (meist metallverarbeitende und handwerkliche Ausbildungshintergründe) und durch die praktische Erfahrung mit dem Produktionsprozess geprägt. Die Beschäftigten beziehen sich sehr häufig auf ihren eigentlichen fachlichen Ausbildungshintergrund und nehmen diesen als Referenz für eine fachlich anspruchsvolle Arbeit, obwohl sie oftmals nicht mehr in dem ursprünglichen Ausbildungsberuf arbeiten. 
Die eigenen Kenntnisse und das technisch-fachliche Verständnis der Arbeit üben eine besonders starke Prägekraft für das gesamte Arbeitsverständnis der Beschäftigten aus und beeinflussen, wie ihrer Meinung nach etwas ,richtig“ gemacht wird. Sie eignen sich ihre Arbeitsaufgaben an, bringen sich mit ihren Vorstellungen, Kompetenzen und Interessen in die Arbeit ein und versuchen, sie zu optimieren. Bei mangelnden offiziellen Beteiligungsmöglichkeiten werden die Beschäftigten auch inoffiziell in dieser Richtung tätig. Die Produktionsbeschäftigten schreiben sich selbst einen Expertenstatus bezüglich der Arbeit und Abläufe ihres Arbeitsbereiches zu und wollen sich als solche auch in den Arbeitsprozess einbringen.

„Natürlich es sind kleine Sachen, die ständig verbessert werden durch das und selber bat man auch eine Idee: Das gehört doch einmal anders gemacht. Und so wird das wirklich schneller gemacht mit dem. Das muss ich schon sagen. Da gibt es immer wieder Dinge. Wie man sagt: Das gebört doch anders gemacht. So hat man dann einen schnelleren $\mathrm{Zu}$ gang zu dem. Dass das auch wirklich dann gemacht wird. Im Endeffekt sollten wir alle so leicht und gut arbeiten wie möglich. Das wird dann doch wieder besser." (Renner, WLF Linie 1, 399ff.)

Das praktische und fachliche Wissen der Beschäftigten ist ein wesentliches Bestimmungsmoment für selbstverantwortliches und selbstbewusstes Handeln. Daraus erwächst ihr Anspruch, eigenverantwortlich zu arbeiten, sich in den Arbeitsprozess einzubringen und an der Gestaltung mitwirken zu wollen.

Die Beteiligung an Optimierung eröffnet den Beschäftigten einen Einblick in übergreifende Prozesse sowie Arbeitsabläufe und schafft Gelegenheiten, ihr eigenes fachliches Wissen zur Behebung von Problemen im Arbeits- und Produktionsprozess anzuwenden.

Zusammenfassend betrachtet ist das Arbeitsverständnis vor dem Hintergrund der eigenen fachlichen Qualifizierung und sich selbst zugeschriebenen Expertenstatus für den eigenen Bereich mit dem Anspruch auf ein selbstbewusstes und eigenverantwortliches Handeln verbunden. Die Beschäftigten generieren aus ihrem alltäglichen Arbeitsprozess heraus Ideen und ergreifen Maßnahmen, um Probleme ihres Arbeitsbereiches (ggf. darüber hinaus) zu beheben, Abläufe zu verbessern und sich mit ihren Fähigkeiten und Fertigkeiten in die Arbeit einzubringen. Die Motivation zur Beteiligung an KVP liegt darauf, die Bedingungen des Arbeits- und Produktionsprozesses so weit zu verbessern, dass die Beschäftigten ihre Aufgabe reibungslos erfüllen können.

Beschäftigte, deren Arbeitsorientierung eine ausgeprägte Bindekraft an arbeitsinhaltliche Ansprüchen aufweist, zeigen grundsätzlich eine hohe Bereit- 
schaft, sich am betrieblichen Angebot von Optimierungsprozessen zu beteiligen.

\subsubsection{Anerkennung}

„Ich beteilige mich, ...

... weil man als Experte des Bereichs ernst genommen wird."

... weil es eine Auszeichnung ist, vom Betrieb zu einem Workshop

ausgewäblt zu werden."

... weil man dem Betrieb zeigen kann, dass man ein fäbiger und

kompetenter Arbeiter ist."

... weil der Vorgesetzte mich fördert."

... weil es dafür Geld gibt."

„Ich beteilige mich nicht, ...

... weil der Betrieb die Veränderungsaktivitäten der Arbeiter nicht unter

stüt:t und sich Engagement nicht lohnt."

... weil der Betrieb nicht auf die Ideen und Probleme der Beschäftigten

eingebt."

... weil man nicht ernst genommen wird."

... weil es kein Geld gibt."

Anerkennung spielt für die Beteiligung der Beschäftigten an betrieblicher Optimierung eine bedeutende Rolle. Ihre eigene Beteiligung an Optimierungsprozessen knüpfen die Beschäftigten daran, ob und inwiefern ihre fachliche Kompetenz und ihre Erfahrungswissen als Arbeiter in ihrem Bereich sowie ihre Leistung und ihr Engagement vom Betrieb wertgeschätzt und anerkannt werden. Die Beschäftigten beziehen sich hauptsächlich aus zwei Perspektiven auf den Anspruch auf Anerkennung.

Erstens verknüpfen sie ihre Beteiligung mit der Erfahrung, dass sie in den betrieblichen Optimierungsprozess eingebunden werden, ihre Bedenken und ihre Ideen vom Betrieb beachtet und ernst genommen werden. Sie empfinden sich von den betrieblichen Akteuren als Subjekt mit Ansprüchen und Bedürfnissen sowie mit fachlichen Kompetenzen wahrgenommen und erhalten die Möglichkeit, an der Gestaltung des eigenen Bereichs mitzuwirken. Von Bedeutung ist dabei nicht nur, dass die Beschäftigten in den Optimierungsprozess einbezogen werden, sondern auch, dass ihre Ideen und Anregungen im Betrieb tatsächlich umgesetzt werden. Vor allem durch die Realisierungen ihrer Ideen 
und erfahren sie ihr Engagement als wirksam und vom Betrieb wertgeschätzt. Eine ausbleibende Umsetzung (ohne ausreichende Erklärungen) kann als fehlende Anerkennung gedeutet werden, die Beschäftigten fühlen sich dann nicht ernst genommen.

Die Gratifikation von Ideen durch das Ideenmanagement stellt für die Beschäftigten zweitens eine materielle Form der Anerkennung dar, die ihnen die Wertschätzung ihres Engagements verdeutlicht. Hierbei geht es meist nicht darum, finanziellen Vorteil durch die Beteiligung an Optimierungsprozessen $\mathrm{zu}$ erlangen, sondern vor allem darum, symbolisch als Leistungsträger und wertvolles Mitglied des betrieblichen Gefüges wahrgenommen zu werden wobei die Beschäftigten eine angemessene monetäre Teilhabe an einer Verbesserung mit hohem finanziellen Nutzen für den Betrieb durchaus als wichtig erachten. Umgekehrt wirkt sich eine komplett ausbleibende finanzielle Beteiligung meist negativ auf die Beteiligungsbereitschaft (insbesondere am Ideenmanagement) aus.

Die Beteiligung wird von den Beschäftigten häufig als eine Leistung betrachtet, die über ihre eigentliche Arbeitspflicht hinausgeht und dem Wohl des Betriebs dient. Die Beschäftigten haben die Erwartung, dass ihr Engagement vom Betrieb anerkannt und belohnt wird.

Ja, freilich. [...] D]as Selbstwertgefübl steigt auch, wenn man weiß, man ist ein bisschen eingebunden. Und nicht wie das alte Schema, wie es war vor 20 Jabren: Du, denken tun wir, und du arbeite. Das war früber so in den Werken. Darum ist auch nichts Besonders hervorgekommen. Dem Arbeiter war es Wurst. ,Die interessiert das sowieso nicht, was ich da mache. 'Dann ist halt gearbeitet worden in einem Trott. Aber heute weiß man ja, dass direkt der, der bei der Arbeit ist, oft die besseren Ideen oder auch mit dem, wenn der Chef da her innen sitzt, der weiß das ja auch nicht die Probleme, was da draußen anfällt. [...] Das hat sich wirklich stark verändert. Also das ist auf jeden Fall bundertprozentig richtig. Auf der anderen Seite hat der Arbeiter auch ein wenig das $\mathrm{Ge}$ fühl, dass er eingebunden ist und was er wert ist. Und nicht nur: Du arbeite, denken tun wir." (Wagner, WLF Linie 1, 429ff.)

Die Orientierung an Anerkennung der eigenen Leistung und der eigenen fachlichen Kompetenzen ist weniger mit einem strategischen Kalkül der Beschäftigten verbunden, sondern eher als Grundlage einer vertrauensvollen Zusammenarbeiten zu verstehen. Dies verdeutlicht die zweite Perspektive des Bezugs auf Anerkennung, bei der die Beschäftigten ihre Nichtbeteiligung an Optimierungsprozessen dadurch begründen, dass ihr Engagement, ihre Zuständigkeit für ihren Bereich und ihre fachlichen Kompetenzen von den anderen betrieblichen Akteure nicht ernst genommen und anerkannt werden. Sie erfahren sich besonders in diesem Punkt als Objekt von Optimierungs- und Rationalisie- 
rungsprozessen und fühlen sich diesbezüglich in ihren Ansprüchen und Bedürfnissen missachtet.

[Rückbezug auf einen Umbau im Arbeitsbereich, B.S.] „Wenn eine Sekunde wer wen gefragt bätte und gesagt bätte, kann man was verbessern; dann bätten wir gesagt, macht ibr ibn [den Kran, B.S.] doch, dass man $20 \mathrm{~cm}$ weiter binauffährt. Das wird einfach, ja, da kommt ein Workshop und der Workshop wird von Mitarbeitern zusammengeset:t, die dort nicht einmal arbeiten. Ich meine, es ist ja das Mindeste eigentlich, dass der Workshop da gemacht wird oder der Mitarbeiter, der dort arbeitet und nicht von der Nacbbarballe, weil dann weiß ich nicht." (Berger, NFZ Montage K T1B, 700ff.)

Dass Anerkennung eine notwendige Bedingung für die Beteiligung der Beschäftigten an Optimierungsprozesses ist, wird durch die durchgängige WeilFormulierung der Motive deutlich. Der Betrieb muss ein Mindestmaß an praktischer Anerkennung leisten, damit die Beschäftigten sich beteiligen. Praktische Anerkennung bedeutet, dass es nicht nur um rhetorische Anerkennung, zum Beispiel in Form von Lob oder „Managementfloskeln“, handeln darf, sondern dass die Beschäftigten praktisch erfahren, dass sie sich beteiligen können und dabei auch ernstgenommen werden.

\subsubsection{Arbeitskraftorientierung}

„Ich beteilige mich, ...

... weil durch die Beteiligung von Arbeitern oft bessere Veränderungen erzielt werden und vermeidliche Verschlechterungen abgewendet werden können."

... weil feblende Mitsprache zu schlechteren Arbeitsbedingungen und

Lösungen für den Betrieb fübrt. "

... um Verschlechterungen der Arbeitssituation zu vermeiden."

... weil sich dadurch die Arbeits- und Leistungssituation verbessert."

... um die Arbeits- und Leistungssituation zu verbessern. "

... um etwas Geld dazu zu verdienen."

... weil man Geld dazu verdienen kann."

„Ich beteilige mich nicht, ...

... um den Arbeitern und einem selbst nicht zu schaden."

... weil man nicht an Rationalisierung von Arbeitsplätzen mitwirken will."

... weil es immer zulasten der Arbeiter geht."

... um keine Arbeitsplätze zu gefährden."

... weil nur der Betrieb davon profitiert." 
Die Motive, die der Arbeitskraftorientierung als der ,eine[n] Weise, in der sich Arbeiter auf ihre stoffliche Tätigkeit im Arbeitsprozeß beziehen [und die] im Status des Arbeiters als Lohnarbeit begründet" (Schumann et al. 1982: 296) ist, zugeordnet werden können, lassen sich in drei Gruppen differenzieren, die als gemeinsamen Bezug das Interesse der Beschäftigten an ihrer Arbeitskraft haben. Erstens beziehen sich diese Motive auf eine konkret erfahrbare Ebene der Arbeits- und Leistungssituation der Beschäftigten. Zweitens eröffnet sich eine abstrakt generalisierende Perspektive auf den Status der abbängigen Arbeitskraft und ihrer habituell eingenommenen Schutzhaltung. Drittens verweisen die Motive auf monetäre Aspekte der Beteiligung an Optimierungen.

Motive, die sich auf die alläglich relevante Arbeits- und Leistungssituation der Beschäftigten beziehen, deuten auf das Bewusstsein der Beschäftigten hin, dass ihre Arbeit zumeist mit physischer - mitunter aufgrund einer stark beanspruchenden Leistungssituation auch psychischer - Verausgabung verbunden ist. Die Arbeit der Beschäftigten ist nicht nur eine arbeitsteilig streng reglementierte und durch leistungsrelevante Vorgabenzeiten fremdbestimmte, sondern sie vor allem auch eine körperlich beanspruchende Tätigkeit. Daher ist der Bezug auf die Beanspruchung der eigenen (vorwiegend physischen) Arbeitskraft und der Schutz eben dieser für alle Beschäftigten von zentraler Bedeutung.

Sämtliche betrieblichen Aktivitäten werden ebenso wie eigene Optimierungsaktivitäten von den Beschäftigten aus der Perspektive betrachtet, welche Auswirkungen diese auf ihre eigene Arbeits- und Leistungssituation haben. Vor allem diejenigen Beschäftigten, die schon einige Jahre in der Produktion arbeiten, betrachten Die Folgen für die Konstitution ihrer eigenen Arbeitskraft wird unter dem Aspekt, ihre Gesundheit und ihr Arbeitsvermögen bis zum Eintritt des Rentenalters erhalten zu können, kritisch, während die Jüngeren hier unbekümmerter sind.

„Wie gesagt, als älterer Mitarbeiter muss ich ganz ebrlich sagen, wird das immer scblimmer, weil der Druck wird immer mehr und da wird keine Rücksicht genommen, ob du jetzt 20 Jabre bist, 50 oder 60 Jabre bist. Du musst denselben Druck mitmachen und das wird für jeden älteren Arbeiter immer schlimmer." (Fischer, NF Montage F_A, 145ff.)

Mit dem Bezug auf die Leistungssituation kann die Beteiligung an Optimierungsprozessen den Beschäftigten die Möglichkeit eröffnen, ungünstige und belastende Arbeitssituationen zu verbessern. Beispielsweise können Arbeitsplätze oder -prozesse ergonomisch so verbessert werden, dass die Tätigkeiten 
weniger belastend sind. Damit ist allerdings auch die fortwährend präsente Gefahr verbunden, dass Tätigkeiten aufgrund von Erleichterungen der Tätigkeiten, etwa mittels Einsatz von Automationstechnik oder anderen technischen und organisatorischen Hilfsmitteln, gänzlich wegfallen und Vorgabezeiten verkürzt werden. Dies kann wiederum zu verschärften Leistungsanforderungen führen:

„[...] Das Rüsten [ist] der einrige Nachteil halt, was das Ganze hat, dass wir so schnell sind mit der Rüstzeit. [...] Wir müssen jeden Tag rüsten. [...] Und die Rüstzeiten sind schon so beruntergesetzt, und jetzt haben wir gebört, jetzt wollen sie die Rüstzeiten noch mehr heruntersetzen, dass man noch schneller ist. Aber das gebt schon jetzt fast nicht mehr, und jetzt wollen sie es noch beruntersetzen, das finde ich ein wenig komisch. Wir keriegen es nicht einmal so jetzt bin, und jetzt wollen sie es noch mebr heruntersetzen. [...]" (Unger, NF Linie 2, 96ff.)

Der Bezug auf die eigene Arbeits- und Leistungssituation kann mit einer starken Ambivalenz verbunden sein. Denn zum einen ermöglicht die Beteiligung an Optimierung eine Verbesserung der eigenen Arbeitssituation, kann aber in ihren Folgen auch eine Verschlechterung der Leistungssituation bedeuten.

Für einige Beschäftigte gehen die Folgen betrieblicher Optimierungs- und Rationalisierungsaktivitäten „immer zulasten der Arbeiter“. Darin spiegelt sich die Wahrnehmung wider, dass man als Arbeiter von den Entscheidungen des Betriebs abhängig, ihnen gar ausgeliefert ist und die Folgen der Rationalisierung unmittelbar zu spüren bekommt - sei es durch eine Verschlechterung der Arbeitssituation oder im Extremfall durch Versetzung und Arbeitsplatzverlust.

[n Bezug auf Kostenrationalisierung, B.S.] „Ich meine, wir haben eine Maschine auf unseren Kanal, da wo sie es probieren schon die ganze Zeit. Wo aber wirklich alles sich querstellt. Sie wollten auch die Maschine schon hinaushauen und wollten eine andere berstellen. Haben sie sich auch quergestellt. Also, da geht es aber um zwei Leute gleich, die was wegfallen würden. [...] Das wäre schon eine Einsparung, so gesehen. Aber das wird nie wer sagen. Da wird nie irgendwer sagen, ja passt, machen wir. Das kann ein Meister sagen, ja. Der Vorarbeiter wird schon Nein sagen. Und die Arbeiter selber sagen sowieso Nein." (Strobl, WLF Linie 2, 852ff.)

Eine Mitwirkung an Optimierungen bedeute, dass man an eben solchen Rationalisierungsfolgen mitwirkt, sich selbst oder den Kollegen Schaden zufügt und somit gegen eigene Interessen und gegen die Interessen der als Solidargemeinschaft empfundenen Arbeiterschaft verstößt. ${ }^{88}$ Genau diese Haltung wurde

${ }^{88}$ In diesem Punkt zeigt sich die Verknüpfung der Arbeitskraftperspektive mit der kollektiven Norm der Arbeitersolidarität. 
von Schumann et al. (1982) als „arbeitspolitischer Konservativismus“ bezeichnet.

Die vorgebrachten Weil-Motive deuten darauf hin, dass diese Haltung auf eigenen oder kollektiven Erfahrungen von Kollegen beruht, die sich im Bewusstsein der Beschäftigten verfestigt haben. Bemerkenswert ist an dieser Stelle, dass diese Haltung keineswegs in direkter Verbindung zu unmittelbaren Negativerfahrungen stehen muss. Einem arbeitspolitischen Konservativismus neigen auch Beschäftigte zu, die keine negativen Erfahrungen gemacht haben. Dies lässt sich dahingehend deuten, dass die Schutzhaltung gegenüber betrieblichen Optimierungen und gar Veränderungen sowohl einen sozialisatorischen als auch habituellen Charakter hat und im Bewusstsein der Industriearbeiter verankert ist.

Die dritte Motivgruppe verbindet die eigene Beteiligung mit monetären Zugewinnen und ist überwiegend bei der Beteiligung am Ideenmanagement relevant. Die Motive werden sowohl als Um-zu- als auch als Weil-Motiv formuliert und verweisen daher auf eine handlungsstrategische und erfahrungsbasierte Perspektive. Die Möglichkeit, sich etwas zum regulären Gehalt hinzuzuverdienen, spricht die Arbeitskraftperspektive der Beschäftigten an, da sie mit der Gratifikation - die je nach Art der Verbesserung sowie ihres Nutzens für den Betrieb unterschiedlich hoch ausfällt - mehr Geld für private Zwecke zur Verfügung haben. Darüber hinaus stellt sie eine Beteiligung an dem für den Betrieb generierten Gewinn dar. Doch nicht nur Perspektive der Arbeitskraft ist hier für die Beschäftigten relevant - eine Gratifikation fungiert zudem als Form der Anerkennung ihrer Beteiligung an Optimierungen, wie in Abschnitt 6.3.2 ausgeführt wurde.

\subsubsection{Erwerbsbiografische Perspektive}

„Ich beteilige mich, ...

... weil es die Karrierechancen verbessert."

... um meine Karrierechancen zu verbessern."

... weil man dem Betrieb zeigen kann, dass man ein fäbiger und

kompetenter Arbeiter ist."

Die erwerbsbiografische Perspektive hat wesentlichen Einfluss auf die Beteiligung an KVP. Sie wirkt sich positiv auf die Beteiligungsdisposition aus, wenn die Beschäftigten Karriereaspirationen verfolgen. Beschäftigte, die nach beruflicher Weiterentwicklung im Betrieb streben, verbinden die Beteiligung mit der Gelegenheit, ihren Vorgesetzten unter Beweis zu stellen, dass sie für „höhere 
Arbeiten" im Betrieb geeignet sind. Dies bezieht sich nicht nur auf eine hierarchische Karriere, die mit dem Aufstieg auf die nächste Hierarchieebene verbunden ist, sondern auch auf fachliche Karrieren, bei denen es um die Weiterentwicklung in technische Abteilungen (Instandhaltung, technischer Service) geht.

"Ja, kein scblecbter Arbeiter, bin ich sicher nicht, aber mir tangt mehr so Serienproduktion, wie kannst du was reinbringen, Abläufe organisieren, viele Sachen wegbringen, umdrehen, da lässt sich einiges machen, ja. Irgendwo so Arbeitsvorbereitung oder so irgendwas, das wär', was mir voll taugen würde, ja. " (Mayr, WLF Linie 2, 99ff.)

Der Wunsch nach beruflicher Weiterentwicklung ist nicht nur allein durch fachliches Interesse und Qualifikation sowie den Wunsch nach mehr Verantwortung für die Produktion und das Personal bedingt, sondern kann auch mit der Strategie verbunden sein, der schichtgängigen und monotonen Produktionsarbeit zu entkommen. Im Falle eines Leiharbeitnehmers kann die Karriereorientierung darin bestehen, in ein unbefristetes Arbeitsverhältnis überführt zu werden.

Eine weitere Ausprägung der erwerbsbiografischen Perspektive ist eher durch die Erwartung eines stabilen Erwerbsverlaufs gekennzeichnet. Angestrebt wird der Erhalt des Status quo angestrebt, also die Beibehaltung der jetzigen Position. Dies kann dadurch bedingt sein, dass man sich in seiner jetzigen Position eingerichtet hat und den Aufwand, den eine berufliche Weiterentwicklung bedeuten würde, nicht aufbringen möchte. Dazu zählen auch Beschäftigte, die zu einem früheren Zeitpunkt in ihrem Erwerbsleben Karriereaspirationen verfolgt, diese aber aufgegeben haben, nachdem sie sich nicht erfüllt haben.
„Nun ja, man hat keine Chancen nicht. Man ist in der Sackgasse. Und das ist das, ich meine, man sieht, dass die [Meister, BS] werden von der FH [Fachhochscbule] heraus bestückt. Das heißt, der Werkesmeister. Irgendwas macht auch keinen Sinn. Ich bätte jetz̨t die Fachakademie für Fertigungs-, Produktionstechnik, wollte ich jetzt gerne ma- chen in [der nächsten größseren Stadt, BS]. Das wäre mit Matura, wie gesagt, ich babe nur gelernt. Das wäre eigentlich auch genau, was herein passen würde. Das kann man wieder nicht machen, weil da kommt man nur erste Schicht, weil die ist auf das ausge- legt, dass man das am Abend [macht]. Das kann man nicht und somit ist das eigentlich Sackgasse."(Berger, NF Montage K T1B, 1850ff.)

Diese Perspektive ist zumeist auch mit dem Vergleich des eigenen Beschäftigungsstatus hinsichtlich Arbeitsplatzsicherheit, Arbeitsbedingungen im Betrieb, Arbeitszeiten und Entgelt mit Arbeitsplätzen außerhalb des Betriebs verbunden. Häufig merken die Beschäftigten an, dass Arbeiter mit vergleichba- 
ren Qualifikationen in der „Privatwirtschaft ${ }^{\text {“89 }}$ deutlich schlechtere Perspektiven hinsichtlich Bezahlung und Arbeitsplatzsicherheit haben.

„Und darum, genau das ist halt das Thema, warum dass keiner einen fixen Arbeitsplatz aufgibt, so wie ich jetzt zum Beispiel. Weil wie sie mich da überstellt haben und nach den ersten zwei Wochen babe ich gesagt, aus, ich kann da nicht arbeiten, das gebt nicht, das ist absolut nicht meins. " Habe ich wirklich schon überlegt, dass ich aufhöre oder kiundige oder wie auch immer. "(Dauner, NF Montage K T2B, 1079ff.)

\subsubsection{Kollegiale Solidarität}

Als kollektive Normen werden in dieser Arbeit Handlungsorientierungen der Beschäftigten verstanden, die als allgemeine Handlungs- und Verhaltenserwartungen im betrieblichen Kontext an die Beschäftigten gerichtet sind und von außen an sie herangetragen werden. Für die Beteiligung an beschäftigtengetragenen Optimierungsprozesse sind zum einen solche Normen relevant, die sich auf die Interessen der als Kollektiv verstandenen Arbeiterschaft beziehen, und zum anderen diejenigen, die als Erwartungshaltung des Betriebs wahrgenommen werden.

„Ich beteilige mich, ...

... weil man etwas Gutes für die Kollegen tun kann."

... um etwas Gutes für die Kollegen zu tun."

... weil sich dadurch die Zusammenarbeit mit den Kollegen verbessert."

... weil es gut für die Arbeiter ist."

... weil es gut für die Arbeiter und den Betrieb ist."

„Ich beteilige mich nicht, ...

... weil Veränderungen oft schlechte Stimmung und Kritik bei den

Kollegen verursachen."

... weil man keinen Kollegen schaden will."

Die genannten Motive verdeutlichen, dass eine mögliche Beteiligung der Beschäftigten an Optimierungsprozessen immer auch im Licht der Auswirkungen auf die Arbeiterschaft im Allgemeinen und auf das Verhältnis zu den direkten Kollegen im Besonderen betrachtet wird. Die Kollegen stellen für die Beschäftigten eine wichtige Referenzgruppe dar. Ein starkes Zugehörigkeitsgefühl zur

${ }^{89}$ Mit Privatwirtschaft sind im Allgemeinen kleine und mittelständische Unternehmen in der Region gemeint. 
Gruppe der Arbeiterschaft erwächst aus dem Bewusstsein, dass man als Arbeiter zu einer eher untergeordneten und abhängigen Gruppe im Betrieb gehört, deren Interessen durch betriebliche Aktivitäten nicht nur individuell, sondern gerade kollektiv gefährdet sein können.

"[Antwort auf die Frage nach guten und scblechten Seiten der Arbeit, BS] Ja die Arbeitskollegen sollen halbwegs passen, die Zusammenarbeit mit den Arbeitskollegen, ja und ja eh, die Kollegialität ist eh kelar mit den Arbeitskollegen, Firmenleitung können wir eh nicht beeinflussen. Die tun eh, was sie wollen. "(Gruber, NF Montage F T2B, 105ff.)

Gruber betont die Wichtigkeit der Zugehörigkeit zur sozialen Gruppe der Arbeiter, die dem Management mit einer gewissen Machtlosigkeit gegenübersteht. Die Wahrnehmung einer potenziellen Gefährdung durch betriebliche Optimierungs- und Rationalisierungsaktivitäten - in Form von Verschlechterung der Arbeits- und Leistungssituation oder gar des Arbeitsplatzverlustes für einige Beschäftigten - ist mit eng der Arbeitskraftorientierung der Beschäftigten und der Haltung des arbeitspolitischen Konservativismus verbunden. Die Norm, sich für das Wohl und den Schutz der Arbeiterinteressen einzusetzen, steht der gemeinsam empfundenen potenziellen Gefahr durch betriebliche Aktivitäten gegenüber. Dies stärkt das Zusammengehörigkeitsgefühl und die Solidarität der Arbeiter untereinander und verleiht den Normen, die sich auf diese kollektive Arbeitersolidarität beziehen, besondere Wirkkraft.

Frage: Macht man sich selbst Gedanken darüber, wie man die Arbeit oder Ablänfe besser machen könnte? BS] „Muss man und hat man auch schon eben durch die Verbesserungsvorschläge da und dann. Es gibt es eh einen murz. Anreiz. Mir ist ja wieder sebr wichtig, wenn man das voneinander haltet, ob man sich nicht selber wegrationalisiert oder den anderen was zu Fleiß tut, sondern/ Aber es gibt überall balt sein Maß und sein Ziel, dort, wo man das sieht. "(Czerni, NF Montage K T3B, 1077ff.)

„Das wäre schon eine Einsparung, so gesehen. Aber das wird nie wer sagen. Da wird nie irgendwer sagen, ja passt, machen wir. Das kann ein Meister sagen, ja. Der Vorarbeiter wird schon Nein sagen. Und die Arbeiter selber sagen sowieso Nein." (Strobl, WLF Linie 2, 852ff.)

Ähnlich wie bei der Arbeitskraftorientierung beziehen sich die Beschäftigten in zweierlei Weise auf die kollegiale Solidarität. Zum einen wird die eigene Beteiligung mit der Intention verbunden, die Arbeits- und Leistungssituation für sich und seine Kollegen zu verbessern, etwa durch Ergonomieverbesserungen. Zum anderen wird durch Nichtbeteiligung an Optimierungsprozessen eine mögliche Verschlechterung der Arbeits- und Leistungssituation sowie eine weitere Benachteiligung der Beschäftigten gegenüber dem Betrieb vermieden. 
Der Schutz des aktuellen Status quo der Beschäftigten ist ein zentraler Bezug im Handeln der Beschäftigten. Eine Verletzung dieser Norm würde sich negativ auf die Kollegialität und Zusammenarbeit der Beschäftigten auswirken und sogar Sanktionen von den Kollegen nach sich ziehen.

„Ich kann sowieso kein Verbesserungsvorschlag machen, wenn ich nicht mit dem Team rede. Ich kann es ja nicht nur für mich besser machen. Wenn es für mich besser ist und fünf andere tun sich schwer durch das, ist keine Verbesserung nicht. Dann ist ein Rückscbritt, das muss ich zuerst abklären und wenn andere sagen: ,Ok, das passt, dann machen wir es. "(Fischer, NF Montage F T1 A, 902ff.)

Umgekehrt wird aufgrund der kollektiven Solidarität bei arbeiterinitiierten Verbesserungen Rücksicht auf die Kollegen genommen, damit für niemanden ein Nachteil entsteht.

Die Wirkkraft der Norm kollektiver Solidarität wird besonders deutlich, wenn man auf das negativ formulierte Beteiligungsmotiv schaut: „Ich beteilige mich nicht, weil Veränderungen oft schlechte Stimmung und Kritik bei den Kollegen verursachen." Veränderungs- und Optimierungsideen können von Beschäftigten zurückgehalten werden, da man dafür eventuell Kritik und Anfeindung von den eigenen Kollegen ernten könnte. Die gute Zusammenarbeit zwischen den Kollegen wird von den Beschäftigten als wichtige Ressource ihrer Arbeitssituation angesehen.

[Beschreibung einer eigenständig vorgenommenen Laufzeitoptimierung von Anlagen um zwei Minuten, BS] „D as war zwar eh ein Kampf am Anfang, aber ich glaub, ich hab' gewonnen, im Großen und Ganzen. Und sie seben es jetzt selber ein, dass die Arbeit eigentlich leichter geworden ist." (Mayr, WLF Linie 2, $113 f f$.

Aufgrund des starken Einflusses der kollektiven Norm der Arbeitersolidarität ist es für Beschäftigte, die sich an Rationalisierungen beteiligen, kaum vermeidbar, sich mit dieser Norm und den potenziellen Auswirkungen ihres Handelns auf die Interessen der kollektiven Arbeiterschaft auseinanderzusetzen.

\subsubsection{Betriebliche Normen}

„Ich beteilige mich, ...

... um die Produktivität ₹u erböhen."

... weil der Betrieb es von mir erwartet."

... um die Erwartungen des Betriebs zu erfüllen."

... weil es gut für die Arbeiter und den Betrieb ist."

... um einen Beitrag zum Erfolg des Betriebs zu leisten."

... weil man einen Teil zum Erfolg des Betriebs leisten kann." 
Auf die Orientierungen an betrieblichen Normen weisen diejenigen Motive hin, die sich auf das vom Betrieb bzw. Management und Vorgesetzten erwartete Verhalten der Beschäftigten richten. Die betrieblichen Normen beziehen sich im engeren Sinn auf Wirtschaftlichkeitsziele des Betriebs, wie Steigerung der Produktivität und Kostensenkung, und verkörpern die Erwartung, dass die Beschäftigten dieses Wirtschaftlichkeitsdenken in ihren Arbeitsalltag einfließen lassen. In einem weiteren Sinne beinhalten die betrieblichen Normen auch Beschäftigteninteressen, wie den Erhalt und die Qualifizierung der Arbeitskraft, da der Betrieb ebenfalls ein starkes Interesse an geringem Krankheitsstand und qualifizierten Beschäftigten hat. Die Beteiligung an Optimierungen kann hierbei als ein vom Betrieb gewünschtes Engagement verstanden werden, dass die betrieblichen Ziele unterstützt.

Diese implizit oder explizit formulierten und von den Beschäftigten wahrgenommenen Verhaltenserwartungen des Betriebs können konkrete Verhaltensaufforderungen durch das Management und vor allem durch den direkten Vorgesetzten sein, wie der Appell zur Beteiligung an KVP, oder auch abstrakter als allgemeine Handlungsempfehlungen für den alltäglichen Arbeitsvollzug daherkommen, wie abstrakte Handlungsanweisungen der Produktionssysteme, wie „richtig von mir" oder „fehlerfrei durch mich“"90, beschreiben.

Die betrieblichen Normen sind für die Beschäftigten auch deswegen von grundlegender Relevanz, da sie als abhängig Beschäftigte im Produktionsprozess auf den Erfolg und Bestand des Betriebs angewiesen sind. Somit entfalten die betrieblichen Normen eine solche Wirkkraft, dass die Beschäftigten den Interessen des Betriebs zumindest nicht absichtlich zuwiderhandeln und Produktionsprozesse oder Arbeitsabläufe sabotieren. Die betrieblichen Normen können allerdings auch im Widerspruch zur Arbeitskraftorientierung der Beschäftigten stehen, da betriebliche Optimierungsaktivitäten ebenfalls mit der Gefahr verbunden werden, sich negativ auf die eigene Arbeits- und Leistungssituation auszuwirken. Aus diesem Grund kann nicht selbstverständlich davon ausgegangen werden, dass die Beschäftigten ihr Handeln unreflektiert an den betrieblichen Normen ausrichten.

90 „Richtig von mir“ und „fehlerfrei durch mich“ stellen jeweils Säulen der Produktionssysteme der Fallbeispiele dar, die den Beschäftigten des ganzen Betriebs eine zentrale Bedeutung für die Qualität und den Erfolg des Produktionsprozesses zuweisen. 
[Frage: Soll man sich als Arbeiter an Optimierung und Kostensenkung beteiligen?, BS] "Ja, wenn es möglich ist, dass ich heute sage, es gibt irgendwelche Sachen, dass das positiv ist, ist bestimmt keine schlechte Idee nicht. Weil es ist ja trotzdem letztendlich deine Arbeit. "(Czerni, NF Montage K, T3B, 1157ff.)

Wenn es um die Beteiligung an Optimierungsprozessen geht, ist die Orientierung an betrieblichen Normen davon abhängig, was der Betrieb als Optimierungspotenzial anerkennt und fördert. Die betriebliche Definition von Optimierungspotenzialen kann sich beispielsweise nur im engen Sinn auf wirtschaftliche Kostensenkungsziele beziehen oder aber in einem breiteren Verständnis auch nicht wirtschaftlich messbare Optimierungsinhalte umfassen, wie ergonomische Verbesserungen oder organisatorische Abläufe, und ebenfalls die Interessen der Beschäftigten einbeziehen. Das Verständnis von Optimierungspotenzialen kann somit von Betrieb zu Betrieb oder von Fallkonstellation zu Fallkonstellation unterschiedlich eng oder weit ausfallen. Konkret erfahrbar wird das betriebliche Verständnis von Optimierungspotenzialen darin, welche Arten von Optimierungen vom Betrieb unterstützt und umgesetzt werden. Die Reichweite der Optimierungsinhalte entscheidet darüber, ob sich betriebliche und kollegiale Normen, arbeitskraftorientierte und arbeitsinhaltliche Ansprüche miteinander vereinbaren lassen oder ob sie in Konflikt zueinanderstehen.

„Ja, ich meine überhaupt generell Arbeiten, also das jetzt als Schichtfübrer gesehen, ist es, weil mir das gefällt, die Materialflusssteuerung91, was man einfach alles steuern kann, und die Einteilung, als das VorausplanenT Voraussehen. Weil es einfach ein großes Spektrum ist, also was ich überschauen soll. [...] Das ganze Drumberum, einfach mit Mitarbeiter einfach ein gutes Ergebnis ervielen - das ist die eigentliche Herausforderung. Gute Schicht, sag ich mal, wo man auch eher kumpelhaft, sage ich einmal freundschaftliches Verbältnis bat." (V aller, WLF Linie 4, 163ff.)

\subsubsection{Rationalisierungsverständnis}

„Ich beteilige mich nicht, ...

... weil es immer zulasten der Arbeiter geht."

... weil nur der Betrieb davon profitiert."

... weil man nicht an der Rationalisierung von Arbeitsplätzen mitwirken will."

... um den Arbeitern und einem selbst nicht zu schaden."

91 Beispiel für Übernahme der Managementsprache. 
„Ich beteilige mich, ...

... weil es gut für die Arbeiter und den Betrieb ist."

... um die Produktivität zu erböhen."

Motive, die sich auf Rationalisierung beziehen, verweisen auf zwei diametrale Haltungen der Beschäftigten. Zum einen verbinden die Beschäftigten mit Rationalisierung negative Folgen für die Arbeiter, zu denen man selbst nicht beitragen möchte; zum anderen wird Rationalisierung im Licht der Wirtschaftlichkeit und letztlich der Beschäftigungssicherheit positiv betrachtet. In den Formulierungen drückt sich eine grundsätzliche Haltung der Beschäftigten aus, da die entsprechenden Aussagen in den Interviews generalisiert und meist wenig konkretisiert geäußert wurden.

In den negativen Bezugnahmen „zulasten der Beschäftigten“ und „nur der Betrieb profitiert" drückt sich aus, dass Beschäftigte sich als Rationalisierungsobjekt sehen, da sich Rationalisierung aus ihrer Sicht vorwiegend negativ auf die Leistungs- und Beschäftigungssituation auswirkt. Die Beschäftigten verbinden Rationalisierungsaktivitäten mit steigenden Leistungsanforderungen, etwa durch die Reduzierung von Vorgabezeiten, oder mit Arbeitsplatzverlust. Diese Aktivitäten wenden sich direkt und unmittelbar gegen die Arbeitskraftorientierungen der Beschäftigten. Etwaige positive Erfahrungen mit beschäftigtengetragenen Prozessoptimierungen haben sich in diesem Rationalisierungsverständnis (bislang) nicht verfestigt.

In der Auseinandersetzung mit dem betrieblichen Beteiligungsangebot zeigen die Beschäftigten beider Betriebe gleich wohl den für Industriearbeiter typischen strukturellen Interessenantagonismus zwischen den betrieblichen Interessen an Profitabilität und Wirtschaftlichkeit einerseits und den Schutzinteressen der Beschäftigten andererseits. Doch selbst wenn Rationalisierung als notwendige Maßnahme zur Wirtschaftlichkeit und Arbeitsplatzsicherheit angesehen wird, ist im Verständnis der meisten Beschäftigten kein vollständiger Rollenwechsel vom Objekt zum Subjekt der Rationalisierung erkennbar. Sogar bei den Beschäftigten, die sich sehr umfangreich mit eigenen Ideen und Problemlösungen in Optimierungsprozesse einbringen, zeigen sich Schutzhaltungen gegen potenzielle betriebliche Rationalisierungen. Die Anwesenheit des wahrgenommenen Interessenantagonismus bedeutet allerdings nicht, dass sich einzelne Beschäftigte nicht auch in Teilen auch als Subjekte von Optimierungsund Rationalisierungsprozesse verstehen. 
[BS: „Sollte man sich an Optimierung und Kostensenkung beteiligen?"] „Ja, freilich. Es beißt eh immer, du sollst eine Qualität liefern, Qualität sind Kosten. Arbeite ich eine gute Qualität, habe ich keine Nacharbeit, sind die Kosten von Haus aus schon mal weniger. Du sollst nichts verschwenden oder sonst was. Aber das machst du dabeim auch. Du lässt ja beim Tag auch nicht das Licht brennen, weil es so lustig ist. Drehst das ja auch ab. Oder wenn du irgendwo gegessen hast, räumst du auch dein Zeug weg, dass es nicht umbersteht oder sonst was. Das ist wieder Charaktersache, der eine tut's, der andere nicht. [...] Ja, wenn sich die Firma Geld spart, verkaufe ich das Produkt vielleicht billiger, bin ich besser im Mark.t. Ich habe weiß Gott was für Kosten. Also die Gedanken mache ich mir schon, ob es sich andere machen, weiß ich nicht. Ich weiß nicht, was andere denken. Gott sei Dank. "(Fischer, NF Montage F T1 A, 851ff.)

Auf jeden Fall. Natürlich, es ist ja im Endeffekt mein Arbeitsplatz dann. Wenn wir wirtschaftlich sind und Gewinne machen, dann ist mein Arbeitsplatr. gesichert, am ehesten. Wenn wir auf Defizit produzieren, dann wird im Endeffekt gesagt: Das sperren wir zu. Wir sind ein Konzern, sagen halt: Ihr in [Ort, BS] ihr, ihr macht keinen Gewinn. Gehen wir in den Ostblock. Sind sie billiger. Es ist aufjeden Fall wichtig, im Endeffek.t unsere Arbeitsplätze. " (Renner, WLF Linie 1, 546ff.)

Wirtschaftlichkeitsaspekte lassen sich im Handeln der Beschäftigten wiederfinden. Die Ausrichtung auf wirtschaftliches Handeln übersteigt aber selten das Ausmaß eines ressourcenschonenden oder nachhaltigen Umgangs mit Material oder Energie, wie man es im Rahmen der privaten, sparsamen Haushalts- und Lebensführung macht. Von Bedeutung ist hierbei vielmehr, dass die Beschäftigten sich bewusst sind, dass die eigene Beschäftigungssicherheit von der Wirtschaftlichkeit des Betriebs abhängig ist, die angesichts des starken Wettbewerbsdrucks, globaler Produktionsnetzwerke und potenzieller Verlagerung der Produktion an Niedriglohnstandorte auch in der Wahrnehmung der Beschäftigten zunehmend unter Druck gerät. Im Vergleich zur „Gefahr von außen“ wird die „Gefahr von innen“ durch betriebliche Rationalisierungs- und Veränderungsmaßnahmen jedoch als unmittelbarer und bedrohlicher wahrgenommen.

Ungeachtet der unterschiedlichen realen Gestaltungsformen beschäftigtengetragener Optimierungsprozesse lässt sich bei fast allen Beschäftigten das Bewusstsein erkennen, als Produktionsbeschäftigter bzw. Lohnarbeiter und insbesondere als Montagearbeiter grundsätzlich Objekt oder gar „Opfer" von betrieblichen Rationalisierungsprozessen zu sein. Die wahrgenommene Abhängigkeit als Lohnarbeiter geht somit mit einer zumindest latent vorhandenen Haltung des arbeitspolitischen Konservativismus einher. Zumeist grenzen sich die Beschäftigten von betrieblichen Rationalisierungsmaßnahmen ab und verweisen die Zuständigkeit für Optimierung und Kosteneinsparung in die Sphäre des Betriebs. 
„Ich glaube, dass so zwiespältig ist, dass man ja nichts Falsches tut oder sagt, ,abm, vielleicht passt das derjenigen Person nicht.'Ja. Ist lieber, man macht seine Arbeit, schaut was geht, redet mit dem Vorarbeiter oder mit dem Meister. Und der kann das weitergeben oder irgendwas. Aber lieber selber seine Arbeit machen, sicher verbessern, einbringen, was wichtig ist. Aber das lieber denen, Leuten lassen, sagen wir so. "(Unger, NF Linie 2, $787 f f$.)

Die einzige Ausnahme hierbei stellt der Fall der reinen Karriereorientierung (Mayr) dar, der sich in seinem Handeln im hohen Maße an betrieblichen Normen, wie Steigerung der Produktivität und Kostensenkung, orientiert und diese nicht als persönliche Bedrohung seiner Arbeitskraft ansieht. Er bezieht nicht nur technisch-funktionale Überlegungen, sondern auch orientiert sich in einem viel deutlicheren Maße an ökonomischen Zielen der Profitabilität und Wirtschaftlichkeit, als es Mitgestaltungsorientierte und schon gar nicht Status-quoOrientierte tun.

Wie stark der Interessengegensatz zwischen Beschäftigten- und Betriebsinteressen wahrgenommen wird und wie sehr der arbeitspolitische Konservativismus im Handeln der Beschäftigten zum Ausdruck kommt, scheint dadurch bedingt, als wie bedrohlich die Beschäftigten vorherrschenden Beschäftigungsbedingungen und ergriffenen bzw. anvisierten Rationalisierungsmaßnahmen und ansehen und welche Erfahrungen sie im Laufe ihres Erwerbslebens mit Rationalisierung gemacht haben. Beschäftigte, die bereits häufiger auf negative Weise von betrieblicher Rationalisierung betroffen waren, etwa Arbeitsplatzverlust oder unfreiwillige Versetzungen erlebt haben, sind gegenüber betrieblicher Rationalisierung kritischer eingestellt und nehmen eine sehr bewusste Schutzhaltung ein.

\subsubsection{Betriebsverständnis}

„Ich beteilige mich, ...

... weil man sich selbst engagieren muss, um Dinge zu verändern. "

... weil man als Experte des Bereichs ernst genommen wird."

... um einen Teil zum Erfolg des Betriebs zu leisten."

„Ich beteilige mich nicht, ...

... weil der Betrieb die Veränderungsaktivitäten der Arbeiter nicht unter

stützt und sich Engagement nicht lohnt."

... weil der Betrieb nicht auf die Ideen und Probleme der Arbeiter eingeht."

... weil man nicht ernst genommen wird." 
Das Betriebsverständnis der Beschäftigten ist eng mit dem Rationalisierungsverständnis und der Arbeitskraftperspektive verknüpft. In der Formulierung der Motive (,weil ...") wird auch hier deutlich, dass Erfahrungen sozialer Situationen in generalisierte Haltungen übergehen. Die Frage, inwiefern sich Beschäftigte als Objekt betrieblicher Rationalisierung und als von Rationalisierung Benachteiligte betrachten, ist Teil des Betriebsverständnisses. Die hier angeführten Motive beziehen sich auf die wahrgenommene Stellung der Beschäftigten innerhalb des Betriebs, die für die Beteiligung an Optimierungsprozessen relevant ist. In der wahrgenommenen existierenden bzw. fehlenden Unterstützung im Zuge beschäftigtengetragener Optimierungsprozesse spiegelt sich die relative Position der Beschäftigten im Verhältnis zu den anderen Beschäftigtengruppen wider. In der Art und Weise, ob und wie der Betrieb auf Ideen und Probleme der Beschäftigten - auch und insbesondere im Arbeitsalltag jenseits des Kontexts von beschäftigtengetragenen Optimierungsprozessen - eingeht und die Beschäftigten ernstnimmt, nehmen sie Über- und Unterordnungsverhältnisse im betrieblichen Sozialgefüge wahr.

„Das Betriebsklima, naja wie sagt man da ja, es ist zwischen Arbeiter und der oberen Ebene ist es nicht so gut, was man so bört. [...] Wie soll man sagen, die Verständigung zwischen Oben und Unten, da gehört ein bisschen mehr her. [I: Verständigung heijs Kommunikation, also es wird nicht genug miteinander geredet, oder?"] "Ja, wie sie es immer sagen, es ist sowieso aufgekommen, aber die Fragen da, denkst du, dass es eigentlich zu eng ist. Die oben sind da und der Arbeiter ist da und der soll arbeiten und die tun das. "(Gruber, NF Montage F T2B, 28ff.)

"Ja, die Stimmungsschwankungen sind immer dann da, wenn wir recht viel Stress haben, wann es vom [Meister, BS] wieder einen auf den Deckel gegeben hat, weil irgendetwas nicht passt. Oder wann eben Leute unscbuldig beschuldigt werden für irgendwelche Sachen, für die sie nicht mal was können. Weil wenn mir bei der Maschine der Roboter irgendeinen Scheiß zusammenfährt. Und auf einmal wo anfährt. Was kann ich dafür. Aber ich werde dafür angespannt. Und das sind Sachen, die was ich nicht verstehe." (Strobl, WLF Linie 2, 942ff.)

Wie für Industriearbeiter charakteristisch, verorten sich die Beschäftigten beider Betriebe als untergeordnete soziale Gruppe innerhalb des betrieblichen Sozialgefüges (Kudera et al. 1979; Kuhlmann/Schumann 2000). Wahrgenommene Dichotomien zwischen „oben“ und „unten“, lassen sich durchgängig bei allen Beschäftigten erkennen. Diese Dichotomien treten für die Beschäftigten besonders im Umgang der einzelnen sozialen Gruppen untereinander, also in Kooperationsbeziehungen und Kommunikationsformen zwischen den Beschäftigten und Führungskräften sowie gegenüber Vertretern unterstützender Bereiche, in Erscheinung. In den sozialen Umgangsformen werden soziale 
Machtdistanzen zwischen den einzelnen betrieblichen Gruppen und besonders in der betrieblichen Hierarchie deutlich. Die Beschäftigten erwarten in den sozialen Interaktionen und alläglichen Aushandlungsprozessen ein Mindest$\mathrm{maß}$ an zwischenmenschlichen Umgangsformen, die zeigen, dass ihnen mit Respekt begegnet wird, zum Beispiel gegenseitiges Grüßen und einen angemessenen Tonfall. Hierzu zählen in den Augen der Beschäftigten aber auch Formen der persönlichen Kontrolle und die Möglichkeit, während der Arbeitszeit menschlichen Bedürfnissen nachgehen zu können, etwa die Toilette aufzusuchen oder etwas zu trinken.

Kontext: Aushandlung über die zeitliche Verwendung des Qualitätsregelmitarbeiters im Team] „Bei uns läuft das so, also wir haben echt diskutieren müssen mit der Segmentleiterin wegen den WC-Pausen und da ist dann balt wirklich die Emotionen schon so boch gekocht teilweise und die baben gesagt, ,hey, dann drücke ich auf den roten Knopf - also auf den NOT-AUS - und gebe auf das Klo, weil das ist ein Grundrecbt und das kann mir nicht verwehrt sein, egal, ob wir Akkord haben oder nicht. 'Das wurde lange diskutiert und jetzt ist es halt so, für Klopausen ist er da, wenn jetzt irgendeiner einen Febler gebaut hat, ist er da, so jal Aber es bängt halt einfach auf den Tag darauf an. "(Berger, NF Montage K T1B, 1202ff.)

„Und das ist auch einfach der Druck in dem ist auch einfach mehr geworden. Und dann, ja die Personalabteilung, teilweise zu wenig Personal, wie es öfters ist, und dann die Unzufriedenheit, wo sich die Leute dann wieder aufregen und Streitereien bier und da sind und auch von den Vorgesetzten her. Das ist eher, was mich schon massiv stört, weil man so, ich nach wie vor der Meinung bin, wenn man die Leute motivieren kann, muss man nicht unbedingt mebr Zeit [geben, BS7, dass man dasselbe rausbringt. Und genau das ist, was hier drinnen den Fübrungskräften abgeht, also der Umgang mit den Leuten selber. Das ist, was mir abgeht, und das ist teilweise schon sehr störend. "(König, NF Montage F T1A, 134ff.)

Die Bedeutung des gegenseitigen Respekts und menschlichen Umgangs miteinander wird insbesondere dann von den Beschäftigten dieses Samples zur Sprache gebracht, wenn sie sich von betrieblichen Vertretern in ihrer Würde verletzt fühlen (vgl. Kratzer et al. (2015). Gerade diese wahrgenommene Missachtung der Beschäftigten trägt zur Bildung großer Machtdistanzen innerhalb des Betriebs bei.

Jenseits der zwischenmenschlichen Umgangsformen hinterfragen die Beschäftigten nicht grundsätzlich die Ordnungsverhältnisse im Betrieb, welche die Dichotomien im Betrieb begründen, sondern akzeptieren größtenteils die im Rahmen der betrieblichen Arbeitsteilung und Spezialisierung in einzelne Fachabteilungen geschaffenen Strukturen: Die „oben“ bestimmen und die Arbeiter „unten“ müssen die Anweisungen ausführen. Häufig formulierte Kritiken der Beschäftigten am Betrieb richten sich eher darauf, inwiefern der 
Betrieb ihrer Meinung nach die Organisation von Arbeitsprozessen sinnvoll und effektiv gestaltet und auf welche Weise die Interessen der Beschäftigten innerhalb der Betriebsorganisation, also von den Vertretern der einzelnen betrieblichen Gruppen und im Rahmen der eigenen Handlungs- und Entscheidungsspielräume der Beschäftigten, Geltung finden bzw. nicht berücksichtigt werden.

Das Verständnis der Beschäftigten von guter und sinnvoller Arbeit ist mit technisch-funktionalen Vorstellungen, wie Arbeits- und Fertigungsprozesse gestaltet werden sollten, damit die Beschäftigten gute Arbeit leisten können und damit auch einen Teil zum Erfolg des Betriebs beitragen zu können. Für das Betriebsverständnis ist dabei relevant, inwiefern in den sozialen Interaktionen mit den betrieblichen Vertretern die Leistungen der Beschäftigten anerkannt werden und ob sie als Träger von Wissen und Erfahrungen verstanden werden, die wichtig für die Gestaltung der Produktionsprozesse sind. Ob im Betrieb diese Rahmenbedingungen hergestellt werden können, ist in hohem Maße von den betrieblichen Kommunikations- und Kooperationsstrukturen abhängig. Eine starke Spezialisierung einzelner Fachbereiche ohne direkten Austausch mit den Produktionsbeschäftigten sowie ausschließliche Top-downEntscheidungsprozesse fördern zum einen große Distanzen zwischen den einzelnen sozialen Gruppen sowie wahrgenommene Dysfunktionalitäten in der Gestaltung von Produktionsprozessen. Kratzer et al. (2015) führen dies unter anderem auf Konflikte zwischen den unterschiedlichen Rationalitätslogiken „technisch-funktional“ vs. „,bürokratisch-prozessual“ und „ökonomisch“ im Betrieb zurück.

\subsubsection{Zusammenfassung}

Die Rekonstruktion der subjektiven Orientierungen der Beschäftigten auf Grundlage der für die Beteiligung an KVP relevanten Motive verdeutlicht, dass generelle, dauerhaft angelegte subjektive Orientierungen für die jeweiligen Beteiligungsdispositionen der Beschäftigten äußerst relevant sind. Die Erfahrungen mit der betrieblichen Organisation von Arbeit können im Sinne eines fortlaufenden Aneignungsprozesses einen prägenden Einfluss auf das eigene Arbeitsverständnis und die Arbeitskraftorientierung haben. So können Erfahrungen, welche die Beschäftigten in restriktiv organisierten Betrieben gemacht haben, eine besonders starke Orientierung auf die Interessen der eigenen Arbeitskraft und kollegialen Solidarität bewirken. Dies wiederum führt oftmals 
dazu, dass sich die Beschäftigten stärker auf den Schutz ihrer Arbeits- und Leistungssituation beziehen als sich auf die Möglichkeit einzulassen, durch Optimierungsideen eine Verbesserung von Arbeitsbedingungen und Produktionsprozess herbeizuführen. Insbesondere die erwerbsbiografische Perspektive ist stark abhängig von den Weiterentwicklungsmöglichkeiten im Betrieb. Wenn diese fehlen oder wenn sich die lebensweltlichen Bedingungen der Beschäftigten ändern (zum Beispiel weniger Zeit durch familiäre Sorgearbeit), können Karriereaspirationen (kurzfristig) aufgeben werden.

Die beschriebenen Orientierungen sind bei fast allen Produktionsbeschäftigten angelegt und können als grundlegendes Orientierungsspektrum einer Typologie des Industriefacharbeiters verstanden werden. Die einzelnen Orientierungen werden jedoch subjektiv unterschiedlich gewichtet, wodurch Variationen der Arbeitsorientierung, des Betriebs- und Rationalisierungsverständnisses entstehen. Zentrale Bezugspunkte für die subjektiven Orientierungsrahmen sind vor allem die Subjekt- und die Arbeitskraftorientierung der Beschäftigten. Bei einzelnen Beschäftigten zeigt sich eine ausgeprägte Karriereorientierung, während bei anderen die Bewahrung einer stabilen Arbeits- und Leistungssituation im Fokus ihres Handelns steht. Subjektive Orientierungen können sich im Laufe des Erwerbslebens verändern, da sie eng mit den Erfahrungen, welche die Beschäftigten sie im Laufe ihres Erwerbslebens - zum Beispiel mit restriktiven oder partizipativen Gestaltungsformen der Arbeit und Optimierungsprozessen - gemacht haben, verknüpft sind. Beispielsweise können Beschäftigte mit vormaligen Karriereorientierungen, diese Aspirationen mittlerweile aufgegeben haben und nun der Status-quo-Orientierung zuneigen.

Die Frage, wie sich die Beschäftigten in konkreten Situationen verhalten, in denen sie mit einer Beteiligungsaufforderung konfrontiert werden, hängt eng damit zusammen, welche Gewichtung der einzelnen subjektiven beteiligungsrelevanten Orientierungen vorliegt und wie stark die damit verbundenen Ansprüche der Beschäftigten in der Gesamtabwägung sind. Aus der unterschiedlichen Gewichtung der einzelnen subjektiven Orientierungen lassen sich drei zentrale Beteiligungstypen rekonstruieren. 


\subsection{Beteiligungstypen}

Die Konzeption der Typen von Beteiligungsorientierungen orientiert sich an der Frage, welche grundlegenden Unterschiede im subjektiven Abwägungsprozess der einzelnen Orientierungen der Beschäftigten für die Beteiligung an KVP relevant sind und welche innere Handlungsorientierung - Triebfeder des Handelns - ersichtlich ist. Die Typologie der subjektiven Beteiligungsorientierungen der Beschäftigten basiert auf den generalisierten handlungsleitenden Orientierungen der Beschäftigten, die sich auf die Beteiligung der Beschäftigten an KVP beziehen.

Es lassen sich drei typische Beteiligungsorientierungen der Beschäftigten identifizieren: Mitgestaltungsorientierung, Karriereorientierung und Status-quoOrientierung. Ähnlich wie beim „Doppelten Bezug auf Arbeit“ (Schumann et al. 1982) lassen sich die einzelnen subjektiven Orientierungen nicht gegeneinander ausspielen, sondern sind gleichzeitig - gleichwohl in unterschiedlicher Stärke relevant. So lassen sich bei allen Beschäftigten sowohl Orientierungen an der Arbeitskraft sowie an arbeitsinhaltlichen Ansprüchen feststellen, die in den Abwägungsprozess für die Beteiligungsdisposition an Optimierungsprozessen ebenso einfließen wie der Einfluss von kollektiven Normen, die Frage der Anerkennung und die jeweilige erwerbsbiografische Perspektive. Bei der Konzeption der Typologie der Beteiligungsorientierung stehen die individuellen Orientierungen der Beschäftigten im Mittelpunkt.

In der Frage der Beteiligung an KVP lassen sich die Beteiligungsorientierungen der Beschäftigten zwischen den Fluchtpunkten der starken arbeitsinhaltlichen, der Arbeitskraft- und der Karriereorientierung verorten. In der Typologie werden die Beteiligungsorientierungen als Idealtypen rekonstruiert, denen sich empirisch Beschäftigte nur selten eindeutig zuordnen lassen. Die Mehrheit der analysierten Fälle befindet sich graduell zwischen den Typen Mitgestaltungsorieniterung und Status-quo-Orientierung (siehe hierzu Abbildung 2). 


\section{Abbildung 2: Typologie der Beteiligungsorientierungen}

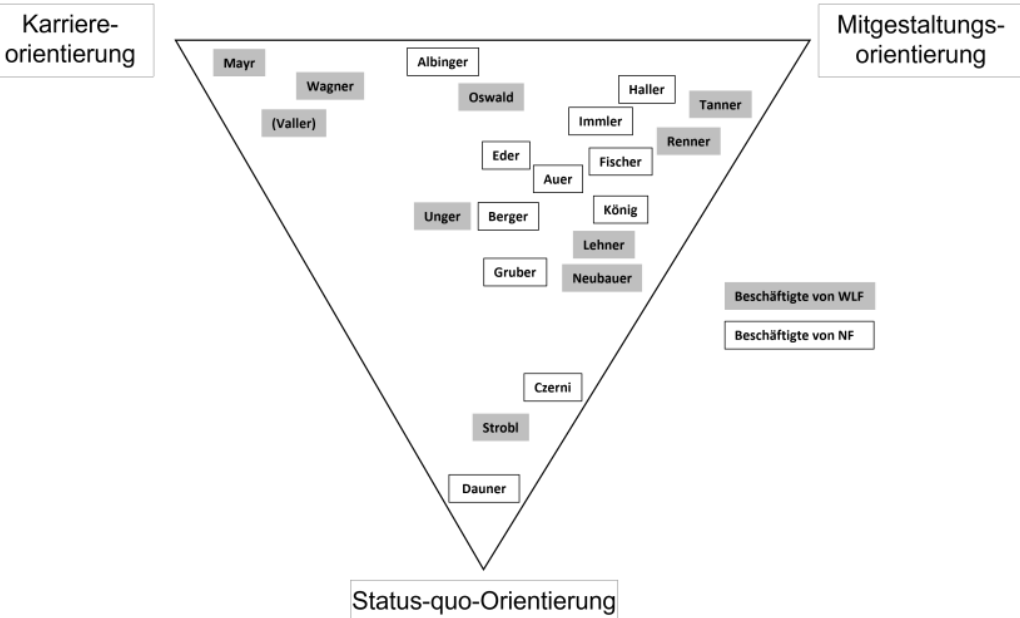

Die Darstellung der einzelnen Typen konzentriert sich auf das Arbeitsorientierung, welches sich aus der jeweiligen Gewichtung der einzelnen subjektiven Orientierung ergibt sowie der Betriebs- und Rationalisierungsverständnis der Beschäftigten. Um Doppelung in der Darstellung der Beteiligungstypen und Aneignungsformen zu vermeiden, werden ausführliche empirische Belege erst in der Darstellung der Aneignungsformen in Kapitel 8 erfolgen.

\subsubsection{Mitgestaltungsorientierung}

Dem Beteiligungstyp „Mitgestaltungsorientierung“ werden Beschäftigte zugeordnet, die ihre Handlungen daran ausrichten, sich gestalterisch in die Arbeit und für den Betrieb einzubringen. Der subjektive Orientierungsrahmen der Mitgestaltungsorientierten ist durch eine konstruktive Problemlöse- und Aufgabenorientierung geprägt. Mitgestaltungsorientierte antizipieren die Anforderungen und Probleme ihrer Arbeit und ihres Arbeitsbereichs, um sich aktiv mit ihren Kompetenzen, eigenen Vorstellungen und Problemlösungen in die Gestaltung von Arbeitsprozessen und organisatorischen Abläufen einzubringen. Konstitutiv für die mitgestaltungsorientierten Beschäftigten ist, dass sie versuchen, Probleme im Arbeitsprozess und in der Arbeitssituation im Rahmen ihrer Handlungs- und Mitsprachemöglichkeiten aktiv zu lösen, sich etwa der 
Behebung von stark belastenden Arbeitsbedingungen annehmen. Mitgestaltungsorientierte Beschäftigte sehen die Beteiligung an Optimierungsprozessen grundsätzlich als Möglichkeit, ihre Vorstellungen von guter und interessanter Arbeit zu verwirklichen und sich selbst darin wiederzufinden.

\section{Arbeitsorientierung der Mitgestaltungsorientierten}

Mitgestaltungsorientierte Beschäftigte beziehen sich in ihrem Handeln vor allem auf Bestimmungsmomente der Subjektorientierung. Die Orientierung an guter und sinnvoller Arbeit verbindet sich hierbei mit einem starken berufsfachlichen Arbeitsverständnis, welches darauf ausgerichtet ist, sich mit den fachlichen, praktischen und sozialen Kompetenzen in den Arbeitsprozess einzubringen.

Die Mitgestaltungsorientierten verweisen häufig darauf, übergreifende technische Zusammenhänge im Arbeitsprozess und von Arbeitsabläufen ebenso gut durchdringen zu können wie betriebliche Experten, und schreiben sich selbst einen Expertenstatus für ihren Arbeitsbereich zu. Untermauert wird die starke fachliche Orientierung durch die im Erwerbsverlauf gesammelten eigenen Erfahrungen und die praktische Routine im Arbeitsprozess. Die Beschäftigten setzen sich tagtäglich mit dem Arbeitsprozess auseinander und lernen mithilfe eigener Strategien mit den aufkommenden Problemen umzugehen.

Die Mitgestaltungsorientierten begreifen sich als ausreichend kompetent, um ihre Aufgaben selbstständig und selbstbewusst auszuführen, ihren eigenen Bereich weiterzuentwickeln und eigene Entscheidungen zu treffen. Besonders deutlich wird dies, wenn ihre selbstzugeschriebene Expertise von den Führungskräften und betrieblichen Experten missachtet wird und sie somit die Orientierung nach Anerkennung der persönlichen Leistung verletzt sehen. Die Beschäftigung mit Optimierungspotenzialen und die Beteiligung an Optimierungen bedeutet für die Mitgestaltungsorientierten eine sehr willkommene Steigerung der Interessantheit der Arbeit und Abwechslung von den zumeist monotonen Arbeitsaufgaben. Ebenso, wenn nicht gar bedeutungsvoller, ist die damit verbundene Möglichkeit, ihre fachlichen und sozialen Kompetenzen, die meist weit über die Anforderungen ihrer eigentlichen Arbeit hinausgehen, sinnvoll anwenden zu können.

Die Orientierung, sich mit der eigenen Subjektivität in den Arbeitsprozess einzubringen, schließt auch den Bezug auf das eigene Leistungsvermögen sein. Das Vermögen, den Leistungsanforderungen gerecht zu werden, ist - nicht 
nur für die Mitgestaltungsorientierten - die Grundvoraussetzung jeglichen Arbeitshandelns. Für die Beschäftigten ist es als elementarer Bestandteil ihres Leistungsverständnisses als Industriearbeiter wichtig, die geforderte Leistung gut zu erbringen und die Anforderungen des Arbeitsprozesses zu erfüllen. Das Ergebnis ihrer Leistung ist ein fertiges Teilprodukt, dessen Qualität an betriebswirtschaftlichen Kennzahlen, wie Qualität, Fehlerquote und Vorgabezeit, gemessen wird. Die Mitgestaltungsorientierten betonen allerdings in besonderem Maße ihre eigene Leistung und die Leistung ihres Arbeitsteams und zeigen eine hohe Identifikation mit der Leistungsfähigkeit des eigenen Bereichs. Dies drückt sich vor allem in einem starken Verantwortlichkeitsgefühl für ihren Aufgabenbereich aus. Auf dieser Identifikation und Verantwortung basiert der Anspruch der Mitgestaltungsorientierten, bei betrieblichen Entscheidungen, die ihren Arbeitsbereich betreffen, mitzureden, mitzuentscheiden und letztlich mitzugestalten.

Das Leistungsbewusstsein der Mitgestaltungsorientierten ist mit dem Bewusstsein verknüpft, einen Teil zum Erfolg und zum Wohl des Betriebs beitragen zu können. In diesem Moment verdeutlicht sich, dass die Mitgestaltungsorientierten betriebliche Normen (Verhaltenserwartungen) als legitim anerkennen und sie für ihr Handeln als relevant erachten.

Die Mitgestaltungsorientierten nehmen allerdings auch wahr, dass die Aussichten auf Realisierung ihrer Ansprüche, sich mit ihren Kompetenzen voll in den Arbeitsprozess und in den Betrieb einzubringen, im Rahmen der Arbeit begrenzt sind. Diese Grenzen sind durch arbeitsteilig vorgegebene Arbeitsumfänge und Zuständigkeiten, betriebliche Vorgaben, Kontrollen und Regularien (Taktzeiten, Zykluszeiten, Aufgabenzuschnitte, Kompetenzbereiche, Kontrolle) bestimmt. Als Teil betrieblicher Verhaltenserwartungen und in Folge der Notwendigkeit, Arbeitsprozesse wirtschaftlich und funktional zu organisieren, werden diese teils akzeptiert, teils kritisiert, wenn sie hinderlich für die Erfüllung der Arbeitsaufgabe sind. Die Mitgestaltungsorientierten versuchen, im Rahmen dieser Grenzen Möglichkeiten zu finden, ihre Ansprüche an Mitgestaltung und Interessantheit der Arbeit zu verwirklichen - auch wenn sie gegebenenfalls Kompromisse eingehen müssen.

Dem Einbringen von sozialen Kompetenzen in und für das Arbeitsteam kommt - vor allem in den Montagearbeitsbereichen - eine große Bedeutung zu. Dies konkretisiert sich darin, dass Mitgestaltungsorientierte sich häufig in der Rolle des Vermittlers zwischen den Kollegen untereinander sowie zwischen Kollegen und Führungskräften sehen. Sie sorgen sich um das gemein- 
same Wohl des Teams, indem sie sich um die Organisation eines optimalen Ablaufs und eine gerechten Aufgabenverteilung im Team kümmern, bei Meinungsverschiedenheiten Ausgleich zu schaffen versuchen, sich sehr um ein gutes soziales Klima im Arbeitsbereich bemühen oder sich auch für die physischen, psychischen und sozialen Bedürfnisse ihrer Kollegen einsetzen. Für diese Aktivitäten sind weitreichende soziale Kompetenzen, wie Kommunikationsfähigkeit und Konfliktlösekompetenzen, von großer Bedeutung. Dies unterstreicht das Arbeitsverständnis der mitgestaltungsorientierten Beschäftigten, sich als „ganzen“ Menschen in die Arbeit und das Arbeitsumfeld einzubringen. Aus diesem Grund übernehmen Mitgestaltungsorientierte häufig Positionen wie Teamsprecher oder Vertrauensmann (soweit vorhanden).

Die besonders hohe Bedeutung, die Mitgestaltungsorientierte insbesondere in den untersuchten Montagebereichen dem Einbringen von sozialen Kompetenzen beimessen, begründet sich darin, dass die technische Komplexität der Produktionsprozesse und somit die fachliche Interessantheit der Arbeit häufig ohnehin nicht sehr hoch ist. Zusätzlich können durch hohe Leistungsvorgaben der taktgebundenen Arbeit, welche die Arbeitsteams zumeist auf sehr engem Raum gemeinsam ausüben, Konflikte zwischen den Beschäftigten und den Vorgesetzten auftreten. Die daraus entstehende Notwendigkeit von Vermittlung, Konfliktlösung und Vertretung der Beschäftigteninteressen gibt den Mitgestaltungsorientierten Raum, sich für das Arbeitsteam einzubringen.

\section{Relevanz sozialer Normen für Mitgestaltungsorientierte}

Verbunden mit dem Einbringen sozialer Kompetenzen ist für die Mitgestaltungsorientierten die soziale Norm der kollegialen Solidarität. Auch wenn die Mitgestaltungsorientierten ihr Handeln an Möglichkeiten orientieren, sich mit ihrer Subjektivität in die Arbeit einzubringen, sind sie sich dennoch bewusst, dass sie einer verschleißenden Tätigkeit (taktgebundene, belastende Montagearbeit, Schichtarbeit) nachgehen und ihre Arbeitskraft durch betriebliche Reorganisationsmaßnahmen sowie den betrieblichen Leistungsanspruch gefährdet werden kann. Mitgestaltungsorientierte sehen sich als Teil der Arbeiterschaft, deren Zugehörige sich latent und ganz allgemein durch betriebliche Aktivitäten in ihrer Arbeitskraft bedroht fühlen. Daher haben über das individuelle Interesse am Schutz der eigenen Arbeitskraft hinaus kollegiale Normen, die sich auf den Schutz der physischen und psychischen Arbeitskraft beziehen, für die Mitgestaltungsorientierten einen zentralen Stellenwert. Die Orientierung an der 
Norm kollegialer Solidarität lässt sich nicht nur dadurch erklären, dass die Mitgestaltungsorientierten sich auf diese Weise mit ihren sozialen Kompetenzen einbringen können, sondern auch dadurch, dass sie mangels Karriereambitionen einen stabilen Erwerbsverlauf in ihrem bisherigen Bereich an der Seite ihrer bisherigen Kollegen erwarten. Die Kollegen sind für die Beschäftigten ein wichtiger und zentraler Bezugspunkt ihres Handelns. Sie „sitzen mit ihnen in einem Boot" und ein gutes Auskommen ist für sie eine wichtige Dimension von guter Zusammenarbeit und Kollegialität. Dass betriebliche und kollegiale Normen häufig in Widerspruch zueinanderstehen können, ist den Mitgestaltungsorientierten ebenfalls bewusst. Sie versuchen, in ihrem Handeln beiden Interessen Rechnung zu tragen und sie in Einklang zu bringen. Bei starken Konflikten sind die kollektiven Normen in der Abwägung meist von höherem Gewicht.

Zusammenfassend betrachtet hat der Anspruch, sich fachlich, leistungsbezogen und sozial einzubringen, für die Mitgestaltungsorientierten eine sinnstiftende Funktion. Inhaltlich ist die Sinnhaftigkeit auf drei Aspekte gerichtet. Erstens orientieren sie sich auf einer persönlichen Ebene an einer facblich interessanten Arbeit, an der Erfüllung des Leistungsanspruches und einer Verbesserung der Arbeitsbedingungen. Diese Punkte sind für die Mitgestaltungsorientierten nicht nur auf der Ebene ihrer eigenen Person relevant, sondern sind auch mit der betrieblich-sozialen Ebene der Arbeiterschaft und den Normen kollektiver Solidarität verbunden. Sinnhafte Handlungen bemessen sich demnach daran, inwiefern sie dem Wohl der Kollegen und auch der Arbeiterschaft insgesamt dienen. Drittens richtet sich die Sinnhaftigkeit des eigenen Handelns auf der betrieblichen Ebene darauf, inwiefern es dem Wobl des Betriebs dient. Die Frage, inwiefern man mit seinem eigenen Handeln zum wirtschaftlichen Erfolg des Betriebs, welcher wiederum mit der eigenen Arbeitsplatzsicherheit verbunden ist, beitragen kann, ist ein wichtiger Bezugspunkt für die Mitgestaltungsorientierungen. Dies drückt sich unter anderem im Wirtschaftlichkeitsdenken aus.

\section{Betriebs- und Rationalisierungsverständnis von Mitgestaltungsorientierten}

Für Mitgestaltungsorientierte ist das Beteiligungsangebot an Optimierungsprozessen grundsätzlich eine sehr willkommene Möglichkeit, sich mit ihren Kompetenzen sinnhaft in die Arbeit und den Arbeitsprozess einzubringen sowie die Interessantheit ihrer Arbeit zu steigern. Durch ihre starke arbeitsinhaltliche Orientierung (Aufgaben- und Problemlösung, technisch-funktionale Rationali- 
tät), ihre Leistungsbereitschaft und ihren Mitgestaltungsanspruch drückt sich aus, dass Mitgestaltungsorientierte tendenziell eine positive Haltung gegenüber der eigenen Beteiligung an Optimierungsprozessen haben. Mitgestaltungsorientierte verstehen sich aber auch als Teil der Arbeiterschaft. Im Vergleich unterschiedlicher mitgestaltungsorientierter Beschäftigter zeigte sich, dass die Antwort auf Fragen, wie sehr und mit welchen Beiträgen sie sich in Optimierungsprozessen einbringen und sich selbst als Subjekt von Rationalisierungsprozessen sehen, sehr stark ihre Wahrnehmung beeinflusst, wie vehement ihre Kollegen sich gegen Optimierungsaktivitäten positionieren. Dabei ist auch entscheidend, wie sehr sich eigene Interessen, zum Beispiel die Verbesserung der Arbeits- und Leistungssituation, sowie das Wohl der Kollegen mit den Interessen des Betriebs vereinbaren lassen. Mitgestaltungsorientierte beteiligen sich in dem Modus pragmatisch gesteuert.

\subsubsection{Karriereorientierung}

Der subjektive Orientierungsrahmen „Karriereorientierung“ bezieht sich auf diejenigen Beschäftigten, die sich im Handeln strategisch auf ihre berufliche Weiterentwicklung ausrichten. Karriereorientierte Beschäftigte streben entweder einen hierarchischen Aufstieg in der Produktion (beispielsweise vom Arbeiter zum Schichtführer und zum Vorarbeiter) oder eine fachliche Karriere in Fachabteilungen des Betriebs, beispielsweise in der Instandhaltung oder Planung, an. Charakteristisch für karriereorientierte Beschäftigte ist, dass sie sich durch ihre überdurchschnittliche Arbeitsleistung und die aktive Übernahme von zusätzlichen, nicht zu ihren eigentlichen definierten Tätigkeiten zählenden Aufgaben von der großen Masse der Arbeiter unterscheiden zu versuchen und sich dem Betrieb als kompetente und engagierte Mitarbeiter präsentieren wollen, die auch für „höhere“ Aufgaben qualifiziert sind. Beschäftigtengetragene Optimierungsprozesse sind daher für Karriereorientierte grundsätzlich eine willkommene Möglichkeit, die Sichtbarkeit ihrer Person als Potenzialkandidaten für hierarchische und fachliche Funktionen zu erhöhen und ihre Kontakte zu Führungskräften und Fachabteilungen zu verbessern.

\section{Arbeitsorientierung der Karriereorientierten}

Die Arbeitsorientierung der karriereorientierten Beschäftigten weist große Gemeinsamkeiten zu jener der Mitgestaltungsorientierten auf. Die Orientierung an sinnhafter und fachlich interessanter Arbeit, an Problemlösungen im 
Arbeitsprozess sowie ein ausgeprägtes Leistungsbewusstsein und bestimmen das Handeln der Karriereorientierten. Ähnlich wie den Mitgestaltungsorientierten ist es ihnen wichtig, sich mit ihren Kompetenzen in ihre Arbeit sowie in die Gestaltung des Arbeitsprozesses und betrieblicher Abläufe einzubringen und daran mitzuwirken. Karriereorientierte zeigen eine hohe Identifikation mit ihrem Bereich und betonen insbesondere ihre Rolle für eine gute Leistung ihres Produktionsbereichs. Zu ihrem Arbeitsverständnis gehört nicht nur, gute Arbeit im Betrieb zu leisten, sondern auch, mit ihrem vorhandenen Fach- und Erfahrungswissen die Produktionsprozesse zu verbessern.

Während das Handeln der Mitgestaltungsorientierten mit einem konsolidierten, fachlich geprägten Arbeitsverständnis verbunden ist, wird das Handeln der Karriereorientierten darüber hinaus durch das strategische Verfolgen beruflicher Weiterentwicklungsperspektiven im Betrieb geleitet. Dies konkretisiert sich darin, dass sie aktiv fachlich anspruchsvolle und für die Organisation und die Leistungsfähigkeit ihres Arbeitsbereiches wichtige und verantwortungsvolle Tätigkeiten übernehmen, um sich als kompetente und engagierte Mitarbeiter zu zeigen. Beispielsweise kümmern sich Karriereorientierte oftmals um Vor- und Nachbereitungstätigkeiten in ihrem Bereich, ersinnen eigenständig Möglichkeiten, die Produktion zu verbessern, probieren Optimierungsmöglichkeiten an der Produktionsanlage aus und nutzen viele Gelegenheiten, um im Rahmen dieser Tätigkeiten enger mit ihren Vorgesetzten und Fachabteilungen in Kontakt zu kommen.

Ob sich Karriereorientierte stärker um fachlich interessante Arbeit bemühen oder eher organisatorische Aufgaben ausführen, richtet sich häufig danach, welche berufliche Weiterentwicklung sie anstreben. ${ }^{92}$

Karriereorientierte begründen ihre Weiterentwicklungsambitionen auf unterschiedliche Weise. Zum einen spielt es eine wichtige Rolle, dass sie ihren Anspruch nach fachlich interessanter Arbeit in der meist als monoton empfundenen und im Betriebskontext untergeordneten Produktionsarbeit nicht befriedigt sehen und die Möglichkeiten, sich mit ihren Kompetenzen in den Arbeitsprozess einzubringen, als sehr begrenzt wahrnehmen. Mit der Arbeit in höheren Positionen, als Vorarbeiter, Meister, Instandhalter oder technischer Planer, verbinden sie einen passgenaueren Einsatz ihrer fachlichen Kompeten-

92 Dies hängt auch sehr stark davon ab, welche Karrierewege im Betrieb für die Beschäftigten ersichtlich sind. 
zen und eine größere Einflussnahme auf die Gestaltung betrieblicher Abläufe und Produktionsprozesse. Karriereorientierte Beschäftigte legitimieren ihre Karriereorientierung in diesem Zusammenhang häufig mit einem allgemeinen und selbstverständlichen Weiterentwicklungsstreben.

Zum anderen verbindet sich mit den Karriereaspirationen auch die Möglichkeit, der zumeist als eintönig, belastend und verschleißend wahrgenommenen Produktionsarbeit zu entkommen. Vermeidung von Schichtarbeit, Schutz vor körperlicher Verausgabung, vor allem aber auch ein deutlich höheres Einkommen sind für Karriereorientierte relevante arbeitskraftorientierte Bezüge für ihre Handlungsorientierung. Dies verdeutlicht, dass in der Karriereorientierung Bestimmungsmomente der Subjektorientierung und der Arbeitskraftorientierung sich mit einer auf Weiterentwicklung ausgerichteten erwerbsbiografischen Perspektive verbinden.

Mit ihren Weiterentwicklungsaspirationen ist die hohe Bedeutung beruflicher Weiterbildung verknüpft. Karriereorientierte betonen in den Gesprächen häufig, technische oder betriebswirtschaftliche Qualifikationsmaßnahmen anzustreben oder bereits absolviert zu haben. Im Mittelpunkt steht zumeist die Qualifikation zum Werkmeister, die oftmals nebenberuflich an der Abendschule mit betrieblicher Unterstützung absolviert wird. Durch die Weiterbildung erhöhen sie nicht nur ihre fachlichen Kompetenzen, sondern sie zeigen dem Betrieb offen ihr Interesse an höheren Aufgaben und Verantwortungsübernahme. Um die Chancen auf eine berufliche Weiterentwicklung im Betrieb zu verbessern, orientierten sie sich an vorgezeichneten Karrierewegen, die sie sie meist durch die Beobachtung der Aufstiege anderer Produktionsbeschäftigte identifiziert haben.

Elemente der Mitgestaltungsorientierung zeigen sich im Handeln der Karriereorientierten als strategisches Moment. Sie versuchen, durch ihr Handeln nicht nur ihr aktuelles Arbeitsumfeld, ihre Arbeitssituation und ihren Bereich zu gestalten, sondern auch, - im Unterschied zu den Mitgestaltungsorientierten - den persönlichen Aufstieg jenseits direkter Produktionsarbeit zu realisieren. Ausdruck findet dies darin, dass Karriereorientierte sich in ihrem Alltagshandeln stärker an betrieblichen als an kollegialen Solidaritätsnormen orientieren. Dadurch wollen sie sich dem Betrieb als kompetenter Mitarbeiter darstellen, der für „höhere“ Aufgaben geeignet ist. 


\section{Relevanz sozialer Normen für Karriereorientierte}

Im Arbeitsverständnis der Karriereorientierten zeigt sich eine hohe Relevanz betrieblicher Normen für das alltägliche Arbeitshandeln. In den Interviews verweisen Karriereorientierte immer wieder nicht nur auf ihr fachliches Interesse, sondern auch darauf, dass sie ihren Bereich und den Betrieb weiterbringen wollen. Dies konkretisiert sich darin, dass die karriereorientierten Beschäftigten sich im alltäglichen Arbeitshandeln an Aspekten der Wirtschaftlichkeit, Effizienz und Kosten von Arbeitsprozessen orientieren (ökonomische Rationalität). Wirtschaftliche Kennzahlen, wie Kosten, Qualität, Liefertreue, Durchlaufzeiten, Ausschussreduzierung und Stückzahloptimierung, sind wichtige Referenzpunkte für ihr Handeln und werden von ihnen viel deutlicher hervorgehoben als von anderen Beteiligungstypen. Auf diese Weise verbindet sich ihr Arbeitsverständnis mit dem vom Betrieb gewünschten Wirtschaftlichkeitsdenken. Obgleich auch andere Beschäftigte sich an diesen Kennzahlen orientieren und dies als wichtig für den betrieblichen Erfolg ansehen, zeigt sich bei den Karriereorientierten deutlich, dass die Orientierung an betrieblichen Normen eine dominantere Rolle einnimmt.

Diese Orientierung an betrieblichen Verhaltenserwartungen veranschaulicht sich in den Interviews insbesondere in dem Verwendung eines betriebswirtschaftlich geprägten Sprachduktus. So verweisen sie auf Begrifflichkeiten wie „Stückzahloptimierung“ oder „Waste-Gespräche“ und bezeichnen ihre Kollegen häufig bereits als Mitarbeiter, so, wie es Führungskräfte in der Regel tun. Anhand der Übernahme des betriebswirtschaftlichen Vokabulars und Denkens verdeutlicht sich die Identifikation mit dem Betrieb sowie mit dem Arbeitsverständnis von Angestellten.

Die Orientierung an betrieblichen Normen ist für die Karriereorientierten insofern relevant, als sie annehmen, dadurch ihre Weiterentwicklungsmöglichkeiten im Betrieb zu verbessern. Wenn sie sich in ihrem alläglichen Arbeitshandeln für die Interessen des Betriebs - teilweise sogar gegen die Interessen ihrer Kollegen - einsetzen, zeigen sie sich als für den Betrieb wertvolle Mitarbeiter. Teil dieses Verständnisses ist es auch, unter Umständen auf die direkte Prämierung von Optimierungs- bzw. Verbesserungsvorschlägen zu verzichten. Mit ihrem Engagement gehen sie gleichsam in Vorleistung und verbinden damit die Erwartung, dass dies vom Betrieb anerkannt und wertgeschätzt wird und sich dieser erkenntlich zeigt, indem er ihren Aufstieg fördert. Zugleich ist den karriereorientierten Beschäftigten bewusst, dass es zahlreiche Interessen- 
ten für eine geringe Anzahl an benötigten Vorarbeitern oder fachlichen Angestellten gibt.

Kollegiale Solidaritätsnormen, die von den Kollegen an sie herangetragen werden, spielen bei den Karriereorientierten eine eher untergeordnete Rolle. Ihnen ist aber bewusst, dass sie durch ihr Arbeitshandeln, welches an Wirtschaftlichkeitsaspekten und Optimierungen von Arbeitsbereichen orientiert ist, gegebenenfalls gegen die Schutzinteressen ihrer Kollegen verstoßen. Optimierungsaktivitäten können beispielsweise eine Verschärfung der Leistungssituation für die übrigen Kollegen bedeuten und von ihnen daher abgelehnt werden. Da sie als Teil der Arbeiterschaft im Produktionsprozess eng mit ihren Kollegen zusammenarbeiten, sind sie mit der kollegialen Solidaritätsnorm unmittelbar konfrontiert, was zu Konflikten führen kann. Für Karriereorientierte ist ein gutes Auskommen mit ihren Kollegen zwar wichtig, die Norm der kollegialen Solidarität entfaltet bei ihnen allerdings nicht die gleiche Wirkkraft wie bei den anderen Beteiligungstypen. Sie setzen sich kritisch mit der als dominant wahrgenommenen Haltung kollegialer Solidarität auseinander und gehen teils rücksichtnehmend, teils distanzierend darauf ein. Letzteres kann bedeuten, dass die Karriereorientierten mit Sanktionen von ihren Kollegen, beispielsweise offener Anfeindung und Streit, rechnen müssen.

Der Stellenwert, den Karriereorientierte Kollegialität beimessen, hängt unter anderem davon ab, ob sie eine fachliche Karriere oder einen hierarchischen Aufstieg anstreben. Beschäftigte, die eher eine fachliche Karriere anvisieren, distanzieren sich unter Umständen sogar sehr stark von ihren Kollegen und deren Arbeitsverständnis. Karriereorientierte, die eher einen hierarchischen Aufstieg, zum Beispiel zum Vorarbeiter, anstreben, nehmen häufig eher eine vermittelnde Rolle zwischen Arbeitern und Betrieb ein, da sie auf die gute Zusammenarbeit mit ihren Kollegen angewiesen sind.

\section{Betriebs- und Rationalisierungsverständnis von Karriereorientierten}

Der Betrieb ist für die Karriereorientierten der Ort der Verwirklichung von Karriereambitionen. Obwohl sie noch Teil der Arbeiterschaft sind, sehen sie sich weniger in ihr verortet, sondern vielmehr auf einer Zwischenstufe hin zum Angestelltenbereich. Wie oben ausführlich dargelegt, bestimmen Optimierungsaktivitäten und die Orientierung an Wirtschaftlichkeitskriterien das Arbeitshandeln der Karriereorientierten. Eine Beteiligung an beschäftigtengetragenen Optimierungsprozessen entspricht grundsätzlich ihrem Arbeitsver- 
ständnis. Darüber hinaus stellt eine Beteiligung eine sehr willkommene und günstige Gelegenheit dar, sich aus der breiten Masse der Arbeiter hervorzuheben. Durch die Beteiligung an Optimierungsprozessen eröffnet sich den Beschäftigten ein Raum, in dem sie als Arbeiter enger in Kontakt zu Angestellten aus Fachabteilungen und Vorgesetzten treten können. Über diesen persönlichen Kontakt können sich die Beschäftigten als engagierte und kompetente Mitarbeiter zeigen und somit aktiv ihre Aufstiegschancen verbessern.

Karriereorientierte beteiligen sich aktiv und vorbehaltlos an beschäftigtengetragenen Optimierungsprozessen. Sie lassen sich in diesem Sample zumeist bei den jüngeren Beschäftigten bis ca. 40 Jahre finden. Der Karriereorientierung lassen sich Mayr, Wagner und Valler zuordnen, die eindeutig eine berufliche Weiterentwicklung anstreben. Bei Eder, Berger und Albinger lassen sich Karriereambitionen erkennen, wobei diese teils stark von den mangelnden Realisierungschancen überlagert werden. 


\subsubsection{Status-quo-Orientierung}

Unter dem Beteiligungstyp „Orientierung am Status-quo“ (kurz: Status-quoOrientierte) werden diejenigen Beschäftigten zusammengefasst, deren dominanter Handlungsbezug vorrangig im Schutz ihrer aktuellen Arbeits- und Leistungsbedingungen besteht. Der subjektive Orientierungsrahmen der am Status quo orientierten Beschäftigten ist durch eine generell interessenantagonistische und reservierte Haltung gegenüber betrieblichen Veränderungsaktivitäten gekennzeichnet, da sie eine Verschlechterung ihrer aktuellen Situation befürchten. Obgleich die Beteiligung an Optimierungsprozessen die Möglichkeit eröffnen, die eigene Arbeitssituation potenziell zu verbessern, wird angesichts des verfestigten Generalverdachts, dass betriebliche Aktivitäten gewöhnlich zulasten der Arbeiter unternommen werden, eine öffentliche Beteiligung an Optimierungen nur dann in Betracht gezogen, wenn negative Folgen eindeutig ausgeschlossen sind.

\section{Arbeitsorientierung der Status-quo-Orientierten}

Die Arbeitsorientierung von Status-quo-orientierten Beschäftigten ist durch ein enges Arbeitsverständnis geprägt, welches sich vorwiegend auf die gute Erfüllung der ihnen im arbeitsteiligen Produktionsprozess zugewiesenen Aufgaben konzentriert. Dieses Arbeitsverständnis wird mitunter auf den ersten Blick mit einem schlichten „Dienst-nach-Vorschrift“-Verhalten in Verbindung gebracht. Charakteristisch für Status-quo-Orientierte ist, dass sie sich in ihrer Arbeitssituation stabil eingerichtet haben.

Status-quo-Orientierte beziehen sich in ihrer Arbeit sehr stark auf Bestimmungsmomente der Arbeitskraftorientierung. Ihr Arbeitsorientierung bezieht sich direkt auf die Arbeits- und Leistungssituation. Ihnen ist sehr deutlich bewusst, dass ihre Arbeit eine körperliche ist, die durch Beanspruchung im Arbeitsprozess und durch die Leistungsanforderungen im Laufe ihres Erwerbslebens verbraucht wird. Die Arbeits- und Leistungssituation wird stärker als bei den anderen beiden Beteiligungstypen unter dem Verschleißkriterium betrachtet. Dezidiert verweisen Status-quo-Orientierte auf die Belastungen der zumeist monotonen und körperlichen Arbeit in Zusammenhang mit den strengen Zeitvorgaben und den häufig als sehr gering wahrgenommenen Handlungsspielräumen und Freiräumen für Regeneration. Status-quoOrientierte betonen, wie wichtig ihnen Freiräume in der Arbeit sind, die ihnen 
ermöglichen, zusätzliche Pausen, zum Beispiel zum Kaffeetrinken und Rauchen, einzulegen, oder die sie als notwendigen Spielraum für die selbstständige Einteilung und Ausführung ihrer Arbeit gemäß der eigenen Bedürfnisse benötigen. Um langfristig mit den Leistungsanforderungen im Betrieb umgehen zu können, müssen sie die Entwicklung der Leistungssituation ihrer Arbeit einschätzen können. Veränderungen, die vom Betrieb einerseits oder von den Arbeitern andererseits herbeigeführt werden, werden primär danach begutachtet, welche Auswirkungen diese auf die Arbeitskraft der Beschäftigten haben.

Neben dem sehr starken Bezug auf die Arbeitskraft spielen Bestimmungsmomente der Subjektperspektive für die Arbeitsorientierung der Statusquo-Orientierten eine wichtige Rolle. Ähnlich wie bei den anderen Beteiligungstypen ist auch hier die Orientierung, die Arbeit gut und in der geforderten Qualität zu erbringen, zentraler Handlungsbezug. In diesem Arbeitsverständnis verbindet sich das spezifische Leistungsbewusstsein der Beschäftigten mit einem sinnhaften Bezug auf Arbeit. Die Beschäftigten wollen ihre Arbeit nicht einfach nur erbringen, sondern verbinden dies mit einer normativen Wertigkeit, die sich zum einen auf das eigene fachliche Verständnis und das eigene Leistungsbewusstsein bezieht und zum anderen durch die betrieblichen Erwartungen definiert ist. Durch die gute Ausführung ihrer Arbeit wollen sie einen Teil zum Erfolg des Betriebs beitragen. Sie haben dezidierte Vorstellungen darüber, wie ihre Arbeit idealerweise zu verrichten ist, und beziehen sich dabei auf ihr berufssozialisatorisch erworbenes Fachwissen sowie die alltägliche Erfahrung des Arbeitslebens, welche sie auch in ihre Arbeit einbringen wollen. Status-quo-Orientierte schreiben sich vor allem eine ausreichende Kompetenz für die selbstständige und eigenverantwortliche Ausführung der ihnen zugewiesenen Arbeiten zu. Diese Kompetenz nutzen sie zum einen, um ihre Arbeit gut und in der geforderten Qualität zu erledigen, und zum anderen, um im Rahmen ihrer Möglichkeiten Tätigkeiten und Abläufe innerhalb ihres Arbeitsbereiches zu optimieren und Belastungen zu kompensieren. Diese Optimierungen werden vorwiegend im Stillen vollzogen und dem Betrieb nicht mitgeteilt, da damit die Gefahr verbunden ist, dass der Betrieb die Leistungsvorgaben verschärfen könnte.

Status-quo-Orientierte betrachten deutlich stärker als die beiden anderen Beteiligungstypen ihre Leistung im Verhältnis zu der vom Betrieb empfangenen Gegenleistung - vorrangig dem Lohn. Sie verweisen sehr häufig darauf, dass man arbeiten muss, um Geld zu haben, um damit wiederum eine Familie zu ernähren, privaten und dauerhaften finanziellen Verpflichtungen nach- 
kommen zu können sowie Annehmlichkeiten und Interessen zu verfolgen. Bedeutsam ist in diesem Untersuchungskontext, dass das Lohnniveau in beiden Betrieben im Vergleich zu anderen Betrieben der Region relativ hoch ist. Dieser gute Lohn wird von den Beschäftigten in Bezug zu ihren materiellen Arbeitsbedingungen gesetzt. Insbesondere die Status-quo-Beschäftigten wiegen die Rahmenbedingungen der Arbeitssituation, etwa einen trockenen, relativ sauberen, stationären und gut bezahlten Arbeitsplatz, häufig kompensatorisch gegen die mangelnde Interessantheit, die belastenden Arbeits- und Leistungsbedingungen sowie die Unterordnung unter einen arbeitsteiligen, fremdbestimmten Produktionsprozess auf. In diesen Kompromiss fließen oftmals die als deutlich schlechter wahrgenommenen Arbeitsbedingungen und Bezahlungen bei anderen, kleineren regionalen Arbeitgebern sowie die prekäre Arbeitsmarktlage für Beschäftigte mit ihrer Qualifikation und Erfahrung als Produktionsarbeiter ein. Status-quo-orientierte Beschäftigte weisen in diesem Zusammenhang auch darauf hin, dass man eine möglichst bequeme Arbeit haben wolle. Mit diesem „man“ beziehen sie sich auf ein vermeintlich allgemeines Arbeitsverständnis, nur das Nötigste zu tun.

\section{Relevanz sozialer Normen für Status-quo-Orientierte}

Die kollektiven Schutznormen haben für die handlungsleitenden Orientierungen der Status-quo-orientierten Beschäftigten eine sehr hohe Wirkkraft. Sie verweisen dezidiert darauf, dass „man“ als Arbeiter durch sein Handeln nicht eigene Interessen und die Interessen der Arbeiter allgemein verletzten dürfe. Die häufige Verwendung der Formulierung „man“ in diesem Kontext deutet auf die Wirkmächtigkeit dieser allgemein anerkannten Norm hin.

Die Wirkkraft der kollektiven Schutznorm ist bei den Status-quoOrientierten sehr stark, weil sie sich zum einen weitgehend mit den eigenen Orientierungen deckt. Zum anderen sind die Kollegen des eigenen Bereichs eine wichtige Referenzgruppe, da sie ebenso wie die materiellen Arbeitsbedingungen ein wichtiger Teil der alltäglichen Arbeit sind. Ein gutes Auskommen mit den Kollegen stellt eine wichtige Arbeitsbedingung sowie Ressource zur Bewältigung von Arbeitsbelastungen dar. Zudem bilden die direkten Kollegen, aber auch die Arbeiterschaft insgesamt eine relevante Bezugsgruppe, da sie unter ähnlichen, ebenfalls belastenden Arbeits- und Leistungsbedingungen arbeiten und sich als Kollektiv im arbeitsteiligen Produktionsprozess gegenüber der betrieblichen Führung sowie den Angestelltengruppen als unterge- 
ordnet und abhängig wahrnehmen. Für die Beschäftigten bedeutet dies, dass grundsätzlich alle Arbeiter von den betrieblichen Reorganisations- und Rationalisierungsaktivitäten betroffen sein können. Dieses wahrgenommene gemeinsame Schicksal ist konstitutiv für die Solidarität untereinander und die Stärke der Schutznorm. Der solidarische Schutz der Arbeiterinteressen - vornehmlich der Schutz vor Verschlechterung der Arbeitssituation, Verschärfung der Leistungsanforderungen und letztlich Verlust des Arbeitsplatzes - wird von den Status-quo-Orientierten als wichtiges Handlungsprinzip explizit betont. Beschäftigte, die gegen diese Schutznorm handeln, wie es beispielsweise Karriereorientierte mitunter tun, müssen mit Sanktionen der Status-quoOrientierten rechnen, etwa in Form von schlechter Stimmung, offener Kritik oder auch Mobbing.

Betriebliche Verhaltenserwartungen spielen für Status-quo-Orientierte insofern eine Rolle, als sie sich auf die Anforderungen beziehen, die von ihrem Arbeitsverhältnis ausgehen. Dies bedeutet, dass sie als Leistung ihres Arbeitsvertrags die ihnen zugewiesenen Arbeiten gut und in der geforderten Qualität ausführen wollen. Dies beinhaltet ebenso, dass sie den Interessen des Betriebs in der Regel nicht zuwiderhandeln, weil sie dadurch ihren Arbeitsplatz gefährden würden. Sich jedoch über ihre Arbeitspflichten hinaus mit mehr Engagement an Rationalisierung und Weiterentwicklung des Betriebs zu beteiligen, steht in Konflikt zu ihren eigenen arbeitskraftorientierten Interessen und zu den Interessen der Solidargemeinschaft.

\section{Betriebs- und Rationalisierungsverständnis von Status-quo-Orientierten}

Durch den betrieblichen Leistungsanspruch, den die Beschäftigten in Form von Kontrolle, fremdbestimmter Aufgabeneinteilung, Optimierung von Durchlaufzeiten, Verkürzung von Vorgabezeiten und insgesamt eine permanente Reorganisation wahrnehmen, sehen sich insbesondere die Status-quoOrientierten in ihrer Arbeitskraft bedroht. Dabei verweisen sie nicht nur auf mögliche Verschlechterungen ihrer Arbeits- und Leistungssituation, sondern auch auf die potenzielle Gefahr, dass ganze Arbeitsplätze wegrationalisiert werden können, was Entlassungen oder Versetzung in andere Bereiche nach sich ziehen kann. Status-quo-Orientierte versuchen im alläglichen Arbeitshandeln ihre Freiräume vor der Entdeckung von Führungskräften und insbesondere von Rationalisierungsexperten, wie beispielsweise aus der Arbeitsvorbereitung, zu schützen und sie auszubauen. 
Häufig erkennen sie zwar das betriebliche Interesse an Gewinn und wirtschaftlichem Erfolg als legitimes Interesse an, von welchem auch letztlich ihr Arbeitsplatz abhängig ist, betonen allerdings deutlich, dass das permanente Rationalisierungsstreben doch Grenzen haben müsse, da es auf Kosten der körperlichen und psychischen Leistungsfähigkeit, also zulasten der Arbeiter, gehe. ${ }^{93}$ Als Grenze definieren die Status-quo-orientierten Beschäftigten die menschliche Würde und das menschliche Leistungsvermögen. So verdeutlichen die Beschäftigten die Grenzüberschreitung der wahrgenommenen Leistungsanforderung dadurch, dass man noch nicht einmal in Ruhe auf die Toilette gehen könne. Dass sich betriebliches Handeln vorwiegend gegen die Interessen der Beschäftigten richtet, hat sich als interessenantagonistische Haltung gegenüber betrieblichen Aktivitäten durch negative Erfahrungen mit betrieblichen Rationalisierungsmaßnahmen dauerhaft im Bewusstsein der Beschäftigten verfestigt. Angesichts der befürchteten Verschlechterungen ihrer persönlichen Arbeitssituation bzw. der Beschäftigungsbedingungen, nehmen die Status-quo-Orientierten eine konservative Schutzhaltung ein, um ihren aktuellen, errungenen Status sowie den damit verbundenen Lohn zu erhalten.

Status-quo-orientierte Beschäftigte stehen der Beteiligung an Optimierungsprozessen aus dem Schutzinteresse hinsichtlich ihrer Arbeitskraft grundsätzlich sehr kritisch gegenüber. Obwohl sie in ihrem Arbeitsverständnis durchaus einen arbeitsinhaltlichen Anspruch an gute Arbeit und zufriedenstellende Leistungserfüllung vertreten, bleibt die Übernahme von Optimierungsaufgaben in das Arbeitsverständnis aus - weil es nicht ihrem Rollenverständnis entspricht, sich mit Rationalisierungsaufgaben womöglich ,gegen die Arbeiter“ zu wenden. Hinsichtlich der eigenen Beteiligung an Optimierungen differenzieren Status-quo-Orientierte sehr stark zwischen den Optimierungsinhalten und den Zuständigkeitssphären. Die als betriebliche Optimierung bzw. Rationalisierung bezeichneten Aktivitäten des Betriebs werden von den Beschäftigten im Interview mit Kostenrationalisierung gleichgesetzt, die sie meist mit Leistungssteigerung in Verbindung bringen. Diese Optimierungsaktivitäten werden als Gefahr für die eigene und kollektive Arbeitskraft angesehen und in die Zuständigkeitssphäre des Betriebs verwiesen. Hierbei sehen die Status-quoOrientierten die Interessen des Betriebs an Profitabilität und die Interessen der Beschäftigten als abhängige Lohnarbeiter als unvereinbar an. Eine Beteiligung

${ }^{3}$ Nicht selten verknüpft sich diese Haltung mit einer allgemeinen Kapitalismuskritik. 
an solchen Optimierungen würde eine massive Verletzung der kollektiven Schutznorm bedeuten, die aus Gründen der kollektiven Solidarität zu vermeiden ist.

Allerdings schließen die Status-quo-Orientierten die Beteiligung an Optimierungen, die den eigenen Arbeitsplatz und die eigenen Tätigkeiten betreffen nicht grundsätzlich aus. eine Mitarbeit an ergonomischen Verbesserungen oder Ablaufoptimierungen, die eine Erleichterung bedeuten können, ziehen sie durchaus in Erwägung. Aufgrund der allgemeinen Gefahr für die Arbeits- und Leistungssituation, die von Veränderungsaktivitäten ausgeht, und der Möglichkeit, dass die eigene Beteiligung eventuell doch Schaden mit sich bringen können, wird häufig eher zurückhaltend gehandelt, um den Status-quo zu bewahren. Status-quo-Orientierte beteiligen sich an beschäftigtengetragenen Optimierungsprozessen entweder nicht oder nur in einem sehr abgesicherten Modus.

Die Beteiligungsdisposition der Status-quo-Orientierten lässt sich mit der von Schumann et al. (1982) identifizierten Haltung des arbeitspolitischen Konservativismus vergleichen.

\subsubsection{Zusammenfassung der Beteiligungstypen}

Zusammengefasst lassen sich die unterschiedlichen Beteiligungstypen wie folgt charakterisieren: Karriereorientierte und Mitgestaltungsorientierte haben grundsätzlich ein weites Arbeitsverständnis, setzen sich aktiv mit den Problemen und Anforderungen des Arbeits- und Produktionsprozesses auseinandersetzten und sind bestrebt, sich mit ihren Kompetenzen und ihrem Erfahrungswissen in den Produktionsprozess und vor allem in Optimierungsprozesse einzubringen. Die beiden Typen unterscheiden sich darin, dass Karriereorientierte durch ihr Handeln vor allem versuchen, ihre persönlichen Weiterentwicklungschancen zu verbessern, und sich daher proaktiv in Optimierungsprozesse einbringen. Mitgestaltungsorientierte suchen hingegen nach Gelegenheiten, ihr fachliches Wissens, ihre Erfahrungen und sozialen Kompetenzen zur Verbesserung ihrer Arbeitsbedingungen und zur Steigerung des Betriebserfolgs einzubringen. Um Verschlechterungen, wie Leistungsintensivierung, zu vermeiden, beteiligen sie sich pragmatisch gesteuert, das heißt, sie erwägen genau, welche Folgen ihre Optimierungsaktivitäten für die Arbeitsbedingungen haben und steuern ihre Aktivitäten so, dass für sie keine Nachteile entstehen. Statusquo-Orientierte hingegen haben ein enges Aufgabenverständnis, welches sich auf die reine Erledigung der ihnen aufgetragenen Arbeit und Erfüllung der 
Leistungsvorgaben beschränkt. Sie setzen sich eher reaktiv mit verändernden Arbeitsbedingungen auseinander und versuchen Veränderungen zu vermeiden, da diese auch ihre Arbeitssituation auch verschlechtern können. An beschäftigtengetragenen Optimierungsprozessen beteiligen sie sich daher nicht oder nur in einer sehr abgesicherten Form, wenn alle negativen Folgen ausgeschlossen sind.

Im Sample dieser Untersuchung gibt es nur wenige Realtypen, die den reinen Beteiligungstypen Mitgestaltungsorientierung, Karriereorientierung und Status-quo-Orientierung entsprechen. Die meisten Beschäftigten bewegen sich zwischen den Polen Mitgestaltungsorientierung und Status-quo-Orientierung. Dies ist evident, da sich die Beschäftigten stets auf unterschiedliche arbeitsinhaltliche, normative und arbeitskraftbezogene Orientierungen zugleich beziehen, diese Orientierungen unterschiedlich gewichtet sein können und sich durch Erfahrungen, welche die Beschäftigten im Laufe ihres Erwerbslebens machen, durchaus verändern können.

Die Beschäftigten zwischen den Polen Mitgestaltungs- und Status-quoOrientierung haben sich in ihrer Arbeit eingerichtet, streben einen stabilen Erwerbsverlauf innerhalb ihres Bereiches oder innerhalb der Produktion an und wollen negative Veränderungen, wie ungewollte Versetzung in andere Bereiche und Verschlechterung ihrer Arbeits- und Beschäftigungsbedingungen, beispielsweise Einkommenseinbußen, vermeiden. Eine Karriereorientierung, die mit einer spezifischen erwerbsbiografischen Perspektive verknüpft ist, lässt sich nur bei wenigen Beschäftigten (Mayr, Wagner, Valler und Albinger) identifizieren. Beschäftigte, die sich in der Mitte des Typendreiecks bewegen, weisen Elemente aller drei Orientierungen auf. Die Karriereorientierung bestimmt jedoch keineswegs vollumfänglich das Handeln der Beschäftigten, da Weiterentwicklungsmöglichkeiten im Betrieb eher begrenzt wahrgenommen werden oder im Laufe des Erwerbslebens in den Hintergrund getreten sind. 
Tabelle 6: Beteiligungstypen und Beteiligungsdisposition ${ }^{94}$

\begin{tabular}{|c|c|c|}
\hline & Charakteristische Merkmale & Beteiligungsdisposition \\
\hline 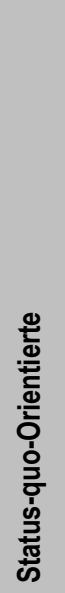 & $\begin{array}{l}\text { Schutz der Arbeitskraft } \\
\text { Enges Aufgabenverständnis: Erfüllung } \\
\text { aufgetragener Arbeiten („Dienst nach Vor- } \\
\text { schrift"), } \\
\text { Optimierungen sind nicht Teil des Aufgaben- } \\
\text { verständnisses } \\
\text { Reaktive Auseinandersetzung mit Arbeitsbe- } \\
\text { dingungen (Vermeidung von Veränderun- } \\
\text { gen) } \\
\text { Hohe Relevanz kollektiver Schutznormen } \\
\text { Rationalisierung richtet sich gegen die } \\
\text { Arbeiter }\end{array}$ & $\begin{array}{l}\text { Abgesichert - keine Beteiligung } \\
\text { Ignorieren, Skepsis, Ablehnung }\end{array}$ \\
\hline 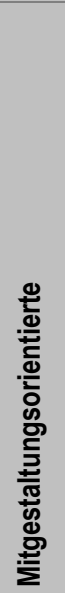 & $\begin{array}{l}\text { Weites Aufgabenverständnis: Problemlöse- } \\
\text { und Aufgabenorientierung (als Element eines } \\
\text { spezifischen Facharbeiterverständnisses) } \\
\text { Aktive Auseinandersetzung mit Anforderun- } \\
\text { gen des Arbeitsprozesses } \\
\text { Einbringen fachlicher und sozialer Kompe- } \\
\text { tenzen } \\
\text { Betriebs- und Rationalisierungsverständnis: } \\
\text { Konsensorientierung zwischen betrieblichen } \\
\text { und kollegialen Interessen } \\
\text { Kollegiale Solidarität } \\
\text { Stabile erwerbsbiografische Perspektive }\end{array}$ & $\begin{array}{l}\text { Aktiv, aber interessensbewusst } \\
\text { abwägend } \\
\text { Willkommene Möglichkeit, sich mit } \\
\text { den eigenen Kompetenzen in die } \\
\text { Arbeit einzubringen (Interessant- } \\
\text { heit/Fachlichkeit) } \\
\text { Verbesserung der Arbeitssituation } \\
\text { Schutz vor Verschlechterung } \\
\text { Steigerung der Interessantheit der } \\
\text { Arbeit }\end{array}$ \\
\hline
\end{tabular}

94 Beteiligungsdisposition ist in dem Sinne zu verstehen, dass in den subjektiven Orientierungen der Beteiligungstypen bzgl. KVP eine Handlungstendenz begründet ist. Da die Typen allgemeingültig ohne direkten Bezug zu objektiven Bedingungen rekonstruiert wurden, lassen sich hier nur Dispositionen benennen, die in subjektiver Auseinandersetzung mit KVP zum Ausdruck kommen können. 


\begin{tabular}{|c|c|c|}
\hline & Charakteristische Merkmale & Beteiligungsdisposition \\
\hline 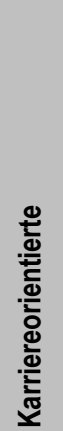 & $\begin{array}{l}\text { Orientierung an beruflicher Weiterentwick- } \\
\text { lung } \\
\text { Weites Aufgabenverständnis: Problemlöse- } \\
\text { und Aufgabenorientierung } \\
\text { Proaktive Auseinandersetzung mit Anforde- } \\
\text { rungen des Arbeitsprozesses } \\
\text { Antizipieren von und Orientierung an betrieb- } \\
\text { lichen Normen Strategisches Handeln, um } \\
\text { eigene Perspektive zu verbessern }\end{array}$ & $\begin{array}{l}\text { Vorbehaltlose Beteiligung } \\
\text { Verbesserung der eigenen Weiter- } \\
\text { entwicklungschancen }\end{array}$ \\
\hline
\end{tabular}

Quelle: eigene Darstellung 



\section{Arbeitspolitische Beteiligungskonstellationen: KVP-Praktiken aus der Perspektive struktureller Beteiligungsbedingungen}

Mit der Analyse der KVP-Praktiken sollen die strukturellen Bedingungen der Gestaltung der einzelnen KVP-Ansätze (Teamarbeit/ProduktionsExzellenz, KVP-Workshop und Ideenmanagement) in Zusammenhang mit der jeweiligen Arbeits- und Betriebsorganisation rekonstruiert werden. Im Zentrum steht hierbei, welche KVP-Praktiken sich in der Umsetzung der KVP-Konzepte in den einzelnen Beteiligungskonstellationen herausgebildet haben und auf welche beteiligungsförderlichen oder -hinderlichen Gestaltungselemente sich diese Praktiken zurückführen lassen. Um die Gestaltungsbedingungen und Wirkungsweisen der KVP-Praktiken zu verdeutlichen, wurden für diese Fallstudie pro Betrieb jeweils zwei Arbeitsbereiche ausgewählt, die sich in dem Grad der Beteiligung an KVP konträr unterscheiden. Zusätzlich werden zu den einzelnen Fallbereichen die typischen KVP-Praktiken - KVP-Workshop (nur bei $\mathrm{NF}$ ) und Ideenmanagement - in den jeweiligen Betrieben hinzugezogen. Diese Praktiken werden im Folgenden porträtiert. 


\subsection{Arbeitspolitische Beteiligungskonstellationen bei NF}

NF befand sich zum Zeitpunkt der Untersuchung in einer sehr volatilen Phase der betrieblichen Restrukturierung. Die häufigen Wechsel im Management, die Einführung flexibler Arbeitszeitmodelle in der Produktion, der starke Wettbewerbs- und Rationalisierungsdruck, ein steter auftragsbedingter Wechsel zwischen Mehr- und Minderarbeit, die Reorganisation der Produktionshierarchie, der Versuch der dezentralen Neuausrichtung der planerischen Bereiche zu Serviceteams sowie letztlich die Einführung der Teamarbeit und KVPWorkshops bewirkten das Aufbrechen etablierter Strukturen im Betrieb. Die einzelnen Akteursgruppen - Führungskräfte, indirekte Bereiche sowie Beschäftigte - waren nun gefordert, entsprechend den neuen Anforderungen des Betriebs neue Aufgaben- und Rollenverständnissen zu entwickeln.

Die beschäftigungspolitischen Maßnahmen, der gestiegene Leistungsdruck sowie strengere Umgangsformen wurden insbesondere von den Produktionsbeschäftigten als massive Verschlechterung ihrer Arbeitsbedingungen wahrgenommen und tragen dominant zu einer durch Unsicherheit geprägten Stimmung im Betrieb bei.

„Ich sage mal so, bis vor vier bis fünf Jabren war das für den Arbeiter ein Vorzeigebetrieb. Draußen in den kleinen Firmen, da musst du wirklich arbeiten. Hier babe ich einen Betriebsrat. Das hast du in einem großen Betrieb, was gut ist; weil du hast Unterstützung. Draußen hast du dich mit dem Chef selbst auseinandersetzen müssen und da gehst du balt zum Betriebsrat, wenn du ein großes Problem hast. Aber die letzten paar Jabren, sagen wir, seitdem wir [übernommen, BS7 wurden, kommt der große Scbnitt. Also gewaltig. Und in letzter Zeit, was sie auffübren, geht unter keine Kubhaut mehr für mich. Das geht an Grenzen von den Leuten. Freilich, man war vorher verwöbnt, so ebrlich bin ich auch. Für einen, der hier drin gelernt hat, muss jetżt eine Welt zusammenbrechen, weil du musst mebr leisten. Im Prinzip kürzen sie deinen Urlaub und so viele Sachen. Wir verstehen es nicht. Wir haben uns am Mittwoch den Chef-Betriebsrat eingeladen, weil es passieren Sachen, die für mich unvorstellbar sind. Durch das ist das Klima so schlecht herinnen. [...] Früber war das Ganze - freilich hat sich die Zeit verändert - kollegial. Es ist mebr auf den Menschen geschaut worden. Heute bekommt man gesagt, BST, Wenn du nicht willst, da ist die Tür, geh binaus und such dir was anderes." (Fischer, NF Montage F_A, 53ff.)

„Die Zeiten sind vorbei. Ich sage, [NF, BS] ist genauso ein Konzern und fäbrt eine brutale Linie. Es ist so, weil sonst kannst nicht überleben. So ist es. Aber das Finanæielle passt und das ist der Grund, warum wir da sind." (Haller, NFZ Montage F T1 A, 30ff.)

Diese allgemeinen beschäftigungspolitischen Veränderungen, die umfangreiche Reorganisation und das schlechte Betriebsklima rufen vielseitig Unsicherheiten 
und Irritationen hervor, aufgrund derer viele Beschäftigte ihren Arbeitsplatz bedroht sehen oder ihrerseits ihr Beschäftigungsverhältnis bei NF infrage stellten.

\subsection{1. „Kampf um gute Arbeit“ (NF Montage F)}

Montage F ist ein manueller Montagebereich von NZ, deren Mitarbeiter sich sehr aktiv an KVP im Rahmen einer selbstorganisierten und engagierten Teamarbeit sowie in KVP-Workshops und in Workshops, die sie selbst mit dem Meister initiiert haben, beteiligen. Die Einführung der Teamarbeit wurde von den Beschäftigten der Montage $\mathrm{F}$ sehr positiv aufgenommen und als Chance ergriffen, die ihnen zugeschriebenen Aufgaben und Kompetenzen aktiv für die eigene Steuerung und Verbesserung des Personaleinsatzes (Abwesenheitsplanung), der Leistungssteuerung und der Arbeitsabläufe zu nutzen. So entwickelten die Teams aus sich heraus mit Beginn der Teamarbeit schnell eine hohe Arbeitseinsatzflexibilität durch Rotation und Anlernen aller Teammitglieder an allen Arbeitsplätzen der Arbeitsbereichs ${ }^{95}$. In Pausen und Teamgesprächen elaborierten die Teams nicht nur gemeinsam Probleme und Verbesserungsmöglichkeiten und diskutierten über Lösungsmöglichkeiten und Nutzen, sondern schöpften gezielt die Mittel der Teamarbeitsstrukturen aus, um ihre Ziele zu verwirklichen. Der Fokus ihrer Optimierungsaktivitäten lag auf der Gestaltung und Erleichterung eines Arbeitsprozesses, für den sich alle Teammitglieder aufgrund der Rotation gemeinsam verantwortlich fühlen. Dies beinhaltete vor allem ergonomische Veränderungen und Ablaufverbesserungen sowie die Vermeidung von Arbeitsunfällen. Beispiele für Optimierungsaktivitäten bis zum Zeitpunkt der Untersuchung (ca. 16 Monate nach Beginn der Teamarbeit) sind: Beschaffung von ergonomischen Matten, selbstinitiierter Ergonomie-Workshop an den Kommissionierungsarbeitsplätzen, Konzipierung eines neuen Montagewagens mit Hebehilfe (mehrwöchiger WS mit Werkzeugbau), Wegzeitoptimierungen.

[BS: ,Überlegt man sich auch, wie man Dinge leichter, besser machen könnte, um besser zurechtzukommen?" "Ja sicher, das machen wir auch. Das brauche ich ja keinen sagen, was ich tue. Für das Team muss es leichter sein. Für den Arbeiter muss es erträglich sein. Ich muss so einfach wie möglich machen. Verschlechtern wird es sich eh von selber. Und das tun wir nach wie vor und wie gesagt, das sind sicher Arbeitshandlungen,

${ }^{95}$ Die Anlernzeit an einem der ca. 7 Arbeitsplätze beträgt ca. 2-3 Tage. 
was wir einfach anders machen, als sie glauben, weil es für den Arbeiter besser ist. " $\mathrm{F} i$ scher, NF Montage F_A, 842ff.]

Für die Beschäftigten sind besonders diejenigen Optimierungsinhalte interessant, die für sie unmittelbar den Vollzug des Arbeitsprozesses erleichtern. Rationalisierungspotenziale werden unter der Annahmen zurückgehalten, dass vom Betrieb Leistungsintensivierungen - unabhängig von den realen Produktionsbedingungen - vorgenommen werden.

- Um ihre Ideen zu realisieren, haben die Teams insbesondere die Teamgespräche für Einladungen des Meisters und Vertreter der indirekten Bereiche genutzt, um mit ihnen im engeren Sinne Optimierungsideen zu diskutieren, aber auch um betriebliche Hintergrundinformationen einzuholen, die für ihre alltägliche Arbeit relevant sind. Dabei stießen die Teams der Montage $\mathrm{F}$ insbesondere zu Beginn der Teamarbeit auf Unverständnis der indirekten Bereiche, die sich noch nicht auf die neuen Kompetenzen, den Aufgabenzuschnitt der Teamarbeit in der Produktion und die damit verbundenen eigenen neuen Rollen eingestellt hatten. Oftmals reagierten diese nicht auf die Einladungen oder fühlten sich für die Anliegen der Teams nicht zuständig.

„Du bist natürlich voll mit einer Energie in die Teamarbeit gegangen, das war so, und wir haben jetæt die Möglichkeit, dass wir was bewirken können. Wir können was umsetzen, wir haben es ja auch vorher schon gedanklich aufgeschrieben, was wir verändern wollen oder dass wir es schon einmal vor der Teamarbeit besprochen haben. Nur bat es dann meistens an der Umsetzung gefehlt. Und jetzt ist es immer so, wenn wir gesehen haben, dass nichts weitergeht, baben wir uns denjenigen eingeladen. Und da hat es schon Teambesprechungen gegeben, wo der [Meister Fuchs, B.S.] auch dabei war, weil es hat dann natürlich der eine auf den anderen geschoben und auf einmal war der nicht zuständig und der nicht zuständig und letztlich ist es wieder an uns herunten bängen geblieben das Ganze. Da habe ich gesagt, ,Das kann es jetzt nicht sein'. Jetzt haben wir so viele [Angestellte], auch Produkttechniker, Prozesstechniker und letatendlich sollen dir die alle helfen und dann lädst du sie ein wegen einem Problem und auf einmal ist der nicht zuständig, weil da musst du zu dem, und der ist nicht zuständig und letztendlich wäre ldie Unternebmenszentrale] quständig. Da babe aber ich nichts davon. Und da muss ich sagen, da ist es schon teilweise voll zur Sache gegangen und das hat sicher was bewirkt [...]. Ich meine, wir haben unsere Veränderungen großteils durchgebracht - bis auf das eine Licht, das wir noch nicht gekriegt haben. "(Immler, NF Montage F_ A, 750ff.)

Die Beschäftigten der Montage F mussten sich mit Hilfe des Meisters beharrlich selbst dafür einsetzen, dass ihre Optimierungsideen, die sie nicht eigenständig in ihrem Bereich umsetzen konnten, realisiert wurden. Dass die indirekten Bereiche, insbesondere die Planungsbereiche, auch ihrerseits die neuen Teamarbeitsstrukturen nicht nutzten, führte dazu, dass im Bereich der Montage F oftmals expertengetriebene Veränderungen durchgeführt wurden, über 
welche die Beschäftigten nicht oder erst sehr spät informiert wurden. Beispielsweise veränderten die Teams hier proaktiv ihren Arbeitsbereich so, dass sich die Wege zwischen dem Montageband und den Bereitstellungsbehältern verkürzten, um die Abläufe zu erleichtern. Diese Veränderungen mussten rückgängig gemacht werden, da sie nicht dem unternehmensweiten standardisierten Konzept der Fertigungsorganisation entsprachen. Standardisierungskonzepte und Konzernrichtlinien waren hier gegenüber den beschäftigtengetragenen Optimierungen vorrangig. Die Dominanz arbeitsprozessferner Reorganisationskonzepte, mangelnde Transparenz und Kommunikation seitens des Betriebs wurden von den Beschäftigten als Einschränkung ihres Handlungsspielraums und als mangelnde Anerkennung ihrer Kompetenzen wahrgenommen.

Der Meister von Montage F (Fuchs) spielte für das Gelingen des KVPs eine wichtige Rolle. Er verstand sich als Unterstützer seiner Beschäftigten, ließ sie im Rahmen der Teamarbeit eigenständig arbeiten und kümmerte sich um die Rahmenbedingungen der Produktion, wie die Beschaffung von Werkzeugen, Hilfsmaterialen und Personal. Er band die Beschäftigten durch die Weitergabe von Informationen über betriebliche Veränderungen und Entscheidungsprozesse in die betrieblichen Abläufe direkt ein. Dabei bestärkte er seine Beschäftigten oftmals deutlich in ihrer Rolle als wertschöpfend Tätige im Betrieb. Die Hervorhebung und Anerkennung der Leistung seiner Teams knüpfte er an die Verantwortung, die seine Beschäftigte für ihren Bereich haben. Auf diese Weise versuchte er die Selbstorganisation, das Verantwortungsgefühl und das Wirtschaftlichkeitsdenken seiner Beschäftigten zu fördern und auf die Notwendigkeit von Veränderungen und Rentabilität hinzuweisen, um den Erfolg des Unternehmens zu sichern.

Mit dem gleichen Engagement setzte sich Meister Fuchs aber auch für die Behandlung der Probleme der Beschäftigten bei den unterstützenden Bereichen ein. Er war bereits vor der Hierarchiereorganisation Meister. Dadurch konnte er seine bislang etablierten Beziehungsstrukturen nutzen, um die dysfunktionale Betriebsorganisation zu umgehen und mit Nachdruck das Management und die indirekten Bereiche (wie Planer, Werkzeugbauer, Einkauf) für die Ideen seiner Beschäftigten zu gewinnen und deren Umsetzung zu beschleunigen.

Dabei differenzierte Meister Fuchs nicht zwischen Optimierungsideen mit einem sich unmittelbar wirtschaftlich rechnenden Nutzen und Arbeitserleichterungen oder Ergonomie, sondern setzte sich offen für die Ideen seiner 
Teams ein. So schaffte er beispielsweise Bodenmatten für sein Team an und unterstützte die ergonomischen Workshops. Indem ihre Optimierungsideen relativ schnell - wenngleich nicht immer ohne Widerstände - umgesetzt wurden, haben die Beschäftigten erfahren, dass ihr Engagement wirksam zur gewünschten Veränderungen führte.

„Aber das, was wir haben mit den Leuten, sind wir hervorragend unterwegs beim Zwischenmenschlichen, das Zweite ist, wir liefern hervorragende, also wir produzieren hervorragende Qualität, und das Dritte ist, wir produzieren auch unsere Stück:zabl, also die Ausbringung, die wir haben. Also uns geht es nicht so schlecht, wir baben Qualität und wir haben unsere Ausbringung, also unsere Leistung. Da lebe ich sorgenfrei. Was will ich noch?" (Meister Fuchs, NF Montage F_A, 1237ff.)

Neben der Unterstützung des Meisters trugen die Teamzusammensetzung und die Praxis einer selbstorganisierten Teamarbeit einen großen Teil zum funktionierenden KVP bei. In Montage F gab es einen hohen Anteil an älteren, erfahreneren Metallfacharbeitern, die teilweise zuvor in nun outgesourcten Montagebereichen als Vorarbeiter oder Vorarbeiterstellvertreter gearbeitet hatten. Diese zumeist mitgestaltungsorientierten Beschäftigten konnten aufgrund ihrer Erfahrungen im Umgang mit betrieblichen Abläufen, ihrer aufgebauten betrieblichen Netzwerke und ihres Bewusstseins, Experte und Leistungsträger des Arbeitsprozesses zu sein, ausreichend Durchhaltevermögen und Mut aufbringen, die Organisation herauszufordern, auf ihre Bedürfnisse einzugehen. Sie waren aktive Träger und Treiber des KVP und in ihren Augen mitverantwortlich für die Qualität des Produktionsprozesses in ihrem Bereich. Mit diesem Bewusstsein agierten die Beschäftigten und zogen unter Nutzung der Teamarbeitskompetenzen und -instrumente die Produktionsleitung, Betriebsrat und Vertreter indirekte Bereiche zur Verantwortung, wenn diese in ihrer Wahrnehmung Fehler machten.

„Bei der] Verlagerung der Prägezange [...] war im Grunde kein Mitarbeiter von uns dabei, sondern das haben größtenteils Fachbochscbüler gemacht und also eher Leute, die noch nicht so lange im Werk sind und eigentlich von der Praxis her - ich will ibnen jetzt nichts unterstellen, aber es ist logisch, wenn ich bis heute noch nie mitgearbeitet habe, kann ich nicht alles wissen. Und da muss ich schon sagen, da baben wir schon, ich glaube, zwei oder drei gravierende Änderungen gemacht, aber erst im Nachbinein, wie wir dann gesagt haben, ,Hallo, so können wir nicht arbeiten'. [...] Das war auch speriell der Fall, wie wir sie dann zu den Teamgesprächen eingeladen haben, weil sonst bätten wir ja überhaupt nichts gewusst, wie das mal in Zukunft ausschaut. Dann ist letztendlich mal beim Teamgespräch ein Plan aufgebängt worden und da sind wir gleich draufgekommen, wenn das so ausschaut wie nach dem Plan, würde der Rabmen auf der verkehrten Seite geprägt werden. [...] Eigentlich ist es ein Wahnsinn, wenn wir da nichts sagen, machen die das. Es kostet ein Schweinegeld und wir wollen dann starten und kommen dann drauf, dass das Fabrzeng verkehrt geprägt ist. [...] Und natürlich haben 
sich alle bedankt, superschön und alles. [...] Aber da waren eigentlich immer wieder wir diejenigen, die gedrängt haben und wieder zum [Abteilungsleiter] gesagt haben, ,Hallo, wieso?" Und dann erst sind wir wieder miteinbezogen worden und dann erst haben wir es wieder mehr oder weniger so hingebracht. Und es hat eh jeder eingesehen, dass es so besser ist, aber wir sind nicht vorher gefragt worden. "(Immler, NF Montage F_A, 1317ff.)

Solche Erlebnisse bestätigten das Bewusstsein der Teammitgliedern, wichtige Erfahrungs- und Kompetenzträger im Betrieb zu sein, und belegten dadurch die Legitimität ihrer Beteiligungsansprüche. Die positive Erfahrung, dass der Betrieb auf ihre Ideen einging - wenn auch auf Nachdruck von Meister und Beschäftigten - und sie auch in die Umsetzung einbezogen wurden, führte dazu, dass sich diese einzelnen Optimierungsmaßnahmen zu kontinuierlichen Optimierungspraktiken verfestigten, während negative Erfahrungen der Ablehnung oder der Rücknahme von eigenen Optimierungen durch den Betrieb in Resignation mündeten.

Für die Beschäftigten in Montage F eröffneten sich durch die Einführung von Teamarbeit neue Wege, die von ihnen für sinnvoll erachteten Veränderungen in ihrem Bereich zu initiieren, selbst an der Umsetzung beteiligt zu sein und sich wirkungsvoll für ihren Bereich zu engagieren. Vor der Teamarbeit gab es außer dem Instrument des Ideenmanagements kaum Möglichkeiten, direkt Veränderungsvorschläge einzubringen, zudem war die Umsetzung von Ideen über das Ideenmanagement mit sehr langen Bearbeitungszeiten verbunden oder funktionierte gar nicht. Verbesserungen des unmittelbaren Arbeitsprozesses wurden nun mit Teamarbeit in Montage F niedrigschwellig direkt umgesetzt. Umfangreichere Ideen, zum Beispiel die neue Konzeption von Montageträgern zur leichteren Montage, wurden im Rahmen von festgelegten Projekten in Teamgesprächen erörtert und in Workshops realisiert, während Verbesserungsideen mit einem hohen finanziellen Nutzen für den Betrieb aufgrund der Prämierung im Rahmen des Ideenmanagements eingebracht wurden - was allerdings nur selten geschieht.

\subsection{2. „Im Stich gelassen“ (NF Montage K)}

In Montage K, wo zum Zeitpunkt der Untersuchung erst seit ca. sechs Monaten in Teamarbeit gearbeitet wird, begann die Teamarbeit zunächst ebenfalls mit motivierten Selbstorganisations- und Optimierungsaktivitäten einiger weniger Teammitglieder (vor allem Teamsprecher und Qualitätsregelkreismitarbeiter). Der Fokus ihrer Aktivitäten lag zu Beginn auf Ordnung und Sauberkeit der Arbeitsplätze, Ergonomie, Umgestaltung von Werkzeug- und Materialwa- 
gen sowie Herstellung von größerer Arbeitseinsatzflexibilität durch Anlernen aller Mitarbeiter an allen Teamarbeitsplätzen.

Bereits nach kurzer Zeit stieß das Engagement der wenigen Teammitglieder auf Hindernisse und Hürden. Auf der Ebene der Arbeitsorganisation behinderten ein hoher Krankenstand und Leistungsdruck das Anlernen neuer Mitarbeiter.
„Wir haben vom ersten Tag an zusammengeräumt, also wir haben alles, was nicht ge- braucht wird und was sinnlos herumgestanden ist, weggeschmissen oder verstaut, zurïck- gegeben im Lager - Und das war eigentlich die größte, also positive Veränderung, weil die Arbeitsplätze sind jetzt sauber, was vorher nicht der Fall war. Also das haben wir selbstständig gemacht, da hat uns keiner dazu aufgefordert, da waren die Leute so ge- scheit und baben gleich von Anfang an gesagt, wir sollen einen sauberen Arbeitsplatz. und sie wollen, dass das alles passt. [...] Und dann baben wir probiert. Dann baben wir uns zusammengesetzt im Teamgespräch und auch in der Mittagspause, haben wir gesagt, ,Wie arbeiten wir zusammen und wie machen wir es?', und am Anfang baben wir genug Personal gehabt, da ist es gleich super gewesen mit dem Wegfabren, da waren die Leute noch begeistert, da bat noch jeder gesagt, Ja, super " [...]. Und jetrt ist es halt so sebr viel Krankenstand. Das ist ein Mangel. Und obne Personal bringst du nichts weiter, das ist so. Wir stagnieren jetzt schon. Jetzt haben wir vier Monate Teamarbeit, wir stagnieren jetzt schon mit dem Qualifizieren der Leute, glaube ich, zwei Monate fast. Weil in den ersten zwei Monaten haben wir die halbe Mannschaft fast überall qua- lifiziert und jetzt stehen wir an. Dann kommen wieder Neue dazu oder wieder weg, dann fängst du wieder von vorne an. "(Albinger, NF Montage K_B, 244ff.)

Verbesserungsideen oder anfallende Probleme wurden mitunter im Teamgespräch, aber zumeist nur zwischen einzelnen Teammitgliedern besprochen.

Maßnahmen, die nicht eigenständig von den Teams umgesetzt werden konnten, weil keine zeitlichen Ressourcen vorhanden, die Unterstützung von Fachbereichen oder Genehmigungen für anfallende Kosten notwendig waren, wurden auf einen Maßnahmenplan - einer Art Tafel im Teamarbeitsbereich geschrieben.

„Man hat eine Idee, man schreibt es auf die Tafel und es braucht ewig und es ist im Endeffekt, man ist halt der Bittsteller. Man kann das hinschreiben, aber ob es dann geschieht oder nicht, das liegt wieder im Ermessen von den Vorgesetzten. Wir haben zum Beispiel drei Arbeitsunfälle gehabt, weil man auf die Transportgestelle oben steht. Und wir haben gesagt, das wäre eine volle Erleichterung auch für die Gesundheit, wenn man dort einfach solche Trittbretter hat, dass man nicht immer auf den Winkeleisen steht. Ja, wir haben das ausgearbeitet, wir haben das ausgemessen, wir haben das bingeschrieben, es waren die Arbeitsunfälle. Es hat geheißen, wir müssen was tun. Im Endeffekt ist es jetzt abgelehnt worden, weil es zu teuer ist. Aber auf der anderen Seite bat man wieder Krankenstandgespräche. Es sind da drei Arbeitsunfälle geschehen und es ist egal. Also es ist, wie soll man sagen. Ja, es resignieren dann viele und sagen, was schreibt man das auf, was tut man das, wenn dann / Und es wird nur das umgesetฉt, was balt wirklich was bringen würde. "(Berger, NF Montage K_B, 547ff.) 
Die Tafel entfaltete allerdings über die reine Funktion einer Sammlung von Verbesserungsideen keine verbindliche Wirkung auf die Führungskräfte und indirekten Bereiche, sich zeitnah und regelmäßig um die Umsetzung zu kümmern. Die Ideen der Beschäftigten werden nur in einzelnen Fällen umgesetzt und nur dann, wenn die Meister oder einzelne Beschäftigte die Umsetzung hartnäckig vorantrieben. Eine direkte Kooperation mit Vertretern von unterstützenden Bereichen, wie es etwa durch die Einladung ins Teamgespräch erfolgen könnte, fand nicht statt.

Oftmals stießen die Initiativen der Teams, wie die von Berger genannte, aus finanziellen und fertigungsorganisatorischen Gründen auf Ablehnung. In der Wahrnehmung der Beschäftigten förderte der Betrieb nur Ideen mit spürbarem finanziellen Nutzen, während für Ideen, bei denen es um die körperliche Versehrtheit (Ergonomie und Vermeidung von Arbeitsunfällen) und Arbeitserleichterungen ging, keine Ressourcen bereitgestellt wurden, diese umzusetzen.

[B.S.: Wird eigentlich auch wirklich vom Betrieb erwartet, dass man sich für Optimierungen einsetzt? ?

„Ja, aber nur in eine Richtung, also die Richtung kennen wir. Das ist, wenn du was einsparst, ist es gut. Es wird zwar immer das Thema Sicherbeit hochgebalten, aber letztendlich sind das nur leere Worte, ja. "(Eder, NF Montage K_B, 774ff.)

Deutlicher noch als in Montage F verbinden die Beschäftigten Veränderungen mit der betrieblichen Intention, zu Lasten der Beschäftigten Kosten zu sparen, und versuchten, Rationalisierungspotenziale ganz bewusst zurückzuhalten, selbst dann, wenn eine Optimierungsmaßnahme mit deutlichen Arbeitserleichterungen verbunden wäre. Einzelne Beschäftigte, die Ideen mit Rationalisierungspotenzial und langfristige Belastungsreduktion einreichen, wurden stark kritisiert und unter Druck gesetzt, solche Vorschläge zu unterlassen.

„Wir haben jetzt wieder einen Verbesserungsvorschlag, einen angehenden, da geht es um eine gravierende Umstellung und wo Arbeitsabläufe rausfallen; und der will einen Verbesserer machen, und ich babe zu dem Kollegen gesagt, sage ich, ,Das musst du selber wissen. Jeder hat das Recht dazu, nur fallen da halt viele Tätigkeiten weg und das musst du halt dann selber abwiegen, weil du arbeitest dann in Zukunft auch wahrscheinlich selber da, ob du das überhaupt willst. " (Albinger, NF Montage K_B, 1004ff.)

Infolge dieser „Warnung“ wurde dieser Verbesserungsvorschlag nicht im Teamgespräch besprochen, sondern von Berger im Ideenmanagement eingereicht. 
Dass das Engagement zu Beginn der Teamarbeit derart ins Leere lief, lag vor allem an der mangelnden Unterstïtzung durch den Meister und die Service-Bereiche. Die fehlende Unterstützung der Beschäftigten war wiederum auf den Rollenzuschnitt des neuen Meisters und die neuen Kooperationsstrukturen zwischen Führung und Servicebereichen zurückzuführen. Die Meister, die vorwiegend aus den Reihen der Vorarbeiter besetzt wurden, hatten in ihrem neuen Führungszuschnitt zwar hohe Verantwortung für eine reibungslose Produktion und ihre Mitarbeiter, aber nur sehr geringe zeitliche Ressourcen und Entscheidungsspielräume.

„Ja gerade, wenn es in Richtung Entscheidungen geht oder Gestaltungsspielraum im eigenen Bereich, hat der [alte, B.S.] Meister sicher mehr - wie soll ich sagen - den sein Wort hat mehr gezäblt. Jetzt als [neuer Meister, B.S.]. Es fehlt zum Teil das Vertrauen von unseren Fübrungskeräften. Ja und auch die Dienstleister oder die, was wir benötigen, die brauchen immer wieder das Wort vom Führungskraft, also von unserer Führungskeraft, vom Modulleiter oder vom Spartenleiter, weil das Wort vom [neuen Meister, B.S.] nicht so - wie soll ich sagen - nicht so schwerwiegend ist oder es greift nicht so." (Aichinger, NF Meister Montage K_A, 44ff.)

Betriebliche Entscheidungen, Aufgaben und Zielvereinbarungen wurde in der Produktionshierarchie der Komponentenfertigung von oben vorgegeben. Die Meister müssen diese Entscheidungen umsetzen und verantworten zugleich den Erfolg des angestrebten Produktionsprogrammes, ohne ausreichend Unterstützung von den indirekten Bereichen zu finden.
„Wir sind sehr viel Laufburschen. Wir machen sehr viele organisatorischen Themen und wir, das ist eben meine Meinung, wir bereiten sebr viel auf für alle anderen Bereiche. Al- so wir übernehmen sehr stark generelle Aufgaben mit, weil teillweise die Verantwortli- chen, also die Verantwortlichkeiten nicht gan z, klar geregelt sind oder es einfach so viele Verantwortliche gibt, dass sich wieder keiner zuständig fühlt und ein bisschen die Kultur da drinnen herrscht, wenn ich es nicht mache, vielleicht macht es dann irgendwer anderes. Weil es dann wieder mühsam macht. Vielleicht sind wir wieder die Leidtragenden, die das alles ausbaden müssen, und bevor es keiner macht, machen wir das lieber selber und damit sind wir dann die Verantwortlichen für das. Also die Fübrungsverantwortung, die wir eigentlich haben sollten, beschränkt sich so ein bisschen auf Informationen weiterge- ben, die wir kurifristig keriegen, wie diesen Zettel jetzt, wo es darum geht, dass der Schichtbus nächste Woche nicht fäbrt am Montag, oder am Dienstag. [...] Wir haben Verantwortung, aber keine Entscheidungsmacht eigentlich, ja. Und das ist auch ein bisschen schade. "(Bäumler, NF Meisterin Montage K_ B, 30ff.)

Obgleich die Neustrukturierung konzeptionell auf Dezentralisierung der Führungsstrukturen und indirekten Bereiche ausgerichtet waren, wurden hier viele Entscheidungen sowie die Aufgabenkoordination vom Abteilungsleiter übernommen. Das reichte so weit, dass die Meister nicht direkt mit ihren zuständigen Planern kooperierten, sondern Anfragen über den Koordinator des Ser- 
vicebereichs erfolgten. Entscheidungen über technische Veränderungen, wie Neuanschaffungen von Werkzeugen, kleinere wie größere (bauliche) Veränderungen im Produktionsbereich, wurden über recht bürokratische Verfahren prozessfern in anderen Bereichen getroffen, so dass die Meister weder Einfluss darauf nehmen können noch ausreichend Informationen über den jeweiligen Bearbeitungsstand hatten. Hinzu kam, dass die Service-Bereiche aufgrund umfangreicher Modernisierungs-, Rationalisierungs- und Standardisierungsprojekte eigene Agenden verfolgten, ohne dass die Beschäftigten rechtzeitig eingebunden wurden, wenn es um die Veränderungen von Fertigungsbereichen ging.

Problembehandlungen und Optimierungspotenziale wurden hier nicht aus den Produktionsbereichen generiert und von den indirekten Bereichen übernommen. Vielmehr hatten von der betrieblichen Führung bestimmte Topdown-Projekte deutlichen Vorrang vor Bottom-up-Veränderungsinitiativen. Die Teamarbeitsorganisation hatte für die Aufgabenbearbeitung der indirekten Bereiche, v.a. den Planungsbereich, kaum Relevanz.

Anders als in Montage $\mathrm{F}$ konnten in Montage $\mathrm{K}$ weder die Teams noch die Führungskräfte gegenüber den indirekten Bereichen die nötige Kraft aufbringen, eine prozessnahe Unterstützung zu „erzwingen“. Die Möglichkeiten des Teamgesprächs wurden nur selten systematisch und eigenständig genutzt. Bislang wurden Vertreter indirekter Bereiche nicht zu Teamgesprächen eingeladen; sie nutzten sie auch umgekehrt nicht, um mit den Beschäftigten direkt in Kontakt zu treten. Dies konnte auch daran liegen, dass innerhalb der Teams, die hier aus wenigen mitgestaltungsorientierten und mehrheitlich Status-quoorientierten Beschäftigten bestehen, kein einheitliches Verständnis darüber vorhanden war, ob und in welcher Art sich das Team an Optimierungen beteiligen sollte.

In Montage $\mathrm{K}$ bemerkten die Beschäftigten trotz der formellen Einführung von Teamarbeit keine substanziell spürbaren Veränderungen. Selbstorganisation und Selbstoptimierungen, die bereits vor der Einführung von Teamarbeit stattfanden, erhielten zwar einen legitimen Rahmen, überließen den Teams aber nur einen schmalen Korridor zur Beteiligung und Gestaltung ihres Arbeitsbereichs. Die Beschäftigten waren nach wie vor Betroffene und keine Akteure von Veränderungs- und Rationalisierungsprozessen und standen Optimierungsprozessen mit deutlichen Ressentiments gegenüber. 


\subsubsection{KVP-Workshops bei NF}

In Montage F und $\mathrm{K}$ wurden mehrere KVP-Workshopreihen durchgeführt, in denen die Fertigungslinien von Anfang bis Ende kleinschrittig auf Optimierungspotenziale hin überprüft wurden. Die Workshops wurden bei NF zugleich genutzt, um das Zeitbemessungssystem von REFA ${ }^{96}$ auf MTM umzustellen und mit den Produktionsbeschäftigten Tätigkeiten im MTM-Datensatz zu erfassen und zu aktualisieren. KVP-Workshops waren für die Beschäftigten in beiden Bereichen neu.

Teilnehmer der Workshops waren der Moderator (hauptamtlicher Produktionssystemtrainer), mindestens ein Vertreter des Betriebsrates, der zuständige Planer, ein MTM-Experte sowie aus jeder Schicht zwei Arbeiter, die umfassende Erfahrungen mit der Arbeit in ihrem Teamabschnitt hatten. Die jeweiligen Meister waren nur punktuell anwesend. Für die Teilnahme am Workshop wurden von den Meistern bewusst erfahrene Beschäftigte ausgewählt, die zudem ein hohes Ansehen bei den Kollegen im Team genossen, um als Träger und Vermittler der Workshopergebnisse zu fungieren. Insbesondere in Montage $\mathrm{K}$, in der betriebliche Rationalisierungsaktivitäten mit großem Argwohn betrachtet wurden, wurden sowohl die Workshops an sich als auch die eigene Teilnahme sowie die Teilnahme von Kollegen teils stark kritisiert.

„Da will ich dann nicht mebr dabei sein, weil wenn du dabei ist, sind die Leute interessiert, dass du irgendwas bringst auch. Und ich sehe das, dass es da nur gegen den Arbeiter geht. So sehe ich das. Und da ist es mir lieber, ich bin nicht dabei, weil ich mir selber nicht weh tun möchte und den anderen Kollegen auch nicht, so muss ich es ebrlich sagen. Und das ist nichts anderes nicht. "(Cžerni, NF Montage K_B, 1249ff.)

„Es ist im Team dann schon oft über das geredet worden, was wir da machen in den Workshops, ob wir da eh nicht nur ,umeinandsitzen" und sowas aber, ja also es ist die einheitliche Meinung, dass man da nur ,umanandsitzt" und nicht recht viel tut. Das war eigentlich ein wenig eine Überraschung. "(Auer, NF Montage K_A, 920ff.)

96 REFA ist die Abkürzung für „Reichsausschuß für Arbeitszeitermittlung“ und bildet einen Methodenkatalog zu Datenerfassung von Montagearbeitszeiten. Im Unterschied zu MTM, wo Montagearbeitszeitvorgaben mittels detaillierter Erfassung von einzelnen Montagetätigkeiten mit festgelegten Zeiten erfasst werden, basieren REFA-Vorgabezeiten - wie im Fall NF - vorwiegend auf Zeitstudien von Arbeitszyklen einzelner verketteter Arbeitshandlungen. 


\section{MTM-Teil}

Der Bearbeitungsteil MTM wurde kooperativ von Beschäftigten und Planern bearbeitet. Durch die Beteiligung an der Datenerhebung für MTM haben die Beschäftigten einen Einblick in die Systematik der Personalbemessung und die Grundlagen für die Definition und Berechnung der Vorgabezeiten erhalten. Dies ist insofern wichtig, als es immer wiederkehrende Kritik und Zweifel an der Richtigkeit betrieblicher Leistungsvorgaben und Personalbemessung gegeben hat.

„Es hat sich zumindest das verändert, dass ich weiß, was ich mache und für was ich das Geld bekomme. Das hat sich gescheit geändert. Weil früher habe ich oft das glauben müssen, was sie mir gesagt haben. Weil ich es nicht kontrollieren habe können, jetst habe ich es eigentlich selber kontrolliert bzw. selber gemacht und was ich selber mache, da weiß ich, was geschiebt und was dabinterliegt, also war das für mich aufschlussreich und auch, glaube ich, für das ganze Team, weil er hat mir es immer wieder gesagt, ,Das, was wir machen, könnt's glauben, das ist drinnen" und wenn wir keinen Febler gemacht, dass wir nichts übersehen baben, ist sicher alles drinnen und ich glaube, wir haben nichts übersehen."(Auer, NF Montage K_A, 1174ff.)

Das generelle Misstrauen der Beschäftigten gegenüber der Richtigkeit der Leistungsbemessung, das den Alltag der Beschäftigten deutlich geprägte, wurde durch diese Maßnahme verringert, war aber angesichts vielseitigen betrieblichen Aktivitäten und Veränderungsmaßnahmen nicht gänzlich erloschen.

\section{Rolle der Beschäftigten im Workshop: Zwischen Kontrolle, Gestaltung und} Ausbruch aus der monotonen Arbeit

Die teilnehmenden Beschäftigten waren sich sehr bewusst, dass die betriebliche Intention des Workshops in der Erzielung von Rationalisierungseffekten lag. Sie verbanden mit ihrer Teilnahme vor allem die Möglichkeit, für sich und ihren Bereich positive Veränderungen zu bewirken. Dabei beziehen sie sich nicht allein auf die Verbesserung von Ergonomie, sondern schließen Optimierungen an Arbeitsprozessen und Abläufen, der Qualität sowie technischen Problemen nicht aus, insofern sie sich nicht negativ auf die Arbeits- und Leistungsbedingungen auswirken. Optimierungspotenziale, die ausschließlich Rationalisierungscharakter hatten, wurden bewusst zurückgehalten. Durch die Teilnahme am Workshop versuchten die Beschäftigten, die Aktivitäten der Planer zu kontrollieren und potenzielle negative Veränderungen abzufangen. Dies galt insbesondere für den MTM-Teil des Workshops.

„Naja, ich habe für mich geschaut, dass man eben nichts übersieht, für die Leute heraußen, dass wirklich alle Zeiten berücksichtigt werden. Das war für mich eigentlich das 
wichtigste. Und dass man keine Verschlechterung qusammenkriegt in dem Sinn. "(Auer, NF Montage K_A, 972ff.)

„Ja, [ich bin, B.S.] der Aufpasser, dass man die MTM richtig rüberbringt, dass man nichts vergisst. Und zu versuchen, einfach was zu verbessern. Ja und ich versuche dann auch nicht einfach nur zu optimieren, dass man, ja ich versuche mich da in dem Bereich zu bewegen und dann umsetzen, was man dann halt immer wieder, ich bin da halt der Umsetzer, sonst passiert nichts, wenn man da nichts macht." (Eder, NF Montage K_B, 1087ff.)

Die Beschäftigten sahen ihre Rolle und Möglichkeiten auch darin, die Zeit im Workshop zu nutzen, um Veränderungen umzusetzen, die während der Arbeit nicht möglich waren. Mit ihrer Teilnahme verfolgten einzelne Beschäftigte, wie Eder, zudem nicht nur einen kollektiven, sondern auch einen individuellen Nutzen. Der Workshop bog grundsätzlich Abwechslung vom monotonen Arbeitsalltag und die Möglichkeit, sich sowohl fachlich mit übergreifenden Themen und betrieblichen Zusammenhängen zu befassen als auch kurzzeitig der belastenden Tätigkeit zu entkommen. Die teilnehmenden Beschäftigten lernten dabei mehr Vertreter unterschiedlicher Funktionen kennen und konnten ihr Beziehungsnetzwerk ausbauen. Darüber hinaus war es potenziell möglich, sich durch die eigene Leistung im Workshop, wie eine hohe Veränderungsbereitschaft und fachliche Eignung, auch über die Wahrnehmung des eigenen Meisters hinaus von der großen Masse der Montagearbeiter abzuheben - wobei zum Zeitpunkt der Untersuchung keinesfalls ersichtlich war, dass die Teilnahmen am Workshop den Beschäftigten irgendeinen Vorteil im Betrieb einbrachte.

Die Workshopteilnehmer mussten die Workshopergebnisse, auf die sie nur teilweise Einfluss haben, allerdings vor ihren Teams rechtfertigen. In dieser Hinsicht ist die Teilnahme der Betriebsräte als Kontrolleure und Unterstützer von großer Bedeutung. Die Betriebsräte und die teilnehmenden Beschäftigten teilten sich gewissermaßen die Verantwortung für die Workshopergebnisse.

\section{Dominanz standardisierter Gestaltungskonzepte gegenüber Problemlösungen im Produktionsprozess}

Im Vorfeld des Workshops überlegte das ganze Team von Montage F, welche Verbesserungen sie in ihrem Bereich im Zuge des Workshops umsetzen wollten, wie zum Beispiel die Neuanordnung der Bereitstellungsbehälter für Montageteile und kleinere Maßnahmen zur Verbesserung der Ergonomie und Abläufe an der Linie. In Montage K gab es lediglich individuelle Vorüberlegungen 
für Optimierungen; innerhalb des Teams fand keine systematische Abstimmung statt. Neben wenigen Verbesserungen an den Kommissionierungswagen und Optimierungen von Arbeitsabläufen wurden vor allem Maßnahmen der Arbeitsplatzgestaltung wie mehr Transparenz und Ordnung direkt während des Workshops vorgenommen.

Die gesammelten Optimierungspotenziale wurden vom Moderator methodisch aufbereitet klassifiziert in: Steigerung der Wertschöpfung, Prozessqualität und Standards. Diese Einordnung wurde zum einen vorgenommen, um das Bewusstsein der Teilnehmer für die unterschiedlichen Arten möglicher Veränderungen zu schaffen und zum anderen Kategorien zu bilden, welche die Leistung des Workshops für das Management, dem am Ende der Workshopwoche die Ergebnisse präsentiert werden, systematisieren. Insgesamt nahm der Moderator als NF-Produktionssystemtrainer als treibende Kraft eine dominante Rolle im Workshop ein, indem er die Verbesserungspotenziale strukturierte und unterstützende Bereiche zur Beratung hinzuzog. In den Workshops wurden die Optimierungspotenziale offen und konstruktiv diskutiert. Durch den Austausch zwischen Planern, Moderator, Betriebsrat und Meister erhielten die Beschäftigten wichtige Informationen über betriebliche Abläufe und Entscheidungen, so dass sie Probleme und Prozesse nachvollziehen konnten.

Die Elaboration und Diskussion der Optimierungspotenziale waren in beiden Bereichen stark orientiert an vorgegebenen standardisierten Konzepten zur Gestaltung des Arbeitsplatzes und des Produktionsprozesses. Bei diesen Gestaltungkonzepten steht die einheitliche und übersichtliche Gestaltung der Arbeitsplätze, wie 5S und das Zonenkonzept ${ }^{97}$ (saubere und transparente Fabrik), im Mittelpunkt. Dabei ging es zum Beispiel um die Beschriftung von Werkzeug- oder Schraubenablageplätzen, Arbeitsanweisungen, genaue Positionen von Bereitstellungsbehältern und Wegezeiten. Diese spezifischen Gestaltungskonzepte waren spürbar dominant. Planer und Produktionssystemtrainer achten im hohen Maße darauf, dass sich die eingebrachten Optimierungspotenziale im Rahmen der vorgegebenen Konzepte bewegen. ${ }^{98}$

${ }^{97}$ Das Zonenkonzept stellt eine räumlich-funktionale Strukturierung der Produktionsbereiche in Montagezonen und Bereitstellungszonen für Montageteile und Kommissionierungswagen da. Das Zonenkonzept dient der Erhöhung der Transparenz, Ordnung und Standardisierung der Montagebereiche dar.

98 Diese Dominanz wird vor allem dadurch verstärkt, dass sehr regelmäßig von Konzernvertretern Audits der Produktionsbereiche stattfinden. Im Fall Montage K war 
„Das ist nicht mehr so leicht, weil du jetzt noch Vorgaben vom [Konzern, B.S.] hast. Da gibt's bestimmte Zonen, wo du was hinstellen darfst, wo du nichts binstellen darfst, das muss einen halben Meter herbeistehen, sonst bist du nicht mehr [Konzern7 Standard. Dann kann ich nichts umstellen, also muss ich es akzeptieren, wie es ist." (Fischer, NF Montage F T_A, 1126ff.)

Verbesserungsideen müssen den betrieblichen Vorgaben standardisierter Arbeitsplatzgestaltung zur Verbesserung der Prozess- und Produktqualität entsprechen.

„Na ja, wir haben da die 5-S-Geschichten, wir müssen da was visualisieren, für mich ist das, ja ich sage, dass man bei dem Workshop beschäftigt ist, man kann natürlich einen Pickel99 ausdrucken. [...] Das nehme ich nicht wirklich ernst, da sieht man wieder, womit andere ibr Geld verdienen den ganzen Monat, ja geht auch. "(Eder, NF Montage F_B, 1245ff.)

Standardisierte Konzepte - bei denen die Anliegen und Bedürfnisse der Beschäftigten hinsichtlich der Gestaltung eines reibungslosen und belastungsreduzierten Arbeitsprozesses in der Regel hintanstehen - dominierten gegenüber prozessnahen Problemlösungen. Auch wenn in den Workshops viele kleinere Verbesserungen vorgenommen wurden, zum Beispiel Klebebandabroller am Montagewagen oder produktqualitätsrelevante Veränderungen im Ablauf einiger Montagetätigkeiten, nahmen sowohl bei der Ideenfindung und in der Diskussion als auch in der Umsetzung Veränderungsmaßnahmen, die sich an den betrieblichen standardisierten Gestaltungskonzepten orientierten, großen Raum ein. Die Umsetzung der Optimierungspotenziale des Workshops oblagen den Meistern, die einem hohen Druck ausgesetzt sind, die Maßnahmen zu realisieren, obwohl sie nur marginal am Workshop beteiligt waren. Aufgrund der Dominanz des Tagesgeschäfts, der schon beschriebenen fehlenden Kompetenzen der Meister und der prozessfernen, eher bürokratischen Arbeitsweise der Planungsbereiche trat die Umsetzung der Maßnahmen häufig in den Hintergrund.

„Es ist zum Teil anstrengend. Den Betrieb oder die, es gibt so Zertifizierungsfirmen, denen liegt viel an der Visualisierung, was wir als Arbeiter draußen eigentlich nicht so sehen. Also ob da jetzt drauf steht, Schrauber" auf dem Schrauber, ist für uns eigentlich unwichtig. Das sind die Sachen, die eigentlich bei den Workshops furchtbar aufhalten, solche Sachen, dass man sich um andere Sachen weniger kümmern kann oder dass man

deutlich erkennbar, dass die KVP-Workshops zur einheitlichen Produktionsgestaltung und Vorbereitung der Audits genutzt werden.

99 Österreichisch für Aufkleber. 
wirklich Verbesserungen für die Leute am Band eher nicht zustande bringt." (Auer, NF Montage K_A, 819ff.)

Dieser Eindruck entstand insbesondere dann, wenn im Workshop erarbeitete Verbesserungsideen in der Umsetzung von einer Reihe betrieblicher Entscheidungen abhängig sind. Sie wurden oftmals erst Wochen oder Monate später realisiert oder gar nicht umgesetzt. Die Beschäftigten erlebten häufig, dass die im Workshop oder in Teamarbeit beschlossenen Maßnahmen durch andere Aktivitäten, die von Planungsteams ohne Mitwirkung von Meister oder Beschäftigten durchgeführt wurden, wieder rückgängig gemacht wurden.

„Also dann ist es mehr so, ja wir können ja bier und picken ${ }^{100}$ noch was an: [Lacht], alles, was wirklich passieren würde, das dauert eh meistens länger, da können wir eb nichts machen und das kostet vielleicht auch Geld, aber es dauert meistens dann, so grundlegende Geschichten, wo man sagt, da kann man was verändern, das passiert eb nicht gleich und das sind dann eh langfristige Geschichten." (Eder, NF Montage F_B, 1262ff.)

„Verändern und verbessern kannst du immer was. Da wirst aber nie fertig und ich muss aber auch nicht wegen alles einen Workshop machen. Was bei uns Workshop drinnen sind zum Teil, da frage ich mich, zu was rennt der wirklich. Da machen wir einen Workshop, der eine stellt Kisten dortbin, zwei Monate drauf wird wieder ein Workshop gemacht. Da räumen sie die Kisten einfach woanders hin. Zum Teil einfach zu viel Workshop. Alles recht und gut, aber/." (Fischer, NF Montage F_A, 1116ff.)

Auch wenn im Workshop kleinere Verbesserungen für die Beschäftigten erzielt werden konnten, wurde die Sinnhaftigkeit des KVP-Workshops im Kontext des angespannten Arbeitsalltags grundsätzlich infrage gestellt. In der Wahrnehmung vieler Beschäftigten gab es nicht nur bei den KVP-Workshops, sondern insgesamt eine hohe Diskrepanz zwischen der rhetorischen Aufforderung zur Beteiligung und der konzeptionellen Neuorientierung auf der einen Seite und der betrieblichen Realität auf der anderen Seite, in der den nicht unmittelbar wirtschaftlichen Verbesserungsideen und Anliegen der Beschäftigten wenig Aufmerksamkeit geschenkt wurde. Zugleich wurden die Arbeitsprozesse und Fertigungsorganisation aufwändig standardisiert, geordnet und visualisiert. Ordnung und Sauberkeit erhielt mehr Gewicht als Arbeitsschutz und die Belastungsreduktion der Beschäftigten

Die Beschäftigten beobachteten, dass der Betrieb viele personelle und finanzielle Ressourcen für Aktivitäten aufwendete, deren Sinnhaftigkeit sich

100 „Picken“ ist ein österreichischer Begriff für Kleben und wird hier im Sinne von Aufkleber kleben verwendet. 
ihnen nicht erschloss, während sie permanent um kleinste Anteile ihrer Vorgabezeiten rangen.

„Schlecbte Seiten, weiß nicht, ob das scblechte Seiten für euch sind, für mich ist einfach das Verbältnis Arbeiter - Angestellte stimmt überbaupt nicht mehr. [...] Bei uns rennen Leute umber, das ist ein Wabnsinn. Der Arbeiter wird immer weniger und ich glaube, die Angestellten werden immer mehr. [...] Ich habe zum Beispiel gesehen bei dem MTM, wo ich dabei war, die haben eine Arbeit, weil die sehe ich auch mit der Mappe herumrennen, weil sie sich was anschauen, aber da weiß ich, was sie tun. Aber es rennen so viele umber, wo man gar nicht weiß, was die tun. Und das wird immer mehr. [...] Beim Arbeiter sparen's und da wird jede Zehntelsekeunde umbergerechnet und bei den Angestellten ist es im Prinzip egal. Und dann fragt man sich wieder, warum wieso weshalb ist das so." (Fischer, NF Montage F T1 A, 173ff.)

Besonders stark wurde das Verhältnis von Produktionsbeschäftigten und Angestellten infrage gestellt, wenn von Angestellten Veränderungsmaßnahmen in den Produktionsbereichen durchgeführt und die Beschäftigten nicht einbezogen wurden. Oftmals führt dies nicht nur zu Verschlechterung der Arbeitssituation, sondern auch zu fachlich-technischen Mängeln. Die Praktiken der KVPWorkshops fokussierten somit sehr stark auf starre Methoden, vorgegebene Gestaltungsrichtlinien und die Einhaltung von Standards.

\subsubsection{Bürokratisches Ideenmanagement bei NF}

Teamarbeit und KVP-Workshops wurden flankiert vom ältesten Beteiligungsinstrument bei NF, dem betrieblichen Ideenmanagement. Ideen, die einen hohen betriebswirtschaftlichen Nutzen ( $>2500$ Euro) generieren, konnten über den Meister oder direkt über das IT-System zur Umsetzung und Prämierung eingereicht werden. Herausragende Gruppen- oder KVP-Workshopideen konnten ebenfalls im Ideenmanagement eingereicht wurden. Kleinere Ideen zur Verbesserung der Ergonomie, des Arbeitsschutzes und der Abläufe hingegen sollten im Rahmen von Teamarbeit direkt von den Beschäftigten umgesetzt werden. Die im betrieblichen Ideenmanagement eingereichten Ideen betrafen - ähnlich wie die Optimierungsvorhaben in Teamarbeit und Workshop - vorwiegend die Themenbereiche Ergonomie, Arbeitsschutz, technische und ablaufbezogene Veränderungen und Erleichterungen der Arbeitssituation im Allgemeinen. Zumeist handelte es sich also um Ideen, die sich positiv oder zumindest neutral auf die jeweilige Arbeitssituation auswirken. Klassische Rationalisierungsvorschläge, etwa die Reduzierung von Arbeitsvorgängen, die eine Kürzung von Vorgabezeiten oder Verschlechterung der Leistungssituati- 
on zur Folge haben könnten, wurden zwar von der Mehrheit der Beschäftigten abgelehnt, aber dennoch vereinzelt ebenfalls eingereicht. ${ }^{101}$

Der Prozess des Ideenmanagements bei NF waren sehr bürokratisch und intransparent gestaltet. Die eingereichten Ideen hatten eine sehr lange Bearbeitungszeit, in deren Bearbeitungsstatus die Beschäftigten keinen Einblick hatten und über deren Bewilligung bzw. Ablehnung sie oftmals keine Rückmeldung von ihrer Führungskraft und zuständigen Fachbereichen erhielten.

[Frage: Macht man sich jetzt noch Gedanken über Verbesserungsideen?] Nein, ich sag mal Nein. Macht man sich nicht, weil die du machst, die meisten werden eh mal abgelehnt und irgendwann kommen sie dann mal drauf, jetzt machen sie es doch so. Du kriegst auch jetzt nichts mehr dafür. "(Fischer, NF Montage $F_{-}$A, 885ff.)

„Ja, ich habe es dann gelassen, wie es am Anfang war, es ist dann drunter und drüber gegangen. Also teilweise ich babe heute noch Verbesserungen, die noch umgesetzt werden teilwweise, von den habe ich nie wieder was gehört, und dass die anderen Geld verdienen, das ist nicht Sinn der Sache. Darum babe ich gesagt, nein, da mache ich jetzt nichts mebr."(Eder, NF Montage K_B, 814ff.)

Gründe für die fehlende Rezeption, Realisierung und Rückmeldung ließen sich auch in den schon beschriebenen dysfunktionalen Strukturen prozessferner Planungsbereiche finden. Die Bearbeitung von Ideenmanagementvorschlägen bedeutete einen Arbeitsaufwand, der zu der eigentlichen Arbeit hinzukam und daher oftmals vernachlässigt wurde.

Neben der finanziellen Prämierung stellten die eigentliche Verbesserung der Arbeits- und Belastungssituation in den meisten Fällen den elementaren Antrieb der Beschäftigten für das Generieren und Einreichen von Ideen dar.

„Also Wertschätzung [durch die Prämie, B.S.], ja. Einigen sicher, ich meine, ich sehe es halt so, ich mache es nicht wegen dem Geld. Freilich ist es ein super Nebeneffekt, wenn ich dann natürlich eine Prämie auch noch kriege für den VV, aber in erster Linie mache ich es eh fuir mich, weil ich arbeite ja bier. Und wenn ich meine Arbeit erleichtern kann, ist ja das schon positiv. Aber viele baben eben gesagt, ,Ja das Geld:" (Immler, NF Montage F_A, 1582ff.)“

Der substanzielle Unterschied zwischen dem Ideenmanagement und den anderen KVP-Instrumenten lag in der Prämierung der Ideen. Die einstige attraktive

101 Die Beschäftigten berichten in Interviews immer nur, dass Kollegen und nicht sie selbst Rationalisierungsideen einbringen. Es wird deutlich, dass innerhalb des Teams kontrovers über die Einreichung dieser Ideen diskutiert wird und der entsprechende Kollege auf die möglichen Folgen für ihre Arbeit aufmerksam gemacht wird. Hierbei zeigt sich deutlich die Dominanz solidarischer Schutzinteressen. 
Prämierung stellte für viele Beschäftigte einen willkommenen Anreiz dar, zu ihrem Gehalt Geld hinzuverdienen zu können. Mit der Prämierung der Beteiligung am Ideenmanagement demonstrierte der Betrieb Anerkennung des Engagements der Beschäftigten und gewährte ihnen (finanzielle) Teilhabe am wirtschaftlichen Erfolg ihrer Ideen.

Die Beschäftigten werteten allerdings den ersatzlosen Wegfall der Prämie für kleinere Verbesserungen unter 2500 Euro Ersparnis als weiteren Ausdruck der von ihnen stark kritisierten betrieblichen Arbeits- und Beschäftigungspolitik, die allein zu ihren Lasten der Beschäftigten gehe, und des fortschreitenden Verlusts von Mitgestaltungsmöglichkeiten sowie mangelnder Anerkennung des eigenen Engagements. Das galt auch dann, wenn die Beschäftigten selbst bekundeten, dass es für kleine Anschaffungen von beispielsweise Mülleimern oder Sicherheitsaufklebern keine Prämie geben müsse. Durch die Neuregelung des betrieblichen Ideenmanagements und den Wegfall kleinerer Prämien machten die Beschäftigten sich mangels Aussicht auf Erfolg nicht mehr die Mühe, Vorschläge im Ideenmanagement einzureichen.

„Ja, das Einzige, was schade ist, letztes Jabr haben wir Veränderung gehabt, dass man nur mal Verbesserungen, die mindestens $2.500 €$ einsparen, dass solche erst übernommen werden. Obwobl es viel andere Sachen, Kleinigkeiten gäbe, wo man sich dann auch leicbter tut. Wo man wirklich auch ein bisschen Zeit herauskriegen würde auch. Aber ja, ist nicht erwünscht und wird nicht mebr angenommen. "(Auer, NF Montage $K \_A$, $754 f f$.)

Die Praxis des Ideenmanagements von NF war durch bürokratische und langwierige Strukturen geprägt, welche die Beschäftigten nicht beeinflussen konnten. Die Bearbeitungsprozesse waren für die Beschäftigten häufig intransparent und schwer nachvollziehbar. Obwohl das Ideenmanagement somit im Vergleich zu Teamarbeit und Workshops das ineffektivste Instrument bei NF war, um Veränderungen in ihrem Produktionsbereich voranzutreiben, war diese Form des Verbesserungswesens bei den Beschäftigten nach wie vor sehr präsent und wurde von ihnen teilweise noch immer genutzt - insbesondere dann, wenn sie sich eine Prämierung ihrer Ideen versprachen und in der Vergangenheit bereits positive Erfahrungen gemacht hatten.

\subsubsection{Resümee: „Eine überforderte Betriebsorganisation“}

Die angestrebte Neuausrichtung der arbeitspolitischen Strukturen hin zu einer besseren Nutzung der Mitarbeiterpotenziale und betriebsweiten Verantwortung für die Leistung und Optimierung des Produktionsprozesses schien bei 
$\mathrm{NF}$ in weiten Teilen missglückt. Im Kern war dies weniger darauf zurückzuführen, dass die Beschäftigten sich nicht in KVP einbringen. Vielmehr schien hier die hohe Reorganisationsdynamik in allen Bereichen des Betriebs zu einer kollektiven Überforderung geführt zu haben. Die nach wie vor hierarchischen Entscheidungsprozesse, Führungsstrukturen und Kooperationsbeziehungen sowie der hohe Profitabilitätsdruck ließen wenig Raum für die Entwicklung von dezentralen und kooperativen Arbeitsstrukturen. Dies führte mehr oder weniger zum Verharren in den etablierten, tayloristisch geprägten Kooperations- und Kommunikationsformen. Nur da, wo sich ein neues Rollenverständnis und prozessnahe Kooperationsstrukturen entwickelt hatte oder auch erkämpft wurden, die Problemlöseprozesse der Beschäftigten in der Produktion unterstützen, ließ sich eine aktive KVP-Praktik feststellen.

\subsection{Arbeitspolitische Beteiligungskonstellation WLF}

Das betriebliche Klima bei WLF war durch eine Arbeits- und Beschäftigungspolitik geprägt, die den Mitarbeiter als gut qualifizierten, verantwortlichen und erfahrenen Kompetenzträger und als einen wichtigen Akteur einer integrierten Betriebsorganisation in den Mittelpunkt stellte. Neben einer auf Selbstständigkeit und Verantwortlichkeit setzenden Arbeitsorganisation legte WLF besonderen Fokus auf ein kooperatives Führungsverständnis, ein breites, allgemeines und fachliches Weiterbildungsangebot für alle Beschäftigten sowie eine umfassende Informationspolitik. Die Beschäftigten hatten über das Intranet, ihre Vorgesetzten und halbjährliche, persönliche Infoveranstaltungen der Geschäftsführung breiten Zugang zu betrieblichen Informationen. ${ }^{102}$ In regelmäBigen Führungskräftetrainings wurde der Fokus auf ein Rollenbild der Führungskräften als wichtige Unterstützer der Beschäftigten gelegt.

Zum Zeitpunkt der Untersuchung erlebten die Beschäftigten die arbeits- und fertigungsorganisatorischen Veränderungen sowie die Einführung von PE nicht - wie bei NF - als Bruch, sondern als inkrementelle Weiterentwicklung bereits bestehender arbeitspolitischer Gestaltungskonzepte und Arbeitsweisen. Auch wenn die Beschäftigten vereinzelt Kritik an betrieblichen Maßnahmen

102 Hierbei werden die Beschäftigten über die wichtigsten wirtschaftlichen Entwicklungen und erwarteten Prognosen informiert und haben die Gelegenheit der Geschäftsführung direkt Fragen zu stellen oder Probleme zu diskutieren. 
äußerten, wird die Gesamtlinie betrieblicher Entscheidungen nicht infrage gestellt und WLF als verlässlicher Arbeitgeber eingeschätzt.

\subsection{1. „Kooperative Beteiligungspartnerschaft“ (WLF Linie 1)}

Linie 1 war derjenige Produktionsbereich im Betrieb WLF, in dem die Beschäftigten das PE-Konzept am umfangreichsten umgesetzt hatten. Sie nutzten als Team die PE-Instrumente sehr aktiv, um Abweichungen und Probleme in ihrem Arbeitsbereich miteinander zu besprechen und gemeinsam mit den Führungskräften und indirekten Bereichen nach Lösungen zu suchen.

Bei Linie 1 handelte es sich um eine verkettete Serienproduktionslinie, in der pro Schicht zehn Mitarbeiter mit einem mitarbeitenden Schichtführer vorwiegend Aufträge in großer Stückzahl fertigen. Die Arbeitsweise der jeweiligen drei Schichtteams ließ sich als teamförmig, selbstorganisiert und kooperativ beschreiben. Besondere Herausforderung und Leistungsanforderung des Teams war das gleichzeitige Rüsten der aus mehreren Anlagen verketteten Produktionslinie, welches oftmals die belastungsreichste Arbeitsphase für etwas 2,2 Stunden einmal pro Schicht darstellt. Die übrige Arbeitszeit war von einer Vielzahl kleinerer, monotonen Tätigkeiten geprägt - dazu gehörten Überwachung, Sichtkontrolle und Endmontage sowie Bestellen und Abholen von Werkzeugen, Organisieren von Arbeitsmitteln, Ordnen und Reinigen des Arbeitsplatzes, Wartung der Anlagen und stichprobenartige Qualitätskontrollen. In Linie 1 waren gut drei Viertel der Beschäftigten an verschiedenen Arbeitsplätzen angelernt ${ }^{103}$ und rotierten regelmäßig zwischen unterschiedlichen, aber nicht allen Arbeitsplätzen. Dadurch wurden nicht nur die (schichtübergreifende) Einsatzflexibilität im Krankheitsfall und der Handlungsspielraum bei der Urlaubsvergabe erhöht, sondern es wurde in hohem Maße das gemeinsame Verantwortungsgefühl sowie das Problembewusstsein der Beschäftigten für übergreifende Verarbeitungsprozesse und Zusammenhänge gefördert. Die Beschäftigten konnten sich eine Leistungsprämie ${ }^{104}$ erarbeiten, die maximal

${ }^{103}$ Die Anlernzeit an einem Anlagenarbeitsplatz beträgt abhängig von der jeweiligen Vorerfahrung ca. 3-6 Monate.

${ }^{104}$ Für die Leistungsprämie sind die Kennzahlen relevant: Rüstzeiten, Ausschuss und Effizienz der Produktionslinie. Sie ist an die Verbesserung von Verlustzeiten (Waste) der Produktionslinie geknüpft. Die Leistungsprämie beträgt maximal 10\% des Grundlohns. Dabei wird dies nicht auf die einzelnen Produktionslinien bezogen, 
zehn Prozent des Grundlohns betrug. Der Vorarbeiter sowie zwei integrierte Instandhalter (Schlosser und Elektriker) waren für zwei weitere Linien zuständig, die in der Verantwortung desselben Meisters lagen.

Die Beschäftigten in Linie 1 nutzten die kurzen täglichen Abweichungssowie die längeren wöchentlichen Practice-Ground-Gespräche, um neu auftretende Probleme bzw. Abweichungen und Problemlösungen gemeinsam zu besprechen. Die Themen umfassten eine breite Spannweite von der Optimierung von Anlagenlaufzeiten, kleineren Verbesserungen am Anlagensystem und der Planung von Reparaturen über ergonomische Arbeitserleichterung (Anschaffung eines Staplers), Mitgestaltung der Produktionsplanung (Änderung von Sequenzlisten) und Ablaufproblemen innerhalb ihres Arbeitsbereichs sowie zwischen angrenzenden Arbeitsbereichen bis hin zu übergreifenden und sozialen Themen. Die Diskussionen waren konsensorientiert. Die Probleme, deren Lösungen sowie das zuständige Teammitglied und die prognostizierte Bearbeitungsdauer wurden auf einer Tafel notiert, so dass die Informationen für alle (Teammitglieder sowie Vorarbeiter, Meister und Indirekte) transparent und sichtbar waren. Ein freiwilliger „Kümmerer“ übernimmt das Nachverfolgen der Problemlösung. Erst wenn das Problem für alle zufriedenstellend gelöst ist, wird es von der Tafel gelöscht.

„Gewisse Sachen liegen nicht bei uns, das können wir nicht entscheiden, aber wir können es so auch hinaufscbreiben. Aber das gebt dann einen anderen Weg. Sicher ist es gut. Ich meine, alles, was binaufleommt, wird bearbeitet, und im Grunde genommen auch, wenn es nur Scheiße ist, müssen sie es auch bearbeiten. Er muss jeden fragen, der was binaufgeschrieben hat: Bist du damit zufrieden? Oder lassen wir es oben stehen?', und wenn er sagt: Nein ok, das ist für mich erledigt', und dann streicht man es runter und aus. Sicher. "(Arbeiter 2/ Gruppendiskussion, WLF Linie 1, 276ff.)

Die Practice-Ground-Tafel wurde nicht nur in den täglichen und wöchentlichen Gesprächen genutzt, sondern war darüber hinaus ein wichtiges Instrument für den Informationsaustausch zwischen den Schichtteams, die Strukturierung der Abweichungs- und Verbesserungsgesprächen und die Artikulation von Problemen und Optimierungspotenzialen sowie für die Kontrolle des Problemlöseprozesses. In regelmäßigen Besprechungen der auf der Tafel notierten Prob-

sondern auf die durchschnittliche Verbesserung von „Waste“ in der ganzen Produktionshalle. Dies wird teilweise von den Beschäftigten der Linie 1 kritisiert, da sie vor der Einführung dieser Regelung zu den besten Linien gehörten. 
leme wurden der Bearbeitungsstand der Lösung abgefragt und gegebenenfalls neue Lösungswege diskutiert. Weil alle drei Schichten mit derselben Tafel arbeiteten, erhielten alle Beschäftigten einen ausführlichen Überblick über Veränderungen und Verbesserungen in ihrem Arbeitsbereich.

„Ich meine, es ist sicher ein Vorteil, weil alles, was du jetzt binaufschreibst, wird behandelt. Früher bist zum Meister gegangen, hast gesagt, ba, wir bräuchten einen Stapler oder so und so, Jajaja! Jetzt steht es oben, jetzt wird es behandelt. Jetzt kriegen wir das. Oder die Hilfsmittel, was du brauchst. Oder wann irgendetwas nicht passt oder mit der Lieferung nicht passt. Das steht alles oben und wird behandelt. Es muss behandelt werden."(Arbeiter 2/ Gruppendiskussion, WLF Linie 1, 242ff.)

$\mathrm{Zu}$ den wöchentlichen Practice-Ground-Gesprächen, die von einem ehrenamtlichen PR-Begleiter moderiert wurden, werden regelmäßig Vertreter aus den indirekten Bereichen hinzugeladen, um Probleme zeitnah und direkt zu lösen oder sich fortan um deren Bearbeitung zu kümmern. Die direkte Kooperation mit den indirekten Bereichen förderte in vielen Fällen eine rasche Bekanntgabe von Abweichungen und Problemen, den unmittelbaren Austausch und prozessnahe Lösungen.

„Ich sage, das ist ein ganz ein gutes Beispiel, weil ich glaube, wenn wir das PProduktionsExzellen₹. BS] nicht gehabt hätten und es will einer durchsetzen, wäre das, glaube ich, nicht so schnell gegangen. Weil da einfach der Kontakt mit dem Zuständigen soweit feblt. Zu dem würdest nicht mal kommen. Und das ist doch innerbalb von ein, zwei Wochen ist das gegangen." (Arbeiter 4/ Gruppendiskussion, WLF Linie 1, 263ff.)

Die besprochenen und auf der Tafel notierten Themen wurden seitens der Beschäftigten und betrieblichen Führung mit hoher Verbindlichkeit behandelt: Die Probleme und Verbesserungsideen der Beschäftigten wurden vom Betrieb aufgegriffen und solange in der Hierarchie nach oben getragen, bis eine nachhaltige Lösung gefunden wurde.

Analog zu den Practice-Ground-Gesprächen fanden auf jeder Hierarchiestufe tägliche Abweichungsgespräche - ebenfalls mit Tafel - statt; auf der ersten Ebene zwischen Meister, Vorarbeitern und wichtigen operativen Indirekten, wie Instandhaltung und Logistik; auf der zweiten Ebene zwischen Produktionsleitung, Meistern und Führungskräften indirekter Bereiche.

In Linie 1 war durch PE eine partnerschaftliche Kooperation zwischen Produktionsbeschäftigten, Führungskräften und indirekten Bereichen entstanden, bei der die Zusammenarbeit selbstverständlich wurde. „Berührungsängste" oder Vorbehalte zwischen den einzelnen betrieblichen Gruppen, wie man sie beispielsweise bei NF deutlich beobachten konnte, wurden weitgehend 
abgebaut und es wurde versucht, problemorientiert und gemeinsam ,an einem Strang" zu ziehen.

„Ja, dass die Probleme gleich gelöst werden. Zum Beispiel in derselben Schicht noch. Oder was war das denn letztes Mal. Da baben wir [...] hinaufgerufen und die sind sofort noch gekommen und das baben wir in derselben Schicht noch heruntergelöscht. Früher wär's verschwunden, da bätt' sich keiner angesprochen gefüblt. Kümmerer und Telefonnummer dazu und ist schon gegangen. "(Oswald, WLF Linie 1, $555 f f$.

Besonders deutlich wurde dies darin, dass die interviewten Beschäftigten aus Linie 1 kaum zwischen Art und Wertigkeit der angesprochenen Themen differenzieren. So unterschieden sie nicht zwischen Problemlösungen, die eine Arbeitserleichterung bedeuten, und denjenigen, die dem Spektrum klassischer Kostenrationalisierung zuzuordnen sind ${ }^{105}$. Ihrer Wahrnehmung nach wurden alle auf der Tafel aufgeführten Punkte vom Betrieb als verbindlich betrachtet und gleichwertig behandelt.

Anteil an dieser Entwicklung hatte mitunter der Meister Wallner, der seinen Beschäftigten in den wöchentlichen Gesprächen einen kurzen Überblick über zentrale, prämienrelevante Kennzahlen und betriebliche Hintergrundinformationen lieferte. Er erklärte je nach Problemlage auch kaufmännische Zusammenhänge, Hintergründe von Entscheidungen und Verfahrensweisen des Betriebs, beispielsweise Entscheidungen über Arbeitszeiten oder Schichtmodelle in Zusammenhang mit Produktionsaufträgen und Lagerbeständen.

„Und dann ist der [Meister, BS] auch dabei und der gibt halt auch Infos über Verkäufe, über Rüstzeiten, über die ganzen Arbeitsunfälle, auf unserer Linie balt praktisch, wie es ausschaut. [...] ist schon eine gute Sache, weil man eigentlich immer am Laufenden ist. Weil wie es früher war, war es, glaube ich, einmal monatlich oder wie es eine Info gegeben hat, eine kurze, und jetzt hat man es eigentlich wöchentlich, das ist eigentlich schon besser. Weil man immer am Laufenden ist. Bei uns im Werk, jetst so alles so kurzlebiger ist, also früher hat man ja praktisch zwei Jahre im Voraus gesehen, wie viel Arbeit man hat, jetzt ist es, weiß ich nicht, auf zwei Monate oder was. Da kann man schneller reagieren und sich schneller drauf einstellen. "(Wagner, WLF Linie 1, 434ff.)

Diese regelmäßigen Erläuterungen des Meisters wurden von den Beschäftigten sehr positiv bewertet. Innerhalb von drei Wochen erhielten die schichtgängigen Beschäftigten relativ aktuell die wichtigsten Informationen, so dass sie selbst abschätzen konnten, wie sich Auftragslage und Auftragsarten entwickeln, die sich auf ihre Arbeitszeiten sowie Arbeitsbelastungen auswirken kön-

105 Wie beispielsweise Optimierung von Rüstzeiten, die zu Veränderung der Personalbemessung führen können. 
nen. Der Meister versetzte seine Beschäftigten durch die Informationen in die Lage, betriebliche Entscheidungen besser nachvollziehen zu können, und gab ihnen in begrenztem Maße die Möglichkeit, sich mit eigenen Vorstellungen und Ideen einzubringen. Ein Beispiel hierfür war, dass die Beschäftigten der Linie 1 den Vorschlag gemacht hatten, gleiche Aufträge in einem engen Zeitraum zusammenzulegen. Dies erleichterte ihnen nicht nur die Arbeit, da weniger Rüstvorgänge weniger Stress bedeuten, sondern ersparte zugleich dem Betrieb Kosten für die Rüstzeiten.

„Informationen transportieren und Rückmeldungen geben. Also da in dieser Funktion sebe ich mich da fast am wichtigsten, also dass ich Eskalationsprozesse annehme, das weitertransportiere, top-down und hin und ber." (Meister Wallner, WLF Linie 1, 196ff.)

Meister Wallner beobachtete genau die Aktivitäten seiner Beschäftigten und unterstützte sie bei der Umsetzung.

„Wenn es Probleme gibt, dann lassen wir es eskalieren, aber im Normalfall funktioniert das. Also da ist die Gewichtung, dass Bewusstsein bei uns in der Firma da berinnen, dass wir wertschöpfend tätig sind in den Linien. Nur der Mitarbeiter an der Maschine ist im Prinzip wertschöpfend, ich bin auch nicht wertschöpfend, nicht, und da ist schon bei vielen der Gedanke, , ok, denen müssen wir das Zeug zur Verfügung stellen, dass sie die Lager produzieren können in der nötigen Qualität, in der nötigen Geschwindigkeit, weil sonst sind wir alle miteinander in zehn Jahren von den Chinesen aufgekauft oder was:" (Meister Wallner, WLF Linie 1, 377ff.)

Das Bewusstsein dafür, dass die Beschäftigten in den Produktionslinien die eigentlich wertschöpfenden Kräfte im Betrieb sind, versuchte der Meister sowohl seinen Mitarbeitern als auch den Beschäftigten in den indirekten Bereichen zu vermitteln.

Meister Wallner verfolgte gemeinsam mit dem PE-Begleiter die langfristige Umsetzung des Business-Excellence-Konzepts in seinem Bereich. Besonders hervorzuheben ist, dass er seinen Beschäftigten immer wieder vor Augen führte, wie viele Veränderungen es in ihrem Arbeitsbereich durch ihr Engagement bei PE bereits gegeben hatte. Weil umgesetzte Verbesserungen und Problemlösungen von der Tafel getilgt wurden und oftmals in Vergessenheit gerieten, führte er - im Gegensatz zu allen anderen Meistern in der Produktion eine Liste, in der er vor allem seinen Beschäftigten sichtbar machte, wieviel sie bereits mit ihren Aktivitäten erreicht hatten. Dadurch versuchte er entgegenzusteuern, dass das Engagement seiner Beschäftigten in Phasen, in denen sie auf langwierige oder kostspielige Veränderungen warten mussten (wie beispielsweise die Anschaffung eines Gabelstaplers), abflaute. 
Der Meister trug durch sein Rollenverständnis als Unterstützer und Vermittler einerseits dazu bei, dass die thematisierten Probleme und Abweichungen schnell und nachhaltig gelöst wurden. Zum anderen förderte er durch regelmäßige wirtschaftliche und betriebliche Hintergrundinformationen das Verständnis für betriebliche Entscheidungen und Verfahren sowie die Verantwortungsübernahme seiner Mitarbeiter.

In Linie 1 hatte sich eine problemorientierte, aktive KVP-Praktik entwickelt, in der die Produktionsbeschäftigten selbstständig und in Kooperation mit den Führungskräften und indirekten Bereichen gemeinsam Verbesserungen erzielen. Die Beschäftigten verstehen sich als Auftraggeber von Problemlösungen und fühlen sich auch von den übrigen betrieblichen Akteuren so behandelt.

\subsection{2. „Desintegrierte Arbeitsgruppe“ (WLF Linie 2)}

Die Beschäftigten der Linie 2 hatten mit der Einführung von PE keine aktive Praxis der gemeinschaftlichen Optimierungen ihrer Linie entwickelt. Die Teams brachten nur wenige Optimierungspotenziale ein und die Treffen waren vorwiegend von Zurückhaltung und Passivität der Beschäftigten geprägt. Generell beteiligten sich lediglich vereinzelte Beschäftigte der Linie aktiv an Verbesserungen im Rahmen von PE.

Linie 2 war eine nicht verkettete Fertigungslinie, in der die ca. 20 Beschäftigten pro Schicht jeweils an Anlagen- und Montagearbeitsplätzen arbeiteten. Die Fertigung von großen Produkten in kleinerer Stückzahl erfolgte an anlagenbezogenen Einzelarbeitsplätzen, zwischen denen jeweils zeitliche Puffer lagen. Die Hauptaufgabe bestand im Rüsten, Einlegen und Kontrollieren der einzelnen mechanischen Fertigungsprozesse und der anschließenden Montage. Der Vorarbeiter kümmerte sich um die vor- und nachbereitenden Aufgaben, wie Bestellen, Abholen und Wegbringen von Werk- und Rüstzeug für die Anlagen.

In Linie 2 hatten sich keine stabilen, teamförmigen Arbeitsstrukturen herausgebildet. Die meisten Beschäftigten arbeiteten schon jahrelang an ,ihrer Maschine" und lehnten das Anlernen an und Rotieren zu anderen Arbeitsplätzen eher ab. Nur wenn sie deutliches Interesse an Rotation zeigten, wurden einzelne Beschäftigte mitunter an anderen Arbeitsplätzen angelernt. Die Beschäftigten arbeiteten weitgehend selbstständig und eigenverantwortlich an ihren Anlagen und verfügten durch die Anlagenlaufzeiten von 3-5 Minuten 
über gewisse zeitliche Handlungsspielräume. Zum Zeitpunkt der Untersuchung wurden von Planern und einzelnen Beschäftigten mehrere Maßnahmen zur Verkürzung der Anlagenzykluszeiten, vorgenommen. Die eklatanten Unterschiede in der Arbeitsorganisation im Vergleich zu Linie 1 ließen sich in Zusammenhang mit der Fertigungsorganisation und der Entwicklungsgeschichte erklären. Während die Aufmerksamkeit der Produktionsleitung zunächst prioritär auf die gewinnträchtigen verketteten Linien und die Qualifizierung der Beschäftigten lagen, wurden in Linie 2 lediglich die Anlagen umgestellt, ohne die Arbeitsorganisation zu ändern. Erst durch PE waren die Beschäftigten systematisch mit Gruppenarbeitsstrukturen in Berührung gekommen.

Die Passivität und Zurückhaltung in den Gesprächen, in denen die Probleme und Optimierungspotenziale hauptsächlich gesammelt wurden, ließen sich durch das Zusammenspiel mehrerer Faktoren auf der Ebene der Arbeitsorganisation, der Aufgaben und des Rollenverständnisses der Vorarbeiter und des Meisters, der Zusammenarbeit mit den indirekten Bereichen und der Rationalisierungserfahrungen der Beschäftigten nachvollziehen.

Die täglichen und wöchentlichen Teambesprechungen fanden in einer Frontalunterrichtsatmosphäre statt. Die Beschäftigten saßen in drei Reihen vor dem moderierenden Vorarbeiter oder PE-Begleiter, der meist nur die an der Tafel notierten Probleme vorlas und über den Stand der Umsetzung berichtet. Vorwiegend wurden Probleme besprochen, die sich übergreifend auf den gesamten Produktionsbereich bezogen, wie Teileanlieferung oder Qualitätsverbesserung. Der Meister war selten anwesend - wenn doch, sprach er eher problematische Themen, wie Reklamationen und Qualitätsprobleme, oder wichtige betriebliche Informationen an. Vertreter der indirekten Bereiche nahmen nur selten an den Treffen teil.

In den Gesprächen herrschte keine spürbar konstruktive und problemorientierte Atmosphäre wie in Linie 1, sondern interessierte Zurückhaltung. Probleme wurden hier nicht als Chance zur Verbesserung, sondern eher als Folge von Fehlern einzelner gesehen, die durch die Artikulation dem Team und dem Betrieb öffentlich gemacht wurden.

„Ja, das Einzige ist natürlich, wenn sie in der Montage wieder hinaufschreiben, dass [wir, B.S.] beim Überstandschleifen [...] zu viel herunterscbleifen müssen, weil die Versetzung nicht passt. Es arbeiten immer drei Arbeiter auf der gleichen Maschine für drei Schichten. Und dann beißst es natürlich, wenn du drinsitzt, und du bist für die Maschine eingeteilt, was hast denn du da zusammengeschliffen? Aber das kann man nicht sagen, dass ich das gemacht habe, weil wir arbeiten zu dritt drauf. Weißt, das ist der einzige 
Nachteil. Dass du natürlich jetzt, weil du gerade da bist, weil du erste Schicht hast, jetz̨t wirst natürlich da angeredet drauf. "(Unger, WLF Linie 2_1, 713ff.)

„Ja, die Stimmungsschwankungen sind immer dann da, wenn wir recht viel Stress haben, wenn es vom [Meister] wieder einen auf den Deckel gegeben hat, weil irgendetwas nicht passt. Oder wenn eben Leute zu unschuldig beschuldigt werden für irgendwelche Sachen, für die sie nicht mal was können. Weil wenn mir bei der Maschine der Roboter irgendeinen Scheiß zusammenfährt. Und auf einmal wo anfährt. Was kann ich dafür. Aber ich werde dafür angespannt. Und das sind Sachen, die was ich nicht verstehe [...] ,Weil wegen dir fallen wir weiter hinter. 'Blablabla. Da gibt es halt dann die Leute, die das einfach schlucken und sagen, ja, rede, was du willst. Dann gibt es aber die anderen Leute, die was sich dann wirklich persönlich angesprochen füblen, und dann gibt es eine Streiterei. [...] Also unser Vorarbeiter selber, der hält sich da eher draus. Der kennt, der kennt alle Leute, er sollte sich mit keinen zerkriegen. Und da ist er still. Da sagt er nichts dafür und nichts dagegen. Das kannst du dir dann selber ausbaden." (Strobl, WLF Linie 2_11, 942ff.)

Dass die einzelnen Beschäftigten sich angegriffen fühlten, begründete sich in der Arbeitsorganisation ohne Rotation zwischen den Einzelarbeitsplätzen. Sie bewirkte, dass die Beschäftigten eine hohe Verantwortung für ihren eigenen kleinen Bereich und das Ergebnis ihrer Arbeit - im Positiven wie im Negativen - empfanden. Anlagen- oder ablaufbedingte Probleme wurden von den Beschäftigten meist nicht angesprochen, um einerseits keine negative Aufmerksamkeit auf sich zu ziehen und andererseits, weil keine ausreichende Unterstützung von den Vorarbeiter erfahren wurde. In Linie 2 gab es zwei ältere Vorarbeiter ${ }^{106}$, die in der Frühschicht arbeiten und keine disziplinarische Verantwortung hatten. In den Gesprächen nahmen sie eine dominante Rolle ein und trugen durch schuldzuweisende und kritische Kommentare zu einer negativen Gesprächskultur bei.

„Bei uns steht fast nichts mehr oben. [...] Weil, es sagt einfach keiner mehr was. Weil es viele Sachen gibt, die was dann in das Lächerliche gezogen werden. Das interessiert halt auch keinen, und wenn man irgendetwas sagt/ es gibt Vorarbeiter, denen was das dann wurscht ist oder die, was das dann belächeln. Für wen soll ich dann etwas sagen?" (Strobl, WLF Linie 2_1, 527ff.)

Die Einzelarbeitsplatzbezogenheit führte einerseits dazu, dass die Beschäftigten ihre Probleme am Arbeitsplatz als irrelevant und uninteressant für die Teamkollegen einschätzen und von ihnen wenig Hilfe bei Problemlösungen erwarten. Andererseits fehlt den Beschäftigten, die nicht zwischen verschiede-

106 Diese Vorarbeiter kann man als „,ausrangierte“ Vorarbeiter sehen, die zwar Teil der Linie 2 sind und sich um „Spezialthemen“ und Sonderanfertigungen kümmern. In ihren Haltungen eher ein tradiertes Führungsverständnis verkörpern. 
nen Arbeitsplätzen und Anlagen rotieren, das gemeinsame Verantwortungsgefühl und Bewusstsein für übergreifenden Anlagen- und Ablaufprobleme, die Quelle neuer Verbesserungsideen sein konnten. Zudem begünstigte die große Gruppengröße die Verantwortungsdiffusion.

Darüber hinaus schient in Linie 2 die Gepflogenheit zu herrschen, denjenigen, der ein Thema in die Gesprächsrunde einbrachte, auch als Kümmerer einzutragen. Viele - nicht alle - Beschäftigte scheuten einerseits den vermeintlichen zusätzlichen Aufwand und zeigten andererseits Berührungsängste, die aus der mangelnden Vertrautheit im Umgang mit neuen Ansprechpartnern aus den indirekten Bereichen resultierten.

„Es schreiben viele nichts hinauf, weil sie glauben dann, sie müssen da irgendwo binrennen oder was. Das ist ja eigentlich irgendwie ein kompletter Blödsinn. Es steht ja dann nur ein Name oben, dass sie wissen, dass sich der vielleicht ein bisschen was umschaut. Aber ich kann ja auch jetst, wenn ich da oben stehe auf der Tafel, ich kann jetzt auch rubig [...] zum Vorarbeiter [bingehen] und sagen: ,K.107, kannst du mir den anrufen, kannst uns den einmal einladen, dass wir wissen, wie das funktioniert?' Ich muss ja selber nicht dort anrufen. Das ist von den Leuten komplett falsch aufgenommen. (Neubaner, WLF Linie 2_2, 869ff.)

Nicht zuletzt herrschte innerhalb der Arbeitsteams von Linie 2 ein hoher, von einigen wenigen Mitarbeitern ausgehender Druck, klassischen Rationalisierungsaktivitäten, wie der Reduzierung von Rüst- und Anlagenlaufzeiten, entgegenzuwirken.

„Sie haben irgendwo eine Angst, dass weiß ich nicht/Keine Ahnung, dass weniger Arbeit da ist oder irgendwas wegen dem vielleicht, dass sie keine Verbesserungen vorschlagen. [...] Aber genau weiß ich es jetzt auch nicht um was/ Ja, da ist balt riemlich noch ein Gruppenzusammenbalt da draußen." (Mayr, WLF Linie 2_2, 598ff.)

Dies betraf nicht nur die Practice-Ground-Gespräche, sondern insgesamt die Beteiligung an Optimierungen in Linie 2. Beschäftigte, die eigenständig an ihren Anlagen Optimierungen vornahmen, wie Mayr, mussten sich der starken Kritik und sogar Anfeindungen stellen, wenn ihre angestoßenen Veränderungen Rationalisierungseffekte, wie Laufzeit- und Rückzeitverkürzungen sowie Leistungsintensivierungen, mit sich brachten.

„Aber ich habe, glaub ich, mittlerweile haben wir eh ein bisschen was auf die Füße gestellt, da draußen. Also die Abläufe sind sicher besser geworden, trau ich mich jetst sagen, sicher Und die Stückzabl ist mit Sicherbeit um 40 Prozent raufgegangen, mit Sicherbeit [...]. Das war zwar ein Kampf am Anfang, aber ich glaub, ich habe gewonnen

107 Anonymisiert. 
im Großen und Ganzen. Und sie sehen es jetzt selber ein, dass die Arbeit eigentlich leichter geworden ist. "(Mayr, WLF Linie 2_2, 109ff.)

Wenn ein Mitarbeiter selbst Verbesserungen der Zeiten vornahm, offenbarte dies, dass generell Rationalisierungspotenziale in der Linie vorhanden waren, und verstieß gegen kollegiale Normen des Schutzes vor Verschlechterung der Arbeits- und Leistungssituation.

$\mathrm{Zu}$ den beteiligungshinderlichen Arbeitsbedingungen kam die langwierige Bearbeitung der gesammelten Optimierungspotenziale hinzu - die Ideen blieben oftmals monatelang auf der Tafel notiert.

Das sind verschiedene Gründe. Entweder wird es nicht weitergegeben oder irgendwie so oder man scheißt darauf, auf Deutsch gesagt. Darum ist es ja jetzt auch gut, dass man, wenn es zu lange oben steht, dass wir dann zur nächsten Instan₹ und das alles gehen. Das ist jetzt vielleicht noch das Gute daran. Aber das ist der Nachteil, wenn das jetzt zum Beispiel zwei Monate oben steht und es ist noch immer nichts passiert. (Neubauer, WLF Linie 2_2, 976ff.)

Das mühsame Vorankommen wurde der mangelhaften Kommunikation der Themen in die Fachbereiche und wieder zurück ins Arbeitsteam zugeschrieben. Die Beschäftigten fühlten sich mit ihren Anliegen nicht ernstgenommen.

"Ja, es werden schon einige Sachen aufgeschrieben, aber ich glaube, mehr oder weniger ist das hier erledigt. [...] Man bat schon das Gefïbl, es wird nicht ernst genommen, was man aufschreibt. [...] Da hat man keine Lust mehr zum Raufscbreiben [...]. Weil ich weiß, es passiert nicht viel. Oder ich habe das schlechte Gewissen und jetzt passiert was, wenn ich was draufschreibe. "(Lehner, WLF Linie 2_2, 136ff.)

Die nichtbehandelten Themen wurden bei jedem Gespräch erneut vorgelesen. Diese Redundanz führte dazu, dass die betriebsseitige Ignoranz den Beschäftigten permanent wieder vor Augen geführt wurde.

Trotz dieser beteiligungshinderlichen Mechanismen wurde PE als Beteiligungsinstrument aufgrund der Zunahme an relevanten Informationen über den Betrieb, den eigenen Bereich und zwischen den Schichten grundsätzlich positiv wahrgenommen. Die Beschäftigten erhielten mehr Kenntnisse über die Probleme der Kollegen, über Zusammenhänge innerhalb des gesamten Fertigungsprozesses und Hintergrundinformationen über betriebliche Entscheidungen.

„Nun ja, gut ist das sicher, wenn man was erfährt, was bei den anderen Schichten auch war. Wenn die irgendwelche Probleme gehabt haben oder wenn irgendwas nicht gepasst hat. Ich weiß nicht, was in der dritten Schicht passiert, ich weiß nicht, was in der ersten Schicht passiert. Und so weit haben wir uns eigentlich geeinigt, die ganzen Schichten, wenn wirklich etwas Gravierendes ist, dass wir uns das auf die Tafel hinaufschreiben. Und wenn jetzt irgendwo einmal, weiß ich nicht, eine Kleinigkeit war, die brauche ich dann nicht hinaufschreiben, weil das reicht, wenn ich es ihm sage. Wenn ich weiß, dass genau die eine Person betroffen ist. "(Neubauer, WLF Linie 2_ 2, 752) 
Einige Beschäftigte wurden durch die drei sich abwechselnden PE-Begleiter darin bestärkt, Abweichungen und Probleme im Produktionsprozess zu benennen und auf die Tafel schreiben zu lassen. Die Anwesenheit externer Moderatoren förderten durch methodisches Nachfragen und Hilfestellungen eine problemlösungsorientierte Kommunikation im Team und bewirkte, dass sich die sonst dominanten, zusätzlichen Vorarbeiter stärker zurückhielten. Die PEBegleiter bekundeten ein glaubhaftes Interesse des Betriebs an den Beiträgen der Beschäftigten, was Vorarbeiter, Meister und indirekte Bereiche nicht in ausreichenden Maße vermitteln konnten. Zudem erhielten die Beschäftigten von einem der drei PE-Begleiter, der Mitglied des Managements war, mehr Informationen über übergeordnete Themen und betriebliche Abläufe.

„Gerade der Z. ${ }^{108}$ ist aber auch einer, der was einen jeden binausziebt aus der Nase. Wenn irgendjemand etwas weiß, der gräbt so lang nach, bis dass er es sagt. Das ist schon nicht schlecht. [...] Es ist gut, weil dann fangen wenigstens die Leute ₹u reden an. Das schon. Und wenn der da ist, redet auch kein Vorarbeiter dagegen. (Strobl, WLF Linie_1, 929ff.)

Wichtiges Instrument für die beschäftigtengetragenen Problemlösungen war auch hier die Tafel, auf der Optimierungspotenziale für alle sichtbar dokumentiert wurden; die dort notierten Vorschläge mussten von den Führungskräften und indirekten Bereichen bearbeitet werden.

„Am Anfang habe ich ebrlich gesagt: ,Für was ist das eigentlich? Was bringt das? Das wird sowieso nicht anders werden. "Aber jetzt mit der Zeit, [...] ich finde, dass es wirklich so super umgesetrt wird und alles. Weil wir scbreiben binauf, und das wird eigentlich innerbalb/ man schreibt die Woche, wo es gemacht wird, und dann bis dabin muss es erledigt sein. [...] Interviewerin: Wodurch ist es jetat anders geworden? Also können Sie jetət mehr Druck ausïben dadurch?] Ja sicher, weil es auf der Tafel steht und egal, was du binaufschreibst. Ich mein, nicht egal, das muss schon wichtig sein, und muss auch behandelt werden. Da kommt kein Vorarbeiter raus, kommt kein Meister. Da kann sich keiner irgendwie ausreden oder irgendetwas. Das wird behandelt und gemacht." (Unger, WLF Linie 2 Schicht 2, $578 f f$. .)

Trotz des Gefühls, nicht ernst genommen zu werden, der empfundenen Schuldzuschreibungen und der Angst vor Rationalisierung zogen selbst Beschäftigte, die PE sehr skeptisch gegenüberstanden, die Verbindlichkeit des PE-Prozesses und insbesondere der dokumentierten Probleme auf der Tafel nicht in Zweifel. Diese Diskrepanz ließ sich angesichts der vorangegangenen

108 Anonymisiert. 
Befunde nur ansatzweise erklären. Zum einen herrschte bei WLF eine Kultur der Wertschätzung und Verbindlichkeit. Das drückte sich auch darin aus, dass PE als Problemlöseprozess auf allen Hierarchieebenen - bis hin zur Betriebsleitung - konsequent praktiziert wurde. Dabei wurde versucht, nicht zwischen der Wertigkeit der einzelnen Optimierungspotenziale nach ihrer Profitabilität zu differenzieren und Ideen, welche Arbeitserleichterungen betrafen, mit der gleichen Ernsthaftigkeit zu behandeln wie Kosteneinsparungsideen.

Insgesamt hatten sich in Linie 2 sowohl auf der Ebene der Arbeitsorganisation als auch auf der Ebene der Führung und in den Kooperationsbeziehungen zu den indirekten Bereichen weniger aktive Beteiligungsstrukturen herausgebildet als in Linie 1. Neben der Gruppengröße ließ sich dies auch darauf zurückführen, dass die Beschäftigten und Vorarbeiter durch ihre jahrzehntelange Tätigkeit in den teils „eigenbrötlerischen“ Strukturen der Einzelarbeitsplätzen wenig Erfahrung mit teamförmiger Arbeit gemacht hatten.

\subsubsection{Dezentrales Ideenmanagement bei WLF}

Parallel und ergänzend zu PE wurden im Rahmen des Ideenmanagements Verbesserungsideen der Beschäftigten prozessnah umgesetzt. Es fungierte als System zur Registrierung und Gratifikation von Verbesserungsideen. WLF verzeichnete eine sehr hohe Beteiligung der Beschäftigten am Ideenmanagement. 2011 hatten sich rund 97 Prozent aller Beschäftigten mit mindestens einer Idee hier eingebracht. Von den eingebrachten Ideen wurden 93 Prozent umgesetzt. ${ }^{109}$

Hinter den hohen Beteiligungs- und Umsetzungsraten bei WLF verbarg sich eine bereits seit Jahren etablierte spezifische Praxis der dezentralen Bearbeitung und Umsetzung von Verbesserungsideen. Vorschläge der Beschäftigten, die im Ideenmanagement registriert wurden, waren bereits zuvor mit dem Vorgesetzten diskutiert und zur Umsetzung freigegeben worden. Dazu zählten auch Verbesserungsideen, die im Rahmen von PE erarbeitet wurden. Seitens des Betriebs wurde nicht zwischen Arten oder der Profitabilität von Ideen differenziert. Die Ideen wurden erst im IT-System des Ideenmanagements erfasst, wenn die Umsetzung erfolgt war.

109 Quelle: Betriebsinterne Statistiken. 
Beim Großteil der Ideen handelte es sich um Veränderungen in kleinem Umfang, die nach Genehmigung in Zusammenarbeit mit dem Vorarbeiter, dem ideengebenden Beschäftigten und der integrierten Instandhaltung umgesetzt wurden. Ideen, deren Umsetzung mit höheren Kosten oder dem Einbezug anderer indirekter Bereiche verbunden war, wurden meist vom Meister bearbeitet und zur Umsetzung gebracht. Die Beschäftigten betonten, dass es wichtig sei, mit den Kollegen aus den anderen Schichten Ideen die Veränderungen an Arbeitsplätzen zufolge haben, vor der Umsetzung direkt abzusprechen. Mitunter waren die Kollegen anderer Meinung. Da die Ideen jedoch häufig der Arbeitserleichterung dienten, konnte in den meisten Fällen ein Kompromiss gefunden werden.

In den einzelnen Bereichen etablierten sich unterschiedliche Praktiken der Ideenumsetzung etabliert. Entweder wurde der integrierte Instandhalter oder betroffene Bereich direkt informiert und kümmerte sich um die Umsetzung oder sie wurden auf ein Ideenmanagement-Flipchart geschrieben und der integrierte Instandhalter kümmerte sich dann um die Umsetzung. Auf dieser Tafel konnte man auch den Fortschritt verfolgen. Abschließend wurde die Idee ins Ideenmanagement eingetragen.

„Wenn man eine Idee hat, gehst du einmal zum Vorarbeiter und besprichst einmal das. Hat es überhaupt einen Sinn, dass wir das machen? Dann, wenn man sich denkt, ja, das hat einen Sinn, dann wird es auf die Tafel hinauf geschrieben. Sobald es auf der Tafel ist, geht das dann weiter. Wird auf jeden Fall bearbeitet und gemacht." (Renner, WLF Linie 1, 597ff.)

„Bei unserer Maschine wird schon seit vier Jabren nur irgendwelche Sachen verbessert. Nie eine Zeit. Ich weiß nicht, was eingetragen wird und was nicht. Ich babe keine $A$ hnung. Ich selber habe noch nie etwas eingetragen. [...] Ich kriege aber auch jedes Jabr meinen Dukaten. [...] Das macht bei uns meist der Scblosser mit. Das sind 60 Ideen im Jabr, da drucket er einem jeden eine binein, und fertig." (Strobl, WLF Linie 2, 669ff.)

Die prozessnahe und niederschwellige Umsetzung der Ideen im Rahmen des Ideenmanagements war eine bereits seit Jahren bestehende Praxis, die sich schon vor der Einführung von PE als Instrument zur Optimierung und Veränderung der Arbeitsbereiche etabliert hatte und an der sich die Produktionsbeschäftigen rege beteiligten.

Die Beschäftigten brachten sich auch im Ideenmanagement mit einer Vielzahl unterschiedlicher Ideen ein. Diese bezogen sich sowohl auf ihren eigenen Arbeitsprozess und -bereich (Ergonomie, Arbeitssicherheit, Ablauforganisation, Anlagenlaufzeiten, Gestaltung der Umgebungsbedingungen wie Lichtver- 
hältnisse und Sauberkeit) als auch auf übergreifende Themen, die nicht unmittelbar die Arbeit betrafen (Beispiele waren: Jacken für den Gang zum Arzt, Uhrenangleichung).

Die Beschäftigten bewerteten sowohl die Praxis der dezentralen und niederschwelligen Absprache mit dem Vorgesetzten als auch die Regelungen des Ideenmanagements überwiegend positiv. Indem sie über den Vorarbeiter und Instandhalter meist in die Umsetzung der Verbesserungsideen eingebunden waren, entstanden Lösungen, mit denen sie zufrieden waren. Im Unterschied zu NF bemängeln die WLF-Beschäftigten weder die Umsetzung, lange Bearbeitungszeiten oder mangelhafte Rückmeldungen durch Vorgesetze, noch haben sie konkrete Verbesserungsvorschläge für das Instrument und die praktische Durchführung des Ideenmanagements.

Bei WLF stellte sich ein selbstverständlicher Umgang ein, mit Vorgesetzen Probleme oder Verbesserungsideen zu diskutieren oder diese sogar direkt mit dem integrierten Instandhalter ohne umfangreiche Rücksprachen umsetzen. Die Beschäftigten nutzten hierbei ihre vom Betrieb gewährten Entscheidungsspielräume. Aufgrund der Diskussion von Ideen mit dem Vorgesetzten im Umfeld erhielten die Beschäftigten eine direkte Rückmeldung, ob die Idee erfolgversprechend war und vom Betrieb umgesetzt wurde. Wurde eine Idee abgelehnt, versuchte der Vorgesetzte - bei sehr ambitionierten Ideen sogar der Ideenmanager selbst - nachvollziehbar zu erklären, aus welchen betrieblichen Gründen die Idee nicht umgesetzt werden konnte.

„Es wird dann schon noch einmal genau bearbeitet. Ist es machbar? Ist es von den Kosten her? Ich habe zum Beispiel einmal eine Idee gehabt, dass man das Ganze mit so Strichcodes über das Magazin macht, mit dem Ausfassen und so. Da ist mir gesagt worden, das ist/ es wäre eigentlich eine super Sache, wo ich genau sebe, was einer ausfasst an Werkzeng, wenn das alles mit Barcode/ das Ganzel Das haben sie zum Beispiel in der [Nachbarfirma, B.S]. Da haben sie gesagt: ,Das ist einfach zu teuer das Ganze. Für unseren Betrieb ist das einfach/ wäre eine gute Sache, aber einfach vom Finanziellen zu teuer.' Abgelehnt, nun ja. Das ist dann so. (Renner, WLF Linie 1, 611ff.)

Durch die persönlichen Rückmeldungen und die Nachvollziehbarkeit der betrieblichen Gründe erfuhren die Beschäftigten trotz Ablehnung Wertschätzung für ihr Engagement und ihre Ideen. Bemerkenswert ist, dass die Beschäftigten bei WLF im Vergleich zu NF kaum negative Beispiele für den Umgang mit ihren Verbesserungsideen thematisierten.

Ideen für größere Kostenersparnisse wurden prozentual zur Einsparung prämiert. Die meisten kleineren Ideen wurden mit einmal jährlich vergebenen 
Goldmünzen ${ }^{110}$ belohnt; für bis zu zehn eingereichte Ideen erhielt ein Beschäftigter eine kleine, ab mehr als zehn Vorschlägen eine große Münze. Diese Gratifikationsregelung mit Staffelung nach Ideenanzahl war nicht unumstritten, da einige Beschäftigte die Spanne zwischen einer und zehn Ideen als zu groß ansahen und ihrer Ansicht nach mittleres Engagement in Relation zum Aufwand nicht gerecht prämiert wurde.

„Wenn ich eine Verbesserung habe, kriege ich so ein Gramm Gold oder irgendwie so. Ich weiß jetzt nicht. Und je mehr Verbesserungen du bast, wenn du jetat deine elf Verbesserungen hast, kriegst halt in großen Golddukaten. Aber sonst/ Wenn das jetzt wirklich eine größere Verbesserung ist, die, was weiß ich nicht, $x$-tausende von Euros einsparen würde, bringt mir das auch nichts. Ja, die Firma freut sich, aber ich selber nicht. (Neubauer, WLF Linie 2_1,953ff.)

Auch die Höhe der Gratifikation von Ideen mit hoher Kostenersparnis ist nicht unumstritten. Viele Beschäftigte bemängeln die niedrige Prämierung:

„Was ich mitgekommen habe, wenn wir da bei den ständigen Verbesserungen sind. [Bei] einer Ersparnis von Scbleifscheiben und solche Sachen. Die stimmen nicht ganz der Realität. Also da fallen die, bei uns da, eher klein aus. Also ich sage jetzt ein Beispiel. Ich habe eine Einsparung gemacht im Jahr von 20.000 Euro und dann keriegst du da einmalig 200 Euro, sage ich einmal. Es kommt mir eher ein bisschen schwach vor. Wo du wirklich ein/ wo du erwiesen die Einsparung machst. Dann könnte man ein bisschen, wie sagt man [I: Großzügiger sein] großzüigiger sein. Und das weiß ich, das sind sie in anderen Werken rum Teil. Was ich weiß von [einem benachbarten Montagewerk]. "(Renner, WLF Linie 1, 3731 ff.)

Nichtdestotrotz schien sich diese Kritik nicht direkt auf das Beteiligungsverhalten der Beschäftigten auszuwirken. In den Interviews war nicht ersichtlich, dass sich die Beschäftigten, welche die Gratifikationspraxis von WLF kritisierten, aus diesem Grund ihre Ideen nicht einreichten oder sich generell weniger in Optimierungsprozesse einbrachten.

\subsection{Zwischen Restriktion und Partizipation}

Im Vergleich der unterschiedlichen strukturellen Gestaltungsbedingungen der einzelnen KVP-Praktiken wird deutlich, welche Bedingungen für gelingende KVP von Bedeutung sind. Insgesamt zeigt sich, dass sich die Gestaltungsbedingungen unterschiedlicher KVP-Praktiken - KVP in Teamarbeit, als Work-

${ }^{110}$ Der Wert der Golfmünzen belief bei den kleinen Münzen um ca. 150 Euro; bei den großen Münzen um ca. 300 Euro. 
shop oder im Ideenmanagement - grundsätzlich sehr ähnlich auf die Beteiligung der Beschäftigten auswirken. Verbesserungsprozesse - wie schon im Aneignungsmodell von KVP (in KapitelKVP - ein Aneignungsmodell) dargestellt - durchlaufen vier zentrale Phasen: Genese der Optimierungspotenziale, Artikulation gegenüber dem Betrieb, Rezeption der Verbesserungsideen und schließlich ihrer Realisierung. Unabhängig von der organisatorischen Form des KVP ist von entscheidender Bedeutung für die Qualität beschäftigtengetragener Optimierungsprozesse, wie die in den Produktionsbereichen generierten und artikulierten Probleme und Optimierungspotenziale durch betriebliche Kooperations- und Kommunikationsprozesse aufgenommen und in Verbesserungen der Arbeitsprozesse überfübrt werden.

In einer synthetisierenden Betrachtung der einzelnen KVP-Praktiken werden die strukturellen, arbeits- und betriebsorganisatorischen Bedingungen der einzelnen Beteiligungskonstellationen unter dem Aspekt betrachtet, inwiefern sie dazu beitragen oder verhindern, dass sich funktionierende Praktiken von beschäftigtengetragenen Optimierungsprozessen herausbilden.

\subsubsection{Arbeitsorganisation}

Wichtiger Referenzpunkt für die Genese von Optimierungspotenzialen durch die Beschäftigten sind ihre alltäglichen Probleme und Herausforderungen im Arbeitsprozess. Die Optimierungspotenziale beziehen sich vor allem auf Aspekte der physischen und psychischen Bedingungen der Arbeit (Ergonomie am Arbeitsplatz, Vermeidung von Arbeitsunfällen), die Qualität des Produkts, der Arbeitsmittel und des Produktionsprozesses, die Verbesserung der Abläufe und die Zusammenarbeit mit vor- und nachgelagerten Bereichen. In der mechanischen Fertigung geht es auch um Anlagenoptimierung.

Im Vergleich zwischen den unterschiedlichen Arbeitsorganisation wird deutlich, dass Gruppengröße, Aufgabenzuschnitt, Rotation, Selbstorganisation sowie Handlungs- und Entscheidungsspielräume eine wichtige Grundlage für die Ideengenese und Artikulation von Optimierungspotenzialen bilden. Aufgabenintegration und systematische Rotation der Beschäftigten über alle Arbeitsplätze des Bereichs hinweg fördern Kenntnisse über Zusammenhänge der Produktionsprozesse. Rotation erweitert nicht nur die Einsatzflexibilität der Beschäftigten innerhalb des Teams, sondern bewirkt, dass alle Beschäftigten innerhalb des Produktionsbereichs gleichermaßen mit den spezifischen, arbeitssituativen Anforderungen und Problemen des Produktionsprozesses vertraut gemacht werden. 
Dies trägt zum einen dazu bei, dass innerhalb des Teams mehr Verantwortung für gemeinsame Gruppenaufgaben übernommen wird und dass sich zum anderen ein gemeinsames Aufgaben- und Problembewusstsein entwickelt. So werden vor allem die gemeinsamen Gesprächszeiten genutzt, um Probleme anzusprechen und nach Lösungen zu suchen, wie in den Fällen Montage F und Linie 1 deutlich geworden ist. Fehler oder Probleme, die durch Anlagenstörungen oder zum Beispiel schlecht kalibrierte Werkzeuge verursacht werden, gelten nicht, wie bei Linie 2, als Fehler einzelner Beschäftigter, sondern als gemeinsames Problem. Diese Beobachtungen zeigen, dass ein fehlendes geteiltes Bewusstsein zu Missverständnissen und falschen Schuldzuweisungen führen kann.

Durch Teamarbeit und PE werden den Beschäftigten zeitliche Ressourcen zur Feststellung von Problemen und Erarbeitung von Optimierungsideen zur Verfügung gestellt. Zusätzliche Funktionen und Aufgaben in den Arbeitsteams, wie Qualitätsregelkreismitarbeiter bei NF oder Kümmerer bei WLF, erweitern nicht nur das Aufgabenspektrum und die Interessantheit der Arbeit, sondern eröffnen den Beschäftigten wichtige Kooperationsmöglichkeiten.

Vorhandende Selbstorganisationsmöglichkeiten und Handlungsspielräume geben den Beschäftigten die Möglichkeit, im Kleinen selbst Optimierungen vorzunehmen, Probleme zu beheben und den eigenen Produktionsbereich mitzugestalten. Bei WLF verfügen die Beschäftigten innerhalb ihres Arbeitsbereichs über weitreichende Spielräume, gemeinsam mit den integrierten Instandhaltern Prozesse zu optimieren. In den Fallkonstellationen von NF schränken zentralistisch eingeführte, standardisierte Gestaltungskonzepte, mit denen die Beschäftigten zudem zu wenig vertraut sind, Handlungsspielräume der Teams für die Gestaltung ihres Produktionsbereichs erheblich ein. Diesen Ausführungen zufolge bilden Formen selbstorganisierter Gruppenarbeit (Gerst 1998a) mit erweitertem Aufgaben- und Funktionszuschnitt, Rotation sowie zur Verfügung stehenden zeitlichen Ressourcen eine wichtige arbeitsorganisatorische Grundlage für dauerhafte beschäftigtengetragene Optimierungsprozesse. Zugleich bilden sie eine wichtige Ressource für die Teams, mit zunehmenden Leistungsanforderungen umzugehen - sei es durch gegenseitiges Aushelfen oder die Suche nach teaminternen Optimierungspotenzialen. Dies zeigte sich in Linie 1, deren Beschäftigte im Vergleich zu Linie 2 zwar höhere Leistungsanforderungen ausgesetzt waren, aber gleichzeitig größere zeitliche Spielräume in ihrer Arbeit hatten. 
Die Bereitstellung von zeitlichen Ressourcen, die das Team für sich, aber auch für die direkte Kommunikation und Kooperation mit anderen Bereichen nutzen kann, trägt maßgeblich dazu bei, dass die Beschäftigten ihre Probleme sowie Optimierungsideen effektiv artikulieren und Hintergrundinformationen über betriebliche Entscheidungen oder Umsetzungsanforderungen erfahren können. In den Beteiligungskonstellationen, in denen die Teamgespräche auch für die Kooperation mit anderen Bereichen genutzt wurden, haben sich lebhaftere KVP-Praktiken herausgebildet.

Nicht zuletzt scheinen die Größe und Zusammensetzung sowie der sperifische Erfahrungshintergrund der einzelnen Beschäftigten in den Teams eine wichtige Rolle für die Beteiligung an KVP zu spielen. Das Ausmaß an kumulativen Erfahrungen eines Teams im Umgang mit betrieblichen Abläufen und Kooperationsstrukturen sowie der Anteil an Status-quo-, Mitgestaltungs- oder Karriereorientierten nehmen offenbar Einfluss darauf nehmen, wie Optimierungsideen im Team diskutiert werden und wie diese umgesetzt werden: Werden mögliche Optimierungspotenziale im Team eher mit einer abwehrenden, schutzorientierten Haltung oder aus einer Mitgestaltungsperspektive diskutiert?

\subsubsection{Rollenverständnis und Funktionszuschnitt der ersten Führungsebene}

Für gelingende Optimierungsprozesse kommt der ersten Führungsebene eine zentrale Bedeutung als Unterstützer der Arbeitsteams zu. Sie ist zumeist die erste betriebliche Instanz, die von Problemen und Optimierungspotenzialen der Teams oder einzelner Beschäftigte erfährt. Die Meister bzw. Vorarbeiter setzen sich mit den Vorschlägen auseinander, beurteilen sie nach ihrem Nutzen und helfen den Teams, Problemlösungen herbeizuführen. Entscheidend für die Beurteilung und Förderung ist hierbei, ob die Meister/Vorarbeiter ein weites oder enges Verständnis von „nützlichen“, förderungswürdigen Problemen und Optimierungsideen haben: Fördern sie lediglich Ideen, die einen Kostenvorteil für den Betrieb bedeuten, oder auch solche, die explizit die Arbeits- und Leistungsbedingungen der Beschäftigten verbessern?

Der Meister fungiert als Bindeglied zwischen Produktionsbeschäftigten und dem Rest der betrieblichen Organisationsstrukturen, indem er Kooperationsbeziehungen $z u$ den richtigen Ansprechpartnern in indirekten Bereichen und $\mathrm{zu}$ betrieblichen Entscheidungsträgern herstellt und sich für die schnelle Realisierung von Optimierungsprozessen einsetzt.(Behrens et al. 1997; Jauch 
1997; Kuhlmann et al. 2004) Dies ist insbesondere dann wichtig, wenn die Betriebsorganisation, wie bei NF, wenig prozessnah und dezentral ausgerichtet ist und sich direkte Kooperationsbeziehungen zwischen den Produktionsbeschäftigten und Vertretern indirekter Bereiche noch nicht etabliert haben. Bei gut etablierten kooperativen Problemlöseprozessen, wie in Linie 1, ist wichtig, dass der Meister einzelne, schwierig zu lösende Probleme zu betrieblichen Entscheidungsträgern auf Führungsebene transportiert und sich für eine nachhaltige Umsetzung stark macht.

Um diesem Rollenverständnis gerecht werden zu können, muss der Meister innerhalb der Betriebsorganisation ausreichend Zeit, Entscheidungs- und Handlungsspielräume für das Ausfüllen der Unterstützerfunktion haben. Wie an den Beispielen der Meister Fuchs (Montage F) und Wallner (Linie1) deutlich wird, nehmen diese Meister sich sehr viel Zeit, Probleme ausführlich zu diskutieren, Problemlösungen herbeizuführen und Hintergrundinformationen über Produkte, betriebliche Prozesse und Entscheidungen zu geben. Der Führungsstil dieser Meister ist weniger durch Kontrolle der Arbeitsergebnisse und Arbeitshandlungen, sondern durch Förderung der Kompetenzen und Selbstständigkeit der Arbeitsteams sowie kooperativer Aushandlungsprozesse geprägt. Durch diesen entwicklungsorientierten Führungsstil erweitern sie bei ihren Mitarbeitern das Verständnis für betriebliche Prozesse und schärfen das Bewusstsein für Optimierungspotenziale.

Diese Scharnierfunktion zwischen Arbeitsteams und Betrieb kann der Meister nur erfüllen, wenn er innerhalb der Betriebsorganisation über ausreichend Durchsetzungskraft und Einfluss verfügt, um Problemlösungen herbeizuführen. Enge hierarchische Steuerungs- und Entscheidungsprozesse sowie eine funktional differenzierte Betriebsorganisation und bürokratische Abläufe verringern deutlich die Handlungs- und Entscheidungsspielräume, wie im Fall Montag K (NF) deutlich wird, in dem man von einem eher „entmachteten“ Meister sprechen kann.

\subsubsection{Prozessnahe Kommunikation und Kooperationsstrukturen zwischen Produktion und indirekten Bereichen}

Kooperations- und Kommunikationsstrukturen zwischen den Produktionsund indirekten Bereichen erfüllen bedeutende Schlüsselfunktion hinsichtlich der Frage, wie die von den Beschäftigten identifizierten und artikulierten Probleme und Optimierungspotenziale aufgenommen (Rezeption) und in reale Ver- 
besserungen überführt (Realisation) werden. Wichtig für gelingende beschäftigtengetragene Optimierungsprozesse sind prozessnahe Kooperationsbeziehungen zwischen Produktion und indirekten Bereichen sowie Kommunikationsformen, welche die Durchlässigkeit relevanter Informationen in beide Richtungen gewährleisten: von den Teams in die indirekten Bereiche und zu den oberen Führungsebenen sowie in einem Rückkopplungsprozess wieder zurück zu den Produktionsteams.

Durch regelmäßige oder punktuell stattfindende direkte Kommunikationsprozesse kann ein direkter Austausch zwischen den erfahrungsbasierten, praktischen Kompetenzen und Fertigkeiten der Beschäftigten auf der einen Seite und dem eher theoretischen Wissen über Produktionsprozesse der Planer auf der anderen Seite erfolgen. Oftmals wird kritisiert, dass praktische Anforderungen des Arbeitsprozesses unzureichend in der Planung berücksichtigt werden. Die Beschäftigten haben hingegen aufgrund ihres Arbeitsalltags genaue Kenntnis über Problemlagen, Prozessstörungen und Auswirkungen auf ergonomische Aspekte ihres Produktionsprozesses.

Regelmäßige Besprechungen mit der ersten Führungsebene und die direkte Kommunikation mit den Produktionsteams bei Bedarf fördern den gemeinsamen Austausch über die alltäglichen Problemen. Bei WLF haben sich durch PE auf allen Hierarchie- und Funktionsebenen netzwerkartige Kommunikations- und Kooperationsstrukturen herausgebildet, in denen zeitnah und problemorientiert an Lösungen gearbeitet wird, wie besonders am Beispiel von Linie 1 deutlich geworden ist. Auf Ebene der Produktionsteams werden bei Bedarf wichtige Unterstützer aus den indirekten Bereichen eingeladen. Die Meister treffen sich täglich mit Vertretern der wichtigsten Funktionen (Logistik, Instandhaltung, Programmplanung, Arbeitsvorbereitung) auf der Sacharbeiterebene. Der Werkleiter trifft sich wöchentlich mit allen produktionsrelevanten Führungskräften. Dieses institutionalisierte System regelmäßiger Gespräche führt zu einer hohen Durchlässigkeit wichtiger Informationen von der Produktionsbasis zur Produktionsleitung. Zusätzlich sind bei WLF Instandhalter direkt dem Meister unterstellt und in die Produktionsbereiche integriert, so dass auf kurzem Weg vor allem technische Probleme behoben und Verbesserungen umgesetzt werden können. Die Übernahme der Kümmererfunktion, also die Kontaktaufnahme zu Problemlösern, das Verfolgen von Umsetzungsfortschritten und regelmäßiges Nachfragen, sorgt für eine weitere Verbindung im Kommunikationsnetzwerk des Betriebs und fördert die direkte Kommunikation zwischen Produktions- und unterstützenden Bereichen. 
Bei NF haben sich zum Zeitpunkt der Untersuchung nur in sehr wenigen Bereichen direkte prozessnahe Kommunikationsstrukturen herausgebildet. Obwohl zwischen den einzelnen Hierarchieebenen der Produktionsbereiche tägliche Shopfloorgespräche stattfinden, an denen Vertreter wichtiger indirekter Bereiche (Logistik, Instandhaltung, Prozesstechniker etc.) teilnehmen, orientieren sich die Gespräche weniger an den artikulierten Problemen der Produktionsprozesse, sondern vor allem an der Einhaltung produktionsrelevanter Kennzahlen (Stückzahl, Fehler, rechtzeitige Anlieferung von Teilen). Prozessnahe Kommunikationsstrukturen lassen sich bei NF nicht in dem Ausmaß finden wie bei WLF.

Die Übernahme von Problemlösungen und Optimierungsaufgaben durch die Produktionsteams erfordert eine Anpassung des Rollenverständnisses und Funktionszuschnitts der indirekten Bereiche zu Unterstützern des Produktionsbereichs. Die einzelnen Funktionsbereiche müssen in der Lage sein, zeitund prozessnah Probleme der Produktionsbereiche zu erfassen und Lösungen zu erarbeiten. Eine Möglichkeit ist die dezentrale Anordnung wichtiger produktionsunterstützender Bereiche direkt in den Produktionsbereichen (Appelbaum et al. 2000; Kuhlmann et al. 2004). In beiden Betrieben lassen sich trotz konzeptioneller Ausrichtung nur ansatzweise dezentrale Organisationsstrukturen erkennen. Bei WLF wurden Instandhalter in die Produktionsbereiche integriert; andere wichtige unterstützende Bereiche waren nicht dezentral angeordnet. Aufgrund der relativ kleinen Größe des Betriebs waren die Wege zu den relativ nahen Produktionsbereichen aber kurz. Insofern wurde die fehlende Dezentralität durch die netzwerkartigen und direkten Kommunikationsstrukturen teilweise kompensiert.

Die schwachen Kommunikations- und Kooperationsstrukturen treffen bei $\mathrm{NF}$ auf ein hohes Maß bürokratisch formalisierter Abläufe und zentralistischhierarchischer Steuerungs- und Entscheidungsprozesse, die zu langen Bearbeitungszeiten, eher prozessfernen Entscheidungen sowie Problemlösungen und expertengetriebenen Optimierungsprozessen führten. Diese Bedingungen, die sich insbesondere in Montage $\mathrm{K}$ zeigten, schränkten prozessnahe Optimierungen in den Produktionsbereichen ein. In Montage F wurden in einzelnen Fällen die etablierten zentral organisierten Strukturen durch direkte Aufforderungen des Meisters und der Teams, bei der Lösung von Problemen zu kooperieren, durchbrochen und neue Formen der prozessnahen Zusammenarbeit entwickelt. Zum Zeitpunkt der Untersuchung war jedoch unklar, inwiefern sich diese punktuellen Kooperationsbeziehungen zu dauerhaften Kooperationspro- 
zessen verstetigen, die sich an der gemeinsamen Optimierung alltäglicher Probleme des Produktionsprozesses orientieren.

Durch den Vergleich der Beteiligungskonstellationen wird deutlich, dass prozessnah ausgerichtete Funktionsbereiche sowie direkte, systematische Kommunikationsstrukturen beschäftigtengetragene Optimierungsprozesse unterstützen, während zentralistisch angeordnete Funktionsbereiche, stark bürokratisch formalisierte Abläufe und hierarchische Steuerungs- und Entscheidungsprozesse einer eher restriktiven Wirkung auf beschäftigtengetragene Optimierungsprozesse haben.

\subsubsection{Arbeitspolitische Ausrichtung von Optimierungsprozessen}

Deutliche Unterschiede lassen sich zwischen den Beteiligungskonstellationen in den einzelnen Gestaltungselementen von KVP erkennen: Sie differieren hinsichtlich der Reichweite der akzeptierten Arten von Optimierungspotenzialen, der Steuerung von Optimierungsprozessen durch Handlungsprinzipien und Standardisierung sowie des Grads der aktiven Einbindung der Beschäftigten.

\section{Inhaltliche Ausrichtung von Optimierungspotenzialen}

Für die KVP-Praktiken ist entscheidend, welche Arten von Optimierungspotenzialen im Betrieb real anerkannt und realisiert werden. Eine enge Definition von Optimierungspotenzialen umfasst wirtschaftliche Optimierungen, die Einsparungen bei Prozessdurchlaufzeiten (Maschinen und Arbeitsabläufe), Verringerung von Fehlern, Nacharbeit und Ausschuss, Material- und Anlagenkosten sowie Personal bewirken. Eine weite Definition schließt darüber hinaus Optimierungen ein, die sich nicht direkt durch betriebliche Kennzahlen messen lassen, wie die Vermeidung von Arbeitsunfällen, die Verringerung ergonomischer Belastungen und Verbesserungen in der Infrastruktur (beispielsweise Pausen- und Waschräume, Raucherbereiche, Sichtbarkeit und Genauigkeit von Uhren).

Bei WLF zeigte sich eher ein weites Verständnis zulässiger Optimierungspotenziale. Vonseiten des Betriebs wurde versucht, alle Probleme und Ideen, die aus Sicht der Beschäftigten eine Verbesserung bedeuten, anzuerkennen und zu bearbeiten. Dies zeigte sich nicht nur auf einer rhetorischen Ebene, sondern vor allem in der konkreten Umsetzung von Optimierungspotenzialen, die nicht unmittelbar einen wirtschaftlichen Nutzen mit sich bringen. Wenn Optimie- 
rungspotenziale nicht umgesetzt wurden, wurden insbesondere in Linie 1 in vielen Fällen persönlich die Gründe erläutert. Die Beschäftigten von Linie 1 sahen überwiegend keine Differenzen in der Behandlung von Optimierungspotenzialen, während die Beschäftigten von Linie 2 oftmals kritisierten, dass durch den Betrieb eine Selektion zugunsten wirtschaftlicher Ideen stattfand. Obwohl das Management bei NF auf konzeptioneller sowie rhetorischer Ebene Ergonomie und Arbeitssicherheit neben wirtschaftlichen Optimierungen hohe Bedeutung zumaß, fanden die Beschäftigten weitgehend lediglich für Ideen mit direktem wirtschaftlichen Nutzen Unterstützung. Verbesserungsideen, die ihnen den Arbeitsalltag erleichterten und dadurch für sie wichtig waren, wurden nicht beachtet oder erst mit größer Verzögerung umgesetzt.

\section{Steuerung von KVP: Zwischen Problem- und Methodenorientierung}

Das betriebliche Verständnis, welche Optimierungspotenziale gefördert werden und welche nicht, ist eng damit verbunden, wie Optimierungsprozesse gesteuert und durchgeführt werden. Bei NF und WLF stehen sich zwei verschiedene Herangehensweisen gegenüber. Bei WLF orientierten sich Optimierungsprozesse vorwiegend an realen Problemen in den Produktionsbereichen (Problemorientierung), während bei NF Methoden standardisierter Gestaltungskonzepte im Mittelpunkt betrieblicher Optimierungs- und Veränderungsprozesse (Methodenorientierung) standen.

Die problemorientierte Ausrichtung der beschäftigtengetragenen Optimierungsprozesse setzte bei WLF direkt an den täglichen Problemen der Beschäftigten im Arbeitsprozess an. Handlungsprinzipien und Verbesserungsmethoden, wie Verschwendungsvermeidung, Standardisierung, prozessgesteuerter Fluss oder Nullfehler, dienten bei WLF eher im Hintergrund als Orientierungshilfe zur Identifizierung und Lösung von Problemen, mit denen die Beschäftigten über Moderationstechniken der BE-Begleiter in Berührung kamen. Auf diese Weise wurden die Beschäftigten in die Lage versetzt, die eigenen Arbeits- und Fertigungsprozesse zu überdenken, zu überarbeiten und gemeinsam neue, verbesserte Regeln in Sinne einer flexiblen Standardisierung zu erarbeiten sowie ein Verständnis für mögliche Prozessverbesserungen zu entwickeln. Die Verbesserungspotenziale wurden stets von den konkreten Alltagsproblemen aus angegangen und bearbeitet.

Optimierungs- und Gestaltungsprozesse wurden bei NF vorrangig mittels standardisierter Methoden und Gestaltungskonzepte durchgeführt. Solche 
Konzepte wurden von betrieblichen Rationalisierungsexperten ohne Einbindung der Beschäftigten erarbeitet und zentralistisch in den Produktionsbereichen umgesetzt. Die Dominanz dieser standardisierten Gestaltungskonzepte bewirkte bei NF, dass den Beschäftigten für die Gestaltung des eigenen Produktionsbereichs nur noch sehr enge Korridore blieben, die noch nicht durchreguliert worden waren. Veränderungs- oder Verbesserungsideen wurden häufig mit dem Verweis zurückgewiesen, dass sie gegen einzelne Standards der Gestaltungskonzepte verstießen. So schränkten diese standardisierten Gestaltungskonzepte auch die Reichweite möglicher Veränderungen ein, die methodenorientierten KVP-Workshops beschlossen haben. Obgleich kleinere Verbesserungen und Problemlösungen in KVP-Workshops erarbeitet wurden, orientierten sich diese häufig weniger an der direkten Herbeiführung von Problemlösungen, sondern an der Umsetzung von zentral festgelegten Standards, wie der Aktualisierung von Visualisierungen an Tafeln und Arbeitsmitteln. Das rigide Festhalten an Standards bei NF bewirkt, dass der Betrieb methoden- und prinzipienfixiert definiert, welche Probleme wichtig sind, was die Gestaltungsmöglichkeiten von Optimierungsprozessen stark einschränkt.

Das im Betrieb praktizierte Verständnis der Reichweite von zulässigen Optimierungspotenzialen sowie die Dominanz von standardisierten Gestaltungskonzepten haben wesentlichen Einfluss darauf, welche Optimierungspotenziale die Beschäftigten artikulieren, welche Realisierungschancen sie diesen beimessen und welchen direkten Nutzen sie davon erwarten.

\section{Grad der Einberiehung von Beschäftigten}

Nicht zuletzt zeigen sich deutliche Differenzen in dem Grad der realen Einbeziehung von Beschäftigten in Optimierungs- und Veränderungsprozesse. Die Spannweite der Beteiligungsformen reicht von einer systematischen Einbindung aller Beschäftigter in Optimierungsprozesse über die punktuelle Beteiligung einzelner ausgewählter Beschäftigter und Stellvertreter in Workshops, welche die Ergebnisse in ihr Team vermitteln sollen, und Beteiligungsformen, in der die Beschäftigten lediglich als Ideengeber einseitig gestalteter Optimierungen fungieren, bis hin zur Nichtbeteiligung an Optimierungsprozessen. Es zeigte sich, dass in den Bereichen, in denen alle Beschäftigte durch problemorientierte Kommunikation innerhalb des Teams und in direkter Kooperation mit unterstützenden Bereichen systematisch in Optimierungsprozesse einge- 
bunden und an ihrer Umsetzung beteiligt wurden, die Akzeptanz von und das Verständnis für KVP bei den Beschäftigten gewachsen ist.

\subsubsection{Finanzielle Gratifizierung}

Finanzielle Gratifikation als Anreiz zur Beteiligung an Optimierungsprozessen, als Teilhabe am wirtschaftlichen Erfolg, der auf beschäftigtengetragene Prozessoptimierungen zurückzuführen ist, und als Anerkennung des Engagements zeigte sich in den vorliegenden Fallkonstellationen als eine wichtige, aber nicht entscheidende Gestaltungsdimension.

Bei NF wurden zwar Überlegungen angestellt, auf Teamebene zusätzliche finanzielle Leistungsanreize auch für Optimierungsaktivitäten zu schaffen, diese wurden aber bis zum Ende des Untersuchungszeitraums nicht realisiert. Die einzige vorgesehene Prämierung war im Ideenmanagement verortet, wobei nur noch hohe Kosteneinsparungen berücksichtigt wurden. Der Wegfall kleiner Verdienstmöglichkeiten durch die Teilnahme am Ideenmanagement wurde zwar deutlich kritisiert; diese Kritik ging aber vorwiegend in der allgemeinen Kritik an sich negativ entwickelnden Beschäftigungsbedingungen auf. Aufgrund der oben beschriebenen Dysfunktionalitäten und der relativ geringen Beteiligung hatte das Ideenmanagement bei NF als Beteiligungsform zur Verbesserung von Prozessen, Arbeitsbedingungen und Kosten ohnehin bereits an Bedeutung verloren.

Bei WLF gibt es zwei Formen der Gratifizierung für die Beteiligung an Optimierungen: das Ideenmanagement und den Prämienlohn. Für die Beschäftigten stellte die zumeist eher symbolische Prämierung im Rahmen des Ideenmanagements einen wichtigen Bezugspunkt ihrer Beteiligung am Ideenmanagement dar. Die Staffelung anhand der Ideenanzahl wurde teilweise kritisiert, grundsätzlich aber stieß diese Form der Anerkennung ihres zusätzlichen Engagements große Akzeptanz.

Dass die Gratifikation wichtig, aber nicht entscheidend ist, lässt sich anhand dieser Befunde so deuten, dass für die Beschäftigten keineswegs nur die konkreten finanziellen Anreize, sondern vor allem die durch ihre Vorschläge bewirkten Veränderungen und Arbeitsverbesserungen wichtige Motivatoren sind. Inwiefern sich unterschiedliche Formen der Gratifikation für die Beteiligung an Optimierungsprozessen als restriktive oder partizipationsfördernde Beteiligungskonstellation auswirken, lässt sich anhand der vorliegenden Fallkonstellationen nicht eindeutig ermitteln. Die Dimensionen Arbeitsorganisati- 
on, betriebliche Kooperations- und Kommunikationsformen, Meisterfunktion, Gestaltungsprinzipien und Kohärenz der Gestaltungskonzepte insgesamt (s. folgenden Absatz) zeigten sich in der Analyse als deutlich stärkere Einflussgrößen. Den positiven Einfluss dieser Faktoren belegten auch bereits Forschungen zu innovativer Arbeitspolitik und High-Performance Work Systems (Appelbaum et al. 2000; Kuhlmann et al. 2004).

\subsubsection{Kohärenz betrieblicher Arbeitspolitik}

Diese vergleichende Untersuchung verdeutlicht, dass beschäftigtengetragene Optimierungsprozesse als Gestaltungskonzept in den Betrieben nicht für sich stehen, sondern nur eine Dimension eines sensiblen, sich wechselseitig bedingenden arbeitspolitischen Gestaltungssystems abbilden. Beschäftigungsbedingungen, Arbeits- und Leistungssituation, die Form der betrieblichen Kooperations- und Kommunikationsprozesse, der Zuschnitt der Führungsebenen sowie die dominante Rationalisierungsstrategie bilden wichtige Rahmenbedingungen für beschäftigtengetragene Optimierungsprozesse. Dieser Anforderung begegneten die Betriebe auf konzeptioneller Ebene meist mit der Erarbeitung weitreichender arbeitspolitischer Organisationskonzepte, deren Gestaltungsdimensionen kompensatorisch zusammenwirken und die Beteiligung der Beschäftigten an Optimierungsaufgaben ermöglichen sollen, wie es sich Ganzheitliche Produktionssysteme als Anspruch gestellt haben. In der Realisierung der Gesamtheit aller Reorganisationskonzepte zeigten sich zwischen den Betrieben deutliche Differenzen, die insbesondere durch Wechselwirkungen einzelner Gestaltungsdimensionen hervorgerufen wurden. Dabei wurde deutlich, dass eine strukturinnovative Arbeits- und Betriebsorganisationsgestaltung in einem deutlichen Zusammenhang mit einer gelingenden KVP-Praktik steht.

In der Alltagspraxis bei WLF ergänzen sich die einzelnen Gestaltungselemente. Dies ist zum einen darauf zurückzuführen, dass in vielen Funktionsbereichen und auf allen Hierarchieebenen PE als ein einheitliches, hierarchieübergreifendes Kommunikations- und Problemlösesystem eingeführt wurde. Zum anderen entfaltet PE seine integrative Wirkung durch die hohe Verbindlichkeit der neu eingeführten Regeln, die nicht nur von den Beschäftigten, sondern auch auf allen Ebenen der betrieblichen Führung sehr bewusst eingehalten werden: festgelegte Gesprächszeiten, Eskalation und Bottom-upProzessorientierung. Obgleich noch nicht in allen Produktionslinien und Angestelltenbereichen PE umfänglich praktiziert wurde und Kommunikations- 
und Kooperationsprozesse nicht optimal abgestimmt waren, wurde das PEKonzept sehr positiv bewertet und als wichtiges Element sich permanent verbessernder Prozesse und Zusammenarbeit im Betrieb angesehen. Nicht zuletzt wurde PE als genuine Weiterentwicklung des bisher eingeschlagenen Weges einer auf Qualifizierung und Mitarbeiterorientierung basierten Arbeitspolitik eingeführt.

Bei NF wurden die detailreichen und intensiv ausgearbeiteten Reorganisationskonzepte (selbstorganisierte Teamarbeit, neue Hierarchie mit Shop-FloorManagement und Dezentralisierung indirekter Bereiche) innerhalb eines kurzen Zeitraums umgesetzt und markierten einen arbeitspolitischen Bruch in dem zuvor eher tayloristisch geprägten Betrieb. Als besonders problematisch erwies sich im Zuge der Einführung von Teamarbeit war, dass lediglich die unmittelbar betroffenen Produktionsbereiche und Hierarchieebenen mit dem Aufgaben- und Funktionszuschnitt der Produktionsteams vertraut waren und wichtige produktionsrelevante Angestelltenbereiche nicht unmittelbar einbezogen wurden, so dass sie auf wichtige Veränderungen, wie zum Beispiel die direkte Kooperation mit den Produktionsteams, nicht vorbereitet waren. Durch weitreichende Restrukturierungsmaßnahmen entstanden Unsicherheiten und unklare Kompetenzzuschnitte einzelner Funktionen und Schnittstellen zwischen produktionsrelevanten, indirekten Bereichen. Um das geplante Produktionsprogramm erfüllen zu können, nahm das Management oftmals per Direktive Einfluss, was zu einem Verharren in alten etablierten Arbeitsweisen und Kooperationsstrukturen sowie einer Zunahme bürokratischstandardisierter Verfahrensweisen führte. Der Entwicklung der Teamarbeit und eines neuen Rollenverständnisses der Meister wurde wenig Raum gegeben und die Reaktionsfähigkeit der indirekten Bereiche auf Unterstützungsanfragen erheblich behindert. ${ }^{111}$ In Bezug auf Rationalisierungs- und Optimierungsprozesse ergaben sich für die Produktionsbeschäftigten aufgrund der Dominanz expertengetriebener Modernisierungs- und Rationalisierungsprojekte sowie der hohen Verbindlichkeit von standardisierten Arbeits- und Fertigungsprozessen auf der einen und dem Ansatz beschäftigtengetragener Optimierungsprozesse

111 Die durch betriebsorganisatorische Dysfunktionalitäten und Startschwierigkeiten von Teamarbeit bedingten Irritationen wurden bei NF auch dadurch verstärkt, dass ein Teil der betrieblichen Interessenvertretung Teamarbeit als Spielball für politische Verhandlungen von Entgeltstrukturen und Standortsicherung genutzt hat. 
auf der anderen Seite widersprüchliche Verhaltenserwartungen und Restriktionen hinsichtlich der im Teamarbeitshandbuch festgelegten Optimierungsaufgaben.

Flankiert wurden die beschriebenen Widersprüchlichkeiten durch eine Verschlechterung des Umgangstons, krisen- und standortbedingte Beschäftigungsunsicherheiten sowie Veränderungen in Entgelt- und Arbeitszeitsystemen, die von den Beschäftigten in hohem Maße als Negativentwicklung ihrer Beschäftigungsbedingungen bei einem zuvor sehr angesehenen und sicheren Arbeitgeber wahrgenommen wurden. Diese Inkohärenz der alltagspraktisch realisierten Arbeitspolitik führte zu einer hohen Verunsicherung und Unzufriedenheit aller Beschäftigten im Betrieb.

\subsubsection{Profile arbeitspolitischer Beteiligungskonstellationen}

Den Ausführungen zufolge entfalten die Gestaltungsdimensionen der arbeitspolitischen Fallkonstellationen im Spannungsfeld sowohl restriktive als auch partizipationsfördernde Wirkungen auf die Beteiligung der Beschäftigten. Im folgenden Diagramm sind die einzelnen Fallkonstellationen nach dem Grad der realen Ausprägungen partizipationsfördernde Gestaltungsbedingungen der einzelnen relevanten Dimensionen von KVP eingeordnet. Die Werte entsprechen keinen absolut ermittelten Kennzahlen, sondern stellen entlang der Rekonstruktion einzelner Ausprägungen in den Fallkonstellationen eine graduelle Einordnung dar.

Im Netzdiagramm werden die vier Beteiligungskonstellationen direkt miteinander verglichen. Dabei wurde die einzelnen arbeitspolitischen Gestaltungsfelder, die für einen „funktionierenden“ KVP relevant sind, jeweils qualitativ für die einzelnen Falle auf den einzelnen Achsen abgebildet. 0 bedeutet ein sehr geringes Maß; 1 ein hohes Maß der jeweiligen Gestaltungsdimension. ${ }^{112}$

112 Teamarbeit mit Selbstorganisation, Rotation, Handlungs- und Entscheidungsspielräume gering (0) oder hoch (1); Meister als Kontrolleur (0) oder als aktiver Unterstützer der Arbeitsteams (Scharnierfunktion) (1); geringes (0) vs. hohes (1) Maß der Einbindung der Beschäftigte in KVP; bürokratische (1) versus prozessnahe (1) Kooperationsstrukturen; rigide Standardisierung (0) oder flexible Standardisierung (1) , keine/geringe finanzielle Gratifikation; Inkohärenz (0) bzw. Kohärenz (1) im Zusammenwirken der einzelnen arbeitspolitischen Gestaltungsfelder 
Abbildung 3: Profil der arbeitspolitischen Gestaltungsbedingungen von KVP
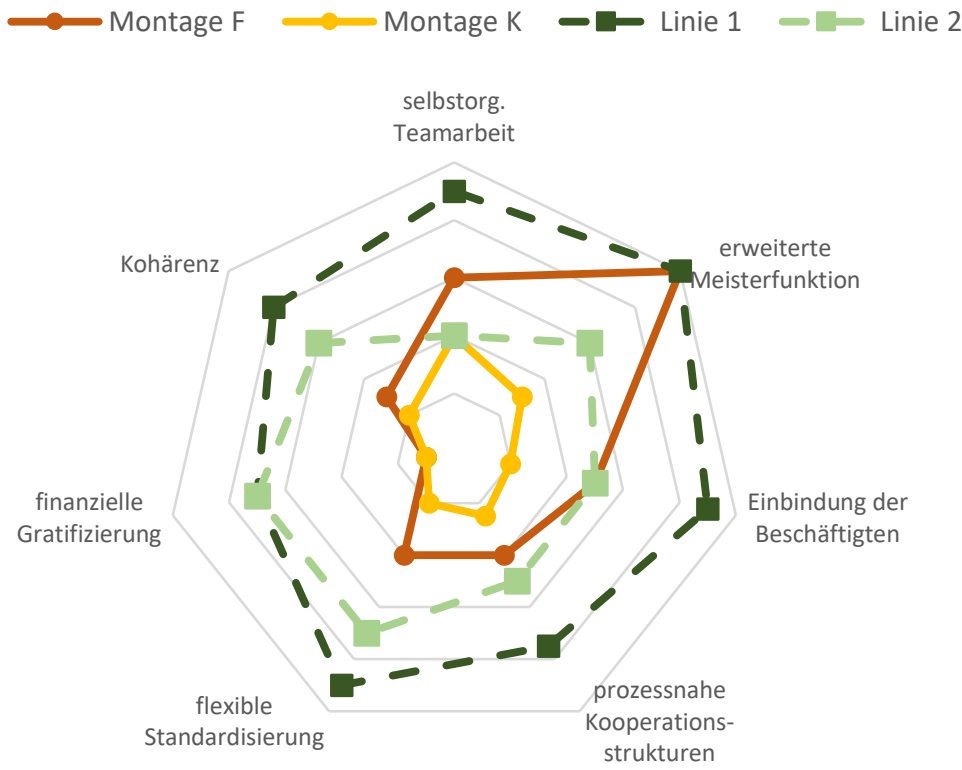

eigene Darstellung

Der direkte Vergleich von Montage F und Linie 1 zeigt deutlich den Wirkungszusammenhang zwischen gelingenden KVP und den strukturinnovativen bzw. partizipativ ausgeprägten Gestaltungsdimensionen, die sich unmittelbar auf die konkreten prozessnahen Strukturen der Arbeitsorganisation, der Führungsrolle, der systematische Kommunikationsprozesse sowie der Kooperation beziehen. In den Fallkonstellationen Montage $\mathrm{K}$ und Linie 2, in denen beschäftigtengetragene Optimierungsprozesse nur in geringem Maße realisiert werden konnten, sind diese Gestaltungsbedingungen - vor allem die Dimension der Arbeitsorganisation und die Meisterfunktion - deutlich restriktiver ausgeprägt und können als strukturkonservativ bezeichnet werden.

Diese Befunde bestätigen die von Appelbaum et al. (2000) und Kuhlmann et al. (2004) herausgestellte negativen Wirkung von restriktiven Gestaltungsbedingungen auf die Beteiligung an beschäftigtengetragenen Optimierungsprozessen auf der einen Seite, während auf der anderen Seite partizipative Bedin- 
gungen die Wahrscheinlichkeit, zusätzliche, über die eigentliche Arbeit hinausgehende Beiträge - in diesem Sinne also die die Beteiligung mit eigenen Ideen und Erfahrungen an Optimierungsprozessen - zu generieren, erhöht wird.

Kumuliert lassen sich die einzelnen Beteiligungskonstellationen für die weitere Analyse der Aneignungs- und Umgangsformen der Beschäftigten mit KVP wie folgt einordnen:

\author{
Linie 1 partizipativ \\ Montage F teils partizipativ, teils restriktiv \\ Linie 2 teils partizipativ, teils restriktiv \\ Montage K restriktiv
}

Restriktive bzw. partizipative Gestaltungsbedingungen bilden jedoch nur ein Bestimmungsmoment für die Realisierungsformen gelingender beschäftigtengetragener Optimierungsprozesse. Sie stellen einen strukturellen Rahmen dar, mit dem sich die Beschäftigten auseinandersetzen und innerhalb dessen die Beschäftigte agieren.

\title{
7.4. Betriebs- und Rationalisierungsverständnis
}

Die restriktiven bzw. partizipativen Bedingungen bilden den Rahmen für die subjektive Auseinandersetzung der Beschäftigten mit ihrer sozialen Rolle im Betrieb und ihrem Stellenwert im real praktizieren, betrieblichen Verwertungskonzept. Dabei fließen nicht nur die aktuellen Wahrnehmungen, sondern auch die kumulativen Erfahrungen, welche die Beschäftigten im Laufe ihrer gesamten betrieblichen Sozialisation mit betrieblichen Beteiligungs- und Arbeitsbedingungen sowie Rationalisierungsprozessen gemacht haben, ein. Wie schon in Kapitel 6.3.8 deutlich wurde, verorten sich die Beschäftigten eher im betrieblichen Unten und sehen sich tendenziell als Leidtragende betrieblicher Rationalisierungsaktivitäten.

Zwischen den Beschäftigten von NF und WLF lassen sich - mit Blick auf die arbeitspolitischen Arbeits- und Beschäftigungsbedingungen wenig überraschend - deutliche Unterschiede erkennen. Diese lassen sich einerseits mit den konkreten Arbeits- und Beteiligungsbedingungen von KVP in Verbindung 
bringen, rühren andererseits aber zu einem bedeutenden Teil aus der allgemeine Beschäftigungspolitik und dem betrieblichen Klima.

\subsubsection{Ausgeprägter Interessenantagonismus bei NF}

Bei NF war in allen Montagebereichen der hohe Rationalisierungsdruck omnipräsent. Hohe Leistungsanforderungen, beschäftigungspolitische Maßnahmen, wie die Einführung von Arbeitszeitkonten und eine Flexibilisierung des Schichtsystems, Dominanz expertengetriebener Optimierungsprojekte und vor allem umfangreiche Einsparmaßnahmen, die sich insbesondere in permanenter Optimierung des Personaleinsatzes in den Produktionsbereichen, aber zum Teil auch in schlechterer Ausstattung mit Werkzeugen und Hilfsmitteln niederschlug, wirkten sich sehr negativ auf die Beschäftigten aus.

Und ich glaube, was momentan das Ärgste ist einfach (2) - wie sage ich das? - (4) der Druck. so. Weiß ich nicht, wie ich es ausdrücken soll? Du füblst dich einfach allein gelassen. Mmb. Du hast nirgends ricbtig Unterstïtzung, sag ich mal, und immer wieder kommt was her, was eigentlich scblecht ist für dich. Du siebst keine Besserung. Kaum glaubst du, der eine Scheiß ist vorüber, kriegst den nächsten Brocken bergescbmissen und wieder was hergeschmissen und was eigentlich wieder nur den Arbeiter betrifft. Die Ungewissheit ist, das glaube ich, was momentan am ärgsten ist, glaube ich. [...] Ja, wie geht's generell weiter da, bleibt das Werk bestehen, bleibts nicht bestehen. Man weiß nicht, wie und was, sag ich mal. Und das schlägt sich einfach, sag ich mal übertrieben, auf die Seele nieder. Durch das lässt jeder den Löffel hängen. Da sind Leute unten, das bätte ich niemals geglaubt, dass sagen: ,Weißst was, mich interessiert's nicht mehr. 'Die haben gelebt für die Arbeit, obwohl das eine Arbeit ist - da bol ich mir einen von der Straße rein, wie ich gesagt habe. So wie ich dazumals gelebt habe, da drüben für die Arbeit, was mir getangt hat, so hat der da herïben gelebt für die Arbeit, und der ist jetzt auch so weit und sagt: ,Weißt was, es interessiert mich nicht mehr.'Das sind aber Leute mit 35 Jahre oder was, wie soll denn der das noch 25 Jabre machen, der dreht irgendwann mal durch, wenn das nicht anders wird. Und es wird nicht besser, es wird, glaube ich, noch schlechter. "(Fischer, NF Montage F_A, 698ff.)

„,Es werden keine betriebsbedingt Leute nicht gekündigt', und sind aber trotzdem ins Personalbüro geholt worden: ,Sie haben so viel Urlaub, mir geben Ihnen die Chance, dass Sie auf Teilzeitding' einvernehmen gehen, so auf die Art. [...] Ich meine, es heißt, man wird nicht gekeïndigt, es stimmen alle dafür, dass der Standort gesichert wird, und auf der anderen Seiten werden dann die berunter gebolt und zwangsverpflichtet irgendwo [...]. Weil es ist dann so eine, ich würde schon fast sagen, Angst. Jeder schaut auf seinen Krankenstand, jeder schaut auf sein Ding. Darum will ja keiner auf das Gespräcb113 hinein, weil alle voll Angst haben, die Kassette114 kommt irgendwo und beim nächsten ist man dabei. "(Berger, NF Montage K_B, 112ff.)

113 Gemeint ist hier die Interviews im Rahmen des SOFI-Forschungsprojekts. In Montage K gab es sehr starke Vorbehalte hinsichtlich der Interviews. Viele Beschäftigte 
Die Beschäftigten nahmen die Gesamtsituation bei NF insgesamt als deutliche Bedrohung wahr. Arbeitsplatzunsicherheit, hoher Leistungsdruck, eine fortschreitende Verschlechterung der Beschäftigungsbedingungen und ein Anstieg der damit einhergehenden psychischen Belastung, ob man in Zukunft noch unter diesen Bedingungen im Betrieb arbeiten wird können, prägten umfassend die Arbeits- und Beschäftigungsbedingungen bei NF.

Obgleich den Beschäftigten durch Teamarbeit und KVP-Workshops mehr Selbstorganisations- und Gestaltungsmöglichkeiten gegeben wurden, was die Beschäftigten meist durchaus positiv bewerteten, überwogen in ihrem Bewusstsein Momente der Fremdbestimmung und Benachteiligung. Die Dominanz expertengetriebener Rationalisierung, rigide Standardisierung sowie der Kampf um Vorgabezeiten und ausreichendes Personal in den Bereichen und letztlich die Fixierung auf Kosteneinsparungspotenziale bei KVP reproduzierte das Rationalisierungsverständnis der Beschäftigten, sich als Objekt von Rationalisierung zu sehen.

„Arbeitssituation, freilich, freilich zwicken's Leute raus. Sie schauen schon, dass Teamarbeit nicht gemacht ist, nur für uns Arbeiter, dass wir mehr Leut' kriegen, das ist schon klar. Das musst verstehen, und sie versuchen schon auch, dass Leute rauszwicken. Dass Produktion mehr wird und mehr wird. Das erhoffen sie sich schon durch die Teamarbeit, weil sonst wäre es nicht gekommen. (751ff.) [...] Aber für die Firma ist es sicher mal das Positive, weil erstens mal Febler werden weniger. Und Febler sind schon mal Kosten, weil Nacharbeit fast weg ist. Und freilich, für uns, für die Teams ist es auch besser geworden, durch das, dass wir uns selber eigentlich organisieren und alles ein wenig machen können. Ist für uns auch ein bisschen besser geworden. Aber in erster Linie ist es schon für die Firma besser geworden. "(Haller, NF Montage F_A, 1337ff.)

Nichtdestotrotz sahen Beschäftigte großes Potenzial für Kosteneinsparungen und Prozessoptimierung jenseits der unmittelbaren Arbeitsplatzrationalisierung und zeigen Bereitschaft, sich daran zu beteiligen.

„Schlechter ist auf jeden Fall einiges geworden. Ich meine, besser, es gäbe Möglichkeiten, einiges besser zu machen, da ist aber das Team noch nicht bereit, da ist auch das Umfeld noch nicht bereit (.) es ist vieles schlechter geworden natürlich, es ist sebr viel mehr Druck, sehr viel, im Prinzip, ,Scher dich. Ibr macht das selber. Ibr seid das Team, kümmert euch darum: Und letztendlich stehst du wieder alleine da, genauso wie vorber. Wenn man nicht sagt, ,Ja, ich engagiere mich', warum auch, man hat ja kein Nutzen

lehnten die Teilnahme ab, da sie das SOFI zunächst auf der Seite des Betriebs verorteten und daher ein starkes Misstrauen hegten, dass das im Interview Gesagte gegen sie verwendet werden könnte.

114 Die Aufnahme des Interviews. 
im Prinzip, das ist ja nur, damit es andere bequemer haben. "(Eder, NF Montage K_B, 696ff.)

Bei den Beschäftigten von NF zeigte sich ein sehr homogenes Rationalisierungsverständnis mit einem ausgeprägten Interessenantagonismus. Angesichts der fortschreitenden Negativentwicklung und Unsicherheiten sowie der nach wie vor hohen Dominanz expertengetriebener Optimierungsprozesse sehen sich die Beschäftigten im Fokus betrieblicher Rationalisierung. Sie differenzierten sehr stark zwischen „Verbesserungen für den Betrieb“ und „Verbesserungen für die Arbeiter". Selbst wenn ein gemeinsamer Nutzen oder ein gegenseitiges Profitieren von Optimierungsprozessen in Betracht gezogen wurde, verwiesen wiederkehrende Formulierungen wie "Wir, die Arbeiter" vs. "die, die Firma" auf interessenantagonistische Positionierungen der Beschäftigten.

Die Beschäftigten sahen sich als Leidtragende der Umwälzungsmaßnahmen im Betrieb. Einsparmaßnahmen wurden vorwiegend im Produktions- und weniger im Angestelltenbereich vollzogen wurden, in dem sogar ein erheblicher Zuwachs stattgefunden hat. Besonderer Kritikpunkt war hierbei die Zunahme von hochqualifizierten Angestellten in Führungspositionen und in den planerischen Bereichen, die aus Sicht der Beschäftigten schlechte Veränderungs- und Rationalisierungsprojekte durchführten, ohne sich ausreichend mit den Problemen und praktischen Anforderungen auseinanderzusetzen.

„Genau. Das sind so Sachen, da sind sie sich zu schade, dass sie sich einen Arbeiter zulegen, der was ein wenig Abnung hat von dem Ganzen. [...] Theorie und Praxis sind zwei Paar Schube. Da kann ich nicht wen hereinholen und sagen: ,So, du machst das Projekt jetzt", dann haut er ab und macht's am Papier wunderschön und dann passt binten und vorne nichts. "(Fischer, NF Montage F_A,199ff.)

„Meine Meinung ist, ich meine, schau sie dir an. Die ganzen Studierten - ich habe jetzt nichts gegen Studenten oder sonst irgendwas, was von einer höheren Schule kommen - aber im Prinzip sind sie da, glauben, sie sind Götter und oben. Druck nach unten in die Hierarchie. Und ich finde, das sind eben Leute, die gescheit sind, aber die was nichts zusammenbringen. Ist meine Meinung über die ganzen Fübrungen da berinnen. Also ich rede jetzt nicht vom Meister, weil ich sage, ein Meister ist eher in meinen Boot als wie einer in einer böheren Ebene." (Haller, NF Montage F_A, 1364ff.)

Bei NF herrschte ein deutliches Machtgefälle zwischen den Führungs- und den Angestelltenbereichen. Die Produktionsbeschäftigten fühlten sich den anderen betrieblichen Gruppen deutlich untergeordnet. Ihrer Wahrnehmung nach schenkten ihnen insbesondere die prozessfernen Bereiche kein Gehör; ihre nützlichen Ideen wurden ignoriert und betriebliche Interessen über ihre Interessen gestellt. Mitunter fühlten die Beschäftigten sich gar in ihren grundlegenden Bedürfnissen missachtet. Angesichts der angespannten wirtschaftlichen 
Lage, des zunehmend schlechten Umgangstons zwischen betrieblichen Vertretern und Produktionsbeschäftigten und begrenzter Einflussmöglichkeiten auf betriebliche Entscheidungen prägten relativ geringe Handlungs- und Gestaltungsspielräume in der Arbeit sowie unzureichende Anerkennung ihrer Erfahrungen und Kompetenzen durch Führungskräfte das Betriebsverständnis der Beschäftigten.

„Wie müsste man die Leute motivieren? Es fängt schon mal heute an mit der Motivation, dass die Fübrungskeräte mebr kollegial mit den Mitarbeitern umgehen. Weil da baben wir sicher große Probleme in der Vergangenheit, ich meine, ich denke nur an ffrïheren Werkleiter, BS], der durchgerannt ist, ich meine, das war für mich das größte Negativbeispiel, wie eine Fübrungskeraft, also wie ich einen Mitarbeiter motivieren kann. [...] Und [das Fahrzeng, B.S.] sollte jeder identifizieren keönnen und da gehört das Lob genauso gut dazu, Jetzt habt ibr wieder mal eine super Arbeit geleistet, Burschen.' Das ist, vielleicht dass wieder mal ein Fest oder irgendwas steigt. Ich meine, alle solche Kleinigkeiten, es muss nicht unbedingt sein. Ich muss nicht dann die große, sicher gebört das Geld auch dazu, aber es ist, sage ich mal, dass mehr miteinander, und nicht nur weil dort, wir wissen, dass der Chef zwar da ist, aber wir wissen nicht, wo er ist. Also das Reden miteinander oder, ,Ich wünsche euch frobe Weihnachten', oder, wie gebt es euch?; das sind lauter so Kleinigkeiten, das sind Kleinigkeiten in meinen Augen, wie man einen Mitarbeiter motivieren kann. Es ist nicht viel, aber es hat große Auswirkungen. "(König, NF, Montage F_A, $775 f f$.

Ungeachtet unterschiedlicher KVP-Praktiken und arbeitssituativer Bedingungen war das Betriebsverständnis durch starke Dichotomien zwischen den einzelnen betrieblichen Gruppen geprägt. Gemeinsam mit den erfahrenen Nachteilen durch betriebliche Rationalisierungsprozesse lässt sich das sehr homogene Rationalisierungs- und Betriebsverständnis in folgender sinngemäß rekonstruierten Aussage resümieren: „Der Betrieb kümmert sich nicht um uns, wir Arbeiter müssen selbst dafür sorgen, dass es uns gut geht."

Die Einführung von Teamarbeit und die Beteiligung an Workshops hat keine grundlegenden Veränderungen im Betriebs- und Rationalisierungsverständnis bewirkt, da die realen Handlungs- und Entscheidungsspielräume sehr begrenzt bleiben. KVP-Workshops, die vom Betrieb als Optimierungsinstrument eingesetzt werden, werden von den Beschäftigten stets unter dem Vorbehalt betrachtet, dass der Betrieb diese hauptsächlich zur Kostenrationalisierung initiiere, was eine Verschlechterung der Arbeits- und Leistungsbedingungen oder Arbeitsplatzrationalisierung nach sich zieht und damit zum Nachteil der Beschäftigten gereiche. Wie sich schon angedeutet hat, eignen sich die Beschäftigten unterschiedliche passive oder aktive Strategien an, um dafür zu sorgen, dass es ihnen gut geht. 
7.4.2. Zwischen latentem und bewusstem Interessenantagonismus bei WLF

Bei den Beschäftigten von WLF ließen sich unterschiedliche Ausprägungen des Betriebs- und Rationalisierungsverständnisses erkennen, das sich zwischen einem bewussten und einem latenten Interessenantagonismus bewegt. Obwohl die meisten Beschäftigten im Gegensatz zu NF dem Betrieb gegenüber insgesamt sehr positiv eingestellt waren und in beschäftigtengetragenen Optimierungsprozessen einen hohen gemeinsamen Nutzen von Betriebs- und Arbeiterinteressen erkannten, blieben die Interessengegensätze meist nunmehr latent bestehen.

Die Fokussierung auf beschäftigtengetragene Problemlöseprozesse, die reale Erfahrung, dass der Betrieb weitgehend alle für die Arbeit und den Produktionsprozess nützlichen Ideen fördert, sowie die Anerkennungsprämie führten dazu, dass die Beschäftigten eine große Schnittmenge zwischen den Interessen beider Seiten wahrgenommen haben.

„Beide. Es profitieren sicher beide davon. Und sicher, wenn das jet:t irgendeine Verbesserung ist, die rigtausende von Euros einspart, wird sicher die Firma mehr davon haben als der Arbeiter. Aber was es jetzt so von den Abweichungen angebt, wird wieder der Arbeiter mehr haben als wie die Firma davon. Denn durch die Abweichungen weiß ja der Arbeiter Bescheid, was er ändern kann. Das kriegt ja die Firma gar nicht mit." (Neubauer, WLF Linie 2, 1008)

Die meisten Beschäftigten von WLF sahen den gegenseitigen Nutzen der Optimierungsprozesse und differenzierten deutlich weniger stark zwischen Arbeiter- und Betriebsinteressen als bei NF. Dies lässt sich zum einen auf die sehr stabile Beschäftigungssituation zurückführen. Zum anderen förderte der Betrieb mithilfe der Problemlösemechanismen von ProduktionsExzellenz und zahlreichen Bemühungen, Optimierungspotenziale zu erschließen, die Realisierung von Problemlösungen und Verbesserungen, die sich unmittelbar und positiv auf die Arbeitssituation der Beschäftigten ausgewirkt haben.

[I: „Wer profitiert denn von BE?"] „Die Mitarbeiter, die Arbeiter, Scbicbtarbeiter, wir. Wir hauptsächlich, naja sicher, warum nicht." [I: „Und warum?"] „Ja, weil mir geholfen wird, wenn ich ein Problem habe, wird mir geholfen, dass ich meine Arbeit leichter mach, meine Tätigkeit oder mein Umfeld. Das schöner wird, sauberer wird, Beispiel: ,mebr Licht" oder so Sachen schreiben wir rauf." II: „Hat denn BE auch eine Bedeutung für die Wirtschaftsfähigkeit oder Leistungsfähigkeit des Betriebes?"] „Das weiß ich nicht. Aber ja, sicher, (2) Beispiel, wenn alles passt in einem Betrieb, kann ich pünktlich die Lager bestellen und der Kunde ist zufrieden. Oder wenn ich nur Schwierigkeiten habe und nichts behandelt wird oder nichts gemacht wird, bring ich keinen Liefertermin nicht zusammen." (Oswald, WLF Linie 1, 636ff.) 
„Ich denke, es wird alles gleich behandelt. W as auch wirtschaftlich mehr bringt, wird für sie sicher auch interessanter sein, als wie wenn sie für die Arbeiter eine Einrichtung machen, die ein Geld kostet. Das kann ich mir vorstellen, aber gemacht wird beides." (Renner, WLF Linie 1, 536)

Dass nur die Vorteile von beschäftigtengetragenen Optimierungsprozessen gesehen und kaum zwischen betrieblichen und beschäftigtenbezogenen Interessen differenziert wurde, traf jedoch nur auf einen Teil der WLFBeschäftigten zu. In Line 2, in der zum einen PE weniger aktiv praktiziert und zugleich einzelne Anlagenoptimierungen durchgeführt wurden, welche die Beschäftigten als deutliche Leistungssteigerung wahrnahmen, wurden negative Auswirkungen von Rationalisierung und teilweise die Wirkungsmechanismen von PE deutlich kritisiert.

„Also für mich rennt das ganze System auf eine Unterwanderung hin. Der eine sage, ,Ja, geht', der andere sagt, ,Nein, geht nicht.' Fängt eine Streiterei an." [I: „Du hast eben Unterwanderung gesagt, oder?"] „Mhm. Der eine sagt, es geht, und der andere sagt, es geht nicht. Weil es kann ja nicht nur jeder eine Maschine, sondern es können ja mehr Leute mehr Maschinen. Und wenn jetzt einer hergeht, und sagt, ,Nein, die Zykluszeiten fahren wir. Weil die sind bei mir auch gegangen. 'Dann ist es eine Unterwanderung für den nächsten. Weil der sagt, ,Ja, wie kommst du auf die Zeiten bin? Auf die komm ich niemals hin.' Da baben wir gestern schon wieder so etwas gehabt. Habe ich recht lachen müssen. Haben sie uns die Zeiten heruntergeschrieben gehabt bei einer $M a-$ schine drinnen. Der fährt jetzt wieder unter die Zeiten. Ja, wohin noch hinunter? [...] Hm. Für die Wirtschaftlichkeit für die Firma ist [PE, B.S.] sicher kein Fehler nicht. Weil Profit schaut durch das mebr heraus, ist so. Aber für die Leute selber gebörte es wieder abgeschafft." (Strobl, WLF Linie 2_1, 1072 ff.)

Für Strobl, der im Sample von WLF die kritischste Haltung einnahm, bedeuteten die Wirkungsmechanismen von PE einen strategischen Versuch, die starke kollegiale Solidarität zu Gunsten des Betriebs zu unterwandern. Trotz der negativen Auswirkung dieser Rationalisierungsmaßnahmen auf die Leistungssituation überwogen aber auch in Line 2 die positiven Effekte von Gestaltungsmöglichkeiten und Handlungsfreiheiten an den einzelnen Arbeitsplätzen. Im Vergleich zu NF waren die Leistungssteigerungen moderat und es gab in der jüngeren Vergangenheit wenig negative Auswirkungen von Rationalisierung auf die Arbeits- und Beschäftigungssituation.

Angesichts der durch PE intensivierten Kooperationsbeziehungen, transparenterer Kommunikationsprozesse, ihrer Einbindung in betriebliche Entscheidungen sowie der Vergrößerung ihrer individuellen Handlungsspielräume am Arbeitsplatz hatten die Beschäftigten das Gefühl, vom Betrieb und den indirekten Bereichen zunehmend ernstgenommen zu werden. Insbesondere durch die Realisierung der von den Beschäftigten artikulierten Probleme und 
Optimierungspotenziale erfuhren sie sowohl die Wirksamkeit des eigenen Engagements für ihre Arbeit und den Betrieb als auch Anerkennung.

Die durch PE gestärkten Kooperationsbeziehungen und die Ausrichtung auf Problemlösungen in den beschäftigtengetragenen Optimierungsprozessen förderte den Informationsfluss und das wechselseitig Verständnis zwischen den betrieblichen Gruppen. Durch diesen Austauschprozess wurde nicht nur das Wissen innerhalb der einzelnen betrieblichen Gruppen erweitert, sondern auch die Genese eines gemeinsamen Verständnisses begünstigt, wie man die Arbeit und die Abläufe effektiv und sinnvoll gestalten kann. Die Beschäftigten verstanden sich mehr als Subjekte und weniger als reine Objekte der betrieblichen Rationalisierung. Das traf insbesondere in Linie $1 \mathrm{zu}$. In Line 2 wurde das Verhältnis zwischen Arbeiter- und Betriebsinteressen insgesamt etwas kritischer betrachtet, so dass die Notwendigkeit, sich vor betrieblicher Rationalisierung schützen zu müssen, höher eingeschätzt wurde.

„Es sind meistens die Leute, die was, da das überbaben, die haben meistens kein Verständnis dafür, was wir da machen. Darum finde ich das nicht korrekt, dass die das machen. Weil das erste, was die sagen dann ist, wir müssen Maß redurieren, dass ich mit der Laufzeit herunterkomme. Aber das ist aber bei vielen Typen, dann tu ich Maß reduzieren, dann bleibt mir der ganze Ring schwary, dann kann ich ibn erst recht weghauen. [...] Ja, das sind einfach Leute, die was studiert haben. Die was, nicht böse gemeint jetrt, aber die was vom Praktischen keine Abnung baben. Die sind mit ibrem Zettel, Papier sind die glücklich, ja, aber was da das andere ist. [...] Die kommen schon zu uns auch her und die reden auch mit dir, wie du das findest. Von dem her, finde ich das schon gut, dass es das gibt. [...] Und dann babe ich balt mit dem so geredet, was der leicht macht, und der hat mir auch gleich erklärt, wie das alles dann funktioniert und das alles. Und habe ich ibn gefragt, ob das wirklich was bringt. Er hat gesagt: "Ja, das bringt was. 'Sicher, ob es dann wirklich irgendwas bringt, das ist dann das nächste. Das bilden sie sich ein, weil sie glauben, dass sie wieder irgendwas ersparen mit der Zeit. Darum, da kannst du als Arbeiter nicht machen. Wenn die sagen, ja, das sind vielleicht drei Sekunden pro Ring, was du dir da ersparst, dann sind das drei Sekunden." (Neubauer, WLF Linie 2, 1142f.)

Ähnlich wie bei NF bildeten die Divergenzen im Rationalisierungsverständnis und in der Arbeitsweise zwischen Produktionsbeschäftigten und Planern deutliche Reibungspunkte und reaktivieren die kollektive Schutzhaltung vor allem der Status-quo-orientierten Beschäftigten. Letztlich fiel in Linie 2 die Wahrnehmung der Auswirkungen von betrieblichen Optimierungs- und Rationalisierungsprozesses jedoch sehr geteilt aus.

"Ja, eigentlich eh nur bei der (), weil der Ablauf dann besser passt, eigentlich eh nur die Firma profitiert davon. Weil im Endeffekt profitiere ich auch davon, weil, ich meine, wie soll ich sagen, die haben ja weniger Probleme dann in der Montage und das, und im Endeffeket ist immer der aufgeschmissen, der in der Montage ist. Weil der kriegt den 
Ring, der muss den zusammenbauen, und ja, wenn das dann durch die einzelnen Sachen besser wird, dann passt das so, ja. " (Unger, WLF Linie 2, 743ff.)

„Ja, freilich. Ich sage mir immer; ja, auf jeden Fall. Man fühlt sich auch/ das Selbstwertgefühl steigt auch, wenn man weiß, man ist ein bisschen eingebunden. Und nicht wie das alte Schema, wie es war vor 20 Jahren: Du, Denken tun wir und du arbeite. Das war ja früher so in den Werken. Darum ist auch nichts besonders hervorgekommen. Dem Arbeiter war es Wurst. Die interessiert das sowieso nicht, was ich da mache. Dann ist halt gearbeitet worden in einem Trott. Aber heute weiß man ja, dass direkt der, der bei der Arbeit ist, oft die besseren Ideen oder auch mit dem/ das wissen/ wenn der Chef da herinnen sitot, der weiß das ja auch nicht, die Probleme, was da draußen anfällt." (Renner, WLF Linie 1, 442ff.)

Indem sie von den anderen betrieblichen Akteuren ernstgenommen werden und unmittelbar in Optimierungsprozesse eingebunden sind, erfuhren die Beschäftigten Anerkennung ihrer Fähigkeiten und eine hohe Selbstwirksamkeit ihres Arbeitshandelns. Die als geringer wahrgenommener Machtdistanzen zu den Führungsebenen und Angestelltenbereichen sowie die Einbindung in betriebliche Entscheidungen und Abläufe führten zu einer Aufwertung ihrer sozialen Stellung im Betrieb - obgleich sie sich zugleich nach wie vor im betrieblichen Unten befanden. Dazu trug auch die auf Entwicklung und Absicherung orientierte Beschäftigungspolitik von WLF bei.

[I: Was ist WLF für ein Betrieb?] „Eher auf sozial, weil ich finde ihn als sebr sozialen Betrieb. Es wird schon auf den Mitarbeiter geachtet. Es ist halt eher eine Geldsache, wo ich sage, ,Nein, den Stapler können wir uns jetzt nicht leisten. Jetə̨t müssen wir sparen. Aber im Großen und Ganzen ist es, ich babe in vielen Betrieben eben gearbeitet vorher, wo es scblimm zugeht, also wo du wirklich eine Nummer bist und/ Ich meine, oben werden sie uns nicht kennen, aber ich sage jetst, es ist halt trotzdem ein anderes Flair, also eher sozial eingestellt. Also man bekommt auch Brillenzuschuss und die Kinder bekommen ein Weihnachtsgeschenk. und so Sachen. "(Valler, ẄLF Linie 4, 46ff.)

„Ja, sicher, man kriegt, wenn man so etwas vorhat, Haus bauen und so, man kriegt jederzeit Urlaub und so, das ist kein Problem. Weil da wird einfach ein anderer eingeset:t oder ein anderer vorgezogen, und du kannst auch Scbicht wechseln, wenn du zum Beispiel, weiß ich nicht, erste Schicht, nein, zweite Schicht, und du mö̈btest lieber erste machen, weil du dann den Nachmittag zu arbeiten hast, so musst Nachmittag in die Arbeit gehen. Du hast schon viele Möglichkeiten, dass du ein wenig variieren kannst. Aber das muss balt abgesprochen werden mit dem Vorarbeiter und mit den einzelnen Arbeitern, weil da muss ja jemand einspringen für dich, muss ja mit dir die Schicht wechseln. [...] Sicher, kann man über alles reden, ja. "(Unger, WLF Linie 2, 1019ff.)

Mit betrieblichen Sozialleistungen, Arrangements bei privaten Problemen (Arbeitszeitregulierungen) und wahrgenommene Arbeitsplatzsichersicherheit trotz fehlender Absicherungsvereinbarungen wie beispielsweise einer Betriebsvereinbarung zu KVP - ging WLF auch auf Bedürfnisse ein, die außerhalb des Betriebs liegen. Zudem stellt der Betrieb seinen Beschäftigten ein weitreichen- 
de Qualifikationsangebot im Rahmen eines internen Colleges zur Verfügung, so dass auch die Produktionsbeschäftigten die Möglichkeiten hatten, sich technisch-fachlich, in Sozial- und Kommunikationskompetenzen oder Sprachen (Englisch) weiterzubilden. Dies war insbesondere für karriereorientierte und mitgestaltungsorientierte Beschäftigte interessant. Insgesamt trugen diese betrieblichen Angebote und Unterstützungsmaßnahmen zu einem Betriebs- und Rationalisierungsverständnis bei, dass dadurch geprägt war, dass der Betrieb sich um seine Mitarbeiter kümmert, ihnen Handlungs- und Gestaltungsmöglichkeiten eröffnet und ihre Interessen beachtete und grundsätzlich nicht zum Nachteil seiner Beschäftigten handelte.

Ungeachtet der wahrgenommenen Vereinbarkeit von Arbeiter- und Betriebsinteressen bei WLF waren die für Industriearbeit typischen Dichotomien zwischen Produktionsbeschäftigten und betrieblichen Vertretern nicht gänzlich verschwunden. Der Schutz ihrer Arbeitskraft vor zu hoher Arbeitsbelastung und psychischer und physischer Verausgabung prägten deutlich das Betriebsund Rationalisierungsverständnis der Beschäftigten. Dass bei vielen WLFBeschäftigten diese Elemente weniger zum Tragen kommen, kann darauf zurückgeführt werden, dass sie einerseits innerhalb ihrer Arbeitsorganisation im Vergleich zu NF deutlich höhere Frei- und Handlungsspielräume hatten und andererseits ihre Situation im Vergleich zu den Arbeits- und Beschäftigungsbedingungen anderer großindustrieller Arbeitgeber in der Region deutlich positiver einschätzten.

[I: Was ist WLF für ein Betrieb?] „Ein weltbekannter Betrieb, würde ich sagen, und in [Standort, B.S.], glaube ich, ein großer, stolzer Betrieb. Es war jahrelang das Thema, dass man bei [WLF, B:S.] anfangen musst, weil du nicht entlassen wirst. Dann ist die Leasingbranche gekommen, da war das auch vorbei. Jet:t hatte man jahrelang einen guten Ruf gehabt, mit meinen Freund zum Beispiel, wie Sie bei [NF] waren drüben. Es war immer so eine Kündigung, ja/nein/ja/nein. Und [bei WLF] bört man nie was." (Oswald, WLF Linie 1, 40ff.)

Die Heterogenität im Betriebs- und Rationalisierungsverständnis zwischen einzelnen Beschäftigten lässt sich in Teilen durch die unterschiedlichen Bereichscharakteristika und Beteiligungsprozesse erklären. Bei einigen Beschäftigten, die vorwiegend in Linie 1 arbeiteten, war ein Rationalisierungsverständnis erkennbar, bei dem der Interessenantagonismus aufgrund des hohen gemeinsamen Nutzens von Optimierungsprozessen und der geringen wahrgenommenen Bedrohung durch den betrieblichen Leistungs- und Rationalisierungsanspruch in den Hintergrund getreten ist, also nur noch latent vorhanden war. Hier verstanden sich die Beschäftigten als Teil einer eher integrierten Betriebs- 
gemeinschaft. Sinngemäß kann dieses Betriebs- und Rationalisierungsverständnis wie folgt auf den Punkt gebracht werden: „Wenn es uns gut geht, geht es dem Betrieb auch gut, und umgekehrt."

Trotz der wahrgenommenen hohen Vereinbarkeit von Arbeiter- und Betriebsinteressen verschwinden auch bei diesen Beschäftigten die Bestimmungsmomente des durch die abhängige Lohnarbeit bedingten strukturellen Interessenantagonismus und der untergeordneten Stellung nicht gänzlich, sondern sind nach wie vor latent vorhanden und können - sollten sich die Bedingungen, insbesondere die subjektiv wahrgenommene Beschäftigungssicherheit und Bedrohung der Arbeitskraft, verschlechtern - wieder reaktiviert werden.

Aufgrund der arbeitspolitischen Gestaltungsbedingungen trat bei einigen Beschäftigten der Linie 1 die Abwehrhaltung des arbeitspolitischen Konservativismus in den Hintergrund. Bei Beschäftigten der Linie 2, die aufgrund von Optimierungsprozessen eine Leistungssteigerung befürchteten, war eine Abwehrhaltung gegenüber betrieblichen Optimierungsprozessen und eine starke Kontrolle hinsichtlich unerwünschter Veränderungen erkennbar. Daher muss das oben formulierte Betriebs- und Rationalisierungsverständnis der Beschäftigten um den Anhang ergänzt werden: „,.. aber wir müssen gemeinsam genau darauf achten, dass daraus kein Nachteil für uns erwächst."

\section{Zusammenfassung}

Im Betriebs- und Rationalisierungsverständnis haben sich die kumulierten Erfahrungen der Beschäftigten mit der Beschäftigungssituation, Rationalisierungen und Auswirkungen betrieblicher Organisation von Arbeit auf die Arbeitskraft sowie Formen sozialer Anerkennung bzw. Missachtung im Betrieb verfestigt. Obgleich sich deutliche Unterschiede im Betriebsverständnis der Beschäftigten von WLF und NF erkennen lassen, prägt die wahrgenommene untergeordnete Position der Produktionsbeschäftigten und die latente bzw. tatsächliche Bedrohung der Arbeitskraft durch den betrieblichen Leistungsund Rationalisierungsanspruch das Bewusstsein der Beschäftigten. Weil sich die strukturellen Bedingungen der Beschäftigten als abhängige Lohnarbeiter nicht - auch nicht durch KVP - verändert haben, bleiben die Interessengegensätze zwischen Betrieb und Arbeiter sowie die Schutzhaltung des arbeitspolitischen Konservativismus weiterhin gültig. 
Der strukturelle Interessengegensatz von Betriebs- und Beschäftigteninteressen kann aber durch eine partizipative Arbeitspolitikgestaltung und beschäftigtengetragene Optimierungsprozesse, wie in Linie 1 bei WLF, und deren hohen wahrgenommenen Nutzen für die Beschäftigten abgeschwächt bzw. in den Hintergrund gedrängt werden. Das hohe $\mathrm{Maß}$ an wahrgenommener Sicherheit, große Handlungs- und Entscheidungsspielräume, prozessnahe Kooperations- und Kommunikationsstrukturen sowie ein weites Verständnis von Optimierungspotenzialen machen es wahrscheinlicher, dass die Beschäftigten betrieblichen Optimierungsprozessen weniger kritisch gegenüberstehen.

Um dies angemessen bewerten zu können, werden im folgenden, letzten empirischen Kapitel die Aneignungsformen der Beschäftigten von KVP unter Bezugnahme der subjektiven Beteiligungsorientierungen, des Betriebs- und Rationalisierungsverständnisses und der strukturellen Bedingungen der KVPPraktiken rekonstruiert. 


\section{Aneignung von KVP}

Das Beteiligungsverhalten der Beschäftigten an KVP lässt sich in der Rekonstruktion - zunächst ungeachtet der unterschiedlichen Gestaltungspraktiken in drei dominante Beteiligungshandlungen kategorisieren: ${ }^{115}$

- aktive Beteiligung

- pragmatisch gesteuerte Beteiligung

- keine Beteiligung.

Unter ,aktiver Beteiligung " an Optimierungsprozessen ist das weitgehend vorbehaltlose Mitwirken an Optimierungen zu verstehen. Dies beinhaltet das Benennen von Optimierungspotenzialen, Initiieren von Veränderungen, die Erarbeitung von Lösungsansätzen und die Umsetzung von Optimierungen, ohne dass die Beschäftigten zwischen Inhalten und Auswirkungen der Optimierungspotenziale differenzieren. Die Einschränkung ,weitgehend vorbehaltlos" ergibt sich daraus - wie sich unten noch ausführlicher zeigen wird -, dass kein Beschäftigter mit vollem Bewusstsein und gezielt seinen Arbeitsplatz oder den eines Kollegen gefährden würde. ${ }^{116}$

115 Selbstoptimierungsleistungen werden in dieser Darstellung nicht berücksichtigt, da sie jenseits von Beteiligungsangeboten des Betriebs vorgenommen werden.

Gleichwohl spielen verdeckte, selbstoptimierende Verbesserungsaktivitäten für die Beschäftigten als Ressource für die Schaffung von individuellen und kollektiven Handlungsspielräumen eine bedeutsame Rolle.

116 Zumindest geht dies nicht aus diesem Sample hervor. 
Die "pragmatisch gesteuerte Beteiligung" ist dadurch gekennzeichnet, dass Beschäftigte sich zwar in Optimierungsprozesse einbringen, dies allerdings bewusst selektiv tun. Die Selektivität bezieht sich auf die Wahl der KVP-Form (Teamarbeit, KVP-Workshop und Ideenmanagement) und auf die Auswahl der Optimierungsinhalte. Beispielsweise lehnen Beschäftigte zwar die Teilnahme an Workshops ab, bringen sich aber in Optimierungen der Arbeitsteams ein; oder sie beteiligen sich an kleineren Verbesserung der Abläufe und Ergonomie, aber nicht an Anlagenoptimierung, die Leistungssteigerungen oder Arbeitsplatzverluste für andere bedeuten könnten.

Auch gibt es Beschäftigte, die sich nicht aktiv in Optimierungsprozesse einbringen, also „keine Beteiligung“zeigen. Dies kann sowohl aus einer eher neutralen Passivhaltung gegenüber Beteiligung als auch aus einer bewussten Ablehnung jeglicher Beteiligung an Optimierungsprozessen rühren. Diesen beiden Ausprägungen ist gemein, dass Beteiligung an Optimierungen nicht als Aufgabe der Beschäftigten verstanden wird.

Das offensichtliche Beteiligungsverhalten der Beschäftigten resultiert aus der Art und Weise, wie sie sich die Anforderungen der KVP-Praktiken und ihre spezifischen strukturellen Gestaltungsbedingungen aneignen. Im abschließenden empirischen Kapitel werden Aneignungsformen von beschäftigtengetragenen Prozessoptimierungen in Anlehnung an die einzelnen Aneignungsebenen - sinnlich-erfahrene Wahrnehmung, subjektive Orientierungen und praktisch-handelnde Aneignung - rekonstruiert. Hierbei wird nachgezeichnet, dass die jeweiligen Beteiligungstypen Stauts-quo-Orientierte, Mitgestaltungsorientierte und Karriereorientierte in bestimmen Phasen des Beteiligungsprozesses unterschiedlich mit den jeweils spezifischen Bedingungen von KVP und Arbeitssituation umgehen.

Aufgrund des Fokus auf Interessen und Bedürfnissen, welcher dem interaktionistischen Aneignungskonzept von Leu zugrunde liegt, ist Aneignung als ein interessenorientierter Abwägungsprozess zu verstehen. Die Beschäftigten setzten sich vor dem Hintergrund ihrer subjektiven Orientierungen und der gegebenen arbeitspolitischen Gestaltungsbedingungen mit der Frage auseinander, inwiefern die Beteiligung an beschäftigtengetragenen Optimierungsprozessen mit Nutzen bzw. Nachteilen für sie verbunden ist. In der folgenden Analyse wer- 
den die subjektiven Umgangs- und Aneignungsformen ${ }^{117}$ nach Arten des Beteiligungshandelns sowie der dahinterliegenden Begründungsmuster differenziert.

Die unterschiedlichen subjektiven Umgangs- und Aneignungsformen sind in Tabelle 7 aufgeführt. Die einzelnen Beteiligungstypen (Status-quoOrientierte, Mitgestaltungsorientierte) und werden nun in ihrem alltagspraktischen Kontext betrachtet. Die Tabelle enthält die Aneignungsmodi sowie deren charakteristische Begründungmuster.

117 Die begriffliche Differenzierung in Umgangs- und Aneignungsformen begründet sich dadurch, dass einige Beschäftigte sich beschäftigtengetragene Optimierungsprozesse aktiv aneignen, diese also in bestimmter Weise instrumentalisieren, während andere hingegen nicht auf das Beteiligungsangebot bzw. die Aufforderung des Betriebs nicht reagieren. 
Tabelle 7: Subjektive Umgangs- und Aneignungsformen von KVP

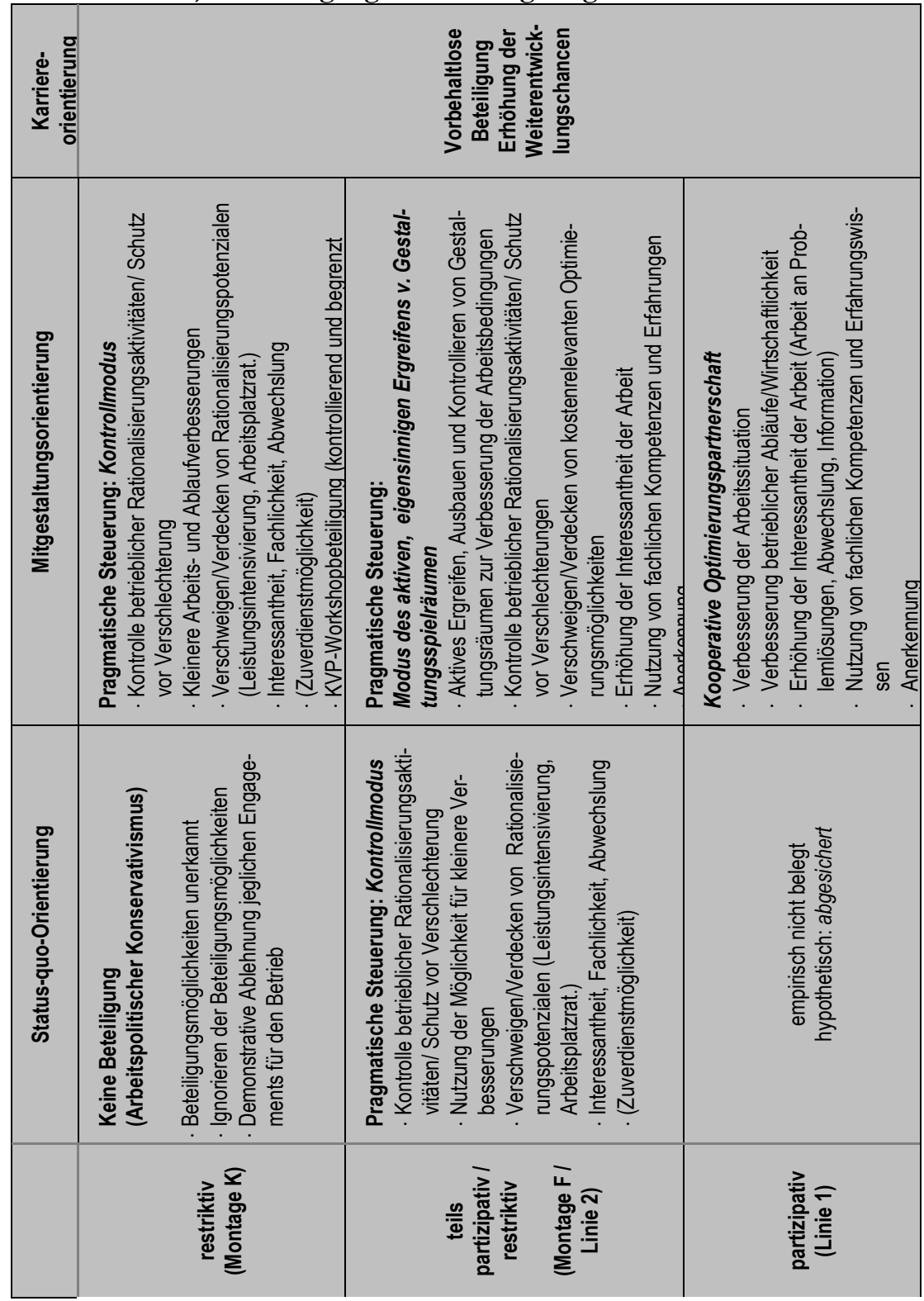




\subsection{Keine Beteiligung}

„Keine Beteiligung“ ist das Beteiligungsverhalten von Status-quo-Orientierten, die vorwiegend unter restriktiven, eher tayloristisch geprägten Gestaltungsbedingungen arbeiten - d.h. unter Bedingungen, die den Beschäftigten aufgrund fehlender betrieblicher Unterstützung und restriktiven Arbeitsbedingungen einerseits wenig Raum lassen, sich an Optimierungen zu beteiligen, und andererseits durch ein Rationalisierungsverständnis geprägt sind, dass Rationalisierung und Wirtschaftlichkeit stets zu Lasten der Beschäftigten errungen werden. Status-quoOrientierte zeigen unter restriktiven Bedingungen einen ausgeprägten Interessenantagonismus hinsichtlich der Vereinbarkeit von betrieblichen und Beschäftigteninteressen. Das Ausbleiben der Beteiligung kann hierbei auf drei Begründungsmuster zurückgeführt werden.

\subsection{1. „Kein Beteiligungsangebot - keine Aneignung“}

Unter den Status-quo-Orientierten gibt es Beschäftigte, welche die Aufforderung und das Angebot des Betriebs, sich an KVP zu beteiligen, nicht bemerkt haben. Obgleich mit der Einführung der Teamarbeitsorganisation intendiert wurde, dass die Teams eigenständig Optimierungen anstoßen und erarbeiten sollen, ist der Betrieb mit diesem Anliegen nicht zu allen Beschäftigten vorgedrungen. Dass die Beteiligungsmöglichkeiten bei restriktiver Gestaltung der beschäftigtengetragenen Optimierungsprozesse - wie bei Montage $\mathrm{K}$ - nicht erkannt wurden, kann darauf zurückzuführen sein, dass sich, über die Zunahme von Selbstorganisation und Rotation durch Teamarbeit hinaus, im Arbeitsalltag keine Veränderungen bemerkbar gemacht haben, die auf die Möglichkeit und die Aufforderung zur Beteiligung an beschäftigtengetragenen Optimierungen schließen lassen. Angesichts ausbleibender Aufforderung zur Optimierungsbeteiligung, unveränderten Kooperationsbeziehungen und Kommunikationsformen zwischen Produktionsbeschäftigten und Führung sowie den unterstützenden Bereichen und expertengetriebener Veränderungsund Rationalisierungsmaßnahmen setzte sich die traditionelle, tayloristische Arbeitsteilung in planende und ausführende Tätigkeiten im Grunde fort. Die Beschäftigten verstanden Teamarbeit hier nicht als Ressource zur Gestaltung der Arbeits- und Produktionsprozesse, sondern lediglich als Instrument für die Urlaubsplanung, das Befüllen von Listen und Arbeitseinsatzabsprachen.

Aufgrund ihrer Nicht-Beteiligung haben Status-quo-Orientierte keine unmittelbaren Erfahrungen mit KVP. Für sie als mittelbar Betroffene der Folgen 
von Optimierungs- und Rationalisierungsprozessen sind die Wirkungen restriktiver Arbeitsgestaltung aber zumeist negativ präsent, wie im Fall NF, wo bei den Beschäftigten die Wahrnehmung der latenten Arbeitsplatzunsicherheit durch expertengetriebene Rationalisierungsaktivitäten, fortschreitende Verschlechterungen der Beschäftigungsbedingungen, Leistungsintensivierung und die damit einhergehenden psychischen Belastungen sehr dominant ist.

„Nein, das ist in puncto Selbstständigkeit, die Leute baben das drinnen - das, was ich vorher grade gesagt habe -, jeder hat seinen Auftrag vom Vorgesetzten gekriegt, wie es frïher war, seine fixe Arbeit, und die hat er gemacht. Und da hat nie irgendwer zusätzliche Tätigkeiten dabeigehabt oder die Anforderung gehabt, dass er selbstständig irgendwas löst oder macht. Und jetzt im Team kommen jetzt Sachen daher, die das Team lösen soll, Verbesserungen oder Umbauten oder Planungen, und das ist noch sehr schwierig, weil die Leute noch immer glauben, dass sie das nichts angebt. Also so: Macht der Teamsprecher, macht der QRK, Danke, Wiederschauen. Und das ist halt das, das noch nicht so funktioniert, das funktioniert aber in keinem Team noch. Denn es ist keinem bewusst, dass jetzt jeder für sich selber, für das Team verantwortlich ist, also sie glauben alle, das läuft von selbst, aber das ist nicht der Fall." (Albinger, NF Montage K T_B, 257ff.)

In einem engen Aufgabenverständnis, demzufolge die Status-quo-Orientierten sich nur für die Erfüllung der ihnen aufgetragenen und festgelegten Tätigkeiten als zuständig betrachten, gelten Optimierungsaufgaben nicht als Teil ihrer Aufgaben und Leistungen, sondern werden in die Zuständigkeitssphäre des Betriebs verwiesen. Dazu zählt auch die Beteiligung am Ideenmanagement.

\subsection{2. „Interessiert mich nicht“}

Diese Zusammenhänge zeigen sich auch, wenn die Status-quo-Orientierten das Beteiligungsangebot zwar zur Kenntnis genommen haben, aber ignorieren. Bei Status-quo-Orientierten, die unter restriktiven Gestaltungsbedingungen arbeiten, dominiert die Ansicht, dass Rationalisierung sich negativ auf die Beschäftigungsund Arbeitssituation auswirkt. Mit diesem Rationalisierungsverständnis ist verbunden, dass Status-quo-Orientierte sich als Opfer von Rationalisierung verstehen und sich vor Verschlechterung der eigenen oder kollektiven Beschäftigungssicherheit sowie der Leistungssituation zu schützen versuchen. Die Ablehnung bezieht sich vor allem auf die Teilnahme an KVP-Workshops, die als betrieblich initiierte Rationalisierung betrachtet werden. Status-quoOrientierte gehen nicht auf das Beteiligungsangebot ein, weil es ihnen für die Wahrung ihrer Interessen nicht zweckdienlich ist, und vermeiden jegliche Beteiligung daran. 
„Da will ich dann nicht mehr dabei sein, weil wenn du dabei ist, sind die Leute interessiert, dass du irgendwas bringst auch. Und ich sehe das, dass es da nur gegen den Arbeiter geht. So sehe ich das. Und da ist es mir lieber, ich bin nicht dabei, weil ich mir selber nicht weh tun möchte und den anderen Kollegen auch nicht, so muss ich es ehrlich sagen. Und das ist nichts anderes nicht. "(Czerni, NF Montage F_B, 1249ff.)

Die Möglichkeit, durch die eigene Beteiligung am Workshop die eigene Arbeitssituation verbessern oder Verschlechterungen verhindern zu können, wird erstens in der Erwartung bzw. unter dem Verdacht abgelehnt, dass Veränderungen, die durch betriebsöffentliche Optimierungsprozesse bewirkt werden, letztlich immer zu Ungunsten der Arbeiter gehen (arbeitspolitischer Konservativismus). Optimierungspotenziale werden bewusst im Verborgenen gehalten, um eigene Freiräume zu schützen und diese nicht der Rationalisierung zum Opfer fallen zu lassen. Im Begründungszusammenhang dieses Verhaltens spielen kollegiale Solidaritätsnormen, der Schutz der Arbeitskraft der Arbeiterschaft insgesamt, eine bedeutende Rolle. So werden vermeidlich unbedachte Verbesserungsideen von Kollegen und die Beteiligung einzelner Kollegen an KVPWorkshops stark kritisiert. Dem arbeitspolitischen Konservativismus entsprechend versuchen Status-quo-Orientierte im Rahmen von verdeckten Selbstoptimierungen, ihre Arbeitsbedingungen für sich selbst so günstig wie möglich zu gestalten.

Zweitens wird die Möglichkeit, als Arbeiter eigene Verbesserungen realisieren zu können, durch die restriktive Gestaltung der beschäftigtengetragenen Optimierungsprozesse ohnehin als gering eingeschätzt, so dass die Beschäftigten aufgrund der Erwartung mangelnder Wirksamkeit und Anerkennung ihres Engagements eine Beteiligung an Optimierungsprozessen nicht in Betracht ziehen.

8.1.3. „Ich will nichts mit dem Betrieb zu tun haben.“ - Demonstrative Ablehnung

Die demonstrative Ablehnung jeglichen Engagements für den Betrieb lässt sich am Beispiel des Montagebeschäftigten Dauner (NF Montage K) verdeutlichen: 
Dauner ist Mitte 30 und wurde bei NF zum Spengler ${ }^{118}$ ausgebildet. Er arbeitete anschließend 14 Jahre im Rohbau von NF. Diesen Bereich musste er infolge von Rationalisierungsmaßnahmen verlassen und wurde seitdem für kurze Zeiten wechselnd in unterschiedlichen Montagebereichen von NF eingesetzt. Die Versetzung vom Rohbau in reine Montagebereiche war für inn mit erheblichen Lohneinbußen verbunden, da Nachtschichtzulagen weggefallen sind. Darüber hinaus hatte er mit Blick auf den engen Tätigkeitszuschnitt der Montagearbeiten, eingeschränktere Freiräume sowie kürzere Taktzyklen erhebliche Anpassungsprobleme in Montage $K$. Durch diese einschneidenden persönlichen Abwertungserlebnisse fühlte sich Dauner vom Betrieb und auch vom Betriebsrat im Stich gelassen und missachtet.

„Ich muss gan₹ ebrlich sagen, ich will da herinnen, also in diesem Werk eigentlich überhaupt nichts mit Verantwortung oder irgendwas zu tun baben. Ich will meine Arbeit machen und wieder nach Hause gehen. Das ist wirklich so. [...] [Hier, BS] rennen so viele wirre Sachen. Wie gesagt, ich bin seit fast 19 Jabren in dem Werk, und ich glaube schon, mich ziemlich gut auszukennen überall, aber es ist halt einfach nicht meine Berufung, dass ich da irgendwie was zu sagen habe. Nicht einmal Teamsprecher [möchte ich werden, BS], wirklich." (Dauner, NF Montage K_B, 268ff.)

„Ich wïrde [die Arbeit bei NF, BS] nicht weiterempfeblen und ich sage es ganz ehrlich, früher war ich mal stoly, dass ich gesagt habe, ja, ich arbeite bei $\left[N F, B S 7^{\circ}\right.$ und so. Aber jetzt ist das schon so. Ja, vielleicht hat das mit dem zu tun, dass ich balt einfach/ Ich komme mir so abgeschoben vor, weißst. "(Dauner, NFZ Montage K T1B, 1020ff.)

Dauner ist nicht mehr bereit, sich für den Betrieb einzubringen, und lehnt zusätzliches Engagement, das über die reine Leistungserbringung als Grundverpflichtung seines Arbeitsverhältnisses hinausgeht, ab. Dennoch zeigt Dauner, dass er sich innerhalb seines Arbeitsbereichs durchaus Gedanken darübermacht, wie man die Arbeit besser gestalten könnte; er probierte sogar gemeinsam mit Kollegen Ideen zur Ablaufverbesserung aus. Die Ablehnung des Engagements für den Betrieb ist unmittelbar mit persönlichen negativen Erfahrungen von Ohnmacht gegenüber betrieblichen Interessen und des Verlusts von Entgelt, von Kollegen im alten Bereich und von Fachlichkeit in der Arbeit verbunden. Kollegiale Solidaritätsnormen haben für Dauner zwar eine hohe Bedeutung, die persönlichen Verlust- und Missachtungserfahrungen führten bei ihm jedoch zu einer „inneren Kündigung“. Aufgrund der schlechten Arbeitsmarktsituation und privater Probleme kann Dauner den

\footnotetext{
118 Süddeutsche und österreichische Bezeichnung für Klempner, Facharbeiter für Metallumformung.
} 
Betrieb nicht verlassen. Ihm bleibt als Reaktion, jegliches Engagement für den Betrieb zu unterlassen. Die demonstrative Ablehnung speist sich vor allem aus dem extrem negativen Erleben seiner Situation, das ein reines, eher neutrales Ignorieren unmöglich macht.

Diese ablehnende und schon fast widerständige Haltung resultiert aus einem massiven Abwertungs- und Missachtungserlebnis, welches über das bloße Ausbleiben von Anerkennung hinausgeht. Dieses einschneidende und wiederholte negative Erfahrung verstärkt und bestätigt die den Status-quoorientierten Beschäftigten auszeichnende Arbeitsorientierung sowie sein Betriebs- und Rationalisierungsverständnis. Man fühlt sich als Beschäftigter herabgewürdigt und als Spielball betrieblichen Wirtschaftlichkeitsstreben, bei dem persönliche Schicksale keine Rolle spielen. Daher bleibt, sofern keine berufliche Alternative in Aussicht ist, sich auf die Minimalanforderungen des Arbeitsvertrags zurückzuziehen.

Die Ablehnung, sich an beschäftigtengetragenen Optimierungen zu beteiligen, schließt Selbstoptimierung an Arbeitshandlungen und -abläufen nicht aus, da sie den Beschäftigten wichtige Freiräume verschaffen. In begrenztem Maße beteiligen sich die Status-quo-Orientierten vereinzelt und versuchsweise am Ideenmanagement. Bei negativer Erfahrung (,ich habe nie wieder was davon gehört") werden keine weiteren Ideen eingereicht. Positive Erfahrungen, wie die Umsetzung der Idee oder hohe Belohnungen, können sich positiv auf die Beteiligungsbereitschaft am Ideenmanagement auswirken.

Insgesamt lassen sich dieser Umgangsform nur wenige Beschäftigte des Samples zuweisen. Mit Blick auf das überwiegend als sehr angespannt wahrgenommene Betriebsklima, die teils schlechten Umgangsformen zwischen den einzelnen betrieblichen Gruppen und der hohen Unsicherheit, die durch die hohe Reorganisationsdynamik bei NF entstanden ist, lässt sich allerdings vermuten, dass diese Aneignungsform nicht nur bei einem Einzelfall anzutreffen ist. Angesichts der freiwillige Teilnahme haben sich mit hoher Wahrscheinlichkeit jedoch eher interessierte und engagierte Beschäftigte zu Interviews bereit erklärt. Beschäftigte, die dem Betrieb und Optimierungsaktivitäten sehr skeptisch gegenüberstehen, haben die Gespräche hingegen häufig vermieden. Während der Arbeitsplatzbeobachtungen haben diese Beschäftigten entweder starke Kritik gegenüber betrieblichen Entscheidungen und ihrer Arbeitssituation geäußert, woraus aber keine Interviewzusage resultierte - nicht zuletzt in der Annahme, dass man als Forscher auf der Seite des Betriebs stehe, wird ver- 
sucht, dem Kontakt eher auszuweichen. Es ist sehr wahrscheinlich, dass unter diesen Beschäftigten, welche die Beteiligungsaufforderung ignorieren oder demonstrativ ablehnen, vor allem Status-quo-Orientierte zu finden sind. ${ }^{119}$

Die Umgangsformen der Nicht-Beteiligung bzw. des mit anderen Worten, „Diensts nach Vorschrift", werden von vielen Beschäftigten als Referenzfolie herangezogen, um ihr eigenes Verhalten positiv abzugrenzen. Vielen Kollegen wird vorgeworfen, dass sie nur ihre Arbeit verrichten, sich keine Gedanken machen und sich nicht einbringen.

"Ja, es ist schwierig, weil vielen ist es komplett egal, was ich ausmache mit den anderen, die nehmen das in Kauf und sagen, ,Ja, ich bin herinnen, damit ich meine Arbeit mache, und alles andere ist mir scheißegal', und ein paar machen sich balt Gedanken darüber und die sagen es mir auch, wenn es ibnen nicht passt, aber das sind halt leider nur drei Leute oder was, die sich was mitreden trauen." (Albinger, NF Montage K T1B, 601ff.)

Ob es diesen Typus von Arbeiter, dem „,alles scheißegal ist", in der Realität wirklich in Reinform gibt, muss an dieser Stelle aufgrund der Verzerrung des Samples offen bleiben.

\subsection{Pragmatisch gesteuerte Beteiligung}

Als pragmatisch gesteuerte Beteiligung werden Aneignungsformen beschrieben, bei denen die Beschäftigten sich aktiv mit den Beteiligungsmöglichkeiten an beschäftigtengetragenen Optimierungsprozessen auseinandersetzen. Sie steuern die Art und Intensität der eigenen Beteiligung bewusst in Abhängigkeit der vorherrschenden Gestaltungsbedingungen und Gelegenheitsstrukturen sowie des erwarteten Nutzens. Die Aneignungsform der pragmatisch gesteuerten Beteiligung lässt sich in zwei Modi differenzen: erstens den Modus der abgesicher-

119 Dieser Hinweis kann durch zahlreiche Beobachtungen während der Untersuchungsphase des SOFI-Projekts untermauert werden. Die Beschäftigten zeigten sich im Vorfeld unserer Erhebungen sehr skeptisch. Sie verweigerten nicht nur die Teilnahme an Interviews, sondern im Vergleich zu empirischen Untersuchungen in anderen Unternehmen in überdurchschnittlich hoher Anzahl auch das Ausfüllen des Fragebogens. Häufig verwiesen die Beschäftigten darauf, dass sich ihr Verhalten nicht gegen uns richte. In der Ablehnung der Teilnahme sehen die Beschäftigten vermutlich vielmehr eine der wenigen Möglichkeiten, ihren Protest gegen den Betrieb zum Ausdruck zu bringen. 
ten, kontrollierenden Beteiligung (Kontrollmodus), zweitens den Modus des aktiven Ergreifens von Handlungsspielräumen (Durchsetzung von Handlungsspielräumen).

\subsection{1. „Nur wenn es gut für den Arbeiter ist“ - Kontrollmodus bzw.} abgesicherte, kontrollierende Beteiligung

Bei der Aneignungsform Kontrollmodus praktizieren die Beschäftigten eine abgesicherte, kontrollierende Beteiligung, deren Hauptintention die Kontrolle betrieblicher Rationalisierungsaktivitäten ist. Die Beschäftigten bringen zwar eigene Ideen ein, versuchen aber jegliche Veränderungen, die eine Verschlechterung der Arbeits- und Leistungssituation bedeuten könnten, zu vermeiden. Diese Aneignungsform lässt sich zum einen bei Status-quo-Orientierten in moderat partiripativen Beteiligungskonstellationen (Beispiel Strobl), zum anderen bei Mitgestaltungsorientierten in restriktiven Beteiligungskonstellationen (Beispiel Eder, Auer) finden.

Strobl (Mitte 40) ist gelernter Spengler und Lackierer. Im Laufe seines Erwerbslebens hat er sowohl die Erfahrung längerer Arbeitslosigkeit als auch wechselnder, kurzfristiger Beschäftigungsverhältnisse in Industriebetrieben der Region gemacht. Vor sechs Jahren ist Strobl als Leiharbeiter zu WLF gekommen und seit zwei Jahren fest bei WLF angestellt. Er arbeitet in Linie 2 (WLF) seit sechs Jahren stets an der gleichen Maschine. Die persönlichen Erfahrungen von Arbeitslosigkeit, Kündigung und unsicherer Beschäftigung prägen die Status-quo-Orientierung sowie das Betriebs- und Rationalisierungsverständnis von Strobl, der sich als abhängig Beschäftigter eher als machtloses Opfer profitorientierter Wirtschaftsunternehmen sieht. Trotz positiver Erfahrungen von Beschäftigungssicherheit und Mitarbeiterorientierung bei WLF überdauern die verfestigten Orientierungen. Das drückt sich darin aus, dass er sich in seinem Handeln in hohem Maße auf den Schutz vor Beschäftigungsverlust und Leistungssteigerung bezieht. Die Orientierung an guter Leistungserfüllung, Interessantheit der Arbeit sowie Mitgestaltung seines Arbeitsplatzes haben für inn Relevanz, werden aber von dem wahrgenommenen Interessenantagonismus überlagert. In den PE-Gesprächen bringt er sich nur sehr begrenzt ein, weil er dies für ein betriebliches Instrument zur Unterwanderung der kollektiven, solidarischen Schutznormen hält. Die Orientierung und das Vertrauen in den kollegialen Schutz sind bei Strobl sehr hoch. 
„Für mich ist das eigentlich nur, dass irgendwelche Verbesserungen wollen in unserem Team. Stehen die Leute dazu, wird etwas verbessert, stehen die Leute nicht dazu, können sie nichts machen. "(Strobl, WLF Linie 2_1, 522ff.)

„Das wäre schon eine Einsparung, so gesehen. Aber das wird nie wer sagen. Da wird nie irgendwer sagen, ja passt, machen wir. Das kann ein Meister sagen, ja. Der Vorarbeiter, wird schon Nein sagen. Und die Arbeiter selber sagen sowieso Nein. (Strobl, WLF Linie 2_1, 852ff.)

Darüber hinaus sind nach Strobls Ansicht die PE-Gespräche im Hinblick auf Optimierungsmöglichkeiten sehr begrenzt, da die von den Beschäftigten benannten Probleme in seinen Augen nicht ernst genommen werden und zu keinen direkten Problemlösungen führen. Expertengetriebene Optimierungen an seiner Anlage versucht er möglichst abzuwehren.

„Ja. Wenn es Sachen sind, die was bringen. Aber ist stelle mich quer bei Sachen, die was mir nichts bringen. "(Strobl, WLF Linie 2/Schicht 1, 767f.)

Strobl beteiligt sich aber an Optimierungen seines Arbeitsplatzes im Rahmen des dezentralen Ideenmanagements von WLF, indem er gemeinsam mit dem integrierten Instandhalter kleine Optimierungen an seiner Anlage und der Organisation der Abläufe unternommen hat. Strobl ist sehr darauf bedacht, dass mögliche Veränderungen für ihn und seine Kollegen einen Vorteil bringen. Aufgrund relativ hoher Handlungs- und Entscheidungsspielräume an seinem Arbeitsplatz sowie mittels Ideenmanagement entscheidet er bewusst, inwiefern er Veränderungen herbeiführt. Materielle Zuverdienstmöglichkeiten sind für Strobl kein direkter Anreiz zur Beteiligung, er bewertet die symbolische Anerkennung aber positiv.

Die Haltung Status-quo-Orientierter in moderat partizipativen Gestaltungsbedingungen ist durch einen ausgeprägten Skeptizismus gegenüber betrieblich organisierten Prozessoptimierungen geprägt. In ihrer Wahrnehmung dominieren negative Aspekte, wie Streit zwischen Kollegen, mangelnde Wirksamkeit des eigenen Engagements aufgrund fehlender Unterstützung durch indirekte Bereichen und die langwierige Umsetzung von Optimierungsideen. Gleichsam einer sich selbst erfüllenden Prophezeiung werden negative Aspekte überbetont. Wahrgenommene Verzögerungen von Problemlösungen oder die Missachtung der artikulierten Probleme suggerieren den Status-quo-Orientierten, dass ihre Beteiligung von den indirekten Bereiche im Grunde wenig beachtenswert ist - „Es wird eh nicht ernst genommen.“ Sogar Berichte anderer Beschäftigter des Bereichs über positive Erfahrungen mit KVP werden von 
den negativen Eindrücken der allgemeinen Arbeits- und Betriebssituation überschattet.

Diese Wahrnehmungen sind gefärbt durch einen starken betrieblichen Interessenantagonismus. Status-quo-Orientierte differenzieren stark zwischen Interessen der Beschäftigten und des Betriebs. Die vom Betrieb initiierten KVP stehen generell unter Verdacht, vom Betrieb einseitig zur Rationalisierung und letztlich zu Lasten der Beschäftigten genutzt zu werden. Daher, so die Ansicht der Beschäftigten, müsse man die Aktivitäten des Betriebs kontrollieren und sich gegebenenfalls gegen potenziale Rationalisierungsmaßnahmen stellen. Hier wird PE nicht als ein geeignetes Instrument betrachtet, um Verbesserungen für die Beschäftigten zu erzielen, sondern als Instrument zur Schwächung der kollegialen Solidarität und Indoktrinierung der Beschäftigten. Der Betrieb ziehe so einzelne Beschäftigte auf seine Seite ziehen und untergrabe die etablierten Schutzmechanismen.

Stattdessen nutzen Status-quo-Orientierte Gelegenheitsstrukturen, die sich unmittelbar am Arbeitsplatz ergeben und die sie direkt beeinflussen und kontrollieren können, zur Verbesserung der Arbeitssituation0 und der Abläufe.

Abgesicherte, kontrollierende Beteiligung an beschäftigtengetragenen Optimierungsprozessen lässt sich zudem bei Mitgestaltungsorientierten in restriktiven Beteiligungskonstellationen erkennen. Mitgestaltungsorientierte wollen sich mit ihren Fachkenntnissen und Erfahrungen in die Arbeit einbringen und durch Problemlösungen und Verbesserung den eigenen Arbeitsbereich aktiv mitgestalten. In restriktiven Beteiligungskonstellationen sind die Möglichkeiten aufgrund mangelnder Unterstützung vom Meister und den indirekten Bereichen, der Dominanz expertengetriebener Veränderungsprozesse und Standardisierungskonzepte sowie der unzureichende Förderung von nicht-wirtschaftlichen Verbesserungsideen begrenzt. Dennoch versuchen Mitgestaltungsorientierte, sich in die Gestaltung ihres Bereichs einzubringen. Zugleich führt die wahrgenommene Bedrohungssituation der Arbeitskraft dazu, dass auch die mitgestaltungsorientierten Beschäftigten darauf bedacht sind, Verschlechterungen der Arbeitsund Beschäftigungssituation für sich und ihre Kollegen aktiv abzuwehren. Im Gegensatz zu Status-quo-Orientierten, die unter vergleichbaren Bedingungen arbeiten und primär darauf fokussiert sind, Veränderungen zu vermeiden, versuchen die Mitgestaltungsorientierten jedoch, durch ihre Beteiligung an beschäftigtengetragenen Optimierungsprozessen ihren Arbeitsbereich mitzugestalten, um Arbeitsverbesserungen zu erzielen. 
Mitgestaltungsorientierte versuchen, im Rahmen von Teamarbeit und im Einvernehmen mit ihren Teamkollegen Probleme zu benennen und diese im Rahmen der begrenzten Ressourcen zu lösen oder im Team Verbesserungen zu erzielen. Ein gutes Einvernehmen zwischen den Kollegen ist Mitgestaltungsorientierten wichtig, da sie die solidarischen Schutznormen in hohem Maße mittragen, nicht gegen sie verstoßen und insgesamt für das ganze Team Verbesserungen der Arbeit erzielen wollen. Aufgrund mangelnder Unterstützung bei Problemlösungen nehmen Mitgestaltungsorientierte nur eine geringe Wirksamkeit ihres Handelns wahr, versuchen aber dennoch immer wieder, sich gestalterisch in die Arbeit einzubringen.

Mit der Teilnahme an KVP-Workshops verbinden Mitgestaltungsorientierte die Möglichkeit, mit Vertretern indirekter Bereiche direkt in Kontakt zu treten, mehr Hintergrundinformationen über übergreifende Fertigungsprozesse, betriebliche Abläufe und Entscheidungen zu erhalten und durch gezielte Mitarbeit an Problemlösungen, bei der sie ihr Wissen und ihre Erfahrungen einbringen können, letztlich Verbesserungen in ihrem Arbeitsbereich zu bewirken. Durch den Zugang zu Hintergrundinformationen können sie Zusammenhänge besser verstehen und nachvollziehen, was für die Mitgestaltungsorientierten einerseits eine Zunahme an Interessantheit der eigenen, sonst eher monotonen Arbeit bedeutet und andererseits ihre Beteiligungschancen an betrieblichen Prozessen erhöht. Zum Zwecke des Schutzes der eigenen Arbeitskraft vor Leistungsintensivierung, der Abwehr von Beschäftigungsgefährdungen sowie aufgrund des hohen Stellenwerts kollegialer Solidaritätsnormen sind Mitgestaltungsorientierte darauf bedacht, bei Optimierungen in Teamarbeit und Workshops dem Betrieb keine Rationalisierungspotenziale zu verraten. In Workshops nehmen sie die Rolle eines Kontrolleurs betrieblicher Optimierungs- und Veränderungsaktivitäten ein und versuchen, negative Auswirkungen zu verhindern.

Diese Aneignungsform lässt sich am Beispiel Eder verdeutlichen:

Eder (Ende 40) ist gelernter Schlosser, hatte vor seiner Beschäftigung bei NF in unterschiedlichen handwerklichen Montagebetrieben gearbeitet und selbst einen kleinen Familienbetrieb in der Dienstleistungsbranche als Meister geleitet. Aus Gründen der finanziellen Absicherung der Familie und geregelter Arbeitszeiten hat er wieder die Arbeit als abhängig Beschäftigter im Industriebetrieb aufgenommen. Eder arbeitete bei NF freiwillig in unterschiedlichen Bereichen der Komponentenmontage. Er beherrscht im Produktionsabschnitt alle Tätigkeiten, so dass er oft in 
andere Teams ausgeliehen wird. Eder sucht sich aktiv neue und interessante Aufgaben, wie beispielsweise die Tätigkeit als QRK oder das Anlernen neuer Kollegen, und versucht, seine Erfahrungen in den Arbeitsprozess einzubringen.

[I: Gibt es auch Aufgaben oder Tätigkeiten, die Spaß machen?] „Ja, auf jeden Fall [...]. Das ist, zum Beispiel, wenn man QRK ist. Das ist das eine interessante Aufgabe, oder man lernt jemanden an. Das ist auch interessant, ja auf jeden Fall. Oder es kommt was Neues und man schaut, dass man mitmachen kann, es gibt immer wieder interessante Geschichten. "(Eder, NF Montage K_B, 117ff.)

Im Rahmen der KVP-MTM-Workshopreihe hat sich Eder bei Meister freiwillig um die Teilnahme bemüht. Die Teilnahme am Workshop ist für ihn eine sehr willkommene und interessante Abwechslung von der monotonen Montagearbeit. Zugleich ist er sich aber auch der Verantwortung bewusst, durch sein Handeln im Workshop keine Verschlechterungen zu begünstigen.

\footnotetext{
„Ich mache ja viele Workshops momentan, ich versuche immer, natürlich babe ich im Hinterkopf, ich werde mich nicht selber einsparen. Ich kann jederzeit einen Kollegen einsparen, ja, klar kann ich auch was verdienen dabei und kann sagen, gut, habe ich was eingespart, dann kriege ich 10.000 Euro: Ja, aber ob das mit dem Gewissen zu vereinbaren ist, ist, erstens mal muss ich dann vielleicht selber [woanders, BS] arbeiten. Und zweitens, ja, wo fängt es denn an und wo hört es auf, wir sind doch alle nur Menschen letatendlich. Ich versuche immer, also wenn ich jetzt im Workshop rede, also wir machen KVPs. Also dass wir Potentiale erkennen, dann versuche ich das so immer hinzusteuern, dass ich sogar mit einem Kollegen aus der anderen Schicht rede, ,he, sei mal vorsichtig, dass wir uns da nicht selber einen Strick draus drehen: Also erst mal muss ich das ja der Mannschaft gegenüber vertreten und sagen, wir machen das, der sagt, he, geht eb: Man muss es mit dem eigenen Gewissen und dem der Kollegen auch noch vertreten, und das geht. Gott sei Dank, bis jetzt haben alle mitgespielt, dass man sagt, ,komm her, ein bisschen runter vom $G$ as, wir verbessern zwar was, dass wir das sehen, dass was verbessert ist", also gewisse Sachen, die wir selber wollen, dass was verbessert ist. " II: Ist da bei Euch noch Potenzial drin?] „Potential ist immer drinnen, ja, aber man muss da wirklich vorsichtig sein. Ich versuche das mit Vorsicht zu genießen, weil ich kann sagen, ich rufe es dir einfach zu, ich habe die Meter verküržt, natürlich kriegt er die Zeit weg." (Eder, NF Montage K, T2B, $779 f f$.
}

Durch die fortwährend präsente Rationalisierungsgefahr achtet Eder genau darauf, welche Folgen sein Handeln im Workshop hat. Als Vertreter seines Teams muss er vor seinen Kollegen, mit denen er nach dem Workshop wieder täglich zusammenarbeiten muss, Rechenschaft über die Workshopergebnisse ablegen. Er versucht pragmatisch und interessenbewusst gesteuert, zum einen die Möglichkeiten der Beteiligung zur Verbesserungen der Arbeitssituation in seinem Bereich sowie zur Steigerung der Interessantheit der eigenen Arbeit zu nutzen. Zum anderen ist er bestrebt, durch die aktive Verschleierung von Rationalisierungspotenzialen und über Einflussnahme im Workshopteam Verschlechterungen für die Beschäftigten zu 
vermeiden bzw. abzuwehren. Eder hat bereits an vielen KVP- und ProblemlöseWorkshops teilgenommen, die vorwiegend vom Betrieb initiiert worden waren. Dabei hat er sehr häufig die Erfahrung gemacht, dass Workshops bei NF sehr ineffektiv sind, da erarbeitete Workshopergebnisse oftmals nicht umgesetzt wurden.

\begin{abstract}
Ja, in den Workshops selber sind sehr engagierte Kollegen, teilweise, und das Problem ist, du scheiterst einfach an dem ganzen System und das frustriert (.) und bremst, das bremst das Ganze ab, warum soll ich mir das antun? Es passiert nichts. Es ist nur ewig, es ist niemand zuständig und ,ja, ja, aber und bla bla; das will ja keiner, es passiert nichts. Wir hätten eine Idee, machen wir mal was, die was engagiert sind. Es ist, ja, aber es ist bei einem großen Betrieb, wo vieles bei Eitelkeiten von gewissen Kollegen oder Machthabern, wie auch immer, dass es daran scheitert. mmb (.) und das ist etwas, was sehr bremst. "(Eder, NF Montage K T2B, 947ff.)
\end{abstract}

Dass Eder trotz dieser Erfahrung nicht resigniert, sondern wiederholt an Workshops teilnimmt, unterstreicht seine hohe Orientierung, die Interessantheit der eigenen Arbeit zu steigern und durch die Beteiligung am Workshop kurzweilig der monotonen Industriearbeit am Band zu entfliehen. Obwohl Eder grundsätzlich an Veränderung und Weiterentwicklung interessiert ist, ist seine Beteiligung nicht unmittelbar mit Karriereorientierung verbunden, da er seine Chancen beruflicher Weiterentwicklung aufgrund fehlender Unterstützung vom Vorgesetzten als schlecht einschätzt. Am Ideenmanagement beteiligt sich Eder nicht mehr, da er in der Vergangenheit negative Erfahrungen gemacht hat. So hat er oftmals keine Rückmeldung zu eingereichten Ideen oder die damit verbundene Gratifikation nicht erhalten.

Der Anspruch der Mitgestaltungsorientierten, die eigene Arbeit und Abläufe ihres Bereichs durch Problemlöseprozesse, in die sie ihr Wissen und ihre Erfahrungen einbringen können, interessanter und sinnvoll zu gestalten, erfüllt sich unter restriktiven Gestaltungsbedingungen nur begrenzt. Angestrebte Verbesserungen der Arbeitssituation scheitern häufig an fehlender Unterstützung durch den Betrieb. Mitgestaltungsorientierte suchen oftmals aktive Möglichkeiten, sich in die Arbeit und für die Beschäftigten einzubringen, sind durch die hohe Rationalisierungsbedrohung in restriktiven Gestaltungspraktiken aber sehr darauf bedacht, Verschlechterungen zu vermeiden. Sich in diesem widersprüchlichen Feld voller Interessenkonflikte zu bewegen, erfordert viel Fingerspitzengefühl. Mitgestaltungsorientierte müssen permanent zwischen ihren Interessen an aktiver Beteiligung, dem Interesse, dem alläglichen Trott monotoner Produktionsarbeit zu entfliehen, den eigenen und den Schutzinteressen der Kollegen sowie den Anforderungen des Betriebs vermitteln. Unter diesen Bedingungen eignen sie sich beschäftigtengetragene Opti- 
mierungsprozesse in einem Modus abgesicherter und kontrollierender Beteiligung an.

\subsection{2. „Wir fordern unser Recht ein“ - Durchsetzung des Beteiligungsanspruchs:}

Der Modus Durchsetzung des Beteiligungsanspruchs unterscheidet sich von der abgesicherten, kontrollierenden Beteiligung darin, dass Beschäftigte die Instrumente der beschäftigtengetragenen Optimierungsprozesse aktiv nutzen, um eigene, relevante Probleme zu artikulieren und nachhaltige Problemlösungen herbeizuführen. Die Beschäftigten nutzen hierbei nicht nur die vorhandenen Gelegenheitsstrukturen der beschäftigtengetragenen Optimierungsprozesse, sondern versuchen auch, sie zu aktiv erweitern. Obgleich die Kontrolle und Vermeidung von Rationalisierung allein zu Gunsten des Betriebs wichtige Handlungsorientierungen sind, ist das aktive Ergreifen von Gestaltungsräumen im Wesentlichen dadurch motiviert, Veränderungen voranzutreiben und gezielte Verbesserungen zu erlangen - kurz: etwas Sinnvolles zu tun. Dieser Gestaltungsmodus lässt sich vor allem bei Mitgestaltungorientierten in (eher) partizipativen Beteiligungskonstellationen finden, deren Betriebs- und Rationalisierungsverständnis durch einen klar erkennbaren Interessenantagonismus geprägt ist (Haller, Immler, Fischer, König). Diese Aneignungsform wird am Beispiel Fischer deutlich.

Fischer (Mitte 50, stellvertretender Teamsprecher) ist gelernter Landmaschinenmechaniker und arbeitet seit 29 Jahren bei NF. 22 Jahre lang war er in der mechanischen Fertigung tätig und wurde infolge betrieblicher Neuausrichtungen vor sieben Jahren in die Fahrzeugmontage versetzt. Seine Arbeit in der mechanischen Fertigung war mit deutlich höheren fachlichen Anforderungen und größerer Verantwortung verbunden als seine jetzige Arbeit. Die Versetzung bedeutete in fachlicher Hinsicht eine deutliche Abwertung der Arbeit; finanzielle Einbußen konnte er durch starke Verhandlungen und betriebsrätliche Unterstützung vermeiden. Positiv bewertet Fischer an seiner jetzigen Arbeit vor allem den sozialen Arbeitszusammenhang und das gute Verhältnis zu seinen Kollegen. Die Arbeitsorientierung von Fischer ist durch eine fachliche, problemorientierte Auseinandersetzung mit den Gestaltungsbedingungen der Arbeit sowie durch ein Leistungsbewusstsein geprägt, gute Arbeit zu leisten und einen Teil zum Erfolg des Betriebs beizutragen. Vor dem Hintergrund wahrgenommener fortschreitender Rationalisierung, Leistungssteigerungen und 
Arbeitsplatzunsicherheit sind für Fischer der Schutz von Freiräumen und die Vermeidung von Leistungssteigerungen von großer Bedeutung, da insbesondere für inn als älteren Arbeitnehmer wichtig ist, seine Arbeit unter den gegebenen Bedingungen bis zu seiner Rente ausführen zu können.

Durch seine frühere Arbeit in der mechanischen Fertigung ist Fischer es gewohnt, mit seinen Fachkenntnissen und Erfahrungen Gehör zu finden und sich gestalterisch in die Arbeit einbringen zu können. Durch die durchregulierte Montagearbeit und die geringen Handlungs- und Entscheidungsspielräume hat er die Erfahrung gemacht, dass sein Engagement für den Betrieb nur noch wenig Wirkung zeigt und nicht anerkannt wird.

Ich habe zuerst geglaubt, wie ich herübergekommen bin, du kannst viel ändern. Weil drüben habe ich schon ein wenig ein Wort gehabt und da haben mich auch gekannt alle. Mich kennen, glaube ich, hier herüben auch schon alle, aber da rennst du gegen Wände. Und dann sagt man irgendwann mal: ,W arum soll ich mich in irgendwas hineinsteigern und aufregen." Ich habe schon Magengeschwür gekriegt und alles Mögliche. Dann sage ich Nein, aus, das steht nicht dafür. Da ist meine Gesundheit schon wichtiger. [...] Genau, du erreichst eh nichts an. Wenn ich sehe, es schaut was raus, dann bin ich jederzeit bereit, das ich mich für etwas aufopfere, aber nicht mehr für irgendwas, wo ich sage: ,Ok, das wird nicht anders werden. '(Fischer, NF Montage F_A 827ff.)

Fischer tendierte dazu, wie hier angedeutet, seine mitgestaltungsorientierten Ansprüche an Arbeit den gegebenen, restriktiven Bedingungen vor der Einführung von Teamarbeit anzupassen und - ähnlich wie Status-quo-Orientierte - seine Arbeit nach Vorschrift zu erledigen. Fischer nimmt - gerade durch die Versetzung in den Montagebereich und den persönlichen Verlust von Einfluss - sehr deutlich die Unterordnung und Machtlosigkeit als Montagearbeiter im betrieblichen Sozialgefüge von NF wahr.

Mit der Einführung von Teamarbeit versuchen Fischer und seine Teamkollegen, die im Teamarbeitskonzept bewilligten Aufgabenbeschreibungen und Handlungsbefugnisse aktiv einzufordern, um sich gestalterisch in die Arbeit einzubringen, ihre Arbeitssituation zu verbessern und sich mehr Handlungsspielräume anzueignen.

„Ja, Teamarbeit ist nicht schlecht, das sage ich nach wie vor. Aber wie gesagt, es muss sich jeder dranhalten, und wenn sich die Herren nicht daranhalten, wird das nie gehen." I: Was waren denn so deine Gedanken, als du börtest, dass es jetzt so Teamarbeit geben sollte?] „W Wa habe ich mir da gedacht, ,Vielleicht bilft es uns weiter. Dass du irgendwas erreichen kannst auch. Es ist zum Teil so. Es hat zwar fürchterlich gek racht bei uns am Anfang. (.) Es war, Schreitöne waren das schon. [I: Woher kam das?] „Woher? Weil wir unser Recht erfahren wollen. Wenn ich Teamarbeit einführe, habe ich Rechte und Pflichten. Genauso auch die Chefs ober uns. Und wenn du so ein Recht ein- 


\begin{abstract}
fordern willst und du bekommst es nicht, dann wird es zum Streiten. Und wenn's dann einen [Meister, BS] hast, der was früher Meister war, der was auch viel zu sagen gehabt hat. Und jetzt auch wieder weniger Sagen hat, und bei der Teamarbeit dann noch ein bisschen weniger zu sagen hat, der füblt sich dann am Schlips getreten, und wenn die Teams unterschiedlich sind, dass dein Team neun Leute hat und das andere Team hat zehn Leute und du willst wissen, warum hat der zehn Leute, und du bekommst keine Antwort nicht drauf, dann ist es halt eskaliert. Da ist fast so weit gegangen, dass man gesagt habt, wir bekommen eine Verwarnung. "(Fischer, NF Montage F_A, 228ff.)
\end{abstract}

Fischer ist einer der mitgestaltungsorientierten Beschäftigten in seinem Bereich, die sich nicht mit den herrschenden restriktiven Bedingungen abgefunden haben, sondern die im Teamarbeitshandbuch beschriebenen Kompetenzen und Handlungsspielräume aktiv einfordern. Das bedeutet, dass sie von den Vorgesetzten und indirekten Bereichen die Bereitstellung ausreichender Ressourcen und Verbindlichkeit der in den Aushandlungsprozessen getroffenen Vereinbarungen verlangen. Fischer und seine Teamkollegen ergreifen aktiv und eigensinnig ihre Handlungsspielräume, um vorrangig die Arbeitssituation in ihrem Bereich zu verbessern. So wurden ausgehend von im Teamgespräch diskutierten Verbesserungsvorschlägen und mit Unterstützung des Meisters die arbeiterinitiierten Workshops zur Verbesserung der Ergonomie eingefordert und durchgeführt. Bedeutende Rolle hierbei spielt auch, dass Fischers Arbeitsteams aus mehreren älteren und erfahreneren Mitgestaltungsorientierten besteht, die sich gegenseitig unterstützen und sich nicht scheuen, gemeinsam gegen den Meister, die Führungsorganisation und indirekte Bereiche in Konflikt zu gehen.

Fischer nutzt aktiv die - durch die Teamarbeitsorganisation zumindest formal erweiterten - Gelegenheitsstrukturen, um die eigene Arbeit gestalten und verbessern zu können. Die in Teamarbeit initiierten Optimierungen betreffen die Gestaltung des eigenen Arbeitsbereichs und die Verbesserung der durch hohe körperliche Belastung geprägten Arbeitsbedingungen. Durch diese Ergonomie-Workshops wurde nicht nur die Arbeit für die Beschäftigten erleichtert, sondern bringen auch indirekt für den Betrieb wirtschaftliche Vorteile, da die geringere körperliche Belastung zu besseren Krankenstandzahlen führt. Verbesserungen der Wirtschaftlichkeit des Betriebs sind hierbei für die Mitgestaltungsorientierten, wie für Fischer, zwar relevant, aber nachrangig, da der Betrieb mit dem Leistungs- und Rationalisierungsanspruch permanent versucht, die Montagebereiche zu rationalisieren.

[I: Überlegt man sich als Team auch, wie man Dinge besser machen kann?] „Ja sicher, das machen wir auch. Das brauche ich ja keinen sagen, was ich tue. Für das Team muss es leichter sein, für den Arbeiter muss es erträglich sein. Ich muss es so einfach wie 
möglich machen. Stark wird es eh von selber. Und das tun wir nach wie vor und wie gesagt, das sind sicher Arbeitssachen, was wir einfach anders machen, als sie glauben. Weil es einfach für den Arbeiter besser ist. " (Fischer, NF Montage F T_A, 842ff.)

Rationalisierungspotenziale werden auch von Fischer bewusst im Verborgenen gehalten.

Mit dem Ideenmanagement hat Fischer eher negative Erfahrungen gemacht, da Ideen oftmals abgelehnt wurden. Infolgedessen beteiligt er sich nicht mehr daran.

\begin{abstract}
„Nein, jetzt nicht mehr. Früher hast dir immens viel Geld verdienen können damit, und das habe ich auch da drüben gemacht. Verbesserungsvorschläge sind ausbezahlt worden. War ein schönes Geld, was man da drüben gemacht hat. Und da herüben ist es, super, danke, aus. " [...] [I: Macht man sich jetzt beim neuen Ideenmanagement noch Gedanken?] „Nein, ich sag mal Nein. Macht man sich nicht, weil die du machst, die meisten werden eh mal abgelehnt, und irgendwann kommen sie dann mal drauf, jet:t machen sie es doch so. Du kriegst auch jetzt nichts mehr dafür. [I: Wie sieht es denn mit Ideen aus, die die eigene Arbeit erleichtern?] „Naja, da muss ich ja keinen Vorschlag machen, die kann ich ja selber machen. Das lässt sich oft durch ein paar Handgriffe nur verändern, und dann mache ich das und aus. " (Fischer, NF Montag F T1 A, 865ff.)
\end{abstract}

Kleinere, arbeitserleichternde Verbesserungen lassen sich in den Augen Fischers im Rahmen der Selbstorganisation und Handlungsspielräume von Teamarbeit vornehmen.

Fischer hat als Stellvertreter des Teamsprechers am KVP-MTM-Workshop teilgenommen. Seine Beteiligung erfolgte, wie bei Eder, tendenziell in abgesicherter Form. Fischer nutzte die Ressourcen der Workshoporganisation, um kleinere Arbeitsverbesserungen in seinem Bereich zu erzielen; gleichzeitig versuchte er, ungewollte Veränderungen, die durch die Workshopaktivität entstehen können, zu verhindern. Anders als Eder verbindet Fischer die Teilnahme am Workshop nicht mit einem persönlichen Nutzen, sondern er versteht sich ein als Vertreter des Teams.

Am Fall Fischers lassen sich zwei Aspekte verdeutlichen. Erstens zeigt Fischer hinsichtlich der unterschiedlichen betrieblichen Beteiligungsangebote unterschiedliche Umgangsweisen: keine Beteiligung am Ideenmanagement wegen schlechter Erfahrungen, breite Beteiligung im Rahmen von Teamarbeit, abgesicherter Modus in Workshops. Pragmatisch suchen sich die Mitgestaltungsorientierten für Problemlösungen und Verbesserungen diejenigen Gelegenheitsstrukturen aus, die in ihren Augen die höchste Realisierungswahrscheinlichkeit haben und die geringsten Nachteile für die Arbeit bewirken. Dort, wo 
Mitgestaltungsorientierte reale Einflussmöglichkeiten haben und Chancen sehen, sich einzubringen, werden sie ergriffen. Dabei stehen vor allem die Interessen der Beschäftigten an Schutz der Arbeitskraft vor Leistungsintensivierung und Beschäftigungsunsicherheit sowie die Gestaltung ihres Arbeitsprozesses und die Organisation der Arbeit im Team im Vordergrund. Dies trifft insbesondere auch auf die mitgestaltungsorientierten Beschäftigten in moderat partizipativen KVP-Praktiken (Linie 2) zu, die aufgrund des geringen Maßes an Teamselbstorganisation, schlechtem Gesprächsklima und eher unterreichender Unterstützung die potenzielle offene Konfrontation im PracticeGround-Gespräch häufig meiden, aber im Rahmen des Ideenmanagements gemeinsam mit den Instandhaltern sehr häufig eigene Optimierungen an ihrem Arbeitsplatz durchführen.

Zweitens verdeutlicht das aktive Ergreifen und Ausbauen der Handlungsund Entscheidungsspielräume den Gestaltungsanspruch der Beschäftigten. Die Übernahme von mehr Verantwortung für die Produktivität und Optimierung des eigenen Bereichs verknüpfen die Mitgestaltungsorientierten mit einer $\mathrm{Zu}$ nahme von Handlungs- und Entscheidungsspielräumen sowie mehr Unterstützung durch die Betriebsorganisation. Die Partizipationsmöglichkeiten, die ihnen durch die Einführung von Teamarbeit versprochen, aber noch von der Betriebsorganisation bislang unzureichend realisiert wurden, werden im Modus des aktiven Ergreifens von Handlungsspielräumen erkämpft. In diesem Fall scheuen die Mitgestaltungsorientierten auch keine offenen Konflikte, um ihre Arbeitsbedingungen zu verbessern und etwas mehr Kontrolle über ihre Arbeitsabläufe zu erlangen.

"Ja, ich finde, ganz wichtig ist, dass das Team entscheidet über Werkzenge. Wir haben das jetzt klargestellt mit dem Chef von der AV120, wegen den neuen Schraubern. Sie haben auch gesagt, wir kriegen die neuen Schrauber, aber wir kriegen die alten Halterungen oben. Haben wir klipp und klar als Team gesagt, wenn wir die alten Aufbängungen lassen, dann braucht er die neuen Schrauben gar nicht aufbängen. Das ist schon/ frïher haben sie dir das hergehängt und dann: ,So, tu damit. 'Und jetzt ist das schon, dass wir fordern: ,Wir wollen die Werkezenge anschauen, wir wollen die Werkezenge probieren, wollen damit arbeiten, schrauben, und dann entscheiden wir Ja oder Nein. "Und das ist jetžt so. Das passt schon jetz̨t. Das ist auf alle Fälle super jetzt. " (Haller, NF Montage F T_A, 1402ff.)

Dabei sind die der Mitgestaltungsorientierten allerdings keineswegs nur auf ihren eigenen Vorteil bedacht oder versuchen, ihre Optimierungsaktivitäten

120 Arbeitsvorbereitung. 
gegen die Wirtschaftlichkeitsinteressen des Betriebs durchsetzen. Sie akzeptieren diese und versuchen, die Wirtschaftlichkeitsziele so gut wie möglich mit den eigenen Interessen zu verbinden.

Dass in Montage $\mathrm{K}$ anders als in Montage $\mathrm{F}$ durch die Einführung von Teamarbeit partizipative Bedingungen entstanden sind, liegt in hohem Maße daran, dass die Beschäftigten sich diese im Rahmen von Teamarbeit in konfrontativer Auseinandersetzung mit dem Meister, den übrigen Vorgesetzten und indirekten Bereichen proaktiv erkämpft haben.

\subsection{Aktive Beteiligung}

Aktive Beteiligung findet sich bei Beschäftigten, die sich relativ frei und bedenkenlos an beschäftigtengetragenen Optimierungsprozessen beteiligen. Die aktive Beteiligung lässt sich bei Mitgestaltungsorientierten in partizipativen Beteiligungskonstellationen und bei Karriereorientierten unabhängig von der Gestaltung der Beteiligungskonstellationen identifizieren.

\subsection{1. „Wenn es uns gut geht, geht es dem Betrieb auch gut“ -} Kooperative Optimierungspartnerschaft

Eine aktive Beteiligung in Form einer kooperativen Optimierungspartnerschaft findet sich bei Mitgestaltungsorientierten in partizipativen Beteiligungskonstellationen (Linie 1), innerhalb derer im Rahmen der Betriebsorganisation die von den Beschäftigten artikulierten Probleme verbindlich und schnell gelöst, Optimierungsideen umgesetzt und den Beschäftigten daraus keine Nachteile erwachsen. Diese Aneignungsform wird hier am Beispiel von Oswald verdeutlicht.

Oswald (Ende 40) ist gelernter Fliesenleger. Nach seiner ersten Ausbildung hat er sich vorwiegend aus finanziellen Gründen entschieden, eine zweite Ausbildung bei WLF als Schlosser zu absolvieren. Er arbeitet seit 23 Jahren bei WLF in unterschiedlichen Produktionslinien. Während dieser Jahre hat Oswald eine deutliche Verbesserung der Arbeitsbedingungen und -strukturen sowie ein hohes Maß an Stabilität der Beschäftigungsbedingungen erlebt. Durch die höhere Automatisierung der verketteten Produktionslinien wurde die Arbeit deutlich erleichtert und die Interessantheit zugleich erhöht. Das Arbeitsverständnis Oswalds ist durch eine hohe Aufgaben- und Problemlöseorientierung geprägt. Er übernimmt über seine eigentliche Aufgabe als Monteur und Anlagenbediener an verschiedenen Arbeitsplätzen 
seiner Produktionslinie hinaus viele organisatorische Aufgaben, die für den reibungslosen Produktionsablauf wichtig sind, wie die Bestellung von Komponenten und Aufklebern oder die Einteilung des Schichtplans. Oswald hat ein sehr hohes fachliches Interesse, „tüftelt" gerne an den Anlagen herum und hilft regelmäßig freiwillig bei der jährlichen, geplanten Instandhaltungspause. Die hohe Mitgestaltungsorientierung drückt sich bei Oswald beispielsweise darin aus, dass er in seiner Freizeit zu Hause eine Montagehilfe gebaut hat, die er im Betrieb nutzen darf und sogar als Idee im Ideenmanagement einreichen konnte.

Die direkte Kommunikation mit Vertretern indirekter Bereiche bildet für Oswald einen selbstverständlichen Teil seiner Arbeit. Die Möglichkeit der Beteiligung an beschäftigtengetragenen Optimierungsprozessen im Rahmen von PE und Ideenmanagement nimmt Oswald ausschließlich positiv wahr und bezieht sich dabei vor allem auf die Verbesserung der Arbeitssituation und der Abläufe. Seine Erfahrungen mit $P E$ beschreibt er wie folgt:

„Probleme, die vorher verschwunden ${ }^{121}$ waren, werden gelöst, damit man mit dem arbeiten kann. Damit jeder seine Sachen aufschreibt und das behandelt wird. Auch wenn es nur eine Kleinigkeit ist. Es ist in vielen Sachen investiert worden, das kommt bis zum Chef hinauf, das brauchen wir auch und das ist wichtig. Sonst wäre es ja verschwunden irgendwann. Das bleibt so lange stehen, bis dass es behandelt worden ist. Früher war's, ja, Pech gebabt.' [...] Wenn irgendwer Probleme mit anderen Abteilungen hat, weil wenn es länger daraufsteht, bole ich mir einen von der Abteilung und der muss sich rechtfertigen dafür, was da los ist. Dann wird das auch sicher behandelt. Es ist auch ein Druck dabinter. "(Oswald, WLF Linie 1, 453ff.)

Die Verbindlichkeit und Transparenz von Problemlöseprozessen und die direkten Kommunikationswege im Rahmen von PE tragen unmittelbar zur Verbesserung seiner Arbeitssituation bei. Die betriebsweite Verbindlichkeit des PE-Prozesses bewirkt, dass Oswald sich im Betrieb ernst genommen fühlt und dass seine Meinungen und Anregungen ein besonderes Gewicht erhalten.

[I: Wer profitiert denn von PE?] „Die Mitarbeiter, die Arbeiter, Schichtarbeiter, wir. Wir hauptsächlich, naja, sicher, warum nicht [...]. Weil mir geholfen wird, wenn ich ein Problem habe, wird mir geholfen, dass ich meine Arbeit leichter mach, meine Tätigkeit oder mein Umfeld. Das schöner wird, sauberer wird, Beispiel ,mehr Licht" oder so Sachen schreiben wir rauf." [I: Hat denn PE auch eine Bedeutung für die Wirtschaftsfähigkeit oder Leistungsfähigkeit des Betriebs?] „Das weiß ich nicht. Aber ja, sicher. Beispiel, wenn alles passt in einem Betrieb, kann ich pünktlich die Lager bestellen und der Kunde ist zufrieden. Oder wenn ich nur Schwierigkeiten habe und nichts behandelt

${ }^{121}$ Dieser Ausdruck ist in dem Sinne zu verstehen, dass dies vorher ignoriert wurde. 
wird oder nichts gemacht wird, bring ich keinen Liefertermin nicht zusammen." (Oswald, WLF Linie 1, 634ff.)

Im Vergleich zu den Beschäftigten der anderen Beteiligungskonstellationen lassen sich im Betriebs- und Rationalisierungsverständnis von Oswald kaum Elemente eines Interessenantagonismus finden. Das konkretisiert sich darin, dass er nicht zwischen Optimierungen für den Betrieb und für die Arbeiter differenziert und erst nach direkten Rückfragen der Interviewerin mögliche Nachteile von Optimierungsprozessen für die Beschäftigten in Erwägung zieht. Gleichwohl rekurriert er in seinen Überlegungen zunächst auf die Vorteile, die PE für die Beschäftigten bringt, und führt erst nach kurzem Nachdenken die Wirtschaftlichkeitsvorteile auf. Oswald sieht in den Optimierungsaktivitäten des Betriebs weder unmittelbare Nachteile noch theoretische Bedrohungen durch Leistungsintensivierungen oder Arbeitsplatzverlust. Dies bedeutet jedoch nicht, dass beschäftigtengetragene Optimierungsprozesse gänzlich frei von Interessenkonflikten und Aushandlungsprozessen wären. So ist es Oswald wichtig, Veränderungen mit seinen Kollegen abzusprechen, um keine Konflikte entstehen zu lassen.

Die vorbehaltlose Beteiligung an beschäftigtengetragenen Optimierungsprozessen ist bei Oswald weniger an der Erfüllung betrieblicher Normen orientiert, sondern mit seinen subjektiven Orientierungen an Fachlichkeit der Arbeit, Anerkennung sowie Arbeitserleichterung sowie dem Leistungsverständnis verbunden, einen Teil zum Erfolg des Betriebs beizutragen.

„Stehenbleiben darf man auf keinen Fall. Nein, sicher nicht. Das ist sicher angemessen, das mit dem Druck und dass man kundenbezogen genau ist und sich keine Fehler erlauben soll, weil der Kunde kauft das Lager woanders genauso schnell. Das soll man sich schon vor Augen halten. "(Oswald, WLF Linie 1, 942ff.)

Das Betriebs- und Rationalisierungsverständnis der Beschäftigten, die sich vorbehaltlos an beschäftigtengetragenen Optimierungsprozessen beteiligen, ist nur in sehr geringem Maß vom eigentlich für Lohnarbeit typischen Interessenantagonismus geprägt. Dies lässt sich möglicherweise damit erlären, dass die Beschäftigten bislang in ihrem Erwerbsleben bzw. während ihrer Beschäftigung bei WLF durchgängig positive Erfahrungen mit betrieblichen Veränderungsprozessen gemacht haben, so dass der strukturelle Interessenantagonismus in den Hintergrund tritt. Dass Leistung intensiviert wird und der Betrieb weiter nach Rationalisierungspotenzialen sucht, wird in diesem Fall als legitimes betriebliches Interesse wahrgenommen, das nicht rücksichtslos gegen die 
Produktionsbeschäftigten durchgesetzt wird. Weil die von den Beschäftigten artikulierten Probleme und Optimierungspotenziale im Rahmen dezentraler Kooperationsstrukturen und funktionierender Bottom-upKommunikationsprozesse schnell, gleichwertig und mit hoher Verbindlichkeit behandelt werden, werden nicht nur die Arbeitssituation und die Abläufe ihres Bereichs verbessert, sondern die Beschäftigten erfahren auch eine hohe Wirksamkeit ihres Engagements. Die Beschäftigten erleben, dass sie mit ihren Problemen ernst genommen werden und eine Stimme im Betrieb haben. Durch die partizipative Gestaltung von beschäftigtengetragenen Optimierungsprozessen erhalten die Mitgestaltungsorientierten die Möglichkeit, ihre subjektiven Ansprüche an Arbeit ein Stück weit zu verwirklichen. Das Arbeitsverständnis der Mitgestaltungsorientierten ist durch ein hohes Aufgaben- und Problemverständnis sowie Interesse an fachlichen Herausforderungen, die über ihre eigentliche Produktionsarbeit hinausgehen, geprägt. Die Beteiligung an Optimierungsprozessen ermöglicht den Mitgestaltungsorientierten, diese Orientierungen in die Arbeit einzubringen.

\subsubsection{Beteiligung als Weiterentwicklungschance}

Karriereorientierte Beschäftigten nutzen die Beteiligung an beschäftigtengetragenen Optimierungsprozessen wird von gezielt, um ihre Weiterentwicklungschancen im Betrieb zu erhöhen. Die Beteiligung bietet ihnen die Möglichkeit, ihre fachliche Fähigkeiten und die Eignung für „,höhere“ Positionen im Betrieb unter Beweis zu stellen und sich von der Masse der Produktionsbeschäftigten positiv abzuheben. Zwar ist die Beteiligung an Optimierungsprozessen auch bei Karriereorientierten mit der unmittelbaren Verbesserung der Produktionsund Arbeitsabläufe verbunden - noch vielmehr allerdings verknüpft sich ihr Beteiligungsverhalten mit dem strategischen Kalkül, als Produktionsbeschäftigte innerhalb der Betriebsorganisation aufzusteigen. Diese Aneignungsform lässt sich am Beispiel Mayr verdeutlichen.

Mayr (Ende 20) hat in einem mittelständischen Betrieb die Lehre als Schlosser absolviert, dort zwei Jahre gearbeitet. Er hat den Betrieb freiwillig aus finanziellen Gründen verlassen, um bei einem großindustriellen Betrieb der Region zu arbeiten. In Folge der Krise 2008 war er zwei Jahre arbeitslos und wurde über eine Leiharbeitsfirma bei WLF beschäftigt. Mayr ist in hohem Maße an technischen Abläufen und mechanischen Prozessen interessiert. Neben seiner eigentlichen Produktions- 
arbeit, die für inn fachlich wenig herausfordernd ist, probiert er eigenständig Optimierungsideen an seiner Anlage aus, tauscht sich mit Anlagenplanern aus und versucht nachhaltig, die Laufzeiten zu optimieren. Er orientiert sich in hohem Maße daran, die Abläufe und Anlagenlaufzeiten an seinen Arbeitsplätzen wirtschaftlich zu gestalten.

„Mir tangt das was. Wo ich sicherlich stark. bin. Ich bin vielleicht nicht so der Arbeit/ Ja, kein schlechter Arbeiter, bin ich sicher nicht, aber mir taugt nicht so Serienproduktion. Wie kannst du was reinbringen, Abläufe organisieren, viele Sachen wegbringen, umtragen, da lässt sich einiges machen, ja. Irgendwo so Arbeitsvorbereitung oder so irgendwas, das wär/ was mir voll tangen würde, ja. Das bätte ich auch gern drüben in der [früherer Betrieb, BS] gemacht, ja. Aber das ist eine andere Geschichte. Würd' ich nicht mehr rübergehen. [...] Drum taugt mir das jetzt grade mit dem ganzen Übergreifenden, jetzt mit der PE, dass sie das alles verketten wollen, und das tangt mir einfach, die Abläufe. [...] Aber ich glaub, mittlerweile haben wir eh ein bisschen was auf die Füße gestellt, ja, da draußen. Also, die Abläufe sind sicher besser geworden, trau ich mich jetzt sagen, sicher. Und die Stückzabl ist mit Sicherbeit um $40 \%$ raufgegangen, mit Sicherbeit. [...] Mhm. Das war zwar eh ein Kampf am Anfang, aber ich glaub, ich hab gewonnen, im Großen und Ganzen. Und sie sehen es jetzt selber ein, dass die Arbeit eigentlich leichter geworden ist. "(Mayr, WLF Linie 2, 97ff.)

Mayr zeigt ein sehr hohes fachliches Interesse. Er orientiert sich in seinem Handeln zwar daran, Erleichterungen der eigenen Arbeit zu erzielen, lehnt die kollegialen Solidaritätsnormen und den Protektionismus der Kollegen in seinem Bereich aber ab. Aufgrund der Laufzeitverkürzung der Anlage, die er unternommen hat, wurde von seinen Kollegen sehr stark kritisiert und sogar angefeindet. ${ }^{122}$ Mayr bringt der Schutzhaltung seiner Kollegen wenig Verständnis entgegen und lässt sich dadurch nicht in seinem Handeln beeinflussen.

„Sie haben irgendwo eine Angst, das weiß ich nicht. Keine Ahnung, dass weniger Arbeit da ist oder irgendwas wegen dem vielleicht, dass sie keine Verbesserungen vorschlagen oder irgendwas. Das/ Vielleicht in die Richtung. Aber genau weiß ich es jetrt auch nicht, um was/ Ja, da ist halt riemlich noch ein Gruppenzusammenhalt da draußen." (Mayr, WLF Linie 2, 598ff.)

Sein Arbeitsverständnis ist dadurch geprägt, gute Arbeit zu leisten und einen Teil dazu beizutragen, seinen Arbeitsbereich wirtschaftlich und effizient zu gestalten. Mayr ist sehr stark leistungs- und karriereorientiert.

122 Während der Untersuchungsphase haben die Kollegen sich in Beobachtungen oder Interviews andeutungsweise auf diese Optimierungsaktivitäten Mayrs bezogen und diese als natives und schädliches Verhalten bewertet. 
„Ich will kein Meister sein, aber so irgendwas, irgendwo so Arbeitsvorbereitung, wo kannst du was rationalisieren und so was, das/ Weiß nicht, das sehe ich einfach/ Das haben wir so gelernt und das ist, wo ich stark bin, ja. Das ist/ Wenn du wo durchgehst, dass du das siehst, das siehst, wie wo gearbeitet wird und solche Sachen, wo falsch gearbeitet wird, und das hab ich irgendwie so drinnen, das haben wir so gelernt und ja." (Mayr, WLF Linie 2, 490ff.)

Mayr erwartet, dass sein Engagement für den Betrieb sich für inn lohnen wird. Zunächst strebt er die Festanstellung bei WLF an und anschließend möchte er durch die Meisterausbildung in den Planungsbereich wechseln. Obwohl Mayr die Beteiligungsmöglichkeiten an Optimierungen durch PE sehr positiv bewertet, unternimmt er Optimierungen meist allein an seinem Arbeitsplatz und bringt nur wenige Optimierungspotenziale in den Gesprächsrunden ein.

Die aktive Beteiligung der karriereorientierten Beschäftigten an beschäftigtengetragenen Optimierungsprozessen scheint in keinem direkten Zusammenhang mit den strukturellen Bedingungen zu stehen. Karriereorientierte Beschäftigte nehmen die Beteiligungsmöglichkeiten in erster Linie als Mittel zur Verbesserung der eigenen Weiterentwicklungschancen im Betrieb wahr. Weil sie beabsichtigen oder sogar fest einplanen, die direkte Produktionsarbeit zu verlassen, müssen sie sich nicht mit den langfristigen Folgen von Optimierung und Rationalisierung im Produktionsbereich auseinandersetzen und haben keine direkten Nachteile daraus zu erwarten. Neben der Möglichkeit, Fachwissen und Erfahrungen in Optimierung einzubringen und diese zu erweitern, bringen eigene Rationalisierungsaktivitäten einen strategischen Vorteil. So kann man sich über die Optierungsleistung nicht nur als geeigneten Aufstiegskandidaten präsentieren, sondern durch die Zusammenarbeit mit indirekten Bereichen auch die innerbetrieblichen Beziehungen stärken. Karriereorientierte sehen den Betrieb als Ort der Verwirklichung ihrer arbeitsinhaltlichen und karrierebezogenen Interessen. Dies führt zu einer starken Orientierung an betrieblichen Verhaltenserwartungen, die Karriereorientierte in Erwartung eines Aufstiegs zu erfüllen versuchen.

Die Hypothese, dass die arbeitspolitischen Bedingungen tatsächlich wenig Einfluss auf die Beteiligungsdisposition der Karriereorientierten haben, muss auch hier vorläufig bleiben. Beschäftigte mit einer hohen Karriereorientierung ließen sich nur im Sample von WLF finden. Bei NF wurden die Aufstiegschancen für Produktionsbeschäftigte angesichts der beobachteten Karriereverläufe als gering eingeschätzt. Es gibt Hinweise darauf, dass einige Beschäftigte 
von NF zwar durchaus Karriereambitionen hegten, diese aufgrund mangelnder Förderung und verschlossener Karrierepfade mittlerweile aber aufgegeben haben.

„Nun, ja, man hat keine Chancen nicht. Man ist in der Sackgasse. Und das ist das, ich meine, man siebt, dass die [Meister] werden von der FH heraus bestïckt. Das heißt der Werksmeister. Irgendwas macht auch keinen Sinn. Ich bätte jetzt die Fachakademie für Fertigungs- und Produktionstechnik. wollte ich jetst gerne machen. Das wäre mit Matura, wie gesagt, ich habe nur gelernt. Das wäre eigentlich auch genau, was berinnen passen würde. Das kann man wieder nicht machen, weil da kommt man nur erste Schicht, weil die ist auf das ausgelegt, dass man das am Abend dran/Das kann man nicht und somit ist das eigentlich eine Sackgasse." (Berger, NF Montage K T_B, 1849ff.)

Die arbeitspolitischen Bedingungen haben hierbei weniger Einfluss auf die Beteiligungsdisposition der Karriereorientierten als vielmehr auf ihre Orientierungen. Wenn seinen Aufstiegsansprüchen auf Aufstieg dauerhaft nicht entsprochen wird, hat der Beschäftigte zwei Möglichkeiten, sich künftig zu verhalten; welche er ergreift, hängt davon ab, wie wichtig ihm die Realisierung seiner Ansprüche ist. ${ }^{123}$ Sind seine Karriereambitionen stark ausgeprägt, kann er den Betrieb verlassen und versuchen, seine Ansprüche außerhalb zu verwirklichen. Falls er den Betrieb nicht verlassen kann oder möchte, wird der eigentlich Karriereorientierte mit dieser starken Diskrepanz umgehen, indem er etwa die die Stärke seiner Ansprüche herunterschraubt und sich mit seiner Situation abfindet. In diesem Fall würde sich die Gewichtung der subjektiven Orientierung eher in Richtung Mitgestaltung oder Status-quo-Orientierung verschieben. Dieser Hypothese folgend lassen sich die Orientierungsmuster einiger Beschäftigter im Sample deuten, die im Laufe des Erwerbslebens ihre Karriereambitionen aufgegeben haben oder aufgrund der als sehr schlecht wahrgenommenen Arbeits- und Beschäftigungsbedingungen bei NF mit dem Gedanken spielen, zu kündigen, vorsichtig deuten. Eine vollständige Prüfung dieser Hypothese bedarf eines tiefergehenden, biografisch-narrativen Zugriffs auf die Orientierungen der Beschäftigten und die Kündigungsgründe ehemaliger Beschäftigter.

123 Siehe zu Disponibilität und Unhintergehbarkeit von Ansprüchen auch Nies (2015). 


\section{Zwischen arbeitspolitischem Konservativismus und Pragmatismus: Zusammenführung und Ausblick}

In der Managementliteratur wird zuhauf das Leitbild des verantwortungsvollen, wirtschaftlich denkenden und agierenden Mitarbeiters gezeichnet. Die Betriebe wiederum tragen diesen Idealtypus an ihre Beschäftigten heran - und setzen deren Beteiligung an betrieblicher Optimierung ohne Blick auf ihre arbeitssituativen „Befindlichkeiten“ gewissermaßen selbstverständlich voraus. Und auch in der arbeits- und industriesoziologischen Forschung wurde wenngleich auf etwas differenziertere Art und Weise - davon ausgegangen, dass Beschäftigte ihrem genuin vorhandenen Beteiligungsanspruch nachkommen, sofern geeignete Arbeits- und Beschäftigungsbedingungen vorhanden sind. Zugleich stand die These des arbeitspolitischen Konservativismus - der Ablehnung von Beteiligung an betrieblicher Rationalisierung zum Zweck der Vermeidung negativer Auswirkungen auf die eigene Arbeitssituation Beschäf- 
tigten - dem vermeintlichen Beteiligungsanspruch der Mitarbeiter und den betrieblichen Beteiligungsaufforderungen unverbunden gegenüber.

Mit der vorliegenden vertieften Fallstudienuntersuchung wurde eine empirische Spurensuche nach differenzierbaren Aneignungs- und Umgangsformen der Beschäftigten mit dem alltagspraktischen Beteiligungsangebot an KVP untergenommen. Ausgangspunkt der Untersuchung waren die beobachteten Varianzen in den Haltungen und dem tatsächlichen Beteiligungsverhalten der Beschäftigten hinsichtlich KVP, die weder linear von den jeweiligen KVPPraktiken noch von den subjektiven Orientierungen der Beschäftigten abgeleitet werden können, sondern in ihrem Zusammenwirken betrachtet werden müssen. Dabei wurde eine subjekt- und interessenorientierte Perspektive gewählt, welche die Rekonstruktion zentraler und differenzierender Bestimmungsmomente sowie ihr spezifisches Zusammenspiel im Aneignungsprozess ermöglicht. Die Subjektivität der Beschäftigten, etwa ihre individuellen Empfindungen, Kompetenzen, Orientierungen, Ansprüche und Interessen, wurden den arbeits- und beschäftigungssituativen sowie betriebsorganisatorischen Bedingungen des KVP gegenübergestellt. Mit diesem konzeptionellen Ansatz werden Beschäftigte nicht rein als auf äußere Anreize reagierende, mehr oder weniger passive Objekte des betrieblichen Verwertungskonzepts betrachtet, sondern als innerhalb ihrer arbeitssituativen und betriebsorganisatorischen Kontextbedingungen relativ eigenständige Akteure wahrgenommen.

Die elaborierte Kernthese dieser Arbeit lautet, dass die Beteiligung der Beschäftigten an KVP Ergebnis eines interessenorientierten Abwägungsprozesses ist. Dieser Aneignungsprozess wird einerseits durch die Ausprägungen der jeweiligen KVP-Praktiken sowie ihres arbeitssituativen und betriebsorganisatorischen Gestaltungkontextes bestimmt, andererseits gehen die Beschäftigten auf Basis unterschiedlicher Orientierungsmuster jeweils unterschiedlich mit den betrieblichen Anforderungen und Bedingungen um. Daher identifizierte diese Studie in analytisch getrennten Schritten zunächst die Verfasstheit der subjektiven Orientierungen und der arbeitspolitischen Gestaltungsbedingungen von KVP, um aus diesen dann synthetisch zu spezifischen Aneignungsformen zu rekonstruieren.

In einem ersten Schritt wurden anhand der Motive, welche die Beschäftigten für ihre Beteiligung oder Nichtbeteiligung an KVP anführen, subjektive Einzelorientierungen der Beschäftigten identifiziert. Von großer Bedeutung für die Beteiligung an betrieblichen Optimierungsprozessen sind drei wesentliche Bestimmungsmomente der Arbeitsorientierungen: erstens die Orientierung der 
Beschäftigten an einer sinnvollen Arbeit, welche mit einem Leistungsverständnis verbunden ist, ihre Arbeit „gut" zu erledigen; zweitens die Orientierung an Schutz vor Verschlechterung der Arbeits-, Leistungs- und Beschäftigungsbedingungen, die im Zusammenspiel mit der kollegialen Solidarität eine starke Wirkung entfaltet; und drittens die erwerbsbiografische Perspektive. Der Wunsch nach beruflicher Weiterentwicklung bzw. beruflichem Aufstieg nimmt insofern Einfluss auf die eigene Mitwirkung an KVP, da eine Beteiligung die Chancen, „entdeckt“ zu werden und sich für eine Karriere zu empfehlen, erhöht. Anerkennung sowie betriebliche und kollegiale Normen sind für die Beteiligung ebenfalls zentrale Handlungsorientierungen, variieren in ihrer Stärke allerdings deutlich zwischen den drei Beteiligungstypen, die sich aus den unterschiedlichen Gewichtungen der Einzeldimensionen empirisch extrahieren lassen.

Mit Blick auf die grundlegende Beteiligungsdisposition lassen sich folgende Beteiligungstypen differenzieren: Status-quo-Orientierte, Karriereorientierte und Mitgestaltungsorientierte. Gemein ist allen drei Beteiligungstypen die Orientierung daran, gute und sinnvolle Arbeit zu leisten, was mit der fachlichen und erfahrungsbasierten Vorstellung eines funktionalen Produktions- und Ablaufprozesses verbunden ist. Differenzen zeigen sich hinsichtlich der (Nicht)Beteiligungsmotive: Für Status-quo-Orientierte stehen potenzielle Verschlechterungen der individuellen und kollektiven Arbeitssituation im Vordergrund des Handelns, weshalb sie Beteiligungen an Optimierungen sehr skeptisch gegenüberstehen. Mitgestaltungsorientierte hingegen streben aktiv nach der Mitwirkung an Problemlösungen und Gestaltung des Arbeits- und Produktionsprozesses - zum Wohl der Arbeiter und des Betriebs. Karriereorientierte fokussieren stärker auf die Verfolgung individueller Ziele. Die beiden letztgenannten Beteiligungstypen sind betrieblichen Optimierungsprozesse gegenüber grundsätzlich aufgeschlossen.

In einem zweiten Schritt wurden anhand eines umfangreichen Vergleichs von vier unterschiedlichen KVP-Praktiken in zwei Fallstudienbetrieben zentrale Gestaltungsbedingungen für funktionierende bzw. nicht funktionierende Kontinuierliche Verbesserungsprozesse analysiert. Hinsichtlich der Gestaltung von KVP im engeren Sinne erwies es sich für eine möglichst hohe Beteiligung der Beschäftigten als förderlich, wenn betriebliche Verbesserungsprozesse sowohl in Form von Workshops als auch via Teamarbeit ein großes Spektrum von Optimierungspotenzialen anvisierten - also auch solche Verbesserungen berücksichtigten, die sich nicht dezidiert auf berechenbare Kosteneinsparun- 
gen beziehen, sondern vor allem Arbeitserleichterungen für die Beschäftigten bedeuten (Kuhlmann et al. 2004; Schwarz-Kocher et al. 2012). Dies ist unmittelbar mit der Bedingung verknüpft, KVP im Betrieb so zu installieren, dass möglichst viele Beschäftigte eingebunden werden und dass die Optimierungen an die konkreten, arbeitsbezogenen Probleme in ihrem Produktionsabschnitt anknüpfen. Nachhaltige Beteiligung kann sich erst dann entfalten, wenn die Beschäftigten durch zeitliche Ressourcen, breitere Tätigkeitszuschnitte und größere Handlungsspielräume befähigt werden, Optimierungspotenziale zu erschließen, und wenn die betrieblichen Kooperations- und Kommunikationsstrukturen zwischen den direkten Produktionsbereichen so ausgerichtet sind, dass die Probleme und Optimierungspotenziale, welche die Beschäftigten anhand der Dysfunktionalitäten des alltäglichen Produktionsprozesses identifizieren und artikulieren, von den Führungskräften und betrieblichen Servicebereichen aufgegriffen und gemeinsam mit den Beschäftigten gelöst bzw. umgesetzt werden.

Diesen partizipativen Strukturen stehen restriktive, beteiligungshinderliche Gestaltungsbedingungen gegenüber: Geringe Handlungsspielräume und enge Tätigkeitszuschnitte, starre methodenzentrierte und bürokratische Prozesse, rigide Standardisierungskonzepte und betrieblich vorangetriebene Rationalisierungsprozesse, in welche die Beschäftigten nicht eingebunden sind, wirken der Beteiligung der Beschäftigten an betrieblicher Optimierung entgegen.

Die Rekonstruktion der Aneignungs- und Umgangsformen der Beschäftigten mit KVP, die im dritten Schritt der empirischen Analyse erfolgte, ergab, dass die verschiedenen Beteiligungstypen von den spezifischen KVP-Praktiken sowie ihren arbeitssituativen und betriebsorganisatorischen Kontextbedingungen jeweils unterschiedlich angesprochen werden (siehe Tabelle 8).

Unter restriktiven Bedingungen beschränkt der Betrieb KVP weitgehend auf eine rhetorische Ansprache, aus der sich keine praktischen Beteiligungsangebote und substanziellen Veränderungen für die Beschäftigten ergeben. Status-quo-Orientierte fühlen sich unter diesen Bedingungen gar nicht angesprochen bzw. motiviert, sich an KVP zu beteiligen. Sie ergreifen - im Unterschied zu Mitgestaltungsorientierten - auch keine Eigeninitiative zur Mitgestaltung. Zudem setzen Status-quo-Orientierte KVP mit Arbeitsplatzrationalisierung und Leistungsintensivierung gleich. Mitgestaltungsorientierte hingegen nutzen aktiv, aber kontrollierend die angebotenen Gestaltungsräume selbst dann, wenn diese unter restriktiven Bedingungen sehr gering sind. Dabei versuchen sie nicht nur, die Möglichkeiten auszuschöpfen, auch kleinere Verbesserungen 
zu realisieren, sondern verfolgen eigensinnige Strategien zur Kontrolle der betrieblichen Optimierungsaktivitäten in KVP-Workshops zum Schutz vor Verschlechterung der Arbeits- und Leistungsbedingungen sowie zur Steigerung der Interessantheit, der Fachlichkeit und des Abwechslungsreichtums der Arbeit.

\section{Tabelle 8: Aneignung von KVP}

\begin{tabular}{|c|c|c|}
\hline \multicolumn{3}{|c|}{$\begin{array}{c}\text { Arbeitspolitische } \\
\text { Gestaltungsbedingungen }\end{array}$} \\
\hline Aneignung & Beteiligungs-orientierungen & Aneignung \\
\hline $\begin{array}{l}\text { Keine Beteiligung } \\
\text { Ignorieren } \\
\text { Demonstrative Ablehnung }\end{array}$ & StATUS-QUO-ORIENTIERTE & selektiv, kontrollierend \\
\hline $\begin{array}{l}\text { Eigensinnige Aneignung } \\
\text { Pragmatisch kontrollierend } \\
\text { Kampf um Gestaltungsspiel- } \\
\text { räume } \\
\text { Abwechslung von der monoto- } \\
\text { nen Arbeit }\end{array}$ & MITGESTALTUNGSORIENTIERTE & $\begin{array}{r}\text { Aktiv, gestaltend } \\
\text { Kooperative Optimierungspart- } \\
\text { nerschaft }\end{array}$ \\
\hline $\begin{array}{l}\text { Erhöhung individueller Auf- } \\
\text { stiegschancen }\end{array}$ & KARRIEREORIENTIERTE & proaktiv, vorbehaltlos \\
\hline
\end{tabular}

Eigene Darstellung

Schließlich - und das ist in diesem Sample die auffälligste eigensinnige Aneignung von betrieblichen Optimierungsprozessen - ergreifen sie Gestaltungsspielräumen aktiv selbst und bauen diese eigenständig aus. So haben Mitgestaltungsorientierte in Montage F aus ihrem Bewusstsein, Experten (beinah „Eigentümer") ihres Arbeitsbereichs zu sein, unter Berufung auf die vom Betrieb verfassten neuen Beteiligungsmöglichkeiten und Organisationsstrukturen (Teamarbeitsorganisation, dezentrale Servicebereiche) sowie durch geschickte Nutzung der Teamgespräche vehement die Kooperation und Unterstützung der Servicebereiche, Führungskräfte und Betriebsräte eingefordert und diese Strukturen und Kanäle zudem nicht nur für Optimierungspotenziale, sondern auch für die Klärung anderer betrieblicher Probleme und Unstimmigkeiten genutzt. Im Unterschied zu den anderen Aneignungsformen versuchten die Mitgestaltungsorientierten in Montage F, die betrieblichen Kooperationsstruk- 
turen im Team zu verändern. Entscheidend war hier, dass zumindest die Führungskraft das Team dahingehend unterstützte, seine Problemlösungen voranzutreiben, indem sie die notwendigen Kooperationsstrukturen zu den Planungsbereichen herstellte. Auf diese Weise konnten erfolgreiche beschäftigtengetragene Problemlöse- und Verbesserungsprozesse stattfinden - die allerdings nicht immer frei von Kritik und Einwänden des Betriebs blieben, da die Eigeninitiativen aufgrund von mangelhaften betrieblichen Informationsaustausch mit anderen expertenzentrierten Projekten kollidierten.

Unter (eher) partizipativen Bedingungen werden zum einen breitere Beteiligungsmöglichkeiten angeboten - das heißt mehr Möglichkeiten zur Artikulation von Optimierungspotenzialen -, und diese zum anderen von prozessnahen Kooperations- und Informationsstrukturen flankiert, welche die Rezeption und Realisierung von Ideen wahrscheinlicher machen als unter stark restriktiven Bedingungen mit bürokratischen Prozessen. Zudem wird ein breites Spektrum an Optimierungspotenzialen zur Verbesserung der Arbeitsbedingungen sowie der Wirtschaftlichkeit des Betriebs gefördert. Unter diesen Bedingungen gehen Status-quo-Orientierte in einem abgesicherten Modus und Mitgestaltungsorientierte aktiv auf das Beteiligungsangebot ein.

Das Beteiligungsverhalten der Karriereorientierten hingegen differiert nicht in Abhängigkeit von den arbeitspolitischen Gestaltungsbedingungen von KVP. Sie nutzen durch proaktives Engagement alle dargebotenen Beteiligungsformen, um sich in die Arbeit einzubringen und dem Betrieb als fähige Aufstiegskandidaten zu präsentieren.

\subsection{Arbeitspolitischer Pragmatismus}

Aus dem komplexen Geflecht von subjektiven Orientierungen und strukturellen Gestaltungsbedingungen stechen im Wesentlichen zwei zentrale Interessen der Beschäftigten heraus. Die Stärke der Beteiligungsbereitschaft changiert in Abhängigkeit von dem subjektiv wahrgenommenen Bedrohungspotenzial, das von KVP für die Beschäftigten ausgeht, und den arbeitsinhaltlichen und mitgestaltungsbezogenen Ansprüchen. Gründe für die Zurückhaltung und Selektion von Optimierungspotenzialen liegen, neben den in Kapitel 7.3.1 beschriebenen Effekten desintegrierter Gruppenarbeit, im wahrgenommenen Interessenantagonismus. Dieser bestimmt sich über das Betriebs- und Rationalisierungsverständnis der Beschäftigten. So ist es von Bedeutung, wie stark sie sich von den Rationa- 
lisierungsfolgen real oder potenziell bedroht fühlen und wie stark die eigenen Bedürfnisse, Ansprüche und Interessen vom Betrieb und seinen Akteuren verletzt oder anerkannt und erfüllt werden.

Obwohl fast alle Beschäftigten diese Diskrepanz zwischen den eigenen und den betrieblichen Interessen deutlich artikuliert haben, führt sie nur bei Status-quo-Orientierten unter (eher) restriktiven Bedingungen $\mathrm{zu}$ einer eindeutigen Haltung des arbeitspolitischen Konservatismus, der mit der strikten Ablehnung der Beteiligung an KVP verbunden ist. Status-quo-Orientierte vermeiden die offene Artikulation von Optimierungspotenzialen und erwarten von betrieblichen Veränderungen stets negative Folgen. Diese pessimistische Erwartungshaltung und das Misstrauen bleiben bei Status-quo-Orientierten und in ihrem kollektiven Bewusstsein im Übrigen auch dann präsent, wenn keine unmittelbare akute Gefahr vorliegt bzw. wenn ihnen partizipative Strukturen offenstehen. Dies kann darauf zurückzuführen sein, dass dieses spezifische Betriebs- und Rationalisierungsverständnis nicht nur aus individuellen, sondern auch aus kollektiv geteilten Erfahrungen gespeist und sozialisatorisch tradiert wird. Aufgrund dieser kumulierten und verfestigten Erfahrungen negativer Effekte von Rationalisierung rechnen Status-quo-Orientierte unter restriktiven, tayloristisch geprägten Arbeits- und Beschäftigungsbedingungen immer damit, dass betrieblich initiierte KVP-Angebote unmittelbar zulasten der Beschäftigten gehen. Dieses Verständnis findet Ausdruck in pauschaler Ablehnung und allgemeinen Vorbehalten gegenüber betrieblichen Optimierungs- und Rationalisierungsaktivitäten. Weil unter restriktiven Bedingungen hohe Leistungsanforderungen bei geringen Handlungs- und Entscheidungsspielräumen und begrenzten Aufgabenumfängen sowie hierarchische Entscheidungsprozesse und Kooperationsstrukturen herrschen, bleibt es bei einer rein rhetorischen Beteiligungsaufforderung betrieblicherseits. Die Beschäftigten erfahren im Betriebsalltag keine substanzielle Veränderung, die eine Revision der konservativistischen Haltung veranlassen könnte.

Jenseits dieser Konstellation (Status-quo-Orientierte unter restriktiven Bedingungen) kann in der Frage der Haltung der Beschäftigten gegenüber KVP allerdings nicht mehr von einem allgemeinen arbeitspolitischen Konservatismus gesprochen werden. Unter der Bedingung, dass sich die aktuellen Arbeitsund Beschäftigungsbedingungen in Summe nicht verschlechtern und mit der Intention, selbige zu verbessern, ergreifen die Beschäftigten die angebotenen Gelegenheitsstrukturen, um Einfluss auf die Arbeits- und Leistungsbedingungen zu nehmen. Mit einer Haltung des arbeitspolitischen Pragmatismus eignen sie 
sich die gegebenen - restriktiven wie partizipativen - Beteiligungsmöglichkeiten an, um zunächst ihre eigenen Interessen zu verfolgen.

Diese Interessen liegen - neben dem Schutz ihrer Arbeitskraft - vornehmlich in der Orientierung an arbeitsinhaltlichen Ansprüchen wie der Verrichtung einer guten und sinnvollen Arbeit sowie der verantwortlichen Erfüllung der ihnen zugewiesenen Arbeitsaufgabe unter den gegebenen stofflichen Bedingungen des Produktionsprozesses. Die Erreichung der Leistungsziele, etwa einer guten Qualität der Arbeitsergebnisse und eingehaltener zeitlicher Vorgaben, sind in der Produktion von der Integration vielgestaltiger arbeitsteiliger und zentral geplanter Prozesse abhängig, bei denen häufig Probleme und Fehler vorkommen können. Durch die Beteiligung an Optimierungen können die Beschäftigten einerseits Probleme im Arbeitsprozess aufzeigen, an deren Lösung mitarbeiten und Arbeitserleichterungen erzielen. Andererseits steigen durch Beteiligung an KVP die Erfahrungen und Kompetenzen der Beschäftigten sowie ihre Möglichkeiten, eigene Ansprüche an Interessantheit und Fachlichkeit der Arbeit zu erfüllen.

Die Beschäftigten befinden sich in einem Spannungsfeld zwischen Bedrohung und Gestaltung. Während Status-quo-Orientierte tendenziell primär auf das Gefährdungspotenzial blicken und sich eher konservativ beteiligen, eignen sich Mitgestaltungorientierte die Gestaltungsspielräume aktiv an und versuchen progressiv, sie auszubauen. Die Haltungen der Beschäftigten bezüglich der Beteiligung an KVP changieren im Spektrum eines konservativen bis progressiven Pragmatismus.

Tabelle 9: Arbeitspolitischer Pragmatismus

\begin{tabular}{|c|c|c|}
\hline \multicolumn{3}{|c|}{ Arbeitspolitischer Pragmatismus } \\
\hline konservativ & & progressiv \\
\hline Schutz & Beteiligungsorientierung & Mitgestaltung \\
\hline Ignorieren/Missachtung & Anerkennungsverhältnisse & Anerkennung \\
\hline stark begrenzt & Handlungsressourcen & Unterstützung \\
\hline gering & Berufliche Entwicklungsmöglichkeiten & Perspektiven \\
\hline
\end{tabular}

Eigene Darstellung 


\subsection{Reziprozität der Anerkennungsverhältnisse: KVP- Beteiligung im Spannungsfeld betrieblicher Anforderungen und subjektiver Orientierungen}

Die Reziprozität der Anerkennungsverbältnisse bildet einen zentralen Wirkmechanismus hinsichtlich der Beteiligungsbereitschaft der Beschäftigten. Reziprozität meint ein Austauschverhältnis von Leistung und Gegenleistung, welches über die im Arbeitsvertrag eingegangen Verpflichtungen hinausgeht. Diese besondere Leistung wird in der Erwartung erbracht, dass sich der Leistungsempfänger moralisch verpflichtet fühlt, eine Gegenleistung zu erbringen (Voswinkel 2008: 239). Diese Reziprozitätsverpflichtungen des Betrieb auf der einen und der Beschäftigten auf der anderen Seite können, so Voswinkel, gegenseitig aktiviert werden. Beispielsweise engagiert sich ein Karriereorientierter in besonderem Maße in der Erwartung, dass der Betrieb ihm dies mit Aufstiegsmöglichkeiten lohnt; oder der Betrieb finanziert seinen Beschäftigten Weiterbildungsmöglichkeiten in der Erwartung, dass diese sich langfristig an den Betrieb binden.

Die bisherige Rekonstruktion der Aneignungsformen hat gezeigt, dass in dem Maße, in dem die Interessen, Bedürfnisse und Ansprüche der Beschäftigten in der betrieblichen Alltagspraxis anerkannt wurden, die Beschäftigten eine höhere Bereitschaft zeigten, sich an KVP zu beteiligen. Präziser formuliert: Dort, wo die Beschäftigten bei der Lösung von Problemen, die bei der Verrichtung der Arbeit, während des Produktionsprozesses oder hinsichtlich der Produktqualität auftreten, nachhaltig und alltäglich Unterstützung von den Vorgesetzten und Servicebereichen erfahren und sich so selbst an den Problemlösungen beteiligen können, werden sie in ihren Ansprüchen und Bedürfnissen ernst genommen. Sie erleben den KVP-Beteiligungsprozess positiv und halten ihre Bereitschaft aufrecht, sich auch künftig einzubringen. Bedeutsam ist hier auch, dass es den Beschäftigten bei ihrer Beteiligung an KVP nicht nur um klassische Arbeitserleichterung durch Verbesserung der Ergonomie geht, sondern auch um die Behebung fachlicher, fertigungstechnischer und prozessualer Dysfunktionalitäten, die den reibungslosen Arbeitsprozess stören und von den Beschäftigten kompensiert werden müssen. Oftmals erfolgt dies durch Mehrarbeit oder Arbeitsintensivierung. Wenn Beschäftigte also von „ernst genommen“ oder „nicht ernst genommen werden“ sprechen, geht es nicht nur um ihre Anerkennung als wichtige Experten ihres Arbeitsbereichs sowie als Leistungs- und Kompetenzträger für den Betrieb, sondern auch um 
die Anerkennung ihrer Problemlagen und Bedürfnisse hinsichtlich der Verrichtung ihrer Arbeit, ihres Leistungsvermögens und der dafür benötigten Ressourcen. Das bedeutet, dass mit dem Beteiligungsanspruch der Beschäftigten unmittelbar arbeitsinhaltliche und Anerkennungsansprüche verknüpft sind.

Umgekehrt nehmen die Beschäftigten die fehlende Beachtung der artikulierten Probleme insbesondere unter restriktiven Bedingungen als Ignoranz oder gar Missachtung wahr. Sie fühlen sich mit ihren Problemen allein gelassen. Verstärkend wirken sich zudem beschäftigungs- und leistungspolitische Verschlechterungen oder Erlebnisse der individuellen Benachteiligung durch den Betrieb auf das subjektive Missachtungsempfinden aus. Der Aneignungsmodus der demonstrativen Ablehnung resultiert aus einer negativen Reziprozität: „Der Betrieb hat mich in meinen wichtigen Ansprüchen verletzt, also verweigere ich jedes Engagement, solange ich mein Beschäftigungsverhältnis nicht selbst gefährde.“

Die Wirtschaftlichkeitsinteressen, die der Betrieb mit den Reorganisationsmaßnahmen und der Einführung von KVP verfolgt, werden von den Beschäftigten als legitime betriebliche Interessen nicht grundsätzlich infrage gestellt. Diese Mindestakzeptanz des Wirtschaftlichkeitsstrebens des Betriebs liegt in der Ansicht begründet, dass der Betrieb so handeln müsse, um sein Fortbestehen und somit auch die Arbeitsplätze zu sichern. Ein Teil der Beschäftigten - Mitgestaltungsorientierte und insbesondere Karriereorientierte nimmt diese Interessen sogar in die eigenen arbeitsinhaltlichen Orientierungen auf. Dies gilt insbesondere für Karriereorientierte, die sich in hohem Maße an der Erfüllung betrieblicher Verhaltenserwartungen orientierten. Dazu zählt nicht nur die Ausrichtung auf Kennzahlen und deren Erreichung, sondern auch die Übernahme und Verfolgung betrieblicher Ziele zur Profitabilitätssteigerung.

Den Blick wieder auf alle Beschäftigte gerichtet, zeigt sich: Bei funktionierenden KVP-Praktiken bildet sich eine große Schnittmenge zwischen den Interessen der Beschäftigten mit jenen des Betriebs - beide Seiten sind interessiert an einem reibungslosen und qualitativ guten Produktionsprozess. Entscheidend ist vielmehr, wie der Betrieb seine Interessen umsetzt: in Anerkennung und Nutzung der Erfabrungen und Kompetenzen der Beschäftigten mittels realer Beteiligung und Unterstützung bei Optimierungen durch prozessnahe Kooperationsstrukturen - oder obne Beteiligung und zulasten der Beschäftigten.

Der Anspruch, den eigenen Arbeitsbereich und die eigenen Arbeitskontext mitzugestalten, um größeren Einfluss auf die Bedingungen der Leistungsver- 
richtung zu nehmen, ist zwar bei den Mitgestaltungsorientierten am stärksten ausgeprägt, lässt sich aber bei allen Beteiligungstypen finden. Die Erfüllung oder Missachtung des Beteiligungsanspruchs durch den Betrieb ist daher von großer Bedeutung, weil dies unmittelbar die eigenen Arbeitsbedingungen betrifft. Eine reale, alltagspraktisch wirksame Anerkennung der Beteiligungsansprüche der Beschäftigten führt dazu, dass diese sich progressiv und konstruktiv in Problemlöseprozesse einbringen.

Die Bereitschaft zur Beteiligung an KVP und das Engagement für Optimierung geht über das minimal erwartete Arbeitspensum hinaus und lässt sich - im Gegensatz zur Erbringung der vertraglich festgelegten Arbeitsleistung nicht erzwingen. Die Beteiligung an betrieblich initiierten KVP wird von den Beschäftigten teils bewusst, teils unbewusst als Verhandlungsmasse im Reziprozitätsaustausch eingesetzt, indem sie die Beteiligung verweigern, ohne direkte Folgen für ihr Beschäftigungsverhältnis befürchten zu müssen.

\section{Wirkung von Anspruchsverletzungen und -erfüllungen}

KVP-Praktiken im Rahmen restriktiver Beteiligungsstrukturen und in Form einseitiger Kostenminimierungsmaßnahmen führen zu einer besonders starken Ambivalenz der Beschäftigtenansprüche - zwischen Schutzbedürfnissen einerseits und Gestaltungswünschen andererseits. In der Frage, wie Beschäftigte mit Anspruchskonflikten umgehen, hat Nies (2015) im Rahmen von Angestelltenuntersuchungen hervorgehoben, dass die Umgangsweise mit diesen Diskrepanzen von der Wichtigkeit der eigenen Ansprüche und wahrgenommenen Legitimität der betrieblichen Anforderungen abhängt. Nicht nachvollziehbare Vorgaben und Handlungsweisen des Betriebs, die zumeist auf ökonomischen und bürokratischen Ordnungsvorstellungen beruhen, kollidieren mit den Vorstellungen der Beschäftigten von einer gut ausgeführten und gestalteten Arbeit. Dieser Widerspruch fordert eigensinnige Reaktionen bei den Beschäftigten heraus. Die technisch-funktionale Rationalität als wichtiger Bezugspunkt des eigenen Handelns ist mit spezifischen Legitimitätsvorstellungen verbunden (Kratzer et al. 2015). Übertragen auf KVP-Praktiken lassen sich diese Zusammenhänge ebenfalls rekonstruieren: Die Diskrepanz zwischen der eher technisch-funktionalen Rationalität der Beschäftigten und der ökonomischen und bürokratischen Funktionalität der Betriebsorganisation in restriktiven KVPPraktiken fordert die Beschäftigten heraus, diesen Widerspruch aufzulösen. 
Infolge andauernder wahrgenommener Ignoranz und Verletzung ihrer Ansprüche durch den Betrieb - insbesondere bei Missachtung der Beteiligungsansprüche - lassen sich zwei typische Entwicklungslinien nachzeichnen, mit dieser Anspruchsverletzung umzugehen: erstens die Revidierung und Zurïckstellung der eigenen Beteiligungsansprüche und eine Fokussierung auf die minimal notwendige Leistungserbringung. Es bildet sich eine instrumentelle bzw. ,privatistische" Arbeitsorientierung heraus, geleistet wird „Dienst nach Vorschrift“. Die sinnstiftenden Orientierungen werden auf Bestimmungsmomente außerhalb der Arbeit und des Betriebs gelenkt (Kratzer et al. 2015; Nies 2015). Dies lässt sich auch bei größeren Verletzungen der Reziprozitätserwartungen der Beschäftigten beobachten. Wenn sie für ihre Beteiligung eine bestimmte Gegenleistung erwarten, etwa eine finanzielle Beteiligung oder, bei Karriereorientierten, den betrieblichen Aufstieg, und diese Erwartungen enttäuscht werden, führt dies dazu, dass die Beschäftigte versuchen, ihre Ansprüche herunterzuschrauben. Fortan erbringen sie nurmehr die nötigsten Arbeitsleistungen. Das bedeutet aber nicht, dass arbeitsinhaltliche Orientierungen an Fachlichkeit verschwinden; wie Nies (2015) am Beispiel von Ingenieuren und Verkaufsberatern herausgearbeitet hat, bleiben sie weiterhin wichtige Bezugsgrößen für die fachlich-berufliche Vorstellung, wie die Arbeitsleistung im Produktionsprozess in richtiger Weise zu erbringen ist. Dies lässt sich in dieser Untersuchung auch an Status-quo-Orientierten nachzeichnen. Selbst wenn die instrumentalistische Arbeitsorientierung im Vordergrund steht, da die Arbeit nicht interessant ist und keinen Spaß macht, man nicht beteiligt wird oder man sich innerlich distanziert, um mit den hohen Leistungsanforderungen zurechtzukommen, bleiben fachliche und erfahrungsbasierte Vorstellungen, etwa professionelle Standards, wie auch schon bei Schumann et al. (1982) gezeigt, wichtige Handlungsorientierungen der Beschäftigten in ihrem Arbeitsvollzug.

Zweitens zeigt sich ein Anstieg des Kritikpotenzials an den betrieblichen Vorgehensweisen: Die Beschäftigten fordern, wie im Fall von Montage F, offen Unterstützung für die Verbesserung ihrer Arbeitssituation ein und erkämpfen sich Handlungsressourcen und Gestaltungsspielräume. Die Mitgestaltungsorientierten aus Montage F haben dabei nicht nur hohe Beteiligungsansprüche und ein Bewusstsein des eigenen Expertenstatus, sondern untermauern die Legitimität ihrer Ansprüche durch die Berufung auf die formalen Regeln und Aufgabenzuschnitte, die der Betrieb im Rahmen der Teamarbeit selbst erlassen und im Handbuch festgehalten hat. Welch hohen Stellenwert die Mitgestaltung der eigenen Arbeitsbedingungen haben kann, zeigt sich da- 
rin, dass die Beschäftigten in Montage F Problemlöseprozesse entwarfen und einforderten, obwobl sie sich durch die Beschäftigungspolitik und das schlechte Betriebsklima stark verletzt und missachtet fühlten. Sie begegneten der wahrgenommenen Bedrohung nicht durch Rückzug ins „Private“, sondern versuchten progressiv-pragmatisch damit umzugehen und die Bedingungen ihrer Arbeit dennoch zu gestalten.

\section{Neue Arbeitserfahrungen - neues Arbeiterbewusstsein?}

Die Wirkungen negativer Arbeitserfahrungen der Beschäftigten unter restriktiven, tayloristischen Arbeitsbedingungen auf das Arbeitsbewusstsein der Beschäftigten, die bereits Gegenstand jahrzehntelanger Arbeiterbewusstseinsforschung gewesen sind, lassen sich auch in dieser Studie eindeutig nachzeichnen. Die Ergebnisse dieser Arbeit geben aber auch Hinweise darauf, dass nicht nur negative arbeitspolitische Erfahrungen Einfluss auf die Orientierungen der Beschäftigten haben, sondern dass positive Erfahrungen mit partizipativer, strukturinnovativer Arbeitsgestaltung sich ebenfalls nachhaltig in veränderten Orientierungsmustern der Beschäftigten ausdrücken können.

Wie anhand von Linie 1 als Prototyp für partizipative, innovative Arbeitsgestaltung dargestellt, werden durch die Beteiligung an Optimierungsprozessen in integrierten und dezentralen Kooperationsstrukturen Distanzen zwischen den einzelnen betrieblichen Akteursgruppen abgebaut. Die großen Handlungsspielräume, die niederschwelligen Beteiligungsmöglichkeiten an Optimierungsprozessen durch PE, die Offenheit des Betriebs, ein breites Spektrum an Optimierungsideen zu fördern, und nicht zuletzt der hohe wahrgenommene Einfluss auf die Gestaltung ihres Arbeitsbereichs haben bei den Beschäftigten bewirkt, dass sie sich zunehmend als Rationalisierungssubjekte wahrnehmen und in einem Betrieb arbeiten, der sich sehr um das Wohl seiner Mitarbeiter kümmert. Beschäftigte empfinden den Interessenantagonismus zwischen sich und dem Betrieb als nicht mehr so eklatant, fühlen sich von den betrieblichen Rationalisierungsaktivitäten weniger stark bedroht und betrachten sich als eher als anerkannte Kompetenz- und Leistungsträger im Betrieb.

Allerdings bleiben wesentliche Bestimmungsmomente für das Bewusstsein der Beschäftigten unverändert. Die Tätigkeiten bleiben in hohem Maße insbesondere durch Schichtarbeit und hohe Leistungsvorgaben körperlich belastend. Betriebliche Verwertungskonzepte in industriellen Betrieben - auch mit innovativer Arbeitspolitik - basieren nach wie vor auf hoch arbeitsteiliger 
Funktionsdifferenzierung unterschiedlicher Aufgaben und Tätigkeiten im Herstellungsprozess, die am Ende in der Fertigung und Montage zu einem Produkt integriert werden müssen. Grundlegende Entscheidungen über Arbeitsteilung, Investitionen und Kosten, Fertigungstechnik, Produktionsprozesse, Aufgabenzuschnitte, Arbeitszeiten etc. werden überwiegend „oben " und ohne die Beteiligung der Beschäftigten getroffen. Die Über- und Unterordnungsbeziehungen zwischen den einzelnen betrieblichen Akteursgruppen bleiben bestehen und werden auch durch die Abhängigkeit der Beschäftigten von Lohnarbeit betont.

Letztlich bleibt auch die Frage, ob und inwiefern die Subjekte ihre Arbeitsorientierung durch positive Erfahrungen verändern (können), in diesem Zusammenhang noch ungeklärt. So ist einerseits denkbar, dass beispielsweise Status-quo-Orientierte durch Erfahrungen mit kooperativen Beteiligungsprozessen und entwicklungsförderlicher, betrieblicher Beschäftigungspolitik ihr innerliches Gewichtsverhältnis zugunsten arbeitsinhaltlicher, mitgestaltungsorientierter Orientierungen verschieben. Andererseits kann das Engagement bzw. das Gestaltungsstreben, welches Mitgestaltungsorientierten zu eigen ist, auch eine stabile individuelle persönliche Eigenschaft sein. Diese kann zwar durch restriktive Bedingungen gebremst, aber nicht aus Beschäftigten hervorgelockt werden, wenn sie gar nicht vorhanden ist.

Diese überaus interessanten Fragen zum Verhältnis von Struktur und Subjekt lassen sich mit dieser Untersuchung nicht abschließend beantworten, da sie sich vor allem mit den aktuellen und arbeitsbezogenen Orientierungsmustern der Produktionsbeschäftigten und weniger mit deren Genese befasst. Es bedarf eines anderen methodischen Zuschnitts und eine erweiterte Fragestellung der Fallstudie - sie müsste mit biografisch-narrativen Interviews arbeiten und als Langzeitstudie angelegt sein, die Veränderungen der Anspruchsmuster einer Person zu verschiedenen Zeitpunkten im Kontext der betrieblichen Bedingungen nachzeichnen kann. 


\subsection{Reichweite und Aussagekraft der Fallstudienergebnisse und weiterführende Perspektiven}

Die vorliegende qualitative Fallstudie kann nur ein zeitlich und örtlich begrenztes sowie von bestimmten Akteursgruppen geprägtes Bild zeichnen, was Reichweite, Übertragbarkeit und Aussagekraft der Befunde einschränkt und offene Fragen hinterlässt. Die Untersuchungsergebnisse gelten zunächst für männliche, festangestellte Produktionsbeschäftigte in Montage- und Fertigungsbereichen, die zur Stammbelegschaft zweier traditionsbewusster Betriebe in Österreich gehören und in internationale Unternehmensstrukturen eingebundenen sind. NF und WLF lassen sich mit Blick auf den arbeitspolitischen Gestaltungsansatz und den Einsatz beteiligungsorientierter Ganzheitlicher Produktionssysteme hinsichtlich der implementierten Arbeitsorganisation, Kooperationsstrukturen und KVP als typische Bespiele für Ganzheitliche Produktionssysteme einordnen (Gerst 2010; 2012b; Schwarz-Kocher et al. 2012; Kötter et al. 2016; Seibold et al. 2016). Ebenso scheint die Vernachlässigung der Entwicklung problemorientierter und prozessnaher Kooperationsund Kommunikationsstrukturen zwischen Produktion und Servicebereichen, um zeitnah und problemadäquat auf die Optimierungspotenziale der Beschäftigten einzugehen, für Industriebetriebe keine Seltenheit zu sein. In neueren subjektorientierten Studien und Untersuchungen zur Auswirkung posttayloristischer Arbeitsgestaltung lassen sich ebenfalls verletzte Beteiligungsansprüche durch unzureichende Beachtung der Erfahrungen und Kompetenzen der Produktionsbeschäftigten (Kratzer et al. 2015), geringe Beteiligungsraten der Beschäftigten an KVP (Kleinwechter 2014) und eine Gleichzeitigkeit von partieller Re-Taylorisierung und Ganzheitlicher Gestaltungsansätze nachzeichnen (Sauer 2005b; 2005a; Hildebrandt et al. 2007; Gerst 2012a; Jürgens, U. 2017). Aufgrund fehlender weiterer Studien zu Verbreitung, Art der Gestaltung und letztlich Auswirkungen von Ganzheitlichen Produktionssystemen lassen sich NF und WLF jedoch (noch) nicht abschließend einordnen. Vielmehr lassen sich an diesen beiden untersuchten Fällen wichtige Zusammenhänge zwischen 
arbeitspolitischer Gestaltung und subjektiver Aneignung einige wichtige $\mathrm{Zu}$ sammenhänge verdeutlichen. ${ }^{124}$

Aufgrund der ohnehin großen Komplexität der Fragestellung wurden die subjektiven Bestimmungsmomente vor allem auf der Mikroebene der spezifische Arbeits- und Betriebsbedingungen betrachtet, während lebensweltliche und biografische Bezüge, die sicherlich ebenfalls bedeutenden Einfluss auf die Aneignungsmodi nehmen können, ${ }^{125}$ kaum berücksichtigt wurden. So steht beispielsweise auch die Frage aus, ob sich durch kulturelle oder regionalen Lebensweisen spezifische Formen des Arbeiterbewusstseins herausgebildet haben und in welcher Weise diese verallgemeinerungsfähig sind. Besonderheiten des Standorts liegen hier zum Beispiel in dem spezifischen Bewusstsein einer über 150 Jahre zurückreichenden Industrie- und Fabrikgeschichte und einer wirkmächtigen sozialdemokratischen Gewerkschafts- und Interessenvertretungskultur. Diese Aspekte werden als Elemente einer spezifischen regionalen Arbeiteridentität von einigen Beschäftigten (aber nicht von der ganzen Belegschaft) hochgehalten und reproduziert. Zudem können die österreichischen Interessenvertretungsstrukturen, bei denen Arbeiter- und Angestelltenbetriebsräume getrennte Körperschaften im Betrieb sind, auch Einfluss auf die Ausgestaltung innerbetrieblicher Verhältnisse haben. So könnte die Aneignungsform des „aktiven Ergreifens von Gestaltungsspielräumen“, welche mit einem hohen Kritikpotenzial und einem starkem Gestaltungswillen der Mitgestaltungsorientierten verbunden ist, auch als eine spezifische Ausprägung verstanden werden, die sich im Kontext einer starken Arbeiter- und Interessenvertretungstradition bei NF herausgebildet hat. Um diese Aneignungsmodi besser verorten zu können, wären weitere subjektorientierte Untersuchungen von Arbeiterorientierungen unter besonderer Berücksichtigung von außerbe-

${ }^{124}$ Dabei gilt es einige Besonderheiten zu beachten. So muss berücksichtigt werden, dass wichtige Veränderungen, wie Arbeitsorganisation, Vermarktlichung und indirekte Steuerung, den etwas eingehegten bzw. abgeschiedenen Standort erst wesentlich später als die deutschen Automobil- und Maschinenbauunternehmen erreicht haben.

125 Am deutlichsten arbeiten Brock/Vetter (1986) regionalspezifische Differenzen im Arbeiterbewusstsein von Industriearbeitern hervor: Im Zuge der Einführung von Robotern in einem niederbayrischen Automobilwerk war im Arbeiterbewusstsein nur ein sehr geringes Kritikpotenzial gegenüber diesem Wandel festzustellen. Die Arbeiter kompensierten den starken Einschnitt mit der Fokussierung auf Landwirtschaft als Nebenerwerb. 
trieblich-lebensweltlichen Orientierungen und gesellschaftlichen Bewusstsein hilfreich. 



\section{Literatur}

Ackroyd, Stephen / Thompson, Paul (1999): Organizational Misbehaviour, London: SAGE.

Antoni, Conny Herbert (Hg.) (1994): Gruppenarbeit in Unternehmen Konzepte, Erfahrungen, Perspektiven, Weinheim: Beltz, PVU. Appelbaum, Eileen / Bailey, Thomas / Berg, Peter / Kalleberg, Arne (2000): Manufacturing Advantage: Why High-Performance Work Systems Pay Off Ithaca (NY): Cornell University Press.

Baethge, Martin (1991): Arbeit, Vergesellschaftung, Identiät - Zur zunehmenden normativen Subjektivierung der Arbeit, In: Soziale Welt, 30.06.1998, Jg. 42, Nr. 1, S. 6-19.

Behrens, Martin / Hardwig, Thomas / Kädtler, Jürgen (1997): Die neue Rolle und Funktion der Meister in Großbetrieben, In: FuchsFrohnhofen / Paul / Henning, Klaus (Hgg.): Die Zukunft des Meisters in modernen Arbeits- und Produktionskonzepten, Band II, München und Mering: Hampp, S. 13-56.

Berger, Johannes (1995): Warum arbeiten die Arbeiter? Neomarxistische und neodurkheimianische Erklärungen, In: Zeitschrift für Soziologie, Jg. 24, Nr. 6, S. 407-421.

Birkmayer, Suzanne / Dannenmaier, Robert / Matlasek, Sabine / Weibert, Wolfgang (2009): six sigma toolkit. Der DMAIC Zkylus in 15 Schritten. Die wichtigsten Werkzeuge in kompakter Form: ifss institut for six sigma.

Boes, Andreas / Pfeiffer, Sabine (Hgg.) (2005): Informationsarbeit neu verstehen. Methoden zur Erfassung informatisierter Arbeit, München: ISF München.

Böhle, Fritz / Pfeiffer, Sabine / Sevsay-Tegethoff, Nese (Hgg.) (2004): Die Bewältigung des Unplanbaren, Wiesbaden: VS Verlag für Sozialwissenschaften.

Böhle, Fritz (2010): Arbeit als Handeln, In: Böhle, Fritz / Voß, G. Günter / Wachtler, Günther (Hgg.): Handbuch Arbeitssoziologie, Wiesbaden: VS Verlag Sozialwissenschaften, S. 151-176. 
Böhle, Fritz (Hg.) (2017): Arbeit als Subjektivierendes Handeln. Handlungsfähigkeit bei Unwägbarkeiten und Ungewisstheiten, Wiesbaden: Springer.

Bohnsack, Ralf (2001): Dokumentarische Methode. Theorie und Praxis wissenssoziologischer Interpretation, In: Hug, Theo (Hg.): Wie kommt Wissenschaft zu Wissen?, Baltmannsweiler: Schneider Verlag, S. 326-345.

Bohnsack, Ralf (2003): Rekonstruktive Sozialforschung. Einführung in qualitative Methoden, 5. Aufl., Opladen: Leske und Budrich.

Bokranz, Rainer / Landau, Kurt (2012): Handbuch Industrial Engineering. Produktivitätsmanagement mit MTM, Stuttgart: Schäffer-Poeschel.

Boxall, Peter (2012): High-performance work systems: what, why, how and for whom?, In: Asia Pacific, Jg. 50, Nr. 2, S. 169-186.

Braverman, Harry (1977): Die Arbeit im modernen Produktionsprozeß, Frankfurt/Main: Campus.

Brock, Ditmar / Vetter, Hans-Rolf (1982): Alltägliche Arbeiterexistenz, Frankfurt/Main: Campus.

Brock, Ditmar / Vetter, Hans-Rolf (1986): Technische Dynamik und soziale Beharrung - Zur subjektiven Perspektive des Robotereinsatzes in einem Automobilwerk, In: Soziale Welt, Jg. 37, Nr. 2/3, S. 208-236.

Bröckling, Ulrich (2007): Das unternehmerische Selbst, Frankfurt/Main: Suhrkamp.

Burawoy, Michael (1979): Manufacturing Consent - Changes in the Labor Process under Monopoly Capitalism, Chicago: University of Chicago Press.

Combs, James / Liu, Yongmei / Hall, Angela / Ketchen, David (2006): How Much Do High-Performance Work Practices Matter? A Meta-Analysis of their Effects on Organizational Performance, In: Personnel Psychology, Jg. 59, Nr. 3, S. 501-528.

Dahm, Markus H. / Haindl, Christoph (2009): Lean Management und Six Sigma - Qualität und Wirtschaftlichkeit in der Wettbewerbsstrategie, Berlin: Erich Schmidt.

Deppe, Frank (1971): Das Bewußtsein der Arbeiter. Studien zur politischen Soziologie des Arbeiterbewußtseins, Köln: PRVPahl-Rugenstein-Verlag 
Deutschmann, Christoph (2002): Postindustrielle Industriesoziologie. Theoretische Grundlagen, Arbeitsverhältnisse und soziale Identitäten, Weinheim: Juventa.

Döhl, Volker / Kratzer, Nick / Sauer, Dieter (2000): Krise der NormalArbeit(s)Politik. Entgrenzung von Arbeit - neue Anforderungen an Arbeitspolitik, In: WSI-Mitteilungen, Jg. 53, Nr. 1, S. 5-17.

Dombrowski, Uwe / Palluck, Markus / Schmidt, Stefan (2006):

Strukturelle Analyse Ganzheitlicher Produktionssysteme, In: Zeitschrift für wirtschaftlichen Fabrikbetrieb, Jg. 101, S. 114118.

Dombrowski, Uwe / Mielke, Tim (Hgg.) (2015): Ganzheitliche Produktionssysteme Berlin: Springer.

Dörre, Klaus / Neubert, Jürgen / Wolf, Harald (1993): „New Deal” im

Betrieb? Unternehmerische Beteiligungskonzepte und ihre Wirkung auf die Austauschbeziehungen zwischen Management, Belegschaften und Interessenvertretung, In: SOFI-Mitteilungen, 25.01.2000, Nr. 20, S. 15-36.

Dörre, Klaus / Neubert, Jürgen (1995): Neue Managementkonzepte: Aushandlungsbedarf statt "Sachzwang Reorganisation", In: Scheyögg, Georg / Sydow, Jörg (Hgg.): Managementforschung 5, Berlin: de Gruyter, S. 167-214.

Dörre, Klaus (2001a): Das deutsche Produktionsmodell unter dem Druck des Shareholder Value, In: Kölner Zeitschrift für Soziologie und Sozialpsychologie, Jg. 53, Nr. 4, S. 675-704.

Dörre, Klaus (2001b): Partizipation im Arbeitsprozess - Alternative oder Ergänzung zur Mittbestimmung?, In: Industrielle Beziehungen, Jg. Jg. 8, Nr. Heft 4, S. 379-407.

Dörre, Klaus (2002): Kampf um Beteiligung. Arbeit, Partizipation und industrielle Beziehungen im flexiblen Kapitalismus, Wiesbaden: Westdeutscher Verlag.

Dörre, Klaus / Happ, Anja / Matuschek, Ingo (2013): Das Gesellschaftsbild der LohnarbeiterInnen. Soziologische Untersuchungen in ost- und westdeutschen Industriebetrieben, Hamburg: VSA.

Edwards, Richard (1981): Herrschaft im modernen Produktionsprozess, Frankfurt: Campus. 
Emery, Fred / Thorsrud, Einar (1982): Industrielle Demokratie. Bericht über das norwegische PRogramm der industriellen Demokratie, Stuttgart: Huber.

Esser, Hartmut (1991): Die Rationalität des Alltagshandelns. Eine Rekonstruktion der Handlungstheorie von Alfred Schütz, In: Zeitschrift für Soziologie, Jg. 20, Nr. 6, S. 430-445.

Faust, Michael (2002): Der "Arbeitskraftunternehmer" - eine Leitidee auf dem ungewissen Weg der Verwirklichung, In: Kuda, Eva / Strauß, Jürgen (Hgg.): Arbeitnehmer als Unternehmer? Herausforderungen für Gewerkschaften und berufliche Bildung, Hamburg: VSA, S. 56-80.

Frerichs, Melanie (2014): Innovationsprozesse und organisationaler Wandel in der Automobilindustrie., Wiesbaden: Springer VS.

Frey, Michael (2009): Autonomie und Aneignung in der Arbeit. Eine soziologische Untersuchung zur Vermarktlichung und Subjektivierung von Arbeit, München: Hampp.

Friedman, Andrew (1977): Industry and Labour - Class Struggle at Work and Monopoly Capitalism, Basingstoke: Macmillan.

Gerst, Detlef / Hardwig, Thomas / Kuhlmann, Martin / Schumann, Michael (1994): Gruppenarbeit in der betrieblichen Erprobung - ein "Modell" kristallisiert sich heraus., In: angewandte Arbeitswissenschaft, 13.05.1998, Nr. 142, S. 5-30.

Gerst, Detlef / Hardwig, Thomas / Kuhlmann, Martin / Schumann, Michael (1995): Gruppenarbeit in den 90ern: Zwischen strukturkonservativer und strukturinnovativer Gestaltungsvariante, In: SOFI-Mitteilungen, Jg. 22, S. 39-64.

Gerst, Detlef (1998a): Selbstorganisierte Gruppenarbeit Gestaltungschancen und Umsetzungsprobleme, Eschborn: RKW.

Gerst, Detlef (1998b): Selbstorganisierte Gruppenarbeit. Gestaltungskonzepte und Umsetzungsprobleme, Eschborn: RKW.

Gerst, Detlef (1999a): Das Ende der selbstorganisierten Gruppenarbeit? Arbeitsgestaltung in der standardisierten Montage, In: SOFIMitteilungen, 14.04.1999, Nr. 27, S. 49-58. 
Gerst, Detlef (1999b): Gestaltungskonzepte für die manuelle Montage: Selbstorganisierte versus standardisierte Gruppenarbeit?, In: angewandte Arbeitswissenschaft, 30.11.1999.

Gerst, Detlef (2000): Arbeitspolitik im Rückwärtsgang? Konzeptionskonkurrenz und Wandel von Kontrolle in der Automobilindustrie, In: WSI-Mitteilungen, 09.05.2001, Jg. 53, Nr. 1, S. 37-45.

Gerst, Detlef (2004): Industrielle Gruppenarbeit und der Leittypus des Arbeitskraftunternehmers, In: Pongratz, Hans / Voß, G. Günterq (Hgg.): Typisch Arbeitskraftunternehmer ?, Berlin: Edition Sigma, S. 187-208.

Gerst, Detlef (2010): Ganzheitliche Produktionssysteme - Mitgestaltung ohne Co-Management, In: Schwitzer, Helga / Ohl, Kay / Rohnert, Richard / Wagner, Hilde (Hgg.): Zeit, dass wir was drehen! Perspektiven der Arbeitszeit- und Leistungspolitik, Hamburg: VS, S. 201-216.

Gerst, Detlef (2012a): Wie nachhaltig sind ganzheitliche Produktionssysteme?, In: Schröder, Lothar / Urban, HansJürgen (Hgg.): Gute Arbeit. Zeitbombe Arbeitsstress Befuunde, Strategien, Regelungsbedarf, Frankfurt/Main: Bund S. 201-216.

Gerst, Detlef (2012b): Produktionssysteme wirtschaftlich und menschengerecht gestalten, In: Arbeitswissenschaft), GfA (Gesellschaft für (Hg.): Angewandte Arbeitswissenschaft für kleine und mittelständische Unternehmen. Tagungsband zur Herbstkonferenz 2012 der Gesellschaft für Arbeitswissenschaft, Dortmund: GfA Press, S. 101-112.

Glaser, Barney G. / Strauss, Anselm L. (1998): Grounded theory. Strategien qualitativer Forschung, Bern, Göttingen, Toronto, Seattle: Huber.

Goldthorpe, John / Lockwood, David / Becherhofer, Frank / Platt, Jennifer (1968): The Affluent Worker: Industrial Attitudes and Behavior, Cambridge.

Goldthorpe, John / Lookwood, David / Bechhofer, Frank / Platt, Jennifer (1970): Der "wohlhabende"Arbeiter in England, München: Goldmann. 
Hack, Lothar / Krause, Wulf / Schmidt, Ute / Wachutka, Werner (1972): Klassenlage und Interessenorientierung. Zum Konstitutionsprozeß der Bewußtseinsstrukturen und Verhaltensmuster junger Industriarbeiter, In: Zeitschrift für Soziologie, Jg. 1, Nr. 1, S. 15-30.

Hack, Lothar / Brose, Hanns-Georg / Czasny, Ingrid / Hager, Frithjof / Moser, Roland / Viesel, Karin (1979): Leistung und Herrschaft. Soziale Strukturzusammenhänge subjektiver Relevanz bei jüngeren Industriearbeitern, Frankfurt/Main: Campus.

Heil, Barbara / Kuhlmann, Martin (2013): Produktionssysteme sind gestaltbar. Von Lean Production zu Produktionssystemen: analytische Perspektiven und eine Fallstudie., In: Mitteilungen aus dem SOFI, Juli 2013, Jg. 7, Nr. 18, S. 1-5.

Heil, Barbara / Kuhlmann, Martin / Menrad, Martin (2014): Perspektiven der Gestaltung von Produktionssystemen konzeptionelle Überlegungen und Fallstudienbefunde, In: Engelhardt-Nowitzki, Corinna / Blahota, Johannes (Hgg.): Forschungsinitiative AGTIL 2014. Beiträge zur Stärkung der Veränderungsfähigkeit von Unternehmen, Shaker Verlag: Aachen, S. 45-63.

Heil, Barbara / Kuhlmann, Martin (2016): "Die da oben, wir hier unten" - Arbeits- und Betriebsverständnis von Industriearbeitern, In: WSI Mitteilungen, Jg. 7, Nr. Schwerpunktheft "Gerechtigkeitsansprüche und ARbeiternehmerbewusstsein heute - neue Ansätze, neue Befunde", S. 521-529.

Heil, Barbara / Wolf, Harald (2017): Arbeiterbewusstsein, In: HirschKreiensen, Hartmut / Minssen, Heiner (Hgg.): Lexikon der Arbeits- und Industriesoziologie, Baden-Baden: Edition Sigma in der Nomos-Verlagsgesellschaft, S. 39-42.

Hildebrandt, Eckart / Jürgens, Ulrich / Oppen, Maria / Teipen, Christina (Hgg.) (2007): Arbeitspolitik im Wandel. Entwicklung und Perspektiven der Arbeitspolitik, Berlin: edition sigma.

Holtgrewe, Ursula / Voswinkel, Stephan / Wagner, Gabriele (Hgg.) (2000): Anerkennung und Arbeit, Konstanz: UVK. 
Hopf, Christel (2009): Qualitative Interviews - ein Überblick, In: Flick, Uwe / von Kardorff, Ernst / Steinke, Ines (Hgg.): Qualitative Forschung. Ein Handbuch, Reinbeck bei Hamburg: Rowohlt Taschenbuch Verlag, S. 349-360.

Howaldt, Jürgen (1993): Vom Objekt zum Subjekt der Rationalisierung? Der kontinuierliche Verbesserungsprozess als beteiligungsorientierter Rationalisierungsansatz, In: Howaldt, Jürgen / Minssen, Heiner (Hgg.): Lean, leaner...? Die Veränderung des Arbeitsmanagements zwischen Humanisierung und Rationalisierung, Dortmund: Montania, S. 125-140.

Howaldt, Jürgen (1994): KVP-Aktivitäten in Deutschland - Eine Befragung von Betriebsräten der Automobil- und Automobilzulieferindustrie, In: Arbeit, 16.01.2001, Jg. 3, Nr. 4, S. 320-330.

Howaldt, Jürgen (1995): Zum Stand der Einführung von KVPAktivitäten. Fünf Fallstudien aus der Atuomobil- und Zuliefererindustrie, Dortmund: Sozialforschungsstelle Dortmund.

Howaldt, Jürgen / Kopp, Ralf (1997): Unterschiedliche KVP-Strategien der Unternehmen der Metall- und Elektroindustrie als Herausforderung für die Betriebsratsarbeit. Endbericht, Dortmund: Sozialforschungsstelle Dortmund.

Howaldt, Jürgen / Kopp, Ralf / Winther, Michael (Hgg.) (1998): Kontinuierlicher Verbesserungsprozeß - KVP als Motor lernender Organisation, Köln: Wirtschaftsverlag Bachem.

Hürtgen, Stefanie / Voswinkel, Stephan (2016): Ansprüche an Arbeit und Leben - Beschäftigte als soziale Akteure, In: WSIMitteilungen, Jg. 7/2016, S. 503-512.

Imai, Masaaki (1992): Kaizen. Der Schlüssel zum Erfolg im Wettbewerb, München: econ.

Iwer, Frank / Ohl, Kay / Wagner, Hilde (2008): Arbeit und Leistung. Entwicklungen und Perspektiven in einem Kernfeld der Betriebs- und Tarifpolitik, In: Wagner, Hilde (Hg.): Arbeit und Leistung - gestern und heute. Ein gewerkschaftliches Politikfeld, Hamburg: VSA, S. 227-249. 
Jauch, Peter (1997): Industriemeister und industrielle Reorganisation, München und Mering: Hampp.

Jürgens, Kerstin (2006a): Arbeits- und Lebenskraft. Reproduktion als eigensinnige Grenzziehung, Wiesbaden: VS.

Jürgens, Ulrich (1997): Rolling Back Cycle Times: The Renaissance of the Classical Assembly Line in Final Assembly, In: Shimokawa, Koichi / Jürgens, Ulrich / Fujimoto, Takahiro (Hgg.): Transforming Automobile Assembly, Berlin: Springer, S. 255273.

Jürgens, Ulrich (2006b): Weltweite Trends in der Arbeitsorganistion, In: Clement, Ute / Lacher, Michael (Hgg.): Produktionssysteme und Kompetenzerwerb. $\mathrm{Zu}$ den Veränderungen moderner Arbeitsorganisation und ihren Auswirkungen auf die berufliche Bildung, Stuttgart: Franz Steiner, S. 15-29.

Jürgens, Ulrich (2013): Aktueller Stand von Produktionssystemen - ein globaler Überblick, In: Angewandte Arbeitswissenschaften, Jg. 176, S. 25-36.

Jürgens, Ulrich (2017): Lean Production/Toyotismus, In: HirschKreiensen, Hartmut / Minssen, Heiner (Hgg.): Lexikon der Arbeits- und Industriesoziologie, Baden-Baden: edition sigma in der Nomos Verlagsgesellschaft, S. 204-207.

Kelle, Udo / Kluge, Susann (2010): Vom Einzelfall zum Typus. Fallvergleich und Fallkontrastierung in der qualitativen Sozialforschung, 2., überarb. Aufl., Wiesbaden VS Verlag für Sozialwissenschaften.

Kern, Horst / Schumann, Michael (1970): Industriearbeit und Arbeiterbewußtsein, Frankfurt/Main: EVA.

Kern, Horst / Schumann, Michael (1977): Industriearbeit und Arbeiterbewusstsein, Frankfurt: Suhrkamp.

Kern, Horst / Schumann, Michael (1984): Das Ende der Arbeitsteilung? Rationalisierung in der industriellen Produktion, München: Beck.

Kirner, Eva / Armbruster, Heidi / Kinkel, Steffen (2006): Kontinuierlicher Verbesserungsprozess - Baustein zur Prozessinnovation in KMU, In: Frauenhofer ISI PI Mitteilung, Jg. 40, S. 1-12. 
Kißler, Leo (Hg.) (1996): Toyotismus in Europa. Schlanke Produktion und Gruppenarbeit in der deutschen und französischen Automobilindustrie, Frankfurt / New York: Campus.

Kleemann, Frank / Matuschek, Ingo / Voß, Günter (1999): Zur Subjektivierung von Arbeit, Berlin: WZB.

Kleemann, Frank / Matuschek, Ingo / Voß, Günter (2002): Subjektivierung von Arbeit. Ein Überblick zum Stand der Diskussion, In: Moldaschl, Manfred / Voß, Günter (Hgg.): Subjektivierung von Arbeit (2., überarb. und erw. Aufl.), München und Mering: Hampp, S. 57-114.

Kleemann, Frank / Voß, Günter (2010): Arbeit und Subjekt, In: Böhle, Fritz / Voß, Günter / Wachtler, Günther (Hgg.): Handbuch Arbeitssoziologie, Wiesbaden: VS, S. 415-450.

Kleemann, Frank (2012): Subjektivierung von Arbeit - Eine Reflexion zum Stand des Diskurses, In: AIS-Studien, Jg. Jg. 5, Nr. Heft 2, S. 6-20.

Kleinwechter, Tobias (2014): Erwerbsorientierung und Teamarbeit: eine qualitative Untersuchung zur Wirkung von subjektiven Dispositionen auf die Akzeptanz von neuen betrieblichen Anforderungen, Jena: Universität.

Kocyba, Hermann / Vormbusch, Uwe (2000): Partizipation als Managementstrategie. Gruppenarbeit und flexible Steuerung in Automobilindustrie und Maschinenbau, Frankfurt: Campus.

Kötter, Wolfgang (2007): Gute Beispiele, wenig Nachahmer. Große ungenutzte potenziale für partizipative Produktionsrationalisierung, In: Peter, Gerd (Hg.): Grenzkonflikte der Arbeit. Die Herausbildung einer neuen europäischen Arbeitspolitik, Hamburg: VSA, S. 117-128.

Kötter, Wolfgang / Schwarz-Kocher, Martin / Zanker, Christoph (Hgg.) (2016): Balanced GSP. Ganzheitliche Produktionssysteme mit stabil-flexiblen Standards und konsequenter Mitarbeiterorientierung, Wiesbaden: Springer.

Kratzer, Nick / Menz, Wolfgang / Nies, Sarah / Sauer, Dieter (2008): Leistungspolitik als Feld "umkämpfter Arbeit", In: Prokla, Nr. 150, S. 11-26.

Kratzer, Nick / Menz, Wolfgang / Tullius, Knut / Wolf, Harald (2015): Legitimationsprobleme in der Erwerbsarbeit: 
Gerechtigkeitsansprüche und Handlungsorientierungen in Arbeit und Betrieb, Baden-Baden: Nomos.

Kudera, Werner / Mangold, Werner / Ruff, Konrad / Schmidt, Rudi / Wentzke, Theodor (1979): Gesellschaftliches und politisches Bewußtsein von Arbeitern, Frankfurt/Main: EVA.

Kudera, Werner / Ruff, Konrad / Schmidt, Rudi (1983): Soziale Lage und Bewußtsein von Arbeitern, In: Littek, Wolfgang / Rammert, Werner / Wachtler, Günther (Hgg.): Einführung in die Arbeits- und Industriesoziologie, Frankfurt/Main: Campus, S. 269-283.

Kuhlmann, Martin / Schumann, Michael (2000): Was bleibt von der Arbeitersolidarität? Zum Arbeits- und Betriebsverständnis bei innovativer Arbeitspolitik, In: WSI-Mitteilungen, 14.02.2000, Jg. 53, Nr. 1, S. 18-27.

Kuhlmann, Martin (2002): Beobachtungsinterview, In: Kühl, Stefan / Strodtholz, Petra (Hgg.): Methoden der Organisationsforschung, Reinbek: Rowohlt, S. 103-138.

Kuhlmann, Martin (2004): Modellwechsel? Die Entwicklung betrieblicher Arbeits- und Sozialstrukturen in der deutschen Automobilindustrie, Berlin: edition sigma.

Kuhlmann, Martin / Sperling, Hans Joachim / Balzert, Sonja (2004): Konzepte innovativer Arbeitspolitik. Good-Practice-Beispiele aus dem Maschinenbau, der Automobil-, Elektro- und Chemischen Industrie, Berlin: edition sigma.

Kuhn, Thomas (1997): Vom Arbeitnehmer zum Mitunternehmer. Anmerkungen zur Intention, Begründung und Umsetzung eines Transformationsvorhabens, In: Zeitschrift für Personalforschung, Jg. 11, Nr. 2, S. 195-220.

Lacher, Michael / Neumann, Dietmar / Rubelt, Jürgen / Schuler, Michael (1989): Leben - Arbeiten - Lernen. Entwurf einer biographisch begründeten Bildungstypologie von Montagearbeitern/innen, In: Meyer-Dohm, Peter / Lacher, Michael / Rubelt, Jürgen (Hgg.): Produktionsarbeiter in angelernten Tätigkeiten Eine Herausforderung für die Bildungsarbeit, Frankfurt am Main: Campus, S. 64-118. 
Langfeldt, Bettina (2009): Subjektorientierung in der Arbeits- und Industriesoziologie. Theorien, Methoden und Instrumente zur Erfassung von Arbeit und Subjektivitität, Wiesbaden: VS.

Lay, Gunter / Dreher, Carsten / Kinkel, Steffen (1996): Neue Produktionskonzepte leisten einen Beitrag zur Sicherung des Standorts Deutschland (PI-Mitteilungen 1), Karlsruhe: Fraunhofer ISI.

Lay, Gunter / Neuhaus, Ralf (2005): Ganzheitliche Produktionssysteme (GPS) - Fortführung von Lean Production?, In: Angewandte Arbeitswissenschaft, Nr. 185, S. 32-47.

Lazonick, William (2006): Corporate Governance, Innovative Enterprise, and Economic Development, In: UNU-WIDER Working-Paper. Research Paper Nr. 2006/71.

Leu, Hans Rudolf (1989): Wechselwirkungen. Die Einbettung von Subjektivität in die Alltagspraxis, In: Brock, Ditmar / Leu, Hans Rudolf / Preiß, Christine / Vetter, Hans-Rolf (Hgg.): Subjektivität im gesellschaftlichen Wandel. Umbrüche im beruflichen Sozialisationsprozess, München: DJI Verlag Deutsches Jugendinstitut, S. 36-58.

Liebold, Renate / Trinczek, Rainer (2002): Experteninterview, In: Kühl, Stefan / Strodtholz, Petra (Hgg.): Methoden der Organisationsforschung. Ein Handbuch, Reinbek bei Hamburg: Rowohlt Taschenbuch, S. 32-71.

Liker, Jeffrey (2004): The Toyota Way. 14 Management Principles from the World's greatest Manufacturer, New York: McGraw-Hill.

Lohr, Karin (2003): Subjektivierung von Arbeit. Ausgangspunkt einer Neuorientierung der Industrie- und Arbeitssoziologie?, In: Berliner Journal für Soziologie, Jg. 13, Nr. 4, S. 511-529.

Lohr, Karin / Nickel, Hildegard (2005): Subjektivierung von Arbeit riskante Chancen: das Paradoxon von erweiterter Teilhabe und sozialen Verwundbarheit, Münster: Westfälisches Dampfboot.

Lohr, Karin (2017a): Subjektivierung von Arbeit, In: Hirsch-Kreiensen, Hartmut / Minssen, Heiner / Bohn, Rainer (Hgg.): Lexikon der Arbeits- und Industriesoziologie, Baden-Baden: Edition Sigma (Nomos), S. 281-285. 
Lohr, Karin (2017b): Subjektivierung von Arbeit In: Hirsch-Kreiensen, Hartmut / Minssen, Heiner (Hgg.): Lexikon der Arbeits- und Industriesoziologie Baden-Baden: Nomos, S. 281-285.

Lutz, Burkart (1988): Qualifizierte Gruppenarbeit - Überlegungen zu einem Orientierungskonzept technisch-organisatorischer Gestaltung., In: Roth, Siegfried / Kohl, Heribert (Hgg.): Perspektive: Gruppenarbeit, Köln: Bund-Verlag, S. 68-78.

Mannheim, Karl (1980): Strukturen des Denkens, Frankfurt am Main: Suhrkamp.

Marrs, Kira (2010): Herrschaft und Kontrolle in der Arbeit, In: Böhle, Fritz / Voß, Günter / Wachtler, Günther (Hgg.): Handbuch Arbeitssoziologie, Wiesbaden: VS, S. 331-356.

Marx, Karl (1867): Das Kapital. Kritik der politischen Ökonomie. Erster Band: Der Produktionsprozess des Kapitals. MarxEngels-Werk (MEK), Berlin: Dietz Verlag [1969].

Marx , Karl (2009 [1872]): Das Kapital. Kritik der politischen Ökonomie, Köln: Anaconda.

Matthiesen, Ulf / Neuendorf, Hartmut (1989): Reproduktionsansatz oder Deutungsmusteranalyse, In: Brock, Ditmar / Leu, Hans Rudolf / Preiß, Christine / Vetter, Hans-Rolf (Hgg.): Subjektivität im gesellschaftlichen Wandel, München: DJI Verlag Deutunges Jugendinstitut, S. 77-99.

Matthöfer, Hans (1980): Humanisierung der Arbeit und Produktivität in der Industriegesellschaft, Köln: Bund.

Menz, Wolfgang (2009): Die Legitimität des Marktregimes: Leistungsund Gerechtigkeitsorientierung in neuen Formen betrieblicher Leistungspolitik, Wiesbaden: VS Verlag für Sozialwissenschaften.

Mikl-Horke, Gertraude (2007): Industrie- und Arbeitssoziologie, München: Oldenbourg.

Minssen, Heiner (1999): Direkte Partizipation contra Mitbestimmung? Herausforderung durch diskursive Koordinierung, In: MüllerJentsch, Walther (Hg.): Konfliktpartnerschaft, München und Mering: Hampp, S. 129-156.

Moldaschl, Manfred / Schultz-Wild, Rainer (1994a): Arbeitsorientierte Rationalisierung, In: Moldaschl, Manfred / Schultz-Wild, Rainer (Hgg.): Arbeitsorientierte Rationalisierung. 
Fertigungsinseln und Gruppenarbeit im Maschinenbau,

Frankfurt/New York: Campus Verlag, S. 9-31.

Moldaschl, Manfred / Schultz-Wild, Rainer (Hgg.) (1994b):

Arbeitsorientierte Rationalisierung - Fertigungsinseln und

Gruppenarbeit im Maschinenbau, Frankfurt/Main: Campus.

Moldaschl, Manfred / Sauer, Dieter (2000): Internalisierung des

Marktes - Zur neuen Dialektik von Kooperation und

Herrschaft, In: Minssen, Heiner (Hg.): Begrenzte

Entgrenzungen - Wandlungen von Organisation und Arbeit, Berlin: edition sigma, S. 205-224.

Moldaschl, Manfred (2002): Das Subjekt als Objekt der Begierde - Die

Perspektive der "Subjektivierung von Arbeit", In:

Managementforschung, Jg. 12, S. 245-280.

Moldaschl, Manfred / Voß, Günter (2002): Subjektivierung von Arbeit, München und Mering: Rainer Hampp.

Moldaschl, Manfred (2010): Organisierung und Organisation von Arbeit, In: Böhle, Fritz / Voß, Günter / Wachtler, Günter (Hgg.): Handbuch Arbeitssoziologie, Wiesbaden: VS Verlag, S. 263-299.

Neuendorf, Hartmut / Sabel, Charles (1976): Zur relativen Autonomie der Deutungsmuster, In: Bolte, Karl Mar'tin (Hg.): Materialien aus der soziologischen Forschung. Verhandlungen des 18. Deutschen Soziologentages vom 28. September bis 1. Oktobr 1976 in Bielefeld. , Darmstad: Luchterhand, S. 842-863.

Nickel, Hildegard / Hünning, Hasko / Frey, Michael (2008): Subjektivierung, Verunsicherung, Eigensinn. Auf der Suche nach Getaltungspotenzialen für eine neue Arbeits- und Geschlechterpolitik, Berlin: Ed.Sigma.

Nies, Sarah (2015): Nützlichkeit und Nutzung von Arbeit, BadenBaden: Edition Sigma/Nomos.

Nohl, Arnd-Michael (2006): Interview und dokumentarische Methode. Anleitungen für die Forschungspraxis, Wiesbaden: VS Verlag für Sozialwissenschaften.

Nohl, Arnd-Michael (2013): Relationale Typenbildung und Mehrebenenvergleich. Neue Wege der dokumentarischen Methode, Wiesbaden: Springer VS. 
Nordhause-Janz, Jürgen / Pekruhl, Ulrich (Hgg.) (2000): Arbeiten in neuen Strukturen? Partizipation, Kooperation, Autonomie und Gruppenarbeit in Deutschland, München und Mering: Hampp. Ohno, Taiichi (1993): Das Toyota-Produktionssystem, Frankfurt/Main: Campus.

Pardi, Tommaso (2007): Redefining the Toyota Production System: the European side of the story, In: New Technology, Work and Employment, Jg. 22, Nr. 1, S. 2-20.

Peters, Klaus / Sauer, Dieter (2005): Indirekte Steuerung - eine neue Herrschaftsform. Zur revolutionären Qualität des gegenwärtigen Umbruchprozesses, In: Wagner, Hilde (Hg.): "Rentier' ich mich noch"? Neue Steuerungskonzepte im Betrieb, Hamburg: VSA, S. 23-58.

Peters, Remco (2009): Shopfloor-Management. Führen am Ort der Wertschöpfung, Stuttgart: LOG_X.

Pfäfflin, Heinz / Schwarz-Kocher, Martin / Seibold, Bettina (2011): Neue Produktionskonzepte: Wirkungen und Gestaltungsoptionen, In: Arbeitsrecht im Betrieb, Jg. 32, Nr. 2, S. 90-94.

Pfeiffer, Sabine (2004): Arbeitsvermögen. Ein Schlüssel zur Analyse (reflexiver) Informatisierung, Wiesbaden: Springer.

Pfeiffer, Sabine (2007): Montage und Erfahrung, München und Mering: Hampp.

Pfeiffer, Sabine (2010): Technisierung von Arbeit, In: Böhle, Fritz / Voß, G. Günter / Wachtler, Günther (Hgg.): Handbuch Arbeitssoziologie, Wiesbaden: VS Verlag, S. 231-261.

Pflüger, Jessica / Pongratz, Hans / Trinczek, Rainer (2010): Fallstudien in der deutschen Arbeits- und Industriesoziologie. Eine Bestandsaufnahme, In: Pongratz, Hans / Trinczek, Rainer (Hgg.): Industriesoziologische Fallstudien, Berlin: Edition Sigma.

Pongratz, Hans / Voß, Günter (2003): Arbeitskraftunternehmer. Erwerbsorientierungen in entgrenzten Arbeitsformen, Berlin: edition sigma.

Popitz, Heinrich / Bahrdt, Hans Paul / Jüres, Ernst August / Kesting, Hanns (1957a): Das Gesellschaftsbild des Arbeiters, Tübingen: Mohr. 
Popitz, Heinrich / Bahrdt, Hans Paul / Jüres, Ernst August / Kesting, Hanns (1957b): Technik und Industriearbeit, Tübingen: Mohr.

Poppitz, Angela (2009): Beruflich Bahnfahren: Aneignung des arbeitsbedingten Bahnalltags bei Pendlern und

Geschäftsreisenden, München: Hampp.

Rammert, Werner (1983): Kapitalistische Rationalität und Organisierung der Arbeit, In: Littek, Wolfgang / Rammert, Werner / Wachtler, Günther (Hgg.): Einführung in die Arbeits- und Industriesoziologie, Frankfurt/Main: Campus, S. 37-61.

Sauer, Dieter / Döhl, Volker (1997): Die Auflösung des Unternehmens? Entwicklungstendenzen der Unternehmensreorganisation in den 90er Jahren, In: ISF München / INIFES Stadtbergen / IfS Frankfurt/Main / SOFI Göttingen (Hgg.): Jahrbuch Sozialwissenschaftliche Technikberichterstattung 1996. Schwerpunkt: Reorganisation, Berlin: edition sigma, S. 19-76.

Sauer, Dieter (2005a): Paradigmenwechsel in der Arbeitspolitik. Gibt es noch Spielräume für anti-tayloristische Deals?, In: Detje, Richard / Pickshaus, Klaus / Urban, Hans-Jürgen (Hgg.): Arbeitspolitik kontrovers. Zwischen Abwehrkräften und Offensivstrategien, Hamburg: VSA, S. 33-55.

Sauer, Dieter (2005b): Arbeit im Übergang. Zeitdiagnose, Hamburg: VSA.

Sauer, Dieter / Boes, Andreas / Kratzer, Nick (2005): Reorganisation des Unternehmens, In: SOFI / IAB / ISF / INIFES (Hgg.): Berichterstattung zur sozioökonomischen Entwicklung in Deutschland, Wiesbaden: VS Verlag, S. 323-350.

Sauer, Dieter (2008): Von "humanisierter" zu guter Arbeit.Paradigmenwechsel in der Arbeitspolitik, In: Wagner, Hilde (Hg.): Arbeit und Leistungs- gestern und heute. Ein gewerkscahftliches Politikfeld, Hamburg: VSA, S. 202-217.

Sauer, Dieter (2010): Vermarktlichung und Vernetzung der Unternehmens- und Betriebsorganisation, In: Böhle, Fritz / Voß, G. Günter / Wachtler, Günther (Hgg.): Handbuch der Arbeitssoziologie, Wiesbaden: VS-Verlag, S. 545-568. 
Schienstock, Gerd (1993): Management als sozialer Prozeß. Theoretische Ansätze zur Institutionalisierung, In: Ganter, Hans-Dieter / Schienstock, Gerd (Hgg.): Management aus soziologischer Sicht, Wiesbaden: Gabler, S. 8-46.

Schmidt, Rudi (2017): Taylorismus, In: Hirsch-Kreiensen, Hartmut / Minssen, Heiner / Bohn, Rainer (Hgg.): Lexikon der Arbeitsund Industriesoziologie, Baden-Baden: edition sigma in der Nomos Verlagsgesellschaft S. 292-296.

Schumann, Michael / Einemann, Edgar / Siebel-Rebell, Christa / Wittemann, Klaus Peter (1982): Rationalisierung, Krise, Arbeiter. Eine empirische Untersuchung der Industrialisierung auf der Werft, Frankfurt am Main: Europäische Verlagsanstalt. Schumann, Michael / Baethge-Kinsky, Volker / Kuhlmann, Martin / Kurz, Constanze / Neumann, Uwe (1994a): Trendreport Rationalisierung. Automobilindustrie, Werkzeugmaschinenbau, Chemische Industrie, 2, Berlin: edition sigma.

Schumann, Michael / Baethge-Kinsky, Volker / Kuhlmann, Martin / Kurz, Constanze / Neumann, Uwe (1994b): Der Wandel der Produktionsarbeit im Zugriff neuer Produktionskonzepte, In: Beckenbach, Niels / Treeck, Werner van (Hgg.): Umbrüche gesellschaftlicher Arbeit (Soziale Welt, Sonderband 9), Göttingen: Schwartz, S. 11-43.

Schumann, Michael / Kuhlmann, Martin / Sanders, Frauke / Sperling, Hans Joachim (2004): Auto 5000 - eine Kampfansage an veraltete Fabrikgestaltung, In: SOFI-Mitteilungen, Nr. 32, S. 23-46.

Schumm, Wilhelm (Hg.) (1988): Arbeit und Subjektivität in der jüngeren soziologischen Forschung, Bonn: Eigenverlag Informationszentrum Sozialwissenschaft.

Schütz, Alfred (1932): Der Sinnhafte Aufbau der Sozialen Welt. Eine Einleitung in die Verstehende Soziologie, Wien: Springer Vienna.

Schütz, Alfred (1971): Gesammelte Aufsätze. Bd. 1: Das Problem der soziale Wirklichkeit, Den Haag: Nijhoff.

Schwarz-Kocher / Seibold, Bettina / Pfäfflin, Heinz / Salm, Rainer (2012): "Gute Arbeit" durch KVP? KVP-Workshops zur 
Verbesserung der Arbeitsbedingungen nutzen, In: IMUBroschüre.

Schwarz-Kocher, Martin / Pfäfflin, Heinz / Salm, Rainer / Seibold, Bettina (2016): Arbeitspolitische Balance in GPS durch umfassende Beteiligung der Beschäftigten, In: Kötter, Wolfgang / Schwarz-Kocher, Martin / Zanker, Christoph (Hgg.): Balanced GPS, Wiesbaden Springer Gabler, S. 63-84.

Schwarz-Kocher, Martin / Salm, Rainer (2016): Industriearbeit im Wandel des aktuellen Rationalisierungsparadigmas, In: Arbeitsund Industriesoziologische Studien, Jg. 9, Nr. 1, S. 5-24.

Seibold, Bettina / Schwarz-Kocher, Martin / Salm, Rainer (2016): Ganzheitlicher Produktionssysteme, Düsseldorf: HansBöckler-Stiftung Online-Publikation. https://www.boeckler.de/pdf/p_study_hbs_mbf_bvd_340.pd $\mathrm{f}$

Springer, Roland (1999a): Rückkehr zum Taylorismus? Arbeitspolitik in der Automobilindustrie am Scheideweg, Frankfurt/Main: Campus.

Springer, Roland (1999b): Von der teilautonomen zur standardisierten Gruppenarbeit - Arbeitspolitische Perspektiven in der Automobilindustrie, In: WSI-Mitteilungen, 19.06.1999, Jg. 52, Nr. 5, S. 309-321.

Sydow, Jörg (1985): Der soziotechnische Ansatz der Arbeits- und Organisationsgestaltung - Darstellung, Kritik, Weiterentwicklung, Frankfurt/Main: Campus.

Taylor, Frederick W. (1995 (1913)): Die Grundsätze wissenschaftlicher Betriebsführung. Reprint, Weinheim: Beltz/Psychologie Verlags Union.

Teufel, Peter (2003): Der Prozess der ständigen Verbesserung (Kaizen) und dessen Einführung, In: Bullinger, Hans-Jörg / Warnecke, Hans-Jürgen / Westkämper, Engelbert (Hgg.): Neue Organisationsformen im Unternehmen. Ein Handbuch für das moderne Management, Berlin: Springer, S. 504-525.

Thompson, Paul / Smith, Chris (2009): Labour Power and Labour Process: Contesting the Marginality of the Sociology of Work, In: Sociology, Jg. 43, Nr. 5, S. 913-930. 
Tullius, Knut (2004): Vertrackte Kontrakte. Formwandel des betrieblichen Steuerungsregimes und die neue Rolle des Meisters, Berlin: edition sigma.

Tullius, Knut / Wolf, Harald (2016): Moderne Arbeitsmoral: Gerechtigkeits- und Rationalitätsansprüche von Erwerbstätigen heute, In: WSI Mitteilungen, Jg. 7, Nr. Schwerpunktheft: Gerechtigkeitsansprüche und Arbeitnehmerbewusstsein heute neue Ansätze, neue Befunde, S. 269-287.

Voß, G. Günter (2010): Was ist Arbeit? Zum Problem eines allgemeinen Arbeitsbegriffs, In: Böhle, Fritz / Voß, G. Günter / Wachtler, Günther (Hgg.): Handbuch der Arbeitssoziologie Wiesbaden: VS-Verlag, S. 23-80.

Voß, Gerd Günter (1980): Arbeitssituation und Bewusstsein : zur Kritik arbeitssoziologischer Erklärungen gesellschaftlichen Bewusstseins, Frankfurt: Campus.

Voß, Günter (1984): Bewußtsein ohne Subjekt? Eine Kritik des industriesoziologischen Bewußtseinsbegriffs, Großhesselohe: Hampp.

Voß, Günter / Pongratz, Hans (1998): Der Arbeitskraftunternehmer. Eine neue Grundform der Ware Arbeitskraft?, In: Kölner Zeitschrift für Soziologie und Sozialpsychologie, 19.02.1998, Jg. 50, Nr. 1, S. 131-168.

Voswinkel, Stephan (2002): Die Anerkennung der Arbeit im Wandel. Zwischen Würdigung und Bewunderung, In: Holtgrewe, Ursula / Voswinkel, Stephan / Wagner, Gabriele (Hgg.): Anerkennung und Arbeit, Konstanz: UVK Universitätsverlag, S. 39-63.

Voswinkel, Stephan (2005): Reziprozität und Anerkennung in Arbeitsbeziehungen, In: Adloff, Frank / Mau, Steffen (Hgg.): Vom geben und Nehmen. Zur Soziologie der Reziprozität, Frankfrut/New York: Campus Verlag, S. 237-256.

Voswinkel, Stephan (2008): Reziprozität und Anerkennung in Arbeitsbeziehungen, In: Adloff, Frank / Mau, Steffen (Hgg.): Vom Geben und Nehmen. Zur Soziologie der Reziprozität, Frankfurt/Main: Campus Verlag, S. 237-256.

Voswinkel, Stephan / Wagner, Gabriele (2013): Vermessung der Anerkennung. Die Bearbeitung unsicherer Anerkennung in 
Organisationen, In: Honneth, Axel / Lindemann, Ophelia /

Voswinkel, Stephan (Hgg.): Strukturwandel der Anerkennung.

Paradoxien sozialer Integration in der Gegenwart,

Frankfurt/Main: Campus, S. 75-120.

Voswinkel, Stephan (2017): Anerkennung, In: Hirsch-Kreiensen,

Hartmut / Minssen, Heiner (Hgg.): Lexikon der Arbeits- und Industriesoziologie, Baden-Baden: Nomos, S. 17-20.

Weber, Max (1921, 1972): Wirtschaft und Gesellschaft, Frankfurt/Main: Zweitausendeins.

Wittemann, Klaus Peter / Kuhlmann, Martin / Schumann, Michael (2010): SOFI-Fallstudien-Ansatz im Wandel. Exemplarische Empirie zur Entwicklung von Industriearbeit, In: Pongratz, Hans / Trinczek, Rainer (Hgg.): Industriesoziologische Fallstudien. Entwicklungspotenziale einer Forschungsstrategie, Berlin: edition sigma, S. 73-117.

Witzel, Andreas (1989): Das problemzentrierte Interview, In: Jüttemann, Gerd (Hg.): Qualitaitve Forschung in der Psychologie. Grundfragen, Verfahrensweisen, Anwendungsfelder, Heidelberg: Asanger, S. 227-256.

Wolf, Harald (1994): Rationalisierung und Partizipation, In: Leviathan, Jg. 22, Nr. 2, S. 243-259.

Wolf, Harald (1999): Arbeit und Autonomie. Ein Versuch über Widersprüche und Metamorphosen kapitalistischer Produktion, Münster.

Wolf, Harald (2003): Partizipatives Management - was bleibt?, Expertise für die Hans-Böckler-Stiftung, Göttingen, http://www.sofigoettingen.de/fileadmin/Harald_Wolf/Material/Wolf_Partizip atives_Management.pdf).

Womack, James / Jones, Daniel / Roos, Daniel (1990): The Machine That Changed the World, New York: Rawson Associates.

Womack, James P. / Jones, Daniel T. / Roos, Daniel (1991): Die zweite Revolution in der Automobilindustrie. Konsequenzen aus der weltweiten Studie aus dem Massachusetts Institute of Technology, Frankfurt/ Main: Campus.

Yin, Robert (2003): Case Study Research. Design and Methods, Thousand Oaks: Sage 



\section{Abbildungen}

Abbildung 1: Aneignungsmodell von KVP............................................ 91

Abbildung 2: Typologie der Beteiligungsorientierungen ...................... 172

Abbildung 3: Profil der arbeitspolitischen Gestaltungsbedingungen

von KVP

\section{Tabellen}

Tabelle 1: Beschäftigtengetragene Prozessoptimierung und

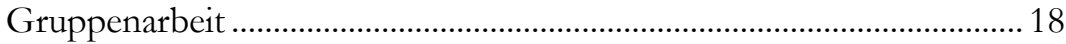

Tabelle 2: Strukturkonservative und -innovative Gestaltung.................. 94

Tabelle 3: Überblick über das Erhebungsprogramm ............................... 99

Tabelle 4: Vergleich der KVP-Konzepte von NF und WLF................ 134

Tabelle 5: Vergleich der arbeitspolitischen Ausgangsbedingungen .... 137

Tabelle 6: Beteiligungstypen und Beteiligungsdisposition..................... 190

Tabelle 7: Subjektive Umgangs- und Aneignungsformen von KVP..258

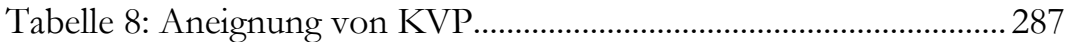

Tabelle 9: Arbeitspolitischer Pragmatismus............................................. 290 



\section{Anhang}

\section{Leitfaden Interviews Beschäftigte ${ }^{126}$}

\section{BERUFLICHER WERDEGANG}

Was haben Sie beruflich gemacht, bevor Sie zu NF/WLF gekommen sind? (Erwerbsbiografischer Hintergrund, Berufswahl, Alternativen)

Warum und wie sind Sie zu NF/WLF gegangen/gekommen?

In welchen Bereichen/Tätigkeiten haben Sie bei NF/WLF bisher gearbeitet?

Was ist ganz allgemein Ihr Eindruck von der Arbeit bei NF/WLF? Was ist das für ein Betrieb?

\section{ARBEIT UND ARBEITSSITUATION}

Wenn man so generell über Arbeit nachdenkt, was ist Ihnen an Arbeit wichtig? Was muss denn Arbeit bringen? (Ansprüche an Arbeit)

Welchen Stellenwert hat Arbeit für Sie - auch wenn man auf Dinge außerhalb der Arbeit schaut?

Können Sie mir Ihre Tätigkeiten im Einzelnen etwas genauer beschreiben? Welche Aufgaben haben Sie? (Interessantheit der Arbeit, Herausforderungen, Spaß, Über- und Unterforderung in der Arbeit)

Was sind aus Ihrer Sicht eigentlich die guten und die schlechten Seiten bei Ihrer jetzigen Arbeit hier?

Wie sieht es mit den Leistungsanforderungen aus? Ist die Arbeit zu schaffen?

Wo liegen heute die größten Belastungen der Arbeit - und hat sich da etwas verändert?

Sind sie zu bewältigen? Wie bewältigt man sie? Schichtarbeit?

Sind die Qualifizierungsangebote ausreichend und sinnvoll? Was fehlt?

${ }^{126}$ Dieser Leitfaden wurde im Betrieb NF eingesetzt. Dabei wurden die unterschiedlichen Erfahrungen der Beschäftigten mit KVP berücksichtigt und diese jeweils einzeln abgefragt. Bei WLF wurde ein an die Gestaltungsbedingungen von Business Excellence angepasster Leitfaden eingesetzt, der im Wesentlichen aber diesem Leitfaden entspricht. 
Passt die Bezahlung - und das Entlohnungssystem? Zuschläge? (Welche Bedeutung?)

Was sind in den letzten Jahren die wichtigsten Veränderungen in Ihrer Arbeit sowie rund um die Arbeit herum?

\section{TEAMARBEIT/PRODUKTIONSEXZELLENZ}

Hatten Sie vor der Einführung bei NF/WLF schon einmal Erfahrungen mit Teamarbeit/ProduktionsExzellenz?

Was waren so Ihre Gedanken, als sie hörten, dass es Teamarbeit/ProduktionsExzellenz geben soll? (Welche Hoffnungen - welche Befürchtungen?)

Welche konkreten Veränderungen hat es bisher durch Teamarbeit/ProduktionsExzellenz gegeben?

- Selbstständigkeit und Entscheidungsspielräume einzelner/des Teams

- Interessantheit der Arbeit

- Wie wird mit Problemen umgegangen?

- Inwieweit wird im Team nun anders gearbeitet? Rotation? Auch besser?

Wie funktioniert die Zusammenarbeit in Ihrem Team/Bereich?

Wie geht das Team mit Problemen um oder wenn es mal Meinungsverschiedenheiten gibt?

Wie laufen die Teamgespräche bei Ihnen ab? (Themen, Teilnehmer, Diskussionskultur, Rolle des Meisters im Teamgespräch)

Welcher Rolle hat der Teamsprecher in Ihrem Team?

Alles in allem: Übernimmt man als Team gemeinsam Verantwortung oder arbeiten viele eher für sich?

Insgesamt: Was läuft bisher gut im Team bzw. bei dem Team - was läuft noch nicht gut?

Was sollte man bezogen auf Teamarbeit noch verändern oder weiterentwickeln?

\section{FÜHRUNGSSITUATION}

Was für Erfahrungen haben Sie bisher mit Ihrem Meister/ Vorarbeiter gemacht?

Lässt der Meister/Vorarbeiter das Team eigenständig arbeiten?

Gibt er ausreichende Unterstützung?

Vertritt er die Gruppe nach außen? („Außenminister“?)

Wie lässt sich der Führungsstil Ihres SL charakterisieren?

\section{VERBESSERUNGSPROZESSE}

\section{Allgemein:}

Macht man sich gewöhnlich selbst Gedanken darüber, wie man die Arbeit oder Abläufe 
anders oder besser machen könnte? Welche? Wie geht man mit diesen Ideen um? Inwieweit werden Sie auch offiziell in die Gestaltung der Arbeit und Produktionsabläufe einbezogen? Inwiefern?

Heutzutage, heißt es, soll man sich ja auch als Arbeiter immer mehr an Optimierungen und Kostensenkung beteiligen?

Wie sieht es denn damit hier bei NF/WLF aus? Wird vom Betrieb erwartet, dass man sich an Optimierung und Kostensenkung beteiligt? (In welcher Form?)

Sollte man sich als Arbeiter überhaupt an Optimierung und Kostensenkung beteiligen? Warum? Warum ja? Warum nein?

Welche Erfahrungen haben Sie mit Verbesserungsprozessen gemacht?

\section{Verbesserung in Teamarbeit}

Wurde in den Teamgesprächen über Verbesserungsmöglichkeiten gesprochen? (Inhalte, Zusammenarbeit mit Kollegen, Führungskraft, Planern, Betriebsrat?)

Was war daran schwierig, was lief gut?

Hat sich dadurch etwas in Ihrer Arbeit/in Ihrem Bereich verändert? Wodurch genau? Ist es sinnvoll, als Team Verbesserungen voranzutreiben? (Vorteile/Nachteile, Veränderungsbedarf?)

\section{Falls Erfahrungen mit KVP-Workshops}

Welche Erfahrungen haben Sie mit KVP-Workshops gemacht? (Optimierungen, KVPWorkshop?)

Wie lief das mit dem Workshop in Ihrem Bereich ab?

Worum ging es da inhaltlich? Ziele?

Waren Kollegen aus Ihrem Bereich dabei? Wie und warum wurdensausgesucht?

Wer war sonst noch dabei? (Führungskraft, MNPS, Planer, BR?)

Wurden die Ergebnisse vorgestellt und im Teamgespräch diskutiert?

Was hat sich durch den Workshop in Ihrem Bereich verändert? (gut/schlecht/sinnvoll)

Was lief bei dem Workshop gut - was weniger gut oder problematisch?

\section{Falls eigene Beteiligung am KVP-Workshop:}

Welche Erfahrungen haben Sie damit gemacht? (Positive/negative Seiten?)

Wie wurden Sie dafür ausgewählt? Freiwillig? Ehre/gute Gelegenheit?

Wie lief der Verbesserungsprozess/-Workshop ab? 
Worum ging es dabei inhaltlich? Themen? Was war Gegenstand der Verbesserung? Wer war an der Verbesserung beteiligt? Welche Aufgabe hatten Sie dabei? Welche Rolle hatten Sie?

Wie war die Zusammenarbeit mit Kollegen, Führungskraft, Experten?

Wo lagen die größten Schwierigkeiten in der Zusammenarbeit?

Konnte man sich als Produktionsarbeiter mit all seinen Ideen und seinem Wissen wirklich in den Workshops einbringen oder war die Verbesserung vom Segmentleiter oder von den Planern bestimmt?

Wurden Sie finanziell an der Verbesserung beteiligt? Gerecht?

Wurden die Ergebnisse des Workshops im Teamgespräch mit den Kollegen besprochen?

Was hat sich in Ihrer Arbeit und in Ihrem Bereich durch den Verbesserungs-WS verändert? Sinnvoll?

Wie sind die Kollegen während des Workshops mit Ihnen umgegangen? Und danach?

Bilanz: Was wurde dabei erreicht? Was nicht? Was lief gut/was schlecht?

Unerwartete/überraschende Dinge?

Was sollte sich an den Verbesserungs-Workshops generell noch verändern? (Themen, in der Zusammenarbeit mit den Indirekten/Führungskräften, Ablauf, Auswahl der Teilnehmer?)

Alles in allem: Was sind die Lehren, die Sie aus der Teilnahme am Workshop ziehen?

Würden Sie sich wieder an Verbesserungs-WS beteiligen?

\section{Ideenmanagement:}

Haben Sie selbst schon einmal Verbesserungsideen im Ideenmanagement eingereicht? Wie viele? Regelmäßig? Gruppenideen/Einzelideen?

Welche Erfahrungen haben sie damit gemacht? (Ablauf, Inhalte, Umsetzung, Beteiligung an der Umsetzung, eigene Rolle, Zusammenarbeit mit Vorgesetzten und Kollegen, Finanzielle Beteiligung)

Was hat sich durch die Verbesserungsidee in Ihrer Arbeit und Ihrem Bereich verändert?

Konnte man sich als Produktionsarbeiter mit all seinen Ideen und seinem Wissen - auch in der Umsetzung - wirklich einbringen oder war die Verbesserung vom SL oder von den Planern bestimmt?

Sollte sich am Ideenmanagement generell noch was ändern?

\section{Bilanzierung Verbesserungsprozesse:}

Insgesamt: Ist die Beteiligung an Optimierungsprozessen etwas, das aus Ihrer Sicht Vortei- 
le oder Nachteile bringt?

Wie hat sich konkret die Arbeit in ihrem Bereich durch KVP verändert? (Berufliche Entwicklungsmöglichkeiten, Zusammenarbeit mit anderen Bereichen/Funktionen, eigene Beteiligung)

Bereichert die Beteiligung an Verbesserungsprozessen die eigene Arbeit oder ist dies eine zusätzliche Belastung? Inwiefern?

Ist die Beteiligung an Verbesserungen in Ihrem Bereich etwas völlig Neues oder haben sich die Arbeiter hier schon immer in Verbesserungsprozesse eingebracht? (Gute oder schlechte Entwicklung?)

Was müsste im optimalen Fall gegeben sein oder was müsste der Betrieb tun, damit man sich als Arbeiter aktiv um Kostensenkungen und Einsparmöglichkeiten bemüht?

Hat sich durch die Erfahrung mit KVP etwas an Ihrem Arbeitsverständnis verändert? Macht man sich heute mehr oder andere Gedanken über die Arbeit und darüber, wie sie sich verbessern lässt?

Hat sich durch die KVP-Erfahrung in Ihrem Verhältnis zum Betrieb etwas verändert? Hat sich etwas untereinander (Kollegen) oder zu anderen Bereichen und betrieblichen Gruppen verändert?

\section{INTERESSENVERTRETUNG}

Welche Aufgaben/Rolle hat der Betriebsrat bei KVP? Ist er direkt in den Verbesserungsprozess eingebunden? Sollte er da mehr tun/mit einbezogen werden?

Sollte der Betriebsrat sich für Wirtschaftlichkeitsfragen und Kostensenkung engagieren? Wie bzw. in welcher Form?

Was bekommt man normalerweise vom Betriebsrat mit? Welche Themen treibt der Betriebsrat voran?

Wird der Betriebsrat bei NF/WLF insgesamt (alles in allem) seiner Aufgabe als Interessenvertretung gerecht?

Was läuft gut? Wo gibt es aus Ihrer Sicht Defizite? Fühlt man sich ausreichend vom BR vertreten?

Wie sollte der BR gegenüber der Betriebsleitung auftreten? Fordernd, auch Konflikte nicht scheuend? Oder sollte der BR das Wohl des Betriebes an die erste Stelle setzen?

\section{ARBEITS- UND BETRIEBSVERSTÄNDNIS}

Wir haben ja am Anfang darüber gesprochen, was Ihnen an Arbeit wichtig ist. Inwieweit entspricht die jetzige Tätigkeit den Ansprüchen, die Sie allgemein an eine Arbeit stellen?

Mal ganz allgemein gefragt: Wie würden Sie denn das Betriebsklima hier im Betrieb beschreiben?

Gibt es hier auch so etwas wie eine besondere Unternehmenskultur, d.h. eine besondere 
Art und Weise, wie mit den Leuten umgegangen wird?

Fühlen Sie sich dem Betrieb gegenüber in besonderer Weise verpflichtet - oder ist diese

Firma ein Arbeitgeber wie andere auch?

Mitunter wird davon geredet, dass man heutzutage alles der Arbeit unterordnen muss, dass das Privatleben und andere Interessend zurückstehen müssen. Wie sieht es bei NFWL damit aus? Wird hier ein besonderer Druck vonseiten der Firma ausgeübt?

Perspektiven

Wollen Sie auf Dauer bei hier bei NZ/WLF bleiben, oder könnten Sie sich auch einen Wechsel vorstellen? Würden Sie im Betrieb gern/lieber etwas anderes machen?

\section{ANGABEN SOZIALSTATISTIK}

Schulabschluss

Berufsabschluss

Berufstätigkeit

Seit wann im Betrieb

Alter

Herkunft 
Überblick über das Interviewsample

\begin{tabular}{|c|c|c|c|c|c|}
\hline Synonym & Betrieb & Bereich & Schicht & $\begin{array}{l}\text { Funktion / ggf. Work- } \\
\text { shopteilnahme }\end{array}$ & Alter ${ }^{127}$ \\
\hline Fuchs & NF & Mont. F & A & MEISTER & \\
\hline Fellner & $\mathrm{NF}$ & Mont. F & $B$ & MEISTER & \\
\hline Fischer & NF & Mont. F & A & $\begin{array}{l}\text { Arbeiter/ stellv. Tspr. }{ }^{128} \text { I } \\
\text { KVP-Workshop }\end{array}$ & $\begin{array}{l}\text { Anfang } \\
50\end{array}$ \\
\hline Gruber & NF & Mont. $F$ & B & $\begin{array}{l}\text { Arbeiter/ QRK129 / KVP- } \\
\text { Workshop }\end{array}$ & Mitte 40 \\
\hline Haller & NF & Mont. F & A & Arbeiter/ Tspr. & Ende 40 \\
\hline Immler & $\mathrm{NF}$ & Mont. $F$ & A & Arbeiter/ Tspr. & Mitte 40 \\
\hline König & $\mathrm{NF}$ & Mont. F & $B$ & Arbeiter & Mitte 40 \\
\hline Aichinger & NF & Mont. K & A & MEISTER & \\
\hline Bäumler & NF & Mont. K & $B$ & MEISTER & \\
\hline Albinger & $\mathrm{NF}$ & Mont. K & B & Arbeiter/ Tspr. & Mitte 30 \\
\hline Auer & $\mathrm{NF}$ & Mont. K & A & $\begin{array}{l}\text { Arbeiter/ Tspr./ Work- } \\
\text { shop }\end{array}$ & Mitte 40 \\
\hline Berger & NF & Mont. K & B & Arbeiter & ca.30 \\
\hline Czerni & NF & Mont. K & B & Arbeiter/ stellv. Tspr. & Mitte 50 \\
\hline Dauner & $\mathrm{NF}$ & Mont. K & B & Arbeiter & Mitte 30 \\
\hline Eder & $N F$ & Mont. K & B & Arbeiter/ Workshop & Ende 40 \\
\hline Wallner & WLF & Linie 1 & schichtübergr. & MEISTER & \\
\hline Wagner & WLF & Linie 1 & & Schichtführer & Mitte 30 \\
\hline Oswald & WLF & Linie 1 & & Arbeiter & Mitte 40 \\
\hline Renner & WLF & Linie 1 & & Arbeiter & Mitte 50 \\
\hline Tanner & WLF & Linie 3 & & Arbeiter & Mitte 50 \\
\hline Steiner & WLF & Linie 2 & schichtübergr. & MEISTER & \\
\hline Lehner & WLF & Linie 2 & 2 & Arbeiter & Mitte 40 \\
\hline Mayr & WLF & Linie 2 & 2 & Arbeiter & Ende 20 \\
\hline Neubauer & WLF & Linie 2 & 2 & Arbeiter & Mitte 20 \\
\hline Unger & WLF & Linie 2 & 1 & Arbeiter & Ende 20 \\
\hline Strobl & WLF & Linie 2 & 1 & Arbeiter & Mitte 40 \\
\hline Valler & WLF & Linie 4 & Linie 4 & Schichtführer & Mitte 30 \\
\hline
\end{tabular}

${ }^{127}$ Das Alter der Beschäftigten wurde in den Gesprächen nicht abgefragt, sondern anhand der im Interview genannten Informationen ermittelt. Daher handelt es sich hierbei nur um ungefähre Angaben.

128 Tspr. $=$ Teamsprecher.

${ }^{129} \mathrm{QRK}=$ Qualitätsregelkreismitarbeiter. Der QRK ist ein Mitarbeiter aus dem Team, der am Ende des Produktionsbereichs das Team Qualitätskontrollen durchführt, Nacharbeiten ausführt und Mitarbeiter im Team qualifiziert. 


\section{Erklärung}

Gemäß \12 der Prüfungsordnung für den Promotionsstudiengang Sozialwissenschaften an der Sozialwissenschaftlichen Fakultät der Georg-August-Universität Göttingen aus dem Jahr 2013 möchte ich folgendes versichern:

1. Die Gelegenheit zum vorliegenden Promotionsvorhaben ist mir nicht kommerziell vermittelt worden. Insbesondere habe ich keine Organisation eingeschaltet, die gegen Entgelt Betreuerinnen und Betreuer für die Anfertigung von Dissertationen sucht oder die mir obliegenden Pflichten hinsichtlich der Prüfungsleistungen für mich ganz oder teilweise erledigt.

2. Ich versichere, dass ich die eingereichte Dissertation ,Arbeitspolitischer Pragmatismus - eine vergleichende Fallstudienuntersuchung zur subjektiven Aneignung von KVP durch Produktionsbeschäftigte "selbstständig und ohne unerlaubte Hilfsmittel verfasst habe; fremde Hilfe habe ich dazu weder unentgeltlich noch entgeltlich entgegengenommen und werde dies auch zukünftig so halten. Anderer als der von mir angegebenen Hilfsmittel und Schriften habe ich mich nicht bedient. Alle wörtlich oder sinngemäß den Schriften anderer Autoren entnommenen Stellen habe ich kenntlich gemacht.

3. Die eingereichte Dissertation habe ich nicht bereits in einem anderen Prüfungsverfahren vorgelegt.

4. Des Weiteren ist mir bekannt, dass Unwahrhaftigkeiten hinsichtlich der vorstehenden Erklärung die Zulassung zur Promotion ausschließen bzw. später zum Verfahrensabbruch oder zur Rücknahme des erlangten Titels berechtigen. 